DEPARTMENT OF THE INTERIOR

UNITED STATES GEOLOGICAL SURVEY

GEORGE OTIS SMITH, DIRECTOR

Water-SUPPLy PAPER 373

\title{
WATER RESOURCES OF HAWAII
}

\section{3}

BY

G. K. LARRISON

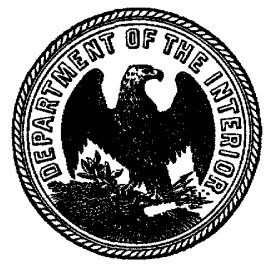

W A S HINGTON

GOVERNMENT PRINTING OFEICE

1915 


\section{CONTENTS.}

Authority for investigations.

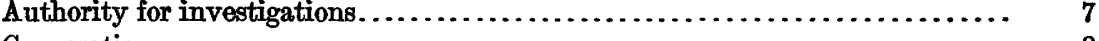

Cooperation............................................... 8

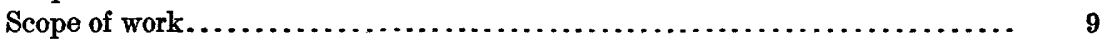

Field methods of measuring stream flow........................... 9

Base data................................................. 9

Weir measurements......................................... 10

Velocity-area method........................................ 11

Definition of terms............................................... 15

Convenient equivalents........................................ 16

Office methods of computing and studying the discharge and run-off........ 17

Explanation of tables.......................................... 20

Accuracy of field data and computed results......................... 21

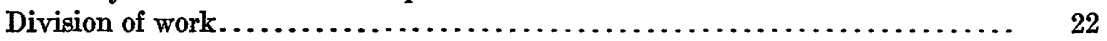

Gaging stations maintained in Hawaii............................ 22

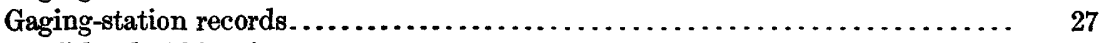

Island of Kauai............................................. 27

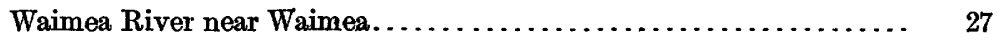

Kawaikoi Stream near Waimea......................... 28

Waialae River near Waimea ............................... 29

Waimea ditch near Waimea............................. 31

Makaweli River near Waimea.......................... 31

Halekua Stream near Waimea......................... 32

Olokele ditch at tunnel No. 12 near Makaweli................. 33

Olokele ditch at weir near Makaweli..................... 35

Poowaiomahaihai ditch near Waimea........................ 36

Hanapepe River at Koula near Eleele.................... 37

Hiloa ditch at Hanapepe Falls near Eleele.................. 38

Hanapepe ditch at Koula near Eleele...................... 40

Hanapepe ditch at weir near Hanapepe................... 41

Huleia River near Lihue.............................. 43

Hanamaulu River at Kapaia near Lihue..................... 45

South Fork of Wailua River above Waiehu Falls near Lihue....... 46

Hanamaulu ditch near Lihue........................... 48

Lihue ditch near Lihue............................ 49

North Fork of Wailua River near Lihue.................... 50

Kanaha ditch near Lihue............................ 51

East Branch of North Fork of Wailua River near Lihue........... 53

Konohiki Stream at Makakualele weir (mauka) near Kealia ........ 55

South Fork of Kaehulua Stream at Wainamunamu weir near Kealia.. $\quad 56$

Kaehulua Stream at Kuhinoa weir near Kealia ............... 57

North Fork of Kaehulua Stream at Kainahola weir, near Kealia ..... 58

Kapaa River near Kealia............................. 59

Akulikuli Spring near Kealia............................ $\quad 60$

Kapahi ditch at Kapahi, near Kealia..................... 61

Kaneha ditch near Kealia............................. 62

Anahola River above dam at Kiokala, near Kealia............ 63 
Gaging-station records-Continued.

Island of Kauai-Continued.

Anahola ditch at Kiokala, near Kealia . Page.

Kalihiwai River near Kilauea........................... $\quad 65$

Hanalei River near Hanalei........................... 67

Kuna ditch near Hanalei........................... 68

Wainiha River (east and west channels) near Wainiha........... 69

Miscellaneous measurements . . . . . . . . . . . . . . . . . . . . . . . . 73

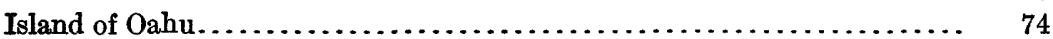

Kalihi Stream at Kioi pool, near Honolulu................. 74

Nuuanu Stream at Luakaha weir, in upper Nuuanu Valley, near Honolulu.

Nuuanu Stream below reservoir No. 2, wasteway, near Honolulu.... Lulumaha ditch at upper Nuuanu reservoir near Honolulu. . . . . ... Pauoa Stream at upper Pauoa Valley, near Honolulu............ Kahuawai Spring at upper Pauoa Valley, near Honolulu. . . . . . . . . Manoa Stream at upper Manoa Valley, near Honolulu............. Manoa Stream at College of Hawaii, near Honolulu................ East Branch of Manoa Stream at upper Manoa Valley, near Honolulu. West Branch of Manoa Stream at upper Manoa Valley, near Honolulu. Waiomao Stream at upper Palolo Valley, near Honolulu............ Waimanalo ditch below main reservoir, near Waimanalo........... Makawao ditch at Makawao flume, near Waimanalo. . .............. Makawao Stream in Kailua Valley, near Kailua.................. Kaimi Stream in Kailua Valley, near Kailua.................. Kamakalepo Stream in Kailua Valley, near Kailua.............. Pohakea Stream in Kailua Valley, near Kailua.................. South Branch of Kahanaiki Stream in Kailua Valley, near Kailua... North Branch of Kahanaiki Stream in Kailua Valley, near Kailua... Waiahole Stream at Manianiaula, near Waikane................. Right Branch of North Fork of Kaukonahua Stream near Wahiawa.. Left Branch of North Fork of Kaukonahua Stream near Wahiawa.... South Fork of Kaukonahua Stream near Wahiawa...............

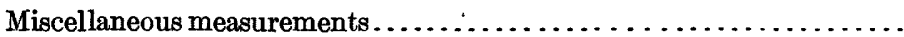

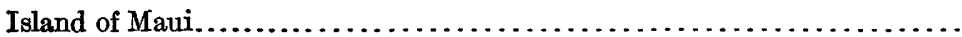

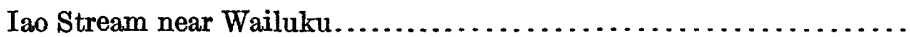

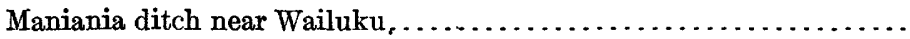

South Waiehu Stream near Wailuku..........................

South Waiehu ditch near Wailuku..........................

North Waiehu Stream near Wailuku.........................

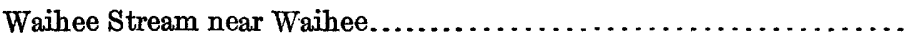

Spreckels ditch near Waihee.............................

Kahakuloa Stream at Kahakuloa, near Waihee..................

Kahakuloa Stream near Honokahau..........................

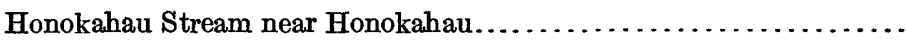

Honokahau ditch at intake near Honokahau.....................

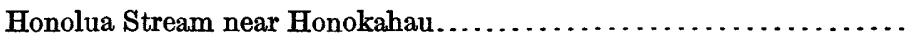

Honokawai Stream near Lahaina. . . . . . . . . . . . . . . . . .

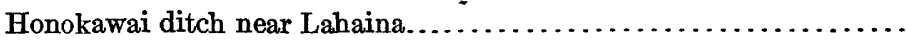

Kahoma ditch at weir near Lahaina............................

Lahainaluna Stream near Lahaina ..............................

Lahainaluna ditch near Lahaina............................

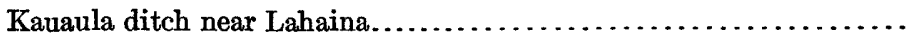

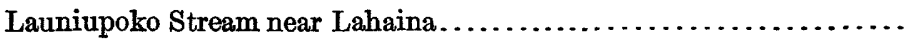


Gaging-station records-Continued.

Island of Maui-Continued. Page.

Olowalu Stream near Olowalu.............................. 128

Olowalu ditch No. 1 near Olowalu......................... 129

Waikapu Stream near Waikapu............................. 131

South Side Waikapu ditch near Waikapu................... 131

Palolo ditch near Waikapu................................ 132

Haipuaena Stream (old station) near Huelo................... 134

Haipuaena Stream (new station) near Huelo.................... 134

Puohakamoa Stream (old station) near Huelo................. 135

Puohakamoa Stream (new station) near Huelo................... 136

Alo Stream near Huelo................................... 137

Waikamoi Stream near Huelo............................... 138

Oopuola Stream near Huelo............................. 139

Spreckels ditch at gage No. 1, near Huelo................... 141

Spreckels ditch at gage No. 2, near Huelo................... 141

Spreckels ditch at gage No. 3, near Huelo..................... 142

Spreckels ditch at gage No. 4, near Huelo. .................. 142

Spreckels ditch at gage No. 5 , near Huelo................... 143

Spreckels ditch at gage No. 6, near Huelo................... 143

Spreckels ditch at gage No. 8, near Huelo.................... 144

Nailiilihaele Stream near Huelo............................. 144

Kailua Stream near Huelo................................ 145

Oanui Stream near Huelo................................ 146

Hoolawanui Stream near Huelo.......................... 147

Hoolawaliilii Stream near Huelo............................ 148

Honopou Stream near Huelo........................... 149

Miscellaneous measurements............................... ' 151

Island of Hawaii.......................................... 152

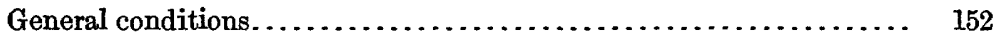

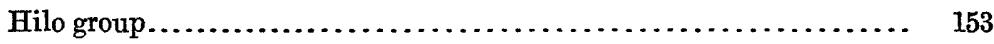

Stations at 2,700-foot level........................... 153

Wailuku River near Hilo.......................... 166

Honolii River at Kaiwiki near Hilo................... 167

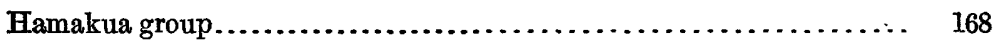

Hamakua ditch at main weir, Puualala, near Waimea.......... 168

New Hamakua ditch at main weir near Kukuihaele......... 169

Kohala group.......................................... 170

Kohala ditch near Kohala.......................... 170

Kehena ditch at Honokane Mauka, near North Kahala........ 173

Miscellaneous measurements................................ 174

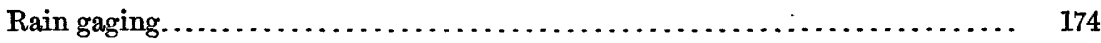

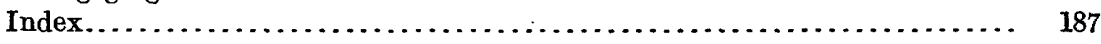




\title{
WATER RESOURCES OF HAWAII, 1913.
}

\author{
By G. K. Larrison.
}

\section{AUTHORITY FOR INVESTIGATIONS.}

This volume contains results of measurements of the flow of certain streams and ditches and rainfall records of the Territory of Hawaii made during the calendar year 1913. The investigations leading to the report were made by the United States Geological Survey in cooperation with the Territory of Hawaii, under the general sanction of the organic law of the Survey (Stat. L., vol. 20, p. 394), which contains the following paragraph:

Provided, That this officer [the Director] shall have the direction of the geological survey and the classification of public lands and examination of the geological structure, mineral resources, and products of the national domain.

As water is the most abundant and most valuable of the minerals, the investigation of water resources is authorized under the provision for examining mineral resources. The work has been supported since the fiscal year ending June 30, 1895, by appropriations in successive sundry civil bills passed by Congress under the following item:

For gaging the streams and determining the water supply of the United States, and for the investigation of underground currents and artesian wells, and for the preparation of reports upon the best methods of utilizing the water resources.

The legislature of the Territory of Hawaii approved on March 22, 1909, "An act to promote the conservation and development of the natural resources of the Territory," which provided in substance as follows: A special tax of 2 per cent shall be levied, assessed, and collected annually on all incomes in excess of $\$ 4,000$; and all amounts so collected shall constitute a special fund to be expended only for the encouragement of immigration and the conservation of natural resources in the proportion of three-fourths for immigration and onefourth for conservation. The conservation fund shall be used for the development, conservation, improvement, and utilization of the natural resources, and shall be available for expenditure at such times and in such manner as a board of three persons appointed in accordance with section 80 of the organic act shall, with the approval of the governor, determine. 
An act of April 26, 1911, amended the original act so as to extend it until December 31, 1913.

On April 4, 1913, the governor of the Territory of Hawaii approved the following acts providing (act 56) for the creation and maintenance of a division of hydrography under the board of agriculture and forestry, and (act 57) appropriating the revenues from water licenses for the use of the board of commissioners of agriculture and forestry toward forest protection and hydrographic surveying.

Section 1 of act 56 reads:

The board of agriculture and forestry is hereby authorized to create and maintain a division of hydrography for the investigation and determination of the water resources of the Territory by the gaging of streams and rainfall and other means, in cooperation with the Enited States Geological Survey or otherwise, and in furtherance thereof to take over and exercise the functions of the Territory in the conduct of the present hydrographic survey of the Territory.

Section 2 provides that this act shall take effect on July 1, 1913.

Section 1 of act 57 reads:

All revenues derived from water licenses, issued by the Territory, during the period beginning July 1, 1913, and ending June 30, 1915, whether by way of rentals or otherwise, shall constitute and be held as a special fund in the treasury of the Territory to be disbursed on warrants of the auditor issued on approved vouchers of the president of the board of commissioners of agriculture and forestry. Such moneys shall be apportioned and applied from time to time by the board of commissioners of agriculture and forestry, acting with the approval of the governor, equally between the division of forestry and the division of hydrography to the following general purposes, and not otherwise:

1. For the protection of forest reservations, established or set apart according to law, against damage by fire, animals, and otherwise by means of fences and any other means whatsoever, and for the expenditures of the division of forestry.

2. For the development and maintenance of the hydrographic survey throughout the Territory.

Each voucher against said fund shall designate the general purpose for which it is drawn.

Section 2 provides that this act also shall take effect on July 1, 1913.

\section{COOPERATION.}

Under the authority conferred by the Federal and Territorial legislation, the Director of the United States Geological Survey and the governor of the Territory of Hawaii entered into a cooperative agreement, dating from July 1, 1910, for "the gaging of streams and the determination of the water supply of the Territory of Hawaii."

The principal features of this agreement are:

1. The United States Geological Survey assumes the responsibility of gathering, analyzing, and publishing the data.

2. During the progress of the work all notes, maps, and data gathered as a result of field studies are at all times open to inspection by

1 The United States Geological Survey is also cooperating with the Territory of Hawaii in mapping the several islands. The whole of the island of Kauai and a part of the island of Hawaii have been mapped. 
the representative of the Territory, and if they are not entirely satisfactory the agreement can be terminated.

3. Accounts for payment of salaries, travel, and subsistence, supplies, or other expenses necessary to the completion of the work shall be rendered in the manner required by the laws and regulations of the contracting parties, and vouchers shall be preferred to either party for payment according as it may be convenient or according to the balance remaining in the respective allotments.

4. The cost of publication is borne entirely by the Geological Survey.

The Territory of Hawaii was represented in the cooperation by the board of conservation appointed by Gov. Walter F. Frear, and consisted of J. P. Cooke (chairman), J. W. Caldwell, and E. V. Wilcox, until June 30,1913. After this date the Territory was represented by the board of commissioners of agriculture and forestry, consisting of W. M. Giffard (president and executive officer), A. Waterhouse, J. M. Dowsett, H. M. von Holt, and A. H. Rice.

\section{SCOPE OF WORK.}

The investigations of stream flow in the Territory are not complete, nor do they include all the streams that might advantageously be studied. They include, however, as many of the streams and ditches on the four larger islands as the available appropriations would allow. It is essential that records of stream flow should be kept during a period of years long enough to determine within reasonable limits the range of flow from the maximum to the minimum. The length of such a period manifestly varies for different streams. Experience has shown that the records should be kept from 20 to 30 years.

In the performance of this work an effort is made to reach the highest degree of precision possible with a rational expenditure of time and money. In all engineering work there is a point beyond which refinement is needless and wasteful, and this statement applies with especial force to stream-measurement work in Hawaii. It has been found, however, that it is possible to obtain data which are sufficiently accurate, although many of those presented in this report are for periods too short to admit of definite conclusions.

\section{FIELD METHODS OF MEASURING STREAM FLOW.}

\section{BASE DATA.}

In making plans for power, irrigation, munioipal water supply, and other projects involving the use of water from surface streams itis necessary to have data from which both the total flow of the stream and its distribution from day to day throughout the year can be obtained. The data necessary for obtaining such information are 
daily gage heights, which give the fluctuations of rise and fall of the stream, and measurements of discharge at various stages, from which a rating eurve and table can be prepared, giving the discharge for any stage. Such a rating is possible from the fact that so long as the conditions at the controlling point in the stream remain the same there will be approximately the same discharge for any given gage height.

The determination of a discharge is termed a discharge measurement, and points at which discharge measurements are made and records of daily fluctuations of stage are kept for determining the daily flow are termed gaging stations.

Gaging stations may be divided into two classes, known as weir stations and velooity-area stations. At weir stations the head of water on the crest of the weir is measured and the discharge computed by means of a formula. The discharge at velocity-area stations is obtained by measuring the velocity of the current and the area of the cross-section, the product of the two giving the discharge.

The data presented in this paper were collected at both weir and velocity-area stations.

\section{WEIR MEASUREMENTS.}

Unquestionably a weir properly constructed and of a type for which accurate coefficients have been determined is one of the most convenient and reliable means of measuring small quantities of water. In practice, however, weirs rarely conform to the requirements imposed by the experimenter who derived the coefficients. If the crest of the weir is sharp and clean and sufficiently high above the bottom of the leading channel and the end contractions are complete and the velocity of approach is wanting, or negligibly small, andif the head on crest is measured at a distance back of the overfall of at least the weir crest length, the Francis formula will give good results. On the other hand, if these essential conditions are not complied with, especially if the velocity of approach is considerable and the contractions are imperfect, the Francis formula will not give accurate results. This is particularly true if the weir is improperly constructed and there is leakage around and under it, as is so frequently the case in practice.

Observations made on various types of weirs in Hawaii show that of the weirs in use in the Territory not all are giving accurate results. If the error is known so that corrections can be made the trouble is largely mitigated, but faulty weir records are too often accepted without investigation as to their accuracy. 
VILOCITY-AREA IIETHOD.

The velocity-area method of measurement consists of determining the mean or average velocity of the water past a given cross-section area. The area of the cross section at right angles to the direction of flow is determined by soundings which are taken at such distances apart as will develop the contour of the stream bed. The depths are recorded and also their distances from some arbitrarily chosen initial point on one side of the stream.

The method of making the soundings depends on the size and stage of the stream. On ditches and small streams, where the depths and velocities are not large, a graduated rod may be used to advantage; on large streams, which must be measured from bridges or cables, a lead weight and sounding line must be used. The weights are of different sizes $-6 \frac{1}{2}, 10$, or 15 pounds-according to the swiftness of the current, and are torpedo shaped, so as to offer as little resistance as possible to the moving water.

On streams with beds which are permanent or nearly so a standard cross section is usually constructed from careful soundings and referred to the zero of the gage, so that the depths for any stage can be found by adding the gage height at that stage to the depths below the zero of the gage. This method is especially useful at high stages, when it is difficult to make accurate soundings.

After the cross-section area of the stream has been measured by soundings and horizontal distances, the velocity is determined at a number of points. These measurements of velocity should be made at frequent intervals across the stream and close enough to take account of any abrupt change in the velocity. For convenience, the velocities are usually observed in the same verticals at which soundings are made. On some streams fairly good measurements of velocities may be made by means of subsurface floats. This method is applicable, however, only to channels of uniform cross-section area over a considerable distance and is very unsatisfactory for use on natural streams like those of Hawaii. ${ }^{1}$

The velocity of flow is best determined by the current meter, which is a form of water wheel actuated by the current, and of such size and shape that it can easily be placed at any point in the stream.

The new type of penta-recording current meter consists of six cups attached to ja vertical shaft which revolves on a conical hardenedsteel point when immersed in moving water. The revolutions are indicated electrically or acoustically. The rating, or relation between the velocity of moving water and the revolutions of the wheel, is determined for each meter by drawing it through still water for a given distance at different speeds and noting the number of revolu-

\footnotetext{
1 Further informstion regarding the float method is given in Water-Supply Paper 95 and in textbooks on stream flow.
} 
tions for each run. From these data a rating table is prepared which gives the velocity in feet per second of moving water for any number of revolutions in a given time interval. The ratio of revolutions per second to velocity of flow in feet per second is very nearly a constant for all speeds and is approximately 0.45 .

Three classes of methods of measuring velocity with current meters are in general use-multiple-point, single-point, and integration.

The two principal multiple-point methods in general use are the vertical velocity curve and 0.2 and 0.8 depth.

In the vertical velocity-curve method a series of velocity determinations are made in each vertical at regular intervals, usually about 10 to 20 per cent of the depth apart. By plotting these velocities as abscissas and their depths as ordinates and drawing a smooth curve among the resulting points, the vertical velocity curve is developed. This ourve shows graphically the magnitude and changes in velocity from the surface to the bottom of the stream. The mean velocity in the vertical is then obtained by dividing the area bounded by this velocity curve and its axis by the depth. This method of obtaining the mean velocity in the vertical is probably the best known, but on account of the length of time required to make a complete measurement its use is largely limited to the determination of coefficients for purposes of comparison.

In the second multiple-point method the meter is held successively at 0.2 and 0.8 depth, and the mean of the velocities at these two points is taken as the mean velocity for that vertical. On the assumption that the vertical velocity curve is a common parabola with horizontal axis, the mean of velocities at 0.22 and 0.79 depth will give (closely) the mean velocity in the vertical. Actual observations under a wide range of conditions show that this multiple-point method gives the mean velocity very closely for open-water conditions and that in a completed measurement it seldom varies as much as 1 per cent from the value given by the vertical velocity-curve method. It is very extensively used in the regular practice of the United States Geological Survey.

The single-point method consists in holding the meter either at the depth of the thread of mean velocity or at an arbitrary depth for which the coefficient for reducing to mean velocity has been determined or must be assumed.

Extensive experiments by means of vertical velocity curves show that the thread of mean velocity generally occurs between 0.5 and 0.7 total depth. In general practice the thread of mean velocity is considered to be at 0.6 depth, and at this point the meter is held in most of the measurements made by the single-point method. A large number of vertical velocity curve measurements, taken on many streams and under varying conditions, show that the average coefficient for reducing the velocity obtained at 0.6 depth to mean 
velocity is practically unity. The variation of the coefficient from unity in individual cases is, however, greater than in the 0.2 and 0.8 method and the general results are not as satisfactory.

In the other principal single-point method the meter is held near the surface, usually 1 foot below, or low enough to be out of the effect of the wind of other disturbing influences. This is known as the subsurface method. The coefficient for reducing the velocity taken at the subsurface to the mean has been found to be in general from about 0.85 to 0.95 , depending on the stage, velocity, and channel conditions. The higher the stage the larger the coefficient. This method is especially adapted for flood measurements, or for measurements when the velocity is so great that the meter can not be kept in the correct position for the other methods.

The vertical integration method consists in moving the meter at a slow but uniform speed from the surface to the bottom and back again to the surface and noting the number of revolutions and the time taken in the operation. This method has the advantage that the velocity at each point of the vertical is measured twice. It is useful as a check on the point methods. In using the Price meter great care should be taken that the vertical movement of the meter is not rapid enough to vitiate the accuracy of the resulting velocity determination.

In practical work on rough streams, such as exist in Hawaii, the meter should be held at 0.6 depth for depths of 1 foot or less. For greater depths the meter should be held at two points in the vertical, 0.2 and 0.8 from the surface.

When the mean velocities in the different verticals have been found, the average of two adjacent means is taken as the mean velocity for that individual section. The area of the section is computed by multiplying the width of the section by the mean depth. The discharge of each section is then the product of the area multiplied by the mean velocity, and the total discharge of the stream results from summing up the discharge of the individual sections. In practice the work is tabulated in such a way as to render the computation very simple. ${ }^{1}$

Current meter measurements are not practicable where there are eddies, cross currents, swirls, or passages for the water underneath stones. It is usually possible, however, to improve the channel by removing bowlders and rocks, so that a satisfactory measuring section may be obtained, even on rough, steep streams such as exist in Hawaii.

Three kinids of velocity-area gaging stations are in general use in Hawaii, according to the means provided for making the observations of depth and velocity. They are wading, bridge, and cable stations.

1 For a discussion of methods of computing the discharge of a stream see Engineering News, June 25, 1908. 
A wading station is one at which measurements are made only by wading; that is, no means exist for getting above the water at any stage except by wading. Such stations are usually on ditches or wide, shallow streams, which do not fluctuate greatly in flow. Frequently, however, measurements are made at low stages by wading, even though other means exist for making measurements at higher stages.

A bridge station is one at which the meter is used from a bridge. In some places highway or other bridges are available from which to make measurements, but generally they are not at the right place on the stream. Special bridges are then built.

A cable station is one at which measurements are made from a cable spanning the stream. Cable stations are used on large streams, such as Hanapepe, Wailua, and Hanalei rivers on the island of Kauai, and Wailuku River on the island of Hawaii. The cable supports the car from which a man works above the water. Distances are marked off on the cable itself or on a small auxiliary cable stretched taut above it.

A suitable place for a gaging station having been selected, a staff gage is set in the edge of the stream, either vertical or inclined, but graduated into tenths, half-tenths, or hundredths of feet vertically. The gage is securely fastened to rocks or trees to prevent displacement by floods and is so placed that the zero, or reference datum, is well below extreme low water. The datum is also referred to a permanent bench mark as an additional precaution. A water-stage recorder is then installed or an observer is engaged to record the heights of water morning and evening, and the mean of the two readings is used as the mean gage height for the day. Owing to the rapid rise and fall of most of the streams in Hawaii, two gage-height readings a day will not as a rule give a true mean for the 24 hours. For this reason, and also owing to the fact that many of the gaging stations are necessarily situated in the mountains at points remote from all habitations and difficult of access, it has generally been found necessary to use water-stage recorders. These instruments are of various types, some requiring weekly visits and others operating for a month without attention.

The essential features of water-stage recorders comprise a float free to rise and fall with fluctuations of the water surface, a device for transferring the motion of the float to the record sheet (either directly or through a reducing mechanism), the recording device, and the clock. The instruments may be designed for any range of stage. Those used by the United States Geological Survey in Hawaii are designed for ranges up to as high as 36 feet, but so far those having a 20 -foot range have been found to be sufficient for any stage. 


\section{DEFINITIOF OF TERMS.}

The volume of water flowing in a stream-the "run-off" or "discharge" - is expressed in various terms, each of which has become associated more or less definitely with a certain class of work. These terms may be divided into two groups: (1) Those which represent a rate of flow, as "second-feet"; "gallons per minute," "gallons per 24 hours," "miner's inches," and "run-off in second-feet per square mile," and (2) those which represent the actual quantity of water, as "run-off in depth in inches," "million gallons," and "acre-feet." They may be defined as follows:

"Second-foot" is an abbreviation for cubic foot per second, and is" the unit for the rate of discharge of water flowing in a stream 1 square foot in cross section at a rate of 1 foot per second. It is generally adopted as the fundamental unit in the measurement of flowing water and is the "natural" unit, as the foot and the second are the units used in making the physical determinations. Other units may be computed from this by the use of factors given in the table of equivalents.

"Gallons per minute" is generally used in connection with pumping and city water supply, the United States gallon of 231 cubic inches being the unit of quantity and 1 minute the unit of time.

The "miner's inch" is the unit for the rate of discharge of water that passes through an orifice 1 inch square under a head which varies locally. It is commonly used by miners and irrigators throughout the West, and is defined by statutes in each State in which it is used.

"Second-feet per square mile" is the average number of cubic feet of water flowing per second from each square mile of area drained, on the assumption that the run-off is distributed uniformly both as regards time and area.

"Run-off in inches" is the depth to which the drainage area would be covered if all the water flowing from it in a given period were conserved and uniformly distributed on the surface. It is used for comparing run-aff with rainfall, which is usually expressed in depth in inches.

An "acre-foot" is equivalent to 43,560 cubic feet, and is the quantity required to cover an acre to the depth of 1 foot. The term is commonly used in connection with storage for irrigation.

In the Territory of Hawaii a unit commonly used in connection with the measurement of water is the "million gallons." This is used with two meanings-(1) to indicate a rate of flow and (2) to express an actual quantity of water. In the former sense "million gallons per 24 hours" is inferred, 1,000,000 gallons being taken as the unit of quantity and 24 hours as the unit of time. With this meaning the term is generally used in connection with pumping and irrigation. In the 
latter sense "million gallons" as an absolute quantity is used in the measurement of storage capacities of reservoirs.

The following convenient approximate relations exist between second-feet, million gallons per 24 hours, and acre-feet: 1 secondfoot flowing 24 hours equals about 2 acre-feet; $1,000,000$ gallons equals about 3 acre-feet; and 1 second-foot equals approximately two-thirds million gallons per 24 hours.

"Man's water" is an irrigator's terms also in common use in Hawaii. It signifies the amount of water that one irrigator can properly handle in the field. It varies greatly, being dependent upon the condition of

- the furrows, the age of the crop, and the skill and individuality of the irrigator.

\section{CONVENIENT EQUIVALENTS.}

The following is a list of convenient equivalents for use in hydraulic computations:

Table for converting discharge in second-feet into run-off in acre-feet.

\begin{tabular}{|c|c|c|c|c|c|}
\hline \multirow{2}{*}{$\begin{array}{l}\text { Discharge } \\
\text { (second- } \\
\text { feet). }\end{array}$} & \multicolumn{5}{|c|}{ Run-off (acre-feet). } \\
\hline & 1 day. & 28 days. & 29 days. & 30 days. & 31 days. \\
\hline $\begin{array}{l}1 \\
2 \\
3 \\
4 \\
5 \\
6 \\
7 \\
8 \\
9\end{array}$ & $\begin{array}{l}1.983 \\
3.967 \\
5.950 \\
7.934 \\
9.917 \\
11.90 \\
13.88 \\
15.87 \\
17.85\end{array}$ & $\begin{array}{l}55.54 \\
111.1 \\
166.6 \\
222.1 \\
277.7 \\
333.2 \\
388.8 \\
444.3 \\
499.8\end{array}$ & $\begin{array}{r}57.52 \\
115.0 \\
172.6 \\
230.1 \\
287.6 \\
345.1 \\
402.6 \\
460.2 \\
517.7\end{array}$ & $\begin{array}{r}59.50 \\
119.0 \\
178.5 \\
238.0 \\
297.5 \\
357.0 \\
416.5 \\
\mathbf{4 7 6 . 0} \\
\mathbf{5 3 5 . 5}\end{array}$ & $\begin{array}{c}61.49 \\
123.0 \\
184.5 \\
246.0 \\
307.4 \\
368.9 \\
430.4 \\
491.9 \\
553.4\end{array}$ \\
\hline
\end{tabular}

Note.-For part of a month multiply values for one day by the number of days.

1 second-foot equals 7.48 United States gallons per seçond; equals 448.8 gallons per minute; equals 646,317 gallons for one day.

1 second-foot for one year (365 days) covers 1 square mile 1.131 feet or 13.572 inches deep.

1 second-foot for one year (365 days) equals 31,536,000 cubic feet.

1 second-foot for one year ( 365 days) equals 724 acre-feet.

1 second-foot equals about 1 acre-inch per hour.

1 second-foot for one day covers 1 square mile 0.03719 inch deep.

1 second-foot for one day equals 1.983 acre-feet.

$1,000,000$ United States gallons per day equals 1.55 second-feet.

$1,000,000$ United States gallons equal 3.07 acre-feet.

$1,000,000$ cubic feet equals 22.95 acre-feet.

1 acre-foot equals 325,850 gallons.

1 inch deep on 1 square mile equals $2,323,200$ cubic feet.

1 inch deep on 1 square mile equals 0.0737 second-foot per year.

1 foot equals 0.3048 meter.

1 mile equals 1.60935 kilometers.

1 mile equals 5,280 feet.

1 acre equals 0.4047 hectare.

1 acre equals 43,560 square feet. 
1 acre equals 209 feet square, nearly.

1 square mile equals 2.59 square kilometers.

1 cubic foot equals 0.0283 cubic meter.

1 cubic foot equals 7.48 gallons.

1 cubic foot of water weighs 62.5 pounds.

1 cubic meter per minute equals 0.5886 second-foot.

1 horsepower equals 550 foot-pounds per second.

1 horsepower equals 76.0 kilogram-meters per second.

1 horsepower equals 746 watts.

1 horsepower equals 1 second-foot falling 8.80 feet.

$1 \frac{1}{3}$ horsepower equals about 1 kilowatt.

To calculate water power quickly: $\frac{\text { Sec.-ft. } \times \text { fall in feet }}{11}=$ net horsepower on water wheel realizing 80 per cent of theoretical power.

\section{OFFICE METHODS OF COMPUTING AND STUDYING DISCHARGE AND RUN-OFF.}

At the end of each year the field or base data for current-meter gaging stations, consisting of water-stage record sheets, daily gage heights, discharge measurements, and notes from observers' books are assembled. The measurements are plotted on cross-section paper and rating curves are drawn wherever feasible. The rating tables prepared from these curves are then applied to the tables of daily gage heights to obtain the daily discharge, and from these applications the tables of monthly discharge and run-off are computed.

Rating curves are drawn and studied with special reference to the class of channels which they represent. The discharge measurements for all classes of stations, when plotted with gage heights in feet as ordinates and discharges in second-feet as abscissas, define rating curves which are generally more or less parabolic in form. For many stations curves of area in square feet and mean velocity in feet per second are also constructed to the same scale of ordinates as the discharge curve. These are used mainly to extend the discharge curves beyond the limits of the plotted discharge measurements, to check the form of the discharge curve, and to determine and eliminate erroneous measurements.

For every rating table the following assumptions are made for the period of application of the table: (a) That the discharge is a function of and increases gradually with the stage; $(b)$ that the discharge is the same whenever the stream is at a given stage, and hence such changes in conditions of flow as may have occurred during the period of application are either compensating or negligible, except that the rating, as stated in the footnote of each table, is not applicable for periods during which the channel was obstructed; $(c)$ that the increased and decreased discharge due to change of slope on rising and falling stages is either negligible or compensating.

$97764^{\circ}-\mathrm{WSP} 373-15-2$ 
As already stated, the gaging stations may be divided into several classes, as indicated in the following paragraphs:

The stations of class 1 represent the most favorable conditions for an accurate rating and are also the most economical to maintain. The bed of the stream is usually composed of rock and is not subject to the deposit of sediment and loose material. This class includes also many stations located in a pool below which is a permanent rocky riffle that controls the flow like a weir. Provided the control is sufficiently high and close to the gage to prevent cut and fill at the gaging point from materially affecting the slope of the water surface, the gage height will for all practical purposes be a true index of the discharge. Discharge measurements made at such stations usually plot within 2 or 3 per cent of the mean discharge curve, and the rating developed from that curve represents a very high degree of accuracy.

Class 2 comprises mainly stations on rough, mountainous streams with steep slopes. The beds of such streams are, as a rule, comparatively permanent during low and medium stages, and when the flow is sufficiently well defined by an adequate number of discharge measurements before and after each flood the stations of this class give nearly as good results as those of class 1 . As it is seldom possible to make measurements covering the time of change at flood stage, the assumption is often made that the curves before and after the flood converged to a common point at the highest gage height recorded during the flood. Hence the only uncertain "period occurs during the period of actual change in conditions of flow.

Class 3 includes those stations where the stream bed is of a shifting character, or the controlling section below the gage frequently changes owing to cutting out by the current and the filling in of sand, gravel, and drift. In some cases in Hawaii changes are caused by the growth of vegetation in the stream bed. No absolute rule can be laid down for stations of this class. Each rating curve must be constructed mainly on the basis of the measurements of the current year, the engineer being guided largely by the history of the station and the following general law: If all measurements ever made at a station of this class are plotted on cross-section paper, they will define a mean curve which may be called a standard curve. It has been found in practice that if after a change caused by high stage a relatively constant condition of flow occurs at medium and low stages, all measurements made after the change will plot on a smooth curve which is practically parallel to the standard curve with respect to ordinates or gage heights. This law of the parallelism of rating curves is the fundamental basis of all ratings and estimates at stations with semipermanent and shifting channels. It is not absolutely correct, but, with few exceptions, answers all the practical requirements 
of estimates made at low and medium stages after a change at a high stage. This law appears to hold equally true whether the change occurs at the measuring section or at some controlling point below. The change is, of course, fundamentally due to change in the channel caused by cut or fill, or both, at or near the measuring section. For all except small streams the changes in section usually occur at the bottom. The following simple but typical examples illustrate this law:

(a) If 0.5 foot of planking were to be nailed on the bottom of a wellrated wooden flume of rectangular section, there would result, other conditions of flow being equal, new curves of discharge, area, and velocity, each plotting 0.5 foot above the original curves when referred to the original gage. In other words, this condition would be analogous to a uniform fill or cut in a river channel which either reduces or increases all three values of discharge, area, and velocity for any gage height. In practice, however, such ideal conditions rarely exist.

(b) In the case of a cut or fill at the measuring section, there is a marked tendency toward decrease or increase, respectively, of the velocity. In other words, the velocity has a compensating effect, and if the compensation is exact at all stages the discharge at a given stage will be the same under both the new and the old conditions.

(c) In the case of uniform change along the crest of a weir or rocky control, the area curve will remain the same as before the change, and it can be shown that here again the change in velocity curve is such that it will produce a new discharge curve essentially parallel to the original discharge curve with respect to their ordinates.

Of course, in actual practice such simple changes of section do not occur. The changes are complicated and lack uniformity, a cut at one place being largely offset by a fill at another, and vice versa. If these changes are very radical and involve large percentages of the total area-as, for example, on small streams-there may result a wide departure from the law of parallelism of rating curves. In complicated changes of section the corresponding changes in velocity which tend to produce a new parallel discharge curve may interfere with each other materially, causing eddies, boils, backwater, and radical changes in slope. In such extreme conditions, however, the measuring section would more properly fall under class 4 and would require very frequent measurements of discharge. Special stress is laid on the fact that in the lack of other data to the contrary the utilization of this law will yield the most probable. results.

Slight changes at low or medium stages of an oscillating character are usually averaged by a mean curve drawn among them parallel to the standard curve, and if the individual measurements do not 
vary more than 5 per cent from the rating curve the results are considered good for stations of this class.

Class 4 comprises stations on streams that have soft, muddy, or sandy beds. Good results can be obtained from such sections only by frequent discharge measurements, the frequency ranging from a measurement every two or three weeks to a measurement every day, according to the rate of diurnal change in conditions of flow. These measurements are plotted and a mean or standard curve drawn among them. It is assumed that there is a different rating curve for every day of the year and that this rating is parallel to the standard curve with respect to their ordinates. On the day of a measurement the rating curve for that day passes through that measurement. For days between successive measurements it is assumed that the rate of change is uniform, and hence the ratings for the intervening days are equally spaced between the ratings passing through the two measurements. This method must be modified "or abandoned altogether under special conditions. Personal judgment and a knowledge of the conditions involved can alone dictate the course to pursue in such cases.

The computations have, as a rule, been carried to three significant figures. Computation machines and the 20-inch slide rule have been generally used. All computations are carefully checked.

After the computations have been completed they are entered in tables and carefully studied and intercompared to eliminate or account for all gross errors so far as possible. Missing periods are filled in, so far as feasible, by means of comparison with records for adjacent streams. The attempt is made to complete years or periods of discharge, thus eliminating fragmentary and disjointed records. Full notes accompanying such estimates follow the daily and monthly discharge tables.

\section{EXPLANATION OF TABLES.}

For each regular current-meter gaging station are given in general the following data: Description of station, list of discharge measurements, table of daily discharge, table of monthly and yearly discharge, and run-off in acre-feet.

All rates of flow are expressed as second-feet, because tistances and depths are measured in feet, and velocities in feet per second. The flow is thus obtained in cubic feet per second, or more briefly in "second-feet." The term "million gallons per 24 hours" is not used except in a few tables where data have been furnished in these units by private cooperations. "Million gallons per 24 hours" is not a primary but a derived unit. To convert second-feet into million gallons per 24 hours multiply by 0.646 . 
In addition to statements regarding the location and installation of current-meter stations, the descriptions give information in regard to any conditions which may affect the constancy of the relation of gage height to discharge, covering such points as shifting channels and backwater; also information regarding diversions which decrease the total flow at the measuring section. Statements are also made regarding the accuracy and reliability of the data.

The discharge-measurement table gives the results of the discharge measurements made during the year, including the date, name of hydrographer, gage height, and discharge in second-feet.

The discharge measurements and gage heights are the base data from which rating tables, daily discharge tables, and monthly discharge tables are computed.

The table of daily discharge gives the discharge in second-feet corresponding to the observed gage height as determined from the rating table, the number of significant figures used varying with the size of the discharge.

In the table of monthly discharge the column headed "Maximum" gives the mean flow, as determined from the rating table, for the day when the mean gage height was highest. As the gage height is the mean for the day, it does not indicate correctly the stage when the water surface was at crest height and the corresponding discharge was consequently larger than given in the maximum column. Likewise in the column of "Minimum" the quantity given is the mean flow for the day when the mean gage height was lowest. The column headed "Mean" gives the average flow in cubic feet for each second during the month. The "run-off in acre-feet" given in the column under that head is computed from the mean discharge in second-feet.

\section{ACCURACY OF FIELD DATA AND COMPUTED RESULTS.}

The accuracy of stream-flow data depends (1) on permanence of the relation between discharge and stage, and (2) on the accuracy of observations of stage, measurements of discharge, and interpretation of data.

The accuracy column in the monthly discharge table does not apply to the maximum or minimum nor to any individual day, but to the monthly mean. It is based on the accuracy of the rating, the probable reliability of the observer, the number of gage readings per day, the range of the fluctuation in stage, and knowledge of local conditions. In this column, $A$ indicates that the mean monthly flow is probably accurate within 5 per cent; $B$, within 10 per cent; $\mathrm{C}$, within 15 per cent; $\mathrm{D}$, within 25 per cent. Special conditions are covered by footnotes.

It should be borne in mind that the observations in each succeeding year may be expected to throw new light on data already collected and published. 


\section{DIVISION OF WORK.}

On account of the isolation of the different islands of the Territory an engineer or employee was assigned to take charge of the field work on each of the four largest islands, with temporary headquarters on those islands.

Persons in charge of feld work.

\begin{tabular}{|c|c|c|}
\hline Island. & In charge. & Headquarters. \\
\hline $\begin{array}{l}\text { Oahu....... } \\
\text { Kauai...... } \\
\text { Maui. } \\
\text { Hawaii..... }\end{array}$ & 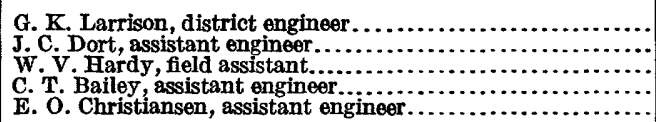 & $\begin{array}{l}\text { Hcnolulu (permanent). } \\
\text { Do. } \\
\text { Waimea (temporary). } \\
\text { Wailuku (temporary). } \\
\text { Hilo a (temporary). }\end{array}$ \\
\hline
\end{tabular}

a Discontinued July 18, 1913.

The ratings, applications, and computations were made by J. C. Dort, C. T. Bailey, W. V. Hardy, E. O. Christiansen, Howard Kimble, H. A. R. Austin, E. E. Goo, G. R. White, John Kaheaku, S. W. Dort, H. M. Kennedy, and R. M. S. Goo. The report was edited by Mrs. B. D. Wood.

\section{GAGING STATIONS MAINTAINED IN HAWATr.}

The following list comprises the gaging stations maintained in Hawaii by the United States Geological Survey and cooperative parties. The stations are arranged by stream basins and appear in systematic order for the several islands, tributaries of main streams being indicated by indention. The date refers to the years or parts of years for which records are available. A dash following the date indicates that the station was being maintained December 31, 1913.

\section{KAUAI ISLAND.}

Waimea River near Waimea, 1910-

Poomau River:

Kawaikoi Stream near Waimea, 1909-

Waiakoali Stream near Waimea, 1909-1912.

Mohihi Stream near Waimea, 1909-1912.

Waialae River near Waimea, 1910-

Kekaha ditch at Weir, Camp No. 1, near Waimea, 1910-1912.

Kekaha ditch at flume No. 3, near Waimea, 1910-1912.

Kekaha ditch at siphon, near Waimea, 1910-1912.

Kekaha ditch at tunnel No. 12 weir, near Waimea, 1910-1912.

Waimea ditch near Waimea, 1911-1913.

Kamenehune ditch near Waimea, 1911-12.

Makaweli River near Waimea, 1911-

Halekua Stream near Waimea, 1912-13.

Olokele River:

Olokele ditch at tunnel No. 12, near Makaweli, 1904

Olokele ditch near Makaweli, 1912-

Poowaiomahaihai ditch near Waimea, 1911-1913. 
Hanapepe River above Hanapepe Falls, near Eleele, 1911-12.

Hanapepe River at Koula, near Eleele, 1910-

Hiloa ditch at Hanapepe Falls, near Eleele, 1911-

East Branch Hanapape River below Hanapepe Falls, near Eleele, 1911-12.

Hanapepe ditch below Hanapepe Falls, near Eleele, 1911-12.

Hanapepe ditch at Koula, near Eleele, 1910-

Hanapepe ditch at weir, near Hanapepe, 1910

Huleia River near Lihue, 1912-

Hanamaulu River at Kapaia, near Lihue, 1911-

Wailua River:

South Fork of Wailua River at siphon, near Lihue, 1910-11.

South Fork of Wailua River above Waiehu Falls, near Lihue, 1911-

Hanamaulu ditch near Lihue, 1910

Lihue ditch near Lihue, 1910-

North Fork of Wailua River near Lihue, 1910-

Kanaha ditch near Lihue, 1910-

East Branch of North Fork of Wailua River, near Lihue, 1912-

Uhau Iole Stream at 750-foot elevation, near Lihue, 1912.

Keahua Stream at 750-foot elevation, near Lihue, 1912.

Kawi Stream at 750-foot elevation, near Lihue, 1912.

Konohiki Stream at Makakualele weir (mauka), near Kapaa, 1911-

Kaehulua Stream at Kuhinoa (mule stable) weir, near Kapaa, 1911-1913.

South Fork of Kaehulua Stream at Wainamuamu weir, near Kapaa, 1911-12.

North Fork of Kaehulua Stream at Wainamuamu weir, near Kapaa, 1911-

Kapaa River, near Kealia, 1910-

Akulikuli Spring near Kealia, 1911-1913.

Kapahi ditch at Kapahi, near Kealia, 1909-

Tunnel ditch at Kapahi, near Kapaa, 1909-1911.

Kapaa ditch at Kapahi, near Kapaa, 1909-1911.

Pipe ditch at Kapahi, near Kapaa, 1909-1911.

Kealia Stream:

Kaneha ditch near Kealia, 1909-

Anahola River at 1,140-foot elevation, near Kealia, 1912.

Anahola River above dam at Kiokala, near Kealia, 1910. 1912-

Anahola River at Kiokala dam, near Kealia, 1910-1912.

Anahola ditch at Kiokala, near Kealia, 1909-

Anahola ditch at Makai weir, near Kealia, 1909-1911.

Kalihiwai River near Kilauea, 1912-

Hanalei River near Hanalei, 191I-

China ditch near Hanalei, 1911-12.

Kuna ditch near Hanalei, 1912-13.

Lumahai River near Wainiha, 1912.

Wainiha River, East Channel, near Wainiha, 1912-

Wainiha River, West Channel, near Wainiha, 1911-

Wainiha canal at intake, near Wainiha, 1910-1912.

Wainiha canal at tunnel No. 18, near Wainiha, 1911.

Wainiha canal at tailrace, near Wainiha, 1911.

\section{OAHU ISLAND.}

Kalihi Stream at Kioi pool near Honolulu, 1913-

Nuuanu Stream at Luakaha weir in upper Nuuanu Valley, near Honolulu, 1903, 1910-13.

Nuuanu Stream below Reservoir No. 2 wasteway, near Honolulu, 1913- 
Nuuanu Stream at Kuakini Street, near Honolulu, 1911-12.

Lulumaha ditch at upper Nuuanu Reservoir, near Honolulu, 1911-1913.

Pauoa Stream at upper Pauoa Valley, near Honolulu, 1911-

Kahuawai Spring at upper Pauoa Valley, near Honolulu, 1912-

Manoa Stream at upper Manoa Valley, near Honolulu, 1910-1913.

Manoa Stream at College of Hawaii, near Honolulu, 1909-

West Branch of Manoa Stream at upper Manoa Valley, near Honolulu, 1913-

East Branch of Manoa Stream at upper Manoa Valley, near Honolulu, 1913-

Palolo Stream.

Pukele Stream at Mahoe springs, near Honolulu, 1912-

Waiomao Stream at upper Palolo Valley, near Honolulu, 1911-13.

Waiomao Stream above Pukele, near Honolulu, 1911-12.

Waimanalo ditch below main reservoir, near Waimanalo, 1912-13.

Pump ditch near Waimanalo, 1912.

Makawao ditch at Makawao flume, near Waimanalo, 1912-

Kailua Stream near Kailua, 1912-

Makawao Stream in Kailua Valley, near Kailua, 1912-

Kaimi Stream in Kailua Valley, near Kailua, 1912-

Kamakalepo Stream in Kailua Valley, near Kailua, 1912-

Pohakea Stream in Kailua Valley, near Kailua, 1912-

Kahanaiki Stream in Kailua Valley, near Kailua, 1912.

South Branch Kahanaiki Stream in Kailua Valley, near Kailua, 1913-

North Branch Kahanaiki Stream in Kailua Valley, near Kailua, 1913-

Kahanaiki ditch in Kailua Valley, near Kailua, 1912-13.

Waiahole Stream at Manianiaula, near Waikane, 1911-

Waiahole Stream at Waiahole, near Waikane, 1911-12.

Waihi Stream, near Waikane, 1911.

Halona Stream, near Waikane, 1911.

Waianu Stream, near Waikane, 1911.

Waikane Stream near Waikane, 1911-12.

Punaluu Stream near Hauula, 1906-7.

Kaluanui Stream near Hauula, 1906-7.

Kaipapau Stream near Hauula, 1906-7.

Kaukonahua Stream:

North Fork of Kaukonahua Stream near Wahiawa, 1911.

Right Branch of North Fork of Kaukonahua Stream near Wahiawa, 1913-

Left Branch of North Fork of Kaukonahua Stream near Wahiawa, 1913-

South Fork of Kaukonahua Stream near Wahiawa, 1911, 1913-

Wahiawa Reservoir ditch near Wahaiwa, 1910- 1 .

\section{MAUI ISLAND.}

Iao Stream near Wailuku, 1910-

West Maul.

Maniania ditch near Wailuku, 1909-1913.

Waiehu Stream:

South Waiehu Stream near Wailuku, 1910-

South Waiehu ditch near Wailuku, 1912-

North Waiehu Stream near Wailuku, 1912-

North Waiehu ditch near Wailuku, 1910-11.

Waihee Stream near Waihee, 1910-1912, 1913-

Waihee canal near Waihee, 1910-1912.

Waihee canal at weir, near Wailuku, 1911-12.

Spreckels ditch near Waihee, 1910-1913.

Spreckels ditch at Waiale weir, near Wailuku, 1910-11. 
Kahakuloa Stream at Kahakuloa, near Waihee, 1912-13.

Kahakuloa Stream near Honokahau, 1913-

Honokahau Stream near Honokahau, 1913-

Honokahau ditch at intake, near Honokahau, 1907-1913.

Honokahau ditch above Honolua Stream, near Honokahau, 1910-11.

Honokahau ditch at Honokawai weir, near Lahaina, 1910-1912.

Honolua Stream at Honolua ranch, 1911.

Honolua Stream near Honokahau, 1913-

Honolua ditch near Honokahau, 1911-12.

Honokawai Stream near Lahaina, 1911; 1912-

Honokawai Stream at weir No. 1, near Lahaina, 1901.

Honokawai ditch near Lahaina, 1912-

Kahoma Stream near Lahaina, 1911-12.

Kahoma Stream at weir No. 1, near Lahaina, 1901.

Kahoma Stream at weir No. 2, near Lahaina, 1901.

Kahoma ditch at weir, near Lahaina, 1911-

Lahainaluna Stream near Lahaina, 1911-

Lahainaluna weir No. 1 near Lahaina, 1901.

Lahainaluna weir No. 2 near Lahaina, 1901.

Lahainaluna ditch near Lahaina, 1913-

Kauaula Stream near Lahaina, 1912.

Kauaula Stream at weir No. 3, near Lahaina, 1901.

Kauaula ditch near Lahaina, 1911-

Kauaula Stream, North Fork, at weir No. 1, near Lahaina, 1901.

Kauaula Stream, South Fork, at weir No. 2, near Lahaina, 1901.

Launiupoko Stream near Lahaina, 1911-

Olowalu Stream near Olowalu, 1913.

Olowalu ditch No. 1 near Olowalu, 1911-

Ukumehame Stream near Olowalu, 1911-12.

Waikapu Stream near Waikapu, 1910-

Palolo (Everett) ditch near Waikapu, 1910-

South Side Waikapu ditch near Waikapu, 1910-

Koolau Ditch region:

East Mand.

Koolau ditch near Keanae, 1910-1912.

Koolau ditch at Alo division weir, near Huelo, 1908-1911.

Spreckels ditch region:

Haipuaena Stream near Huelo, 1910-

Puohakamoa Stream near Huelo, 1910-

Alo Stream near Huelo, 1910-.

Waikamoi Stream near Huelo, 1910-

Oopuola Stream near Huelo, 1910-

Spreckels ditch at station No. 1, near Huelo, 1910-1913.

Spreckels ditch at station No. 2, near Huelo, 1911-1913.

Spreckels ditch at station No. 3, near Huelo, 1910-1913.

Spreckels ditch at station No. 4, near Huelo, 1910-1913.

Spreckels ditch at station No. 5, near Huelo, 1911-1913.

Spreckels ditch at station No. 6, near Huelo, 1911-1913.

Spreckels ditch at station No. 7, near Huelo, 1911-12.

Spreckels ditch at station No. 8, near Huelo, 1911-1913.

Center ditch region:

Center ditch near Huelo, 1910-1912. 
Hamakua ditch region:

Nailiilihaele Stream near Huelo, 1910-1912; 1913-

Kailua Stream near Huelo, 1910-1912; 1913-

Oanui Stream near Huelo, 1910-11; 1913-

Hoolawaliilii Stream near Huelo, 1911-

Hoolawanui Stream near Huelo, 1911-

Honopou Stream near Huelo, 1910

Halehaku Stream at dam, near Huelo, 1910-11.

Halehaku Stream weir near Huelo, 1910-1912.

Opana Stream near Huelo, 1910-1912.

Opana ditch near Huelo, 1910-1912.

New Hamakua ditch at Nailiilihaele weir, near Huelo, 1910-1912.

New Hamakua ditch at Halehaku weir, near Huelo, 1910-1912.

New Hamakua ditch at station No. 1, near Huelo, 1912.

New Hamakua ditch at station No. 2, near Huelo, 1912.

New Hamakua ditch at station No. 3, near Huelo, 1912.

New Hamakua ditch at station No. 4, near Huelo, 1912.

New Hamakua ditch at station No. 5, near Huelo, 1912.

Old Hamakua ditch at Opana weir, near Huelo, 1910-1912.

Kaluanui ditch at Puuomalei, near Hamakuapoko, 1910-1912.

Lowrie ditch at Opana weir, near Huelo, 1910-1912.

Haiku ditch at Peahi weir, near Huelo, 1910-1912.

\section{HAWAII ISLAND.}

Hilo group:

81 stations at 2,700 feet elevation, in forest back of Hilo, 1911-1913.

Wailuku River near Hilo, 1911-1913.

Honolii River at Kaiwiki, near Hilo, 1911-1913.

Honolii ditch at Kaiwiki, near Hilo, 1911.

Kawainui River at Kawainui, near Pepeekeo, 1911-12.

4 stations at Piihonua, near Hilo, 1912.

Hamakua group:

Waipio River below Koiawe, near Waipio, 1911-12.

Waipio River below Waima, near Waipio, 1911-12.

Waipio River at 360 feet elevation, near Waipio, 1901-2.

New Hamakua ditch at Waima Stream, near Waipio, 1912.

New Hamakua ditch at main weir, near Kukiuhaele, 1910-

Hamakua ditch at main weir, at Puualala, Waimea, 1913.

Kawainui Branch of Waipio River, near Waipio, 1911-12.

Kawainui Stream at 2,120 feet elevation, near Waipio, 1901-2.

Kawainui Stream at 1,435 feet elevation, near Waipio, 1901-2.

Kawainui Stream at 775 feet elevation, near Waipio, 1901-2.

Branch No. 3 of Kawainui Stream at 1,700 feet elevation, near Waipio, 1901-2.

Branch No. 2 of Kawainui Stream at 1,405 feet elevation, near Waipio, 1901-2.

Branch No. 1 of Kawainui Stream at 1,380 feet elevation, near Waipio, 1901-2.

Alakahi Stream at 1,200 feet elevation, near Waipio, 1901-2.

Alakahi Stream at 730 feet elevation, near Waipio, 1901-2.

Koiawe Stream at 1,120 feet elevation, near Waipio, 1901-2.

Koiawe Stream at 610 feet elevation, near Waipio, 1901-2.

Waima Stream at 790 feet elevation, near Waipio, 1901-2.

Waima Stream at 385 feet elevation, near Waipio, 1901-2. 
Kohala group:

Honokane Stream:

East Branch of Honokane Stream at 1,300 feet elevation, near Honokane, 1901.

East Branch of Honokane Stream at 770 feet elevation, near Honokane, 1901.

West Branch of Honokane Stream at 1,370 feet elevation,near Honokane,1901.

West Branch of Honokane Stream at 775 feet elevation, near Honokane, 1901.

Kohala ditch near Kohala, 1901-1913.

Kehana ditch at Honokane Mauka, near North Kohala, 1912-13.

\section{GAGING-STATION RECORDS.}

\section{ISLAND OF KAUAI.}

\section{WAIMEA RIVER NEAR WAIMEA, KAUAT.}

Location.-About 2 miles north of Waimea, 250 feet above ford.

Records available.-July 9, 1910, to December 31, 1913.

Drainage area. -58 square miles.

Gage.-Vertical and inclined staff.

-Control.-Shifting.

Discharge measurements.-Made from wire suspension bridge or by wading.

Diversion.-Greater part of low-water flow diverted by irrigation ditches above station.

Accuracy.-Records good except for November and December, when fair results were obtained.

Daily discharge, in second-feet, of Waimea River near Waimea, Kauai, for 1913.

\begin{tabular}{|c|c|c|c|c|c|c|c|c|c|c|c|c|}
\hline Day. & Jan. & Feb. & Mar. & Apr. & May. & June. & July. & Aug. & Sept. & Oct. & Nov. & Dec. \\
\hline $\begin{array}{l}1 . \\
2 . \\
3 . \\
4 .\end{array}$ & $\begin{array}{c}3.2 \\
192 \\
47 \\
7.3 \\
2.4\end{array}$ & $\begin{array}{r}6.6 \\
.2 .2 \\
2.2 \\
2.2 \\
2.2\end{array}$ & $\begin{array}{l}3.2 \\
2.2 \\
3.2 \\
2.2 \\
1.6\end{array}$ & $\begin{array}{c}0.2 \\
42 \\
14 \\
2.2 \\
1.0\end{array}$ & $\begin{array}{c}12 \\
2.2 \\
2.2 \\
1.6 \\
1.0\end{array}$ & $\begin{array}{c}1.0 \\
1.0 \\
186 \\
12 \\
4.1\end{array}$ & $\begin{array}{r}34 \\
\mathbf{3 9 7} \\
184 \\
\mathbf{5 7 2} \\
\mathbf{5 4 5}\end{array}$ & $\begin{array}{l}1.6 \\
1.4 \\
1.1 \\
1.0 \\
1.7\end{array}$ & $\begin{array}{r}373 \\
38 \\
24 \\
20 \\
76\end{array}$ & $\begin{array}{r}0.6 \\
.6 \\
.6 \\
65^{\circ} \\
60\end{array}$ & $\begin{array}{c}283 \\
246 \\
182 \\
6.6 \\
4.1\end{array}$ & $\begin{array}{l}0.6 \\
.4 \\
.4 \\
.2 \\
.2\end{array}$ \\
\hline & $\begin{array}{r}2.2 \\
2.2 \\
2.1 \\
24 \\
6.1\end{array}$ & $\begin{array}{r}2.2 \\
2.2 \\
212 \\
5.4 \\
2.2\end{array}$ & $\begin{array}{r}.6 \\
.2 \\
.2 \\
3.2 \\
2.2\end{array}$ & $\begin{array}{l}1.0 \\
1.0 \\
.2 \\
4.1 \\
8.3\end{array}$ & $\begin{array}{r}1.0 \\
.2 \\
.2 \\
.2 \\
.1\end{array}$ & $\begin{array}{r}4.1 \\
2.2 \\
2.2 \\
1.0 \\
1,490\end{array}$ & $\begin{array}{c}165 \\
151 \\
20 \\
2.2 \\
2.1\end{array}$ & $\begin{array}{l}1.6 \\
1.7 \\
1.4 \\
1.4 \\
1.6\end{array}$ & $\begin{array}{r}3.3 \\
1.5 \\
1.2 \\
1.0 \\
.9\end{array}$ & $\begin{array}{c}57 \\
1.8 \\
3,340 \\
320 \\
277\end{array}$ & $\begin{array}{l}2.8 \\
2.2 \\
2.1 \\
2.1 \\
1.8\end{array}$ & $\begin{array}{l}.2 \\
.2 \\
.1 \\
.1 \\
.1\end{array}$ \\
\hline $\begin{array}{l}14 . \\
15 .\end{array}$ & $\begin{array}{r}186 \\
6.6 \\
489 \\
4.8 \\
6.6\end{array}$ & \begin{tabular}{|r}
255 \\
432 \\
2.2 \\
2.2 \\
2.2
\end{tabular} & $\begin{array}{r}1.0 \\
1.0 \\
.2 \\
.2 \\
.2\end{array}$ & $\begin{array}{l}815 \\
540 \\
320 \\
226 \\
320\end{array}$ & $\begin{array}{c}3,340^{-1} \\
2,190 \\
780 \\
226\end{array}$ & $\begin{array}{c}357 \\
102 \\
47 \\
10 \\
4.1\end{array}$ & $\begin{array}{r}1.8 \\
1.5 \\
47 \\
1,150 \\
182\end{array}$ & \begin{tabular}{r|}
1.6 \\
1.5 \\
6.6 \\
2.2 \\
17
\end{tabular} & $\begin{array}{r}.8 \\
.8 \\
.8 \\
1.0 \\
.9\end{array}$ & $\begin{array}{r}264 \\
78 \\
74 \\
69 \\
65\end{array}$ & $\begin{array}{r}6.6 \\
10,000 \\
10,000 \\
9,300 \\
9,000\end{array}$ & $\begin{array}{r}80 \\
397 \\
255 \\
240 \\
226\end{array}$ \\
\hline $\begin{array}{l}17 \ldots \ldots \\
19 \ldots \ldots \\
20 \ldots \ldots\end{array}$ & $\begin{array}{c}30 \\
4.4 \\
3.2 \\
2.6 \\
441\end{array}$ & $\begin{array}{c}2.2 \\
2.2 \\
2.2 \\
24 \\
2.2\end{array}$ & $\begin{array}{r}.2 \\
1.0 \\
1.0 \\
.6 \\
.2\end{array}$ & $\begin{array}{r}5.4 \\
.1 \\
1.0 \\
1.0 \\
.2\end{array}$ & 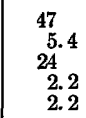 & $\begin{array}{l}2.2 \\
2.2 \\
2.2 \\
3.3 \\
2.1\end{array}$ & $\begin{array}{c}67 \\
2.6 \\
2.2 \\
81\end{array}$ & $\begin{array}{c}3.0 \\
19 \\
14 \\
2.1 \\
1.5\end{array}$ & $\begin{array}{l}.9 \\
1.0 \\
1.0 \\
3.5 \\
3.2\end{array}$ & $\begin{array}{l}53 \\
47 \\
42 \\
41 \\
38\end{array}$ & $\begin{array}{r}8,900 \\
397 \\
320 \\
306 \\
300\end{array}$ & $\begin{array}{r}221 \\
8.0 \\
8.0 \\
8.3 \\
6.4\end{array}$ \\
\hline $\begin{array}{l}22 . . \\
23 . \\
24 . \\
25 . .\end{array}$ & $\begin{array}{r}6.9 \\
5.8 \\
2.2 \\
2.1 \\
.4\end{array}$ & $\begin{array}{l}6.6 \\
4.1 \\
2.2 \\
357 \\
111\end{array}$ & $\begin{array}{r}1.6 \\
1.0 \\
1,940^{2} \\
996\end{array}$ & $\begin{array}{r}255 \\
212 \\
199 \\
94 \\
47\end{array}$ & $\begin{array}{r}1.0 \\
1.0 \\
.2 \\
.1 \\
4.1\end{array}$ & $\begin{array}{l}1.7 \\
1.1 \\
1.0 \\
3.2 \\
1.7\end{array}$ & $\begin{array}{l}4.1 \\
3.3 \\
2.1 \\
1.2 \\
1.1\end{array}$ & $\begin{array}{l}1.6 \\
1.6 \\
1.4 \\
1.6 \\
3.2\end{array}$ & $\begin{array}{l}1.2 \\
2.8 \\
2.2 \\
1.8 \\
1.7\end{array}$ & $\begin{array}{r}36 \\
540 \\
1,310 \\
320 \\
277\end{array}$ & $\begin{array}{r}296 \\
286 \\
58 \\
52 \\
49\end{array}$ & $\begin{array}{l}6.4 \\
6.4 \\
5.8 \\
5.6 \\
5.4\end{array}$ \\
\hline $\begin{array}{l}26 \ldots \\
27 \ldots \\
28 \ldots \\
29 \ldots \\
30 \ldots\end{array}$ & $\begin{array}{r}2.2 \\
2.2 \\
2.2 \\
2.2 \\
2.1 \\
140\end{array}$ & $\begin{array}{r}30 \\
6.6 \\
2.2 \\
\ldots \ldots . \\
\ldots \ldots .\end{array}$ & $\begin{array}{r}270 \\
47 \\
22 \\
6.6 \\
1.0 \\
1.0\end{array}$ & $\begin{array}{r}27 \\
12 \\
1.0 \\
.2 \\
.2\end{array}$ & $\begin{array}{l}4.1 \\
2.2 \\
1.0 \\
1.0 \\
1.0 \\
1.0\end{array}$ & $\begin{array}{c}115 \\
34 \\
21 \\
3.9 \\
2.4\end{array}$ & $\begin{array}{l}2.1 \\
2.2 \\
2.0 \\
1.7 \\
1.8 \\
1.7\end{array}$ & $\begin{array}{r}1.8 \\
1.6 \\
1.5 \\
189 \\
62 \\
174\end{array}$ & $\begin{array}{c}1.7 \\
640 \\
165 \\
158 \\
29\end{array}$ & $\begin{array}{c}258 \\
76 \\
72 \\
68 \\
3.5 \\
3.3\end{array}$ & $\begin{array}{l}47 \\
42 \\
38 \\
.8 \\
.8\end{array}$ & $\begin{array}{l}5.1 \\
4.6 \\
3.5 \\
2.8 \\
2.2 \\
1.6\end{array}$ \\
\hline
\end{tabular}

Note.-No discharge July 17. 
Monthly discharge of Waimea River near Waimea, Kauai, for 1919.

\begin{tabular}{|c|c|c|c|c|c|}
\hline \multirow{2}{*}{ Month. } & \multicolumn{3}{|c|}{ Discharge in second-feet. } & \multirow{2}{*}{$\begin{array}{c}\text { Rum-off } \\
\text { (total in } \\
\text { acre-feet). }\end{array}$} & \multirow{2}{*}{$\begin{array}{l}\text { Accu- } \\
\text { racy. }\end{array}$} \\
\hline & Maximum. & Minimum. & Mean. & & \\
\hline 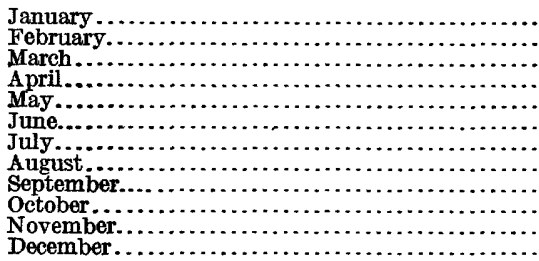 & $\begin{array}{r}489 \\
432 \\
1,940 \\
815 \\
3,340 \\
1,490 \\
1,150 \\
189 \\
640 \\
3,340 \\
10,000 \\
397\end{array}$ & $\begin{array}{r}0.4 \\
2.2 \\
.2 \\
.1 \\
.1 \\
1.0 \\
1.0 \\
.8 \\
.6 \\
.8 \\
.1\end{array}$ & $\begin{array}{c}52.6 \\
53.1 \\
50.5 \\
105 \\
215 \\
80.7 \\
117 \\
16.8 \\
51.9 \\
254 \\
1,670 \\
48.4\end{array}$ & $\begin{array}{r}3,230 \\
2,950 \\
3,110 \\
6,250 \\
13,200 \\
4,800 \\
7,190 \\
1,030 \\
3,090 \\
15,600 \\
99,400 \\
2,980\end{array}$ & $\begin{array}{l}\text { A. } \\
\text { A. } \\
\text { A. } \\
\text { A. } \\
\text { A. } \\
\text { A. } \\
\text { A. } \\
\text { A. } \\
\text { A. } \\
\text { A. } \\
\text { B. } \\
\text { B. }\end{array}$ \\
\hline The year...... & 10,000 & (a) & 225 & 163,000 & \\
\hline
\end{tabular}

a Channel dry.

KAWAIKOI STREAM NEAR WAIMEA, KAUAI.

Location. -8 miles northeast of Knudsen's mountain house, and about 27 miles north of Waimea by horse trail.

Records available.-April 3, 1909, to December 31, 1913.

Drainage area. -4.1 square miles.

Gage.-Barrett \& Lawrence water-stage recorder.

Control.-Probably permanent.

Discharge measurements.-Made from a wire suspension bridge or by wading. Accuracy.-Records good.

Discharge measurements of Kawaikoi Stream near Waimea, Kauai, in 1913.

\begin{tabular}{|c|c|c|c|c|c|c|c|}
\hline Date. & Hydrographer. & $\begin{array}{c}\text { Gage } \\
\text { height. }\end{array}$ & $\begin{array}{l}\text { Dis- } \\
\text { charge. }\end{array}$ & Date. & Hydrographer. & $\begin{array}{c}\text { Gage } \\
\text { height. }\end{array}$ & $\begin{array}{l}\text { Dis- } \\
\text { charge. }\end{array}$ \\
\hline $\begin{array}{ll}\text { Apr. } & 8 \\
\text { May } & 5 \\
\text { June } & 4\end{array}$ & 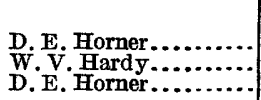 & $\begin{array}{c}\text { Feet. } \\
1.86 \\
1.80 \\
3.49\end{array}$ & $\begin{array}{c}\text { sec. ft. } \\
12.6 \\
8.44 \\
123\end{array}$ & $\begin{array}{ll}\text { June } & 5 \\
\text { July } & 7\end{array}$ & $\begin{array}{l}\text { D. E. Horner........................ } \\
\text { W. V. Hardy }\end{array}$ & $\begin{array}{c}\text { Feet. } \\
2.28 \\
2.68\end{array}$ & $\begin{array}{r}\text { Sec. ft. } \\
44.4 \\
50.3\end{array}$ \\
\hline
\end{tabular}


Daily discharge, in second-feet, of Kawaikoi Stream near Waimea, Kauai, for 1913.

\begin{tabular}{|c|c|c|c|c|c|c|c|c|c|c|c|c|}
\hline Day. & Jan. & Feb. & Mar. & Apr. & May. & June. & July. & Aug. & Sept. & Oct. & Nov. & Dec. \\
\hline 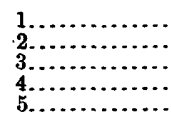 & $\begin{array}{l}18 \\
14\end{array}$ & & $\begin{array}{r}10 \\
9\end{array}$ & & 9 & $\begin{array}{l}13 \\
23 \\
20 \\
68 \\
23\end{array}$ & $\begin{array}{l}14 \\
14 \\
13 \\
20 \\
24\end{array}$ & $\begin{array}{l}9 \\
8 \\
8 \\
7 \\
7\end{array}$ & $\begin{array}{r}34 \\
25 \\
16 \\
8 \\
8\end{array}$ & $\begin{array}{l}8 \\
7 \\
4.5 \\
4 \\
4\end{array}$ & $\begin{array}{l}4.5 \\
69 \\
55 \\
16 \\
12\end{array}$ & $\begin{array}{r}26 \\
20 \\
31 \\
80 \\
110\end{array}$ \\
\hline $\begin{array}{r}6 \ldots \ldots \\
7 \ldots \ldots \ldots \ldots \\
9 \ldots \ldots \ldots \\
10 \ldots \ldots \ldots \ldots\end{array}$ & $\begin{array}{r}14 \\
15 \\
\ldots \ldots\end{array}$ & $\begin{array}{r}15 \\
32 \\
\ldots\end{array}$ & $\cdots$ & & $\begin{array}{l}16 \\
23 \\
15 \\
12 \\
10\end{array}$ & $\begin{array}{l}16 \\
16 \\
14 \\
13 \\
13\end{array}$ & $\begin{array}{l}23 \\
47 \\
22 \\
17 \\
14\end{array}$ & $\begin{array}{l}7 \\
11 \\
20 \\
20 \\
12\end{array}$ & $\begin{array}{l}8 \\
8 \\
8 \\
7 \\
7\end{array}$ & $\begin{array}{l}44 \\
74 \\
82 \\
18 \\
13\end{array}$ & $\begin{array}{r}9 \\
82 \\
27 \\
24 \\
17\end{array}$ & $\begin{array}{r}127 \\
53 \\
50 \\
31 \\
21\end{array}$ \\
\hline $\begin{array}{l}11 \ldots \ldots \ldots \\
12 \ldots \ldots \ldots \\
13 \ldots \ldots \ldots \\
14 . \ldots \ldots \\
15 . \ldots \ldots\end{array}$ & & & & & $\begin{array}{l}63 \\
68 \\
53 \\
16 \\
14\end{array}$ & $\begin{array}{l}13 \\
13 \\
13 \\
13 \\
14\end{array}$ & $\begin{array}{r}13 \\
30 \\
114 \\
63 \\
22\end{array}$ & $\begin{array}{r}9 \\
18 \\
22 \\
39 \\
30\end{array}$ & $\begin{array}{r}7 \\
9 \\
9 \\
14 \\
10\end{array}$ & $\begin{array}{r}12 \\
10 \\
7 \\
7 \\
7\end{array}$ & $\begin{array}{r}80 \\
119 \\
68 \\
21 \\
15\end{array}$ & $\begin{array}{l}18 \\
17 \\
16 \\
15 \\
15\end{array}$ \\
\hline $\begin{array}{l}16 \ldots \ldots . \\
17 \ldots \ldots \ldots \\
18 \ldots \ldots \ldots \\
19 . \ldots \ldots \\
20 . \ldots \ldots\end{array}$ & & & & & $\begin{array}{l}16 \\
55 \\
36 \\
15 \\
13\end{array}$ & $\begin{array}{l}15 \\
14 \\
14 \\
12 \\
11\end{array}$ & $\begin{array}{l}17 \\
15 \\
13 \\
18 \\
33\end{array}$ & $\begin{array}{r}98 \\
26 \\
18 \\
11 \\
9\end{array}$ & $\begin{array}{r}8 \\
7 \\
30 \\
17 \\
10\end{array}$ & $\begin{array}{l}6 \\
5 \\
5 \\
5 \\
5\end{array}$ & $\begin{array}{r}66 \\
109 \\
82 \\
190 \\
75\end{array}$ & $\begin{array}{l}15 \\
14 \\
14 \\
13 \\
12\end{array}$ \\
\hline $\begin{array}{l}21 \ldots \ldots \ldots \\
22 \ldots \ldots \ldots \\
23 \ldots \ldots \\
24 \ldots \ldots \\
25 \ldots \ldots\end{array}$ & & & & & $\begin{array}{r}11 \\
10 \\
8 \\
8 \\
8\end{array}$ & $\begin{array}{l}12 \\
12 \\
12 \\
12 \\
12\end{array}$ & $\begin{array}{l}20 \\
15 \\
13 \\
14 \\
16\end{array}$ & $\begin{array}{r}8 \\
7 \\
7 \\
28 \\
18\end{array}$ & $\begin{array}{c}11 \\
14 \\
8 \\
4.5 \\
6\end{array}$ & $\begin{array}{c}5 \\
5 \\
6.5 \\
12 \\
18\end{array}$ & $\begin{array}{r}31 \\
92 \\
152 \\
172 \\
100\end{array}$ & $\begin{array}{l}11 \\
11 \\
11 \\
10 \\
10\end{array}$ \\
\hline $\begin{array}{l}26 \ldots \\
27 \ldots \\
28 \ldots \\
29 \ldots \\
30 \ldots \\
31 \ldots\end{array}$ & & & & & $\begin{array}{l}8 \\
8 \\
8 \\
8 \\
8 \\
8\end{array}$ & $\begin{array}{r}12 \\
12 \\
12 \\
14 \\
14 \\
\cdots\end{array}$ & $\begin{array}{r}14 \\
12 \\
9 \\
9 \\
9 \\
9\end{array}$ & $\begin{array}{l}19 \\
18 \\
13 \\
22 \\
20 \\
36\end{array}$ & $\begin{array}{l}\mathbf{5} \\
\mathbf{4} .5 \\
\mathbf{5} \\
\mathbf{5} \\
4 \\
\cdots\end{array}$ & $\begin{array}{c}12 \\
11 \\
7 \\
5 \\
4.5 \\
4.5\end{array}$ & $\begin{array}{l}\mathbf{4 4} \\
31 \\
24 \\
28 \\
36\end{array}$ & 11 \\
\hline
\end{tabular}

Notr.-No records Jan. 1-3, Jan. 8 to Feb. 6, Feb. 9 to Mar. 2, and Mar. 5 to May 4.

Monthly discharge of Kawaikoi Stream near Waimea, Kauai, for 1913.

\begin{tabular}{|c|c|c|c|c|c|}
\hline \multirow{2}{*}{ Month. } & \multicolumn{3}{|c|}{ Discharge in second-feet. } & \multirow{2}{*}{$\begin{array}{l}\text { Run-off } \\
\text { (total in } \\
\text { acre-feet). }\end{array}$} & \multirow{2}{*}{$\begin{array}{l}\text { Accu- } \\
\text { racy. }\end{array}$} \\
\hline & Maximum. & Minimum. & Mean. & & \\
\hline \multirow{2}{*}{ 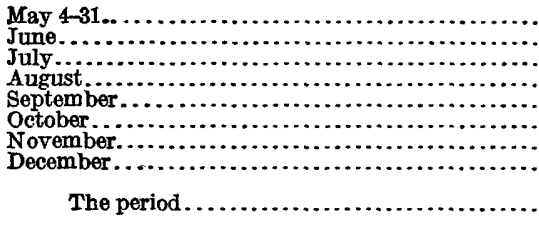 } & $\begin{array}{r}68 \\
114 \\
98 \\
34 \\
82 \\
190 \\
127\end{array}$ & $\begin{array}{l}11 \\
9 \\
7 \\
4 \\
4 \\
4.5 \\
8\end{array}$ & $\begin{array}{l}19.5 \\
15.8 \\
22.1 \\
18.9 \\
10.6 \\
12.2 \\
61.7 \\
26.0\end{array}$ & $\begin{array}{r}1,040 \\
940 \\
1,360 \\
1,160 \\
631 \\
750 \\
3,670 \\
1,600\end{array}$ & \multirow[t]{2}{*}{$\begin{array}{l}\text { A. } \\
\text { A. } \\
\text { A. } \\
\text { A. } \\
\text { A. } \\
\text { A. } \\
\text { A. } \\
\text { A. }\end{array}$} \\
\hline & & & & 11,200 & \\
\hline
\end{tabular}

WAIALAE RIVER NEAR WATMEA, KAUAI.

Iocation. - 3 miles northeast of F. Gay's mountain house, and about 20 miles northeast of Waimea by horse trail.

Records available.-August 1, 1910, to December 31, 1913.

Drainage area. -3.5 square miles.

Gage.-Barrett \& Lawrence water-stage recorder.

Control.-Probaby permanent.

Discharge measurements.-Made from cable or by wading.

Accuracy -Records January to August, good; September to December, fair. 
Discharge measurements of Waialae River near Waimea, Kauai, in $191 s$.

\begin{tabular}{|c|c|c|c|c|c|c|c|}
\hline Date. & Hydrographer. & $\begin{array}{c}\text { Gage } \\
\text { height. }\end{array}$ & $\begin{array}{c}\text { Dis- } \\
\text { charge. }\end{array}$ & Date. & Bydrographer. & $\begin{array}{c}\text { Gage } \\
\text { height. }\end{array}$ & $\begin{array}{c}\text { Dis- } \\
\text { charge. }\end{array}$ \\
\hline $\begin{array}{l}\text { Apr. } \\
\text { May }\end{array}$ & $\begin{array}{c}\text { D. E. Horner.... } \\
\end{array}$ & $\begin{array}{r}\text { Feet. } \\
1.56 \\
1.22\end{array}$ & $\begin{array}{c}\text { Sec. } f t . \\
28.9 \\
8.83\end{array}$ & June 9 & D. E. Horner.. & $\begin{array}{c}\text { Feet. } \\
1.64\end{array}$ & $\begin{array}{r}S e c .-f t . \\
\mathbf{3 5 . 9}\end{array}$ \\
\hline
\end{tabular}

Daily discharge, in second-feet, of Waialae River near Waimea, Kauai, for 191 .

\begin{tabular}{|c|c|c|c|c|c|c|c|c|c|c|c|c|}
\hline Day. & Jan. & Feb. & Mar. & Apr. & May. & June. & July. & Aug. & Sept. & Oct. & Nov. & Dec. \\
\hline & $\begin{array}{l}7.2 \\
7.2 \\
7.2 \\
7.2 \\
7.2\end{array}$ & $\begin{array}{l}3.5 \\
3.6 \\
3.9 \\
4.3 \\
4.3\end{array}$ & $\begin{array}{l}8.0 \\
8.0 \\
7.6 \\
77.6 \\
7.6\end{array}$ & $\begin{array}{c}39 \\
21 \\
11 \\
10 \\
7.6\end{array}$ & $\begin{array}{l}26 \\
23 \\
38 \\
21 \\
16\end{array}$ & $\begin{array}{c}7.2 \\
6.9 \\
8.3 \\
11 \\
11\end{array}$ & $\begin{array}{l}24 \\
50 \\
44 \\
39 \\
50\end{array}$ & $\begin{array}{l}6.9 \\
6.3 \\
6.0 \\
6.0 \\
5.5\end{array}$ & $\begin{array}{l}30 \\
41 \\
31 \\
13 \\
11\end{array}$ & $\begin{array}{l}5.5 \\
5.2 \\
4.3 \\
4.3 \\
4.3\end{array}$ & $\begin{array}{c}7.6 \\
80 \\
30 \\
11 \\
9.9\end{array}$ & $\begin{array}{l}18 \\
11 \\
10 \\
13 \\
23\end{array}$ \\
\hline $\begin{array}{c}6 \ldots \\
7 \ldots \\
8 \% \\
90 \\
10 \ldots \\
\end{array}$ & $\begin{array}{l}7.2 \\
7.2 \\
43 \\
20 \\
50\end{array}$ & $\begin{array}{l}4.3 \\
5.5 \\
34 \\
16 \\
18\end{array}$ & $\begin{array}{l}7.6 \\
7.2 \\
6.6 \\
5.5 \\
5.5\end{array}$ & $\begin{array}{r}6.9 \\
6.6 \\
6.3 \\
9.1 \\
24\end{array}$ & $\begin{array}{c}15 \\
12 \\
11 \\
7.6 \\
7.2\end{array}$ & $\begin{array}{c}11 \\
11 \\
9.1 \\
8.7 \\
36\end{array}$ & $\begin{array}{l}31 \\
24 \\
24 \\
17 \\
11\end{array}$ & $\begin{array}{l}5.8 \\
6.3 \\
6.9 \\
7.2 \\
7.2\end{array}$ & $\begin{array}{c}11 \\
11 \\
10 \\
7.2 \\
7.2\end{array}$ & $\begin{array}{r}4.3 \\
5.8 \\
105 \\
26 \\
11\end{array}$ & $\begin{array}{l}9.9 \\
14 \\
16 \\
41 \\
26\end{array}$ & $\begin{array}{c}48 \\
15 \\
10 \\
8.0 \\
8.0\end{array}$ \\
\hline & $\begin{array}{r}50 \\
16 \\
122 \\
34 \\
27\end{array}$ & $\begin{array}{c}36 \\
16 \\
11 \\
9.5 \\
9.1\end{array}$ & $\begin{array}{l}4.2 \\
3.0 \\
3.0 \\
3.0 \\
3.0\end{array}$ & $\begin{array}{l}34 \\
20 \\
84 \\
51 \\
93\end{array}$ & $\begin{array}{r}24 \\
100 \\
70 \\
29 \\
18\end{array}$ & $\begin{array}{l}41 \\
17 \\
15 \\
23 \\
12\end{array}$ & $\begin{array}{l}9.1 \\
8.0 \\
8.0 \\
14 \\
13\end{array}$ & $\begin{array}{c}7.2 \\
7.2 \\
10 \\
15 \\
17\end{array}$ & $\begin{array}{l}6.0 \\
5.8 \\
5.8 \\
5.8 \\
5.8\end{array}$ & $\begin{array}{l}10 \\
9.1 \\
57 \\
36 \\
26\end{array}$ & $\begin{array}{r}108 \\
122 \\
126 \\
24 \\
21\end{array}$ & $\begin{array}{l}7.2 \\
7.2 \\
7.2 \\
6.3 \\
4.9\end{array}$ \\
\hline $\begin{array}{l}16 . . \\
17 . .\end{array}$ & $\begin{array}{c}32 \\
13 \\
9.5 \\
8.0 \\
7.6\end{array}$ & $\begin{array}{l}8.3 \\
8.0 \\
8.0 \\
8.0 \\
8.0\end{array}$ & $\begin{array}{l}3.0 \\
3.0 \\
4.0 \\
4.0 \\
7.2\end{array}$ & $\begin{array}{l}30 \\
36 \\
44 \\
34 \\
20\end{array}$ & $\begin{array}{l}17 \\
16 \\
16 \\
16 \\
13\end{array}$ & $\begin{array}{c}11 \\
8.3 \\
7.6 \\
7.2 \\
7.2\end{array}$ & $\begin{array}{l}9.9 \\
8.3 \\
8.0 \\
8.3 \\
8.3\end{array}$ & $\begin{array}{l}24 \\
17 \\
16 \\
10 \\
8.0\end{array}$ & $\begin{array}{l}5.8 \\
5.8 \\
5.8 \\
5.8 \\
5.8\end{array}$ & $\begin{array}{l}20 \\
15 \\
14 \\
26 \\
19\end{array}$ & $\begin{array}{l}23 \\
24 \\
17 \\
70 \\
63\end{array}$ & $\begin{array}{l}4.3 \\
4.3 \\
5.5 \\
5.5 \\
5.5\end{array}$ \\
\hline $\begin{array}{l}21 \ldots \\
22 . \ldots \\
24 . \\
25 .\end{array}$ & $\begin{array}{l}7.2 \\
6.9 \\
6.6 \\
6.0 \\
5.8\end{array}$ & $\begin{array}{c}8.0 \\
8.0 \\
7.2 \\
39 \\
20\end{array}$ & $\begin{array}{l}7.6 \\
17 \\
15 \\
54 \\
62\end{array}$ & $\begin{array}{l}18 \\
16 \\
16 \\
16 \\
51\end{array}$ & $\begin{array}{c}11 \\
10 \\
8.0 \\
7.6 \\
7.2\end{array}$ & $\begin{array}{l}7.2 \\
7.2 \\
6.9 \\
6.3 \\
6.3\end{array}$ & $\begin{array}{l}9.1 \\
7.6 \\
7.2 \\
7.2 \\
7.2\end{array}$ & $\begin{array}{l}7.6 \\
7.2 \\
7.2 \\
6.6 \\
6.9\end{array}$ & $\begin{array}{l}5.8 \\
5.5 \\
5.5 \\
5.2 \\
4.9\end{array}$ & $\begin{array}{l}16 \\
17 \\
31 \\
23 \\
30\end{array}$ & $\begin{array}{l}18 \\
25 \\
60 \\
58 \\
61\end{array}$ & $\begin{array}{l}5.8 \\
5.2 \\
4.9 \\
4.9 \\
5.5\end{array}$ \\
\hline $\begin{array}{l}26 \ldots \\
27 \ldots \\
28 \ldots \\
29 . \ldots \\
30 \ldots \\
31 \ldots\end{array}$ & $\begin{array}{l}5.8 \\
5.2 \\
4.2 \\
3.8 \\
3.5 \\
3.5\end{array}$ & $\begin{array}{c}16 \\
11 \\
9.1 \\
\cdots \ldots . .\end{array}$ & $\begin{array}{c}41 \\
14 \\
11 \\
7.2 \\
6.3 \\
6.3\end{array}$ & $\begin{array}{l}28 \\
18 \\
18 \\
45 \\
41\end{array}$ & $\begin{array}{l}7.2 \\
7.2 \\
7.2 \\
7.2 \\
7.2 \\
7.2\end{array}$ & $\begin{array}{c}30 \\
17 \\
18 \\
14 \\
9.1\end{array}$ & $\begin{array}{l}7.2 \\
7.2 \\
7.2 \\
7.2 \\
7.2 \\
6.9\end{array}$ & $\begin{array}{l}6.6 \\
6.3 \\
17 \\
68 \\
18 \\
32\end{array}$ & $\begin{array}{l}4.3 \\
4.0 \\
6.0 \\
5.5 \\
5.5\end{array}$ & $\begin{array}{c}22 \\
28 \\
14 \\
13 \\
11 \\
8.0\end{array}$ & $\begin{array}{l}43 \\
11 \\
18 \\
50 \\
72\end{array}$ & $\begin{array}{l}5.5 \\
5.2 \\
5.8 \\
6.3 \\
6.3 \\
6.6\end{array}$ \\
\hline
\end{tabular}

Monthly discharge of Waialae River near Waimea, Kauai, for 1913.

\begin{tabular}{|c|c|c|c|c|c|}
\hline \multirow{2}{*}{ Month. } & \multicolumn{3}{|c|}{ Discharge in second-feet. } & \multirow{2}{*}{$\begin{array}{c}\text { Run-off } \\
\text { (total in } \\
\text { acre-feet). }\end{array}$} & \multirow{2}{*}{$\begin{array}{l}\text { Accu } \\
\text { racy. }\end{array}$} \\
\hline & Maximum. & Minimum. & Mean. & & \\
\hline 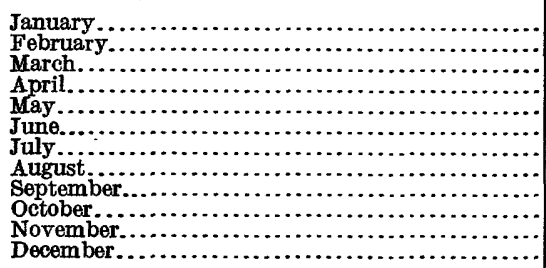 & $\begin{array}{r}122 \\
39 \\
62 \\
93 \\
100 \\
41 \\
50 \\
68 \\
41 \\
105 \\
128 \\
48\end{array}$ & $\begin{array}{l}3.5 \\
3.5 \\
3.0 \\
6.3 \\
7.2 \\
6.3 \\
6.9 \\
5.5 \\
4.0 \\
4.3 \\
7.6 \\
4.3\end{array}$ & $\begin{array}{l}17.5 \\
12.1 \\
11.3 \\
28.5 \\
18.8 \\
13.2 \\
15.8 \\
12.2 \\
9.43 \\
20.0 \\
39.6 \\
9.16\end{array}$ & $\begin{array}{r}1,080 \\
672 \\
695 \\
1,700 \\
1,160 \\
786 \\
972 \\
750 \\
561 \\
1,230 \\
2,360 \\
563\end{array}$ & $\begin{array}{l}\text { A. } \\
\text { A. } \\
\text { A: } \\
\text { A: } \\
\text { A: } \\
\text { A: } \\
\text { A. } \\
\text { B: } \\
\text { B. } \\
\text { B. }\end{array}$ \\
\hline The year.. & $\overline{126}$ & 3.0 & 17.3 & 12,500 & \\
\hline
\end{tabular}


WAMMEA DITCH NEAR WAIMEA, KAUAI.

Location.-About 4 miles north of Waimea and 300 feet below intake.

Records available.-November 4, 1911, to September 30, 1913.

Gage.-Vertical staff.

Control.-Fairly permanent.

Discharge measurements.-Made from a footbridge.

Diversion.-Ditch diverts part of flow of Waimea River.

Accuracy.-Records poor.

Monthly discharge of Waimea ditch near Waimea, Kauai, for 1913.

\begin{tabular}{|c|c|c|c|c|c|}
\hline \multirow{2}{*}{ Month. } & \multicolumn{3}{|c|}{ Discharge in second-feet. } & \multirow{2}{*}{$\begin{array}{c}\text { Run-off } \\
\text { (total in } \\
\text { acre-feet). }\end{array}$} & \multirow{2}{*}{$\begin{array}{l}\text { Accu- } \\
\text { racy. }\end{array}$} \\
\hline & Maximum. & Minimum. & Mean. & & \\
\hline 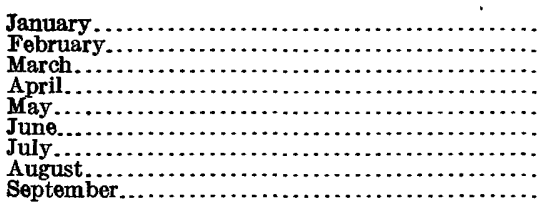 & $\begin{array}{l}12.1 \\
15.3 \\
10.6 \\
27.5 \\
13.7 \\
16.1 \\
16.1 \\
15.3 \\
12.1\end{array}$ & $\begin{array}{l}1.9 \\
1.5 \\
1.0 \\
2.4 \\
3.2 \\
2.4 \\
1.5 \\
2.0 \\
1.7\end{array}$ & $\begin{array}{l}4.97 \\
4.21 \\
3.84 \\
7.93 \\
5.61 \\
6.25 \\
7.16 \\
4.73 \\
4.02\end{array}$ & $\begin{array}{l}306 \\
234 \\
236 \\
472 \\
345 \\
372 \\
440 \\
291 \\
239\end{array}$ & $\begin{array}{l}\text { C. } \\
\mathbf{C .} \\
\mathbf{C .} \\
\mathbf{C .} \\
\mathbf{C .} \\
\mathbf{C .} \\
\mathbf{C .} \\
\mathbf{C .} \\
\mathbf{C .}\end{array}$ \\
\hline The period... & 0 & & ...... & 2,940 & \\
\hline
\end{tabular}

\section{MAKAWELI RIVER NEAR WAIMEA, KAUAI.}

Location.-About 2 miles northeast of Waimea. Reached by wagon road up Makaweli River.

Records available.-October 6, 1911, to December 31, 1913.

Drainage area.-25.8 square miles.

Gage.-Vertical staff.

Control.-Probably slightly shifting.

Discharge measurements.-Made from a wire suspension footbridge or by wading.

Diversions.-Several above station.

Accuracy.-Records fairly good except for November and December, when they were poor.

The following discharge measurement was made by W. V. Hardy: April 30, 1913: Gage height, 4.46 feet; discharge, 195 second-feet. 
Daily discharge, in second-feet, of Makaweli River near Waimea, Kauai, for 1913.

\begin{tabular}{|c|c|c|c|c|c|c|c|c|c|c|c|c|}
\hline Day. & Jan. & Feb. & Mar. & Apr. & May. & June. & July. & Aug. & Sept. & Oct. & Nov. & Dec. \\
\hline $\begin{array}{l}1 \ldots \\
2 \ldots \\
3 \ldots \\
4 \ldots \\
5 \ldots\end{array}$ & $\begin{array}{r}14 \\
14 \\
13 \\
\mathbf{3} \\
\mathbf{3}\end{array}$ & $\begin{array}{l}15 \\
14 \\
14 \\
13 \\
13\end{array}$ & $\begin{array}{l}17 \\
17 \\
14 \\
11 \\
11\end{array}$ & $\begin{array}{r}55 \\
14 \\
12 \\
12 \\
8\end{array}$ & $\begin{array}{r}39 \\
9 \\
6 \\
84 \\
13\end{array}$ & $\begin{array}{r}6 \\
6 \\
226 \\
34 \\
14\end{array}$ & $\begin{array}{l}22 \\
24 \\
72 \\
47 \\
55\end{array}$ & $\begin{array}{l}10 \\
12 \\
11 \\
10 \\
10\end{array}$ & $\begin{array}{l}30 \\
26 \\
24 \\
24 \\
17\end{array}$ & $\begin{array}{l}24 \\
34 \\
25 \\
50 \\
44\end{array}$ & $\begin{array}{l}82 \\
80 \\
78 \\
80 \\
69\end{array}$ & $\begin{array}{l}13 \\
12 \\
12 \\
12 \\
11\end{array}$ \\
\hline $\begin{array}{r}6 \ldots \\
7 \ldots \\
8 \ldots \\
9 \ldots \\
10 \ldots\end{array}$ & $\begin{array}{r}17 \\
14 \\
10 \\
10 \\
3\end{array}$ & $\begin{array}{r}12 \\
10 \\
9 \\
8 \\
84\end{array}$ & $\begin{array}{l}11 \\
10 \\
10 \\
10 \\
10\end{array}$ & $\begin{array}{r}6 \\
6 \\
6 \\
55 \\
15\end{array}$ & $\begin{array}{r}19 \\
17 \\
8 \\
8 \\
6\end{array}$ & $\begin{array}{r}11 \\
6 \\
6 \\
6 \\
39\end{array}$ & $\begin{array}{l}23 \\
18 \\
17 \\
18 \\
18\end{array}$ & $\begin{array}{l}8 \\
8 \\
9 \\
8 \\
8\end{array}$ & $\begin{array}{l}16 \\
16 \\
16 \\
15 \\
14\end{array}$ & $\begin{array}{r}37 \\
35 \\
621 \\
320 \\
260\end{array}$ & $\begin{array}{l}70 \\
74 \\
74 \\
84 \\
93\end{array}$ & $\begin{array}{r}11 \\
10 \\
9 \\
9 \\
8\end{array}$ \\
\hline $\begin{array}{l}14 \ldots \\
15 \ldots\end{array}$ & $\begin{array}{r}190 \\
26 \\
2,660 \\
84 \\
84\end{array}$ & $\begin{array}{l}32 \\
26 \\
20 \\
20 \\
19\end{array}$ & $\begin{array}{r}10 \\
10 \\
6 \\
6 \\
6\end{array}$ & $\begin{array}{r}10 \\
26 \\
383 \\
84 \\
126\end{array}$ & $\begin{array}{r}6 \\
1,280 \\
190 \\
39 \\
34\end{array}$ & $\begin{array}{l}17 \\
15 \\
15 \\
17 \\
17\end{array}$ & $\begin{array}{l}18 \\
18 \\
18 \\
19 \\
19\end{array}$ & $\begin{array}{l}7 \\
7 \\
7 \\
6 \\
6\end{array}$ & $\begin{array}{l}13 \\
13 \\
12 \\
12 \\
11\end{array}$ & $\begin{array}{r}82 \\
78 \\
72 \\
74 \\
148\end{array}$ & $\begin{array}{r}3,160 \\
1,920 \\
255 \\
166 \\
154\end{array}$ & $\begin{array}{l}9 \\
9 \\
8 \\
8 \\
7\end{array}$ \\
\hline $\begin{aligned} & 19 \ldots \ldots \ldots \\
& 20\end{aligned}$ & $\begin{array}{r}143 \\
84 \\
26 \\
26 \\
14\end{array}$ & $\begin{array}{l}17 \\
17 \\
14 \\
14 \\
17\end{array}$ & $\begin{array}{r}6 \\
19 \\
9 \\
9 \\
13\end{array}$ & $\begin{array}{l}84 \\
34 \\
21 \\
20 \\
19\end{array}$ & $\begin{array}{l}20 \\
19 \\
14 \\
14 \\
12\end{array}$ & $\begin{array}{l}14 \\
11 \\
12 \\
10 \\
11\end{array}$ & $\begin{array}{l}18 \\
18 \\
18 \\
18 \\
18\end{array}$ & $\begin{array}{l}6 \\
7 \\
7 \\
7 \\
6\end{array}$ & $\begin{array}{l}10 \\
10 \\
11 \\
12 \\
11\end{array}$ & $\begin{array}{r}143 \\
190 \\
98 \\
93 \\
82\end{array}$ & $\begin{array}{l}118 \\
106 \\
135 \\
139 \\
143\end{array}$ & $\begin{array}{l}7 \\
7 \\
7 \\
7 \\
7\end{array}$ \\
\hline $24 \ldots$ & $\begin{array}{l}14 \\
14 \\
13 \\
13 \\
14\end{array}$ & $\begin{array}{l}26 \\
20 \\
20 \\
19 \\
84\end{array}$ & $\begin{array}{r}12 \\
36 \\
10 \\
44 \\
122\end{array}$ & $\begin{array}{l}19 \\
14 \\
10 \\
11 \\
14\end{array}$ & $\begin{array}{r}6 \\
6 \\
14 \\
10 \\
11\end{array}$ & $\begin{array}{r}10 \\
10 \\
9 \\
9 \\
9\end{array}$ & $\begin{array}{r}16 \\
15 \\
8 \\
7 \\
7\end{array}$ & $\begin{array}{l}6 \\
6 \\
7 \\
7 \\
6\end{array}$ & $\begin{array}{l}10 \\
10 \\
10 \\
11 \\
10\end{array}$ & $\begin{array}{l}80 \\
76 \\
78 \\
75 \\
74\end{array}$ & $\begin{array}{r}135 \\
139 \\
150 \\
154 \\
5,170\end{array}$ & $\begin{array}{l}7 \\
7 \\
7 \\
7 \\
7\end{array}$ \\
\hline $\begin{array}{l}26 \ldots \ldots \ldots \\
27 \ldots \ldots \ldots \ldots \\
28, \ldots \ldots \ldots \ldots \\
30 \ldots \ldots \\
31 \ldots \ldots \\
29\end{array}$ & $\begin{array}{l}19 \\
17 \\
19 \\
19 \\
17 \\
14\end{array}$ & $\begin{array}{l}55 \\
26 \\
20\end{array}$ & $\begin{array}{r}39 \\
10 \\
8 \\
6 \\
6 \\
6\end{array}$ & $\begin{array}{r}14 \\
13 \\
6 \\
122 \\
84\end{array}$ & $\begin{array}{r}10 \\
10 \\
6 \\
6 \\
6 \\
6\end{array}$ & $\begin{array}{r}8 \\
247 \\
104 \\
16 \\
58\end{array}$ & $\begin{array}{l}7 \\
8 \\
9 \\
7 \\
8 \\
8\end{array}$ & $\begin{array}{r}6 \\
6 \\
6 \\
6 \\
70 \\
74\end{array}$ & $\begin{array}{l}9 \\
4 \\
6 \\
6 \\
4\end{array}$ & $\begin{array}{l}72 \\
75 \\
80 \\
80 \\
81 \\
81\end{array}$ & $\begin{array}{r}1,790 \\
289 \\
232 \\
202 \\
13\end{array}$ & $\begin{array}{l}6 \\
6 \\
6 \\
2 \\
2 \\
2\end{array}$ \\
\hline
\end{tabular}

Monthly discharge of Makaweli River near Waimea, Kauai, for 1913.

\begin{tabular}{|c|c|c|c|c|c|}
\hline \multirow{2}{*}{ Month. } & \multicolumn{3}{|c|}{ Discharge in second-feet. } & \multirow{2}{*}{$\begin{array}{l}\text { Run-off } \\
\text { (total in } \\
\text { acre-feet). }\end{array}$} & \multirow{2}{*}{$\begin{array}{l}\text { Accu- } \\
\text { racy. }\end{array}$} \\
\hline & Maximum. & Minimum. & Mean. & & \\
\hline 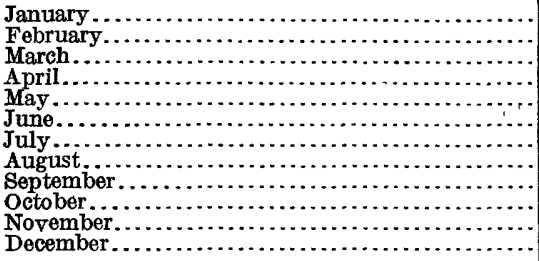 & $\begin{array}{r}2,660 \\
84 \\
122 \\
383 \\
1,280 \\
247 \\
72 \\
74 \\
30 \\
621 \\
5,170 \\
13\end{array}$ & $\begin{array}{r}3 \\
8 \\
6 \\
6 \\
6 \\
6 \\
7 \\
6 \\
4 \\
24 \\
13 \\
2\end{array}$ & $\begin{array}{r}116 \\
23.5 \\
16.6 \\
43.4 \\
62.2 \\
32.4 \\
19.2 \\
11.6 \\
13.1 \\
108 \\
512 \\
7.81\end{array}$ & $\begin{array}{r}7,130 \\
1,310 \\
1,020 \\
2,580 \\
3,820 \\
1,930 \\
1,180 \\
713 \\
780 \\
6,640 \\
30,500 \\
480\end{array}$ & $\begin{array}{l}\text { B. } \\
\text { A. } \\
\text { B. } \\
\text { A. } \\
\text { B. } \\
\text { B. } \\
\text { A. } \\
\text { B. } \\
\text { B. } \\
\text { B. } \\
\text { C. } \\
\text { C. }\end{array}$ \\
\hline The year........ & 5,170 & 2 & 80.3 & 58,100 & \\
\hline
\end{tabular}

HALEKUA STREAM NEAR WAIMEA, KAUAI.

Location.-Station is reached from Waimea via Gay's mountain house by saddle horse; requires two days to make round trip.

Records available.-October 11, 1912, to December 18, 1913.

Drainage area. -0.5 square mile.

Gage.-Barrett \& Lawrence water-stage recorder.

Control.--Probably permanent.

Discharge measuremerits.-Made by wading.

Accuracy.-Records fair.

Cooperation.-Station maintained in cooperation with Hawaiian Sugar Co. 
Discharge measurements of Halekua Stream near Waimea, Kauai, in 1913.

\begin{tabular}{|c|c|c|c|c|c|c|c|}
\hline Date. & Hydrographer. & $\begin{array}{c}\text { Gage } \\
\text { height. }\end{array}$ & $\begin{array}{c}\text { Dis- } \\
\text { charge. }\end{array}$ & Date. & Hydrographer. & $\begin{array}{c}\text { Gage } \\
\text { height. }\end{array}$ & $\begin{array}{c}\text { Dis- } \\
\text { charge. }\end{array}$ \\
\hline $\begin{array}{l}\text { June } 10 \\
\text { Sept. } 11\end{array}$ & 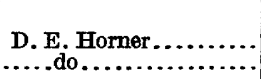 & $\begin{array}{r}\text { Feet. } \\
3.90 \\
3.36\end{array}$ & $\begin{array}{c}\text { Sec.-ft. } \\
10.8 \\
.65\end{array}$ & Oct. 12 & w. V. Hardy..... & $\begin{array}{r}\text { Feet. } \\
\mathbf{3 . 5 0}\end{array}$ & $\begin{array}{r}S e c .-f t . \\
1.68\end{array}$ \\
\hline
\end{tabular}

Daily discharge, in second-feet, of Halekua Stream near Waimea, Kauai, for 1913.

\begin{tabular}{|c|c|c|c|c|c|c|c|c|c|c|c|c|}
\hline Day. & Jan. & Feb. & Mar. & Apr. & May. & June. & July. & Aug. & Sept. & Oct. & Nov. & Dec. \\
\hline $\begin{array}{l}1 \ldots \\
2 \ldots \\
3 \ldots \\
4 \ldots \\
5 \ldots\end{array}$ & & & $\begin{array}{l}2.2 \\
1.9 \\
1.9 \\
1.9 \\
1.9\end{array}$ & $\begin{array}{l}7.8 \\
4.3 \\
4.5 \\
3.3 \\
3.0\end{array}$ & $\begin{array}{l}3.8 \\
9.2 \\
4.2 \\
3.5 \\
3.3\end{array}$ & \begin{tabular}{l|}
2.2 \\
5.0 \\
4.7 \\
3.0 \\
2.9
\end{tabular} & $\cdots$ & & & $\begin{array}{c}1.0 \\
\ldots \ldots\end{array}$ & $\begin{array}{r}1.5 \\
1.5\end{array}$ & $\begin{array}{r}19 \\
1.4 \\
3.0\end{array}$ \\
\hline $\begin{array}{r}6 \ldots . \\
7 \ldots . \\
8 \ldots . \\
9 \ldots \\
10 \ldots\end{array}$ & & & $\begin{array}{l}2.0 \\
2.0 \\
1.8 \\
1.6 \\
1.5\end{array}$ & $\begin{array}{l}2.7 \\
2.6 \\
3.3 \\
5.5 \\
5.8\end{array}$ & 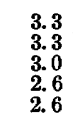 & $\begin{array}{r}\cdots \ldots \ldots \\
\cdots \cdots \ldots\end{array}$ & & $\begin{array}{l}2.5 \\
2.7 \\
2.3\end{array}$ & & & $\begin{array}{r}2.3 \\
3.3 \\
5.0 \\
5.0 \\
35\end{array}$ & $\begin{array}{l}2.7 \\
1.4 \\
1.2 \\
.8 \\
.8\end{array}$ \\
\hline $14 \ldots \ldots$ & & $\begin{array}{l}1.9 \\
1.9 \\
1.9 \\
1.8\end{array}$ & $\begin{array}{l}1.4 \\
1.2 \\
1.1 \\
1.1 \\
1.2\end{array}$ & $\begin{array}{c}4.0 \\
3.5 \\
22 \\
8.2 \\
11\end{array}$ & \begin{tabular}{c|}
20 \\
19 \\
13 \\
4.2 \\
5.8
\end{tabular} & \begin{tabular}{l|}
8.9 \\
3.3 \\
4.2 \\
3.5 \\
3.3
\end{tabular} & $\begin{array}{l}\cdots \\
\cdots \\
\cdots\end{array}$ & $\begin{array}{l}2.0 \\
2.3 \\
2.7 \\
\text { 3. } \\
\text { 3. }\end{array}$ & \begin{tabular}{r}
0.8 \\
.8 \\
.8 \\
\hdashline .8. \\
\end{tabular} & $\begin{array}{l}2.9 \\
2.0 \\
9.2 \\
2.7 \\
1.5\end{array}$ & 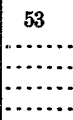 & $\begin{array}{l}.7 \\
.6 \\
.6 \\
.6 \\
.6\end{array}$ \\
\hline 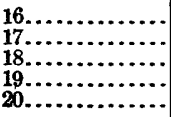 & & $\begin{array}{l}1.7 \\
1.7 \\
1.8 \\
1.8 \\
1.8\end{array}$ & $\begin{array}{l}1.4 \\
1.7 \\
1.9 \\
2.5 \\
4.0\end{array}$ & $\begin{array}{l}4.2 \\
6.0 \\
5.0 \\
5.8 \\
3.3\end{array}$ & \begin{tabular}{l|}
3.8 \\
3.6 \\
4.2 \\
2.9 \\
2.7
\end{tabular} & \begin{tabular}{r}
2.7 \\
2.5 \\
2.9 \\
$\cdots \cdots$ \\
\hdashline$\cdots .$. \\
.2.
\end{tabular} & $\begin{array}{r}2.6 \\
\hdashline\end{array}$ & 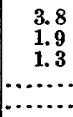 & $\cdots \cdots$ & $\begin{array}{l}1.4 \\
1.4 \\
2.2 \\
1.7 \\
1.3\end{array}$ & ( & $\begin{array}{l}.6 \\
.6 \\
.6 \\
. .\end{array}$ \\
\hline $\begin{array}{l}21 \ldots \ldots . \\
22 \ldots \ldots \ldots \\
23 \ldots \ldots \ldots \\
24 \ldots \ldots \\
25 \ldots \ldots\end{array}$ & & $\begin{array}{l}1.9 \\
1.9 \\
4.2 \\
4.2 \\
3.0\end{array}$ & $\begin{array}{r}3.3 \\
5.0 \\
7.5 \\
9.2 \\
12\end{array}$ & $\begin{array}{l}3.3 \\
2.9 \\
3.3 \\
4.0 \\
8.6\end{array}$ & $\begin{array}{l}2.6 \\
2.6 \\
2.5 \\
2.6 \\
2.6\end{array}$ & 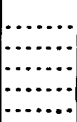 & $\begin{array}{l}2.6 \\
2.6 \\
2.3 \\
2.5 \\
2.5\end{array}$ & \begin{tabular}{l}
$\cdots$ \\
$\cdots \cdots \ldots$ \\
\hdashline$\cdots \cdots$ \\
\hdashline$\ldots \ldots$
\end{tabular} & $\cdots$ & $\begin{array}{l}1.0 \\
1.7 \\
1.9 \\
1.5 \\
3.0\end{array}$ & 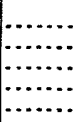 & 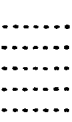 \\
\hline $\begin{array}{l}26 \ldots \ldots \\
27 \ldots \ldots \\
28 \ldots \ldots \\
29 \ldots \\
30 \ldots \\
31 \ldots\end{array}$ & & $\begin{array}{r}2.7 \\
2.6 \\
2.5 \\
\ldots \ldots .\end{array}$ & $\begin{array}{l}5.0 \\
4.3 \\
3.5 \\
3.2 \\
2.9 \\
9.2\end{array}$ & $\begin{array}{c}3.5 \\
3.3 \\
7.0 \\
7.5 \\
6.0 \\
\cdots . . .\end{array}$ & $\begin{array}{l}2.5 \\
2.5 \\
2.5 \\
2.5 \\
2.5 \\
2.5\end{array}$ & 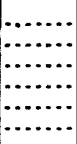 & $\begin{array}{l}2.3 \\
2.2 \\
1.9 \\
2.0 \\
2.0 \\
1.9\end{array}$ & \begin{tabular}{c}
$\cdots$ \\
\hdashline \\
\hdashline \\
\hdashline \\
\hdashline$\ldots \ldots$ \\
$\cdots$
\end{tabular} & $\begin{array}{r}1.0 \\
.8 \\
.8\end{array}$ & 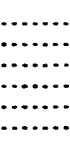 & \begin{tabular}{|c|} 
\\
$\cdots$
\end{tabular}$\ldots$ & $\begin{array}{l}\ldots \ldots \ldots \\
\cdots \ldots \ldots\end{array}$ \\
\hline
\end{tabular}

Nore.-No record for days for which discharge measurements are not given.

OLOKELE DITCH AT TUNNEL NO. 12, NEAR MAKaWELI, KaUAr.

Location. ${ }^{1-A b o u t} 10$ miles northeast of Makaweli.

Records available.-July 24, 1904, to December 31, 1913.

Gage.-Vertical staff.

Control.-Probably permanent.

Discharge measurements.-Made from a plank across ditch.

Diversion.-Ditch diverts all low-water flow of Olokele River.

Accuracy.-Records excellent.

Cooperation.-Gage-height records furnished by Hawaiian Sugar Co.

Discharge measurements of Olokele ditch at tunnel 12, near Makaweli, Kauai, in 1913.

\begin{tabular}{|c|c|c|c|c|c|c|c|}
\hline Date. & Hiydrographer. & $\begin{array}{c}\text { Gage } \\
\text { height. }\end{array}$ & $\begin{array}{c}\text { Dis- } \\
\text { charge. }\end{array}$ & Date. & Hydrographer. & $\begin{array}{c}\text { Gage } \\
\text { height. }\end{array}$ & $\begin{array}{c}\text { Dis- } \\
\text { charge. }\end{array}$ \\
\hline Apr. 27 & 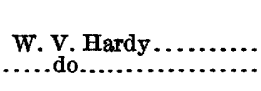 & $\begin{array}{r}\text { Feet. } \\
3.54 \\
\mathbf{3 . 2 4}\end{array}$ & $\begin{array}{r}\text { Sec.-ft. } \\
\mathbf{9 5 . 9} \\
\mathbf{8 6 . 0}\end{array}$ & Apr. 27 & 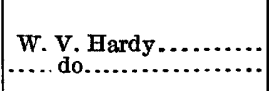 & $\begin{array}{r}\text { Feet. } \\
\mathbf{3 . 0 4} \\
\mathbf{3 . 8 4}\end{array}$ & $\begin{array}{c}\text { Sec.-ft. } \\
78.9 \\
106\end{array}$ \\
\hline
\end{tabular}

1 Described in U. S. Geol. Survey Water-Supply Paper 336 as about "12 miles northeast of Waimea" $97764^{\circ}-$ WsP $373-15-3$ 
Daily discharge, in second-feet, of Olokele ditch at tunnel 12, Makaweli, Kauai, for 1913.

\begin{tabular}{|c|c|c|c|c|c|c|c|c|c|c|c|c|}
\hline Day. & Jan. & Feb. & Mar. & Apr. & May. & June. & July. & Aug. & Sept. & Oct. & Now. & Dec. \\
\hline $\begin{array}{l}1 \ldots \ldots . \\
2 \ldots \ldots \\
3 \ldots \ldots \\
4 \ldots \ldots \\
5 \ldots \ldots\end{array}$ & $\begin{array}{l}64 \\
55 \\
51 \\
51 \\
51\end{array}$ & $\begin{array}{l}51 \\
51 \\
51 \\
47 \\
51\end{array}$ & $\begin{array}{l}51 \\
51 \\
51 \\
51 \\
51\end{array}$ & $\begin{array}{r}105 \\
101 \\
75 \\
72 \\
64\end{array}$ & $\begin{array}{r}81 \\
105 \\
97 \\
81 \\
61\end{array}$ & $\begin{array}{l}51 \\
47 \\
41 \\
41 \\
41\end{array}$ & $\begin{array}{l}105 \\
105 \\
105 \\
105 \\
105\end{array}$ & $\begin{array}{l}51 \\
58 \\
51 \\
51 \\
47\end{array}$ & $\begin{array}{l}91 \\
72 \\
72 \\
61 \\
61\end{array}$ & $\begin{array}{l}55 \\
51 \\
47 \\
47 \\
44\end{array}$ & $\begin{array}{l}47 \\
51 \\
88 \\
61 \\
68\end{array}$ & $\begin{array}{l}38 \\
61 \\
64 \\
68 \\
68\end{array}$ \\
\hline $\begin{array}{r}6 \ldots \ldots \\
7 \ldots \ldots \ldots \\
9 \ldots \ldots \ldots \\
10 \ldots \ldots\end{array}$ & $\begin{array}{r}47 \\
51 \\
101 \\
91 \\
101\end{array}$ & $\begin{array}{l}51 \\
97 \\
78 \\
55 \\
51\end{array}$ & $\begin{array}{l}51 \\
55 \\
55 \\
51 \\
51\end{array}$ & $\begin{array}{l}58 \\
55 \\
51 \\
55 \\
68\end{array}$ & $\begin{array}{l}55 \\
68 \\
94 \\
64 \\
58\end{array}$ & $\begin{array}{r}105 \\
84 \\
61 \\
91 \\
105\end{array}$ & $\begin{array}{l}78 \\
88 \\
68 \\
61 \\
55\end{array}$ & $\begin{array}{l}47 \\
64 \\
78 \\
97 \\
55\end{array}$ & $\begin{array}{l}72 \\
61 \\
55 \\
68 \\
55\end{array}$ & $\begin{array}{r}105 \\
75 \\
105 \\
105 \\
68\end{array}$ & $\begin{array}{r}105 \\
51 \\
88 \\
101 \\
94\end{array}$ & $\begin{array}{r}105 \\
68 \\
55 \\
55 \\
51\end{array}$ \\
\hline $\begin{array}{l}11 \ldots \ldots \\
12 \ldots \ldots \\
13 \ldots \ldots \\
14 \ldots \ldots \\
15 \ldots \ldots\end{array}$ & $\begin{array}{r}101 \\
101 \\
101 \\
101 \\
91\end{array}$ & $\begin{array}{l}88 \\
58 \\
55 \\
51 \\
51\end{array}$ & $\begin{array}{l}47 \\
47 \\
47 \\
47 \\
47\end{array}$ & $\begin{array}{r}97 \\
64 \\
105 \\
101 \\
105\end{array}$ & $\begin{array}{r}51 \\
88 \\
105 \\
78 \\
64\end{array}$ & $\begin{array}{l}97 \\
64 \\
94 \\
78 \\
94\end{array}$ & $\begin{array}{r}55 \\
51 \\
105 \\
72 \\
72\end{array}$ & $\begin{array}{l}55 \\
51 \\
78 \\
84 \\
68\end{array}$ & $\begin{array}{l}\mathbf{5 1} \\
\mathbf{6 8} \\
\mathbf{5 1} \\
\mathbf{5 1} \\
\mathbf{5 1}\end{array}$ & $\begin{array}{r}61 \\
68 \\
105 \\
94 \\
84\end{array}$ & $\begin{array}{r}105 \\
61 \\
105 \\
101 \\
105\end{array}$ & $\begin{array}{l}51 \\
51 \\
51 \\
51 \\
51\end{array}$ \\
\hline $\begin{array}{l}16 \ldots \ldots \ldots \\
17 \ldots \ldots \ldots \\
18 \ldots \ldots \ldots \\
19 \ldots \ldots \ldots\end{array}$ & $\begin{array}{l}91 \\
72 \\
61 \\
58 \\
55\end{array}$ & $\begin{array}{l}58 \\
51 \\
51 \\
72 \\
55\end{array}$ & $\begin{array}{l}55 \\
94 \\
61 \\
72 \\
97\end{array}$ & $\begin{array}{r}84 \\
105 \\
97 \\
101 \\
75\end{array}$ & $\begin{array}{l}68 \\
64 \\
97 \\
61 \\
55\end{array}$ & $\begin{array}{r}101 \\
58 \\
55 \\
75 \\
55\end{array}$ & $\begin{array}{l}64 \\
58 \\
55 \\
58 \\
64\end{array}$ & $\begin{array}{l}58 \\
58 \\
55 \\
51 \\
51\end{array}$ & $\begin{array}{l}55 \\
51 \\
51 \\
51 \\
47\end{array}$ & $\begin{array}{l}97 \\
58 \\
55 \\
64 \\
51\end{array}$ & $\begin{array}{r}105 \\
105 \\
75 \\
105 \\
105\end{array}$ & $\begin{array}{l}55 \\
47 \\
51 \\
51 \\
51\end{array}$ \\
\hline $\begin{array}{l}21 \ldots \ldots \\
22, \ldots \ldots \\
24 \ldots \ldots \\
25 \ldots \ldots\end{array}$ & $\begin{array}{l}58 \\
58 \\
57 \\
55 \\
54\end{array}$ & $\begin{array}{r}68 \\
0 \\
61 \\
101 \\
72\end{array}$ & $\begin{array}{r}91 \\
75 \\
64 \\
97 \\
101\end{array}$ & $\begin{array}{r}72 \\
68 \\
64 \\
72 \\
105\end{array}$ & $\begin{array}{l}51 \\
51 \\
51 \\
51 \\
58\end{array}$ & $\begin{array}{l}97 \\
55 \\
68 \\
72 \\
75\end{array}$ & $\begin{array}{r}55 \\
61 \\
55 \\
68 \\
105\end{array}$ & $\begin{array}{r}105 \\
58 \\
55 \\
51 \\
101\end{array}$ & $\begin{array}{l}47 \\
55 \\
47 \\
47 \\
47\end{array}$ & $\begin{array}{l}51 \\
51 \\
61 \\
51 \\
64\end{array}$ & $\begin{array}{r}94 \\
78 \\
78 \\
105 \\
105\end{array}$ & $\begin{array}{l}47 \\
51 \\
47 \\
47 \\
47\end{array}$ \\
\hline $\begin{array}{l}26 \ldots \ldots \\
27 \ldots \ldots \ldots \\
28 \ldots \ldots \\
29 \ldots \ldots \\
30 \ldots \ldots \\
31 \ldots \ldots\end{array}$ & $\begin{array}{l}52 \\
51 \\
51 \\
51 \\
51 \\
58\end{array}$ & $\begin{array}{r}55 \\
44 \\
55 \\
\cdots \cdots \\
\cdots \cdots \\
\cdots \cdots\end{array}$ & $\begin{array}{l}97 \\
68 \\
78 \\
61 \\
58 \\
61\end{array}$ & $\begin{array}{r}84 \\
68 \\
64 \\
105 \\
101\end{array}$ & $\begin{array}{l}58 \\
51 \\
64 \\
51 \\
97 \\
55\end{array}$ & $\begin{array}{r}105 \\
72 \\
75 \\
75 \\
105 \\
\cdots \cdots\end{array}$ & $\begin{array}{r}105 \\
81 \\
61 \\
105 \\
58 \\
55\end{array}$ & $\begin{array}{r}72 \\
58 \\
68 \\
105 \\
105 \\
105\end{array}$ & $\begin{array}{l}47 \\
47 \\
55 \\
51 \\
47\end{array}$ & $\begin{array}{l}58 \\
58 \\
68 \\
51 \\
47 \\
47\end{array}$ & $\begin{array}{r}105 \\
105 \\
75 \\
68 \\
22\end{array}$ & $\begin{array}{l}47 \\
47 \\
47 \\
47 \\
47 \\
47\end{array}$ \\
\hline
\end{tabular}

Monthly discharge of Olokele ditch at tunnel 12, near Makaweli, Kauai, for 1913.

\begin{tabular}{|c|c|c|c|c|c|}
\hline \multirow{2}{*}{ Month. } & \multicolumn{3}{|c|}{ Discharge in second-feet. } & \multirow{2}{*}{$\begin{array}{c}\text { Run-off } \\
\text { (total in } \\
\text { acre-feet). }\end{array}$} & \multirow{2}{*}{$\begin{array}{l}\text { Accu- } \\
\text { racy. }\end{array}$} \\
\hline & Maximum. & Minimum. & Mean. & & \\
\hline 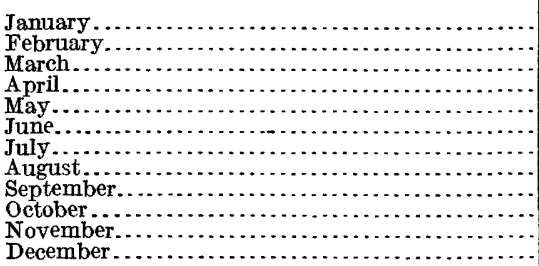 & $\begin{array}{r}101 \\
101 \\
101 \\
105 \\
105 \\
105 \\
105 \\
105 \\
91 \\
105 \\
105 \\
105\end{array}$ & $\begin{array}{r}47 \\
0 \\
47 \\
51 \\
51 \\
41 \\
51 \\
47 \\
47 \\
44 \\
22 \\
38\end{array}$ & $\begin{array}{l}67.5 \\
58.2 \\
64.0 \\
81.4 \\
68.8 \\
74.6 \\
75.4 \\
67.5 \\
56.2 \\
66.0 \\
85.4 \\
53.7\end{array}$ & $\begin{array}{l}4,150 \\
3,230 \\
3,940 \\
4,840 \\
4,230 \\
4,440 \\
4,640 \\
4,150 \\
3,340 \\
4,060 \\
5,080 \\
3,300\end{array}$ & $\begin{array}{l}\text { A. } \\
\text { A. } \\
\text { A. } \\
\text { A. } \\
\text { A. } \\
\text { A. } \\
\text { A. } \\
\text { A. } \\
\text { A. } \\
\text { A. }\end{array}$ \\
\hline The year....... & 105 & 0 & 68.2 & 49,400 & \\
\hline
\end{tabular}




\section{OlOKELE DITCH AT WEIR, NEAR MAKaWELI, KaUAI. ${ }^{1}$}

Location.-About 7 miles northeast of Makaweli and about 3 miles below tunnel Ne. 12 on the same ditch.

Records available.-January 1, 1910, to December 31, 1913.

Gage.-Vertical staff; zero on gage is equal to crest of weir.

Discharge measurements.-Made from plank over ditch. Discharge computed by Francis formula for a 12-foot weir with end contractions; computations checked by current-meter measurements.

Diversion.-Ditch diverts all of low-water flow of Olokele River.

Accuracy.-Records good.

Cooperation.--Records are furnished by the Hawaiian Sugar Co.

Discharge measurements of Olokele ditch at weir, near Makaweli, in 1918.

\begin{tabular}{|c|c|c|c|}
\hline Date. & Hydrographer. & $\begin{array}{c}\text { Gage } \\
\text { height. }\end{array}$ & $\begin{array}{c}\text { Dis- } \\
\text { charge. }\end{array}$ \\
\hline Feb. $\frac{14}{14}$ & $\begin{array}{l}\text { W. v. Hardy } \\
\ldots \ldots\end{array}$ & $\begin{aligned} & \text { Feet. } \\
& 2.21 \\
& 2.21\end{aligned}$ & $\begin{array}{r}\text { Sec. }-f t . \\
51.6 \\
51.8\end{array}$ \\
\hline
\end{tabular}

Daily discharge, in second-feet, of Olokele ditch at weir, near Makaweli, Kauai, for 1013.

\begin{tabular}{|c|c|c|c|c|c|c|c|c|c|c|c|c|}
\hline Day. & Jan. & Feb. & Mar. & Apr. & May. & June. & July. & Aug. & Sept. & Oct. & Nov. & Dec. \\
\hline $\begin{array}{l}1 \ldots \\
2 \ldots \\
3 \ldots \\
4 \ldots \\
5 \ldots\end{array}$ & $\begin{array}{l}73.4 \\
89.3 \\
89.3 \\
95.8 \\
87.6\end{array}$ & $\begin{array}{l}58.7 \\
54.5 \\
54.5 \\
73.4 \\
65.9\end{array}$ & $\begin{array}{l}73.4 \\
58.7 \\
50.4 \\
47.7 \\
49.0\end{array}$ & $\begin{array}{l}87.6 \\
79.6 \\
82.9 \\
70.3 \\
57.3\end{array}$ & $\begin{array}{l}82.9 \\
92.5 \\
90.9 \\
70.3 \\
58.7\end{array}$ & $\begin{array}{l}47.7 \\
57.3 \\
47.7 \\
40.0 \\
43.8\end{array}$ & $\begin{array}{c}95.8 \\
99.1 \\
101 \\
99.1 \\
87.6\end{array}$ & $\begin{array}{l}58.7 \\
53.1 \\
47.7 \\
46.4 \\
47.7\end{array}$ & $\begin{array}{l}79.6 \\
67.4 \\
64.4 \\
57.3 \\
64.4\end{array}$ & $\begin{array}{l}45.1 \\
49.0 \\
45.1 \\
42.5 \\
41.2\end{array}$ & $\begin{array}{l}42.5 \\
42.5 \\
70.3 \\
64.4 \\
61.5\end{array}$ & $\begin{array}{l}41.2 \\
47.7 \\
64.4 \\
73.4 \\
70.3\end{array}$ \\
\hline $\begin{array}{r}6 \ldots \\
7 \ldots \\
8 \ldots \\
9 \ldots \\
10 \ldots\end{array}$ & $\begin{array}{l}82.9 \\
76.5 \\
90.9 \\
86.0 \\
74.9\end{array}$ & $\begin{array}{l}60.1 \\
49.0 \\
49.0 \\
51.7 \\
50.4\end{array}$ & $\begin{array}{l}49.0 \\
51.7 \\
50.4 \\
49.0 \\
60.1\end{array}$ & $\begin{array}{l}51.7 \\
50.4 \\
55.8 \\
76.5 \\
82.9\end{array}$ & $\begin{array}{l}70.3 \\
78.0 \\
74.9 \\
58.7 \\
58.7\end{array}$ & $\begin{array}{l}74.9 \\
63.0 \\
67.4 \\
84.5 \\
84.5\end{array}$ & $\begin{array}{l}78.0 \\
70.3 \\
61.5 \\
57.3 \\
54.5\end{array}$ & $\begin{array}{l}51.7 \\
65.9 \\
65.9 \\
61.5 \\
55.8\end{array}$ & $\begin{array}{l}70.3 \\
61.5 \\
53.1 \\
51.7 \\
47.7\end{array}$ & $\begin{array}{l}46.4 \\
70.3 \\
86.0 \\
90.9 \\
82.9\end{array}$ & $\begin{array}{l}63.0 \\
61.5 \\
61.5 \\
65.9 \\
90.9\end{array}$ & $\begin{array}{l}76.5 \\
79.6 \\
58.7 \\
50.4 \\
49.0\end{array}$ \\
\hline $\begin{array}{l}11 \ldots . \\
12 \ldots \\
13 \ldots \\
14 \ldots \\
15 \ldots\end{array}$ & $\begin{array}{l}64.4 \\
57.3 \\
57.3 \\
60.1 \\
64.4\end{array}$ & $\begin{array}{l}51.7 \\
68.9 \\
64.4 \\
65.9 \\
60.1\end{array}$ & $\begin{array}{l}74.9 \\
76.5 \\
78.0 \\
82.9 \\
86.0\end{array}$ & $\begin{array}{l}78.0 \\
79.6 \\
94.2 \\
95.8 \\
89.3\end{array}$ & $\begin{array}{l}81.2 \\
89.3 \\
89.3 \\
68.9 \\
68.9\end{array}$ & $\begin{array}{l}73.4 \\
70.3 \\
89.3 \\
79.6 \\
73.4\end{array}$ & $\begin{array}{l}54.5 \\
61.5 \\
76.5 \\
70.3 \\
64.4\end{array}$ & $\begin{array}{l}50.4 \\
74.9 \\
76.5 \\
76.5 \\
60.1\end{array}$ & $\begin{array}{l}47.7 \\
49.0 \\
45.1 \\
45.1 \\
47.7\end{array}$ & $\begin{array}{l}68.9 \\
65.9 \\
67.4 \\
86.0 \\
90.9\end{array}$ & $\begin{array}{l}92.5 \\
86.0 \\
89.3 \\
87.6 \\
86.0\end{array}$ & $\begin{array}{l}47.7 \\
47.7 \\
47.7 \\
47.7 \\
47.7\end{array}$ \\
\hline $\begin{array}{l}16 \ldots \\
17 \ldots \\
18 \ldots \\
19 \ldots \\
20 \ldots\end{array}$ & $\begin{array}{l}55.8 \\
51.7 \\
50.4 \\
47.7 \\
47.7\end{array}$ & $\begin{array}{l}60.1 \\
64.4 \\
68.9 \\
57.3 \\
51.7\end{array}$ & $\begin{array}{l}82.9 \\
76.5 \\
76.5 \\
78.0 \\
82.9\end{array}$ & $\begin{array}{l}82.9 \\
90.9 \\
87.6 \\
81.2 \\
65.9\end{array}$ & $\begin{array}{l}64.4 \\
74.9 \\
68.9 \\
57.3 \\
51.7\end{array}$ & $\begin{array}{l}67.4 \\
58.7 \\
57.3 \\
60.1 \\
63.0\end{array}$ & $\begin{array}{l}63.0 \\
58.7 \\
57.3 \\
64.4 \\
58.7\end{array}$ & $\begin{array}{l}61.5 \\
53.1 \\
50.4 \\
50.4 \\
54.5\end{array}$ & $\begin{array}{l}46.4 \\
45.1 \\
45.1 \\
43.8 \\
43.8\end{array}$ & $\begin{array}{l}73.4 \\
61.5 \\
53.1 \\
58.7 \\
53.1\end{array}$ & $\begin{array}{l}90.9 \\
90.9 \\
87.6 \\
92.5 \\
99.1\end{array}$ & $\begin{array}{l}46 . \\
46 . \\
46 . \\
47 . \\
47 .\end{array}$ \\
\hline $\begin{array}{l}21 \ldots \\
22 \ldots \\
23 \ldots \\
24 \ldots \\
25 \ldots \\
\end{array}$ & $\begin{array}{l}49.0 \\
47.7 \\
49.0 \\
51.7 \\
50.4\end{array}$ & $\begin{array}{l}50.4 \\
49.0 \\
47.7 \\
47.7 \\
47.7\end{array}$ & $\begin{array}{l}84.5 \\
82.9 \\
82.9 \\
90.9 \\
94.2\end{array}$ & $\begin{array}{l}65.9 \\
63.0 \\
71.9 \\
74.9 \\
89.3\end{array}$ & $\begin{array}{l}49.0 \\
47.7 \\
51.7 \\
67.4 \\
55.8\end{array}$ & $\begin{array}{l}60.1 \\
57.3 \\
61.5 \\
60.1 \\
86.0\end{array}$ & $\begin{array}{l}54.5 \\
54.5 \\
57.3 \\
67.4 \\
74.9\end{array}$ & $\begin{array}{l}61.5 \\
54.5 \\
55.8 \\
68.9 \\
68.9\end{array}$ & $\begin{array}{l}49.0 \\
46.4 \\
42.5 \\
42.5 \\
41.2\end{array}$ & $\begin{array}{l}47.7 \\
50.4 \\
55.8 \\
53.1 \\
55.8\end{array}$ & $\begin{array}{l}92.5 \\
86.0 \\
92.5 \\
97.5 \\
99.1\end{array}$ & $\begin{array}{l}47.7 \\
49.0 \\
46.4 \\
45.1 \\
45.1\end{array}$ \\
\hline $\begin{array}{l}26 \ldots \\
27 . . \\
28 . . \\
29 . . \\
30 \ldots \\
31 \ldots\end{array}$ & $\begin{array}{l}49.0 \\
50.4 \\
57.3 \\
57.3 \\
61.5 \\
57.3\end{array}$ & $\begin{array}{r}47.7 \\
50.4 \\
74.9 \\
\cdots \ldots . . . \\
\cdots \cdots . . . \\
\cdots \ldots . .\end{array}$ & $\begin{array}{l}84.5 \\
79.6 \\
67.4 \\
64.4 \\
67.4 \\
84.5\end{array}$ & $\begin{array}{l}71.9 \\
71.9 \\
86.0 \\
92.5 \\
90.9\end{array}$ & $\begin{array}{l}53.1 \\
57.3 \\
54.5 \\
57.3 \\
58.7 \\
50.4\end{array}$ & $\begin{array}{l}84.5 \\
87.6 \\
82.9 \\
73.4 \\
90.9\end{array}$ & $\begin{array}{l}79.6 \\
67.4 \\
61.5 \\
67.4 \\
55.8 \\
51.7\end{array}$ & $\begin{array}{l}64.4 \\
71.9 \\
87.6 \\
87.6 \\
82.9 \\
84.5\end{array}$ & $\begin{array}{l}41.2 \\
40.0 \\
42.5 \\
58.7 \\
47.7\end{array}$ & $\begin{array}{l}54.5 \\
50.4 \\
50.4 \\
50.4 \\
45.1 \\
43.8\end{array}$ & $\begin{array}{c}101 \\
92.5 \\
78.0 \\
70.3 \\
53.1 \\
\cdots \cdots\end{array}$ & $\begin{array}{l}45.1 \\
45.1 \\
45.1 \\
43.8 \\
43.8 \\
43.8\end{array}$ \\
\hline
\end{tabular}

1 Described in U. S. Geol. Survey Water-Supply Paper 336 as about 8 miles northeast of Waimea, 
Monthly discharge of Olokele ditch at weir, near Makaweli, Kauai, for 1913.

\begin{tabular}{|c|c|c|c|c|c|}
\hline \multirow{2}{*}{ Month. } & \multicolumn{3}{|c|}{ Discharge in second-feet. } & \multirow{2}{*}{$\begin{array}{c}\text { Run-off } \\
\text { (total in } \\
\text { acre-feet). }\end{array}$} & \multirow{2}{*}{$\begin{array}{l}\text { Accu- } \\
\text { racy. }\end{array}$} \\
\hline & Maximum. & Minimum. & Mean. & & \\
\hline 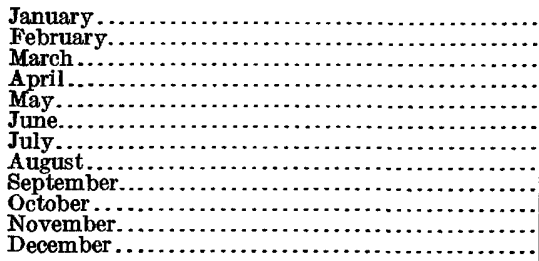 & $\begin{array}{r}95.8 \\
74.9 \\
94.2 \\
95.8 \\
92.5 \\
90.9 \\
101 \\
87.6 \\
79.6 \\
90.9 \\
101 \\
79.6\end{array}$ & $\begin{array}{l}47.7 \\
47.7 \\
47.7 \\
50.4 \\
47.7 \\
40.0 \\
51.7 \\
46.4 \\
40.0 \\
41.2 \\
42.5 \\
41.2\end{array}$ & $\begin{array}{l}64.0 \\
57.0 \\
71.5 \\
77.3 \\
66.2 \\
68.2 \\
68.6 \\
62.9 \\
50.9 \\
59.2 \\
79.6 \\
51.1\end{array}$ & $\begin{array}{l}3,940 \\
3,170 \\
4,400 \\
4,600 \\
4,070 \\
4,060 \\
4,220 \\
3,870 \\
3,030 \\
3,640 \\
4,740 \\
3,140\end{array}$ & $\begin{array}{l}\text { B. } \\
\text { B. } \\
\text { B. } \\
\text { B. } \\
\text { B. } \\
\text { B. } \\
\text { B. } \\
\text { B. } \\
\text { B. }\end{array}$ \\
\hline The year............ & 101 & 40.0 & 64.8 & 46,900 & \\
\hline
\end{tabular}

POOWAIOMAHAIHAI DITCH NEAR WAIMEA, KAUAI.

Location.-About 110 feet below bridge station on Makaweli River, 250 feet below intake, and about 2 miles northeast of Waimea.

Records available.-October 27, 1911, to September 30, 1913.

Gage.-Vertical staff.

Conłrol.-Probably permanent.

Discharge measurements.-Made from timber bridge 15 feet above gage.

Diversion.-Ditch diverts part of flow of Makaweli River.

Accuracy.-Records good.

The following discharge measurement was made by W. V. Hardy:

April 30, 1913: Gage height, 1.67 feet; discharge, 13.5 second-feet.

Daily discharge, in second-feet, of Poowaiomahaihai ditch near Waimea, Kauai, for 1913.

\begin{tabular}{|c|c|c|c|c|c|c|c|c|c|}
\hline Day. & Jan. & Feb. & Mar. & Apr. & May. & June. & July. & Aug. & Sept. \\
\hline $\begin{array}{l}1 \ldots \ldots \ldots \\
2 \ldots \ldots \ldots \\
4 \ldots \ldots \ldots \\
5 \ldots \ldots \ldots\end{array}$ & $\begin{array}{l}4.3 \\
4.3 \\
4.3 \\
3.5 \\
3.5\end{array}$ & $\begin{array}{l}6.2 \\
6.2 \\
6.2 \\
5.2 \\
5.2\end{array}$ & $\begin{array}{l}7.3 \\
7.3 \\
7.3 \\
6.2 \\
6.2\end{array}$ & $\begin{array}{l}8.5 \\
5.2 \\
3.5 \\
3.5 \\
2.8\end{array}$ & $\begin{array}{l}7.3 \\
4.3 \\
4.3 \\
8.5 \\
4.3\end{array}$ & $\begin{array}{l}3.5 \\
2.8 \\
8.5 \\
7.3 \\
5.2\end{array}$ & $\begin{array}{l}6.5 \\
6.8 \\
7.2 \\
6.8 \\
6.2\end{array}$ & $\begin{array}{l}5.7 \\
6.1 \\
6.0 \\
5.8 \\
5.7\end{array}$ & $\begin{array}{l}8.4 \\
7.9 \\
7.5 \\
6.8 \\
6.2\end{array}$ \\
\hline $\begin{array}{r}6 \\
7 \ldots \ldots \\
8 \ldots \ldots \ldots \\
90 \ldots \ldots\end{array}$ & $\begin{array}{l}4.3 \\
4.3 \\
5.2 \\
5.2 \\
3.5\end{array}$ & $\begin{array}{r}5.2 \\
5.2 \\
5.2 \\
4.3 \\
13\end{array}$ & $\begin{array}{l}6.2 \\
5.2 \\
5.2 \\
5.2 \\
5.2\end{array}$ & $\begin{array}{l}2.8 \\
2.8 \\
2.8 \\
8.5 \\
4.3\end{array}$ & $\begin{array}{l}6.2 \\
6.2 \\
4.3 \\
4.3 \\
3.5\end{array}$ & $\begin{array}{l}4.3 \\
3.5 \\
3.5 \\
3.5 \\
7.3\end{array}$ & $\begin{array}{l}6.1 \\
5.9 \\
5.7 \\
5.9 \\
5.8\end{array}$ & $\begin{array}{l}5.5 \\
5.5 \\
5.4 \\
5.2 \\
5.2\end{array}$ & $\begin{array}{l}6.0 \\
6.0 \\
6.1 \\
5.9 \\
5.7\end{array}$ \\
\hline $\begin{array}{l}11 \\
12 \\
13 \\
13 \\
14 \\
15\end{array}$ & $\begin{array}{l}3.5 \\
7.3 \\
39 \\
14 \\
18\end{array}$ & $\begin{array}{l}7.3 \\
7.3 \\
6.2 \\
7.3 \\
7.3\end{array}$ & $\begin{array}{l}5.2 \\
5.2 \\
4.3 \\
4.3 \\
4.3\end{array}$ & $\begin{array}{r}3.5 \\
7.3 \\
18 \\
8.5 \\
9.8\end{array}$ & $\begin{array}{r}3.5 \\
25 \\
8.5 \\
7.3 \\
7.3\end{array}$ & $\begin{array}{l}5.2 \\
5.2 \\
4.3 \\
3.5 \\
3.5\end{array}$ & $\begin{array}{l}5.8 \\
5.7 \\
5.8 \\
5.9 \\
5.9\end{array}$ & $\begin{array}{l}5.1 \\
4.9 \\
4.9 \\
4.8 \\
4.6\end{array}$ & $\begin{array}{l}5.5 \\
5.5 \\
5.3 \\
5.3 \\
5.2\end{array}$ \\
\hline 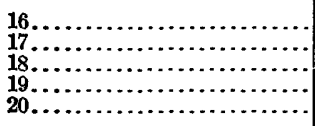 & $\begin{array}{l}18 \\
13 \\
6.2 \\
6.2 \\
4.3\end{array}$ & $\begin{array}{l}7.3 \\
7.3 \\
7.3 \\
6.2 \\
7.3\end{array}$ & $\begin{array}{l}4.3 \\
7.3 \\
4.3 \\
4.3 \\
5.2\end{array}$ & $\begin{array}{l}8.5 \\
7.3 \\
6.2 \\
7.3 \\
6.2\end{array}$ & $\begin{array}{l}6.2 \\
6.2 \\
5.2 \\
5.2 \\
5.2\end{array}$ & $\begin{array}{l}3.5 \\
5.4 \\
5.2 \\
5.2 \\
5.3\end{array}$ & $\begin{array}{l}5.8 \\
\mathbf{5 . 8} \\
\mathbf{5 . 8} \\
\mathbf{5 . 7} \\
\mathbf{5 . 7}\end{array}$ & $\begin{array}{l}4.6 \\
4.6 \\
4.6 \\
4.6 \\
4.5\end{array}$ & $\begin{array}{l}5.1 \\
4.9 \\
5.2 \\
5.3 \\
5.2\end{array}$ \\
\hline $\begin{array}{l}21 \\
22 \\
23 \\
24 \\
25 \\
25 \ldots \ldots \ldots \ldots \ldots \ldots \ldots\end{array}$ & $\begin{array}{l}4.3 \\
4.3 \\
5.2 \\
5.2 \\
4.3\end{array}$ & $\begin{array}{l}8.5 \\
7.3 \\
7.3 \\
7.3 \\
9.8\end{array}$ & $\begin{array}{l}5.2 \\
7.3 \\
4.8 \\
6.2 \\
9.8\end{array}$ & $\begin{array}{l}6.2 \\
6.2 \\
5.2 \\
5.2 \\
6.2\end{array}$ & $\begin{array}{l}3.5 \\
3.5 \\
5.2 \\
4.3 \\
4.3\end{array}$ & $\begin{array}{l}5.3 \\
5.4 \\
5.2 \\
5.1 \\
5.1\end{array}$ & $\begin{array}{l}5.5 \\
5.3 \\
5.0 \\
4.8 \\
4.8\end{array}$ & $\begin{array}{l}4.5 \\
4.6 \\
4.8 \\
4.8 \\
4.6\end{array}$ & $\begin{array}{l}5.1 \\
5.1 \\
5.0 \\
5.2 \\
4.9\end{array}$ \\
\hline 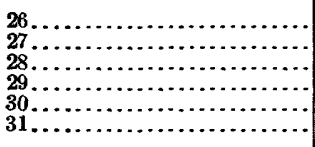 & $\begin{array}{l}5.2 \\
4.3 \\
5.2 \\
4.3 \\
4.3 \\
4.3\end{array}$ & \begin{tabular}{r}
8.5 \\
7.3 \\
7.3 \\
$\cdots$ \\
\hdashline... \\
\end{tabular} & $\begin{array}{l}6.2 \\
4.8 \\
4.3 \\
3.5 \\
3.5 \\
3.5\end{array}$ & $\begin{array}{l}5.2 \\
5.2 \\
4.3 \\
8.5 \\
7.3\end{array}$ & $\begin{array}{l}4.3 \\
3.5 \\
3.5 \\
3.5 \\
3.5 \\
3.5\end{array}$ & \begin{tabular}{c|}
4.9 \\
12 \\
10 \\
6.5 \\
10
\end{tabular} & $\begin{array}{l}5.0 \\
5.1 \\
5.0 \\
5.2 \\
5.3 \\
5.2\end{array}$ & $\begin{array}{l}4.6 \\
4.6 \\
4.5 \\
4.5 \\
7.0 \\
7.2\end{array}$ & $\begin{array}{l}5.9 \\
5.7 \\
6.2 \\
6.1 \\
5.6\end{array}$ \\
\hline
\end{tabular}


Monthly discharge of Poowaimahaihai ditch near Waimea, Kauai, for 1913.

\begin{tabular}{|c|c|c|c|c|c|}
\hline \multirow{2}{*}{ Month. } & \multicolumn{3}{|c|}{ Discharge in second-feet. } & \multirow{2}{*}{$\begin{array}{l}\text { Run-off } \\
\text { (total in } \\
\text { acre-feet). }\end{array}$} & \multirow{2}{*}{$\begin{array}{l}\text { Accu- } \\
\text { racy. }\end{array}$} \\
\hline & Maximum. & Minimum. & Mean. & & \\
\hline \multirow[t]{2}{*}{ 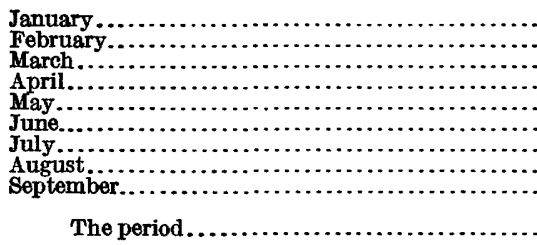 } & $\begin{array}{c}39 \\
13 \\
9.8 \\
18 \\
25 \\
12 \\
7.2 \\
7.2 \\
8.4\end{array}$ & $\begin{array}{l}3.5 \\
4.3 \\
3.5 \\
2.8 \\
3.5 \\
2.8 \\
4.8 \\
4.5 \\
4.9\end{array}$ & $\begin{array}{l}7.20 \\
6.99 \\
5.45 \\
6.23 \\
5.66 \\
5.53 \\
5.71 \\
5.13 \\
5.79\end{array}$ & $\begin{array}{l}\mathbf{4 4 3} \\
388 \\
\mathbf{3 3 5} \\
\mathbf{3 7 1} \\
\mathbf{3 4 8} \\
\mathbf{3 2 9} \\
\mathbf{3 5 1} \\
\mathbf{3 1 5} \\
\mathbf{3 4 5}\end{array}$ & \multirow[t]{2}{*}{$\begin{array}{l}\text { A. } \\
\text { A. } \\
\text { A. } \\
\text { A. } \\
\text { A. } \\
\text { A. } \\
\text { A. } \\
\text { A. } \\
\text { A. }\end{array}$} \\
\hline & & & & 3,220 & \\
\hline
\end{tabular}

HANAPEPE RIVER AT KOULA, NEAR ELEELE, KAUAI.

Location.-About 5 miles north of Eleele.

Records available.-August 18, 1910, to December 31, 1913.

Drainage area.-12.6 square miles.

Gage.-Friez water-stage recorder.

Control.-Fairly permanent.

Discharge measurements.-Made from cable or by wading.

Diversions.-Nearly all low-water flow diverted above station.

Accuracy.-Records fair.

The following discharge measurement was made by D. E. Horner:

October 14, 1913: Gage height, 1.56 feet; discharge, 46.3 second-feet

Daily discharge, in second-feet, of Hanapepe River at Koula, near Eleele, Kauai, for 1913.

\begin{tabular}{|c|c|c|c|c|c|c|c|c|c|c|c|c|}
\hline Day. & Jan. & Feb. & Mar. & Apr. & May. & June. & July. & Aug. & Sept. & Oct. & Nov. & Dec. \\
\hline $\begin{array}{l}1 \ldots \\
2 \ldots \\
3 \ldots \\
4 \ldots \\
5 \ldots\end{array}$ & $\begin{array}{l}24 \\
20 \\
19 \\
18 \\
18\end{array}$ & $\begin{array}{l}16 \\
16 \\
16 \\
15 \\
17\end{array}$ & $\begin{array}{l}13 \\
13 \\
13 \\
13 \\
13\end{array}$ & $\begin{array}{l}78 \\
40 \\
39 \\
29 \\
18\end{array}$ & $\begin{array}{r}55 \\
174 \\
95 \\
58 \\
27\end{array}$ & $\begin{array}{r}14 \\
25 \\
357 \\
108 \\
84\end{array}$ & $\begin{array}{r}87 \\
277 \\
105 \\
118 \\
138\end{array}$ & $\begin{array}{l}67 \\
30 \\
14 \\
13 \\
12\end{array}$ & $\begin{array}{l}51 \\
72 \\
40 \\
16 \\
15\end{array}$ & $\begin{array}{l}15 \\
13 \\
12 \\
12 \\
12\end{array}$ & $\begin{array}{l}14 \\
29 \\
18 \\
13 \\
14\end{array}$ & $\begin{array}{r}126 \\
98 \\
86 \\
66 \\
29\end{array}$ \\
\hline 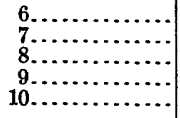 & $\begin{array}{r}19 \\
20 \\
165 \\
50 \\
180\end{array}$ & $\begin{array}{l}50 \\
55 \\
36 \\
17 \\
32\end{array}$ & $\begin{array}{l}13 \\
21 \\
16 \\
13 \\
13\end{array}$ & $\begin{array}{l}14 \\
14 \\
16 \\
55 \\
20\end{array}$ & $\begin{array}{l}40 \\
54 \\
80 \\
32 \\
25\end{array}$ & $\begin{array}{l}38 \\
36 \\
20 \\
46 \\
38\end{array}$ & $\begin{array}{l}50 \\
36 \\
23 \\
22 \\
20\end{array}$ & $\begin{array}{l}13 \\
18 \\
17 \\
14 \\
13\end{array}$ & $\begin{array}{l}43 \\
20 \\
14 \\
14 \\
13\end{array}$ & $\begin{array}{r}53 \\
66 \\
420 \\
196 \\
56\end{array}$ & $\begin{array}{r}20 \\
14 \\
13 \\
94 \\
171\end{array}$ & $\begin{array}{l}53 \\
60 \\
25 \\
23 \\
20\end{array}$ \\
\hline $\begin{array}{l}11 \ldots \ldots \\
12 \ldots \ldots \\
13 \ldots \ldots \\
14 \ldots \ldots \\
15 \ldots \ldots\end{array}$ & $\begin{array}{l}142 \\
142 \\
459 \\
129 \\
264\end{array}$ & $\begin{array}{l}33 \\
16 \\
14 \\
14 \\
14\end{array}$ & $\begin{array}{l}13 \\
13 \\
13 \\
13 \\
13\end{array}$ & $\begin{array}{r}20 \\
15 \\
243 \\
149 \\
142\end{array}$ & $\begin{array}{r}142 \\
246 \\
266 \\
117 \\
67\end{array}$ & $\begin{array}{l}64 \\
24 \\
48 \\
40 \\
34\end{array}$ & $\begin{array}{l}18 \\
17 \\
60 \\
23 \\
22\end{array}$ & $\begin{array}{l}12 \\
26 \\
25 \\
23 \\
15\end{array}$ & $\begin{array}{l}13 \\
13 \\
13 \\
13 \\
14\end{array}$ & $\begin{array}{r}76 \\
53 \\
121 \\
63 \\
43\end{array}$ & $\begin{array}{r}442 \\
603 \\
453 \\
126 \\
88\end{array}$ & $\begin{array}{l}15 \\
14 \\
14 \\
14 \\
13\end{array}$ \\
\hline $\begin{array}{l}16 \ldots \ldots \ldots \\
17 \ldots \ldots \ldots \\
18 \ldots \ldots \\
19 \ldots \ldots \ldots \\
20 . \ldots \ldots \ldots\end{array}$ & $\begin{array}{r}120 \\
59 \\
63 \\
75 \\
24\end{array}$ & $\begin{array}{l}18 \\
15 \\
25 \\
30 \\
29\end{array}$ & $\begin{array}{l}14 \\
23 \\
16 \\
20 \\
22\end{array}$ & \begin{tabular}{r|}
41 \\
112 \\
72 \\
87 \\
30
\end{tabular} & $\begin{array}{l}36 \\
38 \\
56 \\
22 \\
18\end{array}$ & $\begin{array}{l}44 \\
19 \\
23 \\
25 \\
18\end{array}$ & $\begin{array}{l}22 \\
18 \\
18 \\
20 \\
36\end{array}$ & $\begin{array}{l}13 \\
13 \\
12 \\
12 \\
12\end{array}$ & $\begin{array}{l}13 \\
12 \\
12 \\
13 \\
12\end{array}$ & $\begin{array}{l}34 \\
22 \\
29 \\
15 \\
14\end{array}$ & $\begin{array}{r}92 \\
61 \\
52 \\
298 \\
343\end{array}$ & $\begin{array}{l}12 \\
12 \\
13 \\
12 \\
12\end{array}$ \\
\hline $\begin{array}{l}21 \ldots \ldots \ldots \ldots \\
22 \ldots \ldots \ldots \ldots \\
23 \ldots \ldots \ldots \ldots \\
24 \ldots \ldots \ldots \ldots \\
25 \ldots \ldots \ldots\end{array}$ & $\begin{array}{l}29 \\
72 \\
43 \\
80 \\
34\end{array}$ & $\begin{array}{l}19 \\
16 \\
16 \\
48 \\
18\end{array}$ & $\begin{array}{l}22 \\
36 \\
35 \\
28 \\
76\end{array}$ & $\begin{array}{r}24 \\
20 \\
38 \\
18 \\
221\end{array}$ & $\begin{array}{l}17 \\
17 \\
16 \\
26 \\
16\end{array}$ & $\begin{array}{l}26 \\
18 \\
20 \\
16 \\
20\end{array}$ & $\begin{array}{l}18 \\
16 \\
15 \\
17 \\
41\end{array}$ & $\begin{array}{l}32 \\
13 \\
12 \\
13 \\
24\end{array}$ & $\begin{array}{l}12 \\
12 \\
12 \\
12 \\
12\end{array}$ & $\begin{array}{l}18 \\
43 \\
30 \\
60 \\
88\end{array}$ & $\begin{array}{l}125 \\
130 \\
167 \\
184 \\
352\end{array}$ & $\begin{array}{l}12 \\
12 \\
11 \\
11 \\
11\end{array}$ \\
\hline 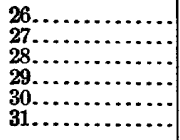 & $\begin{array}{l}20 \\
19 \\
17 \\
16 \\
16 \\
20\end{array}$ & $\begin{array}{r}14 \\
13 \\
13 \\
\cdots \\
\cdots \\
\cdots \\
\cdots\end{array}$ & $\begin{array}{l}67 \\
30 \\
24 \\
29 \\
18 \\
22\end{array}$ & $\begin{array}{r}57 \\
33 \\
29 \\
82 \\
142 \\
\cdots \cdots\end{array}$ & $\begin{array}{r}13 \\
14 \\
14 \\
14 \\
101 \\
24\end{array}$ & $\begin{array}{r}57 \\
120 \\
92 \\
36 \\
78 \\
\hdashline \ldots . .\end{array}$ & $\begin{array}{l}68 \\
25 \\
15 \\
29 \\
14 \\
14\end{array}$ & $\begin{array}{r}18 \\
15 \\
43 \\
143 \\
38 \\
123\end{array}$ & $\begin{array}{l}12 \\
12 \\
26 \\
12 \\
12\end{array}$ & $\begin{array}{l}72 \\
52 \\
34 \\
15 \\
15 \\
15\end{array}$ & $\begin{array}{r}246 \\
132 \\
86 \\
363 \\
225 \\
\cdots \cdots\end{array}$ & $\begin{array}{l}11 \\
11 \\
11 \\
11 \\
11 \\
11\end{array}$ \\
\hline
\end{tabular}


Monthly discharge of Hanapepe River at Koula, near Eleele, Kauai.

\begin{tabular}{|c|c|c|c|c|c|}
\hline \multirow{2}{*}{ Month. } & \multicolumn{3}{|c|}{ Discharge in second-feet. } & \multirow{2}{*}{$\begin{array}{l}\text { Run-off } \\
\text { (total in } \\
\text { acre-feet). }\end{array}$} & \multirow{2}{*}{$\begin{array}{l}\text { Aecu- } \\
\text { racy. }\end{array}$} \\
\hline & Maximum. & Minimum. & Mean. & & \\
\hline 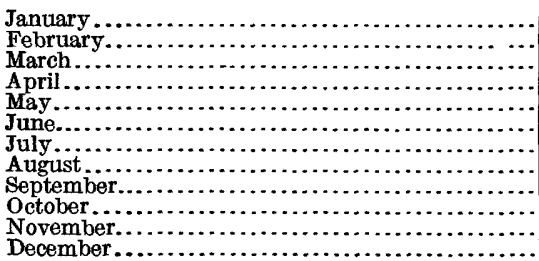 & $\begin{array}{r}459 \\
55 \\
76 \\
243 \\
266 \\
357 \\
277 \\
143 \\
72 \\
420 \\
603 \\
126\end{array}$ & $\begin{array}{l}16 \\
13 \\
13 \\
14 \\
13 \\
14 \\
14 \\
12 \\
12 \\
12 \\
13 \\
11\end{array}$ & $\begin{array}{r}76.6 \\
22.7 \\
22.2 \\
63.3 \\
61.9 \\
52.9 \\
45.2 \\
27.4 \\
18.7 \\
57.0 \\
166 \\
27.1\end{array}$ & $\begin{array}{l}4,710 \\
1,260 \\
1,360 \\
3,770 \\
3,810 \\
3,150 \\
2,780 \\
1,680 \\
1,110 \\
3,500 \\
9,880 \\
1,670\end{array}$ & $\begin{array}{l}\text { B. } \\
\text { B. } \\
\text { B. } \\
\text { B. } \\
\text { B. } \\
\text { B. } \\
\text { B. } \\
\text { B. } \\
\text { B. } \\
\text { B. } \\
\text { B. }\end{array}$ \\
\hline The year....... & 603 & 11 & 53.4 & 38,700 & \\
\hline
\end{tabular}

\section{HILOA DITCH AT haNAPEPE FALLS NEAR ELEELE, KAUAI.}

Location.-About 8 miles north of Eleele and 335 feet below intake, which is just above confluence of the main and east branches of Hanapepe River.

Records available.-November 22, 1911, to December 31, 1913.

Gage.-Vertical staff.

Control.-Probably permanent.

Discharge measurements.-Made from plank across ditch at gage.

Diversion.-Ditch diverts all low-water flow from the main branch of Hanapepe River above Hanapepe Falls.

Accuracy.--Records good.

Discharge measurements of Hiloa ditch at Hanapepe Falls, near Eleele, Kauai, in 1913.

\begin{tabular}{|c|c|c|c|}
\hline Date. & Hydrographer. & $\begin{array}{c}\text { Gage } \\
\text { height. }\end{array}$ & $\begin{array}{c}\text { Dis- } \\
\text { charge. }\end{array}$ \\
\hline $\begin{array}{l}\text { June } 21 \\
\text { Oct. } 14\end{array}$ & $\begin{array}{l}\text { W. V. Hardy ...... } \\
\text { D. E. Horner....... }\end{array}$ & $\begin{array}{r}\text { Feet. } \\
2.09 \\
2.18\end{array}$ & $\begin{array}{r}\text { Sec.-ft. } \\
\quad 41.1 \\
45.5\end{array}$ \\
\hline
\end{tabular}


Daily discharge, in second-feet, of Hiloa ditch at Hanapepe Falls, near Eleele, Kauai, for 1912-13.

\begin{tabular}{|c|c|c|c|c|c|c|c|c|c|c|c|c|}
\hline Day. & Jan. & Feb. & Mar. & Apr. & May. & June. & July. & Aug. & Sept. & Oct. & Nov. & Dec. \\
\hline $\begin{array}{c}1912 . \\
1 \ldots \ldots \cdots \\
2 \ldots \ldots \ldots \\
3 \ldots \ldots \ldots \\
5 \ldots \ldots \ldots\end{array}$ & $\begin{array}{l}\mathbf{3 4} \\
\mathbf{3 5} \\
\mathbf{3 5} \\
\mathbf{3 4} \\
\mathbf{5 0}\end{array}$ & $\begin{array}{l}31 \\
31 \\
30 \\
30 \\
29\end{array}$ & $\begin{array}{l}37 \\
31 \\
32 \\
33 \\
30\end{array}$ & $\begin{array}{l}29 \\
29 \\
28 \\
37 \\
31\end{array}$ & $\begin{array}{l}29 \\
30 \\
43 \\
53 \\
44\end{array}$ & $\begin{array}{l}36 \\
34 \\
33 \\
39 \\
37\end{array}$ & $\begin{array}{l}37 \\
32 \\
30 \\
38 \\
46\end{array}$ & $\begin{array}{l}32 \\
31 \\
30 \\
32 \\
35\end{array}$ & $\begin{array}{l}40 \\
41 \\
40 \\
37 \\
38\end{array}$ & $\begin{array}{l}44 \\
30 \\
39 \\
30 \\
31\end{array}$ & $\begin{array}{l}40 \\
35 \\
34 \\
32 \\
54\end{array}$ & $\begin{array}{l}\mathbf{5 4} \\
62 \\
\mathbf{4 2} \\
\mathbf{4 2} \\
\mathbf{4 2}\end{array}$ \\
\hline $\begin{array}{r}6 \ldots \\
7 \ldots \\
8 \ldots \\
9 \ldots \\
10 \ldots\end{array}$ & $\begin{array}{l}38 \\
41 \\
44 \\
44 \\
40\end{array}$ & $\begin{array}{l}29 \\
29 \\
29 \\
29 \\
32\end{array}$ & $\begin{array}{l}36 \\
32 \\
31 \\
50 \\
54\end{array}$ & $\begin{array}{l}30 \\
37 \\
44 \\
43 \\
40\end{array}$ & $\begin{array}{l}34 \\
34 \\
45 \\
35 \\
31\end{array}$ & $\begin{array}{l}35 \\
32 \\
33 \\
32 \\
31\end{array}$ & $\begin{array}{l}31 \\
31 \\
31 \\
31 \\
43\end{array}$ & $\begin{array}{l}34 \\
45 \\
45 \\
52 \\
36\end{array}$ & $\begin{array}{l}35 \\
40 \\
40 \\
40 \\
36\end{array}$ & $\begin{array}{l}32 \\
34 \\
41 \\
33 \\
35\end{array}$ & $\begin{array}{l}43 \\
35 \\
46 \\
46 \\
40\end{array}$ & $\begin{array}{l}41 \\
40 \\
42 \\
43 \\
40\end{array}$ \\
\hline $\begin{array}{l}11 . . \\
12 \ldots \\
13 . . \\
14 \ldots \\
15 \ldots\end{array}$ & $\begin{array}{l}35 \\
35 \\
39 \\
29 \\
35\end{array}$ & $\begin{array}{l}30 \\
29 \\
28 \\
28 \\
28\end{array}$ & $\begin{array}{l}58 \\
42 \\
45 \\
43 \\
40\end{array}$ & $\begin{array}{l}39 \\
36 \\
33 \\
32 \\
32\end{array}$ & $\begin{array}{l}31 \\
32 \\
32 \\
44 \\
45\end{array}$ & $\begin{array}{l}32 \\
31 \\
54 \\
44 \\
46\end{array}$ & $\begin{array}{l}53 \\
42 \\
48 \\
46 \\
43\end{array}$ & $\begin{array}{l}34 \\
32 \\
32 \\
55 \\
38\end{array}$ & $\begin{array}{l}34 \\
35 \\
34 \\
32 \\
37\end{array}$ & $\begin{array}{l}30 \\
31 \\
32 \\
33 \\
31\end{array}$ & $\begin{array}{l}35 \\
33 \\
31 \\
30 \\
30\end{array}$ & $\begin{array}{l}37 \\
36 \\
36 \\
33 \\
34\end{array}$ \\
\hline $\begin{array}{l}16 \ldots \\
17 \ldots \\
18 \ldots \\
19 . . \\
20 \ldots\end{array}$ & $\begin{array}{l}33 \\
32 \\
32 \\
31 \\
43\end{array}$ & $\begin{array}{l}28 \\
29 \\
28 \\
28 \\
28\end{array}$ & $\begin{array}{l}40 \\
40 \\
40 \\
35 \\
35\end{array}$ & $\begin{array}{l}32 \\
40 \\
39 \\
32 \\
47\end{array}$ & $\begin{array}{l}38 \\
32 \\
31 \\
30 \\
30\end{array}$ & $\begin{array}{l}39 \\
32 \\
41 \\
44 \\
37\end{array}$ & $\begin{array}{l}44 \\
52 \\
41 \\
40 \\
40\end{array}$ & $\begin{array}{l}37 \\
33 \\
32 \\
31 \\
31\end{array}$ & $\begin{array}{l}42 \\
36 \\
31 \\
35 \\
33\end{array}$ & $\begin{array}{l}30 \\
47 \\
35 \\
52 \\
50\end{array}$ & $\begin{array}{l}29 \\
39 \\
49 \\
33 \\
31\end{array}$ & $\begin{array}{l}34 \\
32 \\
31 \\
33 \\
44\end{array}$ \\
\hline $\begin{array}{l}21 \ldots \\
22 \ldots \\
23 \ldots \\
24 \ldots \\
25 \ldots\end{array}$ & $\begin{array}{l}37 \\
31 \\
\mathbf{5 6} \\
18 \\
26\end{array}$ & $\begin{array}{l}29 \\
40 \\
50 \\
39 \\
36\end{array}$ & $\begin{array}{l}34 \\
35 \\
31 \\
36 \\
42\end{array}$ & $\begin{array}{l}46 \\
44 \\
44 \\
32 \\
55\end{array}$ & $\begin{array}{l}34 \\
44 \\
46 \\
49 \\
39\end{array}$ & $\begin{array}{l}50 \\
43 \\
46 \\
49 \\
40\end{array}$ & $\begin{array}{l}40 \\
39 \\
39 \\
51 \\
39\end{array}$ & $\begin{array}{l}31 \\
40 \\
31 \\
39 \\
38\end{array}$ & $\begin{array}{l}32 \\
32 \\
31 \\
30 \\
29\end{array}$ & $\begin{array}{l}48 \\
44 \\
42 \\
35 \\
34\end{array}$ & $\begin{array}{l}34 \\
46 \\
54 \\
42 \\
31\end{array}$ & $\begin{array}{l}61 \\
62 \\
63 \\
50 \\
46\end{array}$ \\
\hline $\begin{array}{l}26 \ldots \ldots \\
27 \ldots \ldots \\
28 \ldots \ldots \\
29 \ldots \ldots \\
30 \ldots \ldots \\
31 \ldots \ldots\end{array}$ & $\begin{array}{l}26 \\
26 \\
28 \\
31 \\
30 \\
33\end{array}$ & $\begin{array}{c}34 \\
32 \\
31 \\
30 \\
\ldots . .\end{array}$ & $\begin{array}{l}33 \\
31 \\
30 \\
30 \\
29 \\
33\end{array}$ & $\begin{array}{c}38 \\
35 \\
33 \\
31 \\
30 \\
\cdots\end{array}$ & $\begin{array}{l}44 \\
48 \\
39 \\
34 \\
42 \\
40\end{array}$ & $\begin{array}{l}34 \\
36 \\
32 \\
31 \\
30\end{array}$ & $\begin{array}{l}38 \\
36 \\
34 \\
33 \\
36 \\
32\end{array}$ & $\begin{array}{l}38 \\
38 \\
41 \\
40 \\
42 \\
40\end{array}$ & $\begin{array}{l}29 \\
28 \\
30 \\
36 \\
42\end{array}$ & $\begin{array}{l}52 \\
49 \\
46 \\
43 \\
45 \\
44\end{array}$ & $\begin{array}{l}29 \\
29 \\
28 \\
32 \\
45\end{array}$ & $\begin{array}{l}42 \\
39 \\
40 \\
39 \\
38\end{array}$ \\
\hline $\begin{array}{c}1913 . \\
1 \ldots \ldots \ldots \\
2 \ldots \ldots \ldots \\
3 \ldots \ldots \ldots \\
5 \ldots \ldots \ldots\end{array}$ & $\begin{array}{l}39 \\
37 \\
34 \\
33 \\
33\end{array}$ & $\begin{array}{l}32 \\
32 \\
31 \\
32 \\
\mathbf{3 4}\end{array}$ & $\begin{array}{l}29 \\
28 \\
28 \\
28 \\
28\end{array}$ & $\begin{array}{l}47 \\
46 \\
48 \\
42 \\
37\end{array}$ & $\begin{array}{l}48 \\
43 \\
44 \\
44 \\
43\end{array}$ & $\begin{array}{l}36 \\
32 \\
48 \\
14 \\
14\end{array}$ & $\begin{array}{l}43 \\
44 \\
44 \\
45 \\
46\end{array}$ & $\begin{array}{l}30 \\
38 \\
35 \\
32 \\
31\end{array}$ & $\begin{array}{l}40 \\
40 \\
42 \\
39 \\
42\end{array}$ & $\begin{array}{l}29 \\
28 \\
27 \\
27 \\
38\end{array}$ & $\begin{array}{l}32 \\
34 \\
37 \\
31 \\
31\end{array}$ & $\begin{array}{l}19 \\
19 \\
18 \\
18 \\
38\end{array}$ \\
\hline $\begin{array}{r}6 \\
7 \\
8 \\
9 \\
10 \ldots \ldots \ldots\end{array}$ & $\begin{array}{l}33 \\
33 \\
56 \\
39 \\
48\end{array}$ & $\begin{array}{l}39 \\
46 \\
45 \\
44 \\
43\end{array}$ & $\begin{array}{l}28 \\
46 \\
29 \\
28 \\
28\end{array}$ & $\begin{array}{l}34 \\
31 \\
33 \\
52 \\
37\end{array}$ & $\begin{array}{l}41 \\
48 \\
50 \\
46 \\
40\end{array}$ & $\begin{array}{l}44 \\
42 \\
44 \\
46 \\
44\end{array}$ & $\begin{array}{l}44 \\
41 \\
39 \\
41 \\
38\end{array}$ & $\begin{array}{l}34 \\
37 \\
36 \\
38 \\
34\end{array}$ & $\begin{array}{l}43 \\
38 \\
34 \\
35 \\
31\end{array}$ & $\begin{array}{l}48 \\
41 \\
45 \\
44 \\
42\end{array}$ & $\begin{array}{l}42 \\
35 \\
30 \\
38 \\
45\end{array}$ & $\begin{array}{r}38 \\
0 \\
35 \\
35 \\
35\end{array}$ \\
\hline $\begin{array}{l}11 \ldots \\
14 \\
15\end{array}$ & $\begin{array}{l}52 \\
30 \\
40 \\
41 \\
49\end{array}$ & $\begin{array}{l}41 \\
35 \\
32 \\
31 \\
31\end{array}$ & $\begin{array}{l}28 \\
27 \\
27 \\
28 \\
27\end{array}$ & $\begin{array}{l}36 \\
33 \\
38 \\
44 \\
45\end{array}$ & $\begin{array}{l}44 \\
47 \\
52 \\
18 \\
39\end{array}$ & $\begin{array}{l}45 \\
40 \\
46 \\
46 \\
46\end{array}$ & $\begin{array}{l}34 \\
34 \\
35 \\
36 \\
37\end{array}$ & $\begin{array}{l}30 \\
45 \\
40 \\
38 \\
35\end{array}$ & $\begin{array}{l}30 \\
35 \\
30 \\
31 \\
32\end{array}$ & $\begin{array}{l}42 \\
43 \\
44 \\
39 \\
42\end{array}$ & $\begin{array}{l}45 \\
39 \\
39 \\
37 \\
40\end{array}$ & $\begin{array}{l}35 \\
34 \\
34 \\
33 \\
32\end{array}$ \\
\hline $\begin{array}{l}16 \ldots \ldots \ldots \ldots \\
17 \ldots \ldots \ldots \\
18 \ldots \ldots \ldots \\
19 \ldots \ldots \ldots\end{array}$ & $\begin{array}{r}37 \\
37 \\
22 \\
0 \\
42\end{array}$ & $\begin{array}{l}30 \\
30 \\
39 \\
47 \\
37\end{array}$ & $\begin{array}{l}40 \\
52 \\
31 \\
34 \\
41\end{array}$ & $\begin{array}{l}39 \\
45 \\
42 \\
42 \\
45\end{array}$ & $\begin{array}{l}39 \\
41 \\
40 \\
40 \\
37\end{array}$ & $\begin{array}{l}45 \\
38 \\
40 \\
41 \\
34\end{array}$ & $\begin{array}{l}43 \\
34 \\
32 \\
33 \\
34\end{array}$ & $\begin{array}{l}31 \\
30 \\
30 \\
28 \\
29\end{array}$ & $\begin{array}{l}29 \\
29 \\
29 \\
29 \\
28\end{array}$ & $\begin{array}{l}45 \\
38 \\
37 \\
35 \\
33\end{array}$ & $\begin{array}{l}38 \\
37 \\
37 \\
39 \\
39\end{array}$ & $\begin{array}{l}31 \\
32 \\
33 \\
32 \\
31\end{array}$ \\
\hline $\begin{array}{l}21 \ldots \ldots \\
22 \ldots \ldots \\
24 \ldots \ldots \\
25 \ldots \ldots \ldots \ldots \ldots\end{array}$ & $\begin{array}{l}42 \\
50 \\
43 \\
44 \\
45\end{array}$ & $\begin{array}{l}37 \\
33 \\
38 \\
43 \\
33\end{array}$ & $\begin{array}{l}40 \\
51 \\
44 \\
38 \\
53\end{array}$ & $\begin{array}{l}48 \\
37 \\
48 \\
37 \\
35\end{array}$ & $\begin{array}{l}35 \\
34 \\
32 \\
48 \\
40\end{array}$ & $\begin{array}{l}46 \\
42 \\
38 \\
33 \\
46\end{array}$ & $\begin{array}{l}34 \\
32 \\
31 \\
35 \\
42\end{array}$ & $\begin{array}{l}44 \\
34 \\
30 \\
37 \\
44\end{array}$ & $\begin{array}{l}28 \\
29 \\
28 \\
27 \\
27\end{array}$ & $\begin{array}{r}32 \\
31 \\
38 \\
38 \\
0\end{array}$ & $\begin{array}{l}37 \\
40 \\
40 \\
41 \\
44\end{array}$ & $\begin{array}{l}31 \\
31 \\
30 \\
30 \\
28\end{array}$ \\
\hline $\begin{array}{l}26 \ldots \ldots \\
27 \ldots \ldots \ldots \\
28 \ldots \ldots \\
29, \ldots \ldots \\
30 \ldots \ldots \\
31 \ldots \ldots\end{array}$ & $\begin{array}{l}40 \\
36 \\
35 \\
\mathbf{3 4} \\
\mathbf{3 3} \\
\mathbf{3 4}\end{array}$ & $\begin{array}{r}31 \\
30 \\
29 \\
\cdots \ldots . . \\
\end{array}$ & $\begin{array}{l}52 \\
40 \\
40 \\
52 \\
48 \\
43\end{array}$ & $\begin{array}{l}40 \\
44 \\
48 \\
48 \\
45 \\
\ldots\end{array}$ & $\begin{array}{l}32 \\
37 \\
37 \\
34 \\
44 \\
39\end{array}$ & $\begin{array}{l}46 \\
53 \\
42 \\
41 \\
40\end{array}$ & $\begin{array}{l}46 \\
40 \\
34 \\
44 \\
33 \\
32\end{array}$ & $\begin{array}{l}39 \\
32 \\
36 \\
48 \\
42 \\
41\end{array}$ & $\begin{array}{l}27 \\
27 \\
28 \\
28 \\
28\end{array}$ & $\begin{array}{r}0 \\
33 \\
40 \\
33 \\
31 \\
31\end{array}$ & $\begin{array}{r}35 \\
33 \\
31 \\
31 \\
25\end{array}$ & $\begin{array}{l}30 \\
29 \\
29 \\
29 \\
29 \\
30\end{array}$ \\
\hline
\end{tabular}

NoTE.-Gage not read on Sundays; discharge estimated. 
Monthly discharge of Hiloa ditch at Hanapepe Falls, near Eleele, Kauai, for 191\%-13.

\begin{tabular}{|c|c|c|c|c|c|}
\hline \multirow{2}{*}{ Month. } & \multicolumn{3}{|c|}{ Discharge in second-feet. } & \multirow{2}{*}{$\begin{array}{l}\text { Run-off } \\
\text { (total in } \\
\text { acre-feet). }\end{array}$} & \multirow{2}{*}{$\begin{array}{l}\text { Accu- } \\
\text { racy. }\end{array}$} \\
\hline & Maximum. & Minimum. & Mean. & & \\
\hline 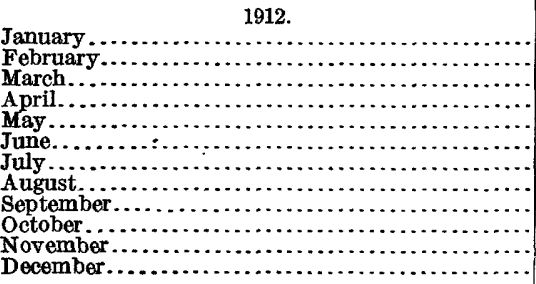 & $\begin{array}{l}56 \\
50 \\
58 \\
55 \\
53 \\
54 \\
53 \\
55 \\
42 \\
52 \\
54 \\
63\end{array}$ & $\begin{array}{l}18 \\
28 \\
29 \\
28 \\
29 \\
30 \\
30 \\
30 \\
28 \\
30 \\
28 \\
31\end{array}$ & $\begin{array}{l}34.9 \\
31.1 \\
37.0 \\
36.6 \\
38.1 \\
37.8 \\
39.2 \\
36.9 \\
35.2 \\
38.8 \\
37.2 \\
42.5\end{array}$ & $\begin{array}{l}2,150 \\
1,790 \\
2,280 \\
2,180 \\
2,340 \\
2,250 \\
2,410 \\
2,270 \\
2,090 \\
2,390 \\
2,210 \\
2,610\end{array}$ & $\begin{array}{l}\text { A. } \\
\text { A. } \\
\text { A. } \\
\text { A. } \\
\text { A. } \\
\text { A. } \\
\text { A. } \\
\text { A. } \\
\text { A. } \\
\text { A. } \\
\text { A. } \\
\text { A. }\end{array}$ \\
\hline 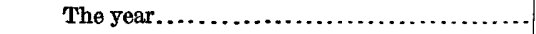 & 63 & 18 & 37.2 & 27,000 & \\
\hline 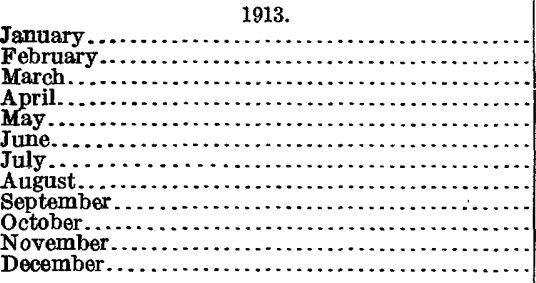 & $\begin{array}{l}56 \\
47 \\
53 \\
52 \\
52 \\
53 \\
46 \\
48 \\
43 \\
48 \\
45 \\
38\end{array}$ & $\begin{array}{r}0 \\
29 \\
27 \\
31 \\
18 \\
14 \\
31 \\
28 \\
27 \\
0 \\
25 \\
0\end{array}$ & $\begin{array}{l}38.3 \\
35.9 \\
36.6 \\
41.5 \\
40.5 \\
40.4 \\
38.1 \\
35.7 \\
32.1 \\
34.6 \\
36.7 \\
29.3\end{array}$ & $\begin{array}{l}2,360 \\
1,990 \\
2,250 \\
2,470 \\
2,490 \\
2,400 \\
2,340 \\
2,200 \\
1,910 \\
2,130 \\
2,180 \\
1,800\end{array}$ & $\begin{array}{l}\text { A. } \\
\text { A. } \\
\text { A. } \\
\text { A. } \\
\text { A. } \\
\text { A. } \\
\text { A. } \\
\text { A. } \\
\text { A. } \\
\text { A. } \\
\text { B. } \\
\text { B. }\end{array}$ \\
\hline The year ...... & 56 & 14 & 36.5 & 26,500 & \\
\hline
\end{tabular}

Note.-Jan. 18, 1913: Gates closed at noon and not opened until the 20th; practically no flow for this period.

May 14, June 4 and 5, 1913, Discharge affected by regulation. Oct. 25-26, no water in ditch; turned in at $4.15 \mathrm{p}$. m. Oct. 27 . Dec. 7,1913 , no water in ditch.

\section{HANAPEPE DITCH AT KOULA, NEAR ELEELE, KAUAI.}

Location.-About 4 miles north of Eleele and about 4 miles below Hanapepe Falls. Records available.-January 1, 1910, to December 31, 1913.

Gage.-Vertical staff.

Control.-Probably permanent.

Discharge measuremonts.-Made from cross timber of flume.

Diversions.-Ditch diverts all low-water flow of East Branch of Hanapepe River.

Accuracy.-Records good.

Cooperation.-Gage-height records furnished by Hawaiian Sugar Co.

The following discharge measurement was made by W. V. Hardy.

June 22, 1913: Gage height, 2.84 feet; discharge, 46.2 second-feet. 
Daily discharge, in second-feet, of Hanapepe ditch at Koula, near Eleele, Kauai, for 1913.

\begin{tabular}{|c|c|c|c|c|c|c|c|c|c|c|c|c|}
\hline Day. & Jan. & Feb. & Mar. & Apr. & May. & June. & July. & Aug. & Sept. & Oct. & Nov. & Dec. \\
\hline $\begin{array}{l}1 \ldots \ldots \\
2 \ldots \ldots \\
3 \ldots \ldots \\
4 \ldots \ldots \\
5 \ldots \ldots\end{array}$ & $\begin{array}{l}50 \\
48 \\
46 \\
44 \\
44\end{array}$ & $\begin{array}{l}57 \\
44 \\
44 \\
43 \\
48\end{array}$ & $\begin{array}{l}38 \\
37 \\
37 \\
36 \\
36\end{array}$ & $\begin{array}{l}52 \\
52 \\
52 \\
52 \\
48\end{array}$ & $\begin{array}{l}52 \\
52 \\
52 \\
51 \\
52\end{array}$ & $\begin{array}{l}46 \\
43 \\
45 \\
47 \\
48\end{array}$ & $\begin{array}{l}51 \\
51 \\
51 \\
51 \\
51\end{array}$ & $\begin{array}{l}38 \\
51 \\
44 \\
41 \\
40\end{array}$ & $\begin{array}{l}51 \\
51 \\
51 \\
50 \\
51\end{array}$ & $\begin{array}{l}37 \\
34 \\
33 \\
30 \\
29\end{array}$ & $\begin{array}{l}38 \\
46 \\
47 \\
40 \\
40\end{array}$ & $\begin{array}{l}93 \\
82 \\
71 \\
60 \\
49\end{array}$ \\
\hline 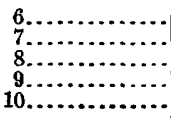 & $\begin{array}{l}44 \\
44 \\
52 \\
52 \\
52\end{array}$ & $\begin{array}{l}50 \\
52 \\
46 \\
45 \\
52\end{array}$ & $\begin{array}{l}36 \\
46 \\
38 \\
37 \\
36\end{array}$ & $\begin{array}{l}43 \\
41 \\
41 \\
44 \\
50\end{array}$ & $\begin{array}{l}52 \\
52 \\
52 \\
52 \\
52\end{array}$ & $\begin{array}{l}50 \\
52 \\
51 \\
51 \\
51\end{array}$ & $\begin{array}{l}51 \\
51 \\
51 \\
51 \\
46\end{array}$ & $\begin{array}{l}39 \\
50 \\
50 \\
46 \\
28\end{array}$ & $\begin{array}{l}51 \\
51 \\
45 \\
44 \\
40\end{array}$ & $\begin{array}{l}49 \\
49 \\
52 \\
51 \\
50\end{array}$ & $\begin{array}{l}51 \\
39 \\
37 \\
49 \\
51\end{array}$ & $\begin{array}{l}50 \\
43 \\
36 \\
36 \\
37\end{array}$ \\
\hline $\begin{array}{l}11 \ldots \ldots \\
12 \ldots \ldots \\
13 \ldots \ldots \\
14 \ldots \ldots \\
15 \ldots \ldots\end{array}$ & $\begin{array}{l}52 \\
52 \\
52 \\
52 \\
52\end{array}$ & $\begin{array}{l}48 \\
45 \\
43 \\
43 \\
43\end{array}$ & $\begin{array}{l}34 \\
\mathbf{3 4} \\
\mathbf{3 4} \\
\mathbf{3 4} \\
\mathbf{3 3}\end{array}$ & $\begin{array}{l}52 \\
42 \\
52 \\
52 \\
52\end{array}$ & $\begin{array}{l}48 \\
50 \\
51 \\
52 \\
52\end{array}$ & $\begin{array}{l}51 \\
51 \\
51 \\
51 \\
51\end{array}$ & $\begin{array}{l}46 \\
45 \\
51 \\
50 \\
49\end{array}$ & $\begin{array}{l}28 \\
38 \\
51 \\
51 \\
47\end{array}$ & $\begin{array}{l}39 \\
43 \\
36 \\
38 \\
41\end{array}$ & $\begin{array}{l}50 \\
51 \\
51 \\
50 \\
50\end{array}$ & $\begin{array}{l}51 \\
51 \\
48 \\
52 \\
52\end{array}$ & $\begin{array}{l}44 \\
44 \\
44 \\
44 \\
43\end{array}$ \\
\hline $\begin{array}{l}16 \ldots \ldots \ldots \\
17 \ldots \ldots \ldots \ldots \\
19 \ldots \ldots \ldots \ldots \\
20 \ldots \ldots \ldots \ldots\end{array}$ & $\begin{array}{l}52 \\
52 \\
17 \\
34 \\
52\end{array}$ & $\begin{array}{l}41 \\
45 \\
52 \\
44 \\
44\end{array}$ & $\begin{array}{l}33 \\
51 \\
39 \\
46 \\
49\end{array}$ & $\begin{array}{l}49 \\
52 \\
52 \\
52 \\
52\end{array}$ & $\begin{array}{l}\mathbf{5 2} \\
\mathbf{5 2} \\
\mathbf{5 2} \\
\mathbf{5 0} \\
\mathbf{5 0}\end{array}$ & $\begin{array}{l}51 \\
50 \\
47 \\
51 \\
45\end{array}$ & $\begin{array}{l}46 \\
48 \\
48 \\
44 \\
46\end{array}$ & $\begin{array}{l}40 \\
38 \\
37 \\
36 \\
27\end{array}$ & $\begin{array}{l}36 \\
35 \\
37 \\
38 \\
44\end{array}$ & $\begin{array}{l}50 \\
48 \\
46 \\
43 \\
42\end{array}$ & $\begin{array}{l}52 \\
52 \\
52 \\
52 \\
51\end{array}$ & $\begin{array}{l}42 \\
42 \\
43 \\
42 \\
41\end{array}$ \\
\hline 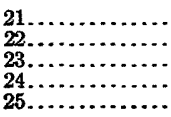 & $\begin{array}{l}\mathbf{5 2} \\
\mathbf{5 2} \\
\mathbf{5 2} \\
\mathbf{5 2} \\
\mathbf{5 2}\end{array}$ & $\begin{array}{l}45 \\
46 \\
52 \\
44 \\
39\end{array}$ & $\begin{array}{l}52 \\
52 \\
49 \\
52 \\
52\end{array}$ & $\begin{array}{l}52 \\
52 \\
52 \\
52 \\
52\end{array}$ & $\begin{array}{l}48 \\
45 \\
44 \\
49 \\
46\end{array}$ & $\begin{array}{l}51 \\
45 \\
46 \\
47 \\
48\end{array}$ & $\begin{array}{l}46 \\
44 \\
39 \\
45 \\
51\end{array}$ & $\begin{array}{l}51 \\
43 \\
38 \\
38 \\
50\end{array}$ & $\begin{array}{l}33 \\
35 \\
33 \\
33 \\
32\end{array}$ & $\begin{array}{l}39 \\
40 \\
51 \\
51 \\
50\end{array}$ & $\begin{array}{l}49 \\
52 \\
52 \\
52 \\
39\end{array}$ & $\begin{array}{l}41 \\
41 \\
40 \\
40 \\
40\end{array}$ \\
\hline 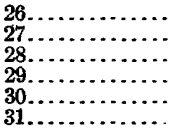 & $\begin{array}{l}52 \\
50 \\
48 \\
45 \\
42 \\
36\end{array}$ & $\begin{array}{r}42 \\
40 \\
39 \\
\cdots \\
\hdashline \ldots\end{array}$ & $\begin{array}{l}52 \\
51 \\
52 \\
52 \\
52 \\
41\end{array}$ & $\begin{array}{l}52 \\
52 \\
52 \\
52 \\
52\end{array}$ & $\begin{array}{l}46 \\
44 \\
47 \\
52 \\
52 \\
49\end{array}$ & $\begin{array}{l}51 \\
48 \\
46 \\
51 \\
51\end{array}$ & $\begin{array}{l}51 \\
51 \\
45 \\
50 \\
43 \\
40\end{array}$ & $\begin{array}{l}49 \\
39 \\
44 \\
51 \\
51 \\
51\end{array}$ & $\begin{array}{l}31 \\
32 \\
48 \\
44 \\
33\end{array}$ & $\begin{array}{l}48 \\
47 \\
46 \\
42 \\
39 \\
40\end{array}$ & $\begin{array}{r}32 \\
34 \\
34 \\
69 \\
104\end{array}$ & $\begin{array}{l}\mathbf{3 9} \\
\mathbf{3 9} \\
\mathbf{3 8} \\
\mathbf{3 8} \\
\mathbf{3 8} \\
\mathbf{3 9}\end{array}$ \\
\hline
\end{tabular}

Note.-Discharge interpolated for Sundays and holidays when no readings were made.

Monthly discharge of Hanapepe ditch at Koula, near Eleele, Kauai, for 1913.

\begin{tabular}{|c|c|c|c|c|c|}
\hline \multirow[b]{2}{*}{ ' } & \multicolumn{3}{|c|}{ Discharge in second-feet. } & \multirow{2}{*}{$\begin{array}{l}\text { Run-off } \\
\text { (total in } \\
\text { acre-feet). }\end{array}$} & \multirow{2}{*}{$\begin{array}{l}\text { Accu- } \\
\text { racy. }\end{array}$} \\
\hline & Maximum. & Minimum. & Mean. & & \\
\hline 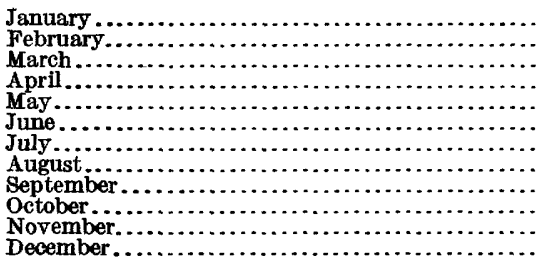 & $\begin{array}{r}52 \\
57 \\
52 \\
52 \\
52 \\
52 \\
51 \\
51 \\
51 \\
52 \\
104 \\
93\end{array}$ & $\begin{array}{l}17 \\
39 \\
33 \\
41 \\
44 \\
43 \\
39 \\
27 \\
31 \\
29 \\
32 \\
36\end{array}$ & $\begin{array}{r}47.6 \\
45.6 \\
42.6 \\
50.1 \\
50.0 \\
48.9 \\
47.8 \\
42.7 \\
40.9 \\
44.8 \\
48.9 \\
45.8\end{array}$ & $\begin{array}{l}\mathbf{2}, 930 \\
\mathbf{2}, 530 \\
\mathbf{2}, 620 \\
\mathbf{2}, 980 \\
\mathbf{3}, 070 \\
2,910 \\
\mathbf{2}, 940 \\
\mathbf{2}, 630 \\
2,430 \\
\mathbf{2}, 750 \\
2,910 \\
2,820\end{array}$ & $\begin{array}{l}\text { A. } \\
\text { A. } \\
\text { A. } \\
\text { A. } \\
\text { A. } \\
\text { A. } \\
\text { A. } \\
\text { A. } \\
\text { A. } \\
\text { A. }\end{array}$ \\
\hline The year... & 104 & 17 & 46.3 & 33,500 & \\
\hline
\end{tabular}

HANAPEPE DITCH AT WEIR NEAR HANAPEPE, KAUAI.

Location.-About $2 \frac{1}{2}$ miles northeast of Hanappe at measuring woir maintained by Hawaiian Sugar Co., on the Hanapepe ditch below the last siphon across Hanapepe River.

Records available.-January, 1910, to December 31, 1913; records show amount of water delivered by the ditch at the cane fields.

Gage.-Vertical staff on right bank; zero on gage equals crest of weir.

Control.-Probably permanent.

Discharge measurements.-Computed from formula for a 12-foot, sharp-crested weir with end contractions; checked by current-meter measurements.

Cooperation.-Gage-height records furnished by Hawaiian Sugar Co. 
Daily discharge, in second-feet, of Hanapepe ditch at weir near Hanapepe, Kauai, for 1913.

\begin{tabular}{|c|c|c|c|c|c|c|c|c|c|c|c|c|}
\hline Day. & Jan. & Feb. & Mar. & Apr. & May. & June. & July. & Aug. & Sept. & Oct. & Nov. & Dec. \\
\hline $\begin{array}{l}1 \ldots \\
2 \ldots \\
3 \ldots \\
4 \ldots \\
5 \ldots\end{array}$ & $\begin{array}{l}46.4 \\
46.4 \\
45.1 \\
43.8 \\
42.5\end{array}$ & $\begin{array}{l}43.8 \\
41.2 \\
41.2 \\
40.0 \\
41.2\end{array}$ & $\begin{array}{l}36.3 \\
35.2 \\
35.2 \\
34.0 \\
32.8\end{array}$ & $\begin{array}{l}41.2 \\
41.2 \\
42.5 \\
41.2 \\
41.2\end{array}$ & $\begin{array}{l}41.2 \\
41.2 \\
41.2 \\
40.0 \\
30.4\end{array}$ & $\begin{array}{r}45.1 \\
42.5 \\
40.0 \\
\ldots \ldots . .\end{array}$ & $\begin{array}{l}45.1 \\
45.1 \\
46.4 \\
45.1 \\
45.1\end{array}$ & $\begin{array}{l}37.5 \\
38.8 \\
42.5 \\
42.5 \\
38.8\end{array}$ & $\begin{array}{l}43.8 \\
43.8 \\
43.8 \\
43.8 \\
42.5\end{array}$ & $\begin{array}{l}34.0 \\
35.2 \\
32.8 \\
30.4 \\
30.4\end{array}$ & $\begin{array}{l}40.0 \\
40.0 \\
40.0 \\
41.2 \\
40.0\end{array}$ & $\begin{array}{l}\ldots \ldots \\
\cdots \ldots . \\
\cdots \ldots . \\
\cdots \ldots \\
\cdots \ldots\end{array}$ \\
\hline $\begin{array}{r}6 \ldots \\
7 \ldots \\
8 \ldots \\
9 \ldots \\
10 . \ldots\end{array}$ & $\begin{array}{l}42.5 \\
42.5 \\
45.1 \\
46.4 \\
45.1\end{array}$ & $\begin{array}{l}42.5 \\
45.1 \\
46.4 \\
45.1 \\
43.8\end{array}$ & $\begin{array}{l}32.8 \\
35.2 \\
38.8 \\
36.3 \\
34.0\end{array}$ & $\begin{array}{l}37.5 \\
36.3 \\
35.2 \\
35.2 \\
37.5\end{array}$ & $\begin{array}{l}37.5 \\
41.2 \\
41.2 \\
41.2 \\
41.2\end{array}$ & $\begin{array}{l}45.1 \\
46.4 \\
45.1 \\
46.4\end{array}$ & $\begin{array}{l}45.1 \\
45.1 \\
42.5 \\
42.5 \\
41.2\end{array}$ & $\begin{array}{l}38.8 \\
40.0 \\
41.2 \\
40.0 \\
38.8\end{array}$ & $\begin{array}{l}42.5 \\
43.8 \\
42.5 \\
40.0 \\
40.0\end{array}$ & $\begin{array}{l}35.2 \\
40.0 \\
43.8 \\
45.1 \\
45.1\end{array}$ & $\begin{array}{l}41.2 \\
42.5 \\
40.0 \\
42.5 \\
46.4\end{array}$ & $\begin{array}{r}40.0 \\
\dddot{21.8} \\
36.3 \\
37.5\end{array}$ \\
\hline $\begin{array}{l}13 \ldots \\
14 \ldots \\
15 \ldots\end{array}$ & $\begin{array}{l}42.5 \\
43.8 \\
45.1 \\
45.1 \\
46.4\end{array}$ & $\begin{array}{l}46.4 \\
46.4 \\
42.5 \\
41.2 \\
41.2\end{array}$ & $\begin{array}{l}32.8 \\
31.6 \\
31.6 \\
31.6 \\
31.6\end{array}$ & $\begin{array}{l}37.5 \\
38.8 \\
38.8 \\
41.2 \\
42.5\end{array}$ & $\begin{array}{r}37.5 \\
40.0 \\
27.1 \\
\ldots \ldots . . \\
\ldots \ldots .\end{array}$ & $\begin{array}{l}46.4 \\
46.4 \\
45.1 \\
45.1 \\
45.1\end{array}$ & $\begin{array}{l}40.0 \\
40.0 \\
40.0 \\
41.2 \\
41.2\end{array}$ & $\begin{array}{l}37.5 \\
37.5 \\
38.8 \\
41.2 \\
41.2\end{array}$ & $\begin{array}{l}37.5 \\
36.3 \\
35.2 \\
35.2 \\
35.2\end{array}$ & $\begin{array}{l}46.4 \\
46.4 \\
46.4 \\
45.1 \\
38.8\end{array}$ & $\begin{array}{l}46.4 \\
46.4 \\
49.0 \\
49.0 \\
45.1\end{array}$ & $\begin{array}{l}38.8 \\
41.2 \\
42.5 \\
42.5 \\
42.5\end{array}$ \\
\hline $\begin{array}{l}16 \ldots . . \\
17 \ldots \ldots \\
18 \ldots \ldots \\
19 \ldots \\
20 \ldots\end{array}$ & $\begin{array}{r}47.7 \\
47.7 \\
28.2 \\
\hdashline 32.8\end{array}$ & $\begin{array}{r}41.2 \\
40.0 \\
41.2 \\
43.8 \\
.45 .1\end{array}$ & $\begin{array}{l}32.8 \\
34.0 \\
40.0 \\
41.2 \\
42.5\end{array}$ & $\begin{array}{l}42.5 \\
42.5 \\
42.5 \\
42.5 \\
42.5\end{array}$ & $\begin{array}{l}30.4 \\
46.4 \\
46.4 \\
46.4 \\
45.1\end{array}$ & $\begin{array}{l}45.1 \\
45.1 \\
45.1 \\
43.8 \\
42.5\end{array}$ & $\begin{array}{l}41.2 \\
41.2 \\
38.8 \\
37.5 \\
40.0\end{array}$ & $\begin{array}{l}38.8 \\
36.3 \\
34.0 \\
32.8 \\
32.8\end{array}$ & $\begin{array}{l}35.2 \\
34.0 \\
32.8 \\
35.2 \\
34.0\end{array}$ & $\begin{array}{l}42.5 \\
43.8 \\
40.0 \\
19.8 \\
35.2\end{array}$ & $\begin{array}{l}40.0 \\
46.4 \\
47.7 \\
49.0 \\
47.7\end{array}$ & $\begin{array}{l}42.5 \\
41.2 \\
40.0 \\
41.2 \\
40.0\end{array}$ \\
\hline $\begin{array}{l}21 \ldots \ldots \\
22 \ldots \ldots \\
23 . \ldots \ldots \\
24 \ldots \ldots \\
25 \ldots \ldots\end{array}$ & $\begin{array}{l}47.7 \\
47.7 \\
47.7 \\
42.5 \\
23.9\end{array}$ & $\begin{array}{l}45.1 \\
42.5 \\
42.5 \\
42.5 \\
43.8\end{array}$ & $\begin{array}{l}43.8 \\
43.8 \\
43.8 \\
42.5 \\
42.5\end{array}$ & $\begin{array}{l}42.5 \\
41.2 \\
41.2 \\
41.2 \\
40.0\end{array}$ & $\begin{array}{l}43.8 \\
42.5 \\
42.5 \\
42.5 \\
43.8\end{array}$ & $\begin{array}{l}43.8 \\
42.5 \\
42.5 \\
41.2 \\
41.2\end{array}$ & $\begin{array}{l}40.0 \\
38.8 \\
37.5 \\
37.5 \\
38.8\end{array}$ & $\begin{array}{l}36.3 \\
37.5 \\
36.3 \\
34.0 \\
35.2\end{array}$ & $\begin{array}{l}32.8 \\
32.8 \\
31.6 \\
30.4 \\
30.4\end{array}$ & $\begin{array}{l}38.8 \\
38.8 \\
41.2 \\
42.5 \\
23.9\end{array}$ & 40.0 & $\begin{array}{l}41.2 \\
41.2 \\
40.0 \\
40.0 \\
40.0\end{array}$ \\
\hline $\begin{array}{l}26 \ldots \\
27 \ldots \\
28 \ldots \\
29 . \ldots \\
30 . \ldots \\
31 . .\end{array}$ & $\begin{array}{l}47.7 \\
47.7 \\
46.4 \\
45.1 \\
43.8 \\
43.8\end{array}$ & $\begin{array}{c}41.2 \\
40.0 \\
37.5 \\
\cdots \cdots\end{array}$ & $\begin{array}{l}43.8 \\
41.2 \\
43.8 \\
42.5 \\
40.0 \\
40.0\end{array}$ & $\begin{array}{r}41.2 \\
41.2 \\
41.2 \\
42.5 \\
42.5 \\
\ldots \ldots\end{array}$ & $\begin{array}{l}42.5 \\
42.5 \\
41.2 \\
41.2 \\
41.2 \\
43.8\end{array}$ & $\begin{array}{l}43.8 \\
43.8 \\
41.2 \\
34.0 \\
45.1 \\
\ldots \ldots .\end{array}$ & $\begin{array}{l}41.2 \\
41.2 \\
41.2 \\
40.0 \\
41.2 \\
38.8\end{array}$ & $\begin{array}{l}38.8 \\
38.8 \\
38.8 \\
40.0 \\
42.5 \\
43.8\end{array}$ & $\begin{array}{l}30.4 \\
30.4 \\
31.6 \\
35.2 \\
32.8 \\
-\cdots . .\end{array}$ & $\begin{array}{l}30.4 \\
40.0 \\
40.0\end{array}$ & $\begin{array}{l}37.5 \\
36.3 \\
36.3 \\
35.2\end{array}$ & $\begin{array}{l}40.0 \\
38.8 \\
38.8 \\
37.5 \\
36.3 \\
35.2\end{array}$ \\
\hline
\end{tabular}

Note.-Ditch dry Jan. 19; May 14-15; June 4-6; Oct. 26-28; Nov. 22-24 and 30; Dec. 1-5 and 7.

Monthly discharge of Hanapepe ditch at weir near Hanapepe, Kauai, for 1913.

\begin{tabular}{|c|c|c|c|c|}
\hline \multirow{2}{*}{ Month. } & \multicolumn{3}{|c|}{ Discharge in second-feet. } & \multirow{2}{*}{$\begin{array}{l}\text { Run-off } \\
\text { (total in } \\
\text { acre-feet). }\end{array}$} \\
\hline & Maximum. & Minimum. & Mean. & \\
\hline 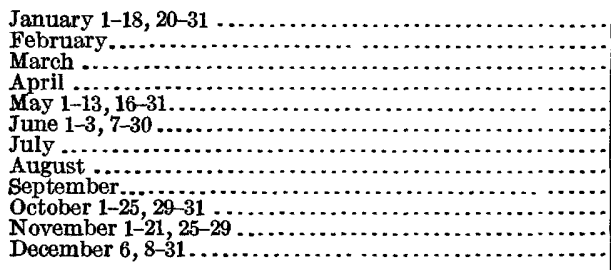 & $\begin{array}{l}47.7 \\
46.4 \\
43.8 \\
42.5 \\
46.4 \\
46.4 \\
46.4 \\
43.8 \\
43.8 \\
46.4 \\
49.0 \\
42.5\end{array}$ & $\begin{array}{l}23.9 \\
37.5 \\
31.6 \\
35.2 \\
27.1 \\
34.0 \\
37.5 \\
32.8 \\
30.4 \\
19.8 \\
35.2 \\
21.8\end{array}$ & $\begin{array}{l}43.6 \\
42.7 \\
37.7 \\
40.5 \\
40.8 \\
43.7 \\
41.3 \\
38.4 \\
36.5 \\
38.3 \\
42.8 \\
39.1\end{array}$ & $\begin{array}{l}2,590 \\
2,370 \\
2,320 \\
2,410 \\
2,350 \\
2,340 \\
2,540 \\
2,360 \\
2,170 \\
2,130 \\
2,200 \\
1,930\end{array}$ \\
\hline The period............ & 49.0 & 19.8 & 40.4 & 27,700 \\
\hline
\end{tabular}

NoTE. - Mean for month and period is for days when ditch carried water. 


\section{HULEIA RIVER NEAR LIHUE, KAUAI.}

Location.-About 300 feet above stone bridge where wagon road from Lihue to the Rice plantation crosses stream; about 4 miles southeast of Lihue.

Records available.=May 8, 1912, to December 31, 1913.

Drainage area.- 17.9 square miles.

Gage.-Vertical low-water staff gage; inclined high-water gage.

Control.-Probably permanent:

Discharge measurements.-Made by wading.

Cooperation.-Station is maintained in cooperation with Mr. Charles Rice.

Diversions.-Several above station.

Accuracy.-Records poor.

Daily discharge, in second-feet, of Huleia River near Lihue, Kauai, for 1912-13.

\begin{tabular}{|c|c|c|c|c|c|c|c|c|}
\hline Day. & May. & June. & July. & Aug. & Sept. & Oet. & Nov. & Dec. \\
\hline 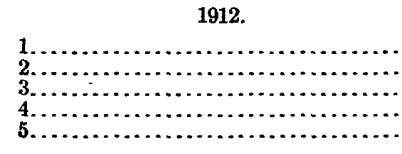 & $\begin{array}{l}\cdots \\
\cdots \cdots \\
\cdots \cdots \\
\cdots \cdots\end{array}$ & $\begin{array}{r}7.2 \\
7.2 \\
7.2 \\
9.0 \\
11\end{array}$ & $\begin{array}{l}6.4 \\
6.2 \\
5.8 \\
5.7 \\
5.5\end{array}$ & $\begin{array}{l}7.0 \\
6.4 \\
6.4 \\
6.2 \\
6.2\end{array}$ & $\begin{array}{l}7.2 \\
6.4 \\
7.2 \\
7.2 \\
7.2\end{array}$ & $\begin{array}{l}\mathbf{5 . 8} \\
\mathbf{5 . 5} \\
\mathbf{5 . 5} \\
\mathbf{5 . 5} \\
\mathbf{5 . 7}\end{array}$ & $\begin{array}{l}7.4 \\
7.4 \\
7.2 \\
7.2 \\
7.2\end{array}$ & $\begin{array}{r}9.0 \\
87^{2} \\
116 \\
55 \\
40\end{array}$ \\
\hline 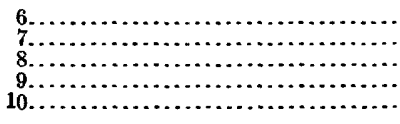 & $\begin{array}{l}7.4 \\
7.2 \\
7.2 \\
7.2\end{array}$ & $\begin{array}{l}9.4 \\
8.3 \\
7.4 \\
7.2 \\
7.2\end{array}$ & $\begin{array}{l}5.5 \\
5.5 \\
5.5 \\
5.5 \\
7.6\end{array}$ & $\begin{array}{l}7.0 \\
6.4 \\
5.8 \\
5.5 \\
5.7\end{array}$ & $\begin{array}{l}61 \\
8.6 \\
7.2 \\
7.2 \\
7.2\end{array}$ & $\begin{array}{l}5.5 \\
5.5 \\
6.0 \\
7.0 \\
7.2\end{array}$ & $\begin{array}{c}7.2 \\
7.2 \\
7.2 \\
40 \\
8.1\end{array}$ & $\begin{array}{l}31 \\
31 \\
31 \\
24 \\
20\end{array}$ \\
\hline $\begin{array}{l}11 \\
12 \\
13 \\
14 \\
15\end{array}$ & $\begin{array}{l}7.2 \\
7.2 \\
7.2 \\
7.2 \\
9.0\end{array}$ & $\begin{array}{l}7.4 \\
7.4 \\
7.2 \\
7.4 \\
7.4\end{array}$ & $\begin{array}{l}9.0 \\
7.4 \\
7.0 \\
40 \\
26\end{array}$ & $\begin{array}{l}5.5 \\
5.5 \\
5.5 \\
7.2 \\
9.2\end{array}$ & $\begin{array}{l}6.4 \\
5.8 \\
5.7 \\
5.5 \\
5.5\end{array}$ & $\begin{array}{l}7.4 \\
7.4 \\
5.8 \\
5.8 \\
5.5\end{array}$ & $\begin{array}{l}8.1 \\
7.7 \\
6.4 \\
5.8 \\
5.8\end{array}$ & $\begin{array}{l}18 \\
18 \\
17 \\
16 \\
15\end{array}$ \\
\hline 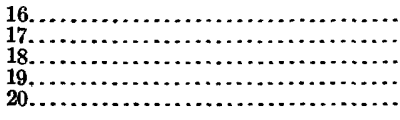 & $\begin{array}{l}8.1 \\
7.4 \\
7.2 \\
7.2 \\
7.0\end{array}$ & $\begin{array}{r}11 \\
7.2 \\
7.2 \\
7.2 \\
7.2\end{array}$ & $\begin{array}{l}7.4 \\
9.0 \\
7.4 \\
7.2 \\
7.2\end{array}$ & $\begin{array}{l}6.5 \\
6.4 \\
5.8 \\
5.8 \\
5.8\end{array}$ & $\begin{array}{l}5.5 \\
5.5 \\
5.5 \\
6.4 \\
5.7\end{array}$ & $\begin{array}{l}5.5 \\
5.5 \\
5.5 \\
6.4 \\
7.2\end{array}$ & $\begin{array}{l}5.7 \\
5.7 \\
7.0 \\
6.2 \\
5.7\end{array}$ & $\begin{array}{l}14 \\
14 \\
14 \\
12 \\
14\end{array}$ \\
\hline 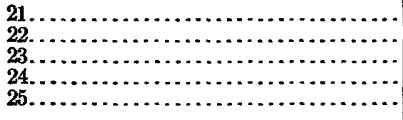 & $\begin{array}{l}6.9 \\
6.5 \\
7.2 \\
7.4 \\
7.2\end{array}$ & $\begin{array}{l}7.2 \\
7.0 \\
6.7 \\
6.4 \\
\mathbf{6 . 4}\end{array}$ & $\begin{array}{l}7.2 \\
7.2 \\
7.0 \\
7.0 \\
7.2\end{array}$ & $\begin{array}{l}5.8 \\
6.2 \\
5.8 \\
7.2 \\
7.0\end{array}$ & $\begin{array}{l}5.5 \\
5.5 \\
5.5 \\
5.5 \\
5.5\end{array}$ & $\begin{array}{l}31 \\
9.0 \\
7.2 \\
7.2 \\
7.0\end{array}$ & $\begin{array}{l}6.4 \\
7.2 \\
8.1 \\
7.4 \\
7.2\end{array}$ & $\begin{array}{l}14 \\
12 \\
55 \\
87 \\
73\end{array}$ \\
\hline $\begin{array}{l}26 \\
27 \\
28 \\
29 \\
30 \\
31\end{array}$ & $\begin{array}{l}7.2 \\
7.2 \\
8.1 \\
8.5 \\
7.6 \\
7.4\end{array}$ & $\begin{array}{l}6.4 \\
6.4 \\
6.4 \\
6.4 \\
6.4\end{array}$ & $\begin{array}{l}7.2 \\
7.2 \\
7.0 \\
7.2 \\
7.2 \\
7.2\end{array}$ & $\begin{array}{r}7.0 \\
52 \\
7.2 \\
7.0 \\
7.2 \\
7.4\end{array}$ & $\begin{array}{r}5.5 \\
+5.5 \\
+5.5 \\
+5.5 \\
5.5\end{array}$ & $\begin{array}{l}24 \\
8.1 \\
7.2 \\
9.0 \\
9.4 \\
8.5\end{array}$ & $\begin{array}{c}6.5 \\
5.7 \\
5.7 \\
20 \\
8.1\end{array}$ & $\begin{array}{l}64 \\
27 \\
16 \\
14 \\
14 \\
14\end{array}$ \\
\hline
\end{tabular}


Daily discharge, in second-feet, of Huleia River near Lihue, for 1912-13-Continued.

\begin{tabular}{|c|c|c|c|c|c|c|c|c|c|c|c|c|}
\hline Day. & Jan. & Feb. & Mar. & Apr. & May. & June. & July. & Aug. & Sept. & Oct. & Nov. & Dec. \\
\hline $\begin{array}{l}1913 . \\
1 \ldots \ldots \ldots \ldots \\
2 \ldots \ldots \ldots \ldots \\
3 \ldots \ldots \ldots \ldots \\
4 \ldots \ldots \ldots \ldots \\
5 \ldots \ldots \ldots \ldots .\end{array}$ & $\begin{array}{c}12 \\
11 \\
9.0 \\
8.1 \\
7.4\end{array}$ & $\begin{array}{l}7.2 \\
6.4 \\
5.7 \\
5.5 \\
7.2\end{array}$ & $\begin{array}{l}11 \\
11 \\
11 \\
10 \\
10\end{array}$ & $\begin{array}{l}26 \\
21 \\
18 \\
16 \\
15\end{array}$ & $\begin{array}{l}11 \\
9.7 \\
9.7 \\
9.7 \\
9.7\end{array}$ & $\begin{array}{c}9.7 \\
12 \\
191 \\
109 \\
80\end{array}$ & $\begin{array}{l}14 \\
46 \\
25 \\
15 \\
12\end{array}$ & $\begin{array}{r}7.7 \\
12 \\
9.7 \\
7.7 \\
7.7\end{array}$ & $\begin{array}{l}14 \\
11 \\
9.7 \\
8.1 \\
7.7\end{array}$ & $\begin{array}{l}6.0 \\
6.0 \\
6.0 \\
7.7 \\
9.9\end{array}$ & $\begin{array}{l}12 \\
67 \\
25 \\
20 \\
18\end{array}$ & $\begin{array}{l}70 \\
67 \\
73 \\
61 \\
55\end{array}$ \\
\hline $\begin{array}{r}6 \ldots \ldots \ldots \ldots \\
7 \ldots \ldots \ldots \\
8 \\
9 \\
90 \ldots \ldots \ldots \ldots\end{array}$ & $\begin{array}{l}7.4 \\
7.2 \\
7.2 \\
7.4 \\
8.1\end{array}$ & $\begin{array}{r}174 \\
\mathbf{5 8} \\
40 \\
37 \\
\mathbf{3 1}\end{array}$ & $\begin{array}{l}9.2 \\
8.3 \\
7.2 \\
7.2 \\
6.4\end{array}$ & $\begin{array}{l}15 \\
15 \\
18 \\
30 \\
25\end{array}$ & $\begin{array}{l}9.9 \\
9.9 \\
9.7 \\
9.7 \\
9.7\end{array}$ & $\begin{array}{r}61 \\
67 \\
124 \\
67 \\
55\end{array}$ & $\begin{array}{l}11 \\
10 \\
9.7 \\
8.7 \\
8.7\end{array}$ & $\begin{array}{l}7.7 \\
7.7 \\
7.7 \\
7.7 \\
7.7\end{array}$ & $\begin{array}{l}7.7 \\
7.7 \\
7.7 \\
7.7 \\
7.7\end{array}$ & $\begin{array}{r}11 \\
12 \\
173 \\
191 \\
44\end{array}$ & $\begin{array}{l}18 \\
21 \\
18 \\
18 \\
31\end{array}$ & $\begin{array}{l}52 \\
80 \\
70 \\
61 \\
52\end{array}$ \\
\hline & $\begin{array}{l}9.2 \\
10 \\
14 \\
14 \\
18\end{array}$ & \begin{tabular}{c|}
17 \\
12 \\
11 \\
9.6 \\
8.1
\end{tabular} & $\begin{array}{l}5.7 \\
5.5 \\
7.2 \\
8.1 \\
7.4\end{array}$ & $\begin{array}{l}23 \\
23 \\
28 \\
21 \\
20\end{array}$ & $\begin{array}{c}9.7 \\
44 \\
101 \\
55 \\
94\end{array}$ & $\begin{array}{l}28 \\
21 \\
18 \\
15 \\
12\end{array}$ & $\begin{array}{l}8.7 \\
7.9 \\
7.7 \\
7.7 \\
7.7\end{array}$ & $\begin{array}{l}7.7 \\
7.7 \\
7.7 \\
7.4 \\
6.8\end{array}$ & $\begin{array}{l}7.7 \\
7.7 \\
7.7 \\
7.7 \\
7.5\end{array}$ & $\begin{array}{l}18 \\
14 \\
12 \\
12 \\
11\end{array}$ & $\begin{array}{l}44 \\
55 \\
35 \\
25 \\
67\end{array}$ & $\begin{array}{l}28 \\
23 \\
18 \\
16 \\
15\end{array}$ \\
\hline $\begin{array}{l}16 \ldots \ldots \\
17 \ldots \ldots \\
18 \ldots \ldots \\
19 \ldots \ldots\end{array}$ & $\begin{array}{l}18 \\
22 \\
24 \\
24 \\
45\end{array}$ & $\begin{array}{l}8.1 \\
7.6 \\
7.2 \\
8.1 \\
9.0\end{array}$ & $\begin{array}{l}6.4 \\
5.8 \\
5.7 \\
5.5 \\
5.5\end{array}$ & $\begin{array}{l}18 \\
16 \\
15 \\
15 \\
14\end{array}$ & $\begin{array}{l}28 \\
25 \\
23 \\
22 \\
20\end{array}$ & $\begin{array}{c}11 \\
11 \\
11 \\
9.9 \\
9.7\end{array}$ & $\begin{array}{l}7.7 \\
77.7 \\
7.7 \\
77.7 \\
7.7\end{array}$ & $\begin{array}{l}6.2 \\
6.2 \\
6.0 \\
6.0 \\
6.0\end{array}$ & $\begin{array}{l}7.5 \\
7.5 \\
7.5 \\
6.8 \\
6.8\end{array}$ & $\begin{array}{l}11 \\
11 \\
23 \\
11 \\
11\end{array}$ & $\begin{array}{r}18 \\
39 \\
39 \\
25 \\
116\end{array}$ & $\begin{array}{l}14 \\
12 \\
12 \\
12 \\
12\end{array}$ \\
\hline $\begin{array}{l}21 \ldots \\
22 \ldots \\
23 \ldots \\
24 \ldots \\
25 \ldots\end{array}$ & $\begin{array}{l}61 \\
55 \\
55 \\
52 \\
17\end{array}$ & $\begin{array}{l}11 \\
12 \\
13 \\
40 \\
17\end{array}$ & $\begin{array}{c}5.5 \\
5.5 \\
272 \\
73 \\
44\end{array}$ & $\begin{array}{l}12 \\
11 \\
10 \\
9.7 \\
9.7\end{array}$ & $\begin{array}{l}19 \\
16 \\
15 \\
13 \\
9.7\end{array}$ & $\begin{array}{l}9.7 \\
9.3 \\
8.7 \\
8.7 \\
8.7\end{array}$ & $\begin{array}{l}7.7 \\
7.7 \\
7.7 \\
7.7 \\
7.7\end{array}$ & $\begin{array}{l}6.0 \\
6.0 \\
6.0 \\
6.0 \\
6.0\end{array}$ & $\begin{array}{l}8.1 \\
7.7 \\
6.8 \\
6.2 \\
6.0\end{array}$ & $\begin{array}{c}9.9 \\
9.9 \\
12 \\
21 \\
74\end{array}$ & $\begin{array}{r}132 \\
73 \\
94 \\
64 \\
108\end{array}$ & $\begin{array}{l}12 \\
12 \\
12 \\
12 \\
12\end{array}$ \\
\hline $\begin{array}{l}26 \ldots \\
27 \ldots \\
27 \ldots \\
29 \ldots \\
30 \ldots \\
31 \ldots\end{array}$ & $\begin{array}{c}16 \\
12 \\
11 \\
9.0 \\
7.4 \\
8.1\end{array}$ & $\begin{array}{c}15 \\
12 \\
12 \\
\cdots . . . \\
\cdots . .\end{array}$ & $\begin{array}{l}25 \\
23 \\
21 \\
22 \\
23 \\
25\end{array}$ & $\begin{array}{r}9.7 \\
9.7 \\
9.7 \\
9.7 \\
28\end{array}$ & $\begin{array}{c}10 \\
9.7 \\
9.7 \\
9.7 \\
9.7 \\
9.7\end{array}$ & $\begin{array}{l}8.1 \\
9.7 \\
11 \\
11 \\
12\end{array}$ & $\begin{array}{l}7.7 \\
7.7 \\
7.7 \\
7.7 \\
7.7 \\
7.7\end{array}$ & $\begin{array}{c}6.0 \\
6.0 \\
6.0 \\
14 \\
12 \\
9.7\end{array}$ & $\begin{array}{l}6.0 \\
6.0 \\
6.0 \\
6.0 \\
6.0\end{array}$ & $\begin{array}{l}70 \\
35 \\
25 \\
16 \\
15 \\
12\end{array}$ & $\begin{array}{l}94 \\
73 \\
61 \\
49 \\
49\end{array}$ & $\begin{array}{l}12 \\
12 \\
12 \\
12 \\
12 \\
12\end{array}$ \\
\hline
\end{tabular}

Monthly discharge of Huleia River near Lihue, Kauai, for 1912-13.

\begin{tabular}{|c|c|c|c|c|c|}
\hline \multirow{2}{*}{ Month. } & \multicolumn{3}{|c|}{ Discharge in second-feet. } & \multirow{2}{*}{$\begin{array}{c}\text { Run-off } \\
\text { (totalal in } \\
\text { acre-feet). }\end{array}$} & \multirow{2}{*}{$\begin{array}{l}\text { Accu- }- \\
\text { racy. }\end{array}$} \\
\hline & Maximum. & Minimum. & Mean. & & \\
\hline 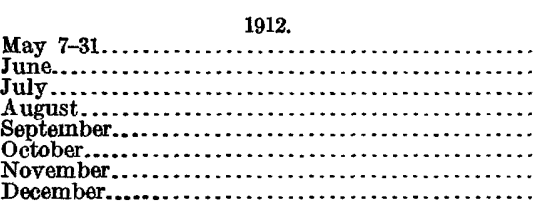 & $\begin{array}{c}9.0 \\
11 \\
40 \\
52 \\
61 \\
31 \\
40 \\
116\end{array}$ & $\begin{array}{l}6.5 \\
6.4 \\
5.5 \\
5.5 \\
5.5 \\
5.5 \\
5.7 \\
9.0\end{array}$ & $\begin{array}{r}7.40 \\
7.45 \\
8.56 \\
7.92 \\
7.96 \\
8.03 \\
8.42 \\
31.8\end{array}$ & $\begin{array}{r}367 \\
443 \\
526 \\
487 \\
474 \\
494 \\
501 \\
1,960 \\
\end{array}$ & $\begin{array}{l}\text { B. } \\
\text { B. } \\
\text { B. } \\
\text { B. } \\
\text { B. } \\
\text { B. } \\
\text { B. } \\
\text { B. }\end{array}$ \\
\hline The period.... & ......... & $\ldots \ldots \ldots$ & …....... & 5,250 & \\
\hline 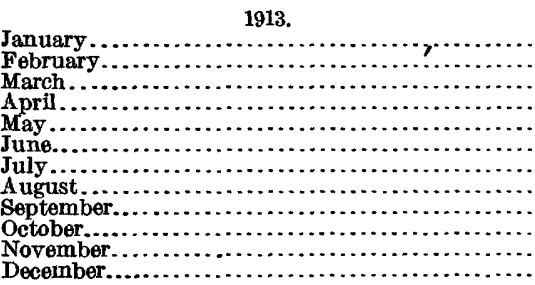 & $\begin{array}{r}61 \\
174 \\
272 \\
30 \\
101 \\
191 \\
46 \\
14 \\
14 \\
191 \\
132 \\
80\end{array}$ & $\begin{array}{r}7.2 \\
5.5 \\
5.5 \\
9.7 \\
9.7 \\
8.1 \\
7.7 \\
6.0 \\
6.0 \\
6.0 \\
12 \\
12\end{array}$ & $\begin{array}{r}18.9 \\
21.5 \\
22.4 \\
17.0 \\
21.0 \\
34.0 \\
10.4 \\
7.6 \\
7.6 \\
29.0 \\
50.3 \\
30.3\end{array}$ & $\begin{array}{r}1,160 \\
1,190 \\
1,380 \\
1,010 \\
\mathbf{1}, 290 \\
\mathbf{2 ,}, 020 \\
640 \\
\mathbf{4 4 0 7} \\
4452 \\
\mathbf{1 , 7 8 0} \\
\mathbf{2 1 , 9 9 0} \\
\mathbf{1 , 9 6 0}\end{array}$ & $\begin{array}{l}\text { C. } \\
\text { C: } \\
\text { C. } \\
\text { C. } \\
\text { C: } \\
C . \\
\text { C. } \\
\text { C. } \\
\text { C. }\end{array}$ \\
\hline The year....... & 272 & 5.5 & 22.4 & 16,200 & \\
\hline
\end{tabular}


HANAMAULU RIVER AT KAPAIA, NEAR LIHUE, KAUAI.

Location.-At wagon-road bridge about 600 feet north of village of Kapaia and about 1.5 miles north of Lihue.

Records available.-September 4, 1911, to December 31, 1913.

Drainage area. -6.41 square miles.

Gage.-Vertical staff.

Control.-Probably permanent.

Discharge measurements.--Made from lower side of bridge during high water and by wading at low-water stages.

Diversions.-Several above station.

Accuracy.-Records for May and June, good; for all other months, only fair.

Discharge measurements of Hanamaulu River at Kapaia, near Lihue, Kauai, in 1913.

\begin{tabular}{|c|c|c|c|c|c|c|c|}
\hline Date. & Hydrographer. & $\begin{array}{c}\text { Gage } \\
\text { height. }\end{array}$ & $\begin{array}{l}\text { Dis- } \\
\text { charge. }\end{array}$ & Date. & Hydrogfapher. & $\begin{array}{c}\text { Gage } \\
\text { height. }\end{array}$ & $\begin{array}{l}\text { Dis- } \\
\text { charge. }\end{array}$ \\
\hline $\begin{array}{l}\text { Jan. } 29 \\
\text { May } 31\end{array}$ & 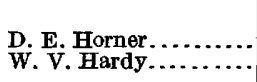 & $\begin{array}{c}\text { Feet. } \\
5.30 \\
5.54\end{array}$ & $\begin{array}{c}\text { Sec.-ft. } \\
12.3 \\
17.6\end{array}$ & $\begin{array}{l}\text { May } 31 \\
\text { May } 31\end{array}$ & $\begin{array}{l}\text { w. v. Hardy. } \\
\ldots . \text { do............. }\end{array}$ & $\begin{array}{r}\text { Feet. } \\
5.69 \\
5.62\end{array}$ & $\begin{array}{r}\text { Sec. } f t . \\
24.2 \\
21.6\end{array}$ \\
\hline
\end{tabular}

Daily discharge, in second-feet, of Hanamaulu River at Kapaia, Lihue, Kauai, for 1913.

\begin{tabular}{|c|c|c|c|c|c|c|c|c|c|c|c|c|}
\hline Day. & Jan. & Feb. & Mar. & Apr. & May. & June. & July. & Aug. & Sept. & Oct. & Nov. & Dec. \\
\hline $\begin{array}{l}1 \ldots \\
2 \ldots \\
3 \ldots \\
4 \ldots \\
5 \ldots\end{array}$ & $\begin{array}{l}25 \\
30 \\
23 \\
23 \\
19\end{array}$ & $\begin{array}{r}9.7 \\
9.2 \\
8.7 \\
11 \\
9.7\end{array}$ & $\begin{array}{c}20 \\
14 \\
8.5 \\
3.0 \\
1.5\end{array}$ & $\begin{array}{l}21 \\
19 \\
20 \\
16 \\
9.8\end{array}$ & $\begin{array}{l}6.5 \\
7.5 \\
8.1 \\
7.3 \\
6.5\end{array}$ & $\begin{array}{l}19 \\
18 \\
82 \\
35 \\
30\end{array}$ & $\begin{array}{l}5 \\
5 \\
8.5 \\
11 \\
14\end{array}$ & $\begin{array}{c}13 \\
13 \\
9.8 \\
6.5 \\
5.8\end{array}$ & $\begin{array}{l}1.8 \\
1.5 \\
4.0 \\
3.0 \\
2.5\end{array}$ & $\begin{array}{l}3.0 \\
3.0 \\
3.0 \\
3.0 \\
2.0\end{array}$ & $\begin{array}{l}47 \\
41 \\
35 \\
35 \\
30\end{array}$ & $\begin{array}{l}25 \\
20 \\
54 \\
25 \\
22\end{array}$ \\
\hline $\begin{array}{r}6 \ldots . . \\
7 \ldots \ldots \\
8 \ldots \ldots \\
9 \ldots \ldots \\
10 \ldots . .\end{array}$ & $\begin{array}{l}15 \\
11 \\
11 \\
8.7 \\
8.7\end{array}$ & $\begin{array}{l}96 \\
47 \\
29 \\
24 \\
18\end{array}$ & $\begin{array}{l}4.2 \\
3.4 \\
4.6 \\
4.7 \\
4.8\end{array}$ & $\begin{array}{r}8.5 \\
8.5 \\
9.8 \\
11 \\
8.5\end{array}$ & $\begin{array}{l}5.0 \\
1.0 \\
5.0 \\
5.0 \\
6.5\end{array}$ & $\begin{array}{l}41 \\
35 \\
33 \\
31 \\
36\end{array}$ & $\begin{array}{r}10 \\
5 \\
4 \\
5 \\
5\end{array}$ & $\begin{array}{l}5.8 \\
5.6 \\
5.3 \\
5.0 \\
3.2\end{array}$ & $\begin{array}{l}4.0 \\
3.0 \\
2.0 \\
1.8 \\
1.8\end{array}$ & $\begin{array}{r}1.0 \\
.5 \\
1.0 \\
21 \\
7.5\end{array}$ & $\begin{array}{r}34 \\
22 \\
21 \\
12 \\
3\end{array}$ & $\begin{array}{l}28 \\
24 \\
20 \\
13 \\
11\end{array}$ \\
\hline $\begin{array}{l}11 \\
12 \\
13 \\
14 \\
15\end{array}$ & $\begin{array}{l}7.7 \\
12 \\
17 \\
12 \\
11\end{array}$ & $\begin{array}{l}9.8 \\
8.1 \\
7.5 \\
14 \\
16\end{array}$ & $\begin{array}{l}3.8 \\
3.8 \\
4.6 \\
5.0 \\
4.6\end{array}$ & $\begin{array}{l}9.8 \\
7.5 \\
6.2 \\
5.0 \\
2.5\end{array}$ & $\begin{array}{l}18 \\
29 \\
41 \\
30 \\
41\end{array}$ & $\begin{array}{l}25 \\
13 \\
14 \\
20 \\
18\end{array}$ & $\begin{array}{c}4 \\
5 \\
10 \\
16 \\
7.5\end{array}$ & $\begin{array}{l}1.5 \\
3.0 \\
3.0 \\
1.5 \\
1.0\end{array}$ & $\begin{array}{l}2.0 \\
2.0 \\
1.8 \\
1.9 \\
2.0\end{array}$ & $\begin{array}{l}2.0 \\
2.5 \\
3.0 \\
1.5 \\
13\end{array}$ & $\begin{array}{l}3.5 \\
9.8 \\
16 \\
11 \\
3\end{array}$ & $\begin{array}{l}11 \\
11 \\
16 \\
14 \\
12\end{array}$ \\
\hline $\begin{array}{l}16 \ldots \ldots \\
17 \ldots \ldots \\
19 \\
20 \ldots \ldots\end{array}$ & $\begin{array}{c}9.7 \\
9.7 \\
11 \\
10 \\
9.7\end{array}$ & $\begin{array}{l}14 \\
13 \\
13 \\
20 \\
13\end{array}$ & $\begin{array}{l}5.6 \\
6.5 \\
5.8 \\
4.2 \\
4.4\end{array}$ & $\begin{array}{c}5.0 \\
16 \\
6.5 \\
5.0 \\
3.8\end{array}$ & $\begin{array}{l}30 \\
41 \\
36 \\
30 \\
24\end{array}$ & $\begin{array}{r}17 \\
16 \\
14 \\
25 \\
5\end{array}$ & $\begin{array}{c}13 \\
17 \\
9.8 \\
6.5 \\
7\end{array}$ & $\begin{array}{l}1.5 \\
2.8 \\
4.0 \\
1.0 \\
3.0\end{array}$ & $\begin{array}{l}2.5 \\
3.0 \\
2.0 \\
1.5 \\
1.0\end{array}$ & $\begin{array}{l}8.5 \\
6.5 \\
25 \\
19 \\
13\end{array}$ & $\begin{array}{r}4 \\
5 \\
20 \\
30 \\
61\end{array}$ & $\begin{array}{l}11 \\
13 \\
8.5 \\
9.8 \\
9.8\end{array}$ \\
\hline $\begin{array}{l}21 \\
22 \\
24 \\
25\end{array}$ & $\begin{array}{l}9.7 \\
15 \\
12 \\
12 \\
11\end{array}$ & $\begin{array}{l}16 \\
16 \\
26 \\
35 \\
16\end{array}$ & $\begin{array}{l}5.0 \\
4.6 \\
12 \\
19 \\
20\end{array}$ & $\begin{array}{l}2.5 \\
2.5 \\
5.0 \\
7.5 \\
8.5\end{array}$ & $\begin{array}{l}25 \\
30 \\
24 \\
30 \\
28\end{array}$ & $\begin{array}{l}1 \\
(a)^{5} \\
(a) \\
(a)\end{array}$ & $\begin{array}{l}7.5 \\
6.5 \\
5 \\
4 \\
5\end{array}$ & $\begin{array}{l}1.0 \\
1.2 \\
1.5 \\
1.5 \\
1.5\end{array}$ & $\begin{array}{l}4.2 \\
7.5 \\
5.8 \\
6.5 \\
2.0\end{array}$ & $\begin{array}{l}14 \\
11 \\
25 \\
54 \\
25\end{array}$ & $\begin{array}{r}168 \\
47 \\
41 \\
35 \\
35\end{array}$ & $\begin{array}{l}7.4 \\
5.0 \\
8.5 \\
9.8 \\
9.2\end{array}$ \\
\hline $\begin{array}{l}26 \ldots \ldots \ldots \\
27 \ldots \ldots \ldots \\
28 \ldots \ldots \ldots \\
30 \ldots \ldots \\
31 \ldots \ldots \\
\end{array}$ & $\begin{array}{c}12 \\
12 \\
9.7 \\
12 \\
12 \\
9.9\end{array}$ & \begin{tabular}{c}
19 \\
25 \\
22 \\
$\cdots \ldots .$. \\
\hdashline$\ldots .$. \\
$\ldots .$.
\end{tabular} & $\begin{array}{l}22 \\
20 \\
12 \\
12 \\
13 \\
19\end{array}$ & $\begin{array}{c}6.5 \\
10 \\
14 \\
7.5 \\
7.5 \\
\cdots \cdots\end{array}$ & $\begin{array}{l}25 \\
22 \\
15 \\
7.5 \\
11 \\
20\end{array}$ & $\begin{array}{c}(a) \\
5 \\
12 \\
8 \\
5\end{array}$ & $\begin{array}{l}7.5 \\
7.5 \\
7.5 \\
7.1 \\
9.2 \\
8.5\end{array}$ & $\begin{array}{c}1.5 \\
1.6 \\
3.5 \\
16 \\
19 \\
10\end{array}$ & $\begin{array}{r}2.5 \\
2.5 \\
7.8 \\
13 \\
4.0\end{array}$ & $\begin{array}{l}25 \\
25 \\
30 \\
25 \\
44 \\
41\end{array}$ & $\begin{array}{l}34 \\
28 \\
30 \\
24 \\
24 \\
\cdots \cdots\end{array}$ & $\begin{array}{l}8.5 \\
7.5 \\
7.0 \\
6.5 \\
11 \\
11\end{array}$ \\
\hline
\end{tabular}

a No discharge June 23-26.

NoтE.-Discharge estimated for Sundays and holidays, on which no observations were made. 
Monthly discharge of Hanamaulu River at Kapaia, near Lihue, Kauai, for 1913.

\begin{tabular}{|c|c|c|c|c|c|}
\hline \multirow{2}{*}{ Month. } & \multicolumn{3}{|c|}{ Discharge in second-feet. } & \multirow{2}{*}{$\begin{array}{c}\text { Rum-off } \\
\text { (total in } \\
\text { acre-feet). }\end{array}$} & \multirow{2}{*}{$\begin{array}{l}\text { Accu } \\
\text { racy. }\end{array}$} \\
\hline & Maximum. & Minimum. & Mean. & & \\
\hline 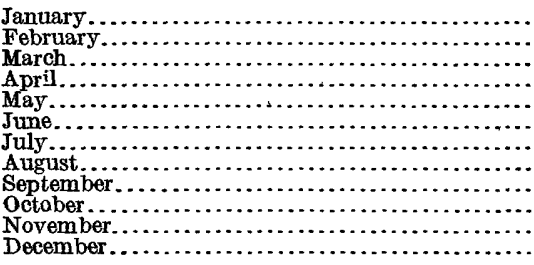 & $\begin{array}{r}30 \\
96 \\
22 \\
21 \\
41 \\
82 \\
17 \\
19 \\
13 \\
54 \\
168 \\
54\end{array}$ & $\begin{array}{r}7.7 \\
7.5 \\
1.5 \\
2.5 \\
1.0 \\
\text { (a) } \\
4.0 \\
1.0 \\
1.0 \\
.5 \\
3.0 \\
5.0\end{array}$ & $\begin{array}{r}13.3 \\
2.2 \\
8.89 \\
9.03 \\
19.9 \\
18.6 \\
7.86 \\
4.95 \\
3.36 \\
14.8 \\
30.3 \\
14.5\end{array}$ & $\begin{array}{r}818 \\
1,120 \\
547 \\
537 \\
1,220 \\
1,110 \\
483 \\
304 \\
200 \\
910 \\
1,800 \\
892\end{array}$ & $\begin{array}{l}\text { B. } \\
\text { B. } \\
\text { B. } \\
\text { A. } \\
\text { A. } \\
\text { B. } \\
\text { B. } \\
\text { B. } \\
\text { C. } \\
\text { C. }\end{array}$ \\
\hline The year....... & 168 & (a) & 13.7 & 9,940 & \\
\hline
\end{tabular}

$a$ Channel dry.

SOUTH FORK OF WAILUA RIVER ABOVE WAIEHU FALLS, NEAR LIHUE, KAUAI.

Location.-One mile above Waiehu Falls and about 7 miles northeast of Lihue.

Records available.-December 10, 1911, to December 31, 1913.

Drainage area.-22.4 square miles.

Gage.-Friez water-stage recorder.

Control.-Probably permanent.

Discharge measurements.-Made from cable or by wading.

Diversions.-Several above station.

Accuracy.-Records good except for November and December, when they were only fair.

Discharge measurements of South Fork of Wailua River above Waiehu Falls, near Lihue, Kauai, in 1913.

\begin{tabular}{|c|c|c|c|c|c|c|c|}
\hline Date. & Hydrographer. & $\begin{array}{c}\text { Gage } \\
\text { height. }\end{array}$ & $\begin{array}{c}\text { Dis- } \\
\text { charge. }\end{array}$ & Date. & Hydrographer. & $\begin{array}{c}\text { Gage } \\
\text { height. }\end{array}$ & $\begin{array}{c}\text { Dis- } \\
\text { charge. }\end{array}$ \\
\hline Feb. $\begin{array}{r}23 \\
23\end{array}$ & $\begin{array}{c}\text { D. E. Horner.. } \\
\text {. do.............. }\end{array}$ & $\begin{array}{r}\text { Feet. } \\
3.61 \\
3.56\end{array}$ & $\begin{array}{r}\text { Sec.-ft. } \\
79.0 \\
76.6\end{array}$ & May 30 & W. V. Hardy ... & $\begin{array}{c}\text { Feet. } \\
4.12\end{array}$ & Sec.-ft. \\
\hline
\end{tabular}


Daily discharge, in second-feet, of South Fork of Wailua River above Waiehu Falls, near Lihue, Kauai, for 1913 .

\begin{tabular}{|c|c|c|c|c|c|c|c|c|c|c|c|c|}
\hline Day. & Jan. & Feb. & Mar. & Apr. & May. & June. & July. & Aug. & Sept. & Oct. & Nov. & Dec. \\
\hline $\begin{array}{l}1 \ldots \ldots . \\
2 \ldots \ldots . \\
3 \ldots \ldots \\
4 \ldots \ldots \\
5 \ldots \ldots\end{array}$ & $\begin{array}{l}87 \\
66 \\
56 \\
\mathbf{5 4} \\
\mathbf{5 4}\end{array}$ & $\begin{array}{l}58 \\
50 \\
42 \\
40 \\
40\end{array}$ & $\begin{array}{l}54 \\
53 \\
51 \\
45 \\
42\end{array}$ & $\begin{array}{r}127 \\
118 \\
.113 \\
111 \\
83\end{array}$ & $\begin{array}{r}116 \\
204 \\
162 \\
131 \\
96\end{array}$ & $\begin{array}{r}58 \\
68 \\
1,150 \\
295 \\
204\end{array}$ & $\begin{array}{l}192 \\
453 \\
162 \\
173 \\
192\end{array}$ & \begin{tabular}{r|}
129 \\
80 \\
45 \\
40 \\
37
\end{tabular} & $\begin{array}{r}204 \\
270 \\
225 \\
98 \\
91\end{array}$ & $\begin{array}{l}15 \\
19 \\
23 \\
25 \\
23\end{array}$ & $\begin{array}{l}109 \\
116 \\
105 \\
105 \\
106\end{array}$ & $\begin{array}{l}\cdots \cdots \cdot \\
\cdots \cdots \cdots \\
\cdots \cdots \cdots \\
\cdots \cdots \cdots\end{array}$ \\
\hline $\begin{array}{r}6 \ldots \ldots \ldots \\
7 \ldots \ldots \ldots \\
8 \ldots \ldots \ldots \\
9 \ldots \ldots \ldots \\
10 \ldots \ldots \ldots\end{array}$ & $\begin{array}{r}66 \\
56 \\
239 \\
116 \\
228\end{array}$ & $\begin{array}{l}608 \\
363 \\
184 \\
109 \\
109\end{array}$ & $\begin{array}{r}40 \\
124 \\
98 \\
74 \\
64\end{array}$ & $\begin{array}{r}72 \\
60 \\
58 \\
157 \\
72\end{array}$ & $\begin{array}{l}173 \\
173 \\
384 \\
150 \\
116\end{array}$ & $\begin{array}{l}189 \\
135 \\
109 \\
127 \\
116\end{array}$ & $\begin{array}{l}98 \\
76 \\
72 \\
76 \\
60\end{array}$ & $\begin{array}{l}37 \\
48 \\
45 \\
43 \\
42\end{array}$ & $\begin{array}{r}201 \\
138 \\
80 \\
66 \\
58\end{array}$ & $\begin{array}{r}109 \\
94 \\
838 \\
774 \\
232\end{array}$ & $\begin{array}{l}107 \\
108 \\
109 \\
335 \\
\mathbf{4 1 8}\end{array}$ & $\begin{array}{l}\cdots \\
1328 \\
128\end{array}$ \\
\hline $\begin{array}{l}11 \ldots \ldots \\
12 \ldots \ldots \\
13 \ldots \ldots \\
14 \ldots \ldots \\
15 . \ldots \ldots\end{array}$ & $\begin{array}{l}184 \\
292 \\
760 \\
242 \\
593\end{array}$ & $\begin{array}{r}120 \\
87 \\
74 \\
68 \\
94\end{array}$ & $\begin{array}{l}60 \\
56 \\
96 \\
96 \\
53\end{array}$ & $\begin{array}{r}72 \\
48 \\
343 \\
256 \\
253\end{array}$ & $\begin{array}{l}295 \\
677 \\
663 \\
225 \\
462\end{array}$ & $\begin{array}{l}154 \\
105 \\
154 \\
138 \\
120\end{array}$ & $\begin{array}{r}56 \\
60 \\
154 \\
83 \\
76\end{array}$ & $\begin{array}{l}40 \\
53 \\
87 \\
80 \\
66\end{array}$ & $\begin{array}{l}53 \\
46 \\
40 \\
42 \\
56\end{array}$ & $\begin{array}{l}195 \\
164 \\
242 \\
167 \\
207\end{array}$ & $\begin{array}{r}792 \\
1,060 \\
925 \\
375 \\
267\end{array}$ & $\begin{array}{l}135 \\
130 \\
130 \\
119 \\
116\end{array}$ \\
\hline $\begin{array}{l}16 \ldots \ldots \\
17 \ldots \ldots \\
18 \ldots \ldots \\
19 \ldots \ldots \\
20 \ldots \ldots\end{array}$ & $\begin{array}{r}458 \\
181 \\
142 \\
120 \\
98\end{array}$ & $\begin{array}{r}116 \\
66 \\
98 \\
133 \\
94\end{array}$ & $\begin{array}{l}45 \\
89 \\
64 \\
68 \\
97\end{array}$ & $\begin{array}{r}118 \\
239 \\
219 \\
150 \\
98\end{array}$ & $\begin{array}{l}288 \\
246 \\
256 \\
152 \\
127\end{array}$ & $\begin{array}{r}160 \\
98 \\
107 \\
184 \\
111\end{array}$ & $\begin{array}{r}72 \\
66 \\
56 \\
68 \\
107\end{array}$ & $\begin{array}{l}46 \\
40 \\
36 \\
38 \\
40\end{array}$ & $\begin{array}{l}46 \\
40 \\
37 \\
40 \\
\mathbf{3 4}\end{array}$ & $\begin{array}{l}152 \\
157 \\
181 \\
131 \\
107\end{array}$ & $\begin{array}{l}253 \\
219 \\
204 \\
962 \\
\ldots \ldots\end{array}$ & $\begin{array}{r}112 \\
110 \\
112 \\
101 \\
88\end{array}$ \\
\hline $\begin{array}{l}21 \ldots \ldots \ldots \ldots \ldots \\
22, \ldots \ldots \ldots \ldots \ldots \\
24 \ldots \ldots \\
25 \ldots \ldots \ldots \ldots\end{array}$ & $\begin{array}{l}144 \\
449 \\
187 \\
359 \\
154\end{array}$ & $\begin{array}{r}80 \\
72 \\
76 \\
162 \\
74\end{array}$ & $\begin{array}{r}98 \\
102 \\
210 \\
142 \\
184\end{array}$ & $\begin{array}{r}94 \\
78 \\
138 \\
80 \\
288\end{array}$ & $\begin{array}{r}116 \\
107 \\
87 \\
100 \\
78\end{array}$ & $\begin{array}{r}105 \\
56 \\
56 \\
48 \\
53\end{array}$ & $\begin{array}{r}70 \\
54 \\
48 \\
54 \\
138\end{array}$ & \begin{tabular}{r|r}
150 & \\
64 & \\
48 & \\
45 & \\
74 &
\end{tabular} & $\begin{array}{l}26 \\
38 \\
13 \\
19 \\
24\end{array}$ & $\begin{array}{l}189 \\
253 \\
127 \\
219 \\
162\end{array}$ & $\therefore$ & $\begin{array}{l}92 \\
92 \\
92 \\
88 \\
85\end{array}$ \\
\hline $\begin{array}{l}26 \ldots \ldots \\
27 \ldots \ldots \\
28 \ldots \\
29 . \ldots \\
30 \ldots \\
31 \ldots\end{array}$ & $\begin{array}{r}105 \\
105 \\
64 \\
100 \\
56 \\
76\end{array}$ & $\begin{array}{r}62 \\
60 \\
74 \\
\ldots \\
\ldots .\end{array}$ & $\begin{array}{r}135 \\
60 \\
66 \\
120 \\
98 \\
87\end{array}$ & $\begin{array}{r}120 \\
83 \\
83 \\
118 \\
225 \\
\\
\end{array}$ & $\begin{array}{r}72 \\
74 \\
74 \\
76 \\
201 \\
76\end{array}$ & $\begin{array}{r}94 \\
201 \\
195 \\
109 \\
113\end{array}$ & $\begin{array}{r}242 \\
120 \\
76 \\
131 \\
78 \\
74\end{array}$ & \begin{tabular}{r|}
60 \\
43 \\
98 \\
278 \\
120 \\
274
\end{tabular} & $\begin{array}{c}24 \\
14 \\
14 \\
14 \\
17 \\
\ldots . .\end{array}$ & $\begin{array}{l}131 \\
109 \\
213 \\
124 \\
131 \\
131\end{array}$ & 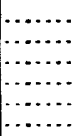 & $\begin{array}{l}88 \\
79 \\
85 \\
66 \\
68 \\
66\end{array}$ \\
\hline
\end{tabular}

Note.-Evidences point to a maximum discharge of about 4,500 second-feet on the night of Nov. 20 . No record Nov. 20 to Dec. 9. Discharge interpolated Nov. 5-7.

Monthly discharge of South Fork of Wailua River above Waiehu Falls, near Lihue, Kauai, for 1913.

\begin{tabular}{|c|c|c|c|c|c|}
\hline \multirow{2}{*}{ Month. } & \multicolumn{3}{|c|}{ Discharge in second-feet. } & \multirow{2}{*}{$\begin{array}{c}\text { Run-off } \\
\text { (total in } \\
\text { acre-feet). }\end{array}$} & \multirow{2}{*}{$\begin{array}{l}\text { Accu- } \\
\text { racy. }\end{array}$} \\
\hline & Maximum. & Minimum. & Mean. & & \\
\hline 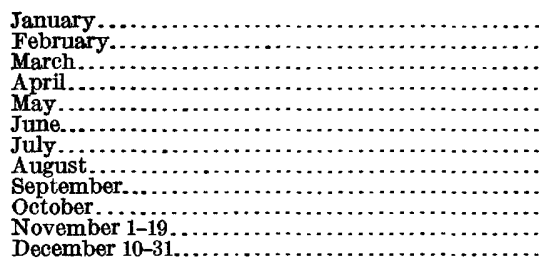 & $\begin{array}{r}760 \\
608 \\
210 \\
343 \\
677 \\
1,150 \\
453 \\
278 \\
270 \\
838 \\
1,060 \\
135\end{array}$ & $\begin{array}{r}54 \\
40 \\
40 \\
48 \\
72 \\
48 \\
48 \\
36 \\
13 \\
15 \\
105 \\
66\end{array}$ & $\begin{array}{l}190 \\
115 \\
85.8 \\
136 \\
199 \\
160 \\
111 \\
75 \\
68.9 \\
182 \\
351 \\
99.2\end{array}$ & $\begin{array}{r}11,700 \\
6,390 \\
5,280 \\
8,090 \\
12,200 \\
9,520 \\
6,820 \\
4,610 \\
4,100 \\
11,200 \\
13,200 \\
4,320\end{array}$ & $\begin{array}{l}\text { A. } \\
\text { A. } \\
\text { A. } \\
\text { A. } \\
\text { A. } \\
\text { A. } \\
\text { A. } \\
\text { A. } \\
\text { B. }\end{array}$ \\
\hline The period... & $\ldots$ & 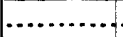 & $\ldots \ldots$ & 97,400 & \\
\hline
\end{tabular}




\section{HANAMAULU DITCH NEAR LIHUE, KaUAI.}

Location.-About 6 miles northwest of Lihue, in flume 180 feet above point where the Kauai Electric Co.'s power line crosses the South Fork of Wailua River.

Records available.-July 1, 1910, to December 31, 1913.

Gage.-Vertical staff installed September 30, 1911, on left side of flume, 18 feet from upper end; new datum.

Control.-Probably permanent.

Discharge measurements.-Made in flume.

Diversion.-Ditch diverts part of flow of the South Fork of Wailua River.

Accuracy.-Records very good.

The following discharge measurement was made by D. E. Horner:

January 31, 1913: Gage height, 2.14 feet; discharge, 38 second-feet.

Daily discharge, in second-feet, of Hanamaulu ditch near Lihue, Kauai, for 1913.

\begin{tabular}{|c|c|c|c|c|c|c|c|c|c|c|c|c|}
\hline Day. & Jan. & Feb. & Mar. & Apr. & May. & June. & July. & Aug. & Sept. & Oct. & Nov. & Dec. \\
\hline $\begin{array}{l}1 \ldots \ldots \\
2 \ldots \ldots \\
3 \ldots \ldots \\
4 \ldots \ldots \\
5 \ldots .\end{array}$ & $\begin{array}{l}38 \\
38 \\
38 \\
27 \\
27\end{array}$ & $\begin{array}{l}35 \\
38 \\
44 \\
42 \\
42\end{array}$ & $\begin{array}{l}28 \\
28 \\
27 \\
27 \\
41\end{array}$ & $\begin{array}{l}32 \\
32 \\
32 \\
32 \\
30\end{array}$ & $\begin{array}{l}32 \\
32 \\
41 \\
41 \\
40\end{array}$ & $\begin{array}{c}41 \\
41 \\
33 \\
9.2 \\
9.2\end{array}$ & $\begin{array}{l}40 \\
39 \\
39 \\
40 \\
40\end{array}$ & $\begin{array}{l}38 \\
36 \\
36 \\
38 \\
38\end{array}$ & $\begin{array}{l}37 \\
38 \\
38 \\
37 \\
37\end{array}$ & $\begin{array}{l}41 \\
38 \\
34 \\
32 \\
32\end{array}$ & $\begin{array}{l}24 \\
23 \\
24 \\
15 \\
15\end{array}$ & 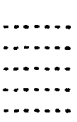 \\
\hline $\begin{array}{r}6 \ldots \ldots \\
7 \ldots \ldots \\
8 \ldots \ldots \\
9 \ldots \ldots \\
10 \ldots \ldots\end{array}$ & $\begin{array}{l}27 \\
32 \\
32 \\
35 \\
35\end{array}$ & $\begin{array}{l}47 \\
41 \\
32 \\
32 \\
14\end{array}$ & $\begin{array}{l}41 \\
42 \\
42 \\
41 \\
41\end{array}$ & $\begin{array}{l}29 \\
29 \\
29 \\
32 \\
32\end{array}$ & $\begin{array}{l}40 \\
41 \\
40 \\
40 \\
40\end{array}$ & $\begin{array}{c}9.2 \\
9.2 \\
40 \\
39 \\
39\end{array}$ & $\begin{array}{l}40 \\
40 \\
40 \\
39 \\
39\end{array}$ & $\begin{array}{l}56 \\
38 \\
38 \\
38 \\
35\end{array}$ & $\begin{array}{l}39 \\
40 \\
40 \\
38 \\
38\end{array}$ & $\begin{array}{l}41 \\
47 \\
47 \\
33 \\
32\end{array}$ & $\begin{array}{l}15 \\
15 \\
15 \\
15 \\
15\end{array}$ & $\begin{array}{r}\cdots \ldots . . \\
\cdots \cdots \cdots \\
\cdots \\
\cdots\end{array}$ \\
\hline $\begin{array}{l}13 \ldots \ldots \\
14 \ldots \ldots \\
15 \ldots \ldots\end{array}$ & $\begin{array}{l}36 \\
41 \\
41 \\
40 \\
40\end{array}$ & $\begin{array}{l}14 \\
32 \\
30 \\
30 \\
30\end{array}$ & $\begin{array}{l}41 \\
38 \\
36 \\
36 \\
36\end{array}$ & $\begin{array}{l}40 \\
40 \\
41 \\
40 \\
40\end{array}$ & $\begin{array}{l}\mathbf{3 2} \\
\mathbf{3 2} \\
\mathbf{3 2} \\
\mathbf{3 2} \\
\mathbf{2 2}\end{array}$ & $\begin{array}{l}38 \\
39 \\
39 \\
39 \\
39\end{array}$ & $\begin{array}{l}38 \\
38 \\
36 \\
36 \\
36\end{array}$ & $\begin{array}{l}35 \\
35 \\
40 \\
40 \\
40\end{array}$ & $\begin{array}{l}38 \\
40 \\
40 \\
36 \\
35\end{array}$ & $\begin{array}{l}41 \\
41 \\
35 \\
34 \\
34\end{array}$ & $\begin{array}{l}15 \\
11 \\
11 \\
12 \\
15\end{array}$ & $\begin{array}{l}12 \\
12 \\
32 \\
32 \\
32\end{array}$ \\
\hline $\begin{array}{l}16 \ldots \ldots \\
17 \ldots \ldots \ldots \\
19 \ldots \ldots \ldots \\
20 \ldots \ldots\end{array}$ & $\begin{array}{l}39 \\
38 \\
38 \\
38 \\
38\end{array}$ & $\begin{array}{l}30 \\
30 \\
30 \\
28 \\
28\end{array}$ & $\begin{array}{l}38 \\
38 \\
40 \\
40 \\
40\end{array}$ & $\begin{array}{l}40 \\
40 \\
40 \\
40 \\
38\end{array}$ & $\begin{array}{l}22 \\
22 \\
15 \\
15 \\
14\end{array}$ & $\begin{array}{l}39 \\
39 \\
39 \\
39 \\
35\end{array}$ & $\begin{array}{l}36 \\
36 \\
36 \\
36 \\
40\end{array}$ & $\begin{array}{l}40 \\
39 \\
39 \\
39 \\
39\end{array}$ & $\begin{array}{l}\mathbf{3 5} \\
35 \\
35 \\
36 \\
\mathbf{3 6}\end{array}$ & $\begin{array}{l}36 \\
36 \\
36 \\
24 \\
35\end{array}$ & $\begin{array}{l}15 \\
32 \\
32 \\
32 \\
33\end{array}$ & $\begin{array}{l}32 \\
32 \\
24 \\
23 \\
23\end{array}$ \\
\hline $\begin{array}{l}21 \ldots \ldots \\
22, \ldots \ldots \\
23 \ldots \ldots \\
24 \ldots \ldots \\
25 \ldots \ldots\end{array}$ & $\begin{array}{l}38 \\
47 \\
47 \\
40 \\
40\end{array}$ & $\begin{array}{l}28 \\
28 \\
28 \\
32 \\
29\end{array}$ & $\begin{array}{l}40 \\
40 \\
42 \\
24 \\
40\end{array}$ & $\begin{array}{l}38 \\
36 \\
38 \\
38 \\
47\end{array}$ & $\begin{array}{l}29 \\
29 \\
29 \\
29 \\
29\end{array}$ & $\begin{array}{l}35 \\
38 \\
38 \\
38 \\
41\end{array}$ & $\begin{array}{l}40 \\
35 \\
35 \\
44 \\
44\end{array}$ & $\begin{array}{l}40 \\
39 \\
38 \\
38 \\
38\end{array}$ & $\begin{array}{l}36 \\
40 \\
40 \\
40 \\
40\end{array}$ & $\begin{array}{l}36 \\
36 \\
36 \\
32 \\
32\end{array}$ & 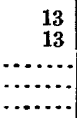 & $\begin{array}{l}23 \\
23 \\
23 \\
23 \\
23\end{array}$ \\
\hline $\begin{array}{l}26 \ldots \ldots \\
27 \ldots \ldots \\
28 \ldots \ldots \\
29 \ldots \ldots \\
30 \ldots \ldots \\
31 \ldots \ldots\end{array}$ & $\begin{array}{l}38 \\
38 \\
38 \\
37 \\
36 \\
36\end{array}$ & $\begin{array}{r}28 \\
28 \\
28 \\
\cdots \cdots \\
\cdots\end{array}$ & $\begin{array}{l}40 \\
\mathbf{3 2} \\
\mathbf{3 2} \\
\mathbf{3 2} \\
\mathbf{3 2} \\
\mathbf{3 2}\end{array}$ & $\begin{array}{l}\mathbf{3 4} \\
\mathbf{3 4} \\
\mathbf{3 2} \\
\mathbf{3 2} \\
\mathbf{3 2}\end{array}$ & $\begin{array}{l}29 \\
\mathbf{3 8} \\
\mathbf{3 8} \\
\mathbf{3 9} \\
\mathbf{4 1} \\
\mathbf{4 1}\end{array}$ & $\begin{array}{l}40 \\
40 \\
40 \\
40 \\
40 \\
\cdots \cdots\end{array}$ & $\begin{array}{l}45 \\
41 \\
41 \\
36 \\
36 \\
36\end{array}$ & $\begin{array}{l}38 \\
38 \\
38 \\
40 \\
37 \\
37\end{array}$ & $\begin{array}{l}39 \\
38 \\
38 \\
39 \\
38\end{array}$ & $\begin{array}{l}32 \\
32 \\
33 \\
32 \\
32 \\
32\end{array}$ & $\begin{array}{r}\cdots \\
\hdashline \\
\hdashline\end{array}$ & $\begin{array}{l}\mathbf{2 3} \\
\mathbf{2 3} \\
\mathbf{3 2} \\
\mathbf{3 2} \\
\mathbf{3 2} \\
\mathbf{3 2}\end{array}$ \\
\hline
\end{tabular}

Note.-Ditch dry, Nov. 23-28 and Dec. 1-9. 
Monthly discharge of Hanamaulu ditch near Lihue, Kauan, for 1913.

\begin{tabular}{|c|c|c|c|c|c|}
\hline \multirow{2}{*}{ Month. } & \multicolumn{3}{|c|}{ Discharge in second-feet. } & \multirow{2}{*}{$\begin{array}{l}\text { Run-off } \\
\text { (total im } \\
\text { acre-feet). }\end{array}$} & \multirow{2}{*}{$\begin{array}{l}\text { Acct- } \\
\text { racy. }\end{array}$} \\
\hline & Maximum. & Minimum. & Mean. & & \\
\hline 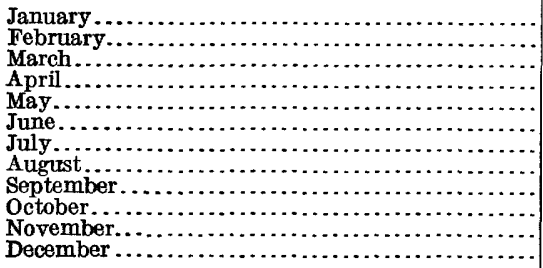 & $\begin{array}{l}47 \\
47 \\
42 \\
47 \\
41 \\
41 \\
45 \\
56 \\
40 \\
47 \\
33 \\
32\end{array}$ & $\begin{array}{l}27 \\
14 \\
24 \\
29 \\
14 \\
9.2 \\
35 \\
35 \\
35 \\
24 \\
0 \\
0\end{array}$ & $\begin{array}{l}37.2 \\
31.4 \\
36.3 \\
35.6 \\
32.1 \\
34.8 \\
38.6 \\
38.6 \\
37.9 \\
35.3 \\
14.0 \\
17.9\end{array}$ & $\begin{array}{r}2,290 \\
1,740 \\
2,230 \\
2,120 \\
1,970 \\
2,070 \\
2,370 \\
2,370 \\
2,260 \\
2,170 \\
832 \\
1,100\end{array}$ & $\begin{array}{l}\text { A. } \\
\text { A. } \\
\text { A. } \\
\text { A. } \\
\text { A. } \\
\text { A. } \\
\text { A. } \\
\text { A. } \\
\text { A. } \\
\text { A. } \\
\text { A. } \\
\text { A. }\end{array}$ \\
\hline The year............ & 56 & 0 & 32.5 & 23,500 & \\
\hline
\end{tabular}

LIHUE DITCH NEAR LIHUE, KAUAI.

Iocation.-About 5 miles northwest of Lihue, at point where Kauai Electric Co.'s power line crosses the ditch.

Records available.-July 1, 1910, to December 31, 1913.

Gage.-Vertical staff.

Control.-Probably permanent.

Discharge measurements.-Made by wading.

Diversion.-Ditch diverts part of flow of the South Fork of Wailua River.

Accuracy.-Records fair.

The following discharge measurement was made by W. V. Hardy and D. E. Forner:

May 29, 1913: Gage height, 1.42 feet; discharge, 8.93 second-feet.

Daily discharge, in second-feet, of Lihue ditch near Lihue, Kauai, for 1913.

\begin{tabular}{|c|c|c|c|c|c|c|c|c|c|c|c|c|}
\hline Day. & Jan. & Feb. & Mar. & Apr. & May. & June. & July. & Aug. & Sept. & Oct. & Nov. & Dec. \\
\hline $\begin{array}{l}1 \ldots \\
2 \ldots \\
3 \ldots \\
4 \ldots \\
5 . .\end{array}$ & $\begin{array}{l}8.8 \\
8.8 \\
8.4 \\
8.4 \\
8.4\end{array}$ & $\begin{array}{l}7.2 \\
7.2 \\
7.1 \\
6.8 \\
6.5\end{array}$ & $\begin{array}{l}3.8 \\
3.8 \\
3.8 \\
3.8 \\
3.8\end{array}$ & $\begin{array}{l}8.8 \\
8.8 \\
8.0 \\
8.0 \\
8.0\end{array}$ & $\begin{array}{l}10.4 \\
10.4 \\
10.4 \\
10.4 \\
10.4\end{array}$ & $\begin{array}{c}9.2 \\
9.6 \\
3.7 \\
7.7\end{array}$ & $\begin{array}{l}9.6 \\
9.6 \\
9.2 \\
9.5 \\
9.4\end{array}$ & $\begin{array}{r}9.8 \\
10.0 \\
9.3 \\
9.1 \\
9.0\end{array}$ & $\begin{array}{l}10.2 \\
10.8 \\
10.7 \\
10.0 \\
11.2\end{array}$ & $\begin{array}{r}10.2 \\
10.0 \\
9.6 \\
9.4 \\
9.4\end{array}$ & $\begin{array}{l}8.8 \\
8.8 \\
9.0 \\
8.8 \\
8.0\end{array}$ & $\begin{array}{l}\cdots \cdot \\
\cdots \cdots \\
\cdots \cdots \\
\cdots \cdots \\
\cdots\end{array}$ \\
\hline $\begin{array}{r}6 . . \\
7 . . \\
8 . . \\
9 . \\
10 .\end{array}$ & $\begin{array}{l}8.4 \\
8.4 \\
9.6 \\
9.6 \\
9.8\end{array}$ & \begin{tabular}{c}
9.6 \\
$\cdots . .$. \\
\hdashline... \\
\end{tabular} & $\begin{array}{l}7.2 \\
7.2 \\
7.2 \\
7.2 \\
7.2\end{array}$ & $\begin{array}{l}7.6 \\
7.6 \\
7.2 \\
7.6 \\
7.6\end{array}$ & $\begin{array}{r}10.4 \\
10.4 \\
10.4 \\
10.4 \\
9.6\end{array}$ & $\begin{array}{l}7.2 \\
7.2 \\
9.0 \\
9.0 \\
9.0\end{array}$ & $\begin{array}{l}9.4 \\
9.4 \\
9.4 \\
9.0 \\
9.0\end{array}$ & $\begin{array}{l}9.0 \\
8.8 \\
8.8 \\
9.8 \\
8.9\end{array}$ & $\begin{array}{l}11.1 \\
10.4 \\
10.4 \\
10.2 \\
10.2\end{array}$ & $\begin{array}{r}9.9 \\
10.9 \\
12.0 \\
10.4 \\
9.9\end{array}$ & $\begin{array}{l}6.6 \\
6.6 \\
6.6 \\
6.5 \\
6.5\end{array}$ & $\begin{array}{l}\cdots \cdot \\
\cdots \cdots \\
\cdots \cdots \\
\cdots \cdots\end{array}$ \\
\hline $\begin{array}{l}13 . . \\
14 . \\
15 . .\end{array}$ & $\begin{array}{r}10.0 \\
10.4 \\
10.6 \\
8.8 \\
8.8\end{array}$ & $\begin{array}{l}4.4 \\
4.4 \\
4.0 \\
3.9\end{array}$ & $\begin{array}{l}7.2 \\
7.2 \\
7.2 \\
7.2 \\
7.2\end{array}$ & $\begin{array}{l}8.0 \\
8.0 \\
8.8 \\
8.8 \\
8.8\end{array}$ & $\begin{array}{r}9.6 \\
10.4 \\
8.0 \\
9.6 \\
9.6\end{array}$ & $\begin{array}{l}9.0 \\
9.8 \\
9.8 \\
9.8 \\
9.8\end{array}$ & $\begin{array}{l}8.8 \\
9.2 \\
9.4 \\
9.4 \\
9.4\end{array}$ & $\begin{array}{r}8.8 \\
8.8 \\
10.0 \\
9.6 \\
9.4\end{array}$ & $\begin{array}{r}9.6 \\
9.6 \\
10.4 \\
10.1 \\
10.1\end{array}$ & $\begin{array}{l}11.4 \\
11.4 \\
11.2 \\
10.6 \\
10.6\end{array}$ & $\begin{array}{l}6.7 \\
7.4 \\
7.5 \\
7.4 \\
7.4\end{array}$ & .... \\
\hline $\begin{array}{l}16 . . \\
17 . \\
18 . . \\
19 . . \\
20 . .\end{array}$ & $\begin{array}{l}8.8 \\
8.8 \\
8.8 \\
8.4 \\
8.4\end{array}$ & $\begin{array}{l}3.8 \\
3.8 \\
3.8 \\
3.6 \\
3.6\end{array}$ & $\begin{array}{l}7.6 \\
7.6 \\
8.0 \\
8.0 \\
8.0\end{array}$ & $\begin{array}{l}8.8 \\
8.8 \\
8.8 \\
8.8 \\
8.0\end{array}$ & $\begin{array}{l}3.7 \\
3.7 \\
3.7 \\
3.7 \\
3.7\end{array}$ & $\begin{array}{l}9.8 \\
9.8 \\
9.8 \\
9.8 \\
9.1\end{array}$ & $\begin{array}{l}9.4 \\
9.4 \\
9.4 \\
9.4 \\
9.8\end{array}$ & $\begin{array}{r}10.0 \\
9.6 \\
9.0 \\
9.6 \\
9.8\end{array}$ & $\begin{array}{l}9.6 \\
9.6 \\
9.6 \\
9.6 \\
9.5\end{array}$ & $\begin{array}{r}10.4 \\
10.6 \\
10.6 \\
3.7 \\
10.4\end{array}$ & $\begin{array}{l}7.4 \\
8.0 \\
8.0 \\
8.0 \\
8.0\end{array}$ & $\begin{array}{l}2.5 \\
2.5 \\
2.5 \\
2.5\end{array}$ \\
\hline $\begin{array}{l}21 \ldots \ldots \\
22 \ldots \ldots \\
23 \ldots \ldots \\
24 \ldots \ldots \\
25 \ldots \ldots\end{array}$ & $\begin{array}{r}8.4 \\
10.4 \\
8.8 \\
8.8 \\
8.0\end{array}$ & $\begin{array}{l}4.0 \\
4.0 \\
3.9 \\
4.4 \\
4.0\end{array}$ & $\begin{array}{l}8.4 \\
8.5 \\
8.8 \\
8.0 \\
8.4\end{array}$ & $\begin{array}{l}8.9 \\
8.0 \\
8.0 \\
8.0 \\
8.8\end{array}$ & $\begin{array}{l}3.7 \\
8.0 \\
8.0 \\
8.0 \\
8.0\end{array}$ & $\begin{array}{l}9.0 \\
9.0 \\
9.0 \\
9.0 \\
9.0\end{array}$ & $\begin{array}{r}9.8 \\
8.9 \\
8.9 \\
9.8 \\
10.4\end{array}$ & $\begin{array}{r}10.4 \\
10.4 \\
10.2 \\
9.6 \\
9.5\end{array}$ & $\begin{array}{r}10.1 \\
10.1 \\
10.1 \\
9.8 \\
9.6\end{array}$ & $\begin{array}{r}10.4 \\
10.9 \\
10.0 \\
9.8 \\
9.9\end{array}$ & $\begin{array}{l}8.0 \\
6.4 \\
6.4 \\
6.4 \\
6.3\end{array}$ & $\begin{array}{l}6.9 \\
6.9 \\
6.9 \\
7.0 \\
7.1\end{array}$ \\
\hline $\begin{array}{l}26 \ldots \ldots \ldots \\
27 \ldots \ldots \\
28 \ldots \ldots \\
29 \ldots \ldots \\
30 \ldots \ldots \\
31 \ldots \ldots\end{array}$ & $\begin{array}{l}7.6 \\
7.2 \\
7.2 \\
7.2 \\
7.2 \\
7.2\end{array}$ & \begin{tabular}{r}
3.8 \\
3.8 \\
3.8 \\
$\cdots \ldots .$. \\
\hdashline$\ldots . .$. \\
$\cdots . .$.
\end{tabular} & $\begin{array}{l}8.0 \\
6.8 \\
6.8 \\
6.8 \\
8.8 \\
8.8\end{array}$ & $\begin{array}{l}10.4 \\
10.4 \\
10.4 \\
10.4 \\
10.4\end{array}$ & $\begin{array}{l}9.6 \\
9.6 \\
9.6 \\
8.8 \\
8.9 \\
9.6\end{array}$ & $\begin{array}{l}9.7 \\
9.7 \\
9.8 \\
9.8 \\
9.6\end{array}$ & $\begin{array}{r}10.4 \\
10.4 \\
10.0 \\
9.9 \\
9.8 \\
9.8\end{array}$ & $\begin{array}{r}9.6 \\
9.6 \\
9.8 \\
10.4 \\
10.8 \\
10.6\end{array}$ & $\begin{array}{r}9.6 \\
9.4 \\
10.2 \\
10.0 \\
10.0\end{array}$ & $\begin{array}{r}10.2 \\
10.4 \\
10.0 \\
10.0 \\
9.6 \\
9.6\end{array}$ & $\begin{array}{l}6.2 \\
6.2 \\
6.4 \\
6.4 \\
7.0 \\
\ldots \ldots\end{array}$ & $\begin{array}{l}7.1 \\
7.2 \\
7.2 \\
7.2 \\
7.2 \\
7.2\end{array}$ \\
\hline
\end{tabular}

Note.-Ditch dry Feb. 7-11, June 4, Dec. 1-16.

$97764^{\circ}-$ wSP $373-15-4$ 
Monthly discharge of Lithue ditch near Lihue, Kauai, for 1913.

\begin{tabular}{|c|c|c|c|c|c|}
\hline \multirow{2}{*}{ Month. } & \multicolumn{3}{|c|}{ Discharge in second-feet. } & \multirow{2}{*}{$\begin{array}{l}\text { Run-off } \\
\text { (total in } \\
\text { acre-feet). }\end{array}$} & \multirow{2}{*}{$\begin{array}{l}\text { Accu- } \\
\text { racy. }\end{array}$} \\
\hline & Maximum. & Minimum. & Mean. & & \\
\hline 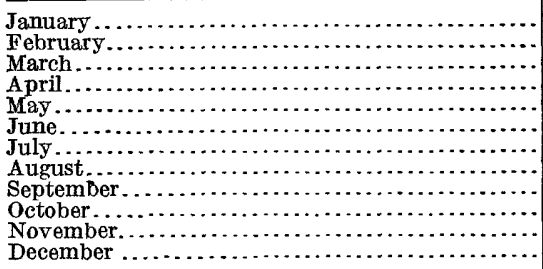 & $\begin{array}{r}10.6 \\
9.6 \\
8.8 \\
10.4 \\
10.4 \\
9.8 \\
10.4 \\
10.8 \\
11.2 \\
12.0 \\
9.0 \\
7.2\end{array}$ & $\begin{array}{l}7.2 \\
0 \\
3.8 \\
7.2 \\
3.7 \\
0 \\
8.8 \\
8.8 \\
9.4 \\
3.7 \\
6.2 \\
0\end{array}$ & $\begin{array}{l}8.66 \\
3.98 \\
7.04 \\
8.59 \\
8.41 \\
8.63 \\
9.52 \\
9.61 \\
10.1 \\
10.1 \\
7.28 \\
2.84\end{array}$ & $\begin{array}{l}532 \\
221 \\
433 \\
511 \\
517 \\
513 \\
585 \\
591 \\
601 \\
621 \\
432 \\
175\end{array}$ & $\begin{array}{l}\text { B. } \\
\text { B. } \\
\text { B. } \\
\text { B. } \\
\text { B. } \\
\text { B. } \\
\text { B. } \\
\text { B. } \\
\text { B. } \\
\text { B. } \\
\text { B. } \\
\text { B. }\end{array}$ \\
\hline The year. . . . . . . . . . . . . . . & 12.0 & 0 & 7.92 & 5,730 & \\
\hline
\end{tabular}

NORTH FORK OF WAILUA RIVER NEAR LIHUE, KAUAI.

Location.-About 12 miles north of Lihue and 300 feet below confluence of main and east branches of the stream.

Records available.-August 1 to October 28, 1910; December 28, 1910, to December 31, 1913.

Drainage area. -14.6 square miles.

Gage.-Friez automatic water-stage recorder. Datum changed.

Control.-Fairly permanent.

Discharge measurements.-Made from wire suspension footbridge.

Diversions. - Kanaha ditch diverts part of flow above the station.

Accuracy.-Records fair from January to May; all other records good.

The following discharge measurement was made by W. V. Hardy:

May 29, 1913: Gage height, 0.52 foot; discharge, 39.7 feet.

Daily discharge, in second-feet, of North Fork of Wailua River near Lihue, Kauai, for 1913.

\begin{tabular}{|c|c|c|c|c|c|c|c|c|c|c|c|c|}
\hline Day. & Jan. & Feb. & Mar. & Apr. & May. & June. & July. & Aug. & Sept. & Oct. & Nov. & Dec. \\
\hline $\begin{array}{l}1 \ldots . \\
2 \ldots \\
3 \ldots \ldots \\
4 \ldots \\
5 \ldots .\end{array}$ & $\begin{array}{l}57 \\
54 \\
43 \\
36 \\
38\end{array}$ & $\begin{array}{l}27 \\
24 \\
23 \\
23 \\
22\end{array}$ & $\begin{array}{l}58 \\
85 \\
84 \\
81 \\
75\end{array}$ & $\begin{array}{l}81 \\
62 \\
64 \\
51 \\
35\end{array}$ & $\begin{array}{r}39 \\
104 \\
54 \\
40 \\
30\end{array}$ & $\begin{array}{r}40 \\
58 \\
.551 \\
146 \\
106\end{array}$ & $\begin{array}{r}95 \\
267 \\
111 \\
119 \\
104\end{array}$ & $\begin{array}{l}42 \\
36 \\
34 \\
32 \\
32\end{array}$ & $\begin{array}{r}95 \\
182 \\
150 \\
77 \\
60\end{array}$ & $\begin{array}{l}33 \\
30 \\
27 \\
26 \\
25\end{array}$ & $\begin{array}{r}54 \\
116 \\
77 \\
57 \\
52\end{array}$ & $\begin{array}{l}202 \\
168 \\
220 \\
213 \\
224\end{array}$ \\
\hline $\begin{array}{r}6 \ldots \\
7 \ldots \\
8 \ldots \\
9 \ldots \\
10 \ldots\end{array}$ & $\begin{array}{r}37 \\
48 \\
130 \\
57 \\
108\end{array}$ & $\begin{array}{r}217 \\
132 \\
67 \\
50 \\
49\end{array}$ & $\begin{array}{r}66 \\
111 \\
44 \\
22 \\
17\end{array}$ & $\begin{array}{l}26 \\
23 \\
23 \\
51 \\
35\end{array}$ & $\begin{array}{r}88 \\
85 \\
106 \\
52 \\
41\end{array}$ & $\begin{array}{r}109 \\
85 \\
70 \\
92 \\
84\end{array}$ & $\begin{array}{l}78 \\
68 \\
56 \\
47 \\
43\end{array}$ & $\begin{array}{l}32 \\
35 \\
38 \\
42 \\
36\end{array}$ & $\begin{array}{r}111 \\
83 \\
60 \\
54 \\
44\end{array}$ & $\begin{array}{r}62 \\
57 \\
368 \\
178 \\
97\end{array}$ & $\begin{array}{r}57 \\
84 \\
62 \\
184 \\
213\end{array}$ & $\begin{array}{l}200 \\
146 \\
124 \\
111 \\
103\end{array}$ \\
\hline $\begin{array}{l}11 \ldots \ldots \\
12 \ldots \ldots \\
13 \ldots \ldots \\
14 \ldots \ldots \\
15 \ldots \ldots\end{array}$ & $\begin{array}{l}111 \\
162 \\
353 \\
128 \\
366\end{array}$ & $\begin{array}{l}50 \\
39 \\
35 \\
33 \\
46\end{array}$ & $\begin{array}{l}16 \\
15 \\
19 \\
18 \\
22\end{array}$ & $\begin{array}{r}43 \\
27 \\
130 \\
156 \\
147\end{array}$ & $\begin{array}{r}140 \\
232 \\
177 \\
111 \\
90\end{array}$ & $\begin{array}{l}92 \\
63 \\
88 \\
70 \\
64\end{array}$ & $\begin{array}{l}42 \\
47 \\
90 \\
63 \\
58\end{array}$ & $\begin{array}{l}32 \\
50 \\
47 \\
64 \\
41\end{array}$ & $\begin{array}{l}41 \\
42 \\
38 \\
36 \\
38\end{array}$ & $\begin{array}{r}120 \\
106 \\
144 \\
111 \\
84\end{array}$ & $\begin{array}{l}394 \\
590 \\
394 \\
173 \\
127\end{array}$ & $\begin{array}{l}92 \\
90 \\
85 \\
78 \\
76\end{array}$ \\
\hline $\begin{array}{l}16 \ldots \\
17 \ldots \\
18 \ldots \\
19 \ldots \\
20 \ldots\end{array}$ & $\begin{array}{r}192 \\
102 \\
78 \\
73 \\
64\end{array}$ & $\begin{array}{r}43 \\
34 \\
66 \\
52 \\
65\end{array}$ & $\begin{array}{l}25 \\
29 \\
32 \\
36 \\
39\end{array}$ & $\begin{array}{r}71 \\
153 \\
106 \\
81 \\
59\end{array}$ & $\begin{array}{r}84 \\
98 \\
109 \\
73 \\
66\end{array}$ & $\begin{array}{l}70 \\
49 \\
54 \\
65 \\
60\end{array}$ & $\begin{array}{l}54 \\
44 \\
36 \\
51 \\
77\end{array}$ & $\begin{array}{l}63 \\
42 \\
37 \\
49 \\
34\end{array}$ & $\begin{array}{l}36 \\
32 \\
32 \\
30 \\
29\end{array}$ & $\begin{array}{l}88 \\
74 \\
77 \\
57 \\
49\end{array}$ & $\begin{array}{r}106 \\
98 \\
100 \\
262 \\
353\end{array}$ & $\begin{array}{l}73 \\
71 \\
71 \\
69 \\
59\end{array}$ \\
\hline $\begin{array}{l}21 \ldots \ldots \\
22 \ldots \\
24 \ldots \ldots \\
25 \ldots \ldots \\
25 \ldots\end{array}$ & $\begin{array}{r}122 \\
196 \\
85 \\
142 \\
84\end{array}$ & $\begin{array}{l}52 \\
43 \\
58 \\
72 \\
42\end{array}$ & $\begin{array}{r}43 \\
53 \\
58 \\
229 \\
222\end{array}$ & $\begin{array}{r}54 \\
51 \\
61 \\
40 \\
132\end{array}$ & $\begin{array}{l}58 \\
49 \\
46 \\
64 \\
49\end{array}$ & $\begin{array}{l}55 \\
38 \\
38 \\
34 \\
42\end{array}$ & $\begin{array}{l}43 \\
37 \\
36 \\
38 \\
83\end{array}$ & $\begin{array}{l}58 \\
42 \\
34 \\
34 \\
45\end{array}$ & $\begin{array}{l}34 \\
40 \\
26 \\
28 \\
26\end{array}$ & $\begin{array}{r}146 \\
132 \\
78 \\
155 \\
83\end{array}$ & $\begin{array}{l}659 \\
424 \\
343 \\
289 \\
504\end{array}$ & $\begin{array}{l}62 \\
54 \\
47 \\
44 \\
42\end{array}$ \\
\hline $\begin{array}{l}26 \ldots \ldots \\
27 \ldots \ldots \\
28 \ldots \\
29 \ldots \\
30 \ldots \\
31 \ldots \ldots\end{array}$ & $\begin{array}{l}70 \\
65 \\
54 \\
47 \\
42 \\
39\end{array}$ & \begin{tabular}{r}
35 \\
31 \\
30 \\
\hdashline$\quad \ldots$ \\
$\quad 3$
\end{tabular} & $\begin{array}{r}172 \\
92 \\
72 \\
65 \\
53 \\
50\end{array}$ & $\begin{array}{l}49 \\
38 \\
34 \\
37 \\
82\end{array}$ & $\begin{array}{l}42 \\
45 \\
47 \\
47 \\
87 \\
45\end{array}$ & $\begin{array}{r}66 \\
97 \\
114 \\
69 \\
98\end{array}$ & $\begin{array}{r}108 \\
71 \\
40 \\
85 \\
43 \\
38\end{array}$ & $\begin{array}{r}39 \\
35 \\
88 \\
171 \\
88 \\
116\end{array}$ & $\begin{array}{l}26 \\
26 \\
58 \\
30 \\
29\end{array}$ & $\begin{array}{r}65 \\
66 \\
112 \\
68 \\
69 \\
63\end{array}$ & $\begin{array}{l}420 \\
257 \\
218 \\
570 \\
303\end{array}$ & $\begin{array}{l}42 \\
41 \\
40 \\
36 \\
32 \\
35\end{array}$ \\
\hline
\end{tabular}

Discharge estimated Mar. 15-20. 
Monthly discharge of North Fork of Wailua River near Lihue, Kauai, for 1913.

\begin{tabular}{|c|c|c|c|c|c|}
\hline \multirow{2}{*}{ Month. } & \multicolumn{3}{|c|}{ Discharge in second-feet. } & \multirow{2}{*}{$\begin{array}{c}\text { Run-off } \\
\text { (total in } \\
\text { acre-feet). }\end{array}$} & \multirow{2}{*}{$\begin{array}{l}\text { Aceu- } \\
\text { racy. }\end{array}$} \\
\hline & Maximum. & Minimum. & Mean. & & \\
\hline 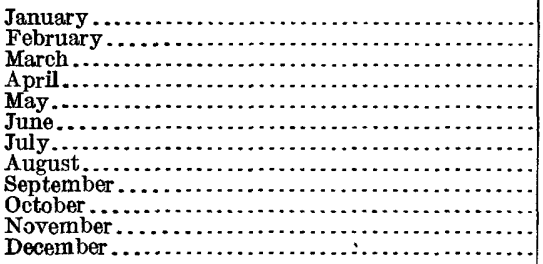 & $\begin{array}{l}366 \\
217 \\
229 \\
156 \\
232 \\
551 \\
267 \\
171 \\
182 \\
368 \\
659 \\
224\end{array}$ & $\begin{array}{l}36 \\
22 \\
15 \\
23 \\
30 \\
34 \\
36 \\
32 \\
26 \\
25 \\
52 \\
32\end{array}$ & $\begin{array}{r}103 \\
52.1 \\
64.6 \\
66.7 \\
77.3 \\
88.9 \\
70.1 \\
50.6 \\
53.1 \\
91.9 \\
251 \\
95.2\end{array}$ & $\begin{array}{r}6,330 \\
\mathbf{2}, 890 \\
3,970 \\
\mathbf{3}, 970 \\
\mathbf{4}, \mathbf{7 5 0} \\
5,290 \\
\mathbf{4}, \mathbf{3 1 0} \\
\mathbf{3}, \mathbf{1 1 0} \\
\mathbf{3}, \mathbf{1 6 0} \\
\mathbf{5}, 650 \\
\mathbf{1 4}, 900 \\
\mathbf{5}, 850\end{array}$ & $\begin{array}{l}\text { B. } \\
\text { B. } \\
\text { B. } \\
\text { B. } \\
\text { B. } \\
\text { A. } \\
\text { A. } \\
\text { A. } \\
\text { A. } \\
\text { A. } \\
\text { A. } \\
\text { A. }\end{array}$ \\
\hline The year.......... & 659 & 15 & 88.7 & 64,200 & \\
\hline
\end{tabular}

\section{KANAHA DITCH NEAR LIHUE, KAUAI.}

Location.-About 9 miles north of Lihue and about 500 feet above the point where the Kauai Electric Co.'s power line crosses the ditch.

Records available.-August 6, 1910, to December 31, 1913.

Gage.-Vertical staff; datum changed.

Discharge measurements.-Made in flume 100 feet above gage.

Diversion.-Diverts part of flow of North Fork of Wailua River.

Accuracy.-Records good.

Discharge measurements of Kanaha ditch near Lihue, Kauai, in 1913.

\begin{tabular}{|c|c|c|c|}
\hline Date. & Hydrographer. & $\begin{array}{c}\text { Gage } \\
\text { height. }\end{array}$ & $\begin{array}{l}\text { Dis- } \\
\text { charge. }\end{array}$ \\
\hline $\begin{array}{l}\text { May } 28 a \\
\text { May } 30 a\end{array}$ & $\begin{array}{l}\text { D. E. Horner. } \\
\text { W. } \\
\text {. Hardy } \\
\text { Hen }\end{array}$ & $\begin{array}{l}\text { Feet. } \\
2.02 \\
2.43\end{array}$ & $\begin{array}{r}\text { Sec. ft. } \\
27.9 \\
\quad 33.2\end{array}$ \\
\hline
\end{tabular}

a Measurements refer to gage installed May 28. 
Daily discharge, in second-feet, of Kanaha ditch near Lihue, Kauai, for 1912-13.

\begin{tabular}{|c|c|c|c|c|c|c|c|c|c|c|c|c|}
\hline Day. & Jan. & Feb. & Mar. & Apr. & May. & June. & July. & Aug. & Sept. & Oct. & Nov. & Dec. \\
\hline $\begin{array}{l}1 \ldots . \\
2 \ldots . \\
3 \ldots . \\
4 \ldots \\
5 \ldots .\end{array}$ & $\begin{array}{l}24 \\
24\end{array}$ & $\begin{array}{l}21 \\
20 \\
20 \\
20 \\
20\end{array}$ & $\begin{array}{l}25 \\
25 \\
28 \\
27 \\
25\end{array}$ & $\begin{array}{l}25 \\
24 \\
24 \\
25 \\
28\end{array}$ & $\begin{array}{l}28 \\
27 \\
28 \\
33 \\
30\end{array}$ & $\begin{array}{l}28 \\
27 \\
27 \\
27 \\
27\end{array}$ & $\begin{array}{l}28 \\
28 \\
28 \\
28 \\
28\end{array}$ & $\begin{array}{l}27 \\
30 \\
30 \\
30 \\
30\end{array}$ & $\begin{array}{l}35 \\
35 \\
33 \\
33 \\
33\end{array}$ & $\begin{array}{l}31 \\
30 \\
31 \\
31 \\
34\end{array}$ & $\begin{array}{l}30 \\
31 \\
31 \\
31 \\
35\end{array}$ & $\begin{array}{l}32 \\
35 \\
33 \\
11 \\
11\end{array}$ \\
\hline $\begin{array}{r}6 \ldots \\
7 \ldots \\
8 \ldots \\
9 \ldots \\
10 \ldots\end{array}$ & $\ldots$ & $\begin{array}{l}19 \\
19 \\
19 \\
25 \\
24\end{array}$ & $\begin{array}{l}25 \\
28 \\
27 \\
28 \\
28\end{array}$ & $\begin{array}{l}27 \\
30 \\
30 \\
30 \\
28\end{array}$ & $\begin{array}{l}28 \\
27 \\
30 \\
28 \\
27\end{array}$ & $\begin{array}{l}28 \\
28 \\
28 \\
28 \\
28\end{array}$ & $\begin{array}{l}28 \\
31 \\
33 \\
28 \\
33\end{array}$ & $\begin{array}{l}39 \\
31 \\
33 \\
33 \\
33\end{array}$ & $\begin{array}{l}35 \\
33 \\
33 \\
33 \\
33\end{array}$ & $\begin{array}{l}32 \\
32 \\
33 \\
32 \\
32\end{array}$ & $\begin{array}{l}35 \\
33 \\
33 \\
35 \\
34\end{array}$ & $\begin{array}{l}11 \\
10 \\
11 \\
11 \\
22\end{array}$ \\
\hline$\cdots$ & $\ldots$ & $\begin{array}{l}24 \\
24 \\
24 \\
22 \\
22\end{array}$ & $\begin{array}{l}31 \\
31 \\
31 \\
30 \\
28\end{array}$ & $\begin{array}{l}27 \\
27 \\
25 \\
25 \\
25\end{array}$ & $\begin{array}{l}26 \\
25 \\
25 \\
28 \\
28\end{array}$ & $\begin{array}{l}28 \\
28 \\
28 \\
28 \\
28\end{array}$ & $\begin{array}{l}33 \\
33 \\
33 \\
35 \\
31\end{array}$ & $\begin{array}{l}33 \\
33 \\
33 \\
35 \\
31\end{array}$ & $\begin{array}{l}33 \\
30 \\
30 \\
30 \\
31\end{array}$ & $\begin{array}{l}33 \\
33 \\
32 \\
31 \\
31\end{array}$ & $\begin{array}{l}32 \\
30 \\
30 \\
30 \\
33\end{array}$ & $\begin{array}{l}22 \\
22 \\
22 \\
21 \\
21\end{array}$ \\
\hline$\cdots$ & $\ldots$ & $\begin{array}{l}22 \\
24 \\
24 \\
24 \\
24\end{array}$ & $\begin{array}{l}27 \\
27 \\
26 \\
25 \\
25\end{array}$ & $\begin{array}{l}27 \\
31 \\
22 \\
21 \\
21\end{array}$ & $\begin{array}{l}28 \\
28 \\
28 \\
28 \\
28\end{array}$ & $\begin{array}{l}28 \\
28 \\
27 \\
27 \\
27\end{array}$ & $\begin{array}{l}28 \\
33 \\
39 \\
31 \\
31\end{array}$ & $\begin{array}{l}33 \\
39 \\
31 \\
31 \\
31\end{array}$ & $\begin{array}{l}32 \\
30 \\
28 \\
28 \\
31\end{array}$ & $\begin{array}{l}30 \\
34 \\
33 \\
34 \\
34\end{array}$ & $\begin{array}{l}29 \\
29 \\
32 \\
33 \\
34\end{array}$ & $\begin{array}{l}21 \\
27 \\
27 \\
30 \\
31\end{array}$ \\
\hline $24 \ldots$ & $\begin{array}{l}21 \\
22 \\
22 \\
22\end{array}$ & $\begin{array}{l}22 \\
25 \\
25 \\
25 \\
28\end{array}$ & $\begin{array}{l}28 \\
28 \\
28 \\
22 \\
24\end{array}$ & $\begin{array}{l}16 \\
16 \\
16 \\
25 \\
28\end{array}$ & $\begin{array}{l}28 \\
28 \\
28 \\
29 \\
29\end{array}$ & $\begin{array}{l}28 \\
30 \\
30 \\
28 \\
27\end{array}$ & $\begin{array}{l}31 \\
28 \\
28 \\
28 \\
30\end{array}$ & $\begin{array}{l}31 \\
39 \\
39 \\
39 \\
35\end{array}$ & $\begin{array}{l}31 \\
32 \\
31 \\
30 \\
28\end{array}$ & $\begin{array}{l}34 \\
34 \\
34 \\
33 \\
33\end{array}$ & $\begin{array}{c}34 \\
35 \\
\ldots . \\
\cdots \\
\cdots\end{array}$ & $\begin{array}{l}29 \\
30 \\
29 \\
30 \\
28\end{array}$ \\
\hline $\begin{array}{l}26 \ldots \ldots \ldots \\
27 \ldots \ldots \ldots \\
28 \ldots \ldots \ldots \\
29 \ldots \ldots \ldots \\
31 \ldots \ldots \ldots \\
31 \ldots \ldots \ldots\end{array}$ & $\begin{array}{l}21 \\
21 \\
21 \\
19 \\
19 \\
19\end{array}$ & $\begin{array}{l}28 \\
28 \\
25 \\
25\end{array}$ & $\begin{array}{l}24 \\
24 \\
22 \\
22 \\
25 \\
25\end{array}$ & $\begin{array}{l}24 \\
27 \\
27 \\
28 \\
28\end{array}$ & $\begin{array}{l}29 \\
29 \\
31 \\
31 \\
30 \\
28\end{array}$ & $\begin{array}{l}27 \\
30 \\
28 \\
27 \\
27\end{array}$ & $\begin{array}{l}31 \\
31 \\
31 \\
39 \\
33 \\
28\end{array}$ & $\begin{array}{l}33 \\
33 \\
33 \\
33 \\
35 \\
35\end{array}$ & $\begin{array}{l}28 \\
28 \\
27 \\
31 \\
32\end{array}$ & $\begin{array}{l}35 \\
34 \\
34 \\
33 \\
31 \\
32\end{array}$ & $\begin{array}{l}11 \\
11 \\
12 \\
33 \\
30\end{array}$ & $\begin{array}{l}27 \\
27 \\
24 \\
22 \\
22 \\
22\end{array}$ \\
\hline 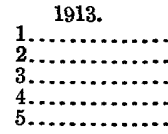 & $\begin{array}{l}22 \\
22 \\
21 \\
22 \\
22\end{array}$ & $\begin{array}{l}24 \\
24 \\
23 \\
23 \\
22\end{array}$ & $\begin{array}{l}12 \\
12 \\
12 \\
11 \\
11\end{array}$ & $\begin{array}{l}31 \\
31 \\
31 \\
31 \\
30\end{array}$ & $\begin{array}{l}31 \\
33 \\
33 \\
33 \\
32\end{array}$ & $\begin{array}{l}26 \\
30 \\
30 \\
26 \\
26\end{array}$ & $\begin{array}{l}32 \\
34 \\
32 \\
31 \\
31\end{array}$ & $\begin{array}{l}29 \\
31 \\
31 \\
28 \\
28\end{array}$ & $\begin{array}{l}34 \\
31 \\
31 \\
31 \\
31\end{array}$ & $\begin{array}{l}28 \\
28 \\
26 \\
26 \\
26\end{array}$ & $\begin{array}{l}31 \\
31 \\
31 \\
26 \\
25\end{array}$ & \\
\hline $\begin{array}{r}6 \ldots \ldots . \\
7 \ldots \ldots . \\
8 \ldots \ldots \\
9 \ldots \ldots \\
10 \ldots \ldots\end{array}$ & $\begin{array}{l}22 \\
22 \\
28 \\
28 \\
29\end{array}$ & $\begin{array}{l}25 \\
30 \\
28 \\
16 \\
16\end{array}$ & $\begin{array}{l}24 \\
33 \\
31 \\
28 \\
28\end{array}$ & $\begin{array}{l}29 \\
28 \\
28 \\
31 \\
31\end{array}$ & $\begin{array}{l}33 \\
32 \\
32 \\
31 \\
30\end{array}$ & $\begin{array}{l}28 \\
24 \\
25 \\
29 \\
25\end{array}$ & $\begin{array}{l}31 \\
31 \\
31 \\
30 \\
30\end{array}$ & $\begin{array}{l}28 \\
28 \\
28 \\
31 \\
28\end{array}$ & $\begin{array}{l}34 \\
32 \\
31 \\
30 \\
30\end{array}$ & $\begin{array}{l}30 \\
32 \\
35 \\
32 \\
30\end{array}$ & $\begin{array}{l}25 \\
25 \\
25 \\
25 \\
26\end{array}$ & (1..... \\
\hline $\begin{array}{l}11 \ldots . \\
12 \ldots . \\
13 \ldots \\
14 \ldots . \\
15 \ldots\end{array}$ & $\begin{array}{l}30 \\
31 \\
33 \\
30 \\
31\end{array}$ & $\begin{array}{l}16 \\
16 \\
16 \\
15 \\
15\end{array}$ & $\begin{array}{l}28 \\
28 \\
28 \\
28 \\
28\end{array}$ & $\begin{array}{l}30 \\
30 \\
31 \\
31 \\
31\end{array}$ & $\begin{array}{l}33 \\
33 \\
33 \\
30 \\
30\end{array}$ & $\begin{array}{l}25 \\
25 \\
31 \\
31 \\
31\end{array}$ & $\begin{array}{l}28 \\
32 \\
32 \\
32 \\
32\end{array}$ & $\begin{array}{l}26 \\
26 \\
32 \\
32 \\
32\end{array}$ & $\begin{array}{l}30 \\
28 \\
31 \\
30 \\
29\end{array}$ & $\begin{array}{l}30 \\
32 \\
34 \\
34 \\
32\end{array}$ & $\begin{array}{l}26 \\
29 \\
26 \\
30 \\
30\end{array}$ & $\begin{array}{r}\cdots \\
\cdots \\
\cdots \\
\quad \text { ii } \\
11 \\
11\end{array}$ \\
\hline $\begin{array}{l}16 \ldots . \\
17 \ldots \\
18 \ldots \\
19 \ldots \ldots \\
20 \ldots\end{array}$ & $\begin{array}{l}33 \\
28 \\
24 \\
22 \\
22\end{array}$ & $\begin{array}{l}15 \\
15 \\
13 \\
12 \\
12\end{array}$ & $\begin{array}{l}28 \\
28 \\
28 \\
30 \\
31\end{array}$ & $\begin{array}{l}31 \\
31 \\
31 \\
31 \\
30\end{array}$ & $\begin{array}{l}30 \\
27 \\
25 \\
24 \\
24\end{array}$ & $\begin{array}{l}31 \\
28 \\
29 \\
31 \\
31\end{array}$ & $\begin{array}{l}30 \\
30 \\
30 \\
30 \\
31\end{array}$ & $\begin{array}{l}34 \\
26 \\
26 \\
29 \\
30\end{array}$ & $\begin{array}{l}29 \\
29 \\
28 \\
28 \\
28\end{array}$ & $\begin{array}{l}32 \\
32 \\
31 \\
31 \\
31\end{array}$ & $\begin{array}{l}30 \\
30 \\
30 \\
30 \\
30\end{array}$ & $\begin{array}{l}11 \\
11 \\
11 \\
14 \\
14\end{array}$ \\
\hline $\begin{array}{l}21 \ldots \ldots . \\
22 \ldots \ldots \\
23 \ldots \ldots \\
24 \ldots \ldots \\
25 \ldots \ldots\end{array}$ & $\begin{array}{l}22 \\
35 \\
31 \\
31 \\
28\end{array}$ & $\begin{array}{l}12 \\
12 \\
12 \\
15 \\
12\end{array}$ & $\begin{array}{l}31 \\
33 \\
33 \\
31 \\
32\end{array}$ & $\begin{array}{l}30 \\
28 \\
30 \\
31 \\
33\end{array}$ & $\begin{array}{l}21 \\
21 \\
21 \\
21 \\
21\end{array}$ & $\begin{array}{l}31 \\
31 \\
31 \\
29 \\
31\end{array}$ & $\begin{array}{l}31 \\
28 \\
29 \\
36 \\
36\end{array}$ & $\begin{array}{l}34 \\
34 \\
32 \\
31 \\
31\end{array}$ & $\begin{array}{l}28 \\
29 \\
28 \\
28 \\
28\end{array}$ & $\begin{array}{l}32 \\
32 \\
32 \\
32 \\
32\end{array}$ & $\begin{array}{l}29 \\
19 \\
19 \\
19 \\
18\end{array}$ & $\begin{array}{l}14 \\
14 \\
16 \\
16 \\
16\end{array}$ \\
\hline 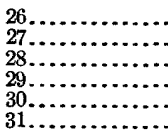 & $\begin{array}{l}28 \\
27 \\
27 \\
24 \\
23 \\
22\end{array}$ & $\begin{array}{l}12 \\
12 \\
12\end{array}$ & $\begin{array}{l}31 \\
30 \\
30 \\
29 \\
29 \\
29\end{array}$ & $\begin{array}{l}33 \\
31 \\
31 \\
31 \\
33\end{array}$ & $\begin{array}{l}27 \\
25 \\
26 \\
29 \\
29 \\
29\end{array}$ & $\begin{array}{l}31 \\
31 \\
32 \\
32 \\
32\end{array}$ & $\begin{array}{l}36 \\
34 \\
32 \\
31 \\
31 \\
31\end{array}$ & $\begin{array}{l}29 \\
29 \\
29 \\
35 \\
34 \\
34\end{array}$ & $\begin{array}{l}28 \\
26 \\
27 \\
29 \\
30\end{array}$ & $\begin{array}{l}32 \\
32 \\
32 \\
32 \\
31 \\
31\end{array}$ & $\begin{array}{l}18 \\
18 \\
18 \\
18 \\
18\end{array}$ & $\begin{array}{l}\mathbf{1 6} \\
16 \\
20 \\
23 \\
\mathbf{2 4} \\
\mathbf{2 4}\end{array}$ \\
\hline
\end{tabular}

Note.-Channel dry Jan. 3-21, Nov. 23-25, 1912; Dec. 1-12, 1913. 
Monthly discharge of Kanaha ditch near Lihue, Kauai, for 1912-13.

\begin{tabular}{|c|c|c|c|c|c|}
\hline \multirow{2}{*}{ Month. } & \multicolumn{3}{|c|}{ Discharge in second-feet. } & \multirow{2}{*}{$\begin{array}{l}\text { Run-off } \\
\text { (total in } \\
\text { acre-feet). }\end{array}$} & \multirow{2}{*}{$\begin{array}{l}\text { Accu- } \\
\text { racy. }\end{array}$} \\
\hline & Maximum. & Minimum. & Mean. & & \\
\hline 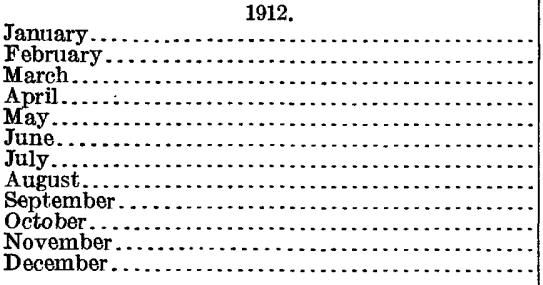 & $\begin{array}{l}24 \\
28 \\
31 \\
30 \\
33 \\
30 \\
39 \\
39 \\
35 \\
35 \\
35 \\
35\end{array}$ & $\begin{array}{r}0 \\
19 \\
22 \\
16 \\
25 \\
27 \\
28 \\
27 \\
27 \\
30 \\
0 \\
10\end{array}$ & $\begin{array}{l}\text { 8. } 23 \\
23.2 \\
26.4 \\
25.2 \\
28.3 \\
27.8 \\
30.9 \\
33.3 \\
31.2 \\
32.6 \\
26.9 \\
23.2\end{array}$ & $\begin{array}{r}506 \\
1,330 \\
1,620 \\
1,500 \\
1,740 \\
1,650 \\
1,900 \\
2,050 \\
1,860 \\
2,000 \\
1,600 \\
1,430\end{array}$ & $\begin{array}{l}\text { A. } \\
\text { A. } \\
\text { A. } \\
\text { A. } \\
\text { A. } \\
\text { A. } \\
\text { A. } \\
\text { A. } \\
\text { A. } \\
\text { A. } \\
\text { A. } \\
\text { A. }\end{array}$ \\
\hline The year... & 39 & 0 & 26.5 & 19,200 & \\
\hline 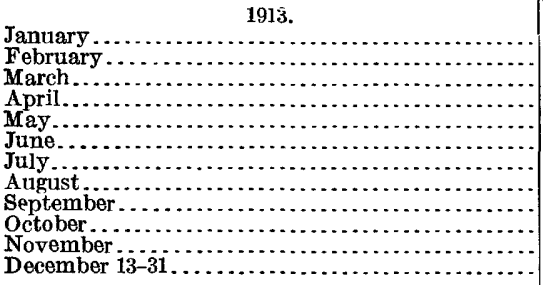 & $\begin{array}{l}35 \\
30 \\
33 \\
33 \\
33 \\
32 \\
36 \\
35 \\
34 \\
35 \\
31 \\
24\end{array}$ & $\begin{array}{l}21 \\
12 \\
11 \\
28 \\
21 \\
24 \\
28 \\
26 \\
26 \\
26 \\
18 \\
0\end{array}$ & $\begin{array}{c}26.4 \\
17.0 \\
26.6 \\
30.6 \\
27.4 \\
29.1 \\
31.5 \\
30.2 \\
29.5 \\
31.0 \\
25.2 \\
9.45\end{array}$ & $\begin{array}{r}1,620 \\
944 \\
1,640 \\
1,820 \\
1,680 \\
1,730 \\
1,940 \\
1,860 \\
1,760 \\
1,910 \\
1,500 \\
581\end{array}$ & $\begin{array}{l}\text { A. } \\
\text { A. } \\
\text { A. } \\
\text { A. } \\
\text { A. } \\
\text { A. } \\
\text { A. } \\
\text { A. } \\
\text { A. } \\
\text { A. } \\
\text { A. } \\
\text { A. }\end{array}$ \\
\hline 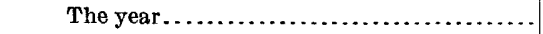 & 36 & 0 & 26.2 & 19,000 & \\
\hline
\end{tabular}

\section{EAST BRANCH OF NORTH FORK OF WAILUA RIVER, NEAR LIHUE, KAUAI.}

Location.-About 8 miles north of Lihue, 600 feet above gaging station on the North Fork of Wailua, and 400 feet above the confluence of the North and East branches of the North Fork of Wailua River.

Records available.-July 27, 1912, to December 31, 1913.

Drainage area. -6.2 square miles.

Gage.-Inclined staff.

Control.-Probably permanent.

Discharge measurements.--Made by wading.

Accuracy.-Records fair except for November, when broken records were obtained.

Discharge measurements of East Branch of North Fork of Wailua River near Lihue, Kauai, in 1913.

\begin{tabular}{|c|c|c|c|}
\hline Date. & Hydrographer. & $\begin{array}{r}\text { Gage } \\
\text { height }\end{array}$ & $\begin{array}{l}\text { Dis- } \\
\text { charge. }\end{array}$ \\
\hline $\begin{array}{ll}\text { Jan. } & 19 \\
\text { May } & 29\end{array}$ & $\begin{array}{l}\text { D. E. Horner..... } \\
\text { w. v. Hardy } \ldots \ldots \ldots \ldots \ldots\end{array}$ & $\begin{array}{r}\text { Feet. } \\
\quad 6.50 \\
\quad 6.30\end{array}$ & $\begin{array}{r}\text { Sec.-ft. } \\
46.7 \\
30.7\end{array}$ \\
\hline
\end{tabular}


Daily discharge, in second-feet, of East Branch of North Fork of Wailua River, near Lihue, Kauai, for 1912-13.

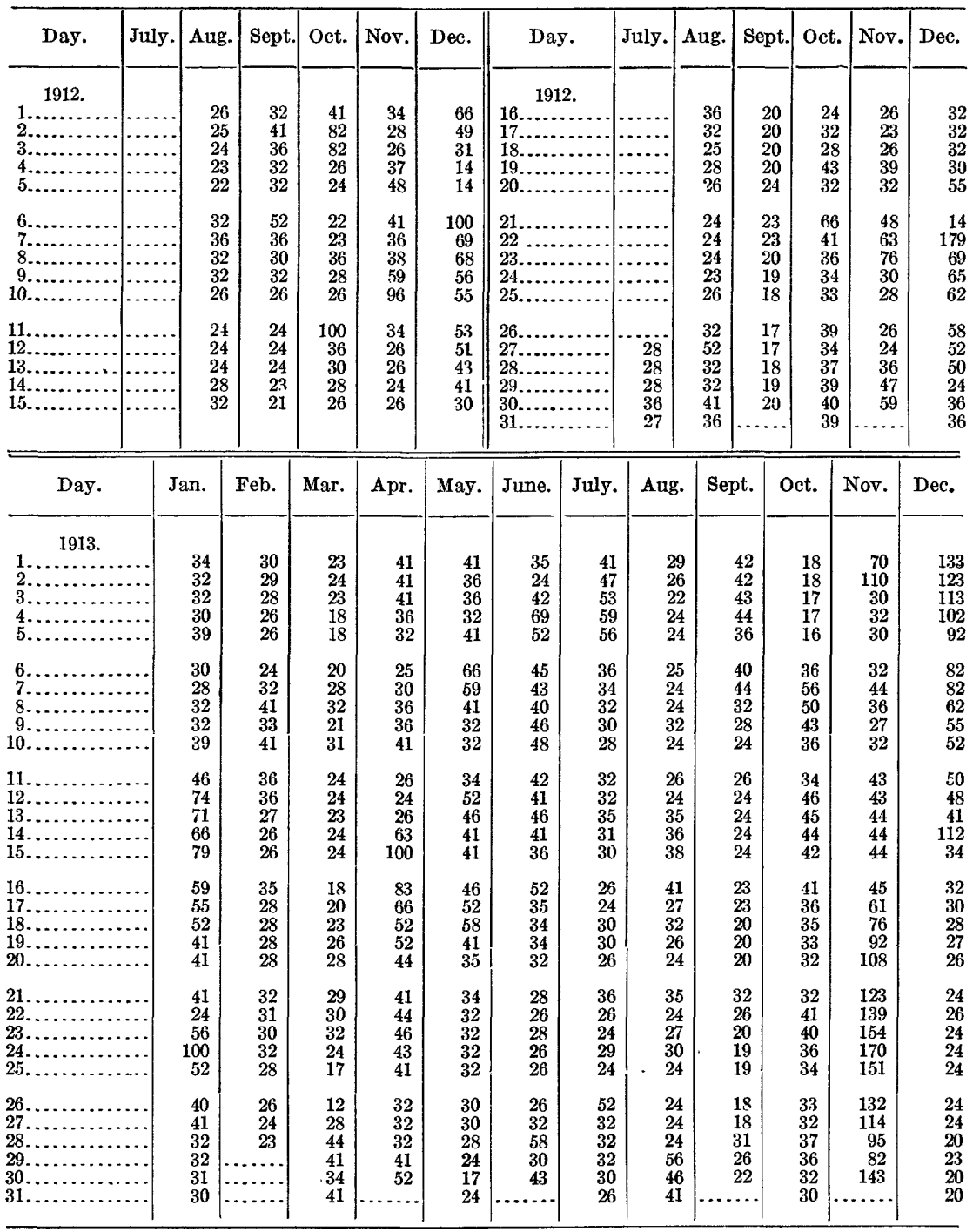

NoTE.-Discharge estimated for following days on which no gage heights were recorded: 1912: Aug. 14; Sept 13. Nov 4, 21, 25, 28, and 29; Dec $23,11,24$ and 25; 1913: Jan 1 10, and 30; Feb. 3, 7, and 19; Mar. 17-19, 24, and 27; Apr. 4, 7, 14, 16, and 22; June 3 and 18; July 2, 3, and 28; Aug. 15 and 23; Sept. 1-3, $6,15,22,29$, and 30; Oct. 6, 15, 20, and 26; Nov. 1, 12-14, 17-23, and 25-27; Dec. 1-5, 16, 19, and 25. 
Monthly discharge of East Branch of North Fork of Wailua River near Lihue, Kauai, for 1912-1.9.

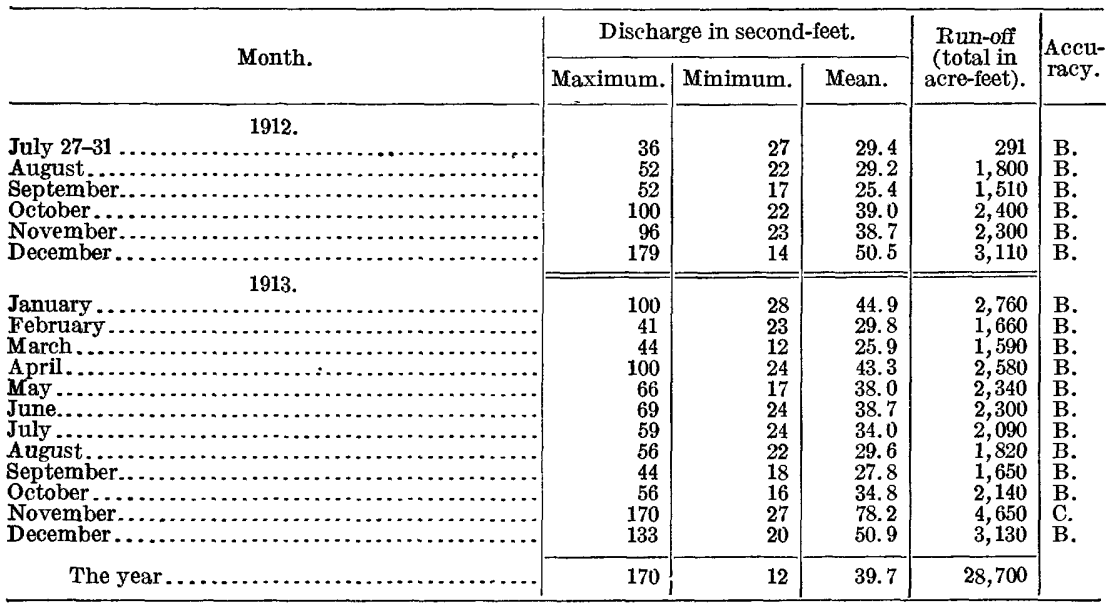

KONOHIKI STREAM AT MAKAKUALELE WEIR (MAUKA), NEAR KEALIA, KAUAI.

Location.-About 5 miles southwest of Kealia.

Records available.-April 1, 1911, to December 31, 1913.

Gage.--Vertical staff.

Discharge measurements.-Made with 3-foot sharp-crested weir.

Cooperation.-Station maintained and daily-discharge record furnished by the Makee Sugar Co.

Discharge, in million gallons per day, of Konohiki Stream at Makakualele weir (mauka), near Kealia, Kauai, for 1913.

\begin{tabular}{|c|c|c|c|c|c|c|c|c|c|c|c|c|}
\hline Day. & Jan. & Feb. & Mar. & Apr. & May. & June. & July. & Aug. & Sept. & Oct. & Nov. & Dec. \\
\hline $\begin{array}{l}1 \ldots . \\
2 \ldots \\
3 \ldots \\
4 \ldots \\
5 \ldots\end{array}$ & $\begin{array}{r}0.38 \\
.38 \\
.38 \\
.38 \\
.38\end{array}$ & $\begin{array}{r}0.38 \\
.38 \\
.38 \\
.38 \\
.38\end{array}$ & $\begin{array}{r}0.45 \\
.45 \\
.45 \\
.41 \\
.41\end{array}$ & $\begin{array}{r}0.54 \\
.54 \\
.54 \\
.49 \\
.49\end{array}$ & $\begin{array}{r}0.49 \\
.49 \\
.49 \\
.49 \\
.49\end{array}$ & $\begin{array}{l}0.54 \\
.54 \\
3.79 \\
2.79 \\
2.21\end{array}$ & $\begin{array}{r}0.86 \\
.86 \\
.86 \\
.86 \\
.86\end{array}$ & $\begin{array}{r}0.67 \\
.67 \\
.67 \\
.67 \\
.67\end{array}$ & $\begin{array}{r}0.67 \\
.67 \\
1.16 \\
.96 \\
.67\end{array}$ & $\begin{array}{r}0.58 \\
.58 \\
.54 \\
.58 \\
.58\end{array}$ & $\begin{array}{r}0.62 \\
.62 \\
.62 \\
.62 \\
.62\end{array}$ & $\begin{array}{l}1.95 \\
2.79 \\
3.01 \\
3.01 \\
2.29\end{array}$ \\
\hline $\begin{array}{r}6 \ldots \ldots \\
7 \ldots \ldots \\
8 \ldots \\
9 \ldots \\
10 \ldots\end{array}$ & $\begin{array}{l}.38 \\
.38 \\
.38 \\
.38 \\
.38\end{array}$ & $\begin{array}{r}1.17 \\
.96 \\
.49 \\
.45 \\
.41\end{array}$ & $\begin{array}{l}.41 \\
.41 \\
.41 \\
.41 \\
.41\end{array}$ & $\begin{array}{l}.49 \\
.49 \\
.49 \\
.49 \\
.49\end{array}$ & $\begin{array}{l}.49 \\
.54 \\
.54 \\
.54 \\
.49\end{array}$ & $\begin{array}{l}1.64 \\
1.28 \\
1.17 \\
1.40 \\
1.17\end{array}$ & $\begin{array}{l}.81 \\
.81 \\
.81 \\
.81 \\
.81\end{array}$ & $\begin{array}{l}.67 \\
.62 \\
.62 \\
.62 \\
.62\end{array}$ & $\begin{array}{l}.67 \\
.67 \\
.67 \\
.67 \\
.62\end{array}$ & $\begin{array}{l}.49 \\
.49 \\
.49 \\
.62 \\
.62\end{array}$ & $\begin{array}{l}.71 \\
.71 \\
.71 \\
.71 \\
.62\end{array}$ & $\begin{array}{l}2.09 \\
2.09 \\
2.71 \\
2.71 \\
2.21\end{array}$ \\
\hline $15 \ldots$ & $\begin{array}{l}.38 \\
.38 \\
.38 \\
.38 \\
.38\end{array}$ & $\begin{array}{l}.45 \\
.41 \\
.41 \\
.41 \\
.41\end{array}$ & $\begin{array}{l}.41 \\
.41 \\
.41 \\
.45 \\
.41\end{array}$ & $\begin{array}{l}.49 \\
.49 \\
.49 \\
.49 \\
.54\end{array}$ & $\begin{array}{r}.49 \\
1.06 \\
1.17 \\
.91 \\
.67\end{array}$ & $\begin{array}{r}1.06 \\
1.01 \\
1.01 \\
.96 \\
.96\end{array}$ & $\begin{array}{l}.81 \\
.81 \\
.96 \\
.86 \\
.86\end{array}$ & $\begin{array}{l}.62 \\
.62 \\
.71 \\
.71 \\
.71\end{array}$ & $\begin{array}{l}.62 \\
.62 \\
.58 \\
.58 \\
.58\end{array}$ & $\begin{array}{l}.54 \\
.54 \\
.54 \\
.54 \\
.54\end{array}$ & $\begin{array}{l}.58 \\
.58 \\
.58 \\
.58 \\
.54\end{array}$ & $\begin{array}{l}2.09 \\
2.02 \\
1.89 \\
1.83 \\
1.83\end{array}$ \\
\hline $20 \ldots$ & $\begin{array}{l}.38 \\
.38 \\
.38 \\
.38 \\
.38\end{array}$ & $\begin{array}{l}.41 \\
.41 \\
.67 \\
.58 \\
.45\end{array}$ & $\begin{array}{l}.41 \\
.41 \\
.41 \\
.41 \\
.41\end{array}$ & $\begin{array}{l}.49 \\
.49 \\
.49 \\
.49 \\
.49\end{array}$ & $\begin{array}{l}.58 \\
.62 \\
.58 \\
.58 \\
.49\end{array}$ & $\begin{array}{l}.96 \\
.96 \\
.96 \\
.96 \\
.96\end{array}$ & $\begin{array}{l}.81 \\
.81 \\
.81 \\
.81 \\
.81\end{array}$ & $\begin{array}{l}.71 \\
.67 \\
.67 \\
.67 \\
.67\end{array}$ & $\begin{array}{l}.58 \\
.58 \\
.58 \\
.58 \\
.58\end{array}$ & $\begin{array}{l}.54 \\
.86 \\
.71 \\
.71 \\
.67\end{array}$ & $\begin{array}{l}.54 \\
.54 \\
.54 \\
.62 \\
.76\end{array}$ & $\begin{array}{l}1.76 \\
1.64 \\
1.52 \\
1.52 \\
1.64\end{array}$ \\
\hline $\begin{array}{l}23 \\
24 \\
25\end{array}$ & $\begin{array}{l}.38 \\
.38 \\
.38 \\
.38 \\
.38\end{array}$ & $\begin{array}{l}.45 \\
.45 \\
.45 \\
.49 \\
.45\end{array}$ & $\begin{array}{r}.41 \\
.41 \\
.58 \\
1.34 \\
.91\end{array}$ & $\begin{array}{l}.49 \\
.49 \\
.49 \\
.49 \\
.49\end{array}$ & $\begin{array}{l}.49 \\
.49 \\
.58 \\
.58 \\
.58\end{array}$ & $\begin{array}{l}.86 \\
.86 \\
.86 \\
.86 \\
.86\end{array}$ & $\begin{array}{r}.81 \\
.81 \\
.76 \\
\ldots .\end{array}$ & $\begin{array}{l}.67 \\
.58 \\
.58 \\
.62 \\
.67\end{array}$ & $\begin{array}{l}.58 \\
.58 \\
.58 \\
.58 \\
.58\end{array}$ & $\begin{array}{l}.58 \\
.58 \\
.62 \\
.76 \\
.67\end{array}$ & $\begin{array}{c}18.3 \\
6.47 \\
2.57 \\
2.57 \\
2.36\end{array}$ & $\begin{array}{l}1.52 \\
1.52 \\
1.46 \\
1.46 \\
1.46\end{array}$ \\
\hline 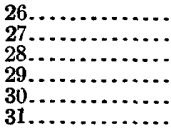 & $\begin{array}{l}.38 \\
.38 \\
.38 \\
.38 \\
.38 \\
.34\end{array}$ & $\begin{array}{r}.45 \\
.45 \\
.45 \\
. . .\end{array}$ & $\begin{array}{l}.81 \\
.71 \\
.62 \\
.62 \\
.62 \\
.54\end{array}$ & $\begin{array}{l}.49 \\
.49 \\
.49 \\
.49 \\
.49 \\
. . .\end{array}$ & $\begin{array}{l}.58 \\
.54 \\
.54 \\
.54 \\
.54 \\
.54\end{array}$ & $\begin{array}{l}.86 \\
.96 \\
.96 \\
.86 \\
.86 \\
. . .\end{array}$ & $\begin{array}{l}\cdots \cdots \\
\cdots .71 \\
.71 \\
.67 \\
.67\end{array}$ & $\begin{array}{l}.58 \\
.67 \\
.67 \\
.67 \\
.67 \\
.67\end{array}$ & $\begin{array}{l}.58 \\
.58 \\
.76 \\
.58 \\
.58\end{array}$ & $\begin{array}{l}.67 \\
.62 \\
.62 \\
.76 \\
.67 \\
.67\end{array}$ & $\begin{array}{r}1.95 \\
1.95 \\
1.95 \\
.62 \\
.62\end{array}$ & $\begin{array}{l}1.40 \\
1.40 \\
1.34 \\
1.34 \\
1.34 \\
1.34\end{array}$ \\
\hline
\end{tabular}

Note.-No record July 24-27. 
Monthly discharge of Konohiki Stream at Makakualele weir (mauka), near Kealia, Kauai, for 1913 .

\begin{tabular}{|c|c|c|c|c|}
\hline \multirow{2}{*}{ Month. } & \multicolumn{3}{|c|}{$\begin{array}{l}\text { Discharge in million gallons per } \\
\text { day. }\end{array}$} & \multirow{2}{*}{$\begin{array}{l}\text { Run-off } \\
\text { (total im } \\
\text { acre-feet). }\end{array}$} \\
\hline & Maximum. & Mimimum. & Mean. & \\
\hline 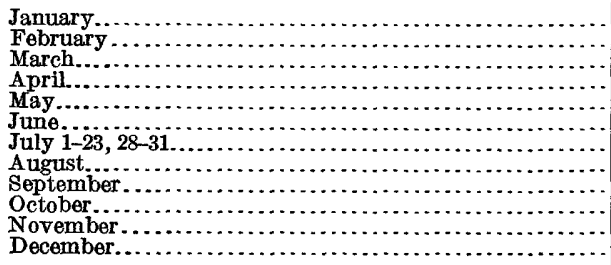 & $\begin{array}{r}0.38 \\
1.17 \\
1.34 \\
.54 \\
1.17 \\
3.79 \\
.86 \\
.71 \\
1.16 \\
.86 \\
18.3 \\
3.01\end{array}$ & $\begin{array}{r}0.34 \\
.38 \\
.41 \\
.49 \\
.49 \\
.54 \\
.67 \\
.58 \\
.58 \\
.49 \\
.54 \\
1.34\end{array}$ & $\begin{array}{r}0.38 \\
.47 \\
.51 \\
.50 \\
.59 \\
1.17 \\
.81 \\
.66 \\
.64 \\
.61 \\
1.72 \\
1.88\end{array}$ & $\begin{array}{r}36 \\
40 \\
49 \\
46 \\
56 \\
108 \\
67 \\
63 \\
59 \\
58 \\
158 \\
179\end{array}$ \\
\hline 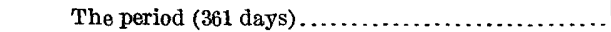 & 18.3 & .34 & .83 & 919 \\
\hline
\end{tabular}

Note.-Mean for July is for 27 days.

SOUTH FORK OF KAEHULUA STREAM AT WAINAMUAMU WEIR, NEAR KEALIA, KAUAI.

Location.-About 5 miles southwest of Kealia.

Records available.-April 1, 1911, to December 31, 1913.

Gage.-Vertical staff.

Discharge measurements.-Made with a 3-foot sharp-crested weir.

Cooperation.-Station maintained and daily-discharge record furnished by the Makee Sugar Co.

Discharge, in million gallons per day, of South Fork of Kaehulua Stream at Wainamuamu weir, near Kealia, Kauai, for 1913.

\begin{tabular}{|c|c|c|c|c|c|c|c|c|c|c|c|c|}
\hline Day. & Jan. & Feb. & Mar. & Apr. & May. & June. & July. & Aug. & Sept. & Oct. & Nov. & Dec. \\
\hline $\begin{array}{l}1 \ldots \\
2 \ldots \\
3 \ldots \\
4 \ldots \\
5 \ldots\end{array}$ & $\begin{array}{r}0.30 \\
.30 \\
.30 \\
.30 \\
.30\end{array}$ & $\begin{array}{r}0.27 \\
.27 \\
.27 \\
.27 \\
.27\end{array}$ & $\begin{array}{r}0.30 \\
.30 \\
.30 \\
.30 \\
.30\end{array}$ & $\begin{array}{l}0.30 \\
.30 \\
.30 \\
.30 \\
.30\end{array}$ & $\begin{array}{l}0.30 \\
.30 \\
.30 \\
.30 \\
.30\end{array}$ & $\begin{array}{r}0.27 \\
.27 \\
1.34 \\
1.06 \\
.86\end{array}$ & $\begin{array}{r}0.38 \\
.38 \\
.38 \\
.38 \\
.38\end{array}$ & $\begin{array}{r}1.64 \\
.91 \\
.38 \\
.38 \\
.38\end{array}$ & $\begin{array}{l}0.58 \\
.67 \\
.67 \\
.67 \\
.58\end{array}$ & $\begin{array}{r}0.49 \\
.49 \\
.45 \\
.41 \\
.41\end{array}$ & $\begin{array}{l}0.62 \\
.67 \\
.58 \\
.58 \\
.58\end{array}$ & $\begin{array}{l}0.58 \\
.58 \\
.58 \\
.58 \\
.58\end{array}$ \\
\hline $\begin{array}{r}6 \ldots . \\
7 \ldots \ldots \\
8 \ldots \ldots \\
9 \ldots \ldots \\
10 \ldots \ldots\end{array}$ & $\begin{array}{l}.30 \\
.30 \\
.30 \\
.30 \\
.30\end{array}$ & $\begin{array}{l}.58 \\
.49 \\
.41 \\
.38 \\
.38\end{array}$ & $\begin{array}{l}.30 \\
.30 \\
.30 \\
.30 \\
.30\end{array}$ & $\begin{array}{l}.30 \\
.30 \\
.30 \\
.30 \\
.30\end{array}$ & $\begin{array}{l}.30 \\
.30 \\
.30 \\
.30 \\
.30\end{array}$ & $\begin{array}{l}.76 \\
.49 \\
.49 \\
.45 \\
.45\end{array}$ & $\begin{array}{c}. .34 \\
\ldots \ldots . . \\
\cdots . . .6 \\
. . .\end{array}$ & $\begin{array}{r}.38 \\
2.29 \\
.38 \\
.38 \\
.38\end{array}$ & $\begin{array}{l}.58 \\
.58 \\
.58 \\
.58 \\
.58\end{array}$ & $\begin{array}{l}.41 \\
.45 \\
.45 \\
.58 \\
.58\end{array}$ & $\begin{array}{l}.58 \\
.58 \\
.58 \\
.58 \\
.58\end{array}$ & $\begin{array}{l}.58 \\
.58 \\
.58 \\
.58 \\
.58\end{array}$ \\
\hline $\begin{array}{l}11 \ldots \ldots \\
12 \ldots \ldots \\
13 \ldots \ldots \\
14 \ldots \ldots \\
15 \ldots \ldots\end{array}$ & $\begin{array}{l}.27 \\
.27 \\
.27 \\
.27 \\
.27\end{array}$ & $\begin{array}{l}.34 \\
.34 \\
.34 \\
.34 \\
.34\end{array}$ & $\begin{array}{l}.30 \\
.30 \\
.30 \\
.30 \\
.30\end{array}$ & $\begin{array}{l}.30 \\
.30 \\
.30 \\
.30 \\
.30\end{array}$ & $\begin{array}{l}.30 \\
.45 \\
.49 \\
.81 \\
.34\end{array}$ & $\begin{array}{l}.41 \\
.41 \\
.41 \\
.38 \\
.38\end{array}$ & $\begin{array}{r}.41 \\
.34\end{array}$ & $\begin{array}{l}.38 \\
.38 \\
.38 \\
.58 \\
.58\end{array}$ & $\begin{array}{l}.58 \\
.58 \\
.54 \\
.54 \\
.54\end{array}$ & $\begin{array}{l}.58 \\
.58 \\
.58 \\
.58 \\
.58\end{array}$ & $\begin{array}{l}.58 \\
.58 \\
.58 \\
.58 \\
.58\end{array}$ & $\begin{array}{l}.58 \\
.58 \\
.58 \\
.58 \\
.58\end{array}$ \\
\hline $\begin{array}{l}16 \ldots \ldots \\
17 \ldots \ldots \\
18 \ldots \ldots \\
19 \ldots \ldots \\
20 \ldots \ldots\end{array}$ & $\begin{array}{l}.27 \\
.27 \\
.27 \\
.27 \\
.27\end{array}$ & $\begin{array}{l}.34 \\
.30 \\
.38 \\
.34 \\
.30\end{array}$ & $\begin{array}{l}.30 \\
.30 \\
.30 \\
.30 \\
.30\end{array}$ & $\begin{array}{l}.30 \\
.30 \\
.30 \\
.30 \\
.30\end{array}$ & $\begin{array}{l}.30 \\
.30 \\
.30 \\
.30 \\
.30\end{array}$ & $\begin{array}{l}.38 \\
.38 \\
.38 \\
.38 \\
.38\end{array}$ & … & $\begin{array}{l}.58 \\
.58 \\
.49 \\
.45 \\
.45\end{array}$ & $\begin{array}{l}.54 \\
.54 \\
.54 \\
.54 \\
.54\end{array}$ & $\begin{array}{l}.58 \\
.96 \\
.76 \\
.76 \\
.71\end{array}$ & $\begin{array}{l}.58 \\
.58 \\
.58 \\
.58 \\
.58\end{array}$ & $\begin{array}{l}.58 \\
.58 \\
.58 \\
.58 \\
.58\end{array}$ \\
\hline $\begin{array}{l}21 \ldots \ldots \ldots \\
22, \ldots \ldots \ldots \\
24 \ldots \ldots \ldots \ldots \\
25 \ldots \ldots \ldots \ldots\end{array}$ & $\begin{array}{l}.27 \\
.27 \\
.27 \\
.27 \\
.27\end{array}$ & $\begin{array}{l}.30 \\
.30 \\
.30 \\
.34 \\
.30\end{array}$ & $\begin{array}{l}.30 \\
.30 \\
.41 \\
.58 \\
.54\end{array}$ & $\begin{array}{l}.30 \\
.30 \\
.30 \\
.30 \\
.30\end{array}$ & $\begin{array}{l}.30 \\
.30 \\
.30 \\
.30 \\
.30\end{array}$ & $\begin{array}{l}.38 \\
.38 \\
.38 \\
.38 \\
.38\end{array}$ & $\begin{array}{r}.30 \\
1.17 \\
.34\end{array}$ & $\begin{array}{l}.45 \\
.62 \\
.62 \\
.62 \\
.62\end{array}$ & $\begin{array}{l}.54 \\
.54 \\
.49 \\
.49 \\
.49\end{array}$ & $\begin{array}{l}.62 \\
.58 \\
.67 \\
.81 \\
.71\end{array}$ & $\begin{array}{l}.58 \\
.58 \\
.58 \\
.58 \\
.58\end{array}$ & $\begin{array}{l}.58 \\
.58 \\
.58 \\
.58 \\
.58\end{array}$ \\
\hline $\begin{array}{l}26 \ldots \ldots \\
27 \ldots \ldots \\
28 \ldots \ldots \\
29 \ldots \ldots \\
30 \ldots \ldots \\
31 \ldots \ldots \\
\end{array}$ & $\begin{array}{l}.27 \\
.27 \\
.27 \\
.27 \\
.27 \\
.27\end{array}$ & $\begin{array}{r}.30 \\
.30 \\
.30 \\
\cdots . . . \\
\cdots . . .\end{array}$ & $\begin{array}{l}.41 \\
.38 \\
.34 \\
.34 \\
.34 \\
.30\end{array}$ & $\begin{array}{l}.30 \\
.30 \\
.30 \\
.30 \\
.30 \\
.30\end{array}$ & $\begin{array}{l}.30 \\
.27 \\
.27 \\
.27 \\
.27 \\
.27\end{array}$ & $\begin{array}{l}.38 \\
.38 \\
.38 \\
.38 \\
.38\end{array}$ & $\begin{array}{r}.34 \\
.34 \\
1.70 \\
.34 \\
.34 \\
.30\end{array}$ & $\begin{array}{l}.58 \\
.58 \\
.58 \\
.58 \\
.58 \\
.58\end{array}$ & $\begin{array}{l}.49 \\
.58 \\
.62 \\
.49 \\
.49\end{array}$ & $\begin{array}{l}.71 \\
.67 \\
.58 \\
.71 \\
.76 \\
.76\end{array}$ & $\begin{array}{l}.58 \\
.58 \\
.58 \\
.58 \\
.58\end{array}$ & $\begin{array}{l}.58 \\
.58 \\
.58 \\
.58 \\
.58 \\
.58\end{array}$ \\
\hline
\end{tabular}

NoTE.-No record July $7-12,15-22$. 
Monthly discharge of South Fork of Kaehulua Stream at Wainamuamu weir, near Kealia, Kauai, for 1913.

\begin{tabular}{|c|c|c|c|c|}
\hline \multirow{2}{*}{ Month. } & \multicolumn{3}{|c|}{$\begin{array}{l}\text { Discharge in million gallons per } \\
\text { day. }\end{array}$} & \multirow{2}{*}{$\begin{array}{l}\text { Run-off } \\
\text { (total in } \\
\text { acre-feet). }\end{array}$} \\
\hline & Maximum. & Minimum. & Mean. & \\
\hline 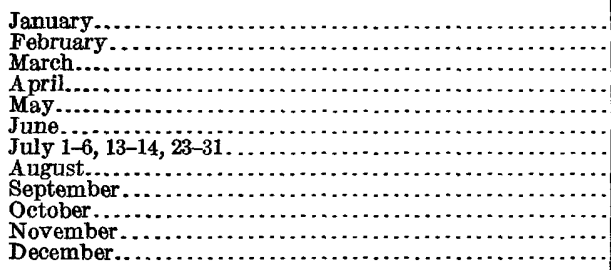 & $\begin{array}{r}0.30 \\
.58 \\
.58 \\
.30 \\
.81 \\
1.34 \\
1.70 \\
2.29 \\
.67 \\
.96 \\
.67 \\
.58\end{array}$ & $\begin{array}{r}0.27 \\
.27 \\
.30 \\
.30 \\
.27 \\
.27 \\
.30 \\
.38 \\
.49 \\
.41 \\
.57 \\
.58\end{array}$ & $\begin{array}{r}0.28 \\
.34 \\
.33 \\
.30 \\
.32 \\
.46 \\
.48 \\
.61 \\
.56 \\
.61 \\
.58 \\
.58\end{array}$ & $\begin{array}{l}27 \\
29 \\
31 \\
28 \\
31 \\
42 \\
25 \\
58 \\
52 \\
58 \\
54 \\
55\end{array}$ \\
\hline The period $(351$ days $) \ldots \ldots \ldots \ldots \ldots \ldots \ldots \ldots \ldots$ & 2.29 & .27 & .45 & 490 \\
\hline
\end{tabular}

\section{KAEHULUA STREAM AT KUHINOA WEIR, NEAR KEALIA, KAUAI.}

Location.-About 3 miles southwest of Kealia.

Records available.-May 1, 1911, to December 31, 1913.

Gage.--Vertical staff.

Discharge measurements.-Made with 6.5-foot sharp-crested weir.

Cooperation.-Station maintained and daily-discharge record furnished by Makee Sugar Co.

Discharge, in million gallons per day, of Kaehulua Stream at Kuhinoa weir, near Kealia, Kauai, for 1913.

\begin{tabular}{|c|c|c|c|c|c|c|c|c|c|c|c|c|}
\hline Day. & Jan. & Feb. & Mar. & Apr. & May. & June. & July. & Aug. & Sept. & Oet. & Nov. & Dec. \\
\hline $\begin{array}{l}1 \ldots \\
2 \ldots \\
3 \ldots \\
4 \ldots \\
5 \ldots\end{array}$ & $\begin{array}{l}0.04 \\
.04 \\
.04 \\
.04 \\
.07\end{array}$ & $\begin{array}{r}0.12 \\
.12 \\
.12 \\
.12 \\
.12\end{array}$ & $\begin{array}{r}0.44 \\
.12 \\
.12 \\
.12 \\
.12\end{array}$ & $\begin{array}{r}0.12 \\
.12 \\
.12 \\
.12 \\
.12\end{array}$ & $\begin{array}{r}0.44 \\
.38 \\
.26 \\
.21 \\
.12\end{array}$ & $\begin{array}{r}0.12 \\
.12 \\
9.14 \\
9.14 \\
11.1\end{array}$ & $\begin{array}{l}0.21 \\
.26 \\
.81 \\
.66 \\
.58\end{array}$ & $\begin{array}{r}0.12 \\
.12 \\
.12 \\
.12 \\
.12\end{array}$ & $\begin{array}{r}0.11 \\
.11 \\
.12 \\
.12 \\
.12\end{array}$ & $\begin{array}{l}0.07 \\
.07 \\
.07 \\
.07 \\
.07\end{array}$ & $\begin{array}{r}0.44 \\
.44 \\
.32 \\
.26 \\
.26\end{array}$ & $\begin{array}{l}6.52 \\
4.96 \\
6.52 \\
5.72 \\
3.55\end{array}$ \\
\hline $\begin{array}{r}6 \ldots \ldots \\
7 \ldots \ldots \\
8 \ldots \ldots \\
9 \ldots \ldots \\
10 \ldots \ldots\end{array}$ & $\begin{array}{l}.07 \\
.11 \\
.12 \\
.07 \\
.12\end{array}$ & $\begin{array}{l}.44 \\
.44 \\
.44 \\
.44 \\
.44\end{array}$ & $\begin{array}{l}.12 \\
.12 \\
.12 \\
.12 \\
.12\end{array}$ & $\begin{array}{l}.12 \\
.12 \\
.12 \\
.12 \\
.12\end{array}$ & $\begin{array}{l}.12 \\
.26 \\
.12 \\
.21 \\
.21\end{array}$ & $\begin{array}{r}3.96 \\
3.82 \\
1.26 \\
1.16 \\
.90\end{array}$ & $\begin{array}{l}.73 \\
.44 \\
.38 \\
.26 \\
.26\end{array}$ & $\begin{array}{l}.12 \\
.12 \\
.12 \\
.12 \\
.12\end{array}$ & $\begin{array}{l}.12 \\
.12 \\
.12 \\
.12 \\
.12\end{array}$ & $\begin{array}{l}.12 \\
.12 \\
.12 \\
.12 \\
.12\end{array}$ & $\begin{array}{l}.26 \\
.44 \\
.51 \\
.51 \\
.44\end{array}$ & $\begin{array}{l}3.55 \\
3.55 \\
4.96 \\
4.23 \\
4.38\end{array}$ \\
\hline $\begin{array}{l}11 \ldots \ldots \\
12 \ldots \ldots \\
14 \ldots \ldots\end{array}$ & $\begin{array}{l}.12 \\
.12 \\
.12 \\
.12 \\
.12\end{array}$ & $\begin{array}{l}.44 \\
.44 \\
.44 \\
.44 \\
.44\end{array}$ & $\begin{array}{l}.12 \\
.12 \\
.12 \\
.12 \\
.12\end{array}$ & $\begin{array}{l}.04 \\
.04 \\
.04 \\
.04 \\
.04\end{array}$ & $\begin{array}{r}.21 \\
.81 \\
2.54 \\
2.08 \\
1.86\end{array}$ & $\begin{array}{l}.58 \\
.44 \\
.38 \\
.32 \\
.38\end{array}$ & $\begin{array}{l}.26 \\
.26 \\
.26 \\
.26 \\
.21\end{array}$ & $\begin{array}{l}.12 \\
.11 \\
.11 \\
.07 \\
.07\end{array}$ & $\begin{array}{l}.11 \\
.11 \\
.07 \\
.07 \\
.07\end{array}$ & $\begin{array}{l}.12 \\
.12 \\
.12 \\
.12 \\
.12\end{array}$ & $\begin{array}{l}.32 \\
.26 \\
.44 \\
.44 \\
.58\end{array}$ & $\begin{array}{l}3.82 \\
2.91 \\
3.55 \\
3.55 \\
3.55\end{array}$ \\
\hline $\begin{array}{l}16 \ldots \ldots \\
17 \ldots \ldots \\
18 \ldots \ldots \\
19 \ldots \ldots \\
20 \ldots \ldots\end{array}$ & $\begin{array}{l}.26 \\
.44 \\
.44 \\
.12 \\
.12\end{array}$ & $\begin{array}{l}.12 \\
.12 \\
.12 \\
.12 \\
.12\end{array}$ & $\begin{array}{l}.12 \\
.12 \\
.12 \\
.12 \\
.12\end{array}$ & $\begin{array}{l}.11 \\
.04 \\
.04 \\
.07 \\
.07\end{array}$ & $\begin{array}{r}1.86 \\
1.86 \\
.81 \\
.81 \\
1.16\end{array}$ & $\begin{array}{l}.51 \\
.32 \\
.32 \\
.32 \\
.32\end{array}$ & $\begin{array}{l}.21 \\
.12 \\
.12 \\
.12 \\
.12\end{array}$ & $\begin{array}{l}.07 \\
.07 \\
.04 \\
.04 \\
.04\end{array}$ & $\begin{array}{l}.07 \\
.07 \\
.07 \\
.07 \\
.07\end{array}$ & $\begin{array}{l}.12 \\
.32 \\
.26 \\
.26 \\
.21\end{array}$ & $\begin{array}{r}.58 \\
.58 \\
.58 \\
.73 \\
1.07\end{array}$ & $\begin{array}{l}2.91 \\
2.91 \\
3.55 \\
6.52 \\
3.55\end{array}$ \\
\hline $\begin{array}{l}21 \ldots \ldots \\
22 \\
24 \ldots \ldots \\
25 \ldots \ldots\end{array}$ & $\begin{array}{l}.12 \\
.12 \\
.58 \\
.12 \\
.12\end{array}$ & $\begin{array}{l}.12 \\
.12 \\
.24 \\
.24 \\
.24\end{array}$ & $\begin{array}{l}.12 \\
.12 \\
.44 \\
.44 \\
.44\end{array}$ & $\begin{array}{l}.07 \\
.12 \\
.12 \\
.32 \\
.26\end{array}$ & $\begin{array}{l}.44 \\
.21 \\
.12 \\
.12 \\
.12\end{array}$ & $\begin{array}{l}.32 \\
.26 \\
.21 \\
.32 \\
.26\end{array}$ & $\begin{array}{l}.12 \\
.21 \\
.12 \\
.12 \\
.12\end{array}$ & $\begin{array}{l}.04 \\
.04 \\
.04 \\
.04 \\
.04\end{array}$ & $\begin{array}{l}.07 \\
.07 \\
.07 \\
.07 \\
.04\end{array}$ & $\begin{array}{l}.21 \\
.21 \\
.44 \\
.44 \\
.44\end{array}$ & $\begin{array}{l}39.7 \\
14.0 \\
6.52 \\
6.52 \\
4.96\end{array}$ & $\begin{array}{l}3.55 \\
3.55 \\
3.55 \\
2.91 \\
2.91\end{array}$ \\
\hline $\begin{array}{l}26 \ldots \ldots \ldots \\
27 \ldots \ldots \ldots \\
28 \ldots \ldots \\
29 \ldots \ldots \ldots \\
30 \ldots \ldots \\
31 \ldots \ldots \ldots\end{array}$ & $\begin{array}{l}.12 \\
.12 \\
.12 \\
.12 \\
.12 \\
.12\end{array}$ & $\begin{array}{r}.24 \\
.24 \\
.24 \\
\cdots . . . \\
\cdots . . .\end{array}$ & $\begin{array}{l}.44 \\
.44 \\
.44 \\
.44 \\
.12 \\
.12\end{array}$ & $\begin{array}{l}.26 \\
.21 \\
.21 \\
.21 \\
.32\end{array}$ & $\begin{array}{l}.12 \\
.12 \\
.12 \\
.12 \\
.12 \\
.12\end{array}$ & $\begin{array}{l}.21 \\
.12 \\
.12 \\
.12 \\
.21\end{array}$ & $\begin{array}{l}.12 \\
.12 \\
.12 \\
.12 \\
.12 \\
.12\end{array}$ & $\begin{array}{l}.04 \\
.04 \\
.04 \\
.04 \\
.04 \\
.04\end{array}$ & $\begin{array}{l}.04 \\
.04 \\
.12 \\
.12 \\
.12\end{array}$ & $\begin{array}{l}.44 \\
.44 \\
.44 \\
.58 \\
.58 \\
.58\end{array}$ & $\begin{array}{r}1.26 \\
.81 \\
.81 \\
.81 \\
.81\end{array}$ & $\begin{array}{l}2.91 \\
.44 \\
.44 \\
.44 \\
.44 \\
.44\end{array}$ \\
\hline
\end{tabular}


Monthly discharge of Kaehulua Stream at Kuhinoa weir, near Kealia, Kauai, for 1913.

\begin{tabular}{|c|c|c|c|c|}
\hline \multirow{2}{*}{ Month. } & \multicolumn{3}{|c|}{$\begin{array}{l}\text { Discharge in million gallons per } \\
\text { day. }\end{array}$} & \multirow{2}{*}{$\begin{array}{l}\text { Run-ofi } \\
\text { (total in } \\
\text { acre-feet). }\end{array}$} \\
\hline & Maximum. & Minimum. & Mean. & \\
\hline 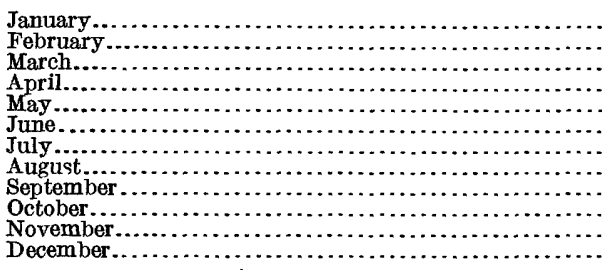 & $\begin{array}{r}0.58 \\
.44 \\
.44 \\
.32 \\
2.54 \\
11.1 \\
.81 \\
.12 \\
.12 \\
.58 \\
39.7 \\
6.52\end{array}$ & $\begin{array}{r}0.04 \\
.12 \\
.12 \\
.04 \\
.12 \\
.12 \\
.12 \\
.04 \\
.04 \\
.07 \\
.26 \\
.44\end{array}$ & $\begin{array}{r}0.14 \\
.26 \\
.20 \\
.13 \\
.58 \\
1.56 \\
.26 \\
.08 \\
.09 \\
.24 \\
2.86 \\
3.43\end{array}$ & $\begin{array}{r}13 \\
22 \\
19 \\
12 \\
55 \\
144 \\
25 \\
8 \\
8 \\
23 \\
263 \\
326\end{array}$ \\
\hline The year.......... & 39.7 & .04 & .82 & 918 \\
\hline
\end{tabular}

\section{NORTH FORK OF KEAHULUA STREAM AT KAINAHOLA WEIR, NEAR} KEALIA, KAUAI.

Location.-About 3 miles southwest of Kealia.

Records available.-April 1, 1911, to December 31, 1913.

Gage.-Vertical staff.

Discharge measurements.-Made with 3-foot sharp-crested weir.

Cooperation.--Station maintained and daily-discharge record furnished by the Makee Sugar Co.

Daily discharge, in million gallons per day, of North Fork of Kaehulua Stream at Kainahola weir, near Kealia, Kauai, for 1913.

\begin{tabular}{|c|c|c|c|c|c|c|c|c|c|c|c|c|}
\hline Day. & Jan. & $\mathrm{Feb}$. & Mar. & Apr. & May. & June. & July. & Aug. & Sept. & Oct. & Nov. & Dec. \\
\hline $\begin{array}{l}1 \ldots \\
2 \ldots \\
3 \ldots \\
4 \ldots \\
5 \ldots\end{array}$ & $\begin{array}{r}0.41 \\
.41 \\
.41 \\
.41 \\
.41\end{array}$ & $\begin{array}{r}0.41 \\
.41 \\
.41 \\
.41 \\
.41\end{array}$ & $\begin{array}{r}0.41 \\
.41 \\
.41 \\
.41 \\
.41\end{array}$ & $\begin{array}{r}0.41 \\
.41 \\
.41 \\
.41 \\
.41\end{array}$ & $\begin{array}{r}0.41 \\
.41 \\
.41 \\
.41 \\
.41\end{array}$ & $\begin{array}{r}0.41 \\
.41 \\
.96 \\
.58 \\
.58\end{array}$ & $\begin{array}{r}0.41 \\
.41 \\
.41 \\
.41 \\
.41\end{array}$ & $\begin{array}{r}0.41 \\
.41 \\
.41 \\
.41 \\
.41\end{array}$ & $\begin{array}{r}0.41 \\
.41 \\
.49 \\
.41 \\
.41\end{array}$ & $\begin{array}{r}0.41 \\
.41 \\
.41 \\
.41 \\
.41\end{array}$ & $\begin{array}{r}0.41 \\
.41 \\
.41 \\
.41 \\
.41\end{array}$ & $\begin{array}{r}0.81 \\
.58 \\
.62 \\
1.01 \\
.81\end{array}$ \\
\hline $\begin{array}{r}6 \ldots \\
7 \ldots \\
8 \ldots \\
9 \ldots \\
10 \ldots\end{array}$ & $\begin{array}{l}.41 \\
.41 \\
.41 \\
.41 \\
.41\end{array}$ & $\begin{array}{l}.49 \\
.54 \\
.45 \\
.41 \\
.41\end{array}$ & $\begin{array}{l}.41 \\
.41 \\
.41 \\
.41 \\
.41\end{array}$ & $\begin{array}{l}.41 \\
.41 \\
.41 \\
.41 \\
.41\end{array}$ & $\begin{array}{l}.41 \\
.41 \\
.41 \\
.49 \\
.41\end{array}$ & $\begin{array}{l}.49 \\
.49 \\
.41 \\
.41 \\
.41\end{array}$ & $\begin{array}{l}.41 \\
.41 \\
.41 \\
.41 \\
.41\end{array}$ & $\begin{array}{l}.41 \\
.41 \\
.41 \\
.41 \\
.41\end{array}$ & $\begin{array}{l}.41 \\
.41 \\
.41 \\
.41 \\
.41\end{array}$ & $\begin{array}{l}.41 \\
.41 \\
.41 \\
.41 \\
.41\end{array}$ & $\begin{array}{l}.41 \\
.41 \\
.41 \\
.49 \\
.41\end{array}$ & $\begin{array}{l}.58 \\
.49 \\
.49 \\
.41 \\
.41\end{array}$ \\
\hline $11 .$. & $\begin{array}{l}.41 \\
.41 \\
.41 \\
.41 \\
.41\end{array}$ & $\begin{array}{l}.41 \\
.41 \\
.41 \\
.41 \\
.41\end{array}$ & $\begin{array}{l}.38 \\
.38 \\
.38 \\
.38 \\
.38\end{array}$ & $\begin{array}{l}.41 \\
.49 \\
.49 \\
.41 \\
.41\end{array}$ & $\begin{array}{l}.41 \\
.45 \\
.41 \\
.41 \\
.41\end{array}$ & $\begin{array}{l}.41 \\
.41 \\
.41 \\
.41 \\
.41\end{array}$ & $\begin{array}{l}.41 \\
.41 \\
.41 \\
.41 \\
.41\end{array}$ & $\begin{array}{l}.41 \\
.41 \\
.41 \\
.41 \\
.41\end{array}$ & $\begin{array}{l}.41 \\
.41 \\
.41 \\
.41 \\
.41\end{array}$ & $\begin{array}{l}.41 \\
.41 \\
.41 \\
.41 \\
.41\end{array}$ & $\begin{array}{l}.41 \\
.41 \\
.41 \\
.41 \\
.41\end{array}$ & $\begin{array}{l}.41 \\
.41 \\
.41 \\
.41 \\
.41\end{array}$ \\
\hline $20 .$. & $\begin{array}{l}.41 \\
.41 \\
.41 \\
.41 \\
.41\end{array}$ & $\begin{array}{l}.41 \\
.41 \\
.58 \\
.45 \\
.41\end{array}$ & $\begin{array}{l}.38 \\
.38 \\
.38 \\
.38 \\
.38\end{array}$ & $\begin{array}{l}.41 \\
.41 \\
.41 \\
.41 \\
.41\end{array}$ & $\begin{array}{l}.41 \\
.41 \\
.41 \\
.41 \\
.41\end{array}$ & $\begin{array}{l}.41 \\
.41 \\
.41 \\
.41 \\
.41\end{array}$ & $\begin{array}{l}.41 \\
.41 \\
.41 \\
.41 \\
.41\end{array}$ & $\begin{array}{l}.41 \\
.41 \\
.41 \\
.41 \\
.41\end{array}$ & $\begin{array}{l}.41 \\
.41 \\
41 \\
.41 \\
.41\end{array}$ & $\begin{array}{l}.41 \\
.41 \\
.41 \\
.41 \\
.41\end{array}$ & $\begin{array}{l}.41 \\
.41 \\
.41 \\
.41 \\
.58\end{array}$ & $\begin{array}{l}.41 \\
.41 \\
.41 \\
.41 \\
.41\end{array}$ \\
\hline 24 & $\begin{array}{l}.41 \\
.41 \\
.41 \\
.41 \\
.41\end{array}$ & $\begin{array}{l}.41 \\
.41 \\
.41 \\
.41 \\
.41\end{array}$ & $\begin{array}{l}.38 \\
.38 \\
.58 \\
.49 \\
.41\end{array}$ & $\begin{array}{l}.41 \\
.41 \\
.41 \\
.41 \\
.41\end{array}$ & $\begin{array}{l}.41 \\
.41 \\
.41 \\
.41 \\
.41\end{array}$ & $\begin{array}{l}.41 \\
.41 \\
.41 \\
.41 \\
.41\end{array}$ & $\begin{array}{l}.41 \\
.41 \\
.41 \\
.41 \\
.41\end{array}$ & $\begin{array}{l}.41 \\
.41 \\
.41 \\
.41 \\
.41\end{array}$ & $\begin{array}{l}.41 \\
.41 \\
.41 \\
.41 \\
.41\end{array}$ & $\begin{array}{l}.41 \\
.41 \\
.41 \\
.41 \\
.41\end{array}$ & $\begin{array}{l}.67 \\
.62 \\
.58 \\
.58 \\
.58\end{array}$ & $\begin{array}{l}.41 \\
.41 \\
.41 \\
.41 \\
.41\end{array}$ \\
\hline $\begin{array}{l}26 \ldots \ldots \\
27 \ldots \ldots \\
28 \ldots \ldots \\
29 \ldots \ldots \\
30 \ldots \ldots \\
31 \ldots \ldots\end{array}$ & $\begin{array}{l}.41 \\
.41 \\
.41 \\
.41 \\
.41 \\
.41\end{array}$ & $\begin{array}{c}.41 \\
.41 \\
.41 \\
\ldots . \cdots \\
\cdots . . .\end{array}$ & $\begin{array}{l}.41 \\
.41 \\
.41 \\
.41 \\
.41 \\
.41\end{array}$ & $\begin{array}{l}.41 \\
.41 \\
.41 \\
.41 \\
.41 \\
.41\end{array}$ & $\begin{array}{l}.41 \\
.41 \\
.41 \\
.41 \\
.41 \\
.41\end{array}$ & $\begin{array}{l}.41 \\
.41 \\
.41 \\
.41 \\
.41\end{array}$ & $\begin{array}{l}.41 \\
.41 \\
.41 \\
.41 \\
.41 \\
.41\end{array}$ & $\begin{array}{l}.41 \\
.41 \\
.41 \\
.41 \\
.41 \\
.41\end{array}$ & $\begin{array}{l}.41 \\
.41 \\
.58 \\
.41 \\
.41\end{array}$ & $\begin{array}{l}.41 \\
.41 \\
.41 \\
.41 \\
.41 \\
.41\end{array}$ & $\begin{array}{l}.49 \\
.41 \\
.41 \\
.41 \\
.41\end{array}$ & $\begin{array}{l}.41 \\
.41 \\
.41 \\
.41 \\
.41 \\
.41\end{array}$ \\
\hline
\end{tabular}


Monthly discharge of North Fork of Kaehulua Stream at Kainahola weir, near Kealia, Kauai, for 1913.

\begin{tabular}{|c|c|c|c|c|}
\hline \multirow{2}{*}{ Month. } & \multicolumn{3}{|c|}{ Discharge in million gallons per day. } & \multirow{2}{*}{$\begin{array}{l}\text { Run-off } \\
\text { (total in } \\
\text { acre-feet). }\end{array}$} \\
\hline & Maximum. & Minimum. & Mean. & \\
\hline 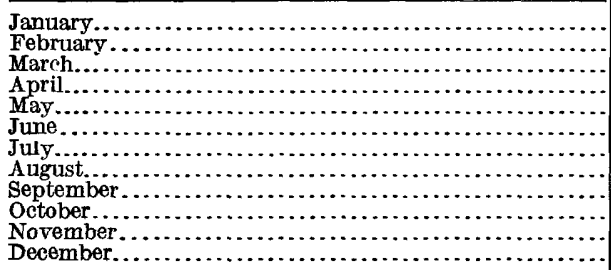 & $\begin{array}{r}0.41 \\
.58 \\
.58 \\
.49 \\
.49 \\
.96 \\
.41 \\
.41 \\
.58 \\
.41 \\
.67 \\
1.01\end{array}$ & $\begin{array}{r}0.41 \\
.41 \\
.38 \\
.41 \\
.41 \\
.41 \\
.41 \\
.41 \\
.41 \\
.41 \\
.41 \\
.41\end{array}$ & $\begin{array}{l}0.41 \\
.43 \\
.41 \\
.42 \\
.41 \\
.44 \\
.41 \\
.41 \\
.42 \\
.41 \\
.45 \\
.48\end{array}$ & $\begin{array}{l}39 \\
37 \\
39 \\
39 \\
39 \\
41 \\
39 \\
39 \\
39 \\
39 \\
41 \\
46\end{array}$ \\
\hline The year.. & 1.01 & .38 & .42 & 477 \\
\hline
\end{tabular}

KAPAA RIVER NEAR KEALIA, KAUAI.

Location.-About 1 mile above intake of Kapahi ditch and 5 miles northwest of Kealia.

Records available.-July 23, 1910, to December 31, 1913.

Drainage area. -3.6 square miles.

Gage.-Vertical staff.

Control.-Fairly permanent.

Discharge measurements.-Made from wire suspension footbridge or by wading.

Accuracy.-Records good except for November and December, when records were fair.

Cooperation.-Station is maintained in cooperation with the Makee Sugar Co.

The following discharge measurement was made by W. V. Hardy:

May 20, 1913: Gage height, 1.54 feet; discharge, 14.1 second-feet.

Daily discharge, in second-feet, of Kapaa River near Kealia, Kauai, for 1918.

\begin{tabular}{|c|c|c|c|c|c|c|c|c|c|c|c|c|}
\hline Day. & Jan. & Feb. & Mar. & Apr. & May. & June. & July. & Aug. & Sept. & Oct. & Nov. & Dec. \\
\hline $\begin{array}{l}1 \ldots \ldots \\
2 \ldots \ldots \\
3 \ldots \ldots \\
4 \ldots \ldots \\
5 . \ldots \ldots\end{array}$ & $\begin{array}{l}17 \\
20 \\
15 \\
13 \\
13\end{array}$ & $\begin{array}{l}12 \\
12 \\
12 \\
12 \\
12\end{array}$ & $\begin{array}{l}12 \\
12 \\
12 \\
11 \\
11\end{array}$ & $\begin{array}{l}22 \\
20 \\
23 \\
20 \\
16\end{array}$ & $\begin{array}{l}13 \\
30 \\
14 \\
16 \\
13\end{array}$ & $\begin{array}{r}16 \\
153 \\
155 \\
78 \\
38\end{array}$ & $\begin{array}{l}23 \\
54 \\
24 \\
33 \\
33\end{array}$ & $\begin{array}{l}14 \\
15 \\
14 \\
13 \\
13\end{array}$ & $\begin{array}{l}32 \\
20 \\
44 \\
18 \\
17\end{array}$ & $\begin{array}{l}13 \\
13 \\
13 \\
13 \\
12\end{array}$ & $\begin{array}{l}15 \\
34 \\
24 \\
18 \\
18\end{array}$ & $\begin{array}{r}27 \\
21 \\
220 \\
139 \\
42\end{array}$ \\
\hline 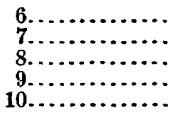 & $\begin{array}{l}14 \\
18 \\
28 \\
19 \\
20\end{array}$ & $\begin{array}{r}158 \\
46 \\
21 \\
16 \\
14\end{array}$ & $\begin{array}{l}12 \\
24 \\
14 \\
13 \\
12\end{array}$ & $\begin{array}{l}14 \\
13 \\
13 \\
24 \\
17\end{array}$ & $\begin{array}{l}32 \\
23 \\
51 \\
27 \\
17\end{array}$ & $\begin{array}{l}38 \\
24 \\
20 \\
42 \\
23\end{array}$ & $\begin{array}{l}19 \\
17 \\
16 \\
17 \\
14\end{array}$ & $\begin{array}{l}13 \\
17 \\
16 \\
21 \\
16\end{array}$ & $\begin{array}{l}32 \\
20 \\
17 \\
16 \\
15\end{array}$ & $\begin{array}{l}23 \\
37 \\
30 \\
48 \\
17\end{array}$ & $\begin{array}{r}16 \\
36 \\
22 \\
115 \\
48\end{array}$ & $\begin{array}{l}60 \\
38 \\
29 \\
22 \\
20\end{array}$ \\
\hline 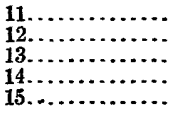 & $\begin{array}{l}17 \\
42 \\
96 \\
26 \\
21\end{array}$ & $\begin{array}{l}15 \\
13 \\
13 \\
12 \\
13\end{array}$ & $\begin{array}{l}11 \\
11 \\
13 \\
12 \\
11\end{array}$ & $\begin{array}{l}22 \\
14 \\
31 \\
45 \\
35\end{array}$ & $\begin{array}{r}16 \\
48 \\
115 \\
36 \\
21\end{array}$ & $\begin{array}{l}32 \\
20 \\
23 \\
22 \\
21\end{array}$ & $\begin{array}{l}15 \\
19 \\
33 \\
24 \\
17\end{array}$ & $\begin{array}{l}14 \\
17 \\
23 \\
41 \\
19\end{array}$ & $\begin{array}{l}14 \\
16 \\
14 \\
14 \\
14\end{array}$ & $\begin{array}{l}20 \\
19 \\
26 \\
19 \\
20\end{array}$ & $\begin{array}{l}44 \\
64 \\
60 \\
26 \\
20\end{array}$ & $\begin{array}{l}18 \\
17 \\
17 \\
16 \\
15\end{array}$ \\
\hline $\begin{array}{l}16 \ldots \ldots \ldots \\
17 \ldots \ldots \ldots \\
18 \ldots \ldots \ldots \\
19, \ldots \ldots \ldots\end{array}$ & $\begin{array}{l}\mathbf{5 1} \\
\mathbf{2 1} \\
17 \\
16 \\
14\end{array}$ & $\begin{array}{l}14 \\
13 \\
86 \\
36 \\
36\end{array}$ & $\begin{array}{l}14 \\
13 \\
13 \\
13 \\
16\end{array}$ & $\begin{array}{l}20 \\
52 \\
29 \\
23 \\
17\end{array}$ & $\begin{array}{l}32 \\
39 \\
33 \\
19 \\
16\end{array}$ & $\begin{array}{l}24 \\
18 \\
18 \\
16 \\
14\end{array}$ & $\begin{array}{l}17 \\
16 \\
14 \\
19 \\
19\end{array}$ & $\begin{array}{l}31 \\
18 \\
14 \\
14 \\
14\end{array}$ & $\begin{array}{l}14 \\
13 \\
13 \\
13 \\
13\end{array}$ & $\begin{array}{l}18 \\
16 \\
36 \\
17 \\
15\end{array}$ & $\begin{array}{r}21 \\
21 \\
31 \\
71 \\
127\end{array}$ & $\begin{array}{l}13 \\
13 \\
14 \\
13 \\
13\end{array}$ \\
\hline 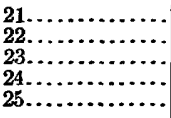 & $\begin{array}{l}64 \\
46 \\
19 \\
20 \\
18\end{array}$ & $\begin{array}{l}18 \\
18 \\
32 \\
28 \\
16\end{array}$ & $\begin{array}{r}19 \\
26 \\
95 \\
105 \\
121\end{array}$ & $\begin{array}{l}17 \\
17 \\
19 \\
15 \\
19\end{array}$ & $\begin{array}{l}15 \\
14 \\
13 \\
16 \\
16\end{array}$ & $\begin{array}{l}14 \\
14 \\
14 \\
13 \\
13\end{array}$ & $\begin{array}{l}17 \\
14 \\
14 \\
16 \\
22\end{array}$ & $\begin{array}{l}21 \\
17 \\
16 \\
17 \\
21\end{array}$ & $\begin{array}{l}15 \\
14 \\
13 \\
13 \\
12\end{array}$ & $\begin{array}{r}38 \\
168 \\
29 \\
20 \\
20\end{array}$ & $\begin{array}{r}631 \\
121 \\
62 \\
76 \\
127\end{array}$ & $\begin{array}{l}13 \\
13 \\
13 \\
12 \\
12\end{array}$ \\
\hline 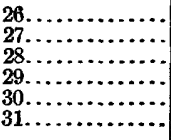 & $\begin{array}{l}16 \\
16 \\
14 \\
14 \\
13 \\
12\end{array}$ & $\begin{array}{r}14 \\
13 \\
13 \\
\cdots \cdots \\
\cdots \cdots\end{array}$ & $\begin{array}{l}62 \\
24 \\
20 \\
18 \\
18 \\
18\end{array}$ & $\begin{array}{l}14 \\
13 \\
13 \\
15 \\
15\end{array}$ & $\begin{array}{l}14 \\
13 \\
16 \\
14 \\
28 \\
15\end{array}$ & $\begin{array}{l}22 \\
49 \\
37 \\
20 \\
37\end{array}$ & $\begin{array}{l}28 \\
27 \\
16 \\
34 \\
18 \\
17\end{array}$ & $\begin{array}{l}21 \\
16 \\
22 \\
44 \\
34 \\
30\end{array}$ & $\begin{array}{l}12 \\
12 \\
48 \\
13 \\
14\end{array}$ & $\begin{array}{l}18 \\
16 \\
41 \\
21 \\
17 \\
16\end{array}$ & $\begin{array}{l}67 \\
51 \\
39 \\
26 \\
62\end{array}$ & $\begin{array}{l}12 \\
12 \\
12 \\
12 \\
12 \\
12\end{array}$ \\
\hline
\end{tabular}


Monthly discharge of Kapaa River near Kealia, Kauai, for 1913.

\begin{tabular}{|c|c|c|c|c|c|}
\hline \multirow{2}{*}{ Month. } & \multicolumn{3}{|c|}{ Discharge in second-feet. } & \multirow{2}{*}{$\begin{array}{l}\text { Run-off } \\
\text { (total in } \\
\text { acre-feet). }\end{array}$} & \multirow{2}{*}{$\begin{array}{l}\text { Accu- } \\
\text { racy. }\end{array}$} \\
\hline & Maximum. & Minimum. & Mean. & & \\
\hline 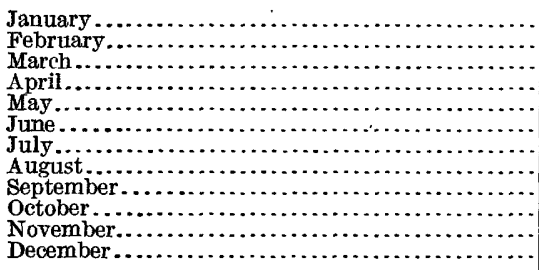 & $\begin{array}{r}96 \\
158 \\
121 \\
52 \\
115 \\
155 \\
54 \\
44 \\
48 \\
168 \\
631 \\
220\end{array}$ & $\begin{array}{l}12 \\
12 \\
11 \\
13 \\
13 \\
13 \\
14 \\
13 \\
12 \\
12 \\
15 \\
12\end{array}$ & $\begin{array}{l}24.2 \\
25.6 \\
25.4 \\
20.9 \\
25.3 \\
34.3 \\
21.5 \\
19.9 \\
17.8 \\
26.7 \\
69.8 \\
29.1\end{array}$ & $\begin{array}{l}1,490 \\
1,420 \\
1,560 \\
1,240 \\
1,560 \\
2,040 \\
1,320 \\
1,220 \\
1,060 \\
1,640 \\
4,150 \\
1,790\end{array}$ & $\begin{array}{l}\text { A. } \\
\text { A. } \\
\text { A. } \\
\text { A. } \\
\text { A. } \\
\text { A. } \\
\text { A. } \\
\text { A. } \\
\text { A. } \\
\text { A. } \\
\text { B. }\end{array}$ \\
\hline The year....... & 631 & 11 & 28.2 & 20,500 & \\
\hline
\end{tabular}

\section{AKULĮKULI SPRING NEAR KEALIA, KAUAI.}

Location.-About 5 miles west of Kealia.

Records available.-April 1, 1911, to December 31, 1913.

Gage.-Vertical staff.

Discharge measurements.-Made with a 3-foot sharp-crested weir.

Cooperation.-Station maintained and daily-discharge record furnished by the Makee Sugar Co.

Discharge, in million gallons per day, of Akulikuli Spring near Kealia, Kauai, for 1913.

\begin{tabular}{|c|c|c|c|c|c|c|c|c|c|c|c|c|}
\hline Day. & Jan. & Feb. & Mar. & Apr. & May. & June. & July. & Aug. & Sept. & Oct. & Nov. & Dec. \\
\hline $\begin{array}{l}1 \ldots \\
2 . \\
3 . \\
4 . \\
5 . .\end{array}$ & $\begin{array}{r}0.67 \\
.67 \\
.67 \\
.67 \\
.67\end{array}$ & $\begin{array}{r}0.62 \\
.62 \\
.62 \\
.62 \\
.62\end{array}$ & $\begin{array}{r}0.67 \\
.67 \\
.62 \\
.58 \\
.58\end{array}$ & $\begin{array}{r}0.67 \\
.67 \\
.67 \\
.67 \\
.67\end{array}$ & $\begin{array}{r}0.67 \\
.67 \\
.67 \\
.67 \\
.67\end{array}$ & $\begin{array}{l}0.58 \\
.58 \\
4.22 \\
3.63 \\
2.64\end{array}$ & $\begin{array}{r}0.67 \\
1.76 \\
1.34 \\
1.34 \\
.96\end{array}$ & $\begin{array}{r}0.67 \\
.67 \\
.67 \\
.67 \\
.67\end{array}$ & $\begin{array}{r}0.76 \\
.71 \\
1.64 \\
1.28 \\
.67\end{array}$ & $\begin{array}{r}0.67 \\
.67 \\
.67 \\
.62 \\
.58\end{array}$ & $\begin{array}{r}0.67 \\
.67 \\
.67 \\
.67 \\
.67\end{array}$ & $\begin{array}{l}1.06 \\
.81 \\
1.64 \\
1.95 \\
1.34\end{array}$ \\
\hline $\begin{array}{r}6 \ldots \\
7 \ldots \\
8 \ldots \\
9 \ldots \\
10 \ldots\end{array}$ & $\begin{array}{l}.67 \\
.67 \\
.67 \\
.67 \\
.67\end{array}$ & $\begin{array}{r}1.59 \\
2.08 \\
.81 \\
.67 \\
.67\end{array}$ & $\begin{array}{l}.58 \\
.58 \\
.58 \\
.58 \\
.58\end{array}$ & $\begin{array}{l}.67 \\
.67 \\
.67 \\
.67 \\
.67\end{array}$ & $\begin{array}{r}.67 \\
1.06 \\
.81 \\
1.34 \\
.81\end{array}$ & $\begin{array}{r}1.95 \\
1.06 \\
.36 \\
1.06 \\
.81\end{array}$ & $\begin{array}{l}.81 \\
.81 \\
.67 \\
.67 \\
.67\end{array}$ & $\begin{array}{l}.67 \\
.67 \\
.67 \\
.67 \\
.67\end{array}$ & $\begin{array}{l}.67 \\
.67 \\
.67 \\
.67 \\
.67\end{array}$ & $\begin{array}{l}.58 \\
.67 \\
.67 \\
.67 \\
.67\end{array}$ & $\begin{array}{l}.67 \\
.76 \\
.67 \\
.71 \\
.67\end{array}$ & $\begin{array}{r}1.17 \\
1.06 \\
.96 \\
.81 \\
.81\end{array}$ \\
\hline$\cdots$ & $\begin{array}{l}.67 \\
.76 \\
.71 \\
.71 \\
.67\end{array}$ & $\begin{array}{l}.67 \\
.67 \\
.67 \\
.67 \\
.67\end{array}$ & $\begin{array}{l}.58 \\
.58 \\
.58 \\
.58 \\
.58\end{array}$ & $\begin{array}{l}.67 \\
.67 \\
.67 \\
.67 \\
.96\end{array}$ & $\begin{array}{r}.67 \\
1.34 \\
1.52 \\
.81 \\
.81\end{array}$ & $\begin{array}{l}.71 \\
.67 \\
.67 \\
.67 \\
.67\end{array}$ & $\begin{array}{l}.67 \\
.67 \\
.81 \\
.76 \\
.67\end{array}$ & $\begin{array}{l}.67 \\
.67 \\
.67 \\
.67 \\
.81\end{array}$ & $\begin{array}{l}.67 \\
.67 \\
.67 \\
.67 \\
.67\end{array}$ & $\begin{array}{l}.67 \\
.67 \\
.67 \\
.67 \\
.67\end{array}$ & $\begin{array}{l}.67 \\
.67 \\
.67 \\
.67 \\
.67\end{array}$ & $\begin{array}{l}.81 \\
.76 \\
.67 \\
.67 \\
.67\end{array}$ \\
\hline $16 \ldots \ldots \ldots$ & $\begin{array}{r}3.39 \\
1.06 \\
.76 \\
.76 \\
.67\end{array}$ & $\begin{array}{r}.67 \\
.67 \\
2.21 \\
.91 \\
.81\end{array}$ & $\begin{array}{l}.58 \\
.58 \\
.58 \\
.58 \\
.58\end{array}$ & $\begin{array}{r}.76 \\
1.52 \\
.86 \\
.67 \\
.67\end{array}$ & $\begin{array}{l}.81 \\
.91 \\
.81 \\
.71 \\
.67\end{array}$ & $\begin{array}{l}.67 \\
.67 \\
.67 \\
.67 \\
.67\end{array}$ & $\begin{array}{l}.67 \\
.67 \\
.67 \\
.67 \\
.67\end{array}$ & $\begin{array}{l}.81 \\
.76 \\
.67 \\
.67 \\
.67\end{array}$ & $\begin{array}{l}.67 \\
.67 \\
.67 \\
.62 \\
.62\end{array}$ & $\begin{array}{l}.67 \\
.67 \\
.67 \\
.67 \\
.67\end{array}$ & $\begin{array}{l}.67 \\
.67 \\
.67 \\
.67 \\
.81\end{array}$ & $\begin{array}{l}.67 \\
.67 \\
.67 \\
.67 \\
.67\end{array}$ \\
\hline $\begin{array}{l}23 . \\
24 . \\
25 .\end{array}$ & $\begin{array}{l}.71 \\
.71 \\
.67 \\
.67 \\
.67\end{array}$ & $\begin{array}{l}.67 \\
.67 \\
.71 \\
.96 \\
.67\end{array}$ & $\begin{array}{r}.58 \\
.71 \\
2.36 \\
1.06 \\
.81\end{array}$ & $\begin{array}{l}.67 \\
.67 \\
.67 \\
.67 \\
.67\end{array}$ & $\begin{array}{l}.67 \\
.67 \\
.67 \\
.67 \\
.67\end{array}$ & $\begin{array}{l}.67 \\
.67 \\
.67 \\
.67 \\
.67\end{array}$ & $\begin{array}{l}.67 \\
.67 \\
.67 \\
.67 \\
.67\end{array}$ & $\begin{array}{l}.67 \\
.67 \\
.67 \\
.67 \\
.67\end{array}$ & $\begin{array}{l}.62 \\
.62 \\
.62 \\
.62 \\
.62\end{array}$ & $\begin{array}{l}.67 \\
.67 \\
.76 \\
.67 \\
.67\end{array}$ & $\begin{array}{l}2.43 \\
1.34 \\
1.06 \\
.96 \\
1.06\end{array}$ & $\begin{array}{l}.67 \\
.67 \\
.67 \\
.67 \\
.67\end{array}$ \\
\hline $\begin{array}{l}26 \ldots \\
27 \ldots \\
28 \ldots \\
29 \ldots \\
30 \ldots \\
31 \ldots \\
\end{array}$ & $\begin{array}{l}.67 \\
.67 \\
.67 \\
.67 \\
.62 \\
.62\end{array}$ & \begin{tabular}{r}
.67 \\
.67 \\
.67 \\
\hdashline .6. \\
$\ldots . .6$
\end{tabular} & $\begin{array}{l}.81 \\
.71 \\
.71 \\
.71 \\
.67 \\
.67\end{array}$ & $\begin{array}{l}.67 \\
.67 \\
.67 \\
.67 \\
.67\end{array}$ & $\begin{array}{l}.67 \\
.58 \\
.58 \\
.58 \\
.58 \\
.58\end{array}$ & $\begin{array}{r}.67 \\
.67 \\
1.23 \\
.91 \\
.81\end{array}$ & $\begin{array}{l}.67 \\
.67 \\
.67 \\
.67 \\
.67 \\
.67\end{array}$ & $\begin{array}{l}.67 \\
.67 \\
.67 \\
.67 \\
.67 \\
.67\end{array}$ & $\begin{array}{l}.62 \\
.62 \\
.96 \\
.67 \\
.67\end{array}$ & $\begin{array}{r}.67 \\
1.67 \\
1.23 \\
1.28 \\
.67 \\
.67\end{array}$ & $\begin{array}{l}.81 \\
.67 \\
.67 \\
.67 \\
.67\end{array}$ & $\begin{array}{l}.67 \\
.67 \\
.67 \\
.67 \\
.67 \\
.67\end{array}$ \\
\hline
\end{tabular}


Monthly discharge of Akulikuli Spring near Kealia, Kauai, for 1913.

\begin{tabular}{|c|c|c|c|c|}
\hline \multirow{2}{*}{ Month. } & \multicolumn{3}{|c|}{$\begin{array}{l}\text { Discharge in million gallons per } \\
\text { day. }\end{array}$} & \multirow{2}{*}{$\begin{array}{l}\text { Rum-off } \\
\text { (total in } \\
\text { acre-feet). }\end{array}$} \\
\hline & Maximum. & Minimum. & Mean. & \\
\hline 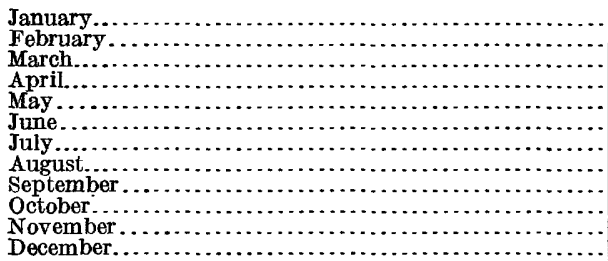 & $\begin{array}{l}\text { 3. } 39 \\
2.21 \\
2.36 \\
1.52 \\
1.52 \\
4.22 \\
1.76 \\
.81 \\
1.64 \\
1.28 \\
2.43 \\
1.95\end{array}$ & $\begin{array}{r}0.62 \\
.62 \\
.58 \\
.67 \\
.58 \\
.58 \\
.67 \\
.67 \\
.62 \\
.58 \\
.67 \\
.67\end{array}$ & $\begin{array}{r}0.78 \\
.83 \\
.70 \\
.72 \\
.78 \\
1.06 \\
.77 \\
.68 \\
.72 \\
.70 \\
.80 \\
.84\end{array}$ & $\begin{array}{l}74 \\
71 \\
67 \\
66 \\
74 \\
\mathbf{9 8} \\
73 \\
65 \\
66 \\
67 \\
74 \\
80\end{array}$ \\
\hline The year $\ldots \ldots \ldots \ldots \ldots \ldots \ldots \ldots \ldots \ldots \ldots \ldots$ & 4. 22 & .58 & .78 & 875 \\
\hline
\end{tabular}

KAPAHI DITCH AT KAPAHI, NEAR KEALIA, KAUAI.

Location.-About 4 miles west of Kealia and 500 feet below diversion dam on Kapaa River.

Records available.-April 15, 1909, to December 31, 1913.

Gage.-Watson water-stage recorder.

Discharge measurements.-There is a 20 -foot sharp-crested weir immediately below the gage, but current-meter measurements give a different rating from the weir formula, and the meter rating is used.

Diversion.-Ditch diverts part of flow of Kapaa River.

Accuracy.-Records good.

Cooperation.-Station is maintained in cooperation with the Makee Sugar Co.

Daily discharge, in second-feet, of Kapahi ditch at Kapahi, near Kealia, Kauai, for 1913.

\begin{tabular}{|c|c|c|c|c|c|c|c|c|c|c|c|c|}
\hline Day. & Jan. & Feb. & Mar. & Apr. & May. & June. & July. & Aug. & Sept. & Oct. & Nov. & Dec. \\
\hline $\begin{array}{l}1 \ldots \\
2 \ldots \\
3 \ldots \\
4 \ldots \\
5 \ldots\end{array}$ & $\begin{array}{l}16.9 \\
27.0 \\
14.8 \\
13.0 \\
12.4\end{array}$ & $\begin{array}{l}10.6 \\
10.6 \\
10.6 \\
10.6 \\
10.6\end{array}$ & $\begin{array}{r}11.2 \\
10.6 \\
10.0 \\
9.0 \\
9.5\end{array}$ & $\begin{array}{l}14.2 \\
17.6 \\
15.5 \\
13.6 \\
14.2\end{array}$ & $\begin{array}{l}10.0 \\
22.4 \\
14.8 \\
14.8 \\
13.0\end{array}$ & $\begin{array}{r}14.8 \\
23.2 \\
93.0 \\
6.0 \\
10.0\end{array}$ & $\begin{array}{l}26.0 \\
71.0 \\
29.0 \\
31.0 \\
27.0\end{array}$ & $\begin{array}{l}14.2 \\
14.2 \\
12.4 \\
11.8 \\
11.2\end{array}$ & $\begin{array}{l}20.0 \\
18.4 \\
16.2 \\
21.6 \\
33.5\end{array}$ & $\begin{array}{l}11.8 \\
11.2 \\
10.6 \\
10.0 \\
10.0\end{array}$ & $\begin{array}{l}12.4 \\
18.4 \\
22.4 \\
16.2 \\
15.5\end{array}$ & $\begin{array}{r}36.0 \\
24.0 \\
10.6 \\
9.5 \\
7.5\end{array}$ \\
\hline $\begin{array}{r}6 \ldots \\
7 \ldots \\
8 \ldots \\
9 \ldots \\
10 \ldots\end{array}$ & $\begin{array}{l}14.2 \\
19.2 \\
27.0 \\
20.0 \\
20.0\end{array}$ & $\begin{array}{c}138 \\
60.0 \\
17.6 \\
16.2 \\
14.8\end{array}$ & $\begin{array}{l}10.0 \\
18.4 \\
16.9 \\
13.0 \\
10.6\end{array}$ & $\begin{array}{l}13.0 \\
12.4 \\
11.8 \\
22.4 \\
16.2\end{array}$ & $\begin{array}{l}45.0 \\
36.0 \\
43.5 \\
30.0 \\
18.4\end{array}$ & $\begin{array}{l}12.4 \\
12.4 \\
18.4 \\
17.6 \\
14.2\end{array}$ & $\begin{array}{l}20.0 \\
16.9 \\
14.8 \\
16.2 \\
13.6\end{array}$ & $\begin{array}{l}11.8 \\
17.6 \\
14.2 \\
18.4 \\
14.2\end{array}$ & $\begin{array}{l}27.0 \\
18.4 \\
15.5 \\
13.6 \\
12.4\end{array}$ & $\begin{array}{l}17.6 \\
24.0 \\
31.0 \\
33.5 \\
35.0\end{array}$ & $\begin{array}{l}14.2 \\
24.0 \\
20.8 \\
17.6 \\
16.2\end{array}$ & $\begin{array}{r}7.5 \\
20.8 \\
36.0 \\
27.0 \\
22.4\end{array}$ \\
\hline $\begin{array}{l}11 \ldots \\
12 \ldots \\
13 \ldots \\
14 \ldots \\
15 \ldots\end{array}$ & $\begin{array}{l}24.0 \\
46.5 \\
72.0 \\
32.5 \\
31.0\end{array}$ & $\begin{array}{l}16.2 \\
13.0 \\
10.6 \\
10.6 \\
13.0\end{array}$ & $\begin{array}{r}9.5 \\
9.5 \\
10.0 \\
10.0 \\
9.5\end{array}$ & $\begin{array}{l}20.0 \\
14.2 \\
42.5 \\
60.0 \\
59.0\end{array}$ & $\begin{array}{r}38.5 \\
18.4 \\
5.0 \\
3.5 \\
3.5\end{array}$ & $\begin{array}{l}17.6 \\
20.0 \\
23.2 \\
23.2 \\
21.6\end{array}$ & $\begin{array}{l}13.6 \\
16.2 \\
37.5 \\
28.0 \\
17.6\end{array}$ & $\begin{array}{l}13.0 \\
16.9 \\
22.4 \\
16.9 \\
15.5\end{array}$ & $\begin{array}{l}12.4 \\
14.2 \\
11.8 \\
12.4 \\
12.4\end{array}$ & $\begin{array}{l}19.2 \\
16.9 \\
21.6 \\
18.4 \\
20.8\end{array}$ & $\begin{array}{l}24.0 \\
20.8 \\
20.8 \\
21.6 \\
18.4\end{array}$ & $\begin{array}{l}20.0 \\
18.4 \\
17.6 \\
16.9 \\
16.2\end{array}$ \\
\hline $\begin{array}{l}16 \ldots \\
17 \ldots \\
18 \ldots \\
19 \ldots \\
20 \ldots\end{array}$ & $\begin{array}{l}51.0 \\
24.0 \\
16.9 \\
14.8 \\
14.2\end{array}$ & $\begin{array}{l}14.2 \\
11.8 \\
66.0 \\
43.5 \\
26.0\end{array}$ & $\begin{array}{l}11.8 \\
11.2 \\
10.6 \\
10.0 \\
13.0\end{array}$ & $\begin{array}{l}21.6 \\
42.5 \\
45.0 \\
27.0 \\
17.6\end{array}$ & $\begin{array}{r}5.5 \\
7.0 \\
7.0 \\
11.2 \\
12.4\end{array}$ & $\begin{array}{l}26.0 \\
18.4 \\
16.9 \\
15.5 \\
14.2\end{array}$ & $\begin{array}{l}16.9 \\
14.8 \\
13.6 \\
26.0 \\
20.8\end{array}$ & $\begin{array}{l}14.8 \\
13.6 \\
13.0 \\
14.2 \\
13.6\end{array}$ & $\begin{array}{l}11.8 \\
11.2 \\
11.2 \\
11.2 \\
11.2\end{array}$ & $\begin{array}{l}16.2 \\
14.8 \\
22.4 \\
16.2 \\
13.0\end{array}$ & $\begin{array}{l}14.8 \\
18.4 \\
18.4 \\
19.2 \\
11.8\end{array}$ & $\begin{array}{l}14.8 \\
14.8 \\
16.2 \\
14.8 \\
14.2\end{array}$ \\
\hline $\begin{array}{l}21 \ldots \\
22 \ldots \\
23 \ldots \\
24 \ldots \\
25 \ldots\end{array}$ & $\begin{array}{l}54.0 \\
26.0 \\
17.6 \\
29.0 \\
25.0\end{array}$ & $\begin{array}{l}20.0 \\
18.4 \\
48.0 \\
36.0 \\
15.5\end{array}$ & $\begin{array}{r}17.6 \\
25.0 \\
74.0 \\
60.0 \\
7.0\end{array}$ & $\begin{array}{l}16.2 \\
16.2 \\
17.6 \\
13.6 \\
20.0\end{array}$ & $\begin{array}{l}12.4 \\
13.6 \\
13.0 \\
16.9 \\
16.2\end{array}$ & $\begin{array}{l}14.2 \\
13.6 \\
13.6 \\
13.0 \\
13.0\end{array}$ & $\begin{array}{l}15.5 \\
13.6 \\
12.4 \\
15.5 \\
21.6\end{array}$ & $\begin{array}{l}32.5 \\
17.6 \\
30.0 \\
17.6 \\
38.5\end{array}$ & $\begin{array}{l}10.6 \\
12.4 \\
11.8 \\
11.2 \\
10.6\end{array}$ & $\begin{array}{l}38.5 \\
82.0 \\
31.0 \\
20.0 \\
18.4\end{array}$ & $\begin{array}{l}14.2 \\
14.8 \\
14.2 \\
13.6 \\
12.4\end{array}$ & $\begin{array}{l}14.8 \\
14.2 \\
13.6 \\
13.6 \\
13.0\end{array}$ \\
\hline $\begin{array}{l}26 \ldots \ldots \\
27 \ldots \ldots \\
28 \ldots \\
29 \ldots \\
30 \ldots \\
31 \ldots \\
\end{array}$ & $\begin{array}{l}17.6 \\
15.5 \\
14.2 \\
13.0 \\
12.4 \\
10.6\end{array}$ & $\begin{array}{l}13.6 \\
12.4 \\
11.8\end{array}$ & $\begin{array}{r}7.5 \\
6.5 \\
7.0 \\
12.4 \\
7.5 \\
13.6\end{array}$ & $\begin{array}{l}14.2 \\
12.4 \\
11.8 \\
14.2 \\
14.2\end{array}$ & $\begin{array}{l}13.6 \\
13.6 \\
15.5 \\
13.6 \\
24.0 \\
14.2\end{array}$ & $\begin{array}{l}19.2 \\
45.0 \\
31.0 \\
25.0 \\
42.5 \\
\cdots . . .\end{array}$ & $\begin{array}{l}23.2 \\
24.0 \\
16.2 \\
36.0 \\
16.9 \\
15.5\end{array}$ & $\begin{array}{l}23.2 \\
43.5 \\
16.9 \\
14.8 \\
23.2 \\
27.0\end{array}$ & $\begin{array}{l}10.6 \\
10.6 \\
40.0 \\
12.4 \\
11.8\end{array}$ & $\begin{array}{l}16.2 \\
13.6 \\
43.5 \\
23.2 \\
15.5 \\
14.2\end{array}$ & $\begin{array}{l}13.0 \\
32.5 \\
53.0 \\
16.2 \\
15.5\end{array}$ & $\begin{array}{l}13.0 \\
13.0 \\
13.0 \\
13.0 \\
13.0 \\
13.0\end{array}$ \\
\hline
\end{tabular}


Monthly discharge of Kapahi ditch at Kapahi, near Kealia, Kauai, for 1913.

\begin{tabular}{|c|c|c|c|c|c|}
\hline \multirow{2}{*}{ Month. } & \multicolumn{3}{|c|}{ Discharge in second-feet. } & \multirow{2}{*}{$\begin{array}{c}\text { Run-off } \\
\text { (total in } \\
\text { acre-feet). }\end{array}$} & \multirow{2}{*}{$\begin{array}{l}\text { Accu- } \\
\text { racy. }\end{array}$} \\
\hline & Maximum. & Minimum. & Mean. & & \\
\hline 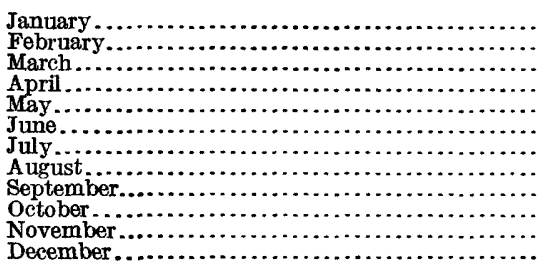 & $\begin{array}{r}72.0 \\
138 \\
74.0 \\
60.0 \\
45.0 \\
93.0 \\
71.0 \\
43.5 \\
40.0 \\
82.0 \\
53.0 \\
36.0\end{array}$ & $\begin{array}{r}10.6 \\
10.6 \\
6.5 \\
11.8 \\
3.5 \\
6.0 \\
12.4 \\
11.2 \\
10.6 \\
10.0 \\
11.8 \\
7.5\end{array}$ & $\begin{array}{l}24.1 \\
25.0 \\
14.9 \\
21.7 \\
17.0 \\
21.6 \\
22.0 \\
18.7 \\
15.3 \\
22.3 \\
18.8 \\
16.5\end{array}$ & $\begin{array}{r}1,480 \\
1,390 \\
916 \\
1,290 \\
1,050 \\
1,290 \\
1,350 \\
1,150 \\
910 \\
1,370 \\
1,120 \\
1,010\end{array}$ & $\begin{array}{l}\text { A. } \\
\text { A. } \\
\text { A. } \\
\text { A. } \\
\text { A. } \\
\text { A. } \\
\text { A. } \\
\text { A. } \\
\text { A. } \\
\text { A. } \\
\text { A. } \\
\text { A. }\end{array}$ \\
\hline The year & 138 & 3.5 & 19.8 & 14,300 & \\
\hline
\end{tabular}

KANEHA DITCH NEAR KEALIA, KAUAI.

Location.-About 5 miles northwest of Kealia, a short distance below the outlet from the Kaneha reservoir.

Records available.-January 1, 1909, to December 31, 1913.

Gage.-Staff.

Discharge measurements.-Made with a 20-foot sharp-crested weir.

Accuracy.-Records poor.

Cooperation.-Station is maintained in cooperation with the Makee Sugar Co.

Daily discharge, in second-feet, of Kaneha ditch near Kealia, Kauai, for 1913.

\begin{tabular}{|c|c|c|c|c|c|c|c|c|c|c|c|c|}
\hline Day. & Jan. & Feb. & Mar. & Apr. & May. & June. & July. & Aug. & Sept. & Oct. & Nov. & Dec. \\
\hline $\begin{array}{l}1 . . \\
2 . . \\
3 . . \\
4 . \\
5 . .\end{array}$ & $\dddot{3} .6$ & $\begin{array}{r}1.9 \\
1.9 \\
2.2 \\
16\end{array}$ & & & & $\begin{array}{l}4.8 \\
2.2 \\
12 \\
35 \\
26\end{array}$ & $\begin{array}{l}5.6 \\
5.2 \\
5.2 \\
5.6 \\
6.4\end{array}$ & $\begin{array}{l}5.2 \\
6.8 \\
7.8 \\
7.3 \\
6.8\end{array}$ & $\begin{array}{l}20 \\
10 \\
8.8 \\
13 \\
22\end{array}$ & $\begin{array}{r}3.6 \\
3.2 \\
3.2 \\
\hdashline \ldots . .\end{array}$ & $\begin{array}{r}6.0 \\
5.6 \\
12 \\
8.3 \\
6.4\end{array}$ & $\begin{array}{r}5.6 \\
5.6 \\
6.8 \\
10 \\
7.3\end{array}$ \\
\hline $\begin{array}{c}6 \ldots \ldots \\
7 \ldots \ldots \\
8 \ldots \ldots \\
9 \ldots \ldots \\
10 \ldots \ldots\end{array}$ & 4.0 & & & & & $\begin{array}{r}8.3 \\
8.8 \\
7.8 \\
10 \\
11\end{array}$ & $\begin{array}{l}6.0 \\
5.6 \\
4.8 \\
4.8 \\
3.2\end{array}$ & $\begin{array}{r}6.8 \\
6.8 \\
8.3 \\
11 \\
7.8\end{array}$ & $\begin{array}{l}13 \\
9.0 \\
7.3 \\
6.4 \\
6.0\end{array}$ & $\begin{array}{l}\ldots \ldots \ldots \\
\cdots \ldots \ldots \\
\cdots \ldots \ldots \\
\cdots \cdots\end{array}$ & $\begin{array}{l}4.8 \\
4.4 \\
5.2 \\
4.0 \\
3.6\end{array}$ & $\begin{array}{l}8.3 \\
7.8 \\
8.8 \\
7.3 \\
6.4\end{array}$ \\
\hline $\begin{array}{l}11 \ldots \ldots \\
12 \ldots \ldots \\
13 \ldots \ldots \\
14 \ldots \ldots \\
15 \ldots .\end{array}$ & $\begin{array}{c}11 \\
6.8 \\
12 \\
6.8\end{array}$ & & & & $\begin{array}{l}\cdots \\
\cdots \\
\cdots\end{array}$ & $\begin{array}{r}11 \\
6.4 \\
6.0 \\
6.0 \\
6.0\end{array}$ & $\begin{array}{c}2.8 \\
3.6 \\
11 \\
9.5 \\
7.3\end{array}$ & $\begin{array}{l}6.0 \\
5.6 \\
4.8 \\
6.0 \\
5.6\end{array}$ & $\begin{array}{l}5.6 \\
6.0 \\
6.4 \\
6.0 \\
6.4\end{array}$ & $\begin{array}{l}6.4 \\
4.4 \\
3.6 \\
12 \\
22\end{array}$ & $\begin{array}{l}5.2 \\
3.2 \\
1.6 \\
7.8 \\
6.0\end{array}$ & $\begin{array}{l}6.0 \\
6.0 \\
6.0 \\
6.4 \\
6.0\end{array}$ \\
\hline $\begin{array}{l}16 \ldots \ldots \\
17 \\
18 \ldots \ldots \ldots \\
19 \ldots \ldots \\
20 \ldots \ldots\end{array}$ & $\begin{array}{r}5.2 \\
4.4 \\
4.4 \\
4.4 \\
4.4\end{array}$ & & & & $\begin{array}{r}13 \\
11 \\
7.8 \\
5.2\end{array}$ & $\begin{array}{l}6.8 \\
6.0 \\
5.6 \\
5.2 \\
4.8\end{array}$ & $\begin{array}{l}7.3 \\
8.8 \\
8.6 \\
8.3 \\
6.8\end{array}$ & $\begin{array}{l}6.0 \\
5.6 \\
4.8 \\
6.4 \\
7.3\end{array}$ & $\begin{array}{l}6.8 \\
3.2 \\
2.8 \\
3.2 \\
3.2\end{array}$ & $\begin{array}{l}21 \\
12 \\
7.3 \\
5.2 \\
6.4\end{array}$ & $\begin{array}{l}6.4 \\
6.4 \\
6.0 \\
8.3 \\
6.4\end{array}$ & $\begin{array}{l}4.8 \\
4.4 \\
4.4 \\
4.4 \\
3.6\end{array}$ \\
\hline $\begin{array}{l}21 \ldots \ldots . \\
22 \ldots \ldots . \\
23 \ldots \ldots \\
24 \ldots \ldots \\
25 \ldots \ldots\end{array}$ & 4.4 & $\begin{array}{l}5.2 \\
4.0 \\
3.6\end{array}$ & & & $\begin{array}{l}4.4 \\
3.6 \\
3.2 \\
3.2 \\
2.8\end{array}$ & $\begin{array}{l}4.4 \\
2.2 \\
4.0 \\
2.2 \\
1.6\end{array}$ & $\begin{array}{l}6.0 \\
4.8 \\
6.0 \\
6.0 \\
5.6\end{array}$ & $\begin{array}{l}7.3 \\
6.4 \\
5.2 \\
6.0 \\
7.8\end{array}$ & $\begin{array}{r}10 \\
4.0 \\
3.6 \\
4.8 \\
5.2\end{array}$ & $\begin{array}{r}7.8 \\
7.3 \\
9.0 \\
10 \\
7.8\end{array}$ & $\begin{array}{l}6.0 \\
5.6 \\
6.0 \\
7.3 \\
5.2\end{array}$ & $\begin{array}{l}3.2 \\
3.2 \\
3.2 \\
4.4 \\
4.8\end{array}$ \\
\hline 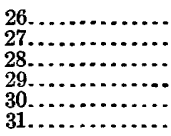 & $\begin{array}{c}4.4 \\
\cdots . . .\end{array}$ & $\begin{array}{l}3.6 \\
3.6\end{array}$ & & & $\begin{array}{l}2.5 \\
2.5 \\
2.5 \\
3.2 \\
4.0 \\
5.2\end{array}$ & $\begin{array}{r}1.6 \\
11 \\
6.4 \\
5.6 \\
6.8\end{array}$ & $\begin{aligned} 9.5 \\
10 \\
8.3 \\
9.5 \\
7.3 \\
5.2\end{aligned}$ & $\begin{array}{c}7.3 \\
7.8 \\
10 \\
9.0 \\
13 \\
8.8\end{array}$ & $\begin{array}{l}4.0 \\
3.2 \\
4.4 \\
4.0 \\
3.6\end{array}$ & \begin{tabular}{|c}
13 \\
14 \\
\hdashline$\ldots$ \\
$\ldots \ldots$ \\
$\ldots \ldots$
\end{tabular} & $\begin{array}{c}3.2 \\
1.9 \\
1.6 \\
20 \\
15 \\
\ldots \ldots\end{array}$ & $\begin{array}{l}5.2 \\
4.8 \\
3.6 \\
2.5 \\
2.5 \\
2.5\end{array}$ \\
\hline
\end{tabular}

Nore.-No record Jan. 1-4, 7-11, 17, 22-24, 27-31; Feb. 5-22; Feb. 28-May 16; Oct. 4-10, 28-31. Discharge interpolated May 18-20 and July 18. 
Monthly discharge of Kaneha ditch near Kealia, Kauai, for 1913.

\begin{tabular}{|c|c|c|c|c|c|}
\hline \multirow{2}{*}{ Month. } & \multicolumn{3}{|c|}{ Discharge in second-feet. } & \multirow{2}{*}{$\begin{array}{l}\text { Run-0fi } \\
\text { (total in } \\
\text { acre-feet). }\end{array}$} & \multirow{2}{*}{$\begin{array}{l}\text { Aceu- } \\
\text { racy. }\end{array}$} \\
\hline & Maximum. & Minimum. & Mean. & & \\
\hline 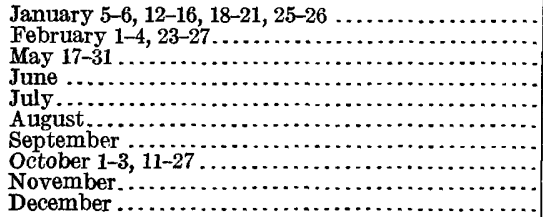 & $\begin{array}{l}12 \\
16 \\
13 \\
35 \\
11 \\
13 \\
22 \\
22 \\
20 \\
10\end{array}$ & $\begin{array}{l}3.6 \\
1.9 \\
2.5 \\
1.6 \\
2.8 \\
4.8 \\
2.8 \\
3.2 \\
1.6 \\
2.5\end{array}$ & $\begin{array}{l}6.13 \\
4.67 \\
4.94 \\
7.85 \\
6.60 \\
7.14 \\
7.06 \\
8.96 \\
6.31 \\
5.35\end{array}$ & $\begin{array}{r}158 \\
83 \\
147 \\
467 \\
406 \\
439 \\
420 \\
355 \\
375 \\
329\end{array}$ & $\begin{array}{l}\text { C. } \\
\text { C. } \\
\text { C. } \\
\text { C. } \\
\text { C. } \\
\text { C. } \\
\text { C. } \\
\text { C. } \\
\text { C. } \\
\text { C. }\end{array}$ \\
\hline The period & & & & 3,180 & \\
\hline
\end{tabular}

ANAHOLA RIVER ABOVE DAM AT KIOKALA, NEAR KEALIA, KAUAI.

Location.-About one-fourth mile above dam at Kiokala and 6 miles northwest of Kealia.

Records available.-August 22 to November 2, 1910; December 28, 1912, to December 31, 1913. December 15, 1910, to December 28, 1912, at dam at Kiokala.

Drainage area. -5.3 square miles.

Gage.-Friez water-stage recorder August 22 to November 2, 1910, and December 28, 1912, to December 31, 1913. From December 15, 1910, to December 28, 1912, an inclined staff gage.

Control.-Permanent.

Discharge measurements.-Made by wading and from foot bridge.

Accuracy.-Records good for January and from May to August; for other months fair.

Cooperation.-Station maintained in cooperation with the Makee Sugar Co.

The following discharge measurement was made by W. V. Hardy:

May 19, 1913: Gage height, 1.24 feet; discharge, 24.5 second-feet.

Daily discharge, in second-feet, of Anahola River above dam at Kiokala, near Kealia, Kauai, for 1913.

\begin{tabular}{|c|c|c|c|c|c|c|c|c|c|c|c|c|}
\hline Day. & Jan. & Feb. & Mar. & Apr. & May. & June. & July. & Aug. & Sept. & Oct. & Nov. & Dec. \\
\hline $\begin{array}{l}1 \ldots \ldots \ldots \ldots \ldots \\
2 \ldots \ldots \ldots \ldots \ldots \ldots \\
3 \ldots \ldots \ldots \ldots \\
4 \ldots \ldots \ldots \ldots \ldots\end{array}$ & $\begin{array}{l}19 \\
56 \\
19 \\
15 \\
20\end{array}$ & $\begin{array}{c}10 \\
9.5 \\
9.2 \\
9.3 \\
10\end{array}$ & $\begin{array}{l}13 \\
13 \\
12 \\
11 \\
11\end{array}$ & $\begin{array}{l}16 \\
16 \\
16 \\
13\end{array}$ & $\begin{array}{l}9.6 \\
17 \\
11 \\
9.6 \\
9.6\end{array}$ & $\begin{array}{l}13 \\
17 \\
97 \\
62 \\
30\end{array}$ & $\begin{array}{l}17 \\
33 \\
18 \\
21 \\
22\end{array}$ & $\begin{array}{l}12 \\
12 \\
11 \\
11 \\
10\end{array}$ & $\begin{array}{l}51 \\
17 \\
17\end{array}$ & $\begin{array}{l}12 \\
13 \\
10 \\
9.6 \\
9.6\end{array}$ & $\begin{array}{l}10 \\
30 \\
15 \\
13 \\
11\end{array}$ & $\begin{array}{r}37 \\
30 \\
211 \\
174 \\
87\end{array}$ \\
\hline $\begin{array}{r}6 \ldots \ldots \ldots \ldots \ldots \\
7 \ldots \ldots \ldots \ldots \ldots \\
8 \ldots \ldots \ldots \ldots \ldots \\
9 \ldots \ldots \ldots \ldots \ldots \\
10 \ldots \ldots \ldots \ldots \ldots\end{array}$ & $\begin{array}{l}16 \\
15 \\
21 \\
15 \\
13\end{array}$ & $\begin{array}{r}142 \\
53 \\
21 \\
16 \\
17\end{array}$ & $\begin{array}{l}11 \\
12 \\
25 \\
17 \\
11\end{array}$ & $\begin{array}{l}12 \\
12 \\
11 \\
15 \\
13\end{array}$ & $\begin{array}{l}34 \\
26 \\
35 \\
22 \\
15\end{array}$ & $\begin{array}{l}23 \\
21 \\
20 \\
24 \\
26\end{array}$ & $\begin{array}{l}13 \\
12 \\
12 \\
12 \\
11 \\
11\end{array}$ & $\begin{array}{l}10 \\
11 \\
12 \\
17 \\
12\end{array}$ & $\begin{array}{l}44 \\
22 \\
15 \\
13 \\
13\end{array}$ & $\begin{array}{l}11 \\
17 \\
23 \\
28 \\
15\end{array}$ & $\begin{array}{l}11 \\
57 \\
23 \\
35 \\
24\end{array}$ & $\begin{array}{l}93 \\
75 \\
53 \\
37 \\
30\end{array}$ \\
\hline $\begin{array}{l}11 \ldots \ldots \\
12 \ldots \ldots \\
13 \ldots \ldots \ldots \\
14 \\
15 \ldots \ldots \ldots\end{array}$ & $\begin{array}{l}21 \\
36 \\
61 \\
30 \\
28\end{array}$ & $\begin{array}{l}17 \\
13 \\
12 \\
11 \\
12\end{array}$ & $\begin{array}{l}11 \\
10 \\
10 \\
10 \\
10\end{array}$ & $\begin{array}{l}13 \\
14 \\
46 \\
61 \\
48\end{array}$ & $\begin{array}{l}67 \\
74 \\
35 \\
45 \\
29\end{array}$ & $\begin{array}{l}29 \\
20 \\
23 \\
18 \\
17\end{array}$ & $\begin{array}{l}10 \\
11 \\
26 \\
20 \\
13\end{array}$ & $\begin{array}{l}10 \\
13 \\
13 \\
13 \\
15\end{array}$ & $\begin{array}{l}12 \\
12 \\
13 \\
18\end{array}$ & $\begin{array}{l}13 \\
13 \\
20 \\
17 \\
20\end{array}$ & $\begin{array}{l}32 \\
26 \\
26 \\
17 \\
15\end{array}$ & $\begin{array}{r}26 \\
22 \\
22 \\
\ldots \ldots \ldots \\
\ldots \ldots \ldots\end{array}$ \\
\hline $\begin{array}{l}\ldots \ldots \ldots \\
\ldots \ldots \ldots \ldots \\
\ldots \ldots \ldots \ldots\end{array}$ & $\begin{array}{l}30 \\
19 \\
17 \\
15 \\
16\end{array}$ & $\begin{array}{l}15 \\
11 \\
63 \\
52 \\
33\end{array}$ & $\begin{array}{l}13 \\
10 \\
10 \\
10 \\
11\end{array}$ & $\begin{array}{l}22 \\
36 \\
34 \\
27 \\
16\end{array}$ & $\begin{array}{l}41 \\
41 \\
40 \\
26 \\
22\end{array}$ & $\begin{array}{l}20 \\
18 \\
17 \\
15 \\
14\end{array}$ & $\begin{array}{l}12 \\
11 \\
11 \\
14 \\
16\end{array}$ & $\begin{array}{l}24 \\
15 \\
14 \\
12 \\
11\end{array}$ & $\begin{array}{l}12 \\
14 \\
13\end{array}$ & $\begin{array}{l}17 \\
13 \\
13 \\
11 \\
11 \\
10\end{array}$ & $\begin{array}{r}15 \\
16 \\
16 \\
46 \\
137\end{array}$ & $\begin{array}{l}\ldots \ldots \\
\ldots \ldots \\
\ldots \ldots \\
\ldots \ldots \\
\ldots \ldots\end{array}$ \\
\hline $\begin{array}{l}21 \ldots \ldots \ldots \\
22 \ldots \ldots \ldots \ldots \\
23 \ldots \ldots \ldots \\
24 \ldots \ldots \ldots \ldots \\
25 \ldots \ldots \ldots \ldots\end{array}$ & $\begin{array}{l}28 \\
36 \\
16 \\
15 \\
19\end{array}$ & $\begin{array}{l}23 \\
22 \\
34 \\
25 \\
20\end{array}$ & $\begin{array}{r}17 \\
20 \\
127 \\
135 \\
136\end{array}$ & $\begin{array}{l}15 \\
18 \\
18 \\
18 \\
13 \\
14\end{array}$ & $\begin{array}{l}20 \\
18 \\
17 \\
18 \\
18\end{array}$ & $\begin{array}{l}13 \\
13 \\
13 \\
13 \\
13\end{array}$ & $\begin{array}{l}12 \\
11 \\
11 \\
14 \\
30\end{array}$ & $\begin{array}{l}16 \\
12 \\
11 \\
13 \\
22\end{array}$ & $\begin{array}{l}13 \\
13 \\
12 \\
12 \\
11\end{array}$ & $\begin{array}{l}12 \\
49 \\
57 \\
22 \\
17\end{array}$ & $\begin{array}{r}176 \\
61 \\
52 \\
57 \\
89\end{array}$ & $\begin{array}{l}\ldots \ldots \\
\ldots \ldots \ldots \\
\ldots \ldots \ldots \\
\ldots \ldots \\
\ldots \ldots\end{array}$ \\
\hline $\begin{array}{l}26 \ldots \ldots \ldots \ldots \ldots \\
27 \ldots \ldots \ldots \ldots \ldots \\
28 \ldots \ldots \ldots \ldots \\
29 \ldots \ldots \ldots \ldots \\
30 \ldots \ldots \ldots \ldots\end{array}$ & $\begin{array}{l}15 \\
15 \\
13 \\
12 \\
12 \\
11\end{array}$ & $\begin{array}{c}17 \\
15 \\
15 \\
\ldots \ldots \ldots \\
\ldots \ldots\end{array}$ & $\begin{array}{l}67 \\
39 \\
33 \\
27 \\
21 \\
19\end{array}$ & $\begin{array}{l}11 \\
9.9 \\
10 \\
9.6 \\
9.6 \\
9 .\end{array}$ & $\begin{array}{l}17 \\
16 \\
15 \\
15 \\
15 \\
14\end{array}$ & $\begin{array}{l}13 \\
28 \\
19 \\
15 \\
18 \\
\cdots\end{array}$ & $\begin{array}{l}18 \\
31 \\
15 \\
18 \\
15 \\
13\end{array}$ & $\begin{array}{l}13 \\
17 \\
17 \\
19 \\
15 \\
21\end{array}$ & $\begin{array}{r}11 \\
11 \\
23 \\
11 \\
10 \\
\cdots\end{array}$ & $\begin{array}{l}15 \\
13 \\
28 \\
14 \\
12 \\
12\end{array}$ & $\begin{array}{r}73 \\
49 \\
43 \\
49 \\
55 \\
\end{array}$ & 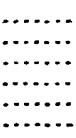 \\
\hline
\end{tabular}

Note.-No record Dec. 14-31. Discharge interpolated Mar. 28-29 and Nov. 29. 
Monthly discharge of Anahola River above dam, at Kiokala, near Kealia, Kauai, for 1913.

\begin{tabular}{|c|c|c|c|c|c|}
\hline \multirow{2}{*}{ Month. } & \multicolumn{3}{|c|}{ Discharge in second-feet. } & \multirow{2}{*}{$\begin{array}{c}\text { Run-off } \\
\text { (total in } \\
\text { acre-feet). }\end{array}$} & \multirow{2}{*}{$\begin{array}{l}\text { Accu- }- \\
\text { racy. }\end{array}$} \\
\hline & Maximum. & Minimum. & Mean. & & \\
\hline 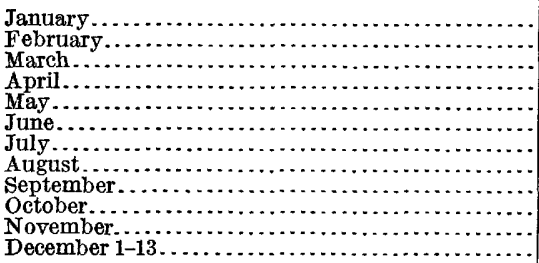 & $\begin{array}{r}61 \\
142 \\
136 \\
61 \\
74 \\
97 \\
33 \\
24 \\
51 \\
57 \\
176 \\
211\end{array}$ & $\begin{array}{l}11 \\
9.2 \\
10 \\
9.6 \\
9.6 \\
13 \\
10 \\
10 \\
10 \\
9.6 \\
10 \\
22\end{array}$ & $\begin{array}{l}21.7 \\
25.2 \\
28.5 \\
19.6 \\
25.5 \\
22.7 \\
16.2 \\
14.0 \\
16.2 \\
17.6 \\
42.6 \\
69.0\end{array}$ & $\begin{array}{r}1,330 \\
1,400 \\
1,750 \\
1,170 \\
1,570 \\
1,350 \\
996 \\
861 \\
964 \\
1,080 \\
2,530 \\
1,780\end{array}$ & $\begin{array}{l}\text { A. } \\
\text { B. } \\
\text { B. } \\
\text { B. } \\
\text { A. } \\
\text { A. } \\
\text { A. } \\
\text { A. } \\
\text { B. } \\
\text { B. } \\
\text { B. }\end{array}$ \\
\hline The period ...... & ...... & & ….. & 16,800 & \\
\hline
\end{tabular}

\section{ANAHOLA DITCH AT KIOKALA, NEAR KEALIA, KAUAI.}

Location.-About 6 miles northwest of Kealia.

Records available.-May 10, 1909, to December 31, 1913.

Gage.-Watson water-stage recorder.

Discharge measurements.-Made with 10-foot sharp-crested weir.

Accuracy.-Records good.

Cooperation.-Station maintained in cooperation with the Makee Sugar Co.

Daily discharge, in second-feet, of Anahola ditch at Kiokala, near Kealia, Kauai, for 1913.

\begin{tabular}{|c|c|c|c|c|c|c|c|c|c|c|c|c|}
\hline Day. & Jan. & Feb. & Mar. & Apr. & May. & June. & July. & Aug. & Sept. & Oet. & Nov. & Dec. \\
\hline $\begin{array}{l}1 \ldots . \\
2 \ldots \\
3 \ldots \\
4 \ldots \\
5 \ldots\end{array}$ & $\begin{array}{l}12.1 \\
13.6 \\
13.2 \\
11.8 \\
11.8\end{array}$ & $\begin{array}{l}6.6 \\
6.3 \\
6.0 \\
6.0 \\
7.5\end{array}$ & $\begin{array}{l}9.4 \\
8.7 \\
8.1 \\
7.8 \\
7.2\end{array}$ & $\begin{array}{l}13.2 \\
12.5 \\
12.5 \\
12.1 \\
11.8\end{array}$ & $\begin{array}{r}8.7 \\
11.8 \\
9.7 \\
8.7 \\
8.1\end{array}$ & $\begin{array}{r}9.4 \\
9.4 \\
14.7 \\
13.6 \\
12.5\end{array}$ & $\begin{array}{l}11.1 \\
12.5 \\
11.8 \\
11.8 \\
12.1\end{array}$ & $\begin{array}{l}7.8 \\
7.8 \\
6.9 \\
6.6 \\
6.0\end{array}$ & $\begin{array}{l}11.1 \\
10.1 \\
17.1 \\
17.2 \\
17.4\end{array}$ & $\begin{array}{l}5.7 \\
6.6 \\
4.2 \\
3.4 \\
3.4\end{array}$ & $\begin{array}{l}4.4 \\
7.8 \\
9.0 \\
7.5 \\
6.6\end{array}$ & \\
\hline $\begin{array}{r}6 \ldots . \\
7 \ldots \ldots \\
8 \ldots \\
9 \ldots \\
10 \ldots\end{array}$ & $\begin{array}{l}12.1 \\
10.7 \\
12.8 \\
11.8 \\
11.8\end{array}$ & $\begin{array}{l}15.9 \\
15.1 \\
12.8 \\
12.1 \\
11.4\end{array}$ & $\begin{array}{r}8.1 \\
8.4 \\
10.7 \\
11.1 \\
.7 .8\end{array}$ & $\begin{array}{l}11.1 \\
10.4 \\
10.1 \\
11.8 \\
10.7\end{array}$ & $\begin{array}{l}11.8 \\
13.2 \\
14.0 \\
12.8 \\
12.1\end{array}$ & $\begin{array}{l}11.8 \\
11.4 \\
11.1 \\
11.4 \\
11.8\end{array}$ & $\begin{array}{r}10.4 \\
10.1 \\
9.4 \\
9.7 \\
8.4\end{array}$ & $\begin{array}{r}5.5 \\
5.7 \\
7.2 \\
11.1 \\
7.8\end{array}$ & $\begin{array}{r}17.5 \\
14.0 \\
10.7 \\
9.0 \\
7.8\end{array}$ & $\begin{array}{r}4.7 \\
8.4 \\
12.1 \\
12.1 \\
8.7\end{array}$ & $\begin{array}{r}5.7 \\
9.7 \\
10.1 \\
10.7 \\
10.1\end{array}$ & \\
\hline $\begin{array}{l}11 \ldots \ldots \\
12 \ldots \ldots \\
13 \ldots \ldots \\
14 \ldots \ldots\end{array}$ & $\begin{array}{l}10.4 \\
13.2 \\
14.3 \\
12.8 \\
12.5\end{array}$ & $\begin{array}{r}12.5 \\
10.1 \\
8.7 \\
11.4 \\
9.0\end{array}$ & $\begin{array}{l}6.9 \\
6.3 \\
6.3 \\
6.0 \\
6.0\end{array}$ & $\begin{array}{l}11.4 \\
10.1 \\
14.0 \\
14.7 \\
15.1\end{array}$ & $\begin{array}{r}12.5 \\
16.7 \\
8.1 \\
\ldots \ldots\end{array}$ & $\begin{array}{l}11.8 \\
11.1 \\
10.7 \\
10.7 \\
10.7\end{array}$ & $\begin{array}{r}7.5 \\
7.5 \\
11.8 \\
12.1 \\
9.7\end{array}$ & $\begin{array}{r}6.0 \\
7.8 \\
9.7 \\
8.4 \\
10.4\end{array}$ & $\begin{array}{r}7.2 \\
6.0 \\
6.0 \\
7.5 \\
11.1\end{array}$ & $\begin{array}{r}6.3 \\
6.3 \\
9.7 \\
8.7 \\
10.4\end{array}$ & $\begin{array}{r}10.4 \\
10.1 \\
10.1 \\
9.0 \\
8.4\end{array}$ & \\
\hline $\begin{array}{l}16 \ldots \ldots \\
17 \ldots \ldots \\
18 \ldots \ldots \\
19 \ldots \ldots \\
20 \ldots\end{array}$ & $\begin{array}{r}13.2 \\
11.4 \\
10.1 \\
9.4 \\
10.1\end{array}$ & $\begin{array}{r}10.4 \\
8.4 \\
14.3 \\
12.5 \\
13.6\end{array}$ & $\begin{array}{l}8.4 \\
6.3 \\
6.0 \\
5.5 \\
6.9\end{array}$ & $\begin{array}{l}13.2 \\
13.6 \\
14.0 \\
13.2 \\
12.1\end{array}$ & $\begin{array}{r}4.2 \\
10.4 \\
10.1 \\
9.7\end{array}$ & $\begin{array}{r}11.1 \\
10.7 \\
10.7 \\
10.4 \\
9.7\end{array}$ & $\begin{array}{r}8.1 \\
7.2 \\
7.2 \\
8.7 \\
10.7\end{array}$ & $\begin{array}{r}12.1 \\
10.4 \\
9.4 \\
6.9 \\
5.5\end{array}$ & $\begin{array}{l}7.2 \\
6.0 \\
5.7 \\
6.9 \\
5.7\end{array}$ & $\begin{array}{l}9.4 \\
6.3 \\
5.2 \\
4.7 \\
4.2\end{array}$ & $\begin{array}{r}8.1 \\
8.7 \\
8.4 \\
10.1 \\
13.2\end{array}$ & \\
\hline $24, \ldots \ldots \ldots$ & $\begin{array}{l}11.4 \\
14.0 \\
11.8 \\
10.7 \\
11.1\end{array}$ & $\begin{array}{l}12.5 \\
12.5 \\
13.2 \\
12.8 \\
12.1\end{array}$ & $\begin{array}{r}10.7 \\
11.4 \\
6.0 \\
.4 \\
.1\end{array}$ & $\begin{array}{l}12.1 \\
12.5 \\
12.1 \\
11.1 \\
11.1\end{array}$ & $\begin{array}{l}9.7 \\
9.7 \\
9.4 \\
9.4 \\
9.4\end{array}$ & $\begin{array}{l}9.0 \\
9.0 \\
8.7 \\
8.4 \\
8.7\end{array}$ & $\begin{array}{r}7.8 \\
6.9 \\
6.9 \\
7.8 \\
14.0\end{array}$ & $\begin{array}{r}8.7 \\
6.0 \\
5.2 \\
6.3 \\
12.5\end{array}$ & $\begin{array}{l}6.3 \\
5.7 \\
5.5 \\
4.7 \\
4.4\end{array}$ & $\begin{array}{r}6.0 \\
9.7 \\
14.7 \\
13.2 \\
11.8\end{array}$ & & \\
\hline $\begin{array}{l}26 \ldots \ldots \\
27 \ldots \ldots \\
28 \ldots \ldots \\
29 \ldots \ldots \\
30 \ldots \ldots \\
31 \ldots \ldots \\
\end{array}$ & $\begin{array}{r}11.1 \\
10.7 \\
8.7 \\
7.8 \\
7.5 \\
-7.2\end{array}$ & $\begin{array}{r}11.4 \\
10.7 \\
9.7 \\
9.9\end{array}$ & $\begin{array}{r}5.5 \\
13.6 \\
13.2\end{array}$ & $\begin{array}{r}10.7 \\
9.4 \\
9.7 \\
9.4 \\
9.0\end{array}$ & $\begin{array}{l}9.4 \\
9.0 \\
9.0 \\
8.7 \\
9.0 \\
8.7\end{array}$ & $\begin{array}{r}8.7 \\
10.1 \\
11.1 \\
10.4 \\
11.1\end{array}$ & $\begin{array}{r}12.8 \\
14.3 \\
10.7 \\
12.5 \\
10.7 \\
9.4\end{array}$ & $\begin{array}{r}8.1 \\
9.0 \\
10.7 \\
12.1 \\
9.4 \\
11.8\end{array}$ & $\begin{array}{l}3.9 \\
4.2 \\
9.7 \\
5.7 \\
4.4\end{array}$ & $\begin{array}{r}9.4 \\
7.2 \\
11.8 \\
8.1 \\
6.0 \\
5.2\end{array}$ & $\begin{array}{l}\cdots \\
\cdots \cdots \\
\cdots\end{array}$ & \\
\hline
\end{tabular}

Note.-No record Mar. 26-28, May 14-16, and Nov. 21 to Dec. 31. Discharge Sept. 4 and 5 interpolated. 
Monthly discharge of Anahola ditch at Kiokala, near Kealia, Kauai, for 1913.

\begin{tabular}{|c|c|c|c|c|c|}
\hline \multirow{2}{*}{ Month. } & \multicolumn{3}{|c|}{ Discharge in second-feet. } & \multirow{2}{*}{$\begin{array}{l}\text { Rum-off } \\
\text { (total in } \\
\text { acre-feet). }\end{array}$} & \multirow{2}{*}{$\begin{array}{l}\text { Accu- } \\
\text { racy. }\end{array}$} \\
\hline & Maximum. & Minimum. & Mean. & & \\
\hline \multirow[t]{2}{*}{ 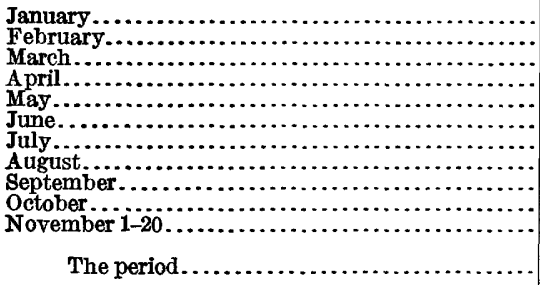 } & $\begin{array}{l}14.3 \\
15.9 \\
13.6 \\
15.1 \\
16.7 \\
14.7 \\
14.3 \\
12.5 \\
17.5 \\
14.7 \\
13.2\end{array}$ & $\begin{array}{l}7.2 \\
6.0 \\
.1 \\
9.0 \\
4.2 \\
8.4 \\
6.9 \\
5.2 \\
3.9 \\
3.4 \\
4.4\end{array}$ & $\begin{array}{c}11.7 \\
10.9 \\
7.60 \\
11.8 \\
10.1 \\
10.7 \\
10.1 \\
8.35 \\
8.52 \\
7.86 \\
8.90\end{array}$ & $\begin{array}{l}719 \\
605 \\
422 \\
702 \\
563 \\
637 \\
621 \\
513 \\
507 \\
483 \\
353\end{array}$ & \multirow[t]{2}{*}{$\begin{array}{l}\text { A. } \\
\text { A. } \\
\text { A. } \\
\text { A. } \\
\text { A. } \\
\text { A. } \\
\text { A. } \\
\text { A. } \\
\text { A. } \\
\text { A. } \\
\text { A. }\end{array}$} \\
\hline & & & & 6,120 & \\
\hline
\end{tabular}

KALIHIWAI RIVER NEAR KILAUEA, KAUAI.

Location.-One-half mile below Hoopouli Falls, about 2 miles west of Kilauea, and about 5 miles above mouth of river. Reached by saddle horse on trail from Princeville ranch.

Records available.-August 21, 1912, to December 31, 1913.

Drainage area. -3.9 square miles.

Gage.-Vertical staff.

Control.-Probably permanent.

Discharge measurements.-Made by wading.

Accuracy.-Records fair except for November and December, 1913, when they were poor.

Cooperation.-Station is maintained in cooperation with the Princeville ranch.

Discharge measurements of Kalihiwai River near Kilauea, Kauai, in' 1919.

\begin{tabular}{|c|c|c|c|c|c|c|c|}
\hline Date. & Hydrographer. & $\begin{array}{c}\text { Gage } \\
\text { height. }\end{array}$ & $\begin{array}{c}\text { Dis- } \\
\text { charge. }\end{array}$ & Date. & Hydrographer. & $\begin{array}{c}\text { Gage } \\
\text { height. }\end{array}$ & $\begin{array}{c}\text { Dis- } \\
\text { charge. }\end{array}$ \\
\hline $\begin{array}{l}\text { Apr. } 18 \\
\text { Apr. } 19\end{array}$ & 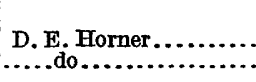 & $\begin{array}{r}\text { Feet. } \\
6.76 \\
6.62\end{array}$ & $\begin{array}{c}\text { Sec. ft. } \\
114 \\
76.8\end{array}$ & $\begin{array}{l}\text { Apr. } 20 \\
\text { May } 23\end{array}$ & $\begin{array}{l}\text { D. E. Horner..... } \\
\text { W. V. Hardy ..... }\end{array}$ & $\begin{array}{c}\text { Feet. } \\
6.44 \\
6.08\end{array}$ & $\begin{array}{r}\text { Sec. ft. } \\
46.8 \\
24.1\end{array}$ \\
\hline
\end{tabular}

Daily discharge, in second-feet, of Kalihiwai River near Kilauea, Kauai, for 1912-13.

\begin{tabular}{|c|c|c|c|c|c|c|c|c|c|c|c|}
\hline Day. & Aug. & Sept. & Oct. & Nov. & Dec. & Day. & Aug. & Sept. & Oct. & Nov. & Dec. \\
\hline $\begin{array}{c}1912 . \\
1 \ldots \ldots \\
2 \ldots \ldots \\
3 . \ldots . \\
4 \ldots \\
5 \ldots \ldots\end{array}$ & {$\left[\begin{array}{c}\cdots \\
\cdots \\
\cdots \\
\cdots\end{array}\right.$} & $\begin{array}{l}29 \\
32 \\
29 \\
29 \\
29\end{array}$ & $\begin{array}{l}24 \\
24 \\
23 \\
22 \\
22\end{array}$ & $\begin{array}{l}25 \\
24 \\
24 \\
24 \\
25\end{array}$ & $\begin{array}{r}28 \\
36 \\
76 \\
2,500 \\
224\end{array}$ & $\begin{array}{r}1912 . \\
16 \ldots \ldots \\
17 \ldots \ldots \\
18 \ldots \ldots \\
19 \ldots \ldots \\
20 \ldots \ldots\end{array}$ & . & $\begin{array}{l}25 \\
25 \\
25 \\
58 \\
58\end{array}$ & $\begin{array}{l}25 \\
24 \\
24 \\
29 \\
46\end{array}$ & $\begin{array}{r}24 \\
24 \\
29 \\
25 \\
111\end{array}$ & $\begin{array}{l}36 \\
39 \\
44 \\
41 \\
36\end{array}$ \\
\hline $\begin{array}{r}6 \ldots \ldots \\
7 \ldots \ldots \\
8 \ldots \ldots \\
10 \ldots \ldots\end{array}$ & 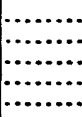 & $\begin{array}{l}46 \\
32 \\
29 \\
29 \\
29\end{array}$ & $\begin{array}{l}23 \\
24 \\
98 \\
36 \\
25\end{array}$ & $\begin{array}{l}32 \\
24 \\
24 \\
32 \\
24\end{array}$ & $\begin{array}{l}76 \\
46 \\
46 \\
41 \\
41\end{array}$ & $\begin{array}{l}21 \ldots \ldots \\
22 \ldots \ldots \\
23 \ldots \ldots \\
24 \ldots . \\
25 \ldots .\end{array}$ & $\begin{array}{r}29 \\
124 \\
36 \\
29 \\
29\end{array}$ & $\begin{array}{l}24 \\
23 \\
23 \\
24 \\
22\end{array}$ & $\begin{array}{l}76 \\
25 \\
29 \\
36 \\
32\end{array}$ & $\begin{array}{l}29 \\
28 \\
25 \\
27 \\
25\end{array}$ & $\begin{array}{l}36 \\
36 \\
52 \\
46 \\
46\end{array}$ \\
\hline $\begin{array}{l}11 \ldots . \\
12 \ldots \\
13 \ldots . \\
14 . \ldots \\
15 . \ldots\end{array}$ & 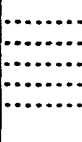 & $\begin{array}{l}29 \\
29 \\
27 \\
25 \\
25\end{array}$ & $\begin{array}{l}24 \\
24 \\
24 \\
24 \\
23\end{array}$ & $\begin{array}{l}24 \\
24 \\
24 \\
24 \\
25\end{array}$ & $\begin{array}{l}38 \\
36 \\
36 \\
46 \\
41\end{array}$ & $\begin{array}{l}26 \ldots \ldots \\
27 \ldots \ldots \\
28 \ldots \\
29 . \ldots \\
30 \ldots \\
31 \ldots \ldots\end{array}$ & $\begin{array}{l}29 \\
98 \\
36 \\
41 \\
36 \\
32\end{array}$ & $\begin{array}{l}22 \\
22 \\
22 \\
22 \\
23\end{array}$ & $\begin{array}{l}34 \\
32 \\
29 \\
28 \\
27 \\
26\end{array}$ & $\begin{array}{l}25 \\
24 \\
27 \\
29 \\
25\end{array}$ & $\begin{array}{l}44 \\
41 \\
36 \\
35 \\
34 \\
29\end{array}$ \\
\hline
\end{tabular}

$97764^{\circ}-\mathrm{WsP} 373-15-5$ 
Daily discharge, in second-feet, of Kalihiwai River near Kilauea, Kauai, for 1912-18-Con.

\begin{tabular}{|c|c|c|c|c|c|c|c|c|c|c|c|c|}
\hline Day. & Jan. & Feb. & Mar. & Apr. & May. & June. & July. & Aug. & Sept. & Oet. & Nov. & Dec. \\
\hline \begin{tabular}{l}
\multicolumn{2}{c}{1913.} \\
$1 . \ldots \ldots \ldots \ldots$ \\
$2 \ldots \ldots \ldots \ldots$ \\
$3 \ldots \ldots \ldots \ldots \ldots$ \\
$4 \ldots \ldots \ldots \ldots \ldots$ \\
$5 \ldots \ldots \ldots \ldots \ldots \ldots$
\end{tabular} & $\begin{array}{l}29 \\
29 \\
28 \\
27 \\
29\end{array}$ & $\begin{array}{l}24 \\
23 \\
23 \\
24 \\
24\end{array}$ & $\begin{array}{l}24 \\
24 \\
24 \\
\mathbf{2 3} \\
\mathbf{2 3}\end{array}$ & $\begin{array}{l}36 \\
36 \\
36 \\
36 \\
27\end{array}$ & $\begin{array}{l}41 \\
87 \\
63 \\
38 \\
25\end{array}$ & $\begin{array}{l}18 \\
18 \\
98 \\
46 \\
29\end{array}$ & $\begin{array}{l}41 \\
36 \\
52 \\
41 \\
35\end{array}$ & $\begin{array}{l}25 \\
24 \\
24 \\
23 \\
23\end{array}$ & $\begin{array}{r}36 \\
1,050 \\
46 \\
38 \\
32\end{array}$ & $\begin{array}{l}22 \\
21 \\
21 \\
21 \\
21\end{array}$ & $\begin{array}{l}25 \\
25 \\
25 \\
24 \\
25\end{array}$ & $\begin{array}{r}400 \\
264 \\
1,450 \\
224 \\
154\end{array}$ \\
\hline $\begin{array}{r}6 \ldots \ldots \ldots \ldots \ldots \\
7 \ldots \ldots \ldots \ldots \ldots \\
8 \ldots \ldots \ldots \ldots \ldots \\
10 \ldots \ldots \ldots \ldots \ldots \ldots\end{array}$ & $\begin{array}{l}36 \\
36 \\
41 \\
36 \\
48\end{array}$ & $\begin{array}{r}750 \\
98 \\
36 \\
29 \\
24\end{array}$ & $\begin{array}{l}23 \\
24 \\
25 \\
23 \\
22\end{array}$ & $\begin{array}{l}26 \\
25 \\
24 \\
32 \\
29\end{array}$ & $\begin{array}{r}264 \\
46 \\
62 \\
41 \\
36\end{array}$ & $\begin{array}{l}25 \\
25 \\
26 \\
32 \\
30\end{array}$ & $\begin{array}{l}35 \\
32 \\
32 \\
31 \\
30\end{array}$ & $\begin{array}{l}23 \\
23 \\
22 \\
24 \\
24\end{array}$ & $\begin{array}{l}30 \\
30 \\
29 \\
32 \\
30\end{array}$ & $\begin{array}{l}21 \\
37 \\
32 \\
31 \\
29\end{array}$ & $\begin{array}{l}26 \\
31 \\
32 \\
37 \\
39\end{array}$ & $\begin{array}{r}187 \\
180 \\
174 \\
50 \\
46\end{array}$ \\
\hline $\begin{array}{l}11 \ldots \ldots \ldots \ldots \\
12 \ldots \ldots \ldots \ldots \ldots \\
13 \ldots \ldots \ldots \ldots \ldots \\
14 \ldots \ldots \ldots \ldots \ldots \\
15 \ldots \ldots \ldots \ldots \ldots \ldots\end{array}$ & $\begin{array}{r}46 \\
187 \\
98 \\
58 \\
52\end{array}$ & $\begin{array}{l}24 \\
23 \\
23 \\
22 \\
23\end{array}$ & $\begin{array}{l}22 \\
22 \\
22 \\
22 \\
22\end{array}$ & $\begin{array}{l}32 \\
32 \\
34 \\
67 \\
62\end{array}$ & $\begin{array}{l}50 \\
80 \\
36 \\
32 \\
46\end{array}$ & $\begin{array}{l}29 \\
29 \\
27 \\
32 \\
36\end{array}$ & $\begin{array}{l}29 \\
29 \\
48 \\
46 \\
38\end{array}$ & $\begin{array}{l}24 \\
41 \\
48 \\
41 \\
36\end{array}$ & $\begin{array}{l}29 \\
27 \\
26 \\
25 \\
25\end{array}$ & $\begin{array}{l}27 \\
25 \\
25 \\
25 \\
24\end{array}$ & $\begin{array}{r}50 \\
400 \\
500 \\
187 \\
154\end{array}$ & $\begin{array}{l}41 \\
39 \\
36 \\
\mathbf{3 4} \\
\mathbf{3 1}\end{array}$ \\
\hline $\begin{array}{l}16 \ldots \ldots \ldots \ldots \\
17 \ldots \ldots \ldots \ldots \ldots \\
18 \ldots \ldots \ldots \ldots \ldots \\
19 \ldots \ldots \ldots \ldots \\
20 \ldots \ldots \ldots \ldots\end{array}$ & $\begin{array}{l}46 \\
41 \\
38 \\
98 \\
98\end{array}$ & $\begin{array}{r}24 \\
24 \\
214 \\
154 \\
46\end{array}$ & $\begin{array}{l}23 \\
23 \\
24 \\
23 \\
23\end{array}$ & $\begin{array}{r}54 \\
111 \\
119 \\
85 \\
52\end{array}$ & $\begin{array}{l}62 \\
80 \\
38 \\
29 \\
29\end{array}$ & $\begin{array}{l}36 \\
36 \\
32 \\
30 \\
29\end{array}$ & $\begin{array}{l}38 \\
36 \\
29 \\
27 \\
26\end{array}$ & $\begin{array}{l}27 \\
26 \\
24 \\
23 \\
23\end{array}$ & $\begin{array}{l}24 \\
32 \\
32 \\
24 \\
32\end{array}$ & $\begin{array}{l}24 \\
24 \\
24 \\
23 \\
36\end{array}$ & $\begin{array}{r}111 \\
76 \\
62 \\
\cdot 700 \\
1,100\end{array}$ & $\begin{array}{l}29 \\
29 \\
29 \\
28 \\
28\end{array}$ \\
\hline $\begin{array}{l}21 \ldots \ldots \ldots \ldots \ldots \\
22 \ldots \ldots \ldots \ldots \ldots \\
23 \ldots \ldots \ldots \ldots \ldots \\
24 \ldots \ldots \ldots \ldots \ldots \\
25 \ldots \ldots \ldots \ldots \ldots \ldots\end{array}$ & $\begin{array}{r}111 \\
98 \\
76 \\
58 \\
41\end{array}$ & $\begin{array}{l}29 \\
29 \\
29 \\
29 \\
24\end{array}$ & $\begin{array}{r}25 \\
32 \\
348 \\
390 \\
400\end{array}$ & $\begin{array}{l}38 \\
38 \\
46 \\
41 \\
80\end{array}$ & $\begin{array}{l}26 \\
25 \\
25 \\
24 \\
23\end{array}$ & $\begin{array}{l}27 \\
27 \\
25 \\
24 \\
24\end{array}$ & $\begin{array}{l}25 \\
32 \\
24 \\
24 \\
52\end{array}$ & $\begin{array}{l}24 \\
24 \\
24 \\
24 \\
24\end{array}$ & $\begin{array}{l}24 \\
24 \\
23 \\
23 \\
23\end{array}$ & $\begin{array}{l}32 \\
29 \\
72 \\
58 \\
36\end{array}$ & $\begin{array}{l}550 \\
650 \\
400 \\
308 \\
600\end{array}$ & $\begin{array}{l}28 \\
27 \\
24 \\
24 \\
24\end{array}$ \\
\hline $\begin{array}{l}26 \ldots \ldots \ldots \\
27 \ldots \ldots \ldots \ldots \\
28 \ldots \ldots \ldots \\
29 \ldots \ldots \ldots \\
30 \ldots \ldots \ldots \ldots \\
31 \ldots \ldots \ldots\end{array}$ & $\begin{array}{l}32 \\
29 \\
25 \\
24 \\
24 \\
24\end{array}$ & $\begin{array}{r}\begin{array}{r}27 \\
25 \\
111 \\
11 \\
\ldots \ldots\end{array} . \\
\hdashline \ldots \ldots . \\
\hdashline \ldots . .\end{array}$ & $\begin{array}{r}308 \\
76 \\
58 \\
41 \\
41 \\
38\end{array}$ & $\begin{array}{r}63 \\
46 \\
41 \\
41 \\
39 \\
\end{array}$ & $\begin{array}{l}22 \\
21 \\
21 \\
20 \\
19 \\
18\end{array}$ & $\begin{array}{r}23 \\
244 \\
41 \\
52 \\
41 \\
\ldots \ldots\end{array}$ & $\begin{array}{l}41 \\
32 \\
29 \\
27 \\
26 \\
25\end{array}$ & $\begin{array}{l}24 \\
24 \\
27 \\
48 \\
39 \\
35\end{array}$ & $\begin{array}{r}23 \\
22 \\
22 \\
22 \\
22 \\
\ldots \ldots\end{array}$ & $\begin{array}{l}29 \\
24 \\
28 \\
27 \\
27 \\
26\end{array}$ & $\begin{array}{r}187 \\
154 \\
124 \\
2,050 \\
600 \\
\ldots \ldots \ldots\end{array}$ & $\begin{array}{l}24 \\
23 \\
23 \\
22 \\
22 \\
22\end{array}$ \\
\hline
\end{tabular}

Monthly discharge of Kalihiwai River near Kilauea, Kauai, for 1912-13.

\begin{tabular}{|c|c|c|c|c|c|}
\hline \multirow{2}{*}{ Month. } & \multicolumn{3}{|c|}{ Discharge in second-feet. } & \multirow{2}{*}{$\begin{array}{l}\text { Run-off (in } \\
\text { acre-feet). }\end{array}$} & \multirow{2}{*}{$\begin{array}{l}\text { Accu- } \\
\text { racy. }\end{array}$} \\
\hline & Maximum. & Minimum. & Mean. & & \\
\hline 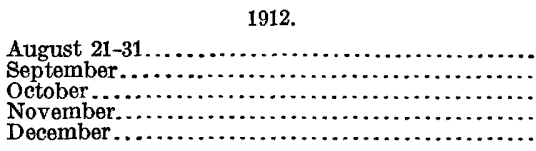 & $\begin{array}{r}124 \\
58 \\
98 \\
111 \\
2,500\end{array}$ & $\begin{array}{l}29 \\
22 \\
22 \\
24 \\
28\end{array}$ & $\begin{array}{r}47.2 \\
28.9 \\
31.0 \\
28.5 \\
127\end{array}$ & $\begin{array}{l}1,030 \\
1,720 \\
1,910 \\
1,700 \\
7,810\end{array}$ & $\begin{array}{l}\text { B. } \\
\text { B. } \\
\text { B. } \\
\text { B. } \\
\text { B. }\end{array}$ \\
\hline 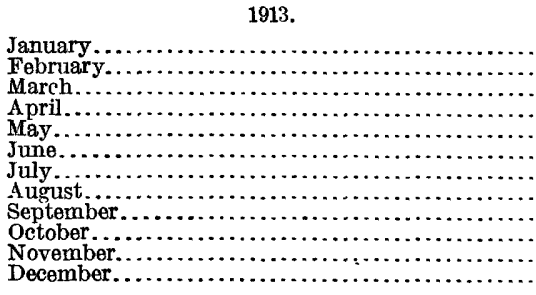 & $\begin{array}{r}187 \\
750 \\
400 \\
119 \\
264 \\
244 \\
52 \\
48 \\
1,050 \\
72 \\
2,050 \\
1,450\end{array}$ & $\begin{array}{l}24 \\
22 \\
22 \\
24 \\
18 \\
18 \\
24 \\
22 \\
22 \\
21 \\
24 \\
22\end{array}$ & $\begin{array}{l}53.0 \\
70.2 \\
71.5 \\
47.6 \\
46.1 \\
39.7 \\
33.8 \\
27.9 \\
61.9 \\
29.1 \\
308 \\
120\end{array}$ & $\begin{array}{r}3,260 \\
3,900 \\
4,400 \\
2,830 \\
2,830 \\
2,360 \\
2,080 \\
1,720 \\
3,680 \\
1,790 \\
18,300 \\
7,380\end{array}$ & $\begin{array}{l}\text { A. } \\
\text { A. } \\
\text { A. } \\
\text { A. } \\
\text { A. } \\
\text { A. } \\
\text { B. } \\
\text { B. } \\
\text { B. } \\
\text { B. } \\
\text { C. } \\
\text { C. }\end{array}$ \\
\hline The year $\ldots \ldots \ldots \ldots \ldots \ldots \ldots \ldots \ldots \ldots \ldots \ldots \ldots \ldots$ & 2,050 & 18 & 75.4 & 54,500 & \\
\hline
\end{tabular}




\section{HANALEI RIVER NEAR HANALEI, KAUAI.}

Location.-About 5 miles up the river from Hanalei.

Records available.-December 28, 1911, to December 31, 1913.

Drainage area.-19.2 square miles.

Gage.-Staff.

Control.-Probably permanent.

Discharge measurements.-Cable and car or by wading.

Accuracy.-Records good for January, February, April, May, August, September, and October; for other months fair.

Discharge measurements of Hanalei River near Hanalei, Kauai, in 1913.

\begin{tabular}{r|c|r|r||c|c|c|c}
\hline Date. & Hydrographer. & $\begin{array}{r}\text { Gage } \\
\text { height. }\end{array}$ & $\begin{array}{c}\text { Dis- } \\
\text { charge. }\end{array}$ & Date. & Hydrographer. & $\begin{array}{c}\text { Gage } \\
\text { height. }\end{array}$ & $\begin{array}{c}\text { Dis- } \\
\text { charge. }\end{array}$ \\
\hline Mar. 25 & Hardy and Horner..... & $\begin{array}{r}\text { Feet. } \\
\mathbf{7 . 9 0}\end{array}$ & $\begin{array}{r}\text { Sec. } f t . \\
\mathbf{1 , 1 6 0} \\
\mathbf{6 . 7 5}\end{array}$ & Nov. 12 & W. V. Hardy.......... & $\begin{array}{r}\text { Feet. } \\
\mathbf{8 . 5 0}\end{array}$ & $\begin{array}{r}\text { Sec. }-f t . \\
\mathbf{1 , 6 2 0}\end{array}$ \\
\hline
\end{tabular}

Daily discharge, in second-feet, of Hanalei River near Hanalei, Kauai, for 1913.

\begin{tabular}{|c|c|c|c|c|c|c|c|c|c|c|c|c|}
\hline Day. & Jan. & Feb. & Mar. & Apr. & May. & June. & July. & Aug. & Sept. & Oct. & Nov. & Dec. \\
\hline $\begin{array}{l}1 \ldots . . \\
2 \ldots \\
3 \ldots \\
4 \ldots \\
5 \ldots\end{array}$ & $\begin{array}{l}124 \\
158 \\
158 \\
124 \\
124\end{array}$ & $\begin{array}{l}124 \\
124 \\
124 \\
124 \\
158\end{array}$ & $\begin{array}{l}93 \\
93 \\
93 \\
93 \\
93\end{array}$ & $\begin{array}{r}93 \\
93 \\
124 \\
124 \\
93\end{array}$ & $\begin{array}{r}93 \\
158 \\
93 \\
93 \\
124\end{array}$ & $\begin{array}{r}93 \\
93 \\
825 \\
124 \\
124\end{array}$ & $\begin{array}{r}124 \\
1,180 \\
364 \\
364 \\
364\end{array}$ & $\begin{array}{l}93 \\
93 \\
93 \\
93 \\
93\end{array}$ & $\begin{array}{l}158 \\
248 \\
158 \\
124 \\
124\end{array}$ & $\begin{array}{r}124 \\
93 \\
93 \\
93 \\
93\end{array}$ & $\begin{array}{r}93 \\
248 \\
158 \\
124 \\
93\end{array}$ & $\begin{array}{l}124 \\
124 \\
825 \\
124 \\
124\end{array}$ \\
\hline $\begin{array}{r}6 \ldots \ldots . \\
7 . \ldots \ldots \\
8 \ldots \ldots \\
9 \ldots \ldots \\
10 \ldots \ldots\end{array}$ & $\begin{array}{l}124 \\
124 \\
124 \\
124 \\
158\end{array}$ & $\begin{array}{l}200 \\
124 \\
124 \\
124 \\
124\end{array}$ & $\begin{array}{r}93 \\
124 \\
124 \\
93 \\
93\end{array}$ & \begin{tabular}{r|}
93 \\
93 \\
124 \\
124 \\
124
\end{tabular} & $\begin{array}{l}124 \\
200 \\
124 \\
124 \\
124\end{array}$ & $\begin{array}{l}124 \\
124 \\
124 \\
158 \\
124\end{array}$ & $\begin{array}{l}124 \\
124 \\
124 \\
124 \\
124\end{array}$ & $\begin{array}{r}124 \\
124 \\
124 \\
124 \\
93\end{array}$ & $\begin{array}{r}124 \\
158 \\
124 \\
124 \\
93\end{array}$ & $\begin{array}{r}93 \\
248 \\
200 \\
124 \\
93\end{array}$ & $\begin{array}{r}93 \\
200 \\
158 \\
158 \\
500\end{array}$ & $\begin{array}{r}124 \\
124 \\
124 \\
93 \\
93\end{array}$ \\
\hline 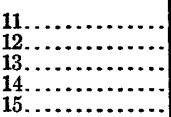 & $\begin{array}{l}124 \\
158 \\
302 \\
158 \\
158\end{array}$ & $\begin{array}{r}124 \\
124 \\
93 \\
93 \\
93\end{array}$ & $\begin{array}{l}93 \\
93 \\
93 \\
93 \\
93\end{array}$ & $\begin{array}{r}124 \\
93 \\
158 \\
124 \\
124\end{array}$ & $\begin{array}{l}124 \\
200 \\
124 \\
124 \\
124\end{array}$ & $\begin{array}{l}124 \\
124 \\
158 \\
124 \\
124\end{array}$ & $\begin{array}{l}124 \\
124 \\
248 \\
200 \\
158\end{array}$ & $\begin{array}{r}93 \\
124 \\
158 \\
158 \\
124\end{array}$ & $\begin{array}{r}93 \\
124 \\
124 \\
124 \\
93\end{array}$ & $\begin{array}{r}93 \\
93 \\
124 \\
124 \\
124 \\
124\end{array}$ & $\begin{array}{l}364 \\
955 \\
200 \\
158 \\
124\end{array}$ & $\begin{array}{l}93 \\
93 \\
93 \\
93 \\
93\end{array}$ \\
\hline $\begin{array}{l}16 \ldots \ldots \ldots \\
17 \ldots \ldots \ldots \ldots \\
18 \ldots \ldots \ldots \ldots \\
19 . \ldots \ldots \ldots \ldots\end{array}$ & $\begin{array}{l}124 \\
124 \\
124 \\
124 \\
158\end{array}$ & $\begin{array}{r}93 \\
124 \\
291 \\
158 \\
124\end{array}$ & $\begin{array}{r}93 \\
93 \\
93 \\
124 \\
124\end{array}$ & $\begin{array}{l}93 \\
93 \\
93 \\
93 \\
93\end{array}$ & $\begin{array}{l}124 \\
124 \\
124 \\
124 \\
124\end{array}$ & $\begin{array}{r}364 \\
124 \\
124 \\
93 \\
93\end{array}$ & $\begin{array}{r}124 \\
124 \\
158 \\
124 \\
93\end{array}$ & $\begin{array}{r}248 \\
158 \\
124 \\
93 \\
93\end{array}$ & $\begin{array}{r}93 \\
93 \\
93 \\
124 \\
93\end{array}$ & $\begin{array}{r}93 \\
93 \\
124 \\
93 \\
93\end{array}$ & $\begin{array}{l}124 \\
124 \\
158 \\
248 \\
910\end{array}$ & $\begin{array}{l}93 \\
93 \\
93 \\
93 \\
93\end{array}$ \\
\hline $\begin{array}{l}21 \ldots \ldots \ldots \ldots \\
22 \ldots \ldots \ldots \ldots \ldots \ldots \\
23 \ldots \ldots \ldots \ldots \ldots \\
24 \ldots \ldots \ldots \ldots \\
25 \ldots \ldots \ldots\end{array}$ & $\begin{array}{l}124 \\
124 \\
124 \\
124 \\
124\end{array}$ & $\begin{array}{r}124 \\
124 \\
93 \\
124 \\
124\end{array}$ & $\begin{array}{l}124 \\
124 \\
124 \\
910 \\
910\end{array}$ & $\begin{array}{r}93 \\
93 \\
93 \\
93 \\
124\end{array}$ & $\begin{array}{r}124 \\
124 \\
124 \\
124 \\
96\end{array}$ & $\begin{array}{l}124 \\
124 \\
124 \\
124 \\
124\end{array}$ & $\begin{array}{r}93 \\
93 \\
124 \\
124 \\
124\end{array}$ & $\begin{array}{r}124 \\
124 \\
93 \\
124 \\
124\end{array}$ & $\begin{array}{l}93 \\
93 \\
93 \\
93 \\
68\end{array}$ & $\begin{array}{r}93 \\
93 \\
93 \\
93 \\
\mathbf{1 2 4}\end{array}$ & $\begin{array}{l}200 \\
158 \\
158 \\
158 \\
200\end{array}$ & $\begin{array}{l}93 \\
93 \\
93 \\
93 \\
93\end{array}$ \\
\hline 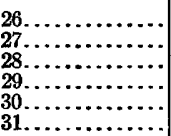 & $\begin{array}{l}124 \\
124 \\
124 \\
124 \\
124 \\
124\end{array}$ & $\begin{array}{r}\mathbf{9 3} \\
\mathbf{9 3} \\
\mathbf{9 3} \\
\ldots \ldots \ldots \\
\ldots \ldots . \\
\end{array}$ & $\begin{array}{r}655 \\
124 \\
224 \\
93 \\
124 \\
124\end{array}$ & $\begin{array}{r}93 \\
124 \\
124 \\
124 \\
158\end{array}$ & $\begin{array}{r}93 \\
93 \\
93 \\
124 \\
124 \\
\mathbf{9 3}\end{array}$ & $\begin{array}{l}124 \\
364 \\
124 \\
124 \\
124\end{array}$ & $\begin{array}{r}93 \\
158 \\
124 \\
93 \\
93 \\
93\end{array}$ & $\begin{array}{l}115 \\
124 \\
364 \\
158 \\
248 \\
200\end{array}$ & $\begin{array}{l}68 \\
.68 \\
158 \\
124 \\
124\end{array}$ & $\begin{array}{r}124 \\
124 \\
124 \\
93 \\
93 \\
\mathbf{9 3} \\
\mathbf{1 2 4}\end{array}$ & $\begin{array}{r}200 \\
124 \\
93 \\
93 \\
158\end{array}$ & $\begin{array}{r}93 \\
93 \\
93 \\
93 \\
93 \\
124\end{array}$ \\
\hline
\end{tabular}


Monthly discharge of Hanalei River near Hanalei, Kauai, for 1918.

\begin{tabular}{|c|c|c|c|c|c|}
\hline \multirow{2}{*}{ - Month. } & \multicolumn{3}{|c|}{ Discharge in second-feet. } & \multirow{2}{*}{$\begin{array}{r}\text { Run-off } \\
\text { (total in } \\
\text { acre-feet). }\end{array}$} & \multirow{2}{*}{$\begin{array}{l}\text { Accu- } \\
\text { racy. }\end{array}$} \\
\hline & Maximum. & Minimum. & Mean. & & \\
\hline 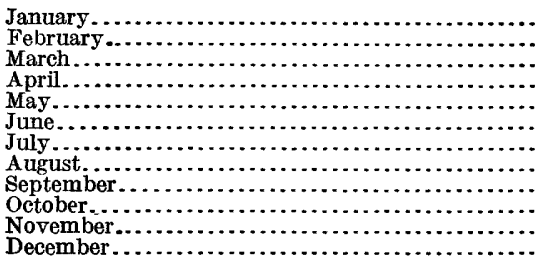 & $\begin{array}{r}302 \\
291 \\
910 \\
158 \\
200 \\
825 \\
1,180 \\
364 \\
248 \\
248 \\
955 \\
825\end{array}$ & $\begin{array}{r}124 \\
93 \\
93 \\
93 \\
93 \\
93 \\
93 \\
93 \\
68 \\
93 \\
93 \\
93\end{array}$ & $\begin{array}{l}137 \\
126 \\
178 \\
110 \\
122 \\
162 \\
184 \\
136 \\
116 \\
112 \\
224 \\
125\end{array}$ & $\begin{array}{r}8,420 \\
7,000 \\
10,900 \\
6,550 \\
7,500 \\
9,640 \\
11,300 \\
8,360 \\
6,900 \\
6,890 \\
13,300 \\
7,690\end{array}$ & $\begin{array}{l}\text { A. } \\
\text { A. } \\
\text { B. } \\
\text { A. } \\
\text { A. } \\
\text { B. } \\
\text { B. } \\
\text { A. } \\
\text { A. } \\
\text { A. } \\
\text { B. } \\
\text { B. }\end{array}$ \\
\hline 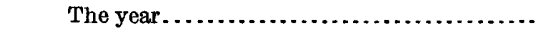 & 1,180 & 68 & 144 & 104,000 & \\
\hline
\end{tabular}

KUNA DITCH NEAR HANALEI, KAUAI.

Location.-About 4 miles up the river from Hanalei and 500 feet below diversion from Hanalei River.

Records available.-January 17, 1912, to September 30, 1913.

Gage.-Vertical staff.

Control.-Probably permanent.

Discharge measurements.-Made from footbridge.

Accuracy.-Records fair.

Discharge measurements of Kuna ditch near Hanalei, Kauai, in 1913.

\begin{tabular}{|c|c|c|c|c|}
\hline Date. & Hydrographer. & • & $\begin{array}{c}\text { Gage } \\
\text { height. }\end{array}$ & $\begin{array}{l}\text { Dis- } \\
\text { charge. }\end{array}$ \\
\hline $\begin{array}{l}\text { Mar. } 26 \\
\text { Aug. } 26\end{array}$ & 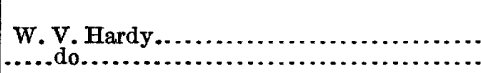 & 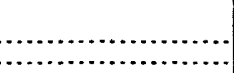 & $\begin{array}{l}\text { Feet. } \\
4.88 \\
4.64\end{array}$ & $\begin{array}{r}\text { Sec. }-f t . \\
23 . \\
19 .\end{array}$ \\
\hline
\end{tabular}

Daily discharge, in second-feet, of Kuna ditch near Hanalei, Kauai, for 1913.

\begin{tabular}{|c|c|c|c|c|c|c|c|c|c|}
\hline Day. & Jan. & Feb. & Mar. & Apr. & May. & June. & July. & Aug. & Sept. \\
\hline $\begin{array}{l}1 \\
1 \\
3 \\
4 \\
4 \\
5, \ldots \ldots \cdots \cdots\end{array}$ & $\begin{array}{l}5.0 \\
5.7 \\
5.7 \\
5.7 \\
5.0\end{array}$ & $\begin{array}{l}4.5 \\
4.5 \\
4.5 \\
4.5 \\
5.7\end{array}$ & $\begin{array}{l}22 \\
22 \\
22 \\
22 \\
22\end{array}$ & $\begin{array}{l}22 \\
22 \\
24 \\
24 \\
22\end{array}$ & $\begin{array}{l}22 \\
24 \\
22 \\
22 \\
22\end{array}$ & $\begin{array}{l}27 \\
26 \\
29 \\
28 \\
28\end{array}$ & $\begin{array}{l}34 \\
48 \\
38 \\
38 \\
38\end{array}$ & $\begin{array}{l}22 \\
22 \\
22 \\
22 \\
23\end{array}$ & $\begin{array}{l}25 \\
29 \\
25 \\
24 \\
24\end{array}$ \\
\hline $\begin{array}{r}6 \\
7 \\
8 \\
9 \\
9 \\
10 \\
\ldots \ldots\end{array}$ & $\begin{array}{l}5.0 \\
5.0 \\
4.5 \\
4.5 \\
5.0\end{array}$ & $\begin{array}{l}7.4 \\
4.5 \\
4.5 \\
4.5 \\
4.5\end{array}$ & $\begin{array}{l}22 \\
24 \\
24 \\
22 \\
22\end{array}$ & $\begin{array}{l}22 \\
22 \\
24 \\
24 \\
24\end{array}$ & $\begin{array}{l}22 \\
24 \\
22 \\
22 \\
22\end{array}$ & $\begin{array}{l}28 \\
27 \\
27 \\
28 \\
28\end{array}$ & $\begin{array}{l}35 \\
35 \\
35 \\
35 \\
34\end{array}$ & $\begin{array}{l}23 \\
23 \\
23 \\
23 \\
22\end{array}$ & $\begin{array}{l}24 \\
25 \\
24 \\
24 \\
23\end{array}$ \\
\hline $\begin{array}{l}11 \\
12 \\
13 \\
14 \\
15\end{array}$ & $\begin{array}{l}4.5 \\
5.0 \\
6.5 \\
5.0 \\
5.0\end{array}$ & $\begin{array}{l}4.5 \\
4.5 \\
22 \\
22 \\
22\end{array}$ & $\begin{array}{l}22 \\
22 \\
22 \\
22 \\
22\end{array}$ & $\begin{array}{l}24 \\
22 \\
26 \\
24 \\
24\end{array}$ & $\begin{array}{l}22 \\
24 \\
22 \\
22 \\
22\end{array}$ & $\begin{array}{l}28 \\
27 \\
28 \\
28 \\
28\end{array}$ & $\begin{array}{l}34 \\
34 \\
35 \\
34 \\
32\end{array}$ & $\begin{array}{l}22 \\
23 \\
24 \\
24 \\
23\end{array}$ & $\begin{array}{l}24 \\
24 \\
24 \\
13 \\
13\end{array}$ \\
\hline $\begin{array}{l}16, \ldots \\
17, \ldots \ldots \\
18, \ldots \ldots \\
190 \ldots \ldots\end{array}$ & $\begin{array}{l}5.0 \\
5.0 \\
5.0 \\
5.0 \\
5.7\end{array}$ & $\begin{array}{l}22 \\
24 \\
24 \\
24 \\
24\end{array}$ & $\begin{array}{l}22 \\
22 \\
22 \\
24 \\
24\end{array}$ & $\begin{array}{l}22 \\
22 \\
22 \\
22 \\
22\end{array}$ & $\begin{array}{l}22 \\
22 \\
22 \\
22 \\
22\end{array}$ & $\begin{array}{l}31 \\
28 \\
28 \\
28 \\
28\end{array}$ & $\begin{array}{l}32 \\
34 \\
36 \\
35 \\
31\end{array}$ & $\begin{array}{l}27 \\
23 \\
24 \\
23 \\
22\end{array}$ & $\begin{array}{l}13 \\
13 \\
13 \\
13 \\
13\end{array}$ \\
\hline $\begin{array}{l}21 \\
22 \\
24 \\
24 \\
25\end{array}$ & $\begin{array}{l}5.0 \\
5.0 \\
5.0 \\
5.0 \\
5.0\end{array}$ & $\begin{array}{l}24 \\
24 \\
22 \\
24 \\
24\end{array}$ & $\begin{array}{l}24 \\
24 \\
24 \\
42 \\
30\end{array}$ & $\begin{array}{l}22 \\
22 \\
22 \\
22 \\
22\end{array}$ & $\begin{array}{l}22 \\
22 \\
22 \\
22 \\
21\end{array}$ & $\begin{array}{l}28 \\
30 \\
30 \\
30 \\
30\end{array}$ & $\begin{array}{l}31 \\
31 \\
32 \\
32 \\
34\end{array}$ & $\begin{array}{l}23 \\
23 \\
22 \\
23 \\
24\end{array}$ & $\begin{array}{l}13 \\
13 \\
13 \\
11 \\
11\end{array}$ \\
\hline 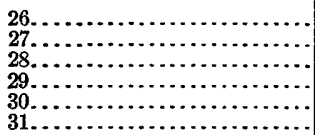 & $\begin{array}{l}5.0 \\
5.0 \\
5.0 \\
5.0 \\
4.5 \\
4.5\end{array}$ & $\begin{array}{c}22 \\
22 \\
22 \\
\cdots \cdots \\
\cdots \cdots \\
\cdots\end{array}$ & $\begin{array}{l}26 \\
24 \\
22 \\
22 \\
24 \\
24\end{array}$ & $\begin{array}{l}22 \\
22 \\
22 \\
22 \\
24\end{array}$ & $\begin{array}{l}21 \\
28 \\
29 \\
28 \\
28 \\
28\end{array}$ & $\begin{array}{l}30 \\
38 \\
32 \\
32 \\
\mathbf{3 2}\end{array}$ & $\begin{array}{l}32 \\
32 \\
26 \\
22 \\
22 \\
22\end{array}$ & $\begin{array}{l}21 \\
24 \\
29 \\
25 \\
27 \\
26\end{array}$ & $\begin{array}{l}11 \\
13 \\
14 \\
13 \\
13 \\
-.\end{array}$ \\
\hline
\end{tabular}


Monthly discharge of Kuna ditch near Hanalei, Kauai, for 1918.

\begin{tabular}{|c|c|c|c|c|c|}
\hline \multirow{2}{*}{ Month. } & \multicolumn{3}{|c|}{ Discharge in second-feet. } & \multirow{2}{*}{$\begin{array}{c}\text { Run-off } \\
\text { (total in } \\
\text { acre-feet). }\end{array}$} & \multirow{2}{*}{$\begin{array}{l}\text { Accu } \\
\text { racy. }\end{array}$} \\
\hline & Maximum. & Minimum. & Mean. & & \\
\hline \multirow[t]{2}{*}{ 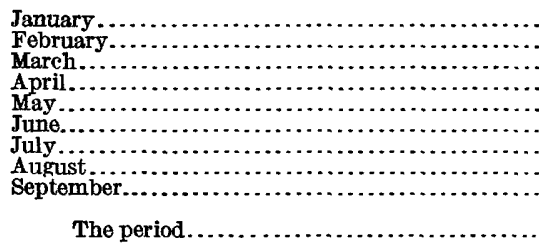 } & $\begin{array}{l}6.5 \\
24 \\
42 \\
26 \\
29 \\
38 \\
48 \\
29 \\
29\end{array}$ & $\begin{array}{l}4.5 \\
4.5 \\
22 \\
22 \\
21 \\
26 \\
22 \\
22 \\
11\end{array}$ & $\begin{array}{l}5.06 \\
15.2 \\
23.7 \\
22.7 \\
23.1 \\
29.0 \\
33.0 \\
23.5 \\
18.2\end{array}$ & $\begin{array}{r}311 \\
844 \\
1,460 \\
1,350 \\
1,420 \\
1,730 \\
2,030 \\
1,440 \\
1,080\end{array}$ & \multirow[t]{2}{*}{$\begin{array}{l}\text { C. } \\
\text { C. } \\
\text { B. } \\
\text { B. } \\
\text { B. } \\
\text { B. } \\
\text { B. } \\
\text { B. } \\
\text { B. }\end{array}$} \\
\hline & & & & 11,600 & \\
\hline
\end{tabular}

WAINIHA RIVER (EAST AND WEST CHANNELS) AT POWER HOUSE, NEAR WAINIHA, KAUAI.

Iocation.-About 2 miles south of Wainiha and just northeast of Kauai Electric Co.'s power house.

Records available.-East Channel, February 25, 1912, to December 31, 1913; West Channel, December 30, 1911, to December 31, 1913.

Drainage area.-14.7 square miles.

Gage.-Two inclined timber gages-one on each channel-to obtain the total flow of the river at this point.

Control.-Two large channels, both rocky and probably permanent. The natives build fish dams at the point where the river divides above the stations, and this shifts the water from one channel to the other.

Discharge measurements.-Made from wire suspension footbridge over each channel, or by wading.

Accuracy.-East Channel, records fair; West Channel, records good.

Cooperation.-Stations maintained in cooperation with the Kauai Electric Co.

Discharge measurements of east channel of Wainiha River near Wainiha, Kauai, in 1913.

\begin{tabular}{|c|c|c|c|c|c|c|c|}
\hline Date. & Hydrographer. & $\begin{array}{c}\text { Gage } \\
\text { height. }\end{array}$ & $\begin{array}{l}\text { Dis- } \\
\text { charge. }\end{array}$ & Date. & Hydrographer. & $\begin{array}{c}\text { Gage } \\
\text { height. }\end{array}$ & $\begin{array}{l}\text { Dis- } \\
\text { charge. }\end{array}$ \\
\hline $\begin{array}{l}\text { Mar. } 31 \\
\text { Apr. 24 } \\
\text { Aug. 23 }\end{array}$ & 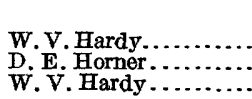 & $\begin{array}{r}\text { Feet. } \\
6.73 \\
6.72 \\
6.59\end{array}$ & $\begin{array}{r}\text { Sec. }-f t . \\
10.3 \\
12.7 \\
.5 .9\end{array}$ & $\begin{array}{l}\text { Nov. } 13 \\
\text { Nov. } 14\end{array}$ & 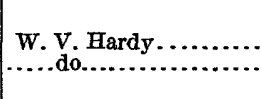 & $\begin{array}{r}\text { Feet. } \\
9.30 \\
8.10\end{array}$ & $\begin{array}{r}\text { Sec.-ft. } \\
407 \\
106\end{array}$ \\
\hline
\end{tabular}


Daily discharge, in sècond-feet, of east channel of Wainiha River near Wainiha, Kauai, for 1912-13.

\begin{tabular}{|c|c|c|c|c|c|c|c|c|c|c|c|c|}
\hline Day. & Jan. & Feb. & Mar. & Apr. & May. & June. & July. & Aug. & Sept. & Oct. & Nov. & Dec. \\
\hline \multicolumn{13}{|l|}{1912.} \\
\hline & & & $\begin{array}{l}94 \\
74\end{array}$ & $\begin{array}{l}28 \\
18\end{array}$ & $\begin{array}{r}8 \\
10\end{array}$ & $\begin{array}{l}14 \\
14\end{array}$ & $\begin{array}{l}12 \\
10\end{array}$ & $\begin{array}{l}7 \\
7\end{array}$ & $\begin{array}{l}46 \\
40\end{array}$ & $\begin{array}{l}16 \\
14\end{array}$ & $\begin{array}{r}14 \\
7\end{array}$ & \multirow{3}{*}{$\begin{array}{r}185 \\
732 \\
1,320 \\
149 \\
34\end{array}$} \\
\hline & & & 94 & 12 & 16 & 14 & 10 & 5 & 34 & 40 & & \\
\hline & & 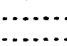 & $\begin{array}{l}57 \\
66\end{array}$ & $\begin{array}{l}31 \\
31\end{array}$ & $\begin{array}{l}18 \\
26\end{array}$ & $\begin{array}{l}18 \\
14\end{array}$ & $\begin{array}{l}10 \\
16\end{array}$ & $\begin{array}{r}6 \\
14\end{array}$ & $\begin{array}{l}18 \\
12\end{array}$ & $\begin{array}{r}8 \\
14\end{array}$ & & \\
\hline & & & 70 & 94 & 23 & 14 & 20 & 20 . & 242 & 12 & 7 & 26 \\
\hline & & & 50 & 256 & 28 & $\begin{array}{l}14 \\
14\end{array}$ & 37 & 26 & 43 & 12 & 10 & 16 \\
\hline & & & $\begin{array}{r}70 \\
734\end{array}$ & $\begin{array}{l}366 \\
3606\end{array}$ & 34 & 14 & 12 & 34 & 18 & 15 & 79 & 20 \\
\hline 10. & & $\cdots$ & $\begin{array}{l}134 \\
256\end{array}$ & $\begin{array}{r}242 \\
74\end{array}$ & $\begin{array}{l}14 \\
10\end{array}$ & $\begin{array}{l}18 \\
14\end{array}$ & 115 & $\begin{array}{r}28 \\
8\end{array}$ & $\begin{array}{l}18 \\
10\end{array}$ & $\begin{array}{l}10 \\
23\end{array}$ & $\begin{array}{r}141 \\
18\end{array}$ & $\begin{array}{l}20 \\
10\end{array}$ \\
\hline 11. & & & 642 & 57 & 10 & 14 & 126 & 14 & 10 & 10 & 12 & 8 \\
\hline & & & 185 & $\begin{array}{l}66 \\
43\end{array}$ & $\begin{array}{l}10 \\
14\end{array}$ & $\begin{array}{l}14 \\
18\end{array}$ & $\begin{array}{r}46 \\
217\end{array}$ & ${ }_{20}^{12}$ & $\begin{array}{l}9 \\
5\end{array}$ & $\begin{array}{l}10 \\
14\end{array}$ & $\begin{array}{l}7 \\
7\end{array}$ & $7_{10}^{7}$ \\
\hline & & & 74 & $\begin{array}{l}70 \\
34\end{array}$ & $\begin{array}{l}14 \\
119\end{array}$ & $\begin{array}{l}18 \\
14\end{array}$ & $\begin{array}{r}211 \\
57\end{array}$ & 230 & $\begin{array}{l}0 \\
3\end{array}$ & $\begin{array}{l}14 \\
14\end{array}$ & 7 & 8 \\
\hline & & & 46 & 37 & 74 & 16 & 40 & 34 & 5 & 10 & 16 & 10 \\
\hline 16. & & & 34 & 20 & 28 & 18 & 17 & 14 & 5 & 16 & 12 & 16 \\
\hline & & $\cdots$ & 20 & 43 & 26 & $\begin{array}{l}14 \\
18\end{array}$ & 106 & 8 & 10 & 35 & 10 & 10 \\
\hline & 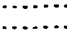 & . & $\begin{array}{r}14 \\
4\end{array}$ & $\begin{array}{r}23 \\
8\end{array}$ & $\begin{array}{l}20 \\
14\end{array}$ & $\begin{array}{l}18 \\
20\end{array}$ & $\begin{array}{l}15 \\
12\end{array}$ & 7 & 5 & 45 & 1 & 12 \\
\hline & & & 28 & 4 & $\begin{array}{l}17 \\
14\end{array}$ & 23 & 12 & 7 & 5 & 46 & 16 & 26 \\
\hline 21. & & & 23 & 5 & 16 & 23 & 16 & 18 & 6 & 106 & 141 & 20 \\
\hline & & $\cdots$ & 10 & 7 & 31 & 16 & 12 & 26 & 16 & $\begin{array}{r}40 \\
40\end{array}$ & 149 & 26 \\
\hline & 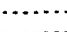 & $\cdots$ & 6 & 8 & 18 & 16 & 12 & 16 & $\begin{array}{l}8 \\
8\end{array}$ & 28 & 100 & $\begin{array}{r}70 \\
175\end{array}$ \\
\hline & $\cdots$ & 100 & $\begin{array}{r}28 \\
349\end{array}$ & $\begin{array}{l}10 \\
12\end{array}$ & $\begin{array}{l}23 \\
16\end{array}$ & 14 & $\begin{array}{l}16 \\
10\end{array}$ & $\begin{array}{r}14 \\
9\end{array}$ & $\begin{array}{l}8 \\
6\end{array}$ & $\begin{array}{l}14 \\
16\end{array}$ & $\begin{array}{l}20 \\
12\end{array}$ & $\begin{array}{l}175 \\
134\end{array}$ \\
\hline & $\ldots .$. & 7 & 37 & 14 & 14 & 14 & 20 & 10 & 5 & 217 & 10 & 100 \\
\hline & … & $\begin{array}{l}14 \\
12\end{array}$ & $\begin{array}{l}23 \\
34\end{array}$ & $\begin{array}{l}8 \\
7\end{array}$ & $\begin{array}{r}23 \\
256\end{array}$ & $\begin{array}{l}14 \\
10\end{array}$ & 12 & $\begin{array}{l}79 \\
66\end{array}$ & $\begin{array}{l}5 \\
8\end{array}$ & $\begin{array}{l}34 \\
34\end{array}$ & 10 & $\begin{array}{l}18 \\
16\end{array}$ \\
\hline & 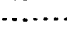 & 16 & 18 & 8 & 89 & 10 & 8 & 66 & 10 & 18 & 119 & 10 \\
\hline & 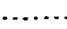 & & 16 & 5 & 28 & 10 & 12 & 119 & 10 & 23 & 141 & 10 \\
\hline & & & 16 & & 18 & & 10 & 94 & & 31 & & 10 \\
\hline 1913. & & & & & & & & & & & & \\
\hline $1 \ldots$ & $\begin{array}{l}10 \\
37\end{array}$ & $\begin{array}{l}6 \\
6\end{array}$ & $\begin{array}{l}7 \\
7\end{array}$ & $\begin{array}{l}50 \\
40\end{array}$ & $\begin{array}{r}7 \\
157\end{array}$ & $\begin{array}{l}5 \\
5\end{array}$ & $\begin{array}{r}18 \\
157\end{array}$ & $\begin{array}{l}7 \\
7\end{array}$ & $\begin{array}{l}84 \\
31\end{array}$ & $\begin{array}{l}7 \\
7\end{array}$ & $\begin{array}{l}18 \\
29\end{array}$ & $\begin{array}{l}61 \\
53\end{array}$ \\
\hline 3. & 20 & 5 & 7 & $\begin{array}{l}40 \\
23\end{array}$ & 37 & 34 & 57 & 5 & $\begin{array}{l}11 \\
18\end{array}$ & 7 & 40 & 464 \\
\hline $4 \ldots \ldots$ & $\begin{array}{l}14 \\
10\end{array}$ & $\begin{array}{l}6 \\
8\end{array}$ & $\begin{array}{l}7 \\
7\end{array}$ & $\begin{array}{l}14 \\
10\end{array}$ & $\begin{array}{l}43 \\
70\end{array}$ & $\begin{array}{l}12 \\
12\end{array}$ & $\begin{array}{l}66 \\
61\end{array}$ & $\begin{array}{l}\mathbf{5} \\
5\end{array}$ & $\begin{array}{l}12 \\
12\end{array}$ & $\begin{array}{l}6 \\
6\end{array}$ & $\begin{array}{l}40 \\
16\end{array}$ & $\begin{array}{l}149 \\
134\end{array}$ \\
\hline $6 .$. & $\begin{array}{c}10 \\
8\end{array}$ & 7 & 7 & 10 & 89 & 19 & 23 & $\begin{array}{l}5 \\
8\end{array}$ & 14 & 10 & 12 & 126 \\
\hline & 84 & 7 & 8 & $\begin{array}{l}10 \\
10\end{array}$ & $\begin{array}{l}43 \\
34\end{array}$ & $\begin{array}{r}14 \\
7\end{array}$ & $\begin{array}{l}20 \\
18\end{array}$ & $16^{\circ}$ & 7 & 242 & 28 & 112 \\
\hline $\begin{array}{r}9 . \ldots \ldots \ldots \\
10 . \ldots \ldots \ldots\end{array}$ & $\begin{array}{l}16 \\
34\end{array}$ & $\begin{array}{l}6 \\
5\end{array}$ & 7 & $\begin{array}{l}18 \\
18\end{array}$ & $\begin{array}{r}12 \\
8\end{array}$ & $\begin{array}{l}14 \\
94\end{array}$ & $\begin{array}{l}11 \\
10\end{array}$ & ${ }_{7}^{23}$ & $\begin{array}{l}8 \\
7\end{array}$ & $\begin{array}{l}23 \\
10\end{array}$ & $\begin{array}{r}141 \\
61\end{array}$ & $\begin{array}{l}89 \\
53\end{array}$ \\
\hline 11.. & 28 & 5 & 7 & 70 & 5 & 53 & 7 & 7 & 7 & 10 & 868 & 57 \\
\hline & $\begin{array}{l}20 \\
50\end{array}$ & 5 & 7 & $\begin{array}{l}53 \\
84\end{array}$ & $\begin{array}{l}50 \\
53\end{array}$ & 18 & $\begin{array}{r}8 \\
50\end{array}$ & ${ }_{20}^{20}$ & $\begin{array}{c}10 \\
7\end{array}$ & $\begin{array}{l}10 \\
61\end{array}$ & $\begin{array}{l}868 \\
485\end{array}$ & $\begin{array}{l}50 \\
43\end{array}$ \\
\hline ... & 9 & 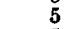 & 9 & 79 & 28 & 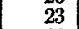 & 7 & 5 & & 18 & 106 & 43 \\
\hline & 37 & 5 & 7 & 206 & 12 & 18 & 20 & 20 & 7 & 10 & 126 & 43 \\
\hline 16. & 112 & 5 & 7 & 46 & 10 & 26 & 16 & 50 & 7 & 18 & 126 & 43 \\
\hline & $\begin{array}{l}12 \\
10\end{array}$ & 10 & 37 & 100 & $\begin{array}{l}46 \\
40\end{array}$ & $\begin{array}{l}8 \\
9\end{array}$ & $\begin{array}{l}14 \\
10\end{array}$ & 18 & 7 & $\begin{array}{l}7 \\
7\end{array}$ & $\begin{array}{r}149 \\
66\end{array}$ & $\begin{array}{l}40 \\
37\end{array}$ \\
\hline$\cdots$ & 8 & 勇4 & $\begin{array}{l}10 \\
16\end{array}$ & $\begin{array}{l}40 \\
66\end{array}$ & $\begin{array}{l}40 \\
12\end{array}$ & 12 & 14 & 10 & 10 & 7 & 2,250 & $\begin{array}{l}37 \\
37\end{array}$ \\
\hline & 10 & 20 & 18 & 37 & 10 & 10 & 14 & 10 & 7 & & 332 & 34 \\
\hline $\begin{array}{l}21 \ldots \ldots \ldots \\
22 \ldots \ldots \ldots\end{array}$ & $\begin{array}{l}10 \\
10\end{array}$ & $\begin{array}{r}16 \\
7\end{array}$ & $\begin{array}{l}16 \\
18\end{array}$ & $\begin{array}{r}18 \\
7\end{array}$ & $\begin{array}{l}8 \\
5\end{array}$ & $\begin{array}{l}12 \\
10\end{array}$ & $\begin{array}{l}8 \\
7\end{array}$ & $\begin{array}{r}10 \\
8\end{array}$ & $\begin{array}{l}7 \\
8\end{array}$ & $\begin{array}{l}8 \\
7\end{array}$ & $\begin{array}{l}100 \\
270\end{array}$ & $\begin{array}{l}35 \\
35\end{array}$ \\
\hline & 8 & 7 & 79 & 8 & 7 & 7 & 7 & 7 & 7 & 7 & 185 & 37 \\
\hline & $\gamma$ & 26 & 89 & 66 & 10 & 18 & $\begin{array}{l}14 \\
23\end{array}$ & 20 & 7 & 7 & 485 & $\begin{array}{l}\text { bu } \\
34\end{array}$ \\
\hline $\begin{array}{l}26 \ldots \ldots \ldots \\
27 \ldots \ldots \ldots\end{array}$ & $\begin{array}{l}7 \\
8\end{array}$ & $\begin{array}{l}9 \\
9 \\
8\end{array}$ & $\begin{array}{r}175 \\
50\end{array}$ & $\begin{array}{l}12 \\
18\end{array}$ & $\begin{array}{r}10 \\
8\end{array}$ & $\begin{array}{l}40 \\
46\end{array}$ & $\begin{array}{l}23 \\
14\end{array}$ & $\begin{array}{l}10 \\
10\end{array}$ & $\begin{array}{l}7 \\
6\end{array}$ & $\begin{array}{l}7 \\
7\end{array}$ & $\begin{array}{r}230 \\
53\end{array}$ & $\begin{array}{l}34 \\
34\end{array}$ \\
\hline $28 \ldots \ldots$ & 7 & 7 & 37 & 31 & 7 & $\begin{array}{l}31 \\
31 \\
14\end{array}$ & 14 & 12 & 18 & 7 & 70 & 34 \\
\hline 30 & 6 & ...... & $\begin{array}{l}54 \\
10\end{array}$ & $\begin{array}{l}58 \\
57\end{array}$ & 18 & 18 & ${ }_{7}^{20}$ & 50 & 7 & 7 & $\begin{array}{r}020 \\
79\end{array}$ & 34 \\
\hline $\mathbf{3 1}$ & 10 & & 10 & & 6 & & 8 & 23 & & 7 & & 34 \\
\hline
\end{tabular}


Monthly discharge of east channel of Wainiha River near Wainiha, Kauai, for 1912-13.

\begin{tabular}{|c|c|c|c|c|c|}
\hline \multirow{2}{*}{ Month. } & \multicolumn{3}{|c|}{ Discharge in second-feet. } & \multirow{2}{*}{$\begin{array}{l}\text { Rum-off } \\
\text { (total in } \\
\text { acre-feet). }\end{array}$} & \multirow{2}{*}{$\begin{array}{l}\text { Aceti- } \\
\text { racy. }\end{array}$} \\
\hline & Maximum. & Minimum. & Mean. & & \\
\hline 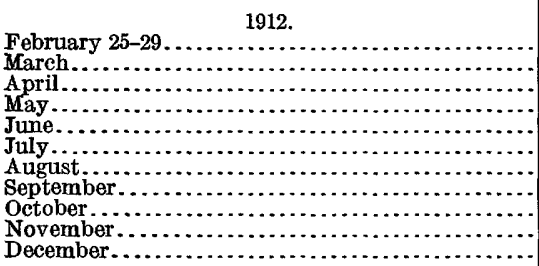 & $\begin{array}{r}16 \\
642 \\
366 \\
256 \\
23 \\
217 \\
230 \\
242 \\
217 \\
149 \\
1,320\end{array}$ & $\begin{array}{r}7 \\
6 \\
4 \\
8 \\
10 \\
7 \\
5 \\
3 \\
8 \\
7 \\
7\end{array}$ & $\begin{array}{l}11.8 \\
86.4 \\
52.4 \\
33.8 \\
15.5 \\
34.6 \\
33.1 \\
20.9 \\
30.9 \\
37.9 \\
104\end{array}$ & $\begin{array}{r}117 \\
5,310 \\
3,120 \\
2,080 \\
922 \\
2,130 \\
2,040 \\
1,240 \\
1,900 \\
2,260 \\
6,400\end{array}$ & $\begin{array}{l}\text { C. } \\
\text { C. } \\
\text { C. } \\
\text { B. } \\
\text { B. } \\
\text { C. } \\
\text { B. } \\
\text { B. }\end{array}$ \\
\hline The period. & $\ldots$ & ... & $\cdots$ & 27,500 & \\
\hline 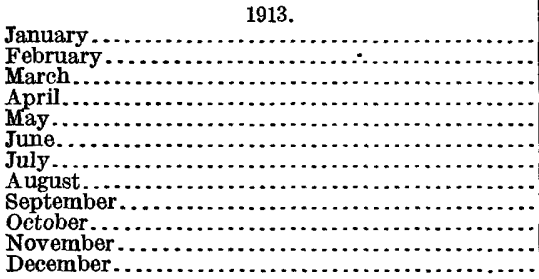 & $\begin{array}{r}112 \\
34 \\
175 \\
206 \\
157 \\
94 \\
157 \\
66 \\
84 \\
242 \\
2,250 \\
464\end{array}$ & $\begin{array}{r}6 \\
5 \\
7 \\
7 \\
5 \\
5 \\
7 \\
5 \\
6 \\
6 \\
12 \\
34\end{array}$ & $\begin{array}{c}21.1 \\
11.1 \\
25.4 \\
42.5 \\
27.7 \\
20.7 \\
26.5 \\
17.0 \\
12.0 \\
18.8 \\
272 \\
70.2\end{array}$ & $\begin{array}{r}1,300 \\
616 \\
1,560 \\
2,530 \\
1,700 \\
1,230 \\
1,630 \\
1,050 \\
714 \\
1,160 \\
16,200 \\
4,320\end{array}$ & $\begin{array}{l}\text { A. } \\
\text { A. } \\
\text { A. } \\
\text { A. } \\
\text { A. } \\
\text { A. } \\
\text { A. } \\
\text { A. } \\
\text { B. } \\
\text { B. } \\
\text { C. } \\
\text { C. }\end{array}$ \\
\hline The year. & 2,250 & 5 & 46.9 & 34,000 & \\
\hline
\end{tabular}

Discharge measurements of west channel of Wainiha River near Wainiha, Kauai, in 1913.

\begin{tabular}{|c|c|c|c|c|c|c|c|}
\hline Date. & Hydrographer. & $\begin{array}{c}\text { Gage } \\
\text { height. }\end{array}$ & $\begin{array}{l}\text { Dis- } \\
\text { eharge. }\end{array}$ & Date. & Hydrographer. & $\begin{array}{c}\text { Gage } \\
\text { height. }\end{array}$ & $\begin{array}{l}\text { Dis- } \\
\text { charge. }\end{array}$ \\
\hline $\begin{array}{l}\text { Aug. } 23 \\
\text { Nov } 13\end{array}$ & $\begin{array}{c}\text { W. V. Hardy ..... } \\
\ldots . . \text { do............ }\end{array}$ & $\begin{array}{r}\text { Feet. } \\
5.50 \\
6.19\end{array}$ & $\begin{array}{r}\text { Sec.-ft. } \\
112 \\
386\end{array}$ & Nov. 13 & W. V. Hardy .... & $\begin{array}{c}\text { Feet. } \\
5.87\end{array}$ & Sec.-ft. \\
\hline
\end{tabular}


Daily discharge, in second-feet, of west channel of Wainiha River near Wainiha, Kauai, for 1912-13.

\begin{tabular}{|c|c|c|c|c|c|c|c|c|c|c|c|c|}
\hline Day. & Jan. & Feb. & Mar. & Apr. & May. & June. & July. & Aug. & Sept. & Oct. & Nov. & Dec. \\
\hline $\begin{array}{r}\quad 1912 . \\
1 \ldots \ldots \ldots \\
2 \ldots \ldots \\
3 \ldots \ldots \\
4 \ldots \ldots \\
5 \ldots \ldots \ldots \\
\end{array}$ & $\begin{array}{r}90 \\
115 \\
140 \\
115 \\
140\end{array}$ & $\begin{array}{l}102 \\
192 \\
210 \\
192 \\
140\end{array}$ & $\begin{array}{l}390 \\
272 \\
365 \\
2550 \\
250\end{array}$ & $\begin{array}{l}272 \\
210 \\
158 \\
250 \\
295\end{array}$ & $\begin{array}{l}140 \\
140 \\
147 \\
158 \\
192\end{array}$ & $\begin{array}{l}140 \\
128 \\
115 \\
140 \\
128\end{array}$ & $\begin{array}{l}128 \\
115 \\
115 \\
115 \\
140\end{array}$ & $\begin{array}{l}115 \\
115 \\
115 \\
90 \\
175\end{array}$ & $\begin{array}{l}340 \\
318 \\
272 \\
210 \\
158\end{array}$ & $\begin{array}{l}135 \\
128 \\
295 \\
115 \\
158\end{array}$ & $\begin{array}{l}158 \\
128 \\
140 \\
128 \\
115\end{array}$ & $\begin{array}{r}115 \\
820 \\
1,310 \\
440 \\
250\end{array}$ \\
\hline $\begin{array}{r}6 \ldots . . \\
7 \ldots . . \\
8 \ldots \ldots \\
9 . \ldots . \\
10 \ldots\end{array}$ & $\begin{array}{l}115 \\
115 \\
175 \\
140 \\
128\end{array}$ & $\begin{array}{l}115 \\
115 \\
128 \\
140 \\
140\end{array}$ & $\begin{array}{l}365 \\
250 \\
295 \\
465 \\
700\end{array}$ & $\begin{array}{l}440 \\
580 \\
670 \\
640 \\
365\end{array}$ & $\begin{array}{l}140 \\
140 \\
175 \\
140 \\
115\end{array}$ & $\begin{array}{l}115 \\
115 \\
115 \\
140 \\
115\end{array}$ & $\begin{array}{l}175 \\
210 \\
158 \\
175 \\
365\end{array}$ & $\begin{array}{l}192 \\
210 \\
272 \\
250 \\
140\end{array}$ & $\begin{array}{l}580 \\
272 \\
210 \\
175 \\
128\end{array}$ & $\begin{array}{l}147 \\
128 \\
140 \\
168 \\
175\end{array}$ & $\begin{array}{l}115 \\
128 \\
272 \\
440 \\
230\end{array}$ & $\begin{array}{l}272 \\
192 \\
210 \\
210 \\
140\end{array}$ \\
\hline $\begin{array}{l}11 \ldots . \\
12 \ldots . \\
13 . . . \\
14 \ldots \\
15 \ldots .\end{array}$ & $\begin{array}{r}90 \\
115 \\
175 \\
140 \\
115\end{array}$ & $\begin{array}{l}158 \\
128 \\
128 \\
128 \\
128\end{array}$ & $\begin{array}{l}790 \\
440 \\
295 \\
250 \\
192\end{array}$ & $\begin{array}{l}250 \\
295 \\
760 \\
250 \\
272\end{array}$ & $\begin{array}{l}115 \\
115 \\
230 \\
415 \\
295\end{array}$ & $\begin{array}{l}115 \\
115 \\
140 \\
115 \\
128\end{array}$ & $\begin{array}{l}318 \\
175 \\
365 \\
272 \\
230\end{array}$ & $\begin{array}{l}158 \\
128 \\
175 \\
520 \\
295\end{array}$ & $\begin{array}{r}115 \\
115 \\
102 \\
90 \\
115\end{array}$ & $\begin{array}{l}115 \\
128 \\
128 \\
128 \\
115\end{array}$ & $\begin{array}{l}158 \\
115 \\
115 \\
115 \\
230\end{array}$ & $\begin{array}{l}140 \\
128 \\
140 \\
140 \\
140\end{array}$ \\
\hline $\begin{array}{l}16 \ldots \\
17 . \ldots \\
18 . \ldots \\
20 \ldots\end{array}$ & $\begin{array}{l}90 \\
90 \\
90 \\
90 \\
90\end{array}$ & $\begin{array}{l}115 \\
115 \\
158 \\
140 \\
158\end{array}$ & $\begin{array}{l}192 \\
192 \\
158 \\
128 \\
272\end{array}$ & $\begin{array}{l}230 \\
390 \\
272 \\
158 \\
158\end{array}$ & $\begin{array}{l}158 \\
158 \\
128 \\
140 \\
140\end{array}$ & $\begin{array}{l}128 \\
115 \\
175 \\
192 \\
175\end{array}$ & $\begin{array}{l}175 \\
318 \\
158 \\
140 \\
128\end{array}$ & $\begin{array}{l}175 \\
128 \\
115 \\
115 \\
115\end{array}$ & $\begin{array}{l}115 \\
140 \\
115 \\
115 \\
115\end{array}$ & $\begin{array}{l}140 \\
230 \\
175 \\
340 \\
365\end{array}$ & $\begin{array}{l}175 \\
140 \\
192 \\
272 \\
192\end{array}$ & $\begin{array}{l}192 \\
140 \\
140 \\
203 \\
390\end{array}$ \\
\hline $\begin{array}{l}21 \ldots \\
22 \ldots \\
24 \ldots \\
25 \ldots\end{array}$ & $\begin{array}{r}90 \\
90 \\
162 \\
175 \\
128\end{array}$ & $\begin{array}{l}272 \\
340 \\
390 \\
340 \\
250\end{array}$ & $\begin{array}{l}295 \\
192 \\
158 \\
2550 \\
580\end{array}$ & $\begin{array}{l}128 \\
128 \\
128 \\
140 \\
158\end{array}$ & $\begin{array}{l}158 \\
192 \\
158 \\
192 \\
158\end{array}$ & $\begin{array}{l}158 \\
128 \\
158 \\
175 \\
128\end{array}$ & $\begin{array}{l}140 \\
140 \\
140 \\
158 \\
140\end{array}$ & $\begin{array}{l}175 \\
210 \\
175 \\
158 \\
175\end{array}$ & $\begin{array}{l}115 \\
175 \\
120 \\
102 \\
102\end{array}$ & $\begin{array}{l}465 \\
272 \\
210 \\
158 \\
175\end{array}$ & $\begin{array}{l}415 \\
415 \\
250 \\
670 \\
158\end{array}$ & $\begin{array}{l}210 \\
272 \\
465 \\
440 \\
318\end{array}$ \\
\hline $\begin{array}{l}26 \ldots \ldots . \\
27 \ldots \ldots \\
28 \ldots \ldots \\
29 . \ldots \ldots \\
30 \ldots \ldots \\
31 . \ldots \ldots\end{array}$ & $\begin{array}{l}102 \\
140 \\
115 \\
115 \\
128 \\
140\end{array}$ & $\begin{array}{l}230 \\
272 \\
192 \\
210\end{array}$ & $\begin{array}{l}272 \\
210 \\
250 \\
210 \\
210 \\
230\end{array}$ & $\begin{array}{l}192 \\
250 \\
175 \\
192 \\
158\end{array}$ & $\begin{array}{l}140 \\
210 \\
440 \\
295 \\
140 \\
140\end{array}$ & $\begin{array}{l}115 \\
135 \\
115 \\
115 \\
115\end{array}$ & $\begin{array}{l}192 \\
140 \\
115 \\
128 \\
128 \\
115\end{array}$ & $\begin{array}{l}140 \\
440 \\
250 \\
192 \\
490 \\
295\end{array}$ & $\begin{array}{r}90 \\
90 \\
140 \\
140 \\
115\end{array}$ & $\begin{array}{l}490 \\
230 \\
272 \\
175 \\
175 \\
230\end{array}$ & $\begin{array}{l}140 \\
115 \\
115 \\
318 \\
390\end{array}$ & $\begin{array}{l}230 \\
147 \\
140 \\
140 \\
140 \\
140\end{array}$ \\
\hline & $\begin{array}{l}140 \\
295 \\
210 \\
175 \\
140\end{array}$ & $\begin{array}{l}115 \\
115 \\
115 \\
115 \\
140\end{array}$ & $\begin{array}{l}115 \\
115 \\
115 \\
115 \\
115\end{array}$ & $\begin{array}{l}272 \\
230 \\
210 \\
110 \\
140\end{array}$ & $\begin{array}{l}128 \\
318 \\
210 \\
115 \\
192\end{array}$ & $\begin{array}{l}115 \\
115 \\
230 \\
140 \\
140\end{array}$ & $\begin{array}{l}158 \\
415 \\
295 \\
318 \\
295\end{array}$ & $\begin{array}{l}115 \\
115 \\
115 \\
115 \\
102\end{array}$ & $\begin{array}{l}365 \\
230 \\
175 \\
140 \\
140\end{array}$ & $\begin{array}{l}115 \\
115 \\
115 \\
102 \\
102\end{array}$ & $\begin{array}{l}160 \\
205 \\
250 \\
250 \\
158\end{array}$ & $\begin{array}{l}140 \\
140 \\
520 \\
295 \\
272\end{array}$ \\
\hline $\begin{array}{r}6 \ldots \ldots . . \\
7 \ldots \ldots . . \\
8 \ldots \ldots . . \\
9 \ldots \ldots \ldots \\
10 \ldots \ldots . .\end{array}$ & $\begin{array}{l}140 \\
128 \\
415 \\
192 \\
340\end{array}$ & $\begin{array}{l}128 \\
175 \\
140 \\
115 \\
115\end{array}$ & $\begin{array}{l}115 \\
140 \\
128 \\
115 \\
115\end{array}$ & $\begin{array}{l}120 \\
115 \\
115 \\
192 \\
175\end{array}$ & $\begin{array}{l}365 \\
230 \\
210 \\
140 \\
115\end{array}$ & $\begin{array}{l}182 \\
175 \\
115 \\
175 \\
318\end{array}$ & $\begin{array}{l}210 \\
210 \\
175 \\
140 \\
128\end{array}$ & $\begin{array}{l}102 \\
128 \\
175 \\
192 \\
115\end{array}$ & $\begin{array}{l}\mathbf{1 7 5} \\
140 \\
115 \\
128 \\
115\end{array}$ & $\begin{array}{l}140 \\
230 \\
580 \\
250 \\
140\end{array}$ & $\begin{array}{l}128 \\
295 \\
230 \\
415 \\
318\end{array}$ & $\begin{array}{l}250 \\
250 \\
230 \\
175 \\
140\end{array}$ \\
\hline $\begin{array}{l}11 \ldots \ldots \\
12 \ldots \ldots \\
13 \ldots \ldots \\
14 \ldots \ldots \\
15 . \ldots \ldots\end{array}$ & $\begin{array}{l}295 \\
2955 \\
465 \\
272 \\
340\end{array}$ & $\begin{array}{l}115 \\
115 \\
102 \\
102 \\
115\end{array}$ & $\begin{array}{l}115 \\
115 \\
115 \\
115 \\
115\end{array}$ & $\begin{array}{l}295 \\
272 \\
520 \\
490 \\
490\end{array}$ & $\begin{array}{l}102 \\
2272 \\
230 \\
140 \\
115\end{array}$ & $\begin{array}{l}272 \\
175 \\
192 \\
210 \\
175\end{array}$ & $\begin{array}{l}115 \\
128 \\
295 \\
340 \\
192\end{array}$ & $\begin{array}{l}115 \\
175 \\
192 \\
272 \\
192\end{array}$ & $\begin{array}{l}115 \\
140 \\
115 \\
115 \\
115\end{array}$ & $\begin{array}{l}140 \\
140 \\
295 \\
175 \\
140\end{array}$ & $\begin{array}{l}730 \\
730 \\
670 \\
230 \\
250\end{array}$ & $\begin{array}{l}140 \\
115 \\
115 \\
115 \\
115\end{array}$ \\
\hline $\begin{array}{l}16 \ldots \ldots . \\
17 \ldots \ldots \ldots \\
18 \ldots \ldots \\
19 . \ldots \ldots \\
20 \ldots \ldots \ldots\end{array}$ & $\begin{array}{l}210 \\
158 \\
140 \\
135 \\
140\end{array}$ & $\begin{array}{l}115 \\
\mathbf{1 4 0} \\
295 \\
295 \\
230\end{array}$ & $\begin{array}{l}115 \\
230 \\
135 \\
158 \\
175\end{array}$ & $\begin{array}{l}340 \\
465 \\
230 \\
192 \\
158\end{array}$ & $\begin{array}{l}128 \\
175 \\
230 \\
115 \\
115\end{array}$ & $\begin{array}{l}210 \\
115 \\
115 \\
140 \\
115\end{array}$ & $\begin{array}{l}158 \\
140 \\
128 \\
140 \\
140\end{array}$ & $\begin{array}{l}295 \\
175 \\
140 \\
120 \\
128\end{array}$ & $\begin{array}{l}115 \\
115 \\
115 \\
140 \\
115\end{array}$ & $\begin{array}{l}175 \\
115 \\
115 \\
115 \\
115\end{array}$ & $\begin{array}{l}250 \\
272 \\
230 \\
580 \\
440\end{array}$ & $\begin{array}{l}115 \\
115 \\
115 \\
115 \\
110\end{array}$ \\
\hline $\begin{array}{l}21 \ldots \ldots \ldots \\
22 \\
23 \ldots \ldots \\
24 \ldots \ldots \\
25 \ldots \ldots \\
\end{array}$ & $\begin{array}{l}140 \\
140 \\
128 \\
115 \\
115\end{array}$ & $\begin{array}{l}192 \\
140 \\
140 \\
272 \\
192\end{array}$ & $\begin{array}{l}158 \\
175 \\
580 \\
640 \\
730\end{array}$ & $\begin{array}{l}158 \\
135 \\
102 \\
128 \\
365\end{array}$ & $\begin{array}{l}128 \\
115 \\
128 \\
140 \\
128\end{array}$ & $\begin{array}{l}128 \\
115 \\
115 \\
115 \\
175\end{array}$ & $\begin{array}{l}128 \\
115 \\
115 \\
140 \\
192\end{array}$ & $\begin{array}{l}140 \\
128 \\
115 \\
128 \\
192\end{array}$ & $\begin{array}{l}115 \\
128 \\
115 \\
115 \\
115\end{array}$ & $\begin{array}{l}128 \\
115 \\
115 \\
128 \\
115\end{array}$ & $\begin{array}{l}210 \\
415 \\
440 \\
440 \\
550\end{array}$ & $\begin{array}{r}115 \\
105 \\
105 \\
102 \\
90\end{array}$ \\
\hline $\begin{array}{l}26 . . \\
27 . \\
28 . . \\
290 . \\
30 .\end{array}$ & $\begin{array}{l}115 \\
128 \\
115 \\
115 \\
115 \\
158\end{array}$ & $\begin{array}{l}135 \\
128 \\
115\end{array}$ & $\begin{array}{l}390 \\
272 \\
220 \\
210 \\
175 \\
175\end{array}$ & $\begin{array}{l}140 \\
175 \\
210 \\
192 \\
250\end{array}$ & $\begin{array}{l}128 \\
128 \\
115 \\
115 \\
175 \\
115\end{array}$ & $\begin{array}{l}250 \\
250 \\
210 \\
140 \\
158\end{array}$ & $\begin{array}{l}192 \\
158 \\
140 \\
210 \\
115 \\
115\end{array}$ & $\begin{array}{l}140 \\
140 \\
158 \\
318 \\
272 \\
210\end{array}$ & $\begin{array}{l}110 \\
102 \\
175 \\
115 \\
115\end{array}$ & $\begin{array}{r}115 \\
115 \\
110 \\
115 \\
115 \\
115\end{array}$ & $\begin{array}{l}365 \\
210 \\
175 \\
490 \\
175\end{array}$ & $\begin{array}{r}90 \\
90 \\
95 \\
102 \\
102 \\
102\end{array}$ \\
\hline
\end{tabular}


Monthly discharge of west channel of Wainiha River at power house, near Wainiha, Kauai, for 1912-13.

\begin{tabular}{|c|c|c|c|c|c|}
\hline \multirow{2}{*}{ Month. } & \multicolumn{3}{|c|}{ Discharge in second-feet. } & \multirow{2}{*}{$\begin{array}{l}\text { Run-off } \\
\text { (total in } \\
\text { acre-feet). }\end{array}$} & \multirow{2}{*}{$\begin{array}{l}\text { Accu- } \\
\text { racy. }\end{array}$} \\
\hline & Maximum. & Minimum. & Mean. & & \\
\hline 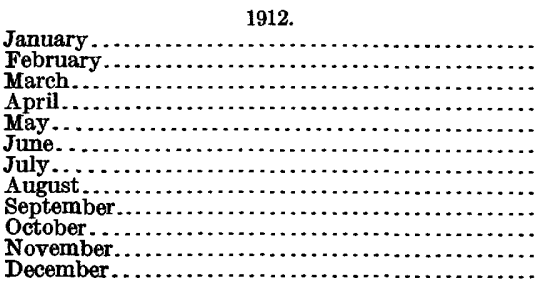 & $\begin{array}{r}175 \\
390 \\
790 \\
760 \\
440 \\
192 \\
365 \\
520 \\
580 \\
490 \\
670 \\
1,310\end{array}$ & $\begin{array}{r}90 \\
102 \\
128 \\
128 \\
115 \\
115 \\
115 \\
90 \\
90 \\
115 \\
115 \\
115\end{array}$ & $\begin{array}{l}119 \\
184 \\
302 \\
285 \\
181 \\
133 \\
178 \\
203 \\
166 \\
203 \\
218 \\
269\end{array}$ & $\begin{array}{r}7,320 \\
10,600 \\
18,600 \\
17,000 \\
11,100 \\
7,910 \\
10,900 \\
12,500 \\
9,880 \\
12,500 \\
13,000 \\
16,500\end{array}$ & $\begin{array}{l}\text { A. } \\
\text { A. } \\
\text { A. } \\
\text { A. } \\
\text { A. } \\
\text { A. } \\
\text { A. } \\
\text { A. } \\
\text { A. } \\
\text { A. } \\
\text { A. }\end{array}$ \\
\hline The year...... & 1,310 & 90 & 204 & 148,000 & \\
\hline 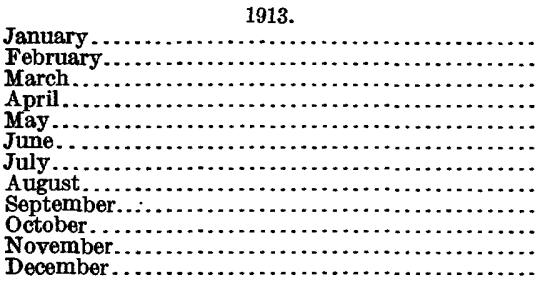 & $\begin{array}{l}465 \\
295 \\
730 \\
520 \\
365 \\
318 \\
365 \\
415 \\
365 \\
580 \\
730 \\
520\end{array}$ & $\begin{array}{l}115 \\
102 \\
115 \\
102 \\
102 \\
115 \\
115 \\
102 \\
102 \\
102 \\
128 \\
90\end{array}$ & $\begin{array}{l}194 \\
150 \\
204 \\
235 \\
165 \\
169 \\
185 \\
162 \\
138 \\
152 \\
342 \\
151\end{array}$ & $\begin{array}{r}11,900 \\
8,300 \\
12,500 \\
14,000 \\
10,100 \\
10,100 \\
11,400 \\
9,960 \\
8,210 \\
9,350 \\
20,400 \\
9,280\end{array}$ & $\begin{array}{l}\text { A. } \\
\text { A. } \\
\text { A. } \\
\text { A. } \\
\text { A. } \\
\text { A. } \\
\text { A. } \\
\text { A. } \\
\text { A. } \\
\text { A. } \\
\text { A. } \\
\text { A. }\end{array}$ \\
\hline The year. $\ldots \ldots \ldots \ldots \ldots$ & 730 & 90 & 187 & 136,000 & \\
\hline
\end{tabular}

MISCELLANEOUS MEASUREMENTS.

Measurements of streams on the island of Kauai at points other than regular gaging stations are listed below:

Miscellaneous discharge measurements on Kauai during the year ending Dec. 31, 1913.

\begin{tabular}{|c|c|c|c|c|c|}
\hline Date. & Stream. & Tributary to- & Locality. & $\begin{array}{c}\text { Gage } \\
\text { height. }\end{array}$ & $\begin{array}{c}\text { Dis- } \\
\text { charge. }\end{array}$ \\
\hline $\begin{array}{lr}\text { Feb. } & 9 \\
\text { Mar. } & 5 \\
\text { July } & 6 \\
\text { Feb. } & 9 \\
\text { Mar. } & 5 \\
\text { July } & 8 \\
\text { July } & 9 \\
\text { Apr. } & 30 \\
\text { July } & 28 \\
\text { Oct. } & 14 \\
\text { June } & 21 \\
\text { Do. } \\
\text { May } & 26 \\
\text { Mar. } 28 \\
\text { Apr. } 26 \\
\text { May } 25 \\
\text { Aug. } 26 \\
\text { Mar. } 22 \\
\text { Mar. } & 30 \\
\text { Sept. } 17 \\
\text { Oct. } 21\end{array}$ & 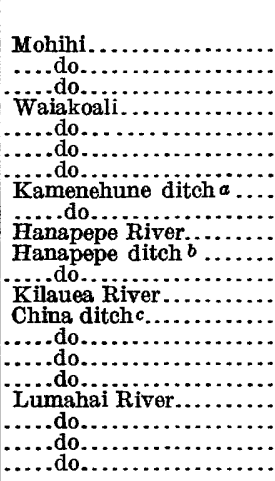 & 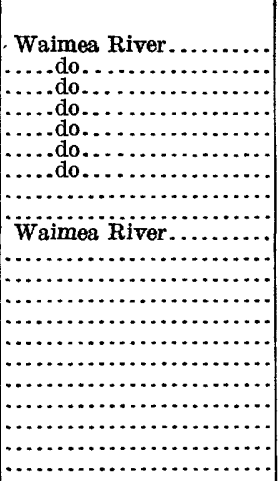 & 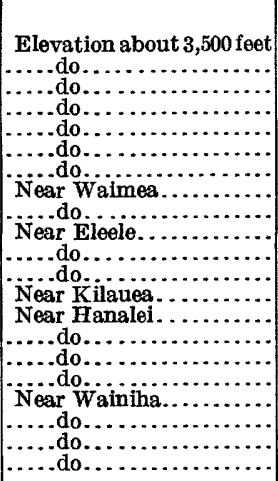 & \begin{tabular}{r} 
Feet. \\
3.81 \\
3.77 \\
3.92 \\
2.52 \\
2.50 \\
2.84 \\
2.57 \\
.60 \\
.37 \\
.38 \\
1.21 \\
1.09 \\
7.70 \\
3.20 \\
3.35 \\
3.64 \\
$\ldots \ldots . .$. \\
\hdashline$\ldots \ldots .$. \\
$\ldots \ldots . .$.
\end{tabular} & \begin{tabular}{|c|} 
Sec. ft. \\
3.04 \\
2.47 \\
4.70 \\
3.03 \\
2.74 \\
10.1 \\
3.53 \\
1.60 \\
.62 \\
29.0 \\
15.5 \\
15.2 \\
4.69 \\
25.1 \\
26.5 \\
30.5 \\
19.8 \\
103 \\
131 \\
63.2 \\
55.2
\end{tabular} \\
\hline
\end{tabular}

a Diverts from Waimea River. $\quad b$ Diverts from Hanapepe River. $\quad c$ Diverts from Hanalei River. 


\section{ISLAND OF OAHU.}

\section{KALIHI STREAM AT KIOI POOL, NEAR HONOLULU, OAHU.}

Location.-At Kioi Pool, about three-eighths mile above Catholic Orphanage, 3 miles up Kalahi Road from King Street car line, and 5 miles north of Honolulu post office.

Records available.-September 8 to December 31, 1913.

Gage.-Gurley weight-driven register installed December 4. September 8 to November 22, 1913, Friez register. No record November 23 to December 3.

Control.-On November 20 a flood lowered the control 0.35 foot; after December 3, probably permanent.

Discharge measurements.-Made by wading or from suspension footbridge about 500 feet above station.

Discharge measurements of Kalihi Stream at Kioi Pool, near Honolulu, Oahu, in 1913.

\begin{tabular}{|c|c|c|c|c|c|c|c|}
\hline Date. & Hydrographer. & $\begin{array}{c}\text { Gage } \\
\text { height. }\end{array}$ & $\begin{array}{c}\text { Dis- } \\
\text { charge. }\end{array}$ & Date. & Hydrographer. & $\begin{array}{c}\text { Gage } \\
\text { height. }\end{array}$ & $\begin{array}{l}\text { Dis- } \\
\text { charge. }\end{array}$ \\
\hline $\begin{array}{lr}\text { Sept. } & 8 \\
\text { Sept. } & 27 \\
\text { Oct. } & 4 \\
\text { Oct. } & 12 \\
\text { Oct. } 25 \\
\text { Nov. } 8\end{array}$ & 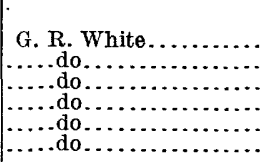 & $\begin{array}{l}\text { Feet. } \\
2.64 \\
2.58 \\
2.58 \\
2.54 \\
2.53 \\
3.41\end{array}$ & $\begin{array}{r}\text { Sec.-ft. } \\
2.37 \\
1.71 \\
1.29 \\
1.06 \\
.98 \\
18.8\end{array}$ & $\begin{array}{l}\text { Nov. } 8 \\
\text { Nov. } 12 \\
\text { Nov. } 22 \\
\text { Dec. } 4 \\
\text { Dec. } 11 \\
\text { Dec. } 23\end{array}$ & 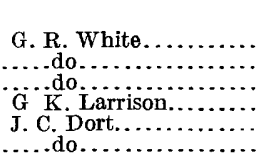 & $\begin{array}{l}\text { Feet. } \\
3.22 \\
3.76 \\
2.88 \\
5.08 \\
2.95 \\
2.50\end{array}$ & $\begin{array}{c}\text { Sec. } f t . \\
13.0 \\
29.0 \\
11.8 \\
96.0 \\
11.0 \\
5.26\end{array}$ \\
\hline
\end{tabular}

Daily discharge, in second-feet, of Kalihi Stream at Kioi Pool, near Honolulu, Oahu, for 1913.

\begin{tabular}{|c|c|c|c|c|c|c|c|c|c|}
\hline Day. & Sept. & Oct. & Nov. & Dec. & Day. & Sept. & Oct. & Nov. & Dec. \\
\hline $\begin{array}{l}1 \ldots \\
2 \ldots \\
3 \ldots \\
4 \ldots \\
5 \ldots\end{array}$ & $\cdots$ & $\begin{array}{l}1.6 \\
1.6 \\
1.6 \\
1.6 \\
1.7\end{array}$ & $\begin{array}{l}0.8 \\
3.1 \\
1.4 \\
1.0 \\
1.0\end{array}$ & $\begin{array}{c}78 \\
46\end{array}$ & 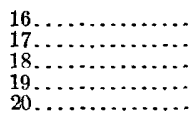 & $\begin{array}{l}2.2 \\
1.9 \\
2.6 \\
2.8 \\
2.2\end{array}$ & $\begin{array}{l}1.7 \\
1.4 \\
1.3 \\
1.2 \\
1.1\end{array}$ & $\begin{array}{l}4.4 \\
4.4 \\
5.0 \\
13 \\
28\end{array}$ & $\begin{array}{l}6.9 \\
7.3 \\
6.6 \\
6.5 \\
6.3\end{array}$ \\
\hline 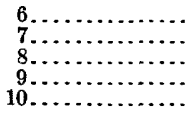 & $\begin{array}{l}2.9 \\
2.6 \\
2.5 \\
2.4 \\
2.5\end{array}$ & $\begin{array}{l}1.9 \\
2.4 \\
3.8 \\
1.8 \\
1.7\end{array}$ & $\begin{array}{c}1.0 \\
8.6 \\
18 \\
12 \\
8.4\end{array}$ & $\begin{array}{l}76 \\
64 \\
28 \\
20 \\
16\end{array}$ & $\begin{array}{l}21 \ldots \ldots \ldots \ldots \ldots \\
22 \ldots \ldots \ldots \\
24 \ldots \ldots \ldots \ldots \ldots \\
25 \ldots \ldots \ldots \ldots \ldots\end{array}$ & $\begin{array}{l}2.2 \\
4.4 \\
2.1 \\
3.1 \\
2.2\end{array}$ & $\begin{array}{l}1.1 \\
1.1 \\
1.1 \\
1.1 \\
1.0\end{array}$ & $\begin{array}{c}18 \\
9.8 \\
\cdots \cdots \\
\cdots \cdots \\
\cdots\end{array}$ & $\begin{array}{r}6.0 \\
5.7 \\
28 \\
5.1 \\
5.0\end{array}$ \\
\hline $\begin{array}{l}11 \\
12 \\
13 \\
14 \\
15 \ldots \ldots\end{array}$ & $\begin{array}{l}2.2 \\
1.9 \\
2.2 \\
3.1 \\
2.4\end{array}$ & $\begin{array}{l}1.5 \\
1.3 \\
1.6 \\
2.1 \\
1.8\end{array}$ & $\begin{array}{c}16 \\
18 \\
12 \\
6.8 \\
5.2 \\
\end{array}$ & $\begin{array}{c}13 \\
10 \\
9.6 \\
10 \\
9.6\end{array}$ & $\begin{array}{l}26 \ldots \ldots \\
27 \ldots \ldots \\
28 \ldots \\
29 \ldots \ldots \\
30 \ldots \ldots \\
31 \ldots \ldots\end{array}$ & $\begin{array}{l}1.9 \\
1.6 \\
1.6 \\
1.6 \\
1.6\end{array}$ & $\begin{array}{r}1.0 \\
1.0 \\
1.0 \\
.9 \\
.9 \\
.8\end{array}$ & $\begin{array}{l}\cdots \cdots \cdots \\
\cdots \cdots \cdots \\
\cdots \cdots \cdots \\
\cdots \cdots \cdots \\
\cdots \cdots \cdots \\
\end{array}$ & $\begin{array}{l}4.9 \\
4.6 \\
4.5 \\
4.4 \\
4.2 \\
4.2\end{array}$ \\
\hline
\end{tabular}

NoTE.-Discharge estimated for days on which no gage heignts were recorded, with the exception of period Nov. 23 to Dec. 3, 1913. Staff gage-height evidence indicated a maximum discharge of about 300 second-feet on night of Dec. 3. 
NUUANU STREAM AT LUAKAHA WEIR IN UPPER NUUANU VALLEY, NEAR HONOLULU, OAHU.

Location.-About 1 mile above reservoir No. 4 (main) in upper Nuuanu Valley, and about 5 miles from Honolulu post office.

Records available._- _ , 1903, to December 31, 1913.

Gage.-Head is measured on iron pipe set about 3.0 feet back from weir crest; read once daily.

Control.-Permanent.

Discharge measurements.-Made by 4-foot sharp-crested weir with end contractions; check measurements made with current meter by wading. At times floods exceed the capacity of the diversion channel and are not measured. Waste water from this station is measured by station on the Nuuanu Stream below reservoir No. 4.

Diversions.-Aqueduct diverts seepage from reservoir No. 4, including inflow from Nuuanu Valley drainage below reservoir No. 4.

Cooperation.-Records furnished by waterworks department of city of Honolulu.

Monthly discharge of Nuuanu Stream at Luakaha weir, in Nuuanu Valley, near Honolulu, Oahu, for 1913 .

\begin{tabular}{|c|c|c|c|c|}
\hline \multirow{2}{*}{ Month. } & \multicolumn{3}{|c|}{ Discharge in second-feet. } & \multirow{2}{*}{$\begin{array}{l}\text { Run-off } \\
\text { (total in } \\
\text { acre-feet). }\end{array}$} \\
\hline & Maximum. & Minimum. & Mean. & \\
\hline 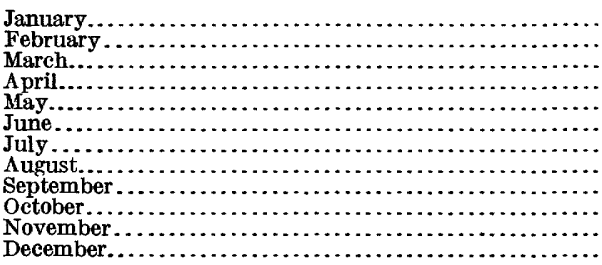 & \begin{tabular}{r|}
11.8 \\
4.71 \\
9.18 \\
9.53 \\
15.0 \\
15.2 \\
5.14 \\
4.85 \\
4.16 \\
2.19 \\
19.8 \\
20.2
\end{tabular} & $\begin{array}{l}2.76 \\
2.41 \\
1.47 \\
2.41 \\
2.41 \\
3.63 \\
2.88 \\
2.64 \\
1.47 \\
.70 \\
.56 \\
6.04\end{array}$ & $\begin{array}{l}4.62 \\
2.60 \\
\text { 3. } 07 \\
3.69 \\
4.96 \\
4.77 \\
3.79 \\
2.94 \\
2.24 \\
1.25 \\
5.60 \\
9.56\end{array}$ & $\begin{array}{r}284 \\
144 \\
189 \\
220 \\
305 \\
284 \\
233 \\
181 \\
133 \\
77 \\
333 \\
588\end{array}$ \\
\hline The year............ & 20.2 & .56 & 4.10 & 2,970 \\
\hline
\end{tabular}

NUUANU STREAM BELOW RESERVOIR NO. 2 WASTEWAY, NEAR HONOLULU, OAHU.

Location.-On Pali road in upper Nuuanu Valley, about 5 miles from Honolulu post office and 1 mile above end of car line.

Records available.-October 21 to December 31, 1913.

Gage.-Inclined staff read to hundredths.

Control.-Permanent.

Discharge measurements.-Low-water discharge measured by 2.0 -foot sharpcrested weir with end contractions. Flood discharge measured by 12.0-foot sharp-crested weir with end contractions, which, with small weir, gives total flood discharge. Both weirs set in concrete structure. Small weir crest is 1.0-foot below large weir crest in elevation.

Diversions.-Irrigation ditch diverts low-water discharge at point 300 feet below gage. Station measures waste water and seepage from reservoirs Nos. 2, 3, and 4 and the Luakaha weir. 
Daily discharge, in second-feet, of Nuuanu Stream below reservoir No. 2 wasteway, near Honolulu, Oahu, for 1918.

\begin{tabular}{|c|c|c|c|c|c|c|c|}
\hline Day. & Oct. & Nov. & Dec. & Day. & Oct. & Nov. & Dec. \\
\hline $\begin{array}{l}1 \\
1 \\
3 \\
4 \ldots \ldots \ldots \ldots \ldots \\
5 \ldots \ldots \ldots \ldots\end{array}$ & & $\begin{array}{l}0.21 \\
.21 \\
.21 \\
.21 \\
.21\end{array}$ & $\begin{array}{l}14.9 \\
45.6 \\
55.4 \\
44.2 \\
30.4\end{array}$ & $\begin{array}{l}16 \ldots \ldots \\
17 \ldots \ldots \\
19 \ldots \ldots\end{array}$ & 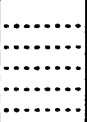 & $\begin{array}{r}3.34 \\
1.89 \\
1.83 \\
2.90 \\
27.1\end{array}$ & $\begin{array}{l}11.4 \\
10.6 \\
10.6 \\
10.6 \\
10.6\end{array}$ \\
\hline $\begin{array}{r}6 \\
7 \\
8 \ldots \ldots \\
9 \\
10 \ldots \ldots\end{array}$ & & $\begin{array}{r}.24 \\
.27 \\
1.08 \\
1.95 \\
1.77\end{array}$ & $\begin{array}{l}48.3 \\
36.5 \\
22.3 \\
18.9 \\
13.2\end{array}$ & $\begin{array}{l}21 \\
22 \\
24 \\
25\end{array}$ & $\begin{array}{r}0.30 \\
.30 \\
.30 \\
.30 \\
.27\end{array}$ & $\begin{array}{l}24.4 \\
17.1 \\
10.3 \\
12.1 \\
29.3\end{array}$ & $\begin{array}{l}9.97 \\
9.01 \\
7.85 \\
7.59 \\
7.35\end{array}$ \\
\hline $\begin{array}{l}11 \ldots \ldots \ldots \\
12 \ldots \ldots \ldots \\
14 \\
15\end{array}$ & $\begin{array}{l}\cdots \cdots \cdots \\
\cdots \cdots \cdots \\
\cdots \cdots \cdots \\
\cdots \cdots \\
\cdots\end{array}$ & $\begin{array}{l}5.05 \\
5.41 \\
6.34 \\
4.38 \\
3.12\end{array}$ & $\begin{array}{c}12.1 \\
10.6 \\
9.64 \\
14.5 \\
12.5\end{array}$ & $\begin{array}{l}26 \ldots \\
27 \ldots \ldots \\
28 \ldots \\
30 \\
31 \ldots \ldots\end{array}$ & $\begin{array}{l}.27 \\
.24 \\
.21 \\
.21 \\
.21 \\
.21\end{array}$ & $\begin{array}{l}12.1 \\
49.4 \\
24.4 \\
17.1 \\
14.1 \\
\end{array}$ & $\begin{array}{l}6.70 \\
6.51 \\
6.51 \\
6.13 \\
6.13 \\
6.13\end{array}$ \\
\hline
\end{tabular}

NoTE.-Mean for November, 9.27 second-feet; minimum for November, 0.21 second-feet. Mean for December, 16.9 second-feet; minimum for December, 6.13 second-feet.

\section{LULUMAHA ${ }^{1}$ DITCH AT UPPER NUUANU RESERVOIR, NEAR HONOLULU, OAHU.}

Location.-About 500 feet east of spillway of upper Nuuanu Valley reservoir, about

7 miles northeast of Honolulu post office.

Records available.-September 2, 1911, to September 30, 1913.

Gage.-Vertical staff.

Control.--Shifting.

Discharge measurements.-Made by wading.

Diversions.-Low-water discharge is diverted from Lulumaha Stream into reservoir

No. 4.

Accuracy.-Records poor.

Discharge measurements of Lulumaha ditch at upper Nuuanu reservoir, near Honolulu, Oahu, in 1913.

\begin{tabular}{|c|c|c|c|}
\hline Date. & Hydrographer. & $\begin{array}{c}\text { Gage } \\
\text { height. }\end{array}$ & $\begin{array}{c}\text { Dis- } \\
\text { charge. }\end{array}$ \\
\hline Apr. $\begin{array}{r}2 \\
14\end{array}$ & 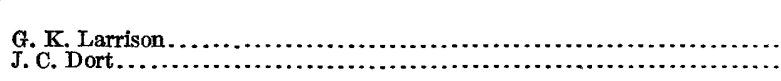 & $\begin{array}{l}\text { Feet. } \\
0.31 \\
.34\end{array}$ & $\begin{array}{r}\text { Sec. } f t . \\
0.58 \\
.77\end{array}$ \\
\hline
\end{tabular}

1 Incorrectly spelled "Lulumaho" in Water-Supply Paper 336. 
ISLAND OF OAHU.

Daily discharge, in second-feet, of Lulumaha ditch at upper Nuuanu reservoir, near Honolulu, Oahu, for 1913.

\begin{tabular}{|c|c|c|c|c|c|c|c|c|c|}
\hline Day. & Jan. & Feb. & Mar. & Apr. & May. & June. & July. & Aug. & Sept. \\
\hline $\begin{array}{l}1 \ldots \\
2 \ldots \\
3 \ldots \\
4 \ldots \\
5 \ldots\end{array}$ & $\begin{array}{r}0.6 \\
.5 \\
.5 \\
.5 \\
.3\end{array}$ & $\begin{array}{r}0.5 \\
.5 \\
.5 \\
.5 \\
.3\end{array}$ & $\begin{array}{r}0.5 \\
.5 \\
.5 \\
.5 \\
.5\end{array}$ & $\begin{array}{r}1.4 \\
.7 \\
1.2 \\
.7 \\
.5\end{array}$ & $\begin{array}{l}0.9 \\
1.9 \\
1.9 \\
1.2 \\
1.2\end{array}$ & $\begin{array}{r}1.2 \\
.9 \\
6.5 \\
.9 \\
.9\end{array}$ & $\begin{array}{l}3.8 \\
3.8 \\
1.9 \\
1.4 \\
1.4\end{array}$ & $\begin{array}{r}0.5 \\
.5 \\
.5 \\
.5 \\
.5\end{array}$ & $\begin{array}{l}3.8 \\
1.4 \\
1.4 \\
1.4\end{array}$ \\
\hline $\begin{array}{c}6 \ldots \\
7 \ldots \\
8 \ldots \\
9 \ldots \\
10 \ldots\end{array}$ & $\begin{array}{r}.3 \\
.3 \\
.9 \\
1.2 \\
2.9\end{array}$ & $\begin{array}{r}2.5 \\
1.4 \\
1.4 \\
.9 \\
.6\end{array}$ & $\begin{array}{l}.5 \\
.5 \\
.5 \\
.5 \\
.5\end{array}$ & $\begin{array}{l}.5 \\
.5 \\
.5 \\
.5 \\
.5\end{array}$ & $\begin{array}{l}.9 \\
.4 .4 \\
2.0 \\
3.1 \\
1.9\end{array}$ & $\begin{array}{r}.9 \\
.9 \\
.9 \\
1.4 \\
3.8\end{array}$ & $\begin{array}{r}1.4 \\
.9 \\
.9 \\
. .2 \\
.9\end{array}$ & $\begin{array}{l}.5 \\
.5 \\
.5 \\
.5 \\
.9\end{array}$ & $\begin{array}{r}1.4 \\
.9 \\
.9\end{array}$ \\
\hline ..... & $\begin{array}{l}8.0 \\
1.7 \\
1.6 \\
1.0 \\
1.2\end{array}$ & $\begin{array}{r}.5 \\
.5 \\
.5 \\
.5 \\
1.4\end{array}$ & $\begin{array}{r}.5 \\
.5 \\
18.5 \\
.9\end{array}$ & $\begin{array}{r}.5 \\
2.9 \\
5.8 \\
1.2 \\
3.8\end{array}$ & $\begin{array}{l}1.4 \\
1.4 \\
4.9 \\
1.2 \\
2.5\end{array}$ & $\begin{array}{r}1.4 \\
1.2 \\
1.2 \\
.9 \\
.9\end{array}$ & $\begin{array}{l}.9 \\
.9 \\
.6 \\
.6 \\
.6\end{array}$ & $\begin{array}{r}.9 \\
1.9 \\
1.4 \\
1.4 \\
1.9\end{array}$ & $\begin{array}{r}.9 \\
.9 \\
.9 \\
1.4 \\
.9\end{array}$ \\
\hline$\cdots$ & $\begin{array}{r}1.9 \\
1.4 \\
1.2 \\
1.0 \\
.8\end{array}$ & $\begin{array}{r}.9 \\
6.2 \\
1.4 \\
.9 \\
.5\end{array}$ & $\begin{array}{l}.6 \\
.5 \\
.5 \\
.5 \\
.5\end{array}$ & $\begin{array}{r}1.2 \\
1.4 \\
1.2 \\
.9 \\
.9\end{array}$ & $\begin{array}{l}1.4 \\
1.4 \\
1.2 \\
1.2 \\
1.2\end{array}$ & $\begin{array}{r}.9 \\
.9 \\
.9 \\
.9 \\
5.4\end{array}$ & $\begin{array}{l}.5 \\
.5 \\
.5 \\
.5 \\
.5\end{array}$ & $\begin{array}{l}1.4 \\
1.4 \\
1.4 \\
1.4 \\
1.4\end{array}$ & .6 \\
\hline $\begin{array}{l}21 \ldots \\
22 \ldots \\
23 \ldots \\
24 \ldots \\
25 \ldots\end{array}$ & $\begin{array}{l}.8 \\
.6 \\
.6 \\
.6 \\
.5\end{array}$ & $\begin{array}{r}.5 \\
.5 \\
.5 \\
2.5 \\
.5\end{array}$ & $\begin{array}{l}1.2 \\
1.2 \\
5.1 \\
1.6 \\
1.0\end{array}$ & $\begin{array}{r}.9 \\
.9 \\
1.1 \\
.9 \\
2.0\end{array}$ & $\begin{array}{r}1.2 \\
1.2 \\
.9 \\
.9 \\
.9\end{array}$ & $\begin{array}{r}1.2 \\
1.2 \\
1.2 \\
.9 \\
.9\end{array}$ & $\begin{array}{l}.5 \\
.5 \\
.5 \\
.5 \\
.9\end{array}$ & $\begin{array}{l}1.4 \\
1.4 \\
1.4 \\
1.4 \\
1.4\end{array}$ & $\begin{array}{l}.5 \\
.5 \\
5\end{array}$ \\
\hline $\begin{array}{l}26 \ldots \ldots \\
27 \ldots \ldots \\
28 \ldots \\
29 \ldots \\
30 \ldots \\
31 \ldots \\
\end{array}$ & $\begin{array}{l}.5 \\
.5 \\
.5 \\
.5 \\
.5 \\
.5\end{array}$ & $\begin{array}{l}.5 \\
.5 \\
.5\end{array}$ & $\begin{array}{r}1.0 \\
.9 \\
.5 \\
.5 \\
.5 \\
3.8\end{array}$ & $\begin{array}{l}1.4 \\
1.2 \\
1.4 \\
1.2 \\
.9\end{array}$ & $\begin{array}{l}.9 \\
1.2 \\
1.2 \\
1.4 \\
1.2 \\
1.2\end{array}$ & $\begin{array}{r}.9 \\
1.2 \\
1.4 \\
1.4 \\
3.8\end{array}$ & $\begin{array}{l}.9 \\
.6 \\
.6 \\
.9 \\
.5\end{array}$ & $\begin{array}{l}1.4 \\
1.9 \\
6.5 \\
1.4 \\
1.4 \\
1.4\end{array}$ & $\begin{array}{r}.5 \\
.5 \\
.5 \\
.5 \\
.5\end{array}$ \\
\hline
\end{tabular}

Monthly discharge of Lulumaha ditch at upper Nuuanu reservoir, near Honolulu, Oahu, for 1913 .

\begin{tabular}{|c|c|c|c|c|c|}
\hline \multirow{2}{*}{. Month. } & \multicolumn{3}{|c|}{ Discharge in second-feet. } & \multirow{2}{*}{$\begin{array}{l}\text { Run-off } \\
\text { (total in } \\
\text { acre-feet). }\end{array}$} & \multirow{2}{*}{$\begin{array}{l}\text { Accu- } \\
\text { racy. }\end{array}$} \\
\hline & Maximum. & Minimuin. & Mean. & & \\
\hline \multirow[t]{2}{*}{ 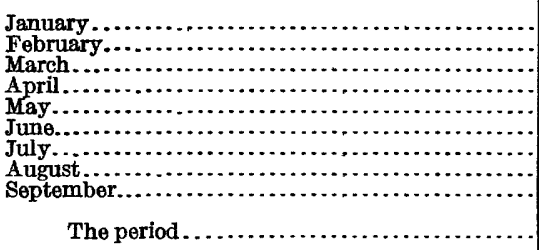 } & $\begin{array}{r}8.0 \\
6.2 \\
18.5 \\
5.8 \\
5.4 \\
6.5 \\
3.8 \\
6.5 \\
3.8\end{array}$ & $\begin{array}{r}0.3 \\
.3 \\
.5 \\
.5 \\
.9 \\
.9 \\
.5 \\
.5 \\
.5\end{array}$ & $\begin{array}{r}1.09 \\
1.01 \\
1.48 \\
1.10 \\
1.63 \\
1.57 \\
.99 \\
1.32 \\
.95\end{array}$ & $\begin{array}{r}67.0 \\
56.1 \\
91.0 \\
65.5 \\
100 \\
93.4 \\
60.9 \\
81.2 \\
56.5\end{array}$ & \multirow[t]{2}{*}{$\begin{array}{l}\text { D. } \\
\text { D. } \\
\text { D. } \\
\text { D. } \\
\text { D. } \\
\text { D. } \\
\text { D. } \\
\text { D. } \\
\text { D. }\end{array}$} \\
\hline & & & & 672 & \\
\hline
\end{tabular}




\section{PaUOA stream at UPPER PaUoa VAlley, Near honolulu, oAhu.}

Location.-In upper Pauoa Valley, 1,000 feet below Kahuawai Spring, about 1 mile above Punchbowl, and about 2.5 miles northeast of Honolulu post office.

Records available.-April 14, 1911, to December 31, 1913.

Gage.-Watson weekly water-stage recorder October 17, 1911, to December 31, 1913; staff gage, graduated to tenths and read to hundredths, April 14 to October 16, 1911.

Control.-Permanent.

Discharge measurements.-April 14 to October 16, 1911, current-meter measure. ments made by wading; October 17, 1911, to December 31, 1913, by 4-foot sharpcrested weir that has been checked with current-meter measurements.

Diversions.-Several small ditches and one pipe line divert part of low-water discharge.

Regulation.-Stream is fed by several perennial springs within 2 miles above station.

Discharge measurements of Pauoa Stream at upper Pauoa Valley, near Honolulu, Oahu, in 1913 .

\begin{tabular}{|c|c|c|c|}
\hline Date. & Hydrographer. & $\begin{array}{c}\text { Gage } \\
\text { height. }\end{array}$ & $\begin{array}{c}\text { Dis- } \\
\text { charge. }\end{array}$ \\
\hline $\begin{array}{l}\text { Mar. } 4 \\
\text { Sept. } 11 \\
\text { Oct. } \quad 2\end{array}$ & 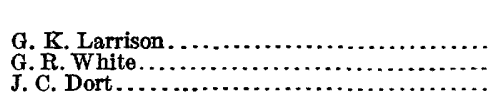 & $\begin{array}{l}\text { Feet. } \\
0.16 \\
.15 \\
.11\end{array}$ & $\begin{array}{r}\text { Sec. ft. } \\
0.91 \\
.54 \\
.48\end{array}$ \\
\hline
\end{tabular}

Daily discharge, in second-feet, of Pauoa Stream at upper Pauoa Valley, near Honolulu, Oahu, for 1913.

\begin{tabular}{|c|c|c|c|c|c|c|c|c|c|c|c|c|}
\hline Day. & Jan. & Feb. & Mar. & Apr. & May. & June. & July. & Aug. & Sept. & Oct. & Nor. & Dee. \\
\hline $\begin{array}{l}1 \ldots \\
2 . . \\
3 . .\end{array}$ & $\begin{array}{l}2.19 \\
2.19 \\
2.08 \\
1.97 \\
1.97\end{array}$ & $\begin{array}{l}1.47 \\
1.37 \\
1.37 \\
1.28 \\
1.28\end{array}$ & $\begin{array}{r}0.93 \\
1.02 \\
.93 \\
.85 \\
.85\end{array}$ & $\begin{array}{l}2.41 \\
1.77 \\
1.97 \\
2.08 \\
1.10\end{array}$ & $\begin{array}{l}8.31 \\
3.50 \\
2.41 \\
5.29 \\
7.64\end{array}$ & $\begin{array}{l}1.77 \\
1.77 \\
1.66 \\
5.14 \\
5.58\end{array}$ & $\begin{array}{l}4.16 \\
6.56 \\
3.50 \\
2.41 \\
3.12\end{array}$ & $\begin{array}{r}0.70 \\
.70 \\
.62 \\
.85 \\
1.02\end{array}$ & $\begin{array}{r}0.89 \\
.90 \\
.91 \\
.92 \\
.93\end{array}$ & $\begin{array}{r}0.55 \\
.55 \\
.55 \\
.55 \\
.55\end{array}$ & $\begin{array}{r}0.55 \\
.55 \\
.55 \\
.55 \\
.55\end{array}$ & $\begin{array}{c}2.88 \\
2.76 \\
12.5 \\
8.14 \\
6.66\end{array}$ \\
\hline $\begin{array}{r}6 \ldots \\
7 \ldots \\
8 \ldots \\
9 \ldots \\
10 \ldots\end{array}$ & $\begin{array}{l}1.97 \\
1.87 \\
1.87 \\
4.85 \\
3.37\end{array}$ & $\begin{array}{l}1.28 \\
1.37 \\
1.37 \\
1.37 \\
1.37\end{array}$ & $\begin{array}{l}.85 \\
.77 \\
.77 \\
.77 \\
.77\end{array}$ & $\begin{array}{r}1.10 \\
1.02 \\
1.02 \\
.93 \\
.85\end{array}$ & $\begin{array}{l}4.57 \\
3.00 \\
3.24 \\
3.63 \\
3.00\end{array}$ & $\begin{array}{l}2.64 \\
2.53 \\
2.30 \\
2.08 \\
3.24\end{array}$ & $\begin{array}{l}1.97 \\
1.57 \\
1.57 \\
1.77 \\
1.57\end{array}$ & $\begin{array}{r}1.02 \\
1.02 \\
1.02 \\
.93 \\
.93\end{array}$ & $\begin{array}{l}.93 \\
.85 \\
.85 \\
.77 \\
.77\end{array}$ & $\begin{array}{l}.55 \\
.55 \\
.55 \\
.55 \\
.55\end{array}$ & $\begin{array}{l}.94 \\
1.32 \\
1.70 \\
2.09 \\
2.48\end{array}$ & $\begin{array}{l}6.98 \\
6.19 \\
5.29 \\
5.29 \\
5.14\end{array}$ \\
\hline $\begin{array}{l}11 \ldots \ldots \\
12 \ldots \ldots \\
13 \ldots \ldots \\
14 \ldots \ldots \\
15 \ldots .\end{array}$ & $\begin{array}{l}5.43 \\
2.53 \\
4.43 \\
3.50 \\
3.00\end{array}$ & $\begin{array}{l}1.37 \\
1.37 \\
1.37 \\
1.37 \\
1.28\end{array}$ & $\begin{array}{r}.70 \\
.62 \\
.62 \\
2.30 \\
1.02\end{array}$ & $\begin{array}{c}.85 \\
3.24 \\
14.1 \\
3.50 \\
2.41\end{array}$ & $\begin{array}{l}2.53 \\
2.19 \\
2.08 \\
2.30 \\
2.19\end{array}$ & $\begin{array}{l}4.16 \\
3.37 \\
2.41 \\
2.19 \\
1.97\end{array}$ & $\begin{array}{l}1.57 \\
1.47 \\
1.47 \\
1.37 \\
1.37\end{array}$ & $\begin{array}{l}.85 \\
.93 \\
.93 \\
.93 \\
.85\end{array}$ & $\begin{array}{l}.77 \\
.77 \\
.77 \\
.85 \\
.85\end{array}$ & $\begin{array}{l}.55 \\
.62 \\
.70 \\
.62 \\
.55\end{array}$ & $\begin{array}{l}2.86 \\
3.24 \\
3.63 \\
2.08 \\
1.47\end{array}$ & $\begin{array}{l}4.57 \\
4.29 \\
4.29 \\
4.29 \\
4.29\end{array}$ \\
\hline $\begin{array}{l}16 \ldots \\
17 \ldots \\
18 . . \\
19 . . \\
20 . .\end{array}$ & $\begin{array}{l}2.64 \\
2.30 \\
2.08 \\
1.97 \\
1.87\end{array}$ & $\begin{array}{l}1.19 \\
1.10 \\
1.10 \\
1.10 \\
1.10\end{array}$ & $\begin{array}{l}.85 \\
.93 \\
.85 \\
.77 \\
.77\end{array}$ & $\begin{array}{l}1.77 \\
3.89 \\
3.76 \\
2.76 \\
2.19\end{array}$ & $\begin{array}{l}7.47 \\
5.43 \\
2.76 \\
2.19 \\
1.97\end{array}$ & $\begin{array}{l}1.97 \\
1.77 \\
1.77 \\
1.77 \\
4.16\end{array}$ & $\begin{array}{l}1.37 \\
1.37 \\
1.37 \\
1.37 \\
1.37\end{array}$ & $\begin{array}{l}.85 \\
.77 \\
.77 \\
.77 \\
.77\end{array}$ & $\begin{array}{r}.77 \\
.77 \\
.85 \\
1.10 \\
.77\end{array}$ & $\begin{array}{l}.55 \\
.55 \\
.55 \\
.55 \\
.55\end{array}$ & $\begin{array}{l}1.19 \\
1.02 \\
2.08 \\
3.24 \\
6.82\end{array}$ & $\begin{array}{l}4.16 \\
4.16 \\
3.76 \\
3.76 \\
3.76\end{array}$ \\
\hline $\begin{array}{l}21 . . \\
22 . \\
23 . \\
24 . \\
25 .\end{array}$ & $\begin{array}{l}1.77 \\
1.77 \\
1.66 \\
1.66 \\
1.66\end{array}$ & $\begin{array}{l}1.02 \\
1.02 \\
1.02 \\
.03 \\
1.02\end{array}$ & $\begin{array}{r}.93 \\
1.19 \\
1.10 \\
1.02 \\
.93\end{array}$ & $\begin{array}{l}1.66 \\
1.47 \\
2.19 \\
3.63 \\
8.48\end{array}$ & $\begin{array}{l}2.08 \\
2.19 \\
2.08 \\
1.87 \\
1.77\end{array}$ & $\begin{array}{l}2.19 \\
1.77 \\
1.57 \\
1.57 \\
1.47\end{array}$ & $\begin{array}{l}1.37 \\
1.19 \\
1.37 \\
1.77 \\
1.37\end{array}$ & $\begin{array}{l}.77 \\
.85 \\
.85 \\
.77 \\
.77\end{array}$ & $\begin{array}{l}.77 \\
.70 \\
.70 \\
.70 \\
.70\end{array}$ & $\begin{array}{l}.55 \\
.55 \\
.55 \\
.55 \\
.55\end{array}$ & $\begin{array}{r}3.76 \\
1.97 \\
1.66 \\
3.76 \\
16.2\end{array}$ & $\begin{array}{l}3.63 \\
3.63 \\
3.54 \\
3.46 \\
3.37\end{array}$ \\
\hline $\begin{array}{l}26 \ldots \ldots \\
27 . \ldots \\
28 \ldots \ldots \\
29 \ldots . . \\
30 \ldots \\
31 \ldots .\end{array}$ & $\begin{array}{l}1.66 \\
1.57 \\
1.57 \\
1.57 \\
1.57 \\
1.47\end{array}$ & $\begin{array}{l}1.02 \\
1.02 \\
1.02\end{array}$ & $\begin{array}{r}.85 \\
.93 \\
.93 \\
1.02 \\
.93 \\
3.63\end{array}$ & $\begin{array}{l}2.41 \\
1.87 \\
3.63 \\
4.29 \\
2.19\end{array}$ & $\begin{array}{l}1.97 \\
1.97 \\
1.97 \\
1.97 \\
1.97 \\
1.87\end{array}$ & $\begin{array}{l}1.47 \\
1.47 \\
3.37 \\
1.97 \\
2.64\end{array}$ & $\begin{array}{l}1.19 \\
1.02 \\
1.37 \\
1.28 \\
1.28 \\
.70\end{array}$ & $\begin{array}{l}.85 \\
.85 \\
.85 \\
.86 \\
.88\end{array}$ & $\begin{array}{l}.62 \\
.62 \\
.55 \\
.62 \\
.62\end{array}$ & $\begin{array}{l}.55 \\
.55 \\
.55 \\
.55 \\
.55 \\
.55\end{array}$ & $\begin{array}{c}12.9 \\
5.58 \\
3.37 \\
2.76 \\
3.24\end{array}$ & $\begin{array}{l}3.24 \\
3.00 \\
2.88 \\
2.88 \\
2.76 \\
2.64\end{array}$ \\
\hline
\end{tabular}


Monthly discharge of Pauoa Stream at upper Pawoa Valley, near Honolulu, Oahu, for 1913.

\begin{tabular}{|c|c|c|c|c|c|}
\hline \multirow{2}{*}{ Month. } & \multicolumn{3}{|c|}{ Discharge in second-feet. } & \multirow{2}{*}{$\begin{array}{l}\text { Run-off } \\
\text { (total in } \\
\text { acre-feet). }\end{array}$} & \multirow{2}{*}{$\begin{array}{l}\text { Accu- } \\
\text { racy. }\end{array}$} \\
\hline & Maximum. & Minimum. & Mean. & & \\
\hline 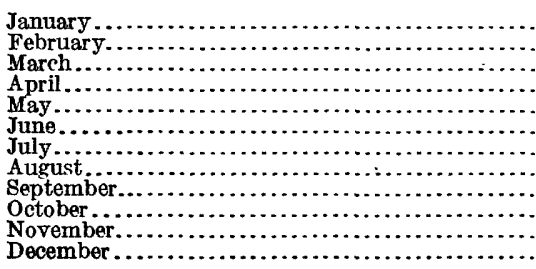 & $\begin{array}{l}5.43 \\
1.47 \\
3.63 \\
14.1 \\
8.31 \\
5.58 \\
6.56 \\
1.02 \\
1.1 \\
16.7 \\
12.5\end{array}$ & $\begin{array}{r}1.47 \\
.93 \\
.62 \\
.85 \\
1.77 \\
1.47 \\
.7 \\
.62 \\
.55 \\
.55 \\
.55 \\
2.64\end{array}$ & $\begin{array}{r}\text { 2. } 32 \\
1.21 \\
1.01 \\
2.82 \\
\text { 3. } 14 \\
2.44 \\
1.81 \\
.85 \\
.79 \\
.56 \\
3.14 \\
4.46\end{array}$ & $\begin{array}{c}143 \\
67.2 \\
62 \\
168 \\
193 \\
146 \\
111 \\
52.3 \\
47 \\
34.4 \\
187 \\
274\end{array}$ & $\begin{array}{l}\text { A. } \\
\text { A. } \\
\text { A. } \\
\text { A. } \\
\text { A. } \\
\text { A. } \\
\text { A. } \\
\text { A. } \\
\text { A. } \\
\text { D. } \\
\text { C. } \\
\text { A. }\end{array}$ \\
\hline 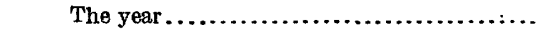 & 16. 2 & .55 & 2.05 & 1,480 & \\
\hline
\end{tabular}

\section{KAHUAWAI SPRING AT UPPER PAUOA VALLEY, NEAR HONOLULU, OAHU.}

Location.-In upper Pauoa Valley, one-fourth mile above Pauoa Stream weir station, about $1 \frac{1}{4}$ miles above Punchbowl, and about $2 \frac{3}{4}$ miles northeast of Honolulu post office.

Records available.-September 23, 1912, to December 31, 1913.

Gage.-A 2 by 2 inch stake driven into pool of spring, with nail in top, 6 feet above weir, to measure head on weir. Head is read twice daily to hundredths.

Control.-Spring boils up vertically in pool about 10 feet in diameter. Weir is built between rock abutments on south side of pool about 10 feet from center.

Discharge.-Computed by Francis formula for sharp-crested weirs with end contractions. Weir has 1.5-foot crest length.

Monthly discharge of Kahuawai Spring at upper Pauoa Valley, near Honolulu, Oahu, for 1913.

\begin{tabular}{|c|c|c|c|c|c|}
\hline \multirow{2}{*}{ Month. } & \multicolumn{3}{|c|}{ Discharge in second-feet. } & \multirow{2}{*}{$\begin{array}{l}\text { Run-off } \\
\text { (total in } \\
\text { acre-feet). }\end{array}$} & \multirow{2}{*}{$\begin{array}{l}\text { Accu- } \\
\text { racy. }\end{array}$} \\
\hline & Maximum. & Minimum. & Mean. & & \\
\hline 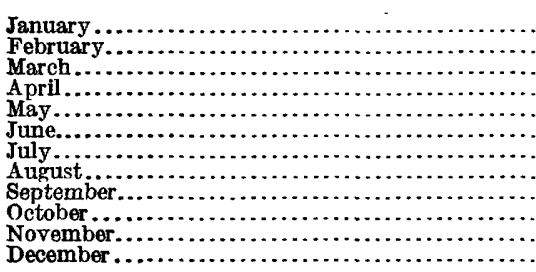 & $\begin{array}{r}0.54 \\
.50 \\
.50 \\
.50 \\
.54 \\
.54 \\
.54 \\
.50 \\
.50 \\
.50 \\
.54 \\
.54\end{array}$ & $\begin{array}{r}0.50 \\
.47 \\
.47 \\
.50 \\
.50 \\
.50 \\
.50 \\
.50 \\
.50 \\
.50 \\
.50 \\
.54\end{array}$ & $\begin{array}{l}0.50 \\
.50 \\
.48 \\
.50 \\
.51 \\
.52 \\
.50 \\
.50 \\
.50 \\
.50 \\
.53 \\
.54\end{array}$ & $\begin{array}{r}30.7 \\
27.8 \\
29.5 \\
29.8 \\
31.4 \\
30.9 \\
30.7 \\
30.7 \\
29.8 \\
30.7 \\
31.5 \\
33.2\end{array}$ & $\begin{array}{l}\text { A. } \\
\text { A. } \\
\text { A. } \\
\text { A. } \\
\text { A. } \\
\text { A. } \\
\text { A. } \\
\text { A. } \\
\text { A. } \\
\text { A. } \\
\text { A. } \\
\text { A. }\end{array}$ \\
\hline The year........ & .54 & .47 & .51 & 367 & \\
\hline
\end{tabular}


MANOA STREAM AT UPPER MANOA VALLEY, NEAR HONOLULU, OAHU.

Location.-Below confluence of two main branches in upper Manoa Valley, about 5 miles east of Honolulu post office.

Records available.-October 6, 1910, to July 5, 1913.

Gage.--Vertical staff.

Control.-Shifting.

Discharge measurements.-Made by wading for low and medium stages up to a gage height of about 4.0 feet; above this stage from footbridge at gage.

Diversions.-Several small irrigation ditches divert a part of the low-water discharge from the branches of the stream above the station. Most of this water returns to the stream by seepage and drains above the station.

Accuracy.-Records poor.

Discharge measurements of Manoa Stream at upper Manoa Valley, near Honolulu, Oahu, in 1913.

\begin{tabular}{|c|c|c|c|c|c|c|c|}
\hline Date. & Hyđrographer. & $\begin{array}{c}\text { Gage } \\
\text { height. }\end{array}$ & $\begin{array}{c}\text { Dis- } \\
\text { charge. }\end{array}$ & Date. & Hydrographer. & $\begin{array}{c}\text { Gage } \\
\text { height. }\end{array}$ & $\begin{array}{l}\text { Dis- } \\
\text { charge. }\end{array}$ \\
\hline $\begin{array}{l}\text { Feb. } 28 \\
\text { Mar. } 21\end{array}$ & 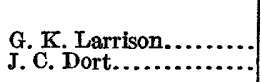 & $\begin{array}{c}\text { Feet. } \\
\mathbf{2 . 9 3} \\
\mathbf{3 . 3 6}\end{array}$ & $\begin{array}{r}\text { Sec.ft. } \\
1.08 \\
6.06\end{array}$ & Apr. 14 & J. C. Dort....... & $\begin{array}{c}\text { Feet. } \\
\mathbf{3 . 7 0}\end{array}$ & $\begin{array}{l}\text { Sec. } f t . \\
18.5\end{array}$ \\
\hline
\end{tabular}

Monthly discharge of Manoa Stream at upper Manoa Valley, near Honolulu, Oahu, for 1913.

\begin{tabular}{|c|c|c|c|c|c|}
\hline \multirow{2}{*}{ Month. } & \multicolumn{3}{|c|}{ Discharge in second-feet. } & \multirow{2}{*}{$\begin{array}{l}\text { Run-off } \\
\text { (total in } \\
\text { acre-feet). }\end{array}$} & \multirow{2}{*}{$\begin{array}{l}\text { Accu- } \\
\text { racy. }\end{array}$} \\
\hline & Maximum. & Minimum. & Mean. & & \\
\hline 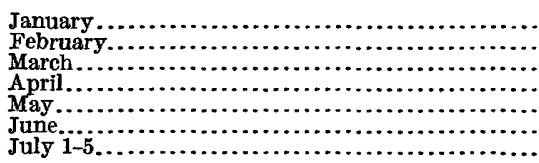 & $\begin{array}{l}\mathbf{5 . 1} \\
12.2 \\
15 \\
33 \\
39 \\
38 \\
13\end{array}$ & $\begin{array}{r}1.2 \\
1.1 \\
1.1 \\
2.5 \\
7.9 \\
7.2 \\
10\end{array}$ & $\begin{array}{l}2.43 \\
2.70 \\
3.40 \\
11.5 \\
13.6 \\
12.2 \\
11.6\end{array}$ & $\begin{array}{l}149 \\
150 \\
209 \\
684 \\
836 \\
726 \\
115\end{array}$ & $\begin{array}{l}\text { D. } \\
\text { D. } \\
D . \\
D \\
\text { D. } \\
D \\
D \\
D\end{array}$ \\
\hline The period. & & & & 2,870 & \\
\hline
\end{tabular}

NoTE.-Two stations were established on the east and west branches of Manoa Stream May 29, 1913. These stations are above all diversions and are intended to supplant the old station, which was discontinued July $5,1913$.

\section{MANOA STREAM AT COLLEGE OF HAWAII, NEAR HONOLULU, OAHU.}

Location.-In gorge about one-half mile southeast of College of Hawaii, and 3 miles east of Honolulu post office.

Records available.-Daily discharge from March 23, 1909, to November 24, 1910;

November 1, 1912, to April 26, 1913; September 10 to December 31, 1913.

Gage.-Vertical staff.

Control.-Probably permanent.

Discharge measurements.-Made by wading.

Diversions.-Several irrigation ditches above station.

Accuracy.-Records poor.

Cooperation.-Maintained in cooperation with the College of Hawaii. 
ISLAND OF OAHU.

Discharge measurements of Manoa Stream at College of Hawaï, near Honolulu, Oahu, in 1913.

\begin{tabular}{|c|c|c|c|c|c|c|c|}
\hline Date. & Hydrographer. & $\begin{array}{c}\text { Gage } \\
\text { height. }\end{array}$ & $\begin{array}{c}\text { Dis- } \\
\text { charge. }\end{array}$ & Date. & Hydrographer. & $\begin{array}{c}\text { Gage } \\
\text { height. }\end{array}$ & $\begin{array}{c}\text { Dis- } \\
\text { charge. }\end{array}$ \\
\hline $\begin{array}{l}\text { Feb. } 28 \\
\text { Mar. } 21\end{array}$ & $\begin{array}{l}\text { G. K. Larrison.. } \\
\text { J. C. Dort............ }\end{array}$ & $\begin{array}{r}\text { Feet. } \\
0.95 \\
2.09\end{array}$ & $\begin{array}{r}\text { Sec. } f t . \\
0.99 \\
26.0\end{array}$ & Apr. $\begin{array}{l}14 \\
17\end{array}$ & $\begin{array}{l}\text { J. C. Dort...... } \\
\text { Dort and white. }\end{array}$ & $\begin{array}{r}\text { Feet. } \\
2.40 \\
2.64\end{array}$ & $\begin{array}{r}\text { Sec. } f t . \\
39.4 \\
56.7\end{array}$ \\
\hline
\end{tabular}

Daily discharge, in second-feet, of Manoa Stream at College of Hawaii, near Honolulu, Oahu, for 1912-13.

\begin{tabular}{|c|c|c|c|c|c|c|c|c|c|c|c|c|}
\hline Day. & Nov. & Dec. & \multicolumn{3}{|c|}{ Day. } & Nov. & Dec. & \multicolumn{3}{|c|}{ Day. } & Nov. & Dec. \\
\hline $\begin{array}{c}1912 . \\
1 \ldots \ldots \ldots \ldots \ldots \\
2 \ldots \ldots \ldots \ldots \ldots \ldots \\
4 \ldots \ldots \ldots \ldots \ldots \ldots \\
5 \ldots \ldots \ldots \ldots \ldots . . .\end{array}$ & $\begin{array}{l}2.6 \\
2.0 \\
3.0 \\
1.1 \\
\text { 3. } 9\end{array}$ & $\begin{array}{r}35 \\
38 \\
169 \\
29 \\
22\end{array}$ & & \multicolumn{2}{|c|}{$\mid$\begin{tabular}{|l}
11 \\
$12 \ldots \ldots \ldots \ldots \ldots \ldots . .$.
\end{tabular}} & $\begin{array}{l}4.8 \\
5.5 \\
2.0 \\
1.2 \\
6.2\end{array}$ & $\begin{array}{l}4.0 \\
2.0 \\
3.5 \\
3.5 \\
2.0\end{array}$ & \multicolumn{3}{|c|}{ 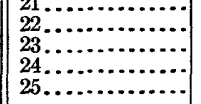 } & $\begin{array}{l}33 \\
35 \\
38 \\
18 \\
11\end{array}$ & $\begin{array}{r}20 \\
106 \\
33 \\
155 \\
20\end{array}$ \\
\hline $\begin{array}{r}6 \ldots \ldots \ldots . \\
7 \ldots \ldots \ldots . \\
7 \ldots \ldots \ldots . \\
90 \ldots \ldots \ldots \\
10 \ldots \ldots . .\end{array}$ & $\begin{array}{r}2.0 \\
7.0 \\
3.0 \\
29 \\
3.8\end{array}$ & $\begin{array}{r}19 \\
17 \\
11 \\
7 \\
7\end{array}$ & \begin{tabular}{l||l}
16 \\
17 \\
18 \\
.0 \\
19 \\
0 & 20
\end{tabular} & $\begin{array}{l}\ldots \ldots . . . \\
\ldots \ldots . . . \\
\ldots \ldots . . . \\
\ldots \ldots . . .\end{array}$ & ......... & $\begin{array}{l}2.5 \\
2.0 \\
2.2 \\
2.3 \\
2.0\end{array}$ & $\begin{array}{r}1 . \\
1 . \\
31 \\
6 .\end{array}$ & \begin{tabular}{l|l}
8 & 26. \\
27 \\
8 \\
88. \\
29 \\
70 \\
31.
\end{tabular} & $\begin{array}{l}\ldots \ldots . . \\
\ldots \ldots . . . \\
\ldots \ldots . . .\end{array}$ & $\begin{array}{l}\ldots \\
\ldots . \\
\cdots \\
\cdots\end{array}$ & $\begin{array}{c}7.0 \\
7.0 \\
62 \\
33 \\
14\end{array}$ & $\begin{array}{l}45 \\
29 \\
20 \\
7.0 \\
7.0 \\
7.0\end{array}$ \\
\hline Day. & Jan. & Feb. & Mar. & Apr. & May. & June. & July. & Aug. & Sept. & Oct. & Nov. & Dec. \\
\hline 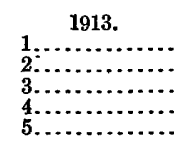 & $\begin{array}{l}7.0 \\
7.0 \\
3.2 \\
2.0 \\
2.0\end{array}$ & $\begin{array}{l}0.9 \\
1.2 \\
1.4 \\
1.3 \\
1.0\end{array}$ & $\begin{array}{r}1.0 \\
.9 \\
.9 \\
1.2 \\
1.4\end{array}$ & $\begin{array}{l}5.8 \\
6.4 \\
4.3 \\
3.1 \\
2.4\end{array}$ & & & & & {$\left[\begin{array}{l}{[\cdots \cdots} \\
\hdashline \cdots \cdots \cdot \\
\hdashline \cdots \cdots \cdots\end{array}\right.$} & $\begin{array}{r}0.5 \\
.5 \\
.5 \\
.5 \\
.5\end{array}$ & $\begin{array}{r}0.6 \\
1.5 \\
2.5 \\
1.0 \\
.8\end{array}$ & $\begin{array}{c}11 \\
8.0 \\
44 \\
102 \\
41\end{array}$ \\
\hline $\begin{array}{r}6 \ldots \ldots . \\
7 \ldots \ldots . \\
8 \ldots \ldots \\
90 \ldots \\
10 \ldots \ldots\end{array}$ & $\begin{array}{l}2.0 \\
2.0 \\
2.0 \\
4.0 \\
7.0\end{array}$ & $\begin{array}{l}1.9 \\
6.7 \\
6.1 \\
2.6 \\
1.8\end{array}$ & $\begin{array}{l}1.2 \\
1.2 \\
1.1 \\
1.2 \\
1.3\end{array}$ & $\begin{array}{r}1.9 \\
1.9 \\
2.6 \\
2.5 \\
20\end{array}$ & & & & & \begin{tabular}{c}
$\cdots$ \\
$\cdots . .$. \\
$\cdots 0.6$ \\
\hdashline 0.6
\end{tabular} & $\begin{array}{l}.5 \\
.5 \\
.5 \\
.5 \\
.5\end{array}$ & $\begin{array}{l}.9 \\
1.0 \\
15 \\
31 \\
32\end{array}$ & $\begin{array}{l}44 \\
65 \\
28 \\
18 \\
12\end{array}$ \\
\hline $\begin{array}{l}11 \ldots \ldots . \\
12 \ldots \ldots \\
13 \ldots \ldots \\
15 \ldots \ldots \\
15 \ldots \ldots\end{array}$ & $\begin{array}{l}10 \\
6.0 \\
14 \\
18 \\
12\end{array}$ & $\begin{array}{r}1.6 \\
1.2 \\
1.0 \\
.9 \\
.9\end{array}$ & $\begin{array}{l}1.2 \\
1.2 \\
1.2 \\
6.4 \\
8.0\end{array}$ & $\begin{array}{l}13 \\
21 \\
57 \\
37 \\
48\end{array}$ & & & & & $\begin{array}{r}.5 \\
.5 \\
.6 \\
1.2 \\
1.0\end{array}$ & $\begin{array}{l}.5 \\
.4 \\
.4 \\
.7 \\
.6\end{array}$ & $\begin{array}{c}36 \\
31 \\
27 \\
12 \\
9.4\end{array}$ & $\begin{array}{l}9.4 \\
7.8 \\
7.0 \\
8.0 \\
6.4\end{array}$ \\
\hline $\begin{array}{l}16 \ldots \ldots \\
17 \ldots \ldots \\
18 \ldots \ldots \\
19 \ldots \ldots \\
20 \ldots . .\end{array}$ & $\begin{array}{l}6.4 \\
4.6 \\
3.6 \\
3.4 \\
2.8\end{array}$ & $\begin{array}{r}.9 \\
1.0 \\
9.0 \\
11 \\
3.2\end{array}$ & $\begin{array}{l}6.4 \\
3.4 \\
2.6 \\
1.8 \\
4.4\end{array}$ & $\begin{array}{l}64 \\
33 \\
24 \\
24 \\
19\end{array}$ & & & & & $\begin{array}{r}.7 \\
.7 \\
.7 \\
4.9 \\
1.1\end{array}$ & $\begin{array}{l}.6 \\
.6 \\
.5 \\
.5 \\
.5\end{array}$ & $\begin{array}{l}3.8 \\
4.6 \\
6.7 \\
23 \\
61\end{array}$ & $\begin{array}{l}4.8 \\
4.6 \\
5.8 \\
3.9 \\
3.1\end{array}$ \\
\hline $\begin{array}{l}21 \ldots \ldots \\
22 \ldots \ldots \\
23 \ldots \\
24 \ldots \\
25 \ldots\end{array}$ & $\begin{array}{l}6.1 \\
4.8 \\
4.9 \\
4.3 \\
3.0\end{array}$ & $\begin{array}{l}1.2 \\
1.0 \\
1.8 \\
2.7 \\
3.2\end{array}$ & $\begin{array}{r}3.6 \\
10 \\
4.6 \\
3.4 \\
3.1\end{array}$ & $\begin{array}{c}13 \\
9.8 \\
18 \\
8.4 \\
67\end{array}$ & & 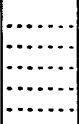 & & & $\begin{array}{l}.8 \\
.8 \\
.8 \\
.7 \\
.7\end{array}$ & $\begin{array}{l}.5 \\
.5 \\
.5 \\
.5 \\
.5\end{array}$ & $\begin{array}{l}31 \\
9.8 \\
9.4 \\
32 \\
75\end{array}$ & $\begin{array}{l}3.1 \\
2.8 \\
1.9 \\
1.4 \\
1.0\end{array}$ \\
\hline $\begin{array}{l}26 \ldots . . \\
27 \ldots \\
28 . \ldots \\
29 . \ldots \\
30 \ldots \\
31 \ldots\end{array}$ & $\begin{array}{l}2.6 \\
2.0 \\
2.0 \\
1.9 \\
.9 \\
2.0\end{array}$ & $\begin{array}{l}2.4 \\
1.6 \\
1.0\end{array}$ & $\begin{array}{l}3.3 \\
2.2 \\
2.8 \\
3.7 \\
15 \\
11\end{array}$ & $\begin{array}{l}18 \\
\cdots \cdots \\
\cdots \cdots\end{array}$ & $\mid \begin{array}{l}\ldots \ldots . \\
\cdots \cdots . . \\
\cdots \cdots \cdots\end{array}$ & & & & $\begin{array}{l}.6 \\
.6 \\
.5 \\
.5 \\
.5\end{array}$ & $\begin{array}{l}.5 \\
.5 \\
.6 \\
.6 \\
.6 \\
.6\end{array}$ & $\begin{array}{r}141 \\
50 \\
27 \\
18 \\
17\end{array}$ & $\begin{array}{r}.9 \\
.9 \\
.9 \\
.8 \\
.8 \\
.7\end{array}$ \\
\hline
\end{tabular}

NoTE. - No record Apr. 27 to Sept. 9, 1913. $97764^{\circ}-$ WSP $373-15-6$ 
Monthly discharge of Manoa Stream at.College of Hawaii, near Honolulu, Oahu, for 1912-13.

\begin{tabular}{|c|c|c|c|c|c|}
\hline \multirow{2}{*}{ Month. } & \multicolumn{3}{|c|}{ Discharge in second-feet. } & \multirow{2}{*}{$\begin{array}{l}\text { Run-off } \\
\text { (total in } \\
\text { acre-feet). }\end{array}$} & \multirow{2}{*}{$\begin{array}{l}\text { Accu- } \\
\text { racy. }\end{array}$} \\
\hline & Maximum. & Minimum. & Mean. & & \\
\hline 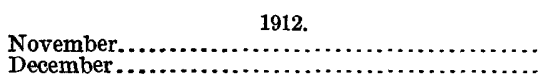 & $\begin{array}{r}62 \\
169\end{array}$ & $\begin{array}{l}1.1 \\
1.5\end{array}$ & $\begin{array}{l}11.5 \\
27.8\end{array}$ & $\begin{array}{r}684 \\
1,710\end{array}$ & $\begin{array}{l}\text { C. } \\
\text { D. }\end{array}$ \\
\hline 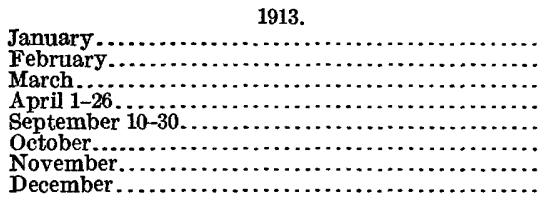 & $\begin{array}{c}18 \\
11 \\
15 \\
67 \\
4.9 \\
141 \\
102\end{array}$ & $\begin{array}{r}0.9 \\
.9 \\
.9 \\
1.9 \\
.5 \\
.4 \\
.6 \\
.7\end{array}$ & $\begin{array}{r}4.95 \\
2.52 \\
3.57 \\
2.02 \\
.90 \\
.52 \\
23.7 \\
14.5\end{array}$ & $\begin{array}{r}304 \\
140 \\
220 \\
1,040 \\
38 \\
32 \\
1,410 \\
892\end{array}$ & $\begin{array}{l}\text { C. } \\
\text { C. } \\
\text { B. } \\
\text { D. } \\
\text { C. } \\
\text { C. }\end{array}$ \\
\hline The period.. & (L) & 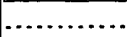 & ..... & 4,080 & \\
\hline
\end{tabular}

EAST BRANCH OF MANOA STREAM AT UPPER MANOA VALLEY, NEAR HONOLULU, OAHU.

Location.-About 4 miles northeast of Honolulu post office, in upper Manoa Valley, about 200 feet above highway bridge, and 600 feetabove confluence of two branches. Records available.-May 29 to December 31, 1913.

Gage.-Vertical staff.

Control.-Somewhat shifting at high water.

Diversions.-One irrigation ditch diverts water above station.

Discharge measurements.-For low and medium stages, made by wading just below highway bridge; for floods, from bridge.

Accuracy.-Records good.

Discharge measurements of East Branch of Manoa Stream at upper Manoa Valley, near Honolulu, Oahu, in 1918.

\begin{tabular}{|c|c|c|c|c|c|c|c|}
\hline Date. & Hydrographer. & $\begin{array}{c}\text { Gage } \\
\text { height. }\end{array}$ & $\begin{array}{c}\text { Dis- } \\
\text { charge. }\end{array}$ & Date. & Hydrographer. & $\begin{array}{c}\text { Gage } \\
\text { height. }\end{array}$ & $\begin{array}{c}\text { Dîs- } \\
\text { charge. }\end{array}$ \\
\hline $\begin{array}{l}\text { June } 23 \\
\text { Sept. 11 } \\
\text { Nov. 10 }\end{array}$ & 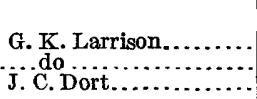 & $\begin{array}{c}\text { Feet. } \\
2.19 \\
2.07 \\
2.54\end{array}$ & $\begin{array}{r}\text { Sec.-ft. } \\
2.41 \\
1.63 \\
4.91\end{array}$ & $\begin{array}{l}\text { Nov. } 12 \\
\text { Dec. } 4\end{array}$ & 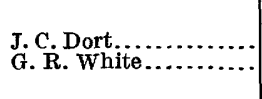 & $\begin{array}{l}\text { Feet. } \\
\mathbf{2 . 8 4} \\
\mathbf{3 . 3 8}\end{array}$ & $\begin{array}{r}\text { Sec. } f t . \\
10.2 \\
24.0\end{array}$ \\
\hline
\end{tabular}


Daily discharge, in second-feet, of East Branch of Manoa Stream at upper Manoa Valley, near Honolulu, Oahu, for 1913.

\begin{tabular}{|c|c|c|c|c|c|c|c|c|}
\hline Day. & May. & June. & July. & Aug. & Sept. & Oct. & Nov. & Dec. \\
\hline $\begin{array}{l}1 \\
2 \ldots \\
3 \ldots \ldots \\
4 \ldots \ldots \\
5 \ldots \ldots \ldots\end{array}$ & & $\begin{array}{c}1.9 \\
1.9 \\
19 \\
3.2 \\
2.6\end{array}$ & $\begin{array}{l}2.1 \\
2.1 \\
2.1 \\
2.1 \\
2.1\end{array}$ & $\begin{array}{l}1.8 \\
1.8 \\
1.7 \\
1.7 \\
1.7\end{array}$ & $\begin{array}{l}1.7 \\
2.0 \\
1.8 \\
1.7 \\
1.7\end{array}$ & $\begin{array}{l}1.6 \\
1.5 \\
1.5 \\
1.5 \\
1.6\end{array}$ & $\begin{array}{l}1.5 \\
1.8 \\
1.6 \\
1.5 \\
1.6\end{array}$ & $\begin{array}{l}3.0 \\
4.5 \\
20 \\
23 \\
14\end{array}$ \\
\hline $\begin{array}{r}6 \\
7 \ldots \ldots \\
8 \ldots \ldots \ldots \ldots \\
9 \\
90 \ldots \ldots \ldots \ldots\end{array}$ & & $\begin{array}{l}2.7 \\
2.6 \\
2.6 \\
3.3 \\
3.7\end{array}$ & $\begin{array}{l}2.0 \\
2.0 \\
2.0 \\
2.0 \\
2.0\end{array}$ & $\begin{array}{l}1.8 \\
1.8 \\
1.7 \\
1.7 \\
2.2\end{array}$ & $\begin{array}{l}5.4 \\
1.7 \\
1.7 \\
1.6 \\
1.6\end{array}$ & $\begin{array}{l}1.6 \\
1.7 \\
2.1 \\
1.6 \\
1.6\end{array}$ & $\begin{array}{l}1.6 \\
15 \\
7.8 \\
7.4 \\
3.8\end{array}$ & $\begin{array}{l}14 \\
18 \\
5.2 \\
7.4 \\
5.8\end{array}$ \\
\hline 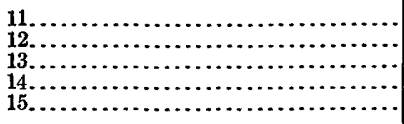 & & $\begin{array}{l}3.0 \\
2.3 \\
2.1 \\
2.0 \\
2.0\end{array}$ & $\begin{array}{l}2.0 \\
2.0 \\
2.2 \\
2.3 \\
2.0\end{array}$ & $\begin{array}{l}1.8 \\
5.6 \\
2.0 \\
1.9 \\
1.8\end{array}$ & $\begin{array}{l}1.6 \\
1.7 \\
1.9 \\
1.7 \\
1.6\end{array}$ & $\begin{array}{l}1.5 \\
1.5 \\
1.9 \\
1.8 \\
1.6\end{array}$ & $\begin{array}{l}4.3 \\
7.4 \\
6.6 \\
4.5 \\
3.2\end{array}$ & $\begin{array}{l}5.7 \\
5.3 \\
5.2 \\
4.8 \\
4.6\end{array}$ \\
\hline $\begin{array}{l}16 \ldots \ldots \ldots \\
17 \ldots \ldots \ldots \ldots \\
18 \ldots \ldots \ldots \ldots \\
19 \ldots \ldots \ldots \ldots\end{array}$ & & $\begin{array}{r}2.0 \\
2.0 \\
2.0 \\
2.3 \\
88\end{array}$ & $\begin{array}{l}1.9 \\
1.9 \\
1.9 \\
1.8 \\
1.8\end{array}$ & $\begin{array}{l}1.8 \\
2.2 \\
1.9 \\
1.8 \\
1.8\end{array}$ & $\begin{array}{l}1.6 \\
1.5 \\
1.7 \\
2.1 \\
1.6\end{array}$ & $\begin{array}{l}2.0 \\
1.6 \\
1.5 \\
1.5 \\
1.5\end{array}$ & $\begin{array}{r}2.4 \\
2.4 \\
4.8 \\
8.3 \\
45\end{array}$ & $\begin{array}{l}4.2 \\
\text { 3. } \\
\text { 3. } 6 \\
\text { 3. } 0 \\
\text { 3. } 4\end{array}$ \\
\hline $\begin{array}{l}21 \\
22 \\
24 \\
24 \\
25\end{array}$ & & $\begin{array}{l}2.2 \\
2.1 \\
2.0 \\
2.0 \\
2.1\end{array}$ & $\begin{array}{l}1.8 \\
1.8 \\
1.8 \\
1.8 \\
1.9\end{array}$ & $\begin{array}{l}1.9 \\
1.9 \\
2.0 \\
2.0 \\
2.0\end{array}$ & $\begin{array}{l}1.8 \\
1.7 \\
1.6 \\
1.6 \\
1.6\end{array}$ & $\begin{array}{l}1.5 \\
1.5 \\
1.5 \\
1.5 \\
1.5\end{array}$ & $\begin{array}{r}8.8 \\
5.6 \\
4.4 \\
4.3 \\
14\end{array}$ & $\begin{array}{l}3.3 \\
3.0 \\
2.8 \\
2.8 \\
2.8\end{array}$ \\
\hline 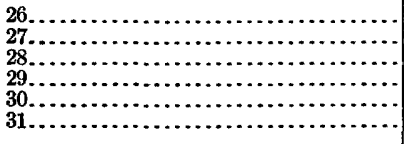 & 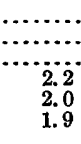 & $\begin{array}{r}2.1 \\
2.1 \\
2.0 \\
2.0 \\
2.1 \\
\cdots\end{array}$ & $\begin{array}{l}2.0 \\
1.8 \\
2.0 \\
1.8 \\
1.8 \\
1.8\end{array}$ & $\begin{array}{l}2.0 \\
1.9 \\
2.2 \\
2.0 \\
2.0 \\
3.6\end{array}$ & $\begin{array}{l}1.5 \\
1.5 \\
1.7 \\
1.6 \\
1.6\end{array}$ & $\begin{array}{l}1.5 \\
1.6 \\
1.6 \\
1.5 \\
1.6 \\
1.5\end{array}$ & $\begin{array}{c}17 \\
15 \\
8.3 \\
6.1 \\
5.8 \\
\ldots \ldots\end{array}$ & $\begin{array}{l}2.8 \\
2.8 \\
2.8 \\
2.8 \\
2.8 \\
2.8\end{array}$ \\
\hline
\end{tabular}

NoTE.-Discharge July 1-10 estimated by comparison with other stream run-off in vicinity.

Monthly discharge of East Branch of Manoa Stream at upper Manoa Valley, near Honolulu, Oahu, for 1913.

\begin{tabular}{|c|c|c|c|c|c|}
\hline \multirow{2}{*}{ Month. } & \multicolumn{3}{|c|}{ Discharge in second-feet. } & \multirow{2}{*}{$\begin{array}{l}\text { Run-off } \\
\text { (total in } \\
\text { acre-feet). }\end{array}$} & \multirow{2}{*}{$\begin{array}{l}\text { Accu- } \\
\text { racy. }\end{array}$} \\
\hline & Maximum. & Minimum. & Mean. & & \\
\hline \multirow[t]{2}{*}{ 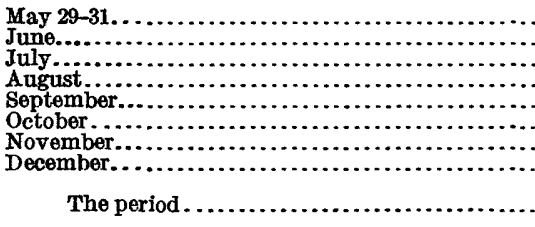 } & $\begin{array}{c}2.2 \\
88 \\
2.3 \\
5.6 \\
5.4 \\
2.1 \\
45 \\
23\end{array}$ & $\begin{array}{l}1.9 \\
1.9 \\
1.8 \\
1.7 \\
1.5 \\
1.5 \\
1.5 \\
2.8\end{array}$ & $\begin{array}{l}2.03 \\
5.72 \\
1.95 \\
2.06 \\
1.80 \\
1.60 \\
7.39 \\
6.13\end{array}$ & $\begin{array}{c}12.1 \\
340 \\
120 \\
127 \\
107 \\
98.4 \\
440 \\
377\end{array}$ & \multirow[t]{2}{*}{$\begin{array}{l}\text { B. } \\
\text { B. } \\
\text { B. } \\
\text { B. } \\
\text { B. } \\
\text { B. } \\
\text { B. } \\
\text { B. }\end{array}$} \\
\hline & $\cdots$ & & ............ & 1,620 & \\
\hline
\end{tabular}


WEST BRANCH OF MANOA STREAM AT UPPER MANOA VALLEY, NEAR HONOLULU, OAHU.

Location.-About 150 feet above highway bridge in upper Manoa Valley, and 4 miles northeast of Honolulu pest office.

Records available.-May 29 to December 31, 1913.

Gage.-Vertical staff.

Control.-Somewhat shifting.

Discharge measurements.-At low and medium stages made by wading; flood measurements made from footbridge.

Diversions.- None above station.

Accuracy.-Records good.

Discharge measurements of West Branch of Manoa Stream at upper Manoa Valley, near Honolulu; Oahu, in 1913.

\begin{tabular}{|c|c|c|c|c|c|c|c|}
\hline Date. & Hydrographer. & $\begin{array}{c}\text { Gage } \\
\text { height. }\end{array}$ & $\begin{array}{c}\text { Dis- } \\
\text { charge. }\end{array}$ & Date. & Hydrographer. & $\begin{array}{c}\text { Gage } \\
\text { height. }\end{array}$ & $\begin{array}{l}\text { Dis- } \\
\text { charge. }\end{array}$ \\
\hline $\begin{array}{l}\text { June } 23 \\
\text { Sept. } 11\end{array}$ & $\begin{array}{c}\text { G. K. Larrison.............. } \\
\text { …do }\end{array}$ & $\begin{array}{r}\text { Feet. } \\
1.84 \\
1.53\end{array}$ & $\begin{array}{r}\text { Sec.ft. } \\
2.44 \\
.68\end{array}$ & $\begin{array}{l}\text { Nov. } 10 \\
\text { Nov. } 12\end{array}$ & $\begin{array}{l}\text { J. C. Dort.......... } \\
\ldots \ldots \text { do }\end{array}$ & $\begin{array}{c}\text { Feet. } \\
2.62 \\
2.95\end{array}$ & $\begin{array}{r}\text { Sec. }-f t . \\
10.1 \\
19.2\end{array}$ \\
\hline
\end{tabular}

Daily discharge, in second-feet, of West Branch of Manoa Stream, at upper Manoa Valley, near Honolulu, Oahu, for 1913.

\begin{tabular}{|c|c|c|c|c|c|c|c|c|}
\hline Day. & May. & June. & July. & Aug. & Sept. & Oct. & Nov. & Dec. \\
\hline 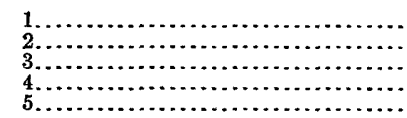 & & $\begin{array}{r}1.4 \\
1.2 \\
14 \\
3.4 \\
2.4\end{array}$ & $\begin{array}{l}1.9 \\
2.0 \\
2.0 \\
2.1 \\
2.1\end{array}$ & $\begin{array}{l}1.3 \\
1.4 \\
1.5 \\
1.4 \\
1.8\end{array}$ & $\begin{array}{l}4.0 \\
2.6 \\
1.4 \\
1.2 \\
1.3\end{array}$ & $\begin{array}{r}0.6 \\
.2 \\
.5 \\
.5 \\
.6\end{array}$ & $\begin{array}{r}0.4 \\
.8 \\
1.0 \\
.7 \\
.6\end{array}$ & $\begin{array}{l}4.7 \\
4.5 \\
14 \\
18 \\
10\end{array}$ \\
\hline 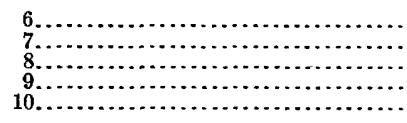 & $\cdots \cdot$ & $\begin{array}{l}2.6 \\
1.8 \\
1.3 \\
4.4 \\
5.4\end{array}$ & $\begin{array}{l}2.2 \\
2.2 \\
2.3 \\
2.3 \\
2.4\end{array}$ & $\begin{array}{l}1.3 \\
1.5 \\
1.1 \\
1.2 \\
3.5\end{array}$ & $\begin{array}{r}1.0 \\
.8 \\
.7 \\
.6 \\
.6\end{array}$ & $\begin{array}{r}.6 \\
.7 \\
3.5 \\
.8 \\
.6\end{array}$ & $\begin{array}{r}.6 \\
6.0 \\
8.2 \\
13 \\
9.2\end{array}$ & $\begin{aligned} 11 \\
8.2 \\
6.8 \\
4.6 \\
4.6\end{aligned}$ \\
\hline $11 \ldots \ldots \ldots \ldots$ & .. & $\begin{array}{l}4.0 \\
3.1 \\
6.4 \\
16 \\
16\end{array}$ & $\begin{array}{l}2.5 \\
2.4 \\
2.3 \\
1.8 \\
2.0\end{array}$ & $\begin{array}{l}1.3 \\
8.0 \\
2.0 \\
1.6 \\
1.2\end{array}$ & $\begin{array}{r}.6 \\
.7 \\
1.6 \\
1.6 \\
.8\end{array}$ & $\begin{array}{l}.5 \\
.5 \\
.8 \\
.4 \\
.7\end{array}$ & $\begin{array}{c}12 \\
15 \\
10 . \\
5.8 \\
4.7\end{array}$ & $\begin{array}{l}4.3 \\
8.6 \\
4.1 \\
3.5 \\
1.3\end{array}$ \\
\hline $\begin{array}{l}16 \ldots \ldots \ldots \ldots \ldots \\
17 \ldots \ldots \ldots \ldots \ldots . . . \\
18 \ldots \ldots \ldots \ldots \ldots \ldots . . \\
20 \ldots \ldots \ldots \ldots \ldots . .\end{array}$ & $\begin{array}{l}\ldots \ldots \\
\cdots \cdots \cdot \\
\cdots \cdots\end{array}$ & $\begin{array}{l}2.3 \\
2.1 \\
2.2 \\
3.5 \\
68\end{array}$ & $\begin{array}{l}1.9 \\
1.3 \\
1.6 \\
1.4 \\
1.6\end{array}$ & $\begin{array}{l}1.2 \\
2.5 \\
1.4 \\
1.1 \\
1.2\end{array}$ & $\begin{array}{r}.7 \\
.6 \\
1.1 \\
3.4 \\
1.6\end{array}$ & $\begin{array}{r}2.2 \\
.6 \\
.5 \\
.5 \\
.5\end{array}$ & $\begin{array}{l}4.1 \\
4.3 \\
5.4 \\
10 \\
22\end{array}$ & $\begin{array}{r}.1 \\
1.4 \\
.1 \\
.8 \\
3.8\end{array}$ \\
\hline $\begin{array}{l}21 \ldots \\
22 \ldots \\
23 \ldots \\
24 \ldots \\
25 \ldots\end{array}$ & $\ldots \ldots$ & $\begin{array}{l}3.4 \\
2.6 \\
2.1 \\
1.9 \\
1.9\end{array}$ & $\begin{array}{l}1.7 \\
1.4 \\
1.8 \\
2.7 \\
1.7\end{array}$ & $\begin{array}{r}1.0 \\
.9 \\
.9 \\
.9 \\
.9\end{array}$ & $\begin{array}{r}1.2 \\
.9 \\
.9 \\
.9 \\
.8\end{array}$ & $\begin{array}{l}.4 \\
.4 \\
.4 \\
.4 \\
.4\end{array}$ & $\begin{array}{l}9.7 \\
6.4 \\
4.3 \\
9.4 \\
8.5\end{array}$ & $\begin{array}{l}4.0 \\
1.8 \\
3.0 \\
2.6 \\
2.5\end{array}$ \\
\hline 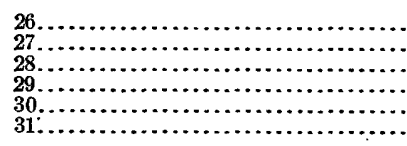 & $\begin{array}{l}1.6 \\
1.6 \\
4.6\end{array}$ & $\begin{array}{l}1.9 \\
1.9 \\
2.6 \\
1.7 \\
1.8\end{array}$ & $\begin{array}{l}1.5 \\
2.0 \\
1.7 \\
2.0 \\
1.9 \\
1.8\end{array}$ & $\begin{array}{r}.7 \\
.7 \\
1.2 \\
1.7 \\
1.1 \\
3.5\end{array}$ & $\begin{array}{l}.7 \\
.6 \\
.5 \\
.8 \\
.6\end{array}$ & $\begin{array}{r}.4 \\
.7 \\
1.4 \\
.6 \\
.4 \\
.4\end{array}$ & $\begin{array}{l}23 \\
17.0 \\
9.2 \\
7.3 \\
5.6\end{array}$ & $\begin{array}{l}2.4 \\
2.3 \\
2.1 \\
2.0 \\
2.0 \\
1.9\end{array}$ \\
\hline
\end{tabular}

NoTE.-Discharge estimated July 1-10. 
Monthly discharge of West Branch of Manoa Stream at upper Manoa Valley, near Honolulu, Oahu, for 1913.

\begin{tabular}{|c|c|c|c|c|c|}
\hline \multirow{2}{*}{ Month. } & \multicolumn{3}{|c|}{ Discharge in second-feet. } & \multirow{2}{*}{$\begin{array}{l}\text { Run-off } \\
\text { (total in } \\
\text { acre-feet). }\end{array}$} & \multirow{2}{*}{$\begin{array}{l}\text { Accu- } \\
\text { racy. }\end{array}$} \\
\hline & Maximum. & Minimum. & Mean. & & \\
\hline \multirow{2}{*}{ 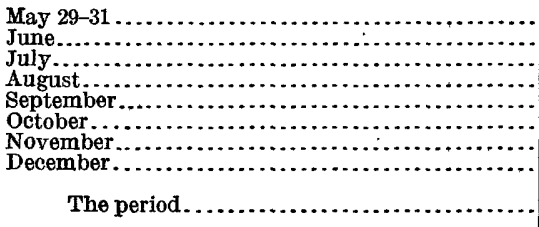 } & $\begin{array}{c}4.6 \\
68 \\
2.7 \\
8.0 \\
4.0 \\
3.5 \\
23 \\
18\end{array}$ & $\begin{array}{r}1.6 \\
1.2 \\
1.3 \\
.7 \\
.5 \\
.2 \\
.4 \\
.1\end{array}$ & $\begin{array}{r}2.60 \\
6.11 \\
1.95 \\
1.65 \\
1.16 \\
.70 \\
.7 .81 \\
4.55\end{array}$ & $\begin{array}{c}15.5 \\
364 \\
120 \\
101 \\
69 \\
43 \\
465 \\
280\end{array}$ & \multirow[t]{2}{*}{$\begin{array}{l}\text { B. } \\
\text { B. } \\
\text { B. } \\
\text { B. } \\
\text { B. } \\
\text { B. } \\
\text { B. } \\
\text { B. }\end{array}$} \\
\hline & & & ....... & 1,460 & \\
\hline
\end{tabular}

WAIOMAO STREAM AT UPPER PALOLO VALLEY, NEAR HONOLULU, OAHU.

Location.-About 3 miles above Pukele in Palolo Valley, and about 9 miles from Honolulu post office, at an elevation of 900 feet.

Records available.-October 10, 1911, to July 12, 1913.

Gage.-Watson water-stage recorder above 3-foot sharp-crested weir; datum uncertain on account of leaks under weir.

Control.-Shifting.

Discharge measurements.-Made by wading.

Diversions. - New pipe line diverted all low-water discharge for Honolulu water supply after July 12, 1913.

Accuracy.-Records fair.

Regulation.-Stream drains Kaau Crater and is regulated by springs.

Discharge measurements of Waiomao Stream at upper Palolo Valley, near Honolulu, Oahu, in 1913.

\begin{tabular}{|c|c|c|c|}
\hline Date., & Hydrographer. & $\begin{array}{c}\text { Gage } \\
\text { height. }\end{array}$ & $\begin{array}{l}\text { Dis- } \\
\text { charge. }\end{array}$ \\
\hline $\begin{array}{l}\text { Feb. } 26 \\
\text { Apr. } 8\end{array}$ & 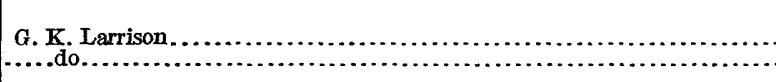 & $\begin{array}{l}\text { Feet. } \\
0.12 \\
.08\end{array}$ & $\begin{array}{r}\text { Sec. } f t_{*} \\
0.49 \\
.29\end{array}$ \\
\hline
\end{tabular}


Daily discharge, in second-feet, of Waiomao Stream at upper Palolo Valley, near Honolulu, Oahu, for 1913 .

\begin{tabular}{|c|c|c|c|c|c|c|c|}
\hline Day. & Jan. & Feb. & Mar. & Apr. & May. & June. & July. \\
\hline 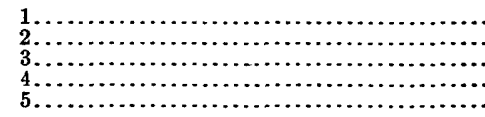 & $\begin{array}{l}0.37 \\
.27 \\
.27 \\
.27 \\
.52\end{array}$ & $\begin{array}{r}0.19 \\
.19 \\
.19 \\
.42 \\
4.08\end{array}$ & $\begin{array}{r}0.37 \\
.37 \\
.37 \\
.32 \\
.32\end{array}$ & $\begin{array}{r}0.96 \\
.70 \\
.70 \\
.58 \\
.47\end{array}$ & $\begin{array}{r}0.58 \\
1.10 \\
1.17 \\
.82 \\
.64\end{array}$ & $\begin{array}{r}0.32 \\
.37 \\
4.30 \\
3.02 \\
1.56\end{array}$ & $\begin{array}{r}1.24 \\
1.56 \\
1.17 \\
.89 \\
.89\end{array}$ \\
\hline $\begin{array}{r}6 \\
8 \\
9 \\
9 \\
10 \\
{ }_{1}\end{array}$ & $\begin{array}{l}.32 \\
.27 \\
.23 \\
.27 \\
.37\end{array}$ & $\begin{array}{r}1.64 \\
1.48 \\
1.72 \\
.82 \\
.58\end{array}$ & $\begin{array}{l}.32 \\
.52 \\
.47 \\
.37 \\
.32\end{array}$ & $\begin{array}{l}.37 \\
.32 \\
.32 \\
.32 \\
.32\end{array}$ & $\begin{array}{l}.47 \\
1.10 \\
3.64 \\
1.64 \\
1.24\end{array}$ & $\begin{array}{r}1.32 \\
.89 \\
.76 \\
.70 \\
.64\end{array}$ & $\begin{array}{l}.64 \\
.70 \\
.52 \\
.52 \\
.42\end{array}$ \\
\hline $\begin{array}{l}11 \\
12 \\
13 \\
14 \\
15\end{array}$ & $\begin{array}{l}.89 \\
.58 \\
.70 \\
.58 \\
.64\end{array}$ & $\begin{array}{r}.47 \\
.37 \\
.32 \\
.27 \\
1.89\end{array}$ & $\begin{array}{r}.32 \\
.27 \\
.27 \\
3.32 \\
2.07\end{array}$ & $\begin{array}{l}.76 \\
1.24 \\
4.08 \\
2.52 \\
1.80\end{array}$ & $\begin{array}{l}1.03 \\
4.88 \\
3.75 \\
2.34 \\
2.16\end{array}$ & $\begin{array}{r}.52 \\
.82 \\
1.03 \\
.76 \\
1.64\end{array}$ & $\begin{array}{r}.42 \\
.52 \\
\cdots+. . \\
\cdots\end{array}$ \\
\hline $\begin{array}{l}16 \ldots \ldots \\
18 \\
19 \\
19 \\
20 \\
20\end{array}$ & $\begin{array}{l}.70 \\
.42 \\
.47 \\
.47 \\
.42\end{array}$ & $\begin{array}{l}2.52 \\
2.16 \\
3.02 \\
2.25 \\
1.32\end{array}$ & $\begin{array}{r}1.17 \\
.89 \\
.64 \\
.52 \\
.47\end{array}$ & $\begin{array}{l}3.22 \\
3.22 \\
1.89 \\
1.40 \\
1.10\end{array}$ & $\begin{array}{r}1.72 \\
1.56 \\
1.17 \\
1.03 \\
.76\end{array}$ & $\begin{array}{l}2.82 \\
2.16 \\
1.24 \\
1.03 \\
1.64\end{array}$ & 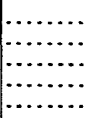 \\
\hline $\begin{array}{l}21 \\
22 \\
23 \\
24 \\
25 \\
25\end{array}$ & $\begin{array}{l}.37 \\
.37 \\
.32 \\
.27 \\
.23\end{array}$ & $\begin{array}{l}.89 \\
.70 \\
.64 \\
.89 \\
.64\end{array}$ & $\begin{array}{l}1.64 \\
1.89 \\
1.32 \\
1.98 \\
1.72\end{array}$ & $\begin{array}{r}.82 \\
.82 \\
1.10 \\
1.03 \\
5.48\end{array}$ & $\begin{array}{l}.64 \\
.58 \\
.53 \\
.48 \\
.42\end{array}$ & $\begin{array}{l}1.03 \\
.76 \\
.64 \\
.52 \\
.47\end{array}$ & $\mid \begin{array}{ll}\cdots \\
\ldots \ldots\end{array}$ \\
\hline $\begin{array}{l}26 \\
27 \\
28 \ldots \ldots \\
29 \\
30 \\
31 \\
\end{array}$ & $\begin{array}{l}.19 \\
.23 \\
.19 \\
.19 \\
.19 \\
.27\end{array}$ & $\begin{array}{r}.58 \\
.52 \\
.42 \\
\cdots \\
\cdots \\
\ldots . \cdots\end{array}$ & $\begin{array}{r}1.17 \\
1.10 \\
.76 \\
.64 \\
1.10 \\
.89\end{array}$ & $\begin{array}{r}1.80 \\
1.10 \\
.96 \\
.82 \\
.64\end{array}$ & $\begin{array}{l}.37 \\
.52 \\
.37 \\
.37 \\
.37 \\
.32\end{array}$ & $\begin{array}{l}.47 \\
1.64 \\
3.12 \\
1.48 \\
1.48\end{array}$ & {$\left[\begin{array}{ll}\cdots \cdots \\
\cdots \cdots \cdots \\
\cdots \cdots \cdots\end{array}\right.$} \\
\hline
\end{tabular}

NotE.-Discharge May 22-24 estimated.

Monthly discharge of Waiomao Stream at upper Palolo Valley, near Honolulu, Oahu, for 1913.

\begin{tabular}{|c|c|c|c|c|c|}
\hline \multirow{2}{*}{ Month. } & \multicolumn{3}{|c|}{ Discharge in second-feet. } & \multirow{2}{*}{$\begin{array}{l}\text { Run-off } \\
\text { (total in } \\
\text { acre-feet). }\end{array}$} & \multirow{2}{*}{$\begin{array}{l}\text { Accu- } \\
\text { racy. }\end{array}$} \\
\hline & Maximum. & Minimum. & Mean. & & \\
\hline \multirow{2}{*}{ 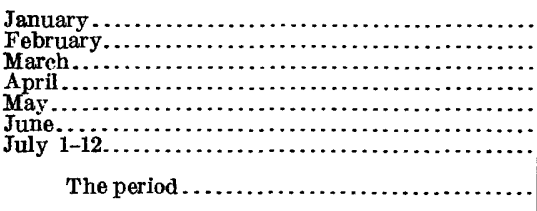 } & $\begin{array}{l}0.89 \\
4.08 \\
3.32 \\
5.48 \\
4.88 \\
4.30 \\
1.56\end{array}$ & $\begin{array}{r}0.19 \\
.19 \\
.27 \\
.32 \\
.32 \\
.32 \\
.42\end{array}$ & $\begin{array}{r}0.38 \\
1.11 \\
.90 \\
1.36 \\
1.22 \\
1.30 \\
.79\end{array}$ & $\begin{array}{l}23.4 \\
61.6 \\
55.3 \\
80.9 \\
75.0 \\
77.4 \\
18.8\end{array}$ & \multirow[t]{2}{*}{$\begin{array}{l}\text { C. } \\
\text { C. } \\
\text { C. } \\
\text { C. } \\
\text { C. } \\
\text { C. } \\
\text { C. }\end{array}$} \\
\hline & $\ldots \ldots \ldots \ldots$ & $\ldots \ldots \ldots \ldots$ & $\ldots \ldots \ldots$ & 390 & \\
\hline
\end{tabular}

\section{WATMANALO DITCH BELOW MAIN RESERVOIR ON THE WAIMANALO PLANTATION, NEAR WAIMANALO, OAHU.}

Iocation.-On main ditch immediately below main reservoir of the Waimanalo plantation, about 2 miles southwest of the plantation headquarters. Waimanalo is about 15 miles east of Honolulu.

Records available.-November 1, 1912, to December 31, 1913.

Gage.-Vertical staff, read twice daily to quarter-tenths.

Control.-Probably permanent.

Discharge measurements.-Made by wading.

Diversions.-Ditch is outlet of the main reservoir on the Waimanalo plantation.

Accuracy.-Records good.

Cooperation.-Maintained in cooperation with the Waimanalo Plantation Co. 
Discharge measurements of Waimanalo ditch below main reservoir on the Waimanalo plantation, near Waimanalo, Oahu, in 1913.

\begin{tabular}{|c|c|c|c|}
\hline Date. & Hydrographer. & $\begin{array}{c}\text { Fage } \\
\text { height. }\end{array}$ & $\begin{array}{c}\text { Dis- } \\
\text { charge. }\end{array}$ \\
\hline $\begin{array}{l}\text { Apr. } 11 \\
\text { Sept. } 9\end{array}$ & 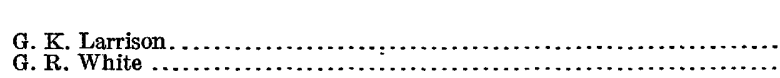 & $\begin{array}{c}\text { Feet. } \\
1.18 \\
1.42\end{array}$ & $\begin{array}{r}\text { Sec.-ft. } \\
6.15 \\
7.53\end{array}$ \\
\hline
\end{tabular}

Daily discharge, in second-feet, of Waimanalo ditch below main reservoir on the Waimanalo plantation, near Waimanalo, Oahu, for 1912-13.

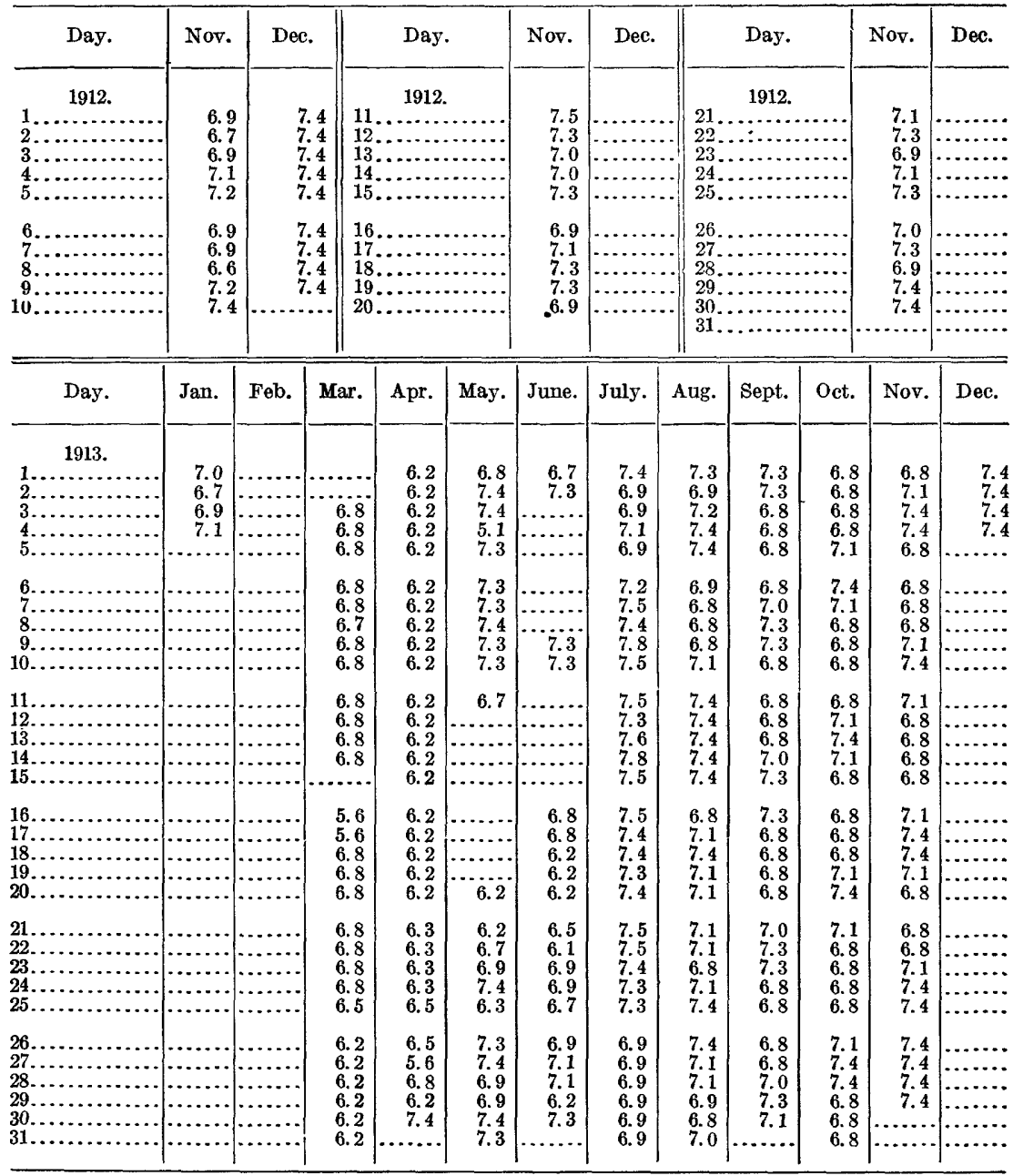

Note.-Ditch dry Dec. 10-31, 1912; Jan. 5 to Mar. 2, May 12-19, June 3-8, 11-15, and Dec. 6-31, 1913. Discharge given is for period of about 10 hours daily. During remaiming 14 hours ditch was dry. Discharge interpolated Sundays from July to December, as gage was not read. 
Monthly discharge of Waimanalo ditch below main reservoir on the Waimanalo plantation, near Waimanalo, Oahu, for 1913.

\begin{tabular}{|c|c|c|c|c|c|}
\hline \multirow{2}{*}{ Month. } & \multicolumn{3}{|c|}{ Discharge in second-feet. } & \multirow{2}{*}{$\begin{array}{c}\text { Run-off } \\
\text { (total in } \\
\text { acre-feet). }\end{array}$} & \multirow{2}{*}{$\begin{array}{l}\text { Accu- } \\
\text { racy. }\end{array}$} \\
\hline & Maximum. & Minimum. & Mean. & & \\
\hline 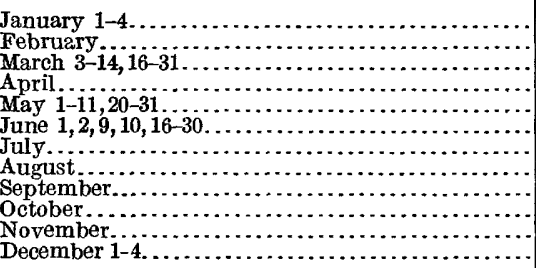 & \begin{tabular}{|c|}
7.1 \\
6.8 \\
7.4 \\
7.4 \\
7.3 \\
7.8 \\
7.4 \\
7.3 \\
7.4 \\
7.4 \\
7.4
\end{tabular} & $\begin{array}{r}6.7 \\
5.6 \\
5.6 \\
5.1 \\
6.1 \\
6.9 \\
6.8 \\
6.8 \\
6.8 \\
6.8 \\
7.4\end{array}$ & $\begin{array}{r}6.92 \\
6.57 \\
6.27 \\
6.97 \\
6.76 \\
7.28 \\
7.13 \\
6.99 \\
6.96 \\
7.09 \\
7.40\end{array}$ & $\begin{array}{c}22.9 \\
152 \\
155 \\
132 \\
106 \\
187 \\
183 \\
173 \\
178 \\
408 \\
58.7\end{array}$ & \multirow[t]{2}{*}{$\begin{array}{l}\text { B. } \\
\text { B. } \\
\text { B. } \\
\text { B. } \\
\text { B. } \\
\text { B. } \\
\text { B. } \\
\text { B. } \\
\text { B. } \\
\text { B. } \\
\text { B. }\end{array}$} \\
\hline The period ... & $\cdots$ & $\ldots$ & & 1,760 & \\
\hline
\end{tabular}

Note.-Ditch dry during February. Water turned out of ditch Dec. 4.

MAKAWAO DITCH AT MAKAWAO FLUME, NEAR WAMMANALO, OAHU.

Location.-At west end of flume in Kailua Valley, 4 miles west of Waimanalo, and 11 miles by road from Honolulu; previous to November 24, 1913, at east end of flume.

Records available.-November 1, 1912, to December 31, 1913.

Gage.--Staff; new datum beginning November 24, 1913.

Control.-Practically permanent.

Discharge measurements.-November 1, 1912, to November 23, 1913, made by current-meter in open flume; November 24 to December 31, 1913, by 2.5 -foot sharp-crested weir with end contractions.

Diversions.-Ditch diverts all low water from headwaters of Kaimi and Makawao streams and discharges into the Waimanalo Reservoir.

Accuracy.-Records poor.

Cooperation.-Observer furnished by Waimanalo Plantation Co.

Discharge measurements of Makawao ditch at Makawao flume, near Waimanalo, Oahu, in 1913.

\begin{tabular}{|c|c|c|c|c|c|c|c|}
\hline Date. & Hydrographer. & $\begin{array}{c}\text { Gage } \\
\text { height. }\end{array}$ & $\begin{array}{c}\text { Dis- } \\
\text { charge. }\end{array}$ & Date. & Hydrographer. & $\begin{array}{c}\text { Gage } \\
\text { height. }\end{array}$ & $\begin{array}{l}\text { Dis- } \\
\text { charge. }\end{array}$ \\
\hline $\begin{array}{l}\text { Apr. } 11 \\
\text { Sept. } 9\end{array}$ & $\begin{array}{l}\text { G. K. Larrison ... } \\
\text { G. R. White .... }\end{array}$ & $\begin{array}{r}\text { Feet. } \\
0.59 \\
.70\end{array}$ & $\begin{array}{r}S e c .-f t . \\
3.03 \\
1.96\end{array}$ & Oct. 20 & J. C. Dort...... & $\begin{array}{c}\text { Feet. } \\
0.65\end{array}$ & $\begin{array}{r}\text { Sec. }-f t . \\
1.79\end{array}$ \\
\hline
\end{tabular}

Monthly discharge of Makawao ditch at Makawao flume, near Waimanalo, Oahu, for 1913.

\begin{tabular}{|c|c|c|c|c|c|}
\hline \multirow{2}{*}{ Month. } & \multicolumn{3}{|c|}{ Discharge in second-feet. } & \multirow{2}{*}{$\begin{array}{l}\text { Run-off } \\
\text { (total in } \\
\text { acre-feet). }\end{array}$} & \multirow{2}{*}{$\begin{array}{l}\text { Aceu- } \\
\text { racy. }\end{array}$} \\
\hline & Maximum. & Minimum. & Mean. & & \\
\hline 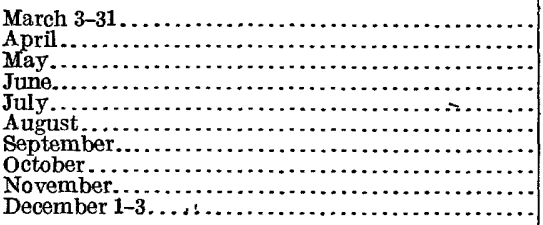 & $\begin{array}{l}5.4 \\
7.3 \\
7.9 \\
4.4 \\
3.2 \\
3.2 \\
2.7 \\
2.1 \\
6.0 \\
3.6\end{array}$ & $\begin{array}{l}2.0 \\
3.0 \\
2.6 \\
2.3 \\
1.9 \\
2.1 \\
1.6 \\
1.6 \\
1.9 \\
1.9\end{array}$ & $\begin{array}{l}3.75 \\
4.08 \\
3.88 \\
3.16 \\
2.43 \\
2.57 \\
1.97 \\
1.95 \\
3.29 \\
2.97\end{array}$ & $\begin{array}{c}216 \\
243 \\
239 \\
188 \\
149 \\
158 \\
117 \\
120 \\
196 \\
17.1\end{array}$ & $\begin{array}{l}\text { D.' } \\
\text { D. } \\
D . \\
D . \\
D . \\
D . \\
D . \\
D . \\
D . \\
D .\end{array}$ \\
\hline The period (276 days) $\ldots \ldots \ldots \ldots \ldots \ldots \ldots$ & 7.9 & 1.6 & 3.00 & 1,640 & \\
\hline
\end{tabular}

Note.-Ditch dry Jan. 1 to Mar. 2 and Dec. 4-31. 
MAKAWAO STREAM IN KAILUA VALLEY, NEAR KAILUA, OAHU.

Location.--One-fourth mile upstream from confluence of Makawao and Kaimi streams, and 100 feet above intake of irrigation ditch on Waimanalo-Honolulu road; about $12 \frac{1}{3}$ miles east by road from Honolulu.

Records available.-November 12, 1912, to December 31, 1913.

Gage.-Wertical staff.

Control.-Probably permanent.

Discharge measurements.-Made by wading and from footbridge.

Diversions.-Low-water discharge of two main branches is diverted into Makawao ditch about three-fourths mile above station. An irrigation ditch diverts most of the low-water discharge at a point about 100 feet below the gage.

Accuracy.-Records fair.

Cooperation.-Maintained in cooperation with the Kaneohe Ranch Co.

Discharge measurements of Makawao Stream in Kailua Valley, near Kailua, Oahu, in 1913.

\begin{tabular}{|c|c|c|c|c|c|c|c|}
\hline Date. & Hydrographer. & $\begin{array}{c}\text { Gage } \\
\text { height. }\end{array}$ & $\begin{array}{l}\text { Dis- } \\
\text { charge. }\end{array}$ & Date. & Hydrographer. & $\begin{array}{c}\text { Gage } \\
\text { height. }\end{array}$ & $\begin{array}{l}\text { Dis- } \\
\text { charge. }\end{array}$ \\
\hline $\begin{array}{l}\text { Apr. } 11 \\
\text { June } 4\end{array}$ & 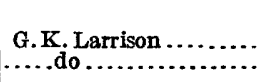 & $\begin{array}{c}\text { Feet. } \\
0.94 \\
1.37\end{array}$ & $\begin{array}{r}\text { Sec.-ft. } \\
1.92 \\
9.11\end{array}$ & $\begin{array}{l}\text { Sept. } 9 \\
\text { Dec. } 10\end{array}$ & $\begin{array}{l}\text { J. C. Dort } \\
\text { G. R. White..................... }\end{array}$ & $\begin{array}{c}\text { Feet. } \\
0.90 \\
1.32\end{array}$ & $\begin{array}{r}\text { Sec. }-f t . \\
1.81 \\
7.09\end{array}$ \\
\hline
\end{tabular}

Daily discharge, in second-feet, of Makawao Stream in Kailua Valley, near Kailua, Oahu, for 1912-13.

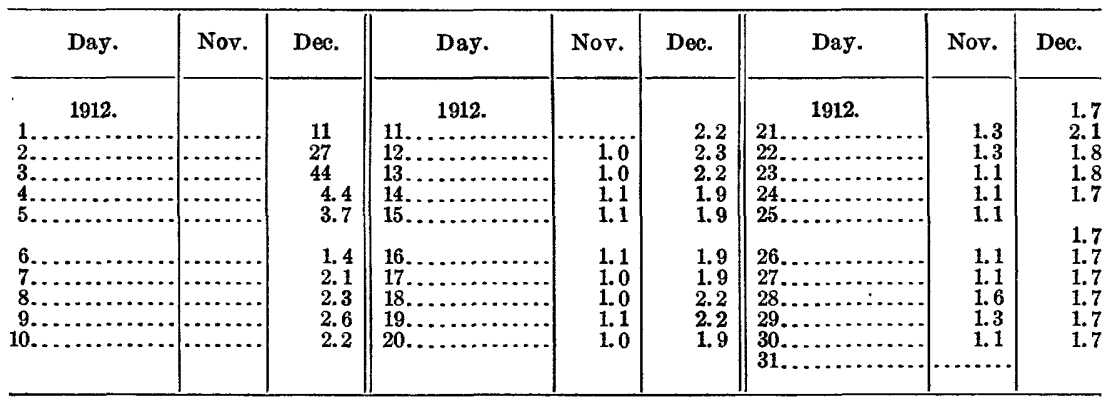


Daily discharge, in second-feet, of Makawao Stream in Kailua Valley, near Kailua, Oahu, for 1912-13-Continued.

\begin{tabular}{|c|c|c|c|c|c|c|c|c|c|c|c|c|}
\hline Day. & Jan. & Feb. & Mar. & Apr. & May. & June. & July. & Aug. & Sept. & Oct. & Nov. & Dec. \\
\hline $\begin{array}{r} \\
1913 . \\
1 \ldots \ldots \ldots \\
2 \ldots \ldots \ldots \\
3 \ldots \ldots \ldots \\
4 \ldots \ldots \ldots \\
5 \ldots \ldots \ldots\end{array}$ & & $\begin{array}{l}2.2 \\
2.2 \\
2.1 \\
1.9 \\
3.1\end{array}$ & $\begin{array}{l}3.1 \\
3.6 \\
2.7 \\
2.9 \\
2.7\end{array}$ & $\begin{array}{l}2.3 \\
2.3 \\
2.5 \\
2.5 \\
2.9\end{array}$ & $\begin{array}{l}1.9 \\
2.5 \\
1.9 \\
1.9 \\
1.7\end{array}$ & $\begin{array}{c}2.7 \\
2.8 \\
20 \\
17 \\
4.3\end{array}$ & $\begin{array}{l}2.5 \\
3.4 \\
2.5 \\
2.5 \\
2.3\end{array}$ & $\begin{array}{l}2.1 \\
1.9 \\
2.1 \\
1.9 \\
1.7\end{array}$ & $\begin{array}{l}2.3 \\
3.4 \\
2.1 \\
2.1 \\
1.7\end{array}$ & $\begin{array}{l}1.6 \\
1.6 \\
1.7 \\
1.6 \\
1.6\end{array}$ & $\begin{array}{l}1.6 \\
1.6 \\
1.6 \\
1.6 \\
1.6\end{array}$ & $\begin{array}{l}2.5 \\
2.1 \\
2.5 \\
27 \\
26\end{array}$ \\
\hline $\begin{array}{r}6 \ldots \\
7 \ldots \\
8 \ldots \\
9 \ldots \\
10 . .\end{array}$ & & $\begin{array}{c}11 \\
6.8 \\
4.1 \\
3.2 \\
2.5\end{array}$ & $\begin{array}{l}2.9 \\
1.9 \\
1.8 \\
1.9 \\
2.2\end{array}$ & $\begin{array}{l}3.4 \\
3.1 \\
2.5 \\
2.3 \\
2.1\end{array}$ & $\begin{array}{l}1.7 \\
2.1 \\
2.1 \\
2.9 \\
3.6\end{array}$ & $\begin{array}{l}3.8 \\
3.7 \\
3.6 \\
3.0 \\
3.1\end{array}$ & $\begin{array}{l}2.1 \\
2.3 \\
2.3 \\
2.3 \\
2.3\end{array}$ & $\begin{array}{l}1.7 \\
1.9 \\
2.1 \\
2.1 \\
1.9\end{array}$ & $\begin{array}{l}1.8 \\
1.7 \\
1.6 \\
1.6 \\
1.6\end{array}$ & $\begin{array}{l}1.6 \\
1.6 \\
1.6 \\
1.6 \\
1.6\end{array}$ & $\begin{array}{l}1.4 \\
1.4 \\
1.7 \\
1.3 \\
1.6\end{array}$ & $\begin{array}{l}9.2 \\
23 \\
13 \\
8.1 \\
6.5\end{array}$ \\
\hline $\begin{array}{l}11 \ldots \ldots \\
12 \ldots \\
14 \ldots \ldots \\
15 \ldots\end{array}$ & $\begin{array}{l}1.1 \\
1.1\end{array}$ & $\begin{array}{l}1.6 \\
1.4 \\
.9 \\
1.6 \\
2.5\end{array}$ & $\begin{array}{l}1.9 \\
1.6 \\
1.1 \\
1.3 \\
1.1\end{array}$ & $\begin{array}{l}2.1 \\
2.2 \\
2.4 \\
2.1 \\
2.1\end{array}$ & $\begin{array}{l}2.0 \\
48 \\
12 \\
15 \\
16\end{array}$ & $\begin{array}{l}3.0 \\
2.9 \\
3.1 \\
3.1 \\
3.1\end{array}$ & $\begin{array}{l}2.3 \\
2.1 \\
2.1 \\
2.5 \\
2.5\end{array}$ & $\begin{array}{l}1.9 \\
4.1 \\
2.9 \\
2.1 \\
1.9\end{array}$ & $\begin{array}{l}1.6 \\
1.6 \\
1.7 \\
1.7 \\
1.7\end{array}$ & $\begin{array}{l}1.6 \\
1.6 \\
1.6 \\
1.6 \\
1.6\end{array}$ & $\begin{array}{l}1.6 \\
1.5 \\
1.6 \\
1.5 \\
1.4\end{array}$ & $\begin{array}{l}5.4 \\
4.8 \\
4.4 \\
3.7 \\
3.6\end{array}$ \\
\hline $\begin{array}{l}16 \ldots \ldots \\
17 \ldots \ldots \\
18 \ldots \ldots \\
19 \ldots \ldots\end{array}$ & $\begin{array}{c}19 \\
1.6 \\
1.4 \\
16 \\
7.6\end{array}$ & $\begin{array}{c}11 \\
14 \\
14 \\
9.9 \\
11\end{array}$ & $\begin{array}{c}1.0 \\
14 \\
5.1 \\
4.6 \\
3.6\end{array}$ & $\begin{array}{l}6.1 \\
2.8 \\
2.1 \\
2.0 \\
1.8\end{array}$ & $\begin{array}{l}3.4 \\
4.4 \\
4.0 \\
3.6 \\
2.5\end{array}$ & $\begin{array}{l}3.2 \\
2.8 \\
2.6 \\
2.7 \\
3.1\end{array}$ & $\begin{array}{l}2.2 \\
2.4 \\
2.3 \\
2.3 \\
2.1\end{array}$ & $\begin{array}{l}1.9 \\
2.3 \\
2.5 \\
2.2 \\
2.1\end{array}$ & $\begin{array}{l}1.6 \\
1.6 \\
1.6 \\
1.6 \\
1.6\end{array}$ & $\begin{array}{l}1.6 \\
1.6 \\
1.6 \\
1.6 \\
1.6\end{array}$ & $\begin{array}{l}1.6 \\
1.6 \\
1.6 \\
1.7 \\
2.3\end{array}$ & $\begin{array}{l}3.1 \\
3.6 \\
4.1 \\
4.1 \\
4.0\end{array}$ \\
\hline $\begin{array}{l}21 \ldots \ldots \ldots \ldots \\
22 \ldots \ldots \ldots \\
23 \ldots \ldots \ldots \\
24 \ldots \ldots \ldots \\
25 \ldots \ldots \ldots\end{array}$ & $\begin{array}{l}15 \\
2.9 \\
2.7 \\
2.5 \\
2.1\end{array}$ & $\begin{array}{l}9.4 \\
6.3 \\
3.2 \\
2.7 \\
4.1\end{array}$ & $\begin{array}{l}3.1 \\
2.3 \\
1.8 \\
3.1 \\
2.8\end{array}$ & $\begin{array}{l}1.9 \\
2.1 \\
1.9 \\
1.9 \\
1.9\end{array}$ & $\begin{array}{l}2.6 \\
2.4 \\
2.3 \\
2.3 \\
2.6\end{array}$ & $\begin{array}{l}2.9 \\
2.5 \\
2.3 \\
2.4 \\
2.4\end{array}$ & $\begin{array}{l}1.9 \\
1.9 \\
1.9 \\
1.9 \\
1.9\end{array}$ & $\begin{array}{l}2.1 \\
1.9 \\
2.1 \\
2.1 \\
2.1\end{array}$ & $\begin{array}{l}1.6 \\
1.7 \\
1.9 \\
1.7 \\
1.7\end{array}$ & $\begin{array}{l}1.6 \\
1.5 \\
1.7 \\
1.8 \\
1.7\end{array}$ & $\begin{array}{l}2.3 \\
2.3 \\
1.9 \\
1.7 \\
2.1\end{array}$ & $\begin{array}{l}3.7 \\
3.6 \\
3.4 \\
3.1 \\
3.1\end{array}$ \\
\hline $\begin{array}{l}26 \ldots \ldots \ldots \ldots \\
27 \\
28 \ldots \ldots \ldots \\
29 \\
30 \ldots \ldots \ldots \ldots \\
31 \ldots \ldots \ldots \\
\end{array}$ & $\begin{array}{l}2.6 \\
1.9 \\
1.9 \\
2.2 \\
2.2 \\
3.2\end{array}$ & \begin{tabular}{r}
4.1 \\
3.6 \\
2.9 \\
$\cdots \cdots$ \\
\hdashline$\ldots . .$. \\
$\ldots .$.
\end{tabular} & $\begin{array}{l}3.2 \\
4.4 \\
3.6 \\
2.6 \\
2.6 \\
2.5\end{array}$ & $\begin{array}{l}1.7 \\
1.6 \\
1.6 \\
1.7 \\
.8\end{array}$ & $\begin{array}{l}2.4 \\
2.5 \\
2.4 \\
2.4 \\
2.4 \\
2.6\end{array}$ & $\begin{array}{l}2.5 \\
2.4 \\
2.5 \\
2.6 \\
2.4\end{array}$ & $\begin{array}{l}1.9 \\
1.9 \\
2.1 \\
2.1 \\
1.9 \\
1.9\end{array}$ & $\begin{array}{l}2.1 \\
2.0 \\
2.0 \\
1.9 \\
2.0 \\
2.1\end{array}$ & $\begin{array}{l}1.6 \\
1.6 \\
1.6 \\
1.6 \\
1.6\end{array}$ & $\begin{array}{l}1.6 \\
1.6 \\
1.7 \\
1.6 \\
1.4 \\
1.4\end{array}$ & $\begin{array}{l}4.8 \\
1.9 \\
1.7 \\
1.6 \\
1.9\end{array}$ & $\begin{array}{l}2.8 \\
3.1 \\
3.1 \\
3.1 \\
3.1 \\
3.1\end{array}$ \\
\hline
\end{tabular}

Note.-No record January 1-13.

Monthly discharge of Makawao Stream in Kailua Valley, near Kailua, Oahu, for 1913.

\begin{tabular}{|c|c|c|c|c|c|}
\hline \multirow{2}{*}{ Month. } & \multicolumn{3}{|c|}{ Discharge in second-feet. } & \multirow{2}{*}{$\begin{array}{l}\text { Run-off } \\
\text { (total in } \\
\text { acre-feet). }\end{array}$} & \multirow{2}{*}{$\begin{array}{l}\text { Accu- } \\
\text { racy. }\end{array}$} \\
\hline & Maximum. & Minimum. & Mean. & & \\
\hline 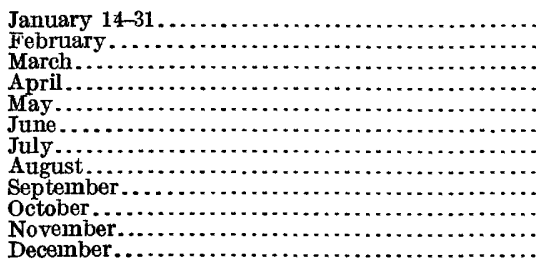 & \begin{tabular}{c|}
19 \\
14 \\
14 \\
6.1 \\
48 \\
20 \\
3.4 \\
4.1 \\
3.4 \\
1.8 \\
4.8 \\
27
\end{tabular} & $\begin{array}{l}1.1 \\
1.9 \\
.8 \\
1.7 \\
2.3 \\
1.9 \\
1.9 \\
1.6 \\
1.4 \\
1.3 \\
2.1\end{array}$ & $\begin{array}{l}4.83 \\
5.14 \\
3.00 \\
2.29 \\
5.16 \\
3.95 \\
2.21 \\
2.12 \\
1.76 \\
1.60 \\
1.80 \\
6.32\end{array}$ & \begin{tabular}{r|}
172 \\
285 \\
184 \\
136 \\
317 \\
235 \\
136 \\
130 \\
105 \\
98 \\
107 \\
389
\end{tabular} & $\begin{array}{l}\text { B. } \\
\text { B. } \\
\text { B. } \\
\text { B. } \\
\text { B. } \\
\text { B. } \\
\text { B. } \\
\text { B. } \\
\text { B. } \\
\text { B. } \\
\text { B. }\end{array}$ \\
\hline The perioc & & & & 2,290 & \\
\hline
\end{tabular}


KAIMI STREAM IN KAILUA VALLEY, NEAR KaILUA, OAHU.

Location.-At highway bridge on Waimanalo-Honolulu government road, about $12 \frac{1}{2}$ miles east of Honolulu.

Records available.-November 12, 1912, to December 31, 1913.

Gage.-Vertical staff, read twice daily. Datum raised 1.00 foot April 10, 1913; that is, 1.50 feet gage height previous to April 10 equals 0.50 foot gage heights after that date.

Control.-Fairly permanent.

Discharge measurements.--During low water, made by wading; during floods, from bridge.

Diversions.-Headwaters diverted by Makawao ditch.

Accuracy.-Records poor.

Cooperation.-Maintained in cooperation with the Kaneohe Ranch Co.

Discharge measurements of Kaimi Stream in Kailua Valley, near Kailua, Oahu, in 1913.

\begin{tabular}{|c|c|c|c|c|c|c|c|}
\hline Date. & Hydrographer. & $\begin{array}{c}\text { Gage } \\
\text { height. }\end{array}$ & $\begin{array}{c}\text { Dis- } \\
\text { charge. }\end{array}$ & Date. & Hydrographer. & $\begin{array}{c}\text { Gage } \\
\text { height. }\end{array}$ & $\begin{array}{l}\text { Dis- } \\
\text { charge. }\end{array}$ \\
\hline $\begin{array}{l}\text { Apr. } 11 \\
\text { June } 4\end{array}$ & 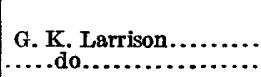 & $\begin{array}{c}\text { Feet. } \\
0.75 \\
1.08\end{array}$ & $\begin{array}{r}\text { Sec.-ft. } \\
1.12 \\
5.55\end{array}$ & Sept. 9 & J. C. Dort.. & $\begin{array}{l}\text { Feet. } \\
0.82\end{array}$ & $\begin{array}{r}S e c .-f t . \\
1.67\end{array}$ \\
\hline
\end{tabular}

Daily discharge, in second-feet, of Kaimi Stream in Kailua Valley, near Kailua, Oahu, for 1912-13.

\begin{tabular}{|c|c|c|c|c|c|c|c|c|}
\hline Day. & Nov. & Dec. & Day. & Nov. & Dec. & Day. & Nov. & Dec. \\
\hline 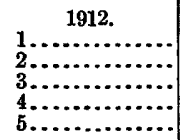 & $\cdots$ & $\begin{array}{l}18 \\
1.7 \\
21 \\
4.3 \\
4.0\end{array}$ & 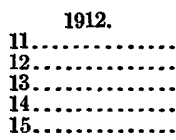 & $\begin{array}{l}1.0 \\
1.0 \\
1.0 \\
1.8\end{array}$ & $\begin{array}{r}1.5 \\
.8 \\
.8 \\
.8 \\
2.4\end{array}$ & $\begin{array}{c}1912 . \\
21 \ldots \ldots \ldots \ldots \ldots \\
22 \ldots \ldots \ldots \ldots \ldots \\
23 \ldots \ldots \ldots \ldots \ldots \ldots \\
25 \ldots \ldots \ldots \ldots \ldots \ldots\end{array}$ & $\begin{array}{l}1.5 \\
1.6 \\
1.3 \\
1.1 \\
1.2\end{array}$ & $\begin{array}{l}2.6 \\
2.5 \\
2.4 \\
2.6 \\
2.6\end{array}$ \\
\hline $\begin{array}{r}6 \ldots \ldots \ldots \\
7 \ldots \ldots \ldots \\
8 \ldots \ldots \ldots \\
10 \ldots \ldots\end{array}$ & & $\begin{array}{l}1.9 \\
2.4 \\
2.5 \\
2.5 \\
2.6\end{array}$ & $\begin{array}{l}16 \ldots \ldots \\
18 \ldots \ldots \\
19 \\
19 \\
20 \ldots \ldots\end{array}$ & $\begin{array}{l}1.0 \\
1.0 \\
1.0 \\
1.1 \\
1.0\end{array}$ & $\begin{array}{l}2.5 \\
2.6 \\
2.5 \\
3.4 \\
2.5\end{array}$ & $\begin{array}{l}26 \\
27 \\
28 \\
29 \\
30 \ldots \ldots \ldots \ldots\end{array}$ & $\begin{array}{l}1.1 \\
1.6 \\
2.8 \\
2.6 \\
1.9 \\
\cdots\end{array}$ & $\begin{array}{l}2.6 \\
2.6 \\
2.0 \\
2.0 \\
2.0 \\
2.4\end{array}$ \\
\hline
\end{tabular}


Daily discharge, in second-feet, of Kaimi Stream in Kailua Valley, near Kailua, Oahu, for 1912-13-Continued.

\begin{tabular}{|c|c|c|c|c|c|c|c|c|c|c|c|c|}
\hline Day. & Jan. & Feb. & Mar. & Apr. & May. & June. & July. & Aug. & Sept. & Oet. & Nov. & Dec. \\
\hline 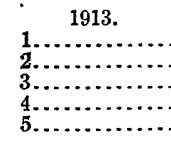 & 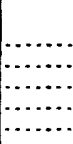 & $\begin{array}{l}2.0 \\
2.0 \\
1.7 \\
1.7 \\
3.2\end{array}$ & $\begin{array}{l}2.9 \\
2.8 \\
2.4 \\
2.4 \\
2.8\end{array}$ & $\begin{array}{l}1.4 \\
1.4 \\
1.4 \\
1.4 \\
1.7\end{array}$ & $\begin{array}{l}1.2 \\
2.2 \\
1.3 \\
1.2 \\
1.5\end{array}$ & $\begin{array}{r}1.5 \\
1.5 \\
12 \\
9.6 \\
3.6\end{array}$ & $\begin{array}{l}1.9 \\
2.4 \\
1.7 \\
1.8 \\
1.8\end{array}$ & $\begin{array}{l}1.6 \\
1.5 \\
1.5 \\
1.5 \\
1.5\end{array}$ & $\begin{array}{l}3.2 \\
4.3 \\
1.9 \\
1.5 \\
1.7\end{array}$ & $\begin{array}{l}1.4 \\
1.4 \\
1.4 \\
1.4 \\
1.4\end{array}$ & $\begin{array}{l}1.4 \\
1.5 \\
1.5 \\
1.5 \\
1.5\end{array}$ & $\begin{array}{l}2.9 \\
2.5 \\
2.6 \\
23 \\
24\end{array}$ \\
\hline $\begin{array}{l}6 \ldots \ldots \\
7 \ldots \ldots \\
8 \ldots \ldots \\
9 \ldots \ldots \\
0 \ldots \ldots\end{array}$ & & $\begin{array}{l}10 \\
8.4 \\
3.4 \\
2.4 \\
1.5\end{array}$ & $\begin{array}{l}2.4 \\
1.9 \\
1.6 \\
1.4 \\
2.4\end{array}$ & $\begin{array}{l}2.2 \\
1.7 \\
1.2 \\
.9 \\
1.0\end{array}$ & $\begin{array}{l}1.2 \\
1.4 \\
15 \\
16 \\
14\end{array}$ & $\begin{array}{l}4.8 \\
4.1 \\
3.6 \\
2.4 \\
2.5\end{array}$ & $\begin{array}{l}1.7 \\
1.5 \\
1.7 \\
1.7 \\
1.5\end{array}$ & $\begin{array}{l}1.4 \\
1.4 \\
1.5 \\
1.4 \\
1.5\end{array}$ & $\begin{array}{l}1.6 \\
1.7 \\
1.6 \\
1.4 \\
1.5\end{array}$ & $\begin{array}{l}1.4 \\
1.5 \\
1.5 \\
1.4 \\
1.5\end{array}$ & $\begin{array}{l}1.4 \\
1.5 \\
2.9 \\
2.4 \\
1.7\end{array}$ & $\begin{array}{r}15 \\
25 \\
13 \\
7.8 \\
5.5\end{array}$ \\
\hline $\begin{array}{l}11 \ldots \ldots \\
12 \ldots \ldots \\
14 \ldots \ldots\end{array}$ & $\begin{array}{r}0.8 \\
0.8\end{array}$ & $\begin{array}{r}.9 \\
1.2 \\
.7 \\
1.4 \\
2.6\end{array}$ & $\begin{array}{r}2.4 \\
1.7 \\
1.6 \\
1.4 \\
.9\end{array}$ & $\begin{array}{l}1.2 \\
1.7 \\
3.8 \\
1.2 \\
1.2\end{array}$ & $\begin{array}{l}1.4 \\
26 \\
10 \\
18 \\
19\end{array}$ & $\begin{array}{l}2.8 \\
2.9 \\
4.0 \\
4.1 \\
4.5\end{array}$ & $\begin{array}{l}1.5 \\
1.5 \\
1.5 \\
1.5 \\
1.5\end{array}$ & $\begin{array}{l}1.4 \\
2.9 \\
1.9 \\
1.4 \\
1.4\end{array}$ & $\begin{array}{l}1.5 \\
1.4 \\
1.5 \\
1.6 \\
1.5\end{array}$ & $\begin{array}{l}1.5 \\
1.5 \\
1.5 \\
1.5 \\
1.5\end{array}$ & $\begin{array}{l}1.7 \\
1.7 \\
1.7 \\
1.5 \\
1.5\end{array}$ & $\begin{array}{l}5.2 \\
4.1 \\
4.0 \\
3.6 \\
3.5\end{array}$ \\
\hline $\begin{array}{l}16 \ldots \ldots \\
17 \ldots \ldots \ldots \\
19 \ldots \ldots \\
20 \ldots \ldots\end{array}$ & $\begin{array}{l}10 \\
1.2 \\
1.2 \\
10 \\
2.6\end{array}$ & $\begin{array}{r}12 \\
7.0 \\
5.0 \\
3.8 \\
3.8\end{array}$ & $\begin{array}{r}14.8 \\
5.0 \\
3.8 \\
2.8\end{array}$ & $\begin{array}{r}4.8 \\
1.3 \\
1.1 \\
.9 \\
1.1\end{array}$ & $\begin{array}{l}3.5 \\
5.0 \\
4.1 \\
3.8 \\
3.6\end{array}$ & $\begin{array}{l}4.1 \\
2.5 \\
1.9 \\
2.0 \\
7.6\end{array}$ & $\begin{array}{l}1.5 \\
1.6 \\
1.5 \\
1.5 \\
1.4\end{array}$ & $\begin{array}{l}1.5 \\
2.2 \\
2.2 \\
1.5 \\
1.6\end{array}$ & $\begin{array}{l}1.4 \\
1.4 \\
1.4 \\
1.7 \\
1.7\end{array}$ & $\begin{array}{l}1.5 \\
1.4 \\
1.5 \\
1.4 \\
1.4\end{array}$ & $\begin{array}{l}1.6 \\
1.7 \\
1.7 \\
2.2 \\
3.2\end{array}$ & $\begin{array}{l}3.2 \\
4.3 \\
5.2 \\
5.2 \\
5.2\end{array}$ \\
\hline $\begin{array}{l}21 \ldots \ldots \ldots \ldots \\
22, \ldots \ldots \ldots \ldots \\
24 \ldots \ldots \ldots \ldots \\
25 \ldots \ldots \ldots \ldots\end{array}$ & $\begin{array}{l}2.6 \\
2.6 \\
2.1 \\
2.6 \\
2.4\end{array}$ & $\begin{array}{l}5.0 \\
3.8 \\
3.5 \\
2.6 \\
5.0\end{array}$ & $\begin{array}{l}2.2 \\
1.4 \\
.9 \\
1.7 \\
1.2\end{array}$ & $\begin{array}{r}.9 \\
.9 \\
1.2 \\
1.2 \\
1.0\end{array}$ & $\begin{array}{l}1.9 \\
1.7 \\
1.7 \\
1.7 \\
1.6\end{array}$ & $\begin{array}{l}2.2 \\
2.2 \\
1.9 \\
1.6 \\
1.6\end{array}$ & $\begin{array}{l}1.5 \\
1.4 \\
1.4 \\
1.4 \\
1.4\end{array}$ & $\begin{array}{l}1.5 \\
1.3 \\
1.4 \\
1.4 \\
1.4\end{array}$ & $\begin{array}{l}1.7 \\
1.7 \\
1.9 \\
1.5 \\
1.9\end{array}$ & $\begin{array}{l}1.5 \\
1.5 \\
1.5 \\
1.5 \\
1.5\end{array}$ & $\begin{array}{l}3.2 \\
3.4 \\
2.9 \\
2.2 \\
2.9\end{array}$ & $\begin{array}{l}5.2 \\
4.8 \\
4.5 \\
4.5 \\
4.3\end{array}$ \\
\hline $\begin{array}{l}26 \ldots \ldots \\
27 \ldots \ldots \\
28 \ldots \\
29 \ldots \\
30 \ldots \\
31 \ldots\end{array}$ & $\begin{array}{l}3.4 \\
2.9 \\
2.6 \\
2.8 \\
2.6 \\
3.4\end{array}$ & $\begin{array}{r}4.8 \\
3.8 \\
3.2 \\
\hdashline \ldots . .\end{array}$ & $\begin{array}{l}1.5 \\
3.8 \\
3.5 \\
2.4 \\
2.0 \\
1.6\end{array}$ & $\begin{array}{l}1.4 \\
1.4 \\
1.4 \\
1.2 \\
1.1\end{array}$ & $\begin{array}{l}1.4 \\
1.4 \\
1.5 \\
1.4 \\
1.4 \\
1.4\end{array}$ & $\begin{array}{l}1.7 \\
1.7 \\
1.7 \\
1.9 \\
1.8\end{array}$ & $\begin{array}{l}1.4 \\
1.2 \\
1.5 \\
1.5 \\
1.5 \\
1.5\end{array}$ & $\begin{array}{l}1.4 \\
1.4 \\
1.4 \\
1.4 \\
1.4 \\
1.4\end{array}$ & $\begin{array}{l}1.6 \\
1.6 \\
1.2 \\
1.3 \\
1.2\end{array}$ & $\begin{array}{l}1.4 \\
1.4 \\
1.5 \\
1.5 \\
1.4 \\
1.4\end{array}$ & $\begin{array}{l}9.0 \\
2.4 \\
2.5 \\
2.3 \\
2.4\end{array}$ & $\begin{array}{l}4.1 \\
4.0 \\
4.1 \\
3.8 \\
4.0 \\
4.1\end{array}$ \\
\hline
\end{tabular}

Nore.-No record Jan. 1-13, 1913.

Monthly discharge of Kaimi Stream in Kailua Valley, near Kailua, Oahu, for 1913.

\begin{tabular}{|c|c|c|c|c|c|}
\hline \multirow{2}{*}{ Month. } & \multicolumn{3}{|c|}{ Discharge in second-feet. } & \multirow{2}{*}{$\begin{array}{c}\text { Run-off } \\
\text { (total in } \\
\text { acre-feet). }\end{array}$} & \multirow{2}{*}{$\begin{array}{l}\text { Accu- } \\
\text { racy. }\end{array}$} \\
\hline & Maximum. & Minimum. & Mean. & & \\
\hline \multirow[t]{2}{*}{ 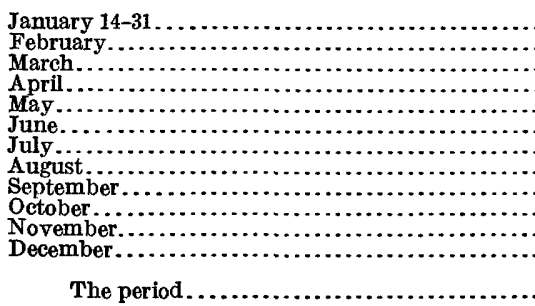 } & $\begin{array}{c}10 \\
12 \\
14 \\
4.8 \\
26 \\
12 \\
2.4 \\
2.9 \\
4.3 \\
1.5 \\
9.0 \\
25\end{array}$ & $\begin{array}{r}0.8 \\
.7 \\
.8 \\
.9 \\
1.2 \\
1.5 \\
1.2 \\
1.3 \\
1.2 \\
1.4 \\
1.4 \\
2.5\end{array}$ & $\begin{array}{l}3.15 \\
3.80 \\
2.57 \\
1.48 \\
5.37 \\
3.34 \\
1.56 \\
1.55 \\
1.70 \\
1.45 \\
2.30 \\
6.85\end{array}$ & $\begin{array}{c}112 \\
211 \\
158 \\
88.1 \\
330 \\
199 \\
95.9 \\
95.3 \\
101 \\
89.2 \\
137 \\
421\end{array}$ & \multirow[t]{2}{*}{$\begin{array}{l}\text { C. } \\
\mathbf{c} . \\
\mathbf{C} . \\
\mathbf{c} . \\
\mathbf{c} . \\
\mathbf{c} . \\
\mathbf{c} . \\
\mathbf{c} . \\
\mathbf{C} . \\
\mathbf{C} . \\
\mathbf{c} . \\
\mathbf{c} .\end{array}$} \\
\hline & & & & 2,040 & \\
\hline
\end{tabular}


KAMAKALEPO STREAM IN KAILUA VALLEY, NEAR KAILUA, OAHU.

Location.-At highway bridge on Waimanalo-Honolulu government road, and about $3 \frac{1}{2}$ miles from Waimanalo.

Records available.-November 12 to December 3, 1912; April 9 to December 31, 1913.

Gage.-Vertical staff bolted to left abutment of bridge, installed April 10, 1913, to replace original gage washed out December 3, 1912; new datum.

Control.-Shifting.

Discharge measurements.-Made by wading or from highway bridge.

Diversions.-None above station.

Accuracy.-Records good.

Cooperation.-Station maintained in cooperation with Kaneohe Ranch Co.

Discharge measurements of Kamakalepo Stream in Kailua Valley, near Kailua, Oahu, in 1913.

\begin{tabular}{|c|c|c|c|c|c|c|c|}
\hline Date. & Hydrographer. & $\begin{array}{c}\text { Gage } \\
\text { height. }\end{array}$ & $\begin{array}{c}\text { Dis- } \\
\text { charge. }\end{array}$ & Date. & Hydrographer. & $\begin{array}{c}\text { Gage } \\
\text { height. }\end{array}$ & $\begin{array}{l}\text { Dis- } \\
\text { charge. }\end{array}$ \\
\hline $\begin{array}{l}\text { Apr. } 11 \\
\text { June } 4\end{array}$ & 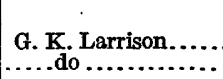 & $\begin{array}{l}\text { Feet. } \\
1.29 \\
1.50\end{array}$ & $\begin{array}{r}\text { Sec.-ft. } \\
1.33 \\
4.91\end{array}$ & $\begin{array}{l}\text { Sept. } 9 \\
\text { Dec. } 10\end{array}$ & $\begin{array}{l}\text { J. C. Dort } \\
\text { G. R. White.. }\end{array}$ & $\begin{array}{c}\text { Feet. } \\
1.30 \\
1.60\end{array}$ & $\begin{array}{r}\text { Sec.-ft. } \\
1.7 \\
7.3 t\end{array}$ \\
\hline
\end{tabular}

Daily discharge, in second-feet, of Kamakalepo Stream in Kailua Valley, near Kailua, Oahu, for 1913.

\begin{tabular}{|c|c|c|c|c|c|c|c|c|c|}
\hline Day. & Apr. & May. & June. & July. & Aug. & Sept. & Oct. & Nov. & Dee. \\
\hline $\begin{array}{l}1 \\
4 \\
4 \\
5\end{array}$ & ……... & $\begin{array}{l}1.3 \\
2.1 \\
1.4 \\
1.5 \\
1.5\end{array}$ & $\begin{array}{c}1.7 \\
1.9 \\
17 \\
19 \\
2.4\end{array}$ & $\begin{array}{l}2.4 \\
3.9 \\
2.4 \\
1.9 \\
1.7\end{array}$ & $\begin{array}{r}2.1 \\
2.0 \\
1.9 \\
1.9 \\
-1.9\end{array}$ & $\begin{array}{l}2.6 \\
6.3 \\
2.2 \\
1.6 \\
1.6\end{array}$ & $\begin{array}{l}0.9 \\
.8 \\
.7 \\
.7 \\
.7\end{array}$ & $\begin{array}{r}0.5 \\
.6 \\
.5 \\
.5 \\
.5\end{array}$ & $\begin{array}{l}3.7 \\
3.1 \\
4.3 \\
12 \\
13\end{array}$ \\
\hline $\begin{array}{r}6 \\
7 \\
8 \\
9 \\
9 \\
10 \ldots \ldots \ldots \ldots\end{array}$ & $\begin{array}{l}1.6 \\
1.4\end{array}$ & $\begin{array}{c}1.2 \\
1.5 \\
12 \\
16 \\
6.1\end{array}$ & $\begin{array}{l}2.6 \\
2.8 \\
2.5 \\
3.1 \\
3.3\end{array}$ & $\begin{array}{l}1.7 \\
1.6 \\
1.6 \\
1.9 \\
1.6\end{array}$ & $\begin{array}{l}1.6 \\
1.9 \\
1.6 \\
1.4 \\
1.5\end{array}$ & $\begin{array}{l}1.6 \\
1.4 \\
1.6 \\
1.5 \\
1.5\end{array}$ & $\begin{array}{l}.7 \\
.8 \\
.7 \\
.6 \\
.8\end{array}$ & $\begin{array}{r}.5 \\
.5 \\
1.4 \\
1.1 \\
.6\end{array}$ & $\begin{array}{c}19 \\
26 \\
13 \\
7.8 \\
5.4\end{array}$ \\
\hline $\begin{array}{l}11 \ldots \ldots \ldots \ldots \\
12 \ldots \ldots \ldots \ldots \ldots \\
13 \ldots \ldots \ldots \ldots \ldots \\
15 \ldots \ldots \ldots \ldots \ldots\end{array}$ & $\begin{array}{l}1.5 \\
2.1 \\
4.5 \\
1.5 \\
1.7\end{array}$ & $\begin{array}{l}2.2 \\
36 \\
18 \\
18 \\
16\end{array}$ & $\begin{array}{l}3.1 \\
3.1 \\
3.1 \\
2.9 \\
2.9\end{array}$ & $\begin{array}{l}1.5 \\
1.6 \\
1.6 \\
1.9 \\
2.1\end{array}$ & $\begin{aligned} 1.6 \\
.2 .4 \\
\cdot 1.9 \\
1.3 \\
1.4\end{aligned}$ & $\begin{array}{l}1.4 \\
1.4 \\
1.4 \\
1.6 \\
1.6\end{array}$ & $\begin{array}{l}.9 \\
.7 \\
.7 \\
.6\end{array}$ & $\begin{array}{l}.7 \\
.7 \\
.7 \\
.7 \\
.8\end{array}$ & $\begin{array}{l}3.3 \\
2.8 \\
2.4 \\
1.9 \\
1.3\end{array}$ \\
\hline $\begin{array}{l}16 \\
17 \ldots \ldots \ldots \ldots \ldots \ldots \ldots \ldots \ldots \ldots \ldots \ldots\end{array}$ & $\begin{array}{l}2.2 \\
2.1 \\
2.0 \\
1.6 \\
1.7\end{array}$ & $\begin{array}{l}2.4 \\
4.3 \\
3.3 \\
3.3 \\
2.9\end{array}$ & $\begin{array}{l}3.3 \\
2.9 \\
2.8 \\
2.9 \\
9.5\end{array}$ & $\begin{array}{l}1.7 \\
1.7 \\
1.9 \\
2.1 \\
1.6\end{array}$ & $\begin{array}{r}1.6 \\
-2.2 \\
2.4 \\
1.6 \\
1.6\end{array}$ & $\begin{array}{l}1.4 \\
1.3 \\
1.2 \\
1.6 \\
1.6\end{array}$ & $\begin{array}{l}.6 \\
.6 \\
.6 \\
.6 \\
.6\end{array}$ & $\begin{array}{r}.9 \\
.9 \\
1.2 \\
1.2 \\
2.0\end{array}$ & $\begin{array}{l}1.2 \\
2.1 \\
2.9 \\
2.6 \\
2.4\end{array}$ \\
\hline $\begin{array}{l}21 \ldots \ldots \ldots \ldots \\
22, \ldots \ldots \ldots \ldots \ldots \\
23 \ldots \ldots \ldots \ldots \ldots \\
25 \ldots \ldots \ldots \ldots \ldots\end{array}$ & $\begin{array}{l}1.4 \\
1.4 \\
1.7 \\
1.6 \\
1.7\end{array}$ & $\begin{array}{l}2.8 \\
2.6 \\
2.8 \\
2.6 \\
2.5\end{array}$ & $\begin{array}{l}3.1 \\
2.6 \\
2.5 \\
2.6 \\
2.4\end{array}$ & $\begin{array}{l}1.6 \\
1.5 \\
1.4 \\
1.3 \\
1.4\end{array}$ & $\begin{array}{l}1.6 \\
1.5 \\
1.5 \\
1.6 \\
1.4\end{array}$ & $\begin{array}{l}1.4 \\
1.5 \\
1.7 \\
1.3 \\
1.6\end{array}$ & $\begin{array}{l}.6 \\
.6 \\
.7 \\
.8 \\
.9\end{array}$ & $\begin{array}{l}1.2 \\
1.2 \\
1.2 \\
1.6 \\
4.1\end{array}$ & $\begin{array}{l}2.4 \\
2.2 \\
2.1 \\
2.1 \\
1.9\end{array}$ \\
\hline $\begin{array}{l}26 \ldots \\
27 \ldots \\
28 \ldots \\
29 \ldots \\
30 \ldots \\
31 \ldots\end{array}$ & $\begin{array}{l}1.5 \\
1.5 \\
1.5 \\
1.5 \\
1.4\end{array}$ & $\begin{array}{l}2.2 \\
1.9 \\
2.0 \\
2.0 \\
2.0 \\
1.9\end{array}$ & $\begin{array}{l}2.5 \\
2.4 \\
2.4 \\
2.5 \\
2.4\end{array}$ & $\begin{array}{l}1.4 \\
1.4 \\
1.5 \\
1.5 \\
1.5 \\
1.6\end{array}$ & $\begin{array}{l}1.6 \\
1.6 \\
1.4 \\
1.6 \\
1.4 \\
1.4\end{array}$ & $\begin{array}{l}1.5 \\
1.4 \\
1.0 \\
1.1 \\
1.1\end{array}$ & $\begin{array}{l}.6 \\
.6 \\
.6 \\
.6\end{array}$ & $\begin{array}{l}8.9 \\
4.5 \\
3.7 \\
2.4 \\
3.3\end{array}$ & $\begin{array}{l}1.6 \\
1.6 \\
1.6 \\
1.5 \\
1.5 \\
1.6\end{array}$ \\
\hline
\end{tabular}


Monthly discharge of Kamakalepo Stream in Kailua Valley, near Kailua, Oahu, for 1913.

\begin{tabular}{|c|c|c|c|c|c|}
\hline \multirow{2}{*}{ Month. } & \multicolumn{3}{|c|}{ Discharge in second-feet. } & \multirow{2}{*}{$\begin{array}{l}\text { Run-off } \\
\text { (total in } \\
\text { acre-fe日t). }\end{array}$} & \multirow{2}{*}{$\begin{array}{l}\text { Accu- } \\
\text { racy. }\end{array}$} \\
\hline & Maximum. & Minimum. & Mean. & & \\
\hline 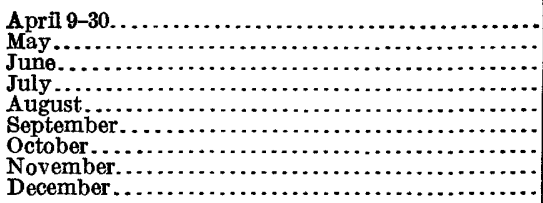 & \begin{tabular}{l|}
4.5 \\
36 \\
19 \\
3.9 \\
2.4 \\
6.3 \\
.9 \\
8.9
\end{tabular} & $\begin{array}{r}1.4 \\
1.2 \\
1.7 \\
1.3 \\
1.3 \\
1.0 \\
.6 \\
.5 \\
1.2\end{array}$ & $\begin{array}{l}1.78 \\
5.69 \\
3.94 \\
1.76 \\
1.69 \\
1.67 \\
.68 \\
1.62 \\
4.85\end{array}$ & $\begin{array}{l}77.6 \\
350 \\
234 \\
108 \\
104 \\
99.4 \\
41.8 \\
96.4 \\
298\end{array}$ & \multirow[t]{2}{*}{$\begin{array}{l}\mathbf{B} . \\
\mathbf{B} . \\
\mathbf{B} . \\
\mathbf{B} . \\
\mathbf{B} . \\
\mathbf{B} . \\
\mathbf{B} . \\
\mathbf{B} . \\
\mathbf{B} .\end{array}$} \\
\hline The period ...... & . & $\ldots .$. & $\because$ & 1,410 & \\
\hline
\end{tabular}

POHAKEA STREAM IN KAILUA VALLEY, NEAR KAILUA, OAHU.

Location.-One-half mile above highway bridge on the Waimanalo road.

Records available.-November 12, 1912, to December 31, 1913.

Gage.-Vertical staff, read twice daily; datum changed November 24, when 1-foot sharp-crested weir was established. Gage heights from November 25 to December 31 show head on crest of weir.

Control.-Fairly permanent.

Discharge measurements.-Made by wading with current-meter until November 24 , after which low-water discharge was measured by sharp-crested weir (1-foot crest) with end contractions.

Diversions.-None above station; two small ditches below.

Accuracy.-Records poor.

Cooperation.-Maintained in cooperation with the Kaneohe Ranch Co.

Discharge measurements of Pohakea Stream in Kailua Valley, near Kailua, Oahu, in 1913.

\begin{tabular}{|c|c|c|c|c|c|c|c|}
\hline Date. & Hydrographer. & $\begin{array}{c}\text { Gage } \\
\text { height. }\end{array}$ & $\begin{array}{l}\text { Dis- } \\
\text { charge. }\end{array}$ & Date. & Hydrographer. & $\begin{array}{c}\text { Gage } \\
\text { height. }\end{array}$ & Dis- \\
\hline$\underset{\text { June }}{\text { Apr. }} 11$ & 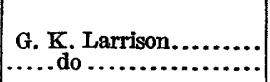 & $\begin{array}{c}\text { Feet. } \\
1.20 \\
1.68\end{array}$ & $\begin{array}{r}\text { Sec.-ft. } \\
0.24 \\
1.50\end{array}$ & Sept. 9 & J. C. Dort...... & $\begin{array}{c}\text { Feet. } \\
1.16\end{array}$ & $\begin{array}{r}\text { Sec. fft. } \\
0.22\end{array}$ \\
\hline
\end{tabular}

Daily discharge, in second-feet, of Pohakea Stream in Kailua Valley, near Kailua, Oahu, for 1912-13.

\begin{tabular}{|c|c|c|c|c|c|c|c|c|}
\hline Day. & Nov. & Dec. & Day. & Nov. & Dec. & Day. & Nov. & Dec. \\
\hline 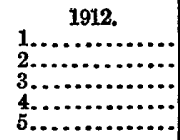 & $\begin{array}{c}\cdots \\
\cdots \\
\cdots\end{array}$ & $\begin{array}{r}1.34 \\
.24 \\
1.51 \\
.90 \\
.82\end{array}$ & $\begin{array}{l}1912 . \\
11 \ldots \ldots \ldots \\
12 \ldots \ldots \ldots \\
13 \ldots \ldots \ldots \ldots \\
14 \ldots \ldots \ldots \ldots \ldots\end{array}$ & $\begin{array}{r}0.08 \\
.08 \\
.06 \\
.12\end{array}$ & $\begin{array}{l}0.33 \\
.28 \\
.33 \\
.28 \\
.25\end{array}$ & 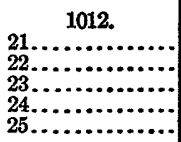 & $\begin{array}{r}0.19 \\
.17 \\
.14 \\
.11 \\
.11\end{array}$ & $\begin{array}{l}0.25 \\
.24 \\
.23 \\
.23 \\
.23\end{array}$ \\
\hline $\begin{array}{r}6 \ldots \ldots . \\
7 \ldots \ldots \ldots \\
9 \ldots \ldots \\
10 \ldots \ldots\end{array}$ & $\cdots$ & $\begin{array}{l}.49 \\
.49 \\
.42 \\
.37 \\
.34\end{array}$ & $\begin{array}{l}16 \ldots \ldots \\
17 \ldots \ldots \\
19 \ldots \ldots \\
20 \ldots \ldots\end{array}$ & $\begin{array}{l}.08 \\
.08 \\
.06 \\
.07 \\
.07\end{array}$ & $\begin{array}{l}.25 \\
.25 \\
.24 \\
.24 \\
.23\end{array}$ & $\begin{array}{l}26 \ldots \ldots \ldots \\
27 \ldots \ldots \ldots \ldots \\
28 \ldots \ldots \ldots \ldots \\
29 \ldots \ldots \ldots \ldots \\
30 \ldots \ldots\end{array}$ & $\begin{array}{l}.10 \\
.10 \\
.11 \\
.23 \\
.13\end{array}$ & $\begin{array}{l}.23 \\
.20 \\
.20 \\
.20 \\
.20 \\
.20\end{array}$ \\
\hline
\end{tabular}


ISLAND OF OAHU.

Daily discharge, in second-feet, of Pohakea Stream in Kailua Valley. near Kailua, Oahu, for 1912-13-Continued.

\begin{tabular}{|c|c|c|c|c|c|c|c|c|c|c|c|c|}
\hline Day. & Jan. & Feb. & Mar. & Apr. & May. & June. & July. & Aug. & Sept. & Oct. & Nov. & Dec. \\
\hline $\begin{array}{c} \\
191 \\
1 \ldots \ldots \ldots \\
2 \ldots \ldots \ldots \\
3 \ldots \ldots \ldots \\
4 \ldots \ldots \ldots \\
5 \ldots \ldots \ldots\end{array}$ & & $\begin{array}{r}0.13 \\
.13 \\
.12 \\
.11 \\
.15\end{array}$ & $\begin{array}{l}0.24 \\
.25 \\
.24 \\
.25 \\
.23\end{array}$ & $\begin{array}{l}0.29 \\
.33 \\
.34 \\
.33 \\
.38\end{array}$ & $\begin{array}{l}0.20 \\
.23 \\
.22 \\
.20 \\
.20\end{array}$ & $\begin{array}{r}0.28 \\
.29 \\
1.72 \\
2.02 \\
.74\end{array}$ & $\begin{array}{r}0.28 \\
.29 \\
.23 \\
.23 \\
.23\end{array}$ & $\begin{array}{l}0.20 \\
.19 \\
.17 \\
.20 \\
.20\end{array}$ & $\begin{array}{l}0.28 \\
.45 \\
.20 \\
.19 \\
.20\end{array}$ & $\begin{array}{l}0.19 \\
.17 \\
.17 \\
.17 \\
.17\end{array}$ & $\begin{array}{l}0.15 \\
.15 \\
.15 \\
.14 \\
.12\end{array}$ & $\begin{array}{r}0.44 \\
.39 \\
.72 \\
.66 \\
.72\end{array}$ \\
\hline $\begin{array}{r}6 \ldots \ldots \\
7 \ldots \ldots \\
9 \ldots \ldots \ldots \\
10 \ldots \ldots\end{array}$ & 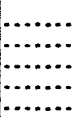 & $\begin{array}{l}.90 \\
.98 \\
.44 \\
.29 \\
.22\end{array}$ & $\begin{array}{l}.20 \\
.15 \\
.14 \\
.16 \\
.19\end{array}$ & $\begin{array}{l}.45 \\
.38 \\
.33 \\
.28 \\
.28\end{array}$ & $\begin{array}{r}.20 \\
.19 \\
.23 \\
2.02 \\
1.44\end{array}$ & $\begin{array}{l}.31 \\
.52 \\
.42 \\
.35 \\
.40\end{array}$ & $\begin{array}{l}.22 \\
.23 \\
.23 \\
.23 \\
.22\end{array}$ & $\begin{array}{l}.18 \\
.18 \\
.19 \\
.19 \\
.19\end{array}$ & $\begin{array}{l}.20 \\
.22 \\
.22 \\
.20 \\
.20\end{array}$ & $\begin{array}{l}.17 \\
.17 \\
.17 \\
.17 \\
.17\end{array}$ & $\begin{array}{l}.12 \\
.15 \\
.25 \\
.22 \\
.16\end{array}$ & $\begin{array}{r}.66 \\
.66 \\
.84 \\
.91 \\
1.11\end{array}$ \\
\hline $\begin{array}{l}11 \\
13 \ldots \\
14 \ldots \ldots\end{array}$ & $\begin{array}{r}0.16 \\
.16\end{array}$ & $\begin{array}{l}.15 \\
.17 \\
.15 \\
.16 \\
.17\end{array}$ & $\begin{array}{l}.17 \\
.15 \\
.11 \\
.11 \\
.11\end{array}$ & $\begin{array}{l}.22 \\
.25 \\
.42 \\
.22 \\
.23\end{array}$ & $\begin{array}{r}.35 \\
1.72 \\
1.07 \\
.98 \\
1.11\end{array}$ & $\begin{array}{l}.42 \\
.42 \\
.42 \\
.42 \\
.42\end{array}$ & $\begin{array}{l}.22 \\
.20 \\
.20 \\
.22 \\
.22\end{array}$ & $\begin{array}{l}.17 \\
.28 \\
.19 \\
.18 \\
.17\end{array}$ & $\begin{array}{l}.19 \\
.19 \\
.19 \\
.20 \\
.20\end{array}$ & $\begin{array}{l}.17 \\
.17 \\
.17 \\
.16 \\
.15\end{array}$ & $\begin{array}{l}.15 \\
.15 \\
.15 \\
.14 \\
.14\end{array}$ & $\begin{array}{r}1.04 \\
.94 \\
.91 \\
.78 \\
.63\end{array}$ \\
\hline $\begin{array}{l}16 \ldots \ldots \\
18 \ldots \ldots \\
19 \ldots \ldots \\
20 \ldots \ldots\end{array}$ & $\begin{array}{l}.64 \\
.22 \\
.20 \\
.22 \\
.22\end{array}$ & $\begin{array}{l}1.20 \\
1.72 \\
1.54 \\
1.30 \\
1.04\end{array}$ & $\begin{array}{r}.11 \\
1.54 \\
.71 \\
.49 \\
.38\end{array}$ & $\begin{array}{l}.30 \\
.20 \\
.23 \\
.22 \\
.23\end{array}$ & $\begin{array}{l}.45 \\
.59 \\
.45 \\
.38 \\
.40\end{array}$ & $\begin{array}{l}.37 \\
.35 \\
.31 \\
.33 \\
.50\end{array}$ & $\begin{array}{l}.20 \\
.20 \\
.20 \\
.20 \\
.19\end{array}$ & $\begin{array}{l}.22 \\
.23 \\
.25 \\
.19 \\
.19\end{array}$ & $\begin{array}{l}.19 \\
.18 \\
.19 \\
.22 \\
.22\end{array}$ & $\begin{array}{l}.15 \\
.15 \\
.15 \\
.14 \\
.14\end{array}$ & $\begin{array}{l}.14 \\
.14 \\
.15 \\
.19 \\
.28\end{array}$ & $\begin{array}{l}.57 \\
.49 \\
.44 \\
.44 \\
.44\end{array}$ \\
\hline $\begin{array}{l}21 \ldots \ldots \\
22, \ldots \ldots \\
24 \ldots \ldots \\
25 \ldots \ldots \ldots\end{array}$ & $\begin{array}{l}.23 \\
.25 \\
.20 \\
.20 \\
.17\end{array}$ & $\begin{array}{l}.90 \\
.76 \\
.49 \\
.35 \\
.52\end{array}$ & $\begin{array}{l}.35 \\
.28 \\
.24 \\
.38 \\
.33\end{array}$ & $\begin{array}{l}.23 \\
.22 \\
.22 \\
.22 \\
.24\end{array}$ & $\begin{array}{l}.34 \\
.28 \\
.28 \\
.25 \\
.25\end{array}$ & $\begin{array}{l}.38 \\
.31 \\
.30 \\
.31 \\
.33\end{array}$ & $\begin{array}{l}.20 \\
.19 \\
.20 \\
.17 \\
.19\end{array}$ & $\begin{array}{l}.21 \\
.17 \\
.19 \\
.17 \\
.17\end{array}$ & $\begin{array}{l}.22 \\
.21 \\
.22 \\
.20 \\
.19\end{array}$ & $\begin{array}{l}.15 \\
.15 \\
.16 \\
.17 \\
.16\end{array}$ & $\begin{array}{l}.23 \\
.28 \\
.23 \\
.22 \\
.47\end{array}$ & $\begin{array}{l}.39 \\
.39 \\
.39 \\
.34 \\
.30\end{array}$ \\
\hline $\begin{array}{l}26 \ldots \ldots \ldots \\
27 \ldots \ldots \ldots \\
28 \ldots \ldots \ldots \\
29 \ldots \ldots \\
30 \ldots \ldots \\
31 \ldots \ldots\end{array}$ & $\begin{array}{l}.20 \\
.15 \\
.11 \\
.13 \\
.12 \\
.20\end{array}$ & $\begin{array}{r}.35 \\
.33 \\
.29 \\
\ldots \ldots . \\
\ldots . .\end{array}$ & $\begin{array}{r}.44 \\
1.54 \\
1.40 \\
.87 \\
.54 \\
.34\end{array}$ & $\begin{array}{l}.22 \\
.22 \\
.22 \\
.22 \\
.20\end{array}$ & $\begin{array}{l}.23 \\
.23 \\
.23 \\
.23 \\
.23 \\
.29\end{array}$ & $\begin{array}{l}.30 \\
.27 \\
.27 \\
.28 \\
.28\end{array}$ & $\begin{array}{l}.19 \\
.20 \\
.21 \\
.19 \\
.19 \\
.19\end{array}$ & $\begin{array}{l}.17 \\
.20 \\
.20 \\
.20 \\
.20 \\
.20\end{array}$ & $\begin{array}{l}.19 \\
.19 \\
.19 \\
.19 \\
.17\end{array}$ & $\begin{array}{l}.15 \\
.15 \\
.16 \\
.17 \\
.16 \\
.15\end{array}$ & $\begin{array}{l}.66 \\
.49 \\
.39 \\
.30 \\
.37\end{array}$ & $\begin{array}{l}.32 \\
.30 \\
.30 \\
.30 \\
.28 \\
.25\end{array}$ \\
\hline
\end{tabular}

NoTE.-No record Jan. 1-13, 1913.

Monthly discharge of Pohakea Stream in Kailua Valley, near Kailua, Oahu, for 1913.

\begin{tabular}{|c|c|c|c|c|c|}
\hline \multirow{2}{*}{ Month. } & \multicolumn{3}{|c|}{ Discharge in second-feet. } & \multirow{2}{*}{$\begin{array}{l}\text { Run-off } \\
\text { (total in } \\
\text { acre-feet). }\end{array}$} & \multirow{2}{*}{$\begin{array}{l}\text { Accu- } \\
\text { racy. }\end{array}$} \\
\hline & Maximum. & Minimum. & Mean. & & \\
\hline 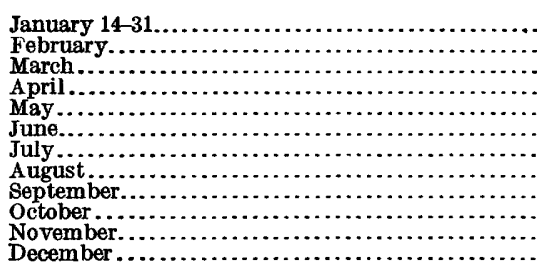 & $\begin{array}{r}0.64 \\
1.72 \\
1.54 \\
.45 \\
2.02 \\
2.02 \\
.29 \\
.28 \\
.45 \\
.19 \\
.66 \\
1.11\end{array}$ & $\begin{array}{r}0.11 \\
.11 \\
.11 \\
.20 \\
.19 \\
.27 \\
.19 \\
.17 \\
.17 \\
.14 \\
.12 \\
.25\end{array}$ & $\begin{array}{r}0.21 \\
.54 \\
.41 \\
.27 \\
.50 \\
.47 \\
.21 \\
.19 \\
.21 \\
.16 \\
.23 \\
.56\end{array}$ & $\begin{array}{c}7.49 \\
30.0 \\
25.2 \\
16.1 \\
30.7 \\
28.0 \\
12.9 \\
11.7 \\
12.5 \\
9.84 \\
13.7 \\
34.4\end{array}$ & $\begin{array}{l}\text { c. } \\
\text { c. } \\
\text { c. } \\
\text { c. } \\
\text { c. } \\
\text { c. } \\
\text { c. } \\
\text { c. } \\
\text { c. } \\
\text { c. } \\
\text { c. } \\
\text { c. }\end{array}$ \\
\hline The period. & & 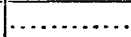 & $\ldots$ & 233 & \\
\hline
\end{tabular}


SOUTH BRANCH OF KAHANAIKI STREAM IN KAILUA VALLEY, NEAR KAILUA, OAHU.

Location.-About 300 feet above junction of two main branches, 600 feet above bridge on government road, and 4 miles northwest (by road) from Waimanalo.

Records available.-April 10 to December 31, 1913.

Gage.--Vertical staff.

Control.-Probably permanent.

Discharge measurements.-Made by wading.

Diversions.-None above station; small ditch 100 feet below station.

Accuracy.-Records poor.

Cooperation.-Station maintained in cooperation with the Kaneohe Ranch Co.

Discharge measurements of South Branch of Kahanaiki Stream in Kailua Valley, near Kailua, Oahu, in 1913.

\begin{tabular}{|c|c|c|c|c|c|c|c|}
\hline Date. & Made by- & $\begin{array}{c}\text { Gage } \\
\text { height. }\end{array}$ & $\begin{array}{l}\text { Dis- } \\
\text { charge. }\end{array}$ & Date. & Made by- & $\begin{array}{c}\text { Gage } \\
\text { height. }\end{array}$ & $\begin{array}{l}\text { Dis- } \\
\text { charge. }\end{array}$ \\
\hline $\begin{array}{l}\text { Apr. } 11 \\
\text { June } 4\end{array}$ & 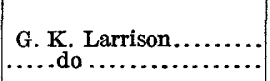 & $\begin{array}{r}\text { Feet. } \\
0.47 \\
.84\end{array}$ & $\begin{array}{r}\text { Sec.-ft. } \\
0.19 \\
1.79\end{array}$ & Sept. 9 & G. K. Larrison. . & Feet. & $\begin{array}{l}S e c . f t \text {. } \\
0.20\end{array}$ \\
\hline
\end{tabular}

Monthly discharge of South Branch of Kahanaiki Stream in Kailua Valley, near Kailua, Oahu, for 1913 .

\begin{tabular}{|c|c|c|c|c|c|}
\hline \multirow{2}{*}{ Month. } & \multicolumn{3}{|c|}{ Discharge in second-feet. } & \multirow{2}{*}{$\begin{array}{c}\dot{\text { Run-off }} \\
\text { (total in } \\
\text { acre-feet). }\end{array}$} & \multirow{2}{*}{$\begin{array}{l}\text { Accu- } \\
\text { racy. }\end{array}$} \\
\hline & Maximum. & Minimum. & Mean. & & \\
\hline 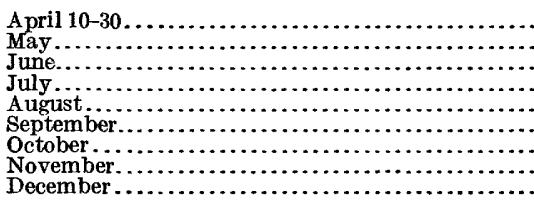 & $\begin{array}{l}0.6 \\
4.4 \\
3.4 \\
.5 \\
1.1 \\
2.0 \\
.1 \\
1.2 \\
4.3\end{array}$ & $\begin{array}{l}0.3 \\
.2 \\
.2 \\
.2 \\
.2 \\
.1 \\
.05 \\
.05 \\
.2\end{array}$ & $\begin{array}{r}0.41 \\
1.01 \\
.70 \\
.27 \\
.54 \\
.32 \\
.07 \\
.30 \\
.88\end{array}$ & $\begin{array}{r}17.0 \\
62.1 \\
41.7 \\
16.6 \\
33.2 \\
19.0 \\
4.3 \\
17.9 \\
54.1\end{array}$ & $\begin{array}{l}\text { D. } \\
\text { D: } \\
\text { D: } \\
\text { D. } \\
\text { D. } \\
\text { D. } \\
\text { D: } \\
\text { D: } \\
\text { D. }\end{array}$ \\
\hline The period..... & & & $\ldots$ & 266 & \\
\hline
\end{tabular}

\section{NORTH BRANCH OF KAHANAIKI STREAM IN KAILUA VALLEY, NEAR} KAILUA, OAFU.

Location.-About 400 feet above junction of two main branches, 700 feet above bridge on government road, and 4 miles northwest of Waimanalo.

Records available.-April 11 to December 31, 1913.

Gage.-Vertical staff up to November 25; November 26 to December 31, 1913, head read on weir crest.

Control.-Probably permanent.

Diversions.-Small ditch diverts about 0.3 second-foot at point about 400 feet above station, to left.

Discharge measurements.-Made by current-meter until November 25; November 25 to December 31 by 1.0-foot sharp-crested weir.

Accuracy.-Records poor until November 25; November 26 to December 31, good. Cooperation.-Station maintained in cooperation with the Kaneohe Ranch Co. 
Discharge measurements of North Branch of Kahanaiki Stream in Kailua Valley, near Kailua, Oahu, in 1918.

\begin{tabular}{|c|c|c|c|c|c|c|c|}
\hline Date. & Hydrographer. & $\begin{array}{c}\text { Gage } \\
\text { height. }\end{array}$ & $\begin{array}{l}\text { Dis- } \\
\text { charge. }\end{array}$ & Date. & Hydrographer. & $\begin{array}{c}\text { Gage } \\
\text { height. }\end{array}$ & $\begin{array}{l}\text { Dis- } \\
\text { charge. }\end{array}$ \\
\hline $\begin{array}{l}\text { Apr. } 11 \\
\text { June } 4\end{array}$ & $\begin{array}{l}\text { G. K. Larrison. } \\
\text {....do................ }\end{array}$ & $\begin{array}{c}\text { Feet. } \\
0.95 \\
1.28\end{array}$ & $\begin{array}{r}\text { Sec.-ft } \\
0.11 \\
2.76\end{array}$ & Sept. 9 & J. C. Dort..... & $\begin{array}{c}\text { Feet. } \\
1.05\end{array}$ & $\begin{array}{r}\text { Sec.ft. } \\
\mathbf{0 . 5 4}\end{array}$ \\
\hline
\end{tabular}

Monthly discharge of North Branch of Kahanaiki Stream in Kailua Valley, near Kailua, Oahu, for 1913.

\begin{tabular}{|c|c|c|c|c|c|}
\hline \multirow{2}{*}{ Month. } & \multicolumn{3}{|c|}{ Discharge in second-feet. } & \multirow{2}{*}{$\begin{array}{l}\text { Run-off } \\
\text { (total in } \\
\text { acre-feet). }\end{array}$} & \multirow{2}{*}{$\begin{array}{l}\text { Accu } \\
\text { racy. }\end{array}$} \\
\hline & Maximum. & Minimum. & Mean. & & \\
\hline \multirow[t]{2}{*}{ 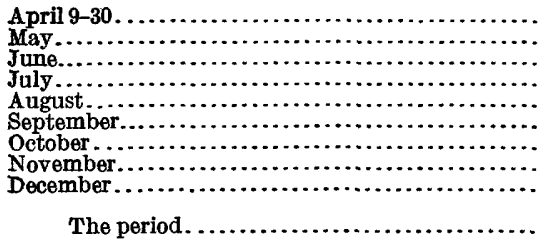 } & $\begin{array}{r}0.40 \\
3.00 \\
8.20 \\
.30 \\
.20 \\
1.50 \\
.15 \\
.75 \\
2.20\end{array}$ & $\begin{array}{l}0.01 \\
.05 \\
.10 \\
.03 \\
.04 \\
.05 \\
.07 \\
.07 \\
.45\end{array}$ & $\begin{array}{r}0.17 \\
+49 \\
1.04 \\
.17 \\
.06 \\
.43 \\
.09 \\
.24 \\
1.07\end{array}$ & $\begin{array}{c}7.42 \\
30.1 \\
61.9 \\
10.5 \\
3.69 \\
25.6 \\
5.53 \\
14.3 \\
65.8\end{array}$ & \multirow[t]{2}{*}{$\begin{array}{l}\text { c. } \\
\text { C. } \\
\text { C. } \\
\text { C. } \\
\text { C. } \\
\text { C. } \\
\text { C. } \\
\text { B. }\end{array}$} \\
\hline & & & & 225 & \\
\hline
\end{tabular}

Note-Apr. 9 to Aug. 31, Sept. 3-4, and Sept. 24 to Nov. 7 , the Kahanaiki ditch diverted aboui 0.3 second-foot from the stream above the station. Water overflowed weir Dec. 10-18; discharge estimated.

\section{WAIAHOLE STREAM AT MANIANIAULA, NEAR WAIKANE, OAHU.}

Location.-In upper Waiahole Valley, at boundary line of government and private land, $3 \frac{1}{2}$ miles southwest of Waikane.

Records available.-September 25, 1911, to December 31, 1913.

Gage.-Vertical staff; read twice daily.

Control.-Probably shifting.

Discharge measurements.-Made by wading.

Diversions.-None above gage.

Regulation.-Flow regulated by large springs at head of Waiahole Valley.

Accuracy.-Records good.

Discharge measurements of Waiahole Stream at Manianiaula, near Waikane, Oahu, in 1913.

\begin{tabular}{|c|c|c|c|c|c|c|c|}
\hline Date. & Made by- & $\begin{array}{c}\text { Gage } \\
\text { height. }\end{array}$ & $\begin{array}{c}\text { Dis- } \\
\text { charge. }\end{array}$ & Date. & Made by- & $\begin{array}{c}\text { Gage } \\
\text { height. }\end{array}$ & $\begin{array}{c}\text { Dis- } \\
\text { charge. }\end{array}$ \\
\hline $\begin{array}{lr}\text { Mar. } & 1 \\
\text { May } & 3 \\
\text { June } & \mathbf{3 0} \\
\text { Aug. } & 8\end{array}$ & 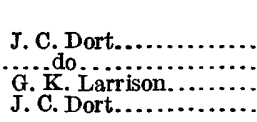 & $\begin{array}{r}\text { Feet. } \\
0.96 \\
.96 \\
.99 \\
1.26\end{array}$ & $\begin{aligned} \text { Sec. }-f t . \\
23.4 \\
20.4 \\
22.3 \\
37.6\end{aligned}$ & $\begin{array}{l}\text { Aug. } 15 \\
\text { Sept. } 12 \\
\text { Oct. } 1 \\
\text { Dec. } 9\end{array}$ & 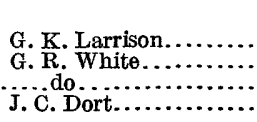 & $\begin{array}{r}\text { Feet. } \\
1.29 \\
1.39 \\
1.30 \\
1.30\end{array}$ & $\begin{array}{r}\text { Sec. }-f t . \\
37.2 \\
43.0 \\
40.1 \\
39.5\end{array}$ \\
\hline
\end{tabular}

$97764^{\circ}-$ WSP $373-15-7$ 
Daily discharge, in second-feet, of Waiahole Stream at Manianiaula, near Waikane, Oahu, for 1913.

\begin{tabular}{|c|c|c|c|c|c|c|c|c|c|c|c|c|}
\hline Day. & Jan. & Feb. & Mar. & Apr. & May. & June. & July. & Aug. & Sept. & Oct. & Nov. & Dec. \\
\hline $\begin{array}{l}1 \ldots \ldots \\
2 \ldots \ldots \\
3 \ldots \ldots \\
4 \ldots \ldots \\
5 \ldots \ldots\end{array}$ & $\begin{array}{l}18 \\
18 \\
18 \\
18 \\
18\end{array}$ & $\begin{array}{l}19 \\
20 \\
20 \\
20 \\
20\end{array}$ & $\begin{array}{l}20 \\
20 \\
20 \\
20 \\
20\end{array}$ & $\begin{array}{l}20 \\
20 \\
20 \\
20 \\
20\end{array}$ & $\begin{array}{l}20 \\
20 \\
20 \\
20 \\
20\end{array}$ & $\begin{array}{l}21 \\
21 \\
30 \\
27 \\
22\end{array}$ & $\begin{array}{l}22 \\
23 \\
22 \\
22 \\
22\end{array}$ & $\begin{array}{l}26 \\
32 \\
34 \\
35 \\
35\end{array}$ & $\begin{array}{l}47 \\
49 \\
49 \\
47 \\
46\end{array}$ & $\begin{array}{l}44 \\
44 \\
44 \\
44 \\
44\end{array}$ & $\begin{array}{l}37 \\
37 \\
37 \\
37 \\
37\end{array}$ & $\begin{array}{l}37 \\
37 \\
38 \\
37 \\
37\end{array}$ \\
\hline $\begin{array}{r}6 \ldots \ldots . \\
7 \ldots \ldots \\
8 \ldots \ldots \\
9 \ldots \ldots \\
10 \ldots \ldots\end{array}$ & $\begin{array}{l}18 \\
18 \\
18 \\
18 \\
18\end{array}$ & $\begin{array}{l}28 \\
22 \\
22 \\
22 \\
21\end{array}$ & $\begin{array}{l}20 \\
20 \\
20 \\
20 \\
20\end{array}$ & $\begin{array}{l}20 \\
20 \\
19 \\
19 \\
19\end{array}$ & $\begin{array}{l}20 \\
20 \\
20 \\
20 \\
21\end{array}$ & $\begin{array}{l}22 \\
22 \\
22 \\
22 \\
22\end{array}$ & $\begin{array}{l}22 \\
22 \\
22 \\
21 \\
21\end{array}$ & $\begin{array}{l}35 \\
37 \\
37 \\
37 \\
37\end{array}$ & $\begin{array}{l}46 \\
46 \\
46 \\
46 \\
45\end{array}$ & $\begin{array}{l}44 \\
43 \\
42 \\
41 \\
40\end{array}$ & $\begin{array}{l}37 \\
37 \\
38 \\
37 \\
37\end{array}$ & $\begin{array}{l}37 \\
37 \\
38 \\
39 \\
39\end{array}$ \\
\hline $\begin{array}{l}11 \ldots \ldots \ldots \\
12 \ldots \ldots \ldots \\
13 \ldots \ldots \\
14 \ldots \ldots\end{array}$ & $\begin{array}{l}18 \\
18 \\
18 \\
18 \\
18\end{array}$ & $\begin{array}{l}21 \\
20 \\
20 \\
20 \\
21\end{array}$ & $\begin{array}{l}20 \\
20 \\
20 \\
20 \\
20\end{array}$ & $\begin{array}{l}19 \\
20 \\
21 \\
21 \\
21\end{array}$ & $\begin{array}{l}20 \\
22 \\
22 \\
46 \\
23\end{array}$ & $\begin{array}{l}22 \\
22 \\
24 \\
22 \\
22\end{array}$ & $\begin{array}{l}21 \\
21 \\
21 \\
21 \\
21\end{array}$ & $\begin{array}{l}38 \\
39 \\
38 \\
39 \\
38\end{array}$ & $\begin{array}{l}44 \\
44 \\
44 \\
44 \\
44\end{array}$ & $\begin{array}{l}39 \\
39 \\
39 \\
39 \\
39\end{array}$ & $\begin{array}{l}37 \\
37 \\
37 \\
37 \\
37\end{array}$ & $\begin{array}{l}39 \\
39 \\
39 \\
39 \\
39\end{array}$ \\
\hline $\begin{array}{l}16 \ldots \ldots \\
17 \ldots \ldots \\
18 \ldots \ldots \\
19 \ldots \ldots \\
20 \ldots \ldots\end{array}$ & $\begin{array}{r}19 \\
19 \\
19 \\
100 \\
22\end{array}$ & $\begin{array}{l}21 \\
21 \\
21 \\
21 \\
21\end{array}$ & $\begin{array}{l}20 \\
20 \\
20 \\
20 \\
20\end{array}$ & $\begin{array}{l}20 \\
20 \\
20 \\
20 \\
20\end{array}$ & $\begin{array}{l}22 \\
22 \\
21 \\
21 \\
21\end{array}$ & $\begin{array}{l}22 \\
22 \\
22 \\
22 \\
22\end{array}$ & $\begin{array}{l}21 \\
22 \\
22 \\
22 \\
22\end{array}$ & $\begin{array}{l}41 \\
91 \\
41 \\
43 \\
43\end{array}$ & $\begin{array}{l}45 \\
45 \\
45 \\
49 \\
47\end{array}$ & $\begin{array}{l}39 \\
39 \\
39 \\
39 \\
38\end{array}$ & $\begin{array}{l}37 \\
37 \\
38 \\
38 \\
38\end{array}$ & $\begin{array}{l}39 \\
38 \\
38 \\
38 \\
38\end{array}$ \\
\hline $\begin{array}{l}21 \ldots \ldots \\
22 \ldots \ldots \\
23 \ldots \ldots \\
24 \ldots \ldots \\
25 \ldots \ldots \\
\end{array}$ & $\begin{array}{l}22 \\
22 \\
19 \\
19 \\
19\end{array}$ & $\begin{array}{l}21 \\
21 \\
21 \\
21 \\
21\end{array}$ & $\begin{array}{l}20 \\
20 \\
20 \\
21 \\
21\end{array}$ & $\begin{array}{l}20 \\
20 \\
20 \\
20 \\
20\end{array}$ & $\begin{array}{l}21 \\
21 \\
21 \\
20 \\
20\end{array}$ & $\begin{array}{l}22 \\
22 \\
22 \\
22 \\
22\end{array}$ & $\begin{array}{l}22 \\
21 \\
21 \\
21 \\
22\end{array}$ & $\begin{array}{l}43 \\
44 \\
45 \\
46 \\
47\end{array}$ & $\begin{array}{l}47 \\
47 \\
46 \\
45 \\
45\end{array}$ & $\begin{array}{l}38 \\
38 \\
39 \\
38 \\
38\end{array}$ & $\begin{array}{l}38 \\
38 \\
37 \\
37 \\
37\end{array}$ & $\begin{array}{l}38 \\
38 \\
38 \\
38 \\
38\end{array}$ \\
\hline $\begin{array}{l}26 \ldots \ldots \ldots \\
27 \ldots \ldots \ldots \ldots \\
28 \ldots \ldots \ldots \\
29 \ldots \ldots \ldots \ldots \\
30 \ldots \ldots \ldots \ldots \\
31 \ldots \ldots \ldots \ldots\end{array}$ & $\begin{array}{l}19 \\
19 \\
19 \\
19 \\
19 \\
19\end{array}$ & $\begin{array}{c}21 \\
21 \\
21 \\
\cdots \ldots \\
\ldots \ldots\end{array}$ & $\begin{array}{l}21 \\
21 \\
21 \\
20 \\
20 \\
20\end{array}$ & $\begin{array}{l}20 \\
20 \\
20 \\
20 \\
20\end{array}$ & $\begin{array}{l}20 \\
21 \\
21 \\
21 \\
21 \\
21\end{array}$ & $\begin{array}{l}22 \\
22 \\
22 \\
22 \\
22\end{array}$ & $\begin{array}{l}22 \\
22 \\
22 \\
22 \\
22 \\
22\end{array}$ & $\begin{array}{l}47 \\
47 \\
47 \\
47 \\
47 \\
47\end{array}$ & $\begin{array}{l}45 \\
45 \\
45 \\
45 \\
45 \\
\cdots \cdots\end{array}$ & $\begin{array}{l}38 \\
37 \\
37 \\
37 \\
37 \\
37\end{array}$ & \begin{tabular}{c|}
37 \\
37 \\
37 \\
37 \\
37 \\
$\cdots$
\end{tabular} & $\begin{array}{l}38 \\
38 \\
38 \\
38 \\
38 \\
38\end{array}$ \\
\hline
\end{tabular}

NoTe.-Beginning Aug. 1, water developed in the Waiahole Tunnel was wasted into this stream.

Monthly discharge of Waiahole Stream at Manianiaula, near Waikane, Oahu, for 1913.

\begin{tabular}{|c|c|c|c|c|c|}
\hline \multirow{2}{*}{ Month. } & \multicolumn{3}{|c|}{ Discharge in second-feet. } & \multirow{2}{*}{$\begin{array}{c}\text { Run-off } \\
\text { (total in } \\
\text { acre-feet). }\end{array}$} & \multirow{2}{*}{$\begin{array}{l}\text { Accu- } \\
\text { racy. }\end{array}$} \\
\hline & Maximum. & Minimum. & Mean. & & \\
\hline 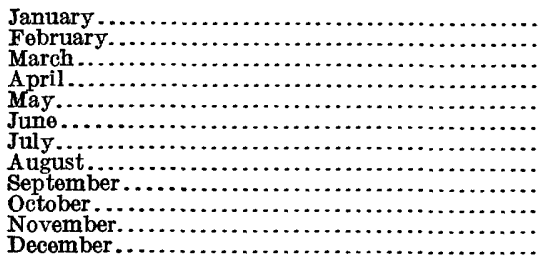 & $\begin{array}{r}100 \\
28 \\
21 \\
20 \\
46 \\
30 \\
23 \\
91 \\
49 \\
44 \\
38 \\
39\end{array}$ & $\begin{array}{l}18 \\
19 \\
20 \\
19 \\
20 \\
21 \\
21 \\
26 \\
44 \\
37 \\
37 \\
37\end{array}$ & $\begin{array}{l}21.4 \\
21.0 \\
20.2 \\
20.0 \\
21.6 \\
22.4 \\
21.7 \\
42.0 \\
45.8 \\
39.8 \\
37.2 \\
38.1\end{array}$ & $\begin{array}{l}1,320 \\
1,170 \\
1,240 \\
1,190 \\
1,330 \\
1,330 \\
1,330 \\
2,580 \\
2,730 \\
2,450 \\
2,210 \\
2,340\end{array}$ & $\begin{array}{l}\text { A. } \\
\text { A. } \\
\text { A. } \\
\text { A. } \\
\text { A. } \\
\text { A. } \\
\text { A. } \\
\text { A. } \\
\text { A. } \\
\text { A. } \\
\text { A. }\end{array}$ \\
\hline The year...$\ldots \ldots \ldots \ldots \ldots \ldots \ldots$ & 100 & 18 & 29.3 & 21,200 & \\
\hline
\end{tabular}


RIGHT BRANCH OF NORTH FORK OF KAUKONAHUA STREAM NEAR WAHIAWA, OAHU.

Location.-About 8 miles northeast of Wahiawa. and 200 feet up the Right Branch from the intake of the Waialua Agricultural Co. tunnel, which is at the confluence of the Right and Left Branches or two main branches of the North Fork.

Records available.-May 29 to December 31, 1913.

Gage.-Stevens continuous water-stage recorder.

Control.-Probably permanent.

Discharge measurements.-Made by wading or from a footbridge about 20 feet above gage.

Diversions.-None above station.

Accuracy.-Records fair.

Cooperation.-The Waialua Agricultural Co. appropriated sufficient funds to cover cost of equipment and installation of station.

Discharge measurements of Right Branch of North Fork of Kaukonahua Stream, near Wahiawa, Oahu, in 1913.

\begin{tabular}{|c|c|c|c|c|c|c|c|}
\hline Date. & Hydrographer. & $\begin{array}{c}\text { Gage } \\
\text { height. }\end{array}$ & $\begin{array}{c}\text { Dis- } \\
\text { charge. }\end{array}$ & Date. & Hydrographer. & $\begin{array}{c}\text { Gage } \\
\text { height. }\end{array}$ & $\begin{array}{c}\text { Dis- } \\
\text { charge. }\end{array}$ \\
\hline May 30 & J. C. Dort . & $\begin{array}{c}\text { Feet. } \\
2.00\end{array}$ & $\begin{array}{c}\text { Sec.-ft. } \\
10.8\end{array}$ & Oct. & ite... & $\begin{array}{c}\text { Feet. } \\
1.30\end{array}$ & $\begin{array}{r}\text { Sec.-ft. } \\
0.54\end{array}$ \\
\hline $\begin{array}{ll} & 30 \\
\text { July } & 11 \\
\text { Aug. } 21 \\
\text { Sept. } 1\end{array}$ & $\begin{array}{l}\text { G. K Larrison } \\
\text { J. C. Dort . }\end{array}$ & $\begin{array}{l}1.78 \\
1.57 \\
1.55 \\
3.18\end{array}$ & $\begin{array}{c}5.76 \\
2.89 \\
2.79 \\
92.8\end{array}$ & Nov. $\begin{array}{r}31 \\
20 \\
26\end{array}$ & $\begin{array}{l}\text { J. C. Dort. } \\
\text { G. R. White } \\
\text { J. c. Dort. }\end{array}$ & $\begin{array}{l}1.35 \\
3.44 \\
2.49 \\
3.88\end{array}$ & $\begin{array}{l}120^{\circ} \\
34.7 \\
190\end{array}$ \\
\hline
\end{tabular}

Daily discharge, in second-feet, of Right Branch of North Fork of Kaukonahua Stream, near Waihiawa, Oahu, for 191 s.

\begin{tabular}{|c|c|c|c|c|c|c|c|c|}
\hline Day. & May. & June. & July. & Aug. & Sept. & Oct. & Nov. & Dec. \\
\hline $\begin{array}{l}1 \ldots \ldots \\
2 \ldots \ldots \\
3 \ldots \ldots \\
4 \ldots \ldots \\
5 \ldots \ldots\end{array}$ & & $\begin{array}{c}4.0 \\
3.7 \\
4.2 \\
30 \\
6.8\end{array}$ & $\begin{array}{l}11 \\
36 \\
27 \\
10 \\
86\end{array}$ & $\begin{array}{l}1.4 \\
1.3 \\
1.3 \\
1.2 \\
1.2\end{array}$ & $\begin{array}{l}6.2 \\
3.8 \\
3.2 \\
2.2 \\
2.1\end{array}$ & $\begin{array}{r}0.8 \\
.8 \\
.8 \\
.8 \\
.8\end{array}$ & $\begin{array}{l}1.1 \\
3.7 \\
3.3 \\
1.6 \\
1.4\end{array}$ & $\begin{array}{l}8.5 \\
7.4 \\
40 \\
55 \\
21\end{array}$ \\
\hline 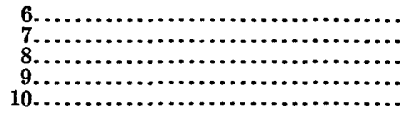 & & $\begin{array}{l}5.0 \\
4.8 \\
4.7 \\
4.5 \\
4.0\end{array}$ & $\begin{array}{l}9.1 \\
7.4 \\
6.8 \\
5.4 \\
4.2\end{array}$ & $\begin{array}{l}1.2 \\
1.2 \\
1.2 \\
1.4 \\
3.8\end{array}$ & $\begin{array}{l}2.0 \\
1.8 \\
1.6 \\
1.6 \\
1.6\end{array}$ & $\begin{array}{l}.8 \\
.8 \\
.7 \\
.6 \\
.6\end{array}$ & $\begin{array}{l}1.4 \\
4.5 \\
15 \\
34 \\
45\end{array}$ & $\begin{array}{c}43 \\
27 \\
14 \\
11 \\
9.1\end{array}$ \\
\hline $\begin{array}{l}11 \\
12 \\
13 \\
14 \\
15 \\
\ldots \ldots \ldots \ldots\end{array}$ & & $\begin{array}{l}3.5 \\
3.6 \\
4.7 \\
10 \\
25\end{array}$ & $\begin{array}{l}3.1 \\
2.9 \\
2.9 \\
2.8 \\
3.1\end{array}$ & $\begin{array}{l}1.8 \\
67 \\
2.5 \\
2.1 \\
1.7\end{array}$ & $\begin{array}{l}1.4 \\
1.4 \\
1.4 \\
1.5 \\
2.2\end{array}$ & $\begin{array}{r}1.1 \\
28 \\
9.6 \\
2.7 \\
1.7\end{array}$ & $\begin{array}{r}87 \\
114 \\
72 \\
20 \\
14\end{array}$ & $\begin{array}{l}8.0 \\
7.4 \\
6.8 \\
5.7 \\
5.4\end{array}$ \\
\hline $\begin{array}{l}16 \ldots \ldots \\
18 \ldots \ldots \\
190 \ldots\end{array}$ & & $\begin{array}{l}5.9 \\
9.9 \\
5.9 \\
4.8 \\
4.0\end{array}$ & $\begin{array}{l}2.3 \\
2.2 \\
2.3 \\
2.4 \\
2.0\end{array}$ & $\begin{array}{l}2.8 \\
8.3 \\
3.2 \\
2.2 \\
2.0\end{array}$ & $\begin{array}{l}2.1 \\
1.6 \\
1.6 \\
4.1 \\
1.8\end{array}$ & $\begin{array}{l}1.3 \\
1.2 \\
1.0 \\
1.0 \\
1.3\end{array}$ & $\begin{array}{r}9.9 \\
8.1 \\
7.2 \\
16 \\
104\end{array}$ & $\begin{array}{l}5.0 \\
5.2 \\
5.6 \\
5.0 \\
3.9\end{array}$ \\
\hline 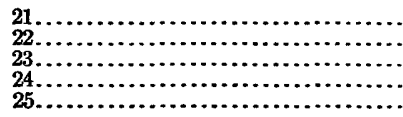 & & $\begin{array}{r}6.8 \\
9.1 \\
35 \\
9.1 \\
6.3\end{array}$ & $\begin{array}{l}1.9 \\
1.7 \\
1.8 \\
1.8 \\
2.0\end{array}$ & $\begin{array}{l}2.9 \\
2.5 \\
2.2 \\
2.5 \\
1.7\end{array}$ & $\begin{array}{r}1.6 \\
1.9 \\
.7 \\
.7 \\
.7\end{array}$ & $\begin{array}{l}2.8 \\
3.7 \\
2.8 \\
1.8 \\
3.2\end{array}$ & $\begin{array}{l}56 \\
20 \\
15 \\
16 \\
80\end{array}$ & $\begin{array}{l}3.8 \\
3.6 \\
3.1 \\
2.7 \\
2.5\end{array}$ \\
\hline $\begin{array}{r}26 \\
27 \\
28 \ldots \ldots \\
29 \\
30 \ldots \ldots \\
31 . \ldots\end{array}$ & $\begin{array}{r}\ldots \ldots . . \\
\cdots \\
9.6 \\
8.1 \\
4.4\end{array}$ & $\begin{array}{l}5.2 \\
4.9 \\
5.0 \\
10 \\
15\end{array}$ & $\begin{array}{l}2.3 \\
4.0 \\
2.8 \\
2.6 \\
3.3 \\
1.8\end{array}$ & $\begin{array}{l}1.4 \\
1.4 \\
3.2 \\
3.3 \\
2.1 \\
4.4\end{array}$ & $\begin{array}{l}.7 \\
.8 \\
.8 \\
.8 \\
.8\end{array}$ & $\begin{array}{l}3.6 \\
1.6 \\
1.3 \\
1.2 \\
1.0 \\
1.0\end{array}$ & $\begin{array}{c}79 \\
46 \\
32 \\
20 \\
16 \\
\ldots\end{array}$ & $\begin{array}{l}2.5 \\
2.4 \\
2.3 \\
2.2 \\
2.2 \\
1.9\end{array}$ \\
\hline
\end{tabular}

Note.-No records June 21-July 10, Sept. 24-30, and Dec. 1-30; discharge estimated by comparison with records of station on Left Branch of North Fork of the Kaukonahua. 
Monthly discharge of Right Branch of North Fork of Kaukonahua Stream near Wahiawa, Oahu, for 1913 .

\begin{tabular}{|c|c|c|c|c|c|}
\hline \multirow{2}{*}{ Month. } & \multicolumn{3}{|c|}{ Discharge in second-feet. } & \multirow{2}{*}{$\begin{array}{l}\text { Run-off } \\
\text { (total in } \\
\text { acre-feet). }\end{array}$} & \multirow{2}{*}{$\begin{array}{l}\text { Accu } \\
\text { racy }\end{array}$} \\
\hline & Maximum. & Minimum. & Mean. & & \\
\hline \multirow[t]{2}{*}{ 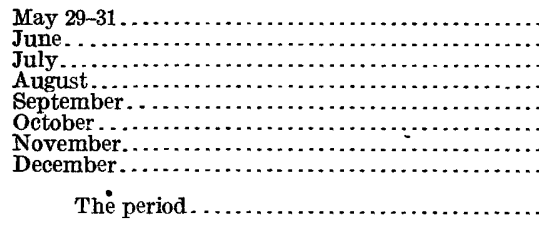 } & $\begin{array}{c}9.6 \\
35 \\
86 \\
67 \\
6.2 \\
28 \\
141 \\
55\end{array}$ & $\begin{array}{r}4.4 \\
3.5 \\
1.7 \\
1.2 \\
.7 \\
.6 \\
1.1 \\
1.9\end{array}$ & $\begin{array}{l}7.37 \\
8.51 \\
8.22 \\
4.40 \\
1.82 \\
2.59 \\
32.3 \\
10.4\end{array}$ & $\begin{array}{c}43.8 \\
506 \\
504 \\
271 \\
108 \\
159 \\
1,920 \\
640\end{array}$ & \multirow[t]{2}{*}{$\begin{array}{l}\text { C. } \\
\text { C. } \\
\text { B. } \\
\text { B. } \\
\text { B. } \\
\text { A. } \\
\text { C. }\end{array}$} \\
\hline & & & & 4,150 & \\
\hline
\end{tabular}

Note.-Actual maximum, Nov. 20, 10 p. m., 600 second-feet., actual minimum, Sept. 27, 29, and Oct. 11, 0.6 second-feet.

\section{LEFT BRANCH OF NORTH FORK OF KAUKONAHUA STREAM NEAR WAHIAWA, OAHU.}

Location.-About 8 miles east by north of Wahiawa and 100 feet above the intake of the Waialua Agricultural Co 's tunnel, which is at the confluence of the right and left branches or the two main branches of the North Fork.

Records available.-May 25 to December 31, 1913.

Gage.-Stevens continuous water-stage recorder.

Control.-Probably permanent.

Diversions.-None above station.

Discharge measurements.-Made by wading or from cable.

Accuracy.-Records good.

Cooperation.-The Waialua Agricultural Co. appropriated sufficient funds to cover the cost of equipment and installation of station.

Discharge measurements of Left Branch of North Fork of Kaukonahua Stream near Wahiawa, Oahu, in 1913 .

\begin{tabular}{|c|c|c|c|c|c|c|c|}
\hline Date. & Made by- & $\begin{array}{c}\text { Gage } \\
\text { height. }\end{array}$ & $\begin{array}{c}\text { Dis- } \\
\text { charge. }\end{array}$ & Date. & Made by- & $\begin{array}{c}\text { Gage } \\
\text { height. }\end{array}$ & $\begin{array}{c}\text { Dis- } \\
\text { charge. }\end{array}$ \\
\hline $\begin{array}{lr}\text { May } & 23 \\
& 24 \\
& 24 \\
& 27 \\
\text { July } & 11 \\
\text { Aug. } & 31 \\
\text { Sept. } & 1\end{array}$ & 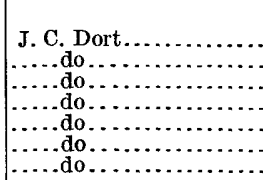 & $\begin{array}{r}\text { Feet. } \\
1.39 \\
1.62 \\
1.78 \\
1.40 \\
1.39 \\
1.60 \\
1.25\end{array}$ & $\begin{array}{c}\text { Sec. ft. } \\
6.31 \\
14.9 \\
20.5 \\
7.26 \\
6.01 \\
12.1 \\
2.95\end{array}$ & $\begin{array}{lr}\text { Sept. } & 1 \\
& 1 \\
\text { Oct. } & 1 \\
31 \\
\text { Nov. } 20 \\
20 \\
26\end{array}$ & 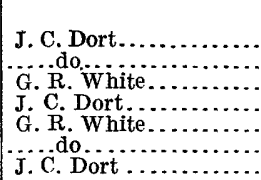 & $\begin{array}{c}\text { Feet. } \\
2.08 \\
2.52 \\
1.08 \\
1.00 \\
2.70 \\
2.27 \\
3.00\end{array}$ & $\begin{array}{r}\text { Sec. ft. } \\
41.6 \\
94.6 \\
.93 \\
.99 \\
94.1 \\
60.2 \\
167\end{array}$ \\
\hline
\end{tabular}


Daily discharge, in second-feet, of Left Branch of North Fork of Kaukonahua Stream near Wahiawa, Oahu, for 1913.

\begin{tabular}{|c|c|c|c|c|c|c|c|c|}
\hline Day. & May. & June. & July. & Aug. & Sept. & Oct. & Nov. & Dec. \\
\hline $\begin{array}{l}1 \\
2 \\
3 \\
4\end{array} \ldots \ldots \ldots$ & & $\begin{array}{r}4.6 \\
4.2 \\
4.8 \\
26 \\
7.7\end{array}$ & $\begin{array}{l}20 \\
63 \\
47 \\
18 \\
15\end{array}$ & $\begin{array}{l}3.6 \\
3.3 \\
3.1 \\
3.0 \\
2.8\end{array}$ & $\begin{array}{r}13 \\
6.9 \\
7.7 \\
5.1 \\
4.2\end{array}$ & $\begin{array}{l}1.3 \\
1.3 \\
1.1 \\
1.1 \\
1.1\end{array}$ & $\begin{array}{r}0.9 \\
14 \\
1.4 \\
1.4 \\
1.4\end{array}$ & $\begin{array}{l}15 \\
13 \\
70 \\
96 \\
37\end{array}$ \\
\hline $\begin{array}{r}6 \\
7 \\
8 \\
9 \\
10 \\
10\end{array}$ & & $\begin{array}{l}10 \\
9.1 \\
7.2 \\
6.2 \\
5.9\end{array}$ & $\begin{array}{c}16 \\
13 \\
12 \\
9.4 \\
7.4\end{array}$ & $\begin{array}{r}3.0 \\
3.0 \\
2.8 \\
3.8 \\
12\end{array}$ & $\begin{array}{l}4.1 \\
3.3 \\
3.0 \\
2.8 \\
2.8\end{array}$ & $\begin{array}{l}1.1 \\
1.1 \\
21 \\
33 \\
7.2\end{array}$ & $\begin{array}{l}1.4 \\
1.4 \\
1.2 \\
1.0 \\
1.0\end{array}$ & $\begin{array}{l}75 \\
47 \\
24 \\
19 \\
16\end{array}$ \\
\hline 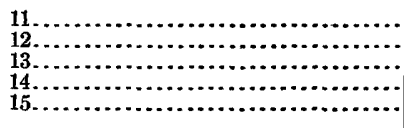 & & $\begin{array}{r}7.4 \\
6.9 \\
6.2 \\
11 \\
21\end{array}$ & $\begin{array}{l}6.4 \\
6.2 \\
5.9 \\
5.7 \\
5.7\end{array}$ & $\begin{array}{r}4.1 \\
40 \\
7.2 \\
8.3 \\
5.7\end{array}$ & $\begin{array}{l}2.7 \\
2.8 \\
2.5 \\
2.7 \\
3.4\end{array}$ & $\begin{array}{l}4.1 \\
3.3 \\
2.7 \\
2.3 \\
2.3\end{array}$ & $\begin{array}{l}1 . \dot{9} \\
49 \\
17 \\
4.7 \\
3.0\end{array}$ & $\begin{array}{c}14 \\
13 \\
12 \\
10 \\
9.4\end{array}$ \\
\hline $\begin{array}{l}16 \\
18 \\
19 \\
19 \\
20\end{array}$ & & $\begin{array}{c}12 \\
30 \\
15 \\
9.1 \\
9.4\end{array}$ & $\begin{array}{l}5.5 \\
4.4 \\
4.4 \\
4.4 \\
4.2\end{array}$ & $\begin{array}{r}7.2 \\
20 \\
8.3 \\
6.2 \\
4.8\end{array}$ & $\begin{array}{r}2.7 \\
2.3 \\
2.2 \\
11 \\
3.4\end{array}$ & $\begin{array}{l}2.8 \\
8.3 \\
5.1 \\
3.6 \\
3.8\end{array}$ & $\begin{array}{l}2.3 \\
2.1 \\
1.7 \\
1.7 \\
2.3\end{array}$ & $\begin{array}{l}8.8 \\
9.1 \\
9.8 \\
8.8 \\
6.9\end{array}$ \\
\hline 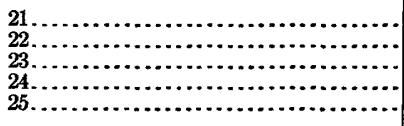 & צ.7. & $\begin{array}{l}12 \\
16 \\
61 \\
16 \\
11\end{array}$ & $\begin{array}{l}4.1 \\
3.4 \\
3.4 \\
3.6 \\
6.9\end{array}$ & $\begin{array}{r}11 \\
5.9 \\
5.3 \\
5.3 \\
4.2\end{array}$ & $\begin{array}{l}2.4 \\
2.3 \\
3.9 \\
1.5 \\
1.5\end{array}$ & $\begin{array}{l}9.8 \\
3.9 \\
2.8 \\
2.7 \\
6.6\end{array}$ & $\begin{array}{l}4.9 \\
6.5 \\
4.9 \\
3.2 \\
5.6\end{array}$ & $\begin{array}{l}6.6 \\
6.4 \\
5.5 \\
4.8 \\
4.4\end{array}$ \\
\hline 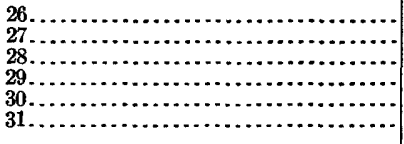 & $\begin{array}{l}5.5 \\
5.5 \\
5.3 \\
4.4 \\
7.2 \\
5.3\end{array}$ & $\begin{array}{c}9.1 \\
8.6 \\
8.8 \\
18 \\
27 \\
-\cdots\end{array}$ & \begin{tabular}{c|c}
9.8 \\
13 \\
17 \\
12 \\
6.2 \\
4.4
\end{tabular} & $\begin{array}{l}3.9 \\
3.8 \\
4.1 \\
3.4 \\
2.8 \\
8.3\end{array}$ & $\begin{array}{l}1.3 \\
1.2 \\
1.3 \\
1.2 \\
1.3 \\
\cdots\end{array}$ & $\begin{array}{l}2.8 \\
2.3 \\
1.9 \\
1.8 \\
1.5 \\
1.6\end{array}$ & $\begin{array}{r}137 \\
46 \\
28 \\
20 \\
20 \\
20\end{array}$ & $\begin{array}{l}4.4 \\
4.2 \\
4.1 \\
3.8 \\
3.8 \\
3.4\end{array}$ \\
\hline
\end{tabular}

Note.-Discharge Nov. 3-25 estimated by comparison with records of the station on the Right Branch of the North Fork of the Kaukonahua.

Monthly discharge of Left Branch of North Fork of Kaukonahua Stream near Wahiawa, Oahu, for 1913.

\begin{tabular}{|c|c|c|c|c|c|}
\hline \multirow{2}{*}{ Month. } & \multicolumn{3}{|c|}{ Discharge in second-feet. } & \multirow{2}{*}{$\begin{array}{l}\text { Run-off } \\
\text { (total in } \\
\text { acre-feet). }\end{array}$} & \multirow{2}{*}{$\begin{array}{l}\text { Aceu- } \\
\text { racy. }\end{array}$} \\
\hline & Maximum. & Minimum. & Mean. & & \\
\hline \multirow[t]{2}{*}{ 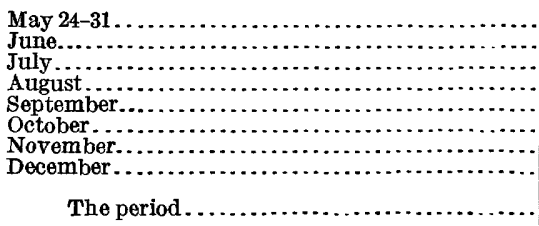 } & $\begin{array}{c}7.2 \\
61 \\
63 \\
40 \\
13 \\
33 \\
137 \\
96\end{array}$ & $\begin{array}{r}4.4 \\
4.2 \\
3.4 \\
2.8 \\
1.2 \\
1.1 \\
.9 \\
3.4\end{array}$ & $\begin{array}{c}5.56 \\
13.4 \\
11.5 \\
6.78 \\
3.54 \\
4.69 \\
12.9 \\
18.2\end{array}$ & $\begin{array}{r}77.1 \\
797 \\
707 \\
417 \\
211 \\
288 \\
768 \\
1,120\end{array}$ & \multirow[t]{2}{*}{$\begin{array}{l}\text { A. } \\
\text { A. } \\
\text { A. } \\
\text { B. } \\
\text { B. } \\
\text { C. } \\
\text { A. }\end{array}$} \\
\hline & $\cdots$ & $\ldots \ldots$ & $\ldots$ & 4,390 & \\
\hline
\end{tabular}

Note.-Actual maximum for year, Nov. 20,10 p. m., 750 second-feet; actual minimum for year, Nov. $1,0.9$ second-feet. 


\section{SOUTH FORK OF KAUKONAHUA STREAM NEAR WAHIAWA, OAHU.}

Location.-About 10 miles east of Wahiawa by main road to United States Army reservoir and trail up ditch bank to about one-eighth mile above the United States Army ditch intake.

Records available.-June 18 to December 31, 1913.

Gage.-Stevens continuous water-stage recorder.

Control.-Probably permanent.

Discharge measurements.-Made by wading or from cable just above gage house. Diversions.- None above station. Small ditch diverts water one-eighth mile below station for the supply of Schofield Barracks.

Accuracy.-Records fair.

Cooperation.-The Quartermaster Department, United States Army, appropriated sufficient funds to cover the cost of equipment and installation of this station.

Discharge measurements of South Fork of Kaukonahua Stream near Wahiawa, Oahu, in 1913.

\begin{tabular}{|c|c|c|c|c|c|c|c|}
\hline Date. & Hydrographer. & $\begin{array}{c}\text { Gage } \\
\text { height. }\end{array}$ & $\begin{array}{c}\text { Dis- } \\
\text { charge. }\end{array}$ & Date. & Hydrographer. & $\begin{array}{c}\text { Gage } \\
\text { height. }\end{array}$ & $\begin{array}{c}\text { Dis- } \\
\text { charge. }\end{array}$ \\
\hline $\begin{array}{lr}\text { June } & 18 \\
\text { July } & 9 \\
\text { Sept. } 13 \\
\text { Sept. } 24\end{array}$ & 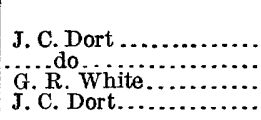 & $\begin{aligned} \text { Feet. } \\
1.66 \\
1.63 \\
1.47 \\
1.46\end{aligned}$ & $\begin{array}{r}S e c .-f t . \\
4.27 \\
5.42 \\
1.41 \\
1.14\end{array}$ & $\begin{array}{l}\text { Oct. } 9 \\
\text { Nov. } 11 \\
\text { Nov. } 21 \\
\text { Dec. } 17\end{array}$ & 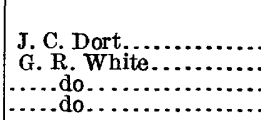 & $\begin{array}{l}\text { Feet. } \\
1.55 \\
2.21 \\
2.00 \\
1.58\end{array}$ & $\begin{array}{c}\text { Sec.-ft. } \\
3.08 \\
39.2 \\
29.6 \\
6.66\end{array}$ \\
\hline
\end{tabular}

Daily discharge, in second-feet, of South Fork of Kaukonahua Stream near Wahiawa, Oahu, for 1913 .

\begin{tabular}{|c|c|c|c|c|c|c|c|c|c|c|c|c|c|c|c|}
\hline Day. & June. & July. & Aug. & Sept. & Oct. & Nov. & Dec. & Day. & June. & July. & Aug. & Sept. & Oct. & Nov. & Dec. \\
\hline & & $\begin{array}{l}27 \\
43 \\
28 \\
11 \\
15\end{array}$ & $\begin{array}{r}1.1 \\
.9 \\
.7 \\
.7 \\
.7\end{array}$ & $\begin{array}{l}2.0 \\
2.0 \\
2.0 \\
2.0 \\
2.0\end{array}$ & $\begin{array}{r}1.2 \\
.8 \\
.8 \\
.7 \\
.7\end{array}$ & $\begin{array}{l}0.6 \\
.7 \\
9.6 \\
2.2 \\
1.1\end{array}$ & $\begin{array}{l}63 \\
57 \\
51 \\
45 \\
39\end{array}$ & $\begin{array}{l}16 . . \\
17 . \\
18 . \\
19 . . \\
20 . .\end{array}$ & & $\begin{array}{l}4.8 \\
4.5 \\
4.3 \\
4.0 \\
3.8\end{array}$ & $\begin{array}{l}3.8 \\
3.8 \\
4.0 \\
4.3 \\
4.5\end{array}$ & \begin{tabular}{|r}
2.0 \\
4.8 \\
16 \\
4.5 \\
3.2
\end{tabular} & $\begin{array}{l}1.2 \\
1.1 \\
1.2 \\
1.1 \\
.7\end{array}$ & $\begin{array}{r}7.7 \\
7.0 \\
7.4 \\
114 \\
111\end{array}$ & $\begin{array}{l}7.8 \\
6.7 \\
5.9 \\
5.2 \\
4.9\end{array}$ \\
\hline & & \begin{tabular}{c|}
11 \\
7.7 \\
8.0 \\
5.8 \\
5.1
\end{tabular} & $\begin{array}{r}.8 \\
1.2 \\
1.2 \\
.9 \\
1.8\end{array}$ & $\begin{array}{l}2.0 \\
2.0 \\
2.0 \\
2.0 \\
2.2\end{array}$ & $\begin{array}{r}.9 \\
2.2 \\
18 \\
6.4 \\
2.0\end{array}$ & \begin{tabular}{|l}
1.0 \\
1.1 \\
13 \\
88 \\
37
\end{tabular} & $\begin{array}{l}33 \\
30 \\
26 \\
22 \\
20\end{array}$ & $\begin{array}{l}21 \ldots \ldots \\
22 \ldots \\
23 \ldots \\
24 \ldots \\
25 \ldots\end{array}$ & $\begin{array}{l}9.6 \\
7.0 \\
6.1 \\
5.4 \\
5.4\end{array}$ & $\begin{array}{l}3.2 \\
3.0 \\
2.7 \\
2.5 \\
5.4\end{array}$ & $\begin{array}{r}2.2 \\
3.0 \\
12 \\
2.2 \\
1.8\end{array}$ & $\begin{array}{l}2.5 \\
7.4 \\
3.8 \\
1.8 \\
2.2\end{array}$ & $\begin{array}{r}.7 \\
.7 \\
.7 \\
1.1 \\
4.8\end{array}$ & $\begin{array}{c}43 \\
9.8 \\
41 \\
119 \\
29\end{array}$ & $\begin{array}{l}4.9 \\
4.3 \\
4.0 \\
4.0 \\
3.8\end{array}$ \\
\hline $\begin{array}{l}11 . \\
12 . \\
13 .\end{array}$ & & $\begin{array}{l}4.8 \\
4.8 \\
4.8 \\
4.8 \\
4.8\end{array}$ & $\begin{array}{c}2.2 \\
35 \\
7.7 \\
3.0 \\
3.2\end{array}$ & $\begin{array}{l}2.5 \\
3.0 \\
2.0 \\
1.8 \\
2.7\end{array}$ & $\begin{array}{l}1.4 \\
1.1 \\
2.0 \\
3.5 \\
1.6\end{array}$ & \begin{tabular}{|c|}
28 \\
66 \\
35 \\
13 \\
8.8
\end{tabular} & $\begin{array}{c}17 \\
15 \\
13 \\
11 \\
9.4\end{array}$ & $\begin{array}{l}26 \ldots \ldots \\
27 \ldots \\
28 \ldots \\
29 \ldots \\
30 \ldots \\
31 \ldots\end{array}$ & \begin{tabular}{|c|}
5.1 \\
11 \\
17 \\
7.4 \\
9.6
\end{tabular} & $\begin{array}{r}4.3 \\
7.4 \\
12 \\
6.4 \\
2.7 \\
2.0\end{array}$ & $\begin{array}{l}2.0 \\
2.2 \\
1.4 \\
2.7 \\
2.2 \\
2.0\end{array}$ & $\begin{array}{r}1.2 \\
1.0 \\
.9 \\
1.0\end{array}$ & $\begin{array}{r}1.1 \\
.8 \\
.8 \\
1.2 \\
.7 \\
.7\end{array}$ & \begin{tabular}{|l|}
92 \\
86 \\
80 \\
74 \\
68
\end{tabular} & $\begin{array}{l}3.6 \\
3.3 \\
3.1 \\
2.9 \\
2.7 \\
2.5\end{array}$ \\
\hline
\end{tabular}

Note.-Discharge estimated Nov. 27 to Dec. 5, and Dec. 18.

Monthly discharge of South Fork of Kaukonahua Stream near Wahiawa, Oahu, for 1913.

\begin{tabular}{|c|c|c|c|c|c|}
\hline \multirow{2}{*}{ Month. } & \multicolumn{3}{|c|}{ Discharge in second-feet. } & \multirow{2}{*}{$\begin{array}{l}\text { Run-off } \\
\text { (total in } \\
\text { acre-feet). }\end{array}$} & \multirow{2}{*}{$\begin{array}{l}\text { Accu- } \\
\text { racy. }\end{array}$} \\
\hline & Maximum. & Minimum. & Mean. & & \\
\hline 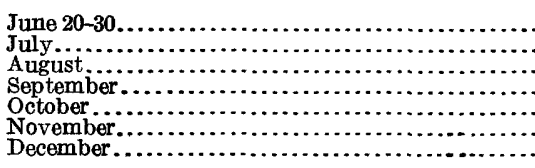 & $\begin{array}{r}23 \\
43 \\
35 \\
16 \\
18 \\
119 \\
63\end{array}$ & $\begin{array}{r}5.1 \\
2.0 \\
.7 \\
.9 \\
.7 \\
.6 \\
2.5\end{array}$ & $\begin{array}{r}9.69 \\
8.34 \\
3.72 \\
2.85 \\
2.00 \\
36.5 \\
16.8\end{array}$ & $\begin{array}{r}211 \\
513 \\
229 \\
170 \\
123 \\
2,170 \\
1,030\end{array}$ & $\begin{array}{l}\text { B. } \\
\text { B. } \\
\text { B. } \\
\text { B. } \\
\text { C.: } \\
\text { C. }\end{array}$ \\
\hline The period.. & & & $\cdots$ & 4,450 & \\
\hline
\end{tabular}


MISCELLANEOUS MEASUREMENTS.

Measurements of streams on the island of Oahu at points other than regular gaging stations are listed below:

Miscellaneous discharge measurements on Oahu in 1918.

\begin{tabular}{|c|c|c|c|c|}
\hline Late. & Stream. & Locality. & $\begin{array}{c}\text { Gage } \\
\text { height. }\end{array}$ & $\begin{array}{c}\text { Dis- } \\
\text { charge. }\end{array}$ \\
\hline & & & et. & Sec.-ft. \\
\hline $\begin{array}{l}\text { Max. } 27 \\
\text { Do.... }\end{array}$ & Wajalua artesian well, in flu & Waialua.. & & 0.79 \\
\hline & Hauula artesian well.. & Ḧauula....... & & 1.02 \\
\hline Mar. 28 & Lung Do $\mathrm{Y}$ artesian we & Honolulu... & & 1.28 \\
\hline Apr. 4 & Drain from Young Hote & .....do.... & & .60 \\
\hline Do.... & Alakea Street flood sewe & Alakea and Merchant St & & .75 \\
\hline Do. & .....do. & Alakea and Queen Streets, & & .59 \\
\hline Do. & .....do. & Opposite Hawaii Electric Co. & & 4.25 \\
\hline Do.... & do. & 100 feet aboveoutlet & & 5.36 \\
\hline Apr. 11 & Main Kahanaiki. & Kailua Valley. & 0.92 & 1.00 \\
\hline Do.... & Kahanaiki ditcho & .....do... & 82 & .31 \\
\hline Do.. & Wong Leong's ditc & ......do... & 1.92 & 4.66 \\
\hline May 15 & Paumalu Gulch. & Three-fourths mile below in- & & .13 \\
\hline Do. & ....do. & A bove intake, Pupuke & & .11 \\
\hline Do.... & Paumalu Stream & Below Pupukeapipe, P & & .08 \\
\hline$\underset{\text { Do.... }}{\operatorname{May}} 30$ & Waiomao Stream & A bove outlet of Kaau Crater.. & & .05 \\
\hline Do.... & do & $\begin{array}{l}\text { Crater. } \\
\text { Top of lower falls. }\end{array}$ & & 29 \\
\hline Do..... & .......do. & 900 feet elevation. & & .29 \\
\hline June 2 & Kalihi Stream. & Upper Kalihi & & 2.36 \\
\hline June 3 & Artesian well, on Bishop estate lan & Below Kamehameha Sch & & 1.88 \\
\hline June 4 & Kahanaiki ditch $a \ldots$ & Kailua Valley. & .60 & .27 \\
\hline July 1 & Branch Kahana Strean & Weir No. $12 \mathrm{~K}$ & & .74 \\
\hline Do.... & Main Branch Kahana Stream & Weir No. $5 \mathrm{~K}$ & & 5.91 \\
\hline Do.... & Branch Kahana Stream. & Weir No. 2 K & & .35 \\
\hline July 2 & Waih & Upper Waia & & 3.74 \\
\hline Do.... & Waianu Stream $c$. & Weir No. 2 Waianu Valles & & 3. 72 \\
\hline Do.... & Uwau Stream $d$. & At weir, Waianu Valles & & .48 \\
\hline Do..... & Waiswa Stream & Above intake, & & 2.80 \\
\hline $\begin{array}{l}\text { Aug. } 8 \\
\text { Aug. } 15\end{array}$ & Portal of tunnel....... & Waiahole... & 1.94 & 25.5 \\
\hline $\begin{array}{l}\text { Aug. } 15 \\
\text { Sept. } 9\end{array}$ & Wong Leong's ditch $b$ & Käailua Väliey...... & $\begin{array}{l}2.10 \\
1.49\end{array}$ & $\begin{array}{l}20.0 \\
4.22\end{array}$ \\
\hline Sept. 12 & Portal of tunnel.... & Waiahole.............. & 2.20 & 31.7 \\
\hline Oet. 22 & .....do. & .....do........ & 1.96 & 25.8 \\
\hline Oet. 31 & $\ldots . . . \mathrm{d}$ & & 2.03 & 22.4 \\
\hline Dec. 5 & Keaahala Spring $e$ & Kane & & .48 \\
\hline Jan. 25 & Pukele Stream $f$. & Below Mahoe Springs, Palolo .. & .62 & .50 \\
\hline Feb. 26 & .....do. & .....do. & .66 & .54 \\
\hline Mar. 29 & .....do. & .....do....... & .64 & .62 \\
\hline Apr. 13 & $\ldots .$. & .....do. & 1.58 & 19.2 \\
\hline June 24 & $\ldots . . . \mathrm{dc}$ & 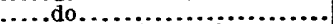 & .74 & 1.01 \\
\hline Feb. 26 & Waioma stream $f$. & A bove Pukele, Palolo.. & 1.10 & .42 \\
\hline Mar. 29 & .....do. & .....do......... & 1.12 & \\
\hline Apr. 13 & 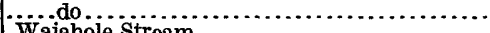 & $\ldots$ do & 1.80 & 17.8 \\
\hline Fêb. 1 & Waiahole Stream & At Waiahole, near Waik & 1.08 & 28.8 \\
\hline $\begin{array}{ll}\text { Aug. } & 8 \\
\text { Apr. } & 2\end{array}$ & Lulumaha Stream $g$ & Near Honoluiu.......... & 10.00 & $\begin{array}{l}8.4 \\
.51\end{array}$ \\
\hline Apr. 14 & ....do.. & ....do............ & 1.66 & .62 \\
\hline May 23 & ..... do. & .....do.......... & 1.61 & .70 \\
\hline $\begin{array}{l}\text { Sept. } 12 \\
\text { Nov. } 10\end{array}$ & a..d & …..ddo.. & 1.79 & $\dot{0.78}$ \\
\hline & & & & \\
\hline
\end{tabular}

$a$ Diverts from Kahanaiki Stream.

$b$ Diverts from Kailua Stream.

c Tributary to Waiahole Stream.

d Tributary to Waianu Stream. e Tributary to Kaneohe Stream. f Tributary to Palolo Stream. $g$ Tributary to Nuuanu Stream. 


\section{ISLAND OF MAUI.}

\section{IAO STREAM NEAR WAILUKU, MAUI.}

Location.-About 3 miles west of Wailuku and one-fourth mile below the forks of the stream.

Records available.-May 7, 1910, to December 31, 1913.

Gage.-Friez water-stage recorder.

Control.-Fairly permanent.

Discharge measurements.--Made by wading or from cable.

Diversions.-None above station.

Accuracy.--Records good.

Discharge measurements of Iao Stream near Wailuku, Maui, in 1913.

\begin{tabular}{|c|c|c|c|c|c|c|c|}
\hline Date. & Hydrographer. & $\begin{array}{l}\text { Gage } \\
\text { height. }\end{array}$ & $\begin{array}{c}\text { Dis- } \\
\text { charge. }\end{array}$ & Date. & Hydrographer. & $\begin{array}{c}\text { Gage } \\
\text { height. }\end{array}$ & $\begin{array}{l}\text { Dis- } \\
\text { charge. }\end{array}$ \\
\hline $\begin{array}{l}\text { Mar. } 17 \\
\text { Aug. } 12\end{array}$ & $\begin{array}{l}\text { C. T. Bailey ................ } \\
\text { Bailey and Christiansen }\end{array}$ & $\begin{array}{r}\text { Feet. } \\
2.86 \\
4.08\end{array}$ & $\begin{array}{c}\text { Sec.-ft. } \\
33.0 \\
213\end{array}$ & Dec. 4 & C. T. Bailey & $\begin{array}{c}\text { Feet. } \\
5.25\end{array}$ & Sec.-ft. \\
\hline
\end{tabular}

Daily discharge, in second-feet, of Iao Stream near Wailuku, Maui, for 1913.

\begin{tabular}{|c|c|c|c|c|c|c|c|c|c|c|c|c|}
\hline Day. & Jan. & Feb. & Mar. & Apr. & May. & June. & July. & Aug. & Sept. & Oct. & Nov. & Dec. \\
\hline $\begin{array}{l}3 \ldots . . \\
4 \ldots . .\end{array}$ & $\begin{array}{l}20 \\
19\end{array}$ & $\begin{array}{l}18 \\
12 \\
12 \\
16 \\
54\end{array}$ & $\begin{array}{l}64 \\
65 \\
65 \\
60 \\
61\end{array}$ & $\begin{array}{l}29 \\
25 \\
22 \\
22\end{array}$ & 76 & $\begin{array}{l}59 \\
65 \\
60 \\
56 \\
64\end{array}$ & $\begin{array}{r}92 \\
93 \\
72 \\
54 \\
118\end{array}$ & $\begin{array}{l}22 \\
22 \\
25 \\
23 \\
28\end{array}$ & $\begin{array}{l}35 \\
56 \\
40 \\
28 \\
26\end{array}$ & $\begin{array}{l}18 \\
15 \\
16 \\
19 \\
40\end{array}$ & $\begin{array}{r}12 \\
92 \\
138 \\
66 \\
35\end{array}$ & $\begin{array}{r}54 \\
46 \\
164 \\
553 \\
270\end{array}$ \\
\hline $\begin{array}{r}6 \ldots \ldots \\
7 \ldots \ldots \\
9 \ldots \ldots \ldots \\
10 \ldots \ldots\end{array}$ & $\begin{array}{l}18 \\
18 \\
18 \\
20 \\
20\end{array}$ & $\begin{array}{r}85 \\
131 \\
141 \\
47 \\
39\end{array}$ & $\begin{array}{l}62 \\
66 \\
65 \\
64 \\
59\end{array}$ & $\cdots$ & $\ldots$. & $\begin{array}{l}66 \\
60 \\
49 \\
90 \\
59\end{array}$ & $\begin{array}{l}58 \\
44 \\
31 \\
31 \\
31\end{array}$ & $\begin{array}{l}28 \\
30 \\
27 \\
51 \\
32\end{array}$ & $\begin{array}{l}26 \\
30 \\
30 \\
26 \\
30\end{array}$ & $\begin{array}{l}40 \\
43 \\
36 \\
26 \\
25\end{array}$ & $\begin{array}{r}25 \\
54 \\
341 \\
530 \\
173\end{array}$ & $\begin{array}{l}254 \\
148 \\
108 \\
83 \\
66\end{array}$ \\
\hline $\begin{array}{l}11 \\
12 \\
13 \ldots \ldots \\
14 \ldots \ldots\end{array}$ & $\begin{array}{r}19 \\
40 \\
145 \\
157 \\
124\end{array}$ & $\begin{array}{l}33 \\
27 \\
26 \\
26 \\
74\end{array}$ & $\begin{array}{r}56 \\
52 \\
51 \\
70 \\
108\end{array}$ & $\begin{array}{r}\ldots \ldots . . \\
\cdots 338 \\
308 \\
326\end{array}$ & $\begin{array}{r}38 \\
134 \\
124 \\
290 \\
177\end{array}$ & $\begin{array}{r}61 \\
105 \\
72 \\
100 \\
122\end{array}$ & $\begin{array}{l}31 \\
54 \\
42 \\
37 \\
34\end{array}$ & $\begin{array}{r}212 \\
170 \\
75 \\
64 \\
82\end{array}$ & $\begin{array}{l}25 \\
27 \\
27 \\
22 \\
22\end{array}$ & $\begin{array}{l}25 \\
11 \\
71 \\
30 \\
18\end{array}$ & $\begin{array}{r}159 \\
146 \\
108 \\
69 \\
55\end{array}$ & $\begin{array}{l}59 \\
52 \\
51 \\
89 \\
69\end{array}$ \\
\hline $\begin{array}{l}16 \ldots \ldots \ldots \\
17 \ldots \ldots \ldots \\
18 \ldots \ldots \ldots \\
19 \ldots \ldots \ldots \\
20 \ldots \ldots\end{array}$ & $\begin{array}{l}44 \\
32 \\
26\end{array}$ & $\begin{array}{r}65 \\
60 \\
244 \\
236 \\
141\end{array}$ & $\begin{array}{r}132 \\
54 \\
29 \\
26 \\
26\end{array}$ & $\begin{array}{r}216 \\
181 \\
122 \\
72 \\
66\end{array}$ & $\begin{array}{r}119 \\
159 \\
132 \\
138 \\
94\end{array}$ & $\begin{array}{l}92 \\
75 \\
67 \\
65 \\
66\end{array}$ & $\begin{array}{l}32 \\
32 \\
30 \\
75 \\
78\end{array}$ & $\begin{array}{r}183 \\
93 \\
65 \\
54 \\
43\end{array}$ & $\begin{array}{l}25 \\
60 \\
66 \\
36 \\
30\end{array}$ & $\begin{array}{l}13 \\
11 \\
11 \\
10 \\
10\end{array}$ & $\begin{array}{l}111 \\
104 \\
183 \\
121 \\
111\end{array}$ & $\begin{array}{l}54 \\
66 \\
93 \\
60 \\
54\end{array}$ \\
\hline $\begin{array}{l}21 \ldots \\
22, \ldots \\
23 \ldots \\
25 \ldots\end{array}$ & & $\begin{array}{r}105 \\
76 \\
65 \\
82 \\
141\end{array}$ & $\begin{array}{l}28 \\
79 \\
28 \\
25 \\
34\end{array}$ & \begin{tabular}{r}
76 \\
71 \\
$7 \ldots$ \\
\hdashline$\ldots .$. \\
\end{tabular} & $\begin{array}{l}82 \\
74 \\
79 \\
79 \\
69\end{array}$ & $\begin{array}{l}66 \\
78 \\
82 \\
76 \\
83\end{array}$ & $\begin{array}{l}45 \\
37 \\
49 \\
47 \\
40\end{array}$ & $\begin{array}{l}60 \\
45 \\
42 \\
34 \\
27\end{array}$ & $\begin{array}{l}33 \\
30 \\
30 \\
28 \\
\mathbf{2 7}\end{array}$ & $\begin{array}{r}10 \\
10 \\
9 \\
9 \\
9\end{array}$ & $\begin{array}{r}108 \\
66 \\
49 \\
126 \\
159\end{array}$ & $\begin{array}{l}58 \\
56 \\
58 \\
44 \\
49\end{array}$ \\
\hline 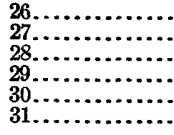 & 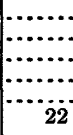 & $\begin{array}{l}82 \\
69 \\
64 \\
\cdots \cdots\end{array}$ & $\begin{array}{l}25 \\
25 \\
40 \\
22 \\
27 \\
47\end{array}$ & 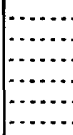 & $\begin{array}{l}96 \\
85 \\
85 \\
69 \\
65 \\
60\end{array}$ & $\begin{array}{r}79 \\
254 \\
155 \\
79 \\
150\end{array}$ & $\begin{array}{l}35 \\
24 \\
25 \\
23 \\
22 \\
21\end{array}$ & $\begin{array}{l}34 \\
27 \\
44 \\
30 \\
19 \\
13\end{array}$ & $\begin{array}{l}27 \\
27 \\
21 \\
19 \\
19\end{array}$ & $\begin{array}{r}10 \\
10 \\
8 \\
7 \\
6 \\
6\end{array}$ & $\begin{array}{r}141 \\
54 \\
44 \\
183 \\
64\end{array}$ & $\begin{array}{l}54 \\
51 \\
55 \\
54 \\
52 \\
51\end{array}$ \\
\hline
\end{tabular}

NoTE.-No record Jan. 1-3, 19-30; Apr. 5-12, 23-30; May 1, 3-10. 
Monthly discharge of Iao Stream near Wailuku, Maui, for 1913.

\begin{tabular}{|c|c|c|c|c|c|}
\hline \multirow{2}{*}{ Month. } & \multicolumn{3}{|c|}{ Discharge in second-feet. } & \multirow{2}{*}{$\begin{array}{l}\text { Run-off } \\
\text { (total in } \\
\text { acre-feet). }\end{array}$} & \multirow{2}{*}{$\begin{array}{l}\text { Accu- } \\
\text { racy. }\end{array}$} \\
\hline & Maximum. & Minimum. & Mean. & & \\
\hline \multirow[t]{2}{*}{ 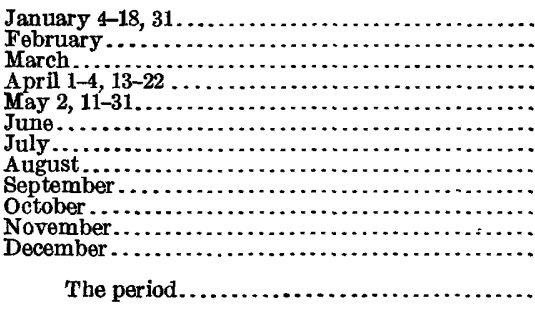 } & $\begin{array}{r}157 \\
244 \\
132 \\
338 \\
290 \\
254 \\
118 \\
212 \\
66 \\
71 \\
530 \\
553\end{array}$ & $\begin{array}{r}18 \\
12 \\
22 \\
22 \\
38 \\
49 \\
21 \\
13 \\
19 \\
6 \\
12 \\
44\end{array}$ & $\begin{array}{c}46.3 \\
77.5 \\
52.1 \\
134 \\
106 \\
85.2 \\
46.3 \\
55.0 \\
30.9 \\
18.8 \\
121 \\
95.9\end{array}$ & $\begin{array}{l}1,470 \\
4,300 \\
3,200 \\
3,720 \\
4,620 \\
5,070 \\
2,850 \\
3,380 \\
1,840 \\
1,160 \\
7,200 \\
5,900\end{array}$ & \multirow[t]{2}{*}{$\begin{array}{l}\mathbf{B} . \\
\mathbf{B} . \\
\mathbf{B} . \\
\mathbf{B} . \\
\mathbf{B} . \\
\mathbf{B} . \\
\mathbf{B} . \\
\mathbf{B} . \\
\mathbf{B} . \\
\text { B. } \\
\text { B. } \\
\text { B }\end{array}$} \\
\hline & $\cdots$ & $\ldots \ldots \ldots \ldots$ & $\ldots \ldots \ldots$ & 44,700 & \\
\hline
\end{tabular}

\section{MANIANIA DITCH NEAR WAILUKU, MAUI.}

Location.-About $2 \frac{1}{2}$ miles west of Wailuku and 800 feet below intake.

Records available.-October 18, 1910, to September 30, 1913.

Gage.-Vertical staff.

Control.-Probably permanent.

Discharge measurements.--Made from plank footbridge above 50 feet above gage. Accuracy.-Records fair.

Discharge measurements of Maniania ditch near Wailuku, Maui, in 1918.

[C. T. Bailey, hydrographer.]

\begin{tabular}{|c|c|c|c|c|c|}
\hline Date. & $\begin{array}{c}\text { Gage } \\
\text { height. }\end{array}$ & $\begin{array}{c}\text { Dis- } \\
\text { charge. }\end{array}$ & Date. & $\begin{array}{r}\text { Gage } \\
\text { height. }\end{array}$ & $\begin{array}{c}\text { Dis- } \\
\text { charg } \theta .\end{array}$ \\
\hline $\begin{array}{l}\text { Mar. } 17 \\
\text { Apr. } 8 \ldots \ldots \ldots \ldots \ldots \ldots\end{array}$ & $\begin{array}{r}\text { Feet. } \\
1.07 \\
.74\end{array}$ & $\begin{array}{r}\text { Sec.-ft. } \\
22.8 \\
15.8\end{array}$ & May $1 . . . .$. & $\begin{array}{c}\text { Feet. } \\
1.56\end{array}$ & $\begin{array}{r}\text { Sec.-ft. } \\
\quad 33.2\end{array}$ \\
\hline
\end{tabular}

Monthly discharge of Maniania ditch near Wailuku, Maui, for 1918.

\begin{tabular}{|c|c|c|c|c|c|}
\hline \multirow{2}{*}{ Month. } & \multicolumn{3}{|c|}{ Discharge in second-feet. } & \multirow{2}{*}{$\begin{array}{c}\text { Run-off } \\
\text { (total in } \\
\text { acre-feet). }\end{array}$} & \multirow{2}{*}{$\begin{array}{l}\text { Accu- } \\
\text { racy. }\end{array}$} \\
\hline & Maximum. & Minimum. & Mean. & & \\
\hline \multirow[t]{2}{*}{$\begin{array}{l}\text { January } \\
\text { February } \\
\text { March } \\
\text { April } \\
\text { May } \\
\text { June } \\
\text { July } \\
\text { August } \\
\text { September }\end{array}$} & $\begin{array}{l}28 \\
26 \\
28 \\
31 \\
30 \\
31 \\
32 \\
32 \\
34\end{array}$ & $\begin{array}{c}14 \\
5.0 \\
15 \\
17 \\
10 \\
22 \\
23 \\
20 \\
17\end{array}$ & $\begin{array}{l}19.5 \\
13.5 \\
21.7 \\
24.1 \\
23.9 \\
26.6 \\
27.5 \\
26.5 \\
22.8\end{array}$ & $\begin{array}{r}1,200 \\
750 \\
1,330 \\
1,430 \\
1,470 \\
1,580 \\
1,690 \\
1,630 \\
1,360\end{array}$ & \multirow[t]{2}{*}{$\begin{array}{l}\text { C. } \\
\text { C. } \\
\text { C. } \\
\text { C. } \\
\text { C. } \\
\text { C. } \\
\text { C. } \\
\text { C. } \\
\text { C. }\end{array}$} \\
\hline & .. & & & 12,400 & \\
\hline
\end{tabular}




\section{SOUTH WAIEHU STREAM NEAR WAILUKU, MAUI.}

Location.-About 3 miles west of Wailuku and 300 feet above South Waiehu ditch intake.

Records available.-March 19 to December 31, 1913.

Gage.-Vertical staff.

Control.-Shifting.

Discharge measurements.-Made from footbridge or by wading.

Diversions.- One small taro ditch, carrying about 0.2 second-foot at ordinary stages, diverts water above the gage.

Accuracy.-Records for March, July, August, and October, good; for other months, poor.

Discharge measurements of South Waiehu Stream near Wailuku, Maui, in 1913.

[C. T. Bailey, hydrographer.]

\begin{tabular}{|c|c|c|c|c|c|}
\hline Date. & $\begin{array}{c}\text { Gage } \\
\text { height. }\end{array}$ & $\begin{array}{l}\text { Dis- } \\
\text { charge. }\end{array}$ & Date. & $\begin{array}{c}\text { Gage } \\
\text { hoight. }\end{array}$ & $\begin{array}{l}\text { Dis- } \\
\text { charge. }\end{array}$ \\
\hline $\begin{array}{l}\text { Mar. } 18 \ldots \ldots \ldots \\
\text { Mar. } 21 \ldots \ldots \ldots \ldots\end{array}$ & $\begin{array}{r}\text { Feet. } \\
0.58 \\
.76\end{array}$ & $\begin{array}{r}\text { Sec. }-f t \\
4.91 \\
8.01\end{array}$ & $\begin{array}{l}\text { June } 28, \ldots \ldots \ldots \\
\text { July } 31, \ldots \ldots \ldots \ldots\end{array}$ & $\begin{array}{r}\text { Feet. } \\
0.54 \\
.53\end{array}$ & $\begin{array}{r}\text { sec. }-f t \\
\text { 3. } \\
2.73 \\
\text { 2. }\end{array}$ \\
\hline
\end{tabular}

Daily discharge, in second-feet, of South Waiehu Stream near Wailuku, Maui, for 1913.

\begin{tabular}{|c|c|c|c|c|c|c|c|c|c|c|}
\hline Day. & Mar. & Apr. & May. & June. & July. & Aug. & Sept. & Oct. & Nov. & Dec. \\
\hline $\begin{array}{l}1 \ldots \ldots \\
2 \ldots \ldots \\
4 \ldots \ldots \\
5 \ldots \ldots\end{array}$ & & $\begin{array}{l}3.8 \\
4.2 \\
3.8 \\
4.2 \\
\text { 3. } 8\end{array}$ & $\begin{array}{l}3.8 \\
4.4 \\
3.8 \\
3.8 \\
3.8\end{array}$ & $\begin{array}{l}3.8 \\
3.8 \\
4.2 \\
3.8 \\
3.8\end{array}$ & $\begin{array}{l}2.4 \\
2.8 \\
3.0 \\
3.0 \\
3.0\end{array}$ & $\begin{array}{l}3.4 \\
6.0 \\
7.4 \\
3.4 \\
4.7\end{array}$ & $\begin{array}{r}3.6 \\
92 \\
6.5 \\
3.6 \\
3.6\end{array}$ & $\begin{array}{l}6.3 \\
6.3 \\
7.1 \\
4.4 \\
5.6\end{array}$ & $\begin{array}{r}5.6 \\
8.8 \\
58 \\
5.1 \\
4.0\end{array}$ & $\begin{array}{r}7.1 \\
7.7 \\
76 \\
177 \\
185\end{array}$ \\
\hline $\begin{array}{r}6 \ldots \ldots \\
7 \ldots \ldots \\
8 \ldots \ldots \\
10 \ldots \ldots\end{array}$ & & $\begin{array}{l}\text { 3. } 8 \\
\text { 3. } 8 \\
\text { 3. } 8 \\
\text { 3. } 8 \\
\text { 3. } 8\end{array}$ & $\begin{array}{l}3.8 \\
3.8 \\
3.8 \\
3.8 \\
3.8\end{array}$ & $\begin{array}{l}3.8 \\
3.8 \\
3.8 \\
3.8 \\
4.7\end{array}$ & $\begin{array}{l}3.0 \\
2.8 \\
2.8 \\
3.0 \\
2.8\end{array}$ & $\begin{array}{l}4.9 \\
5.4 \\
4.7 \\
5.6 \\
5.8\end{array}$ & $\begin{array}{c}3.8 \\
20 \\
6.0 \\
3.8 \\
3.8\end{array}$ & $\begin{array}{l}8.8 \\
4.7 \\
4.7 \\
4.7 \\
4.7\end{array}$ & $\begin{array}{c}4.0 \\
76 \\
137 \\
58 \\
8.8\end{array}$ & $\begin{array}{r}143 \\
44 \\
8.0 \\
5.6 \\
4.9\end{array}$ \\
\hline $\begin{array}{l}11 \ldots \ldots \\
12 \ldots \ldots \\
13 \ldots \ldots \\
14 \ldots . \\
15 \ldots .\end{array}$ & $\cdots$ & $\begin{array}{c}30 \\
14 \\
49 \\
5.6 \\
17\end{array}$ & $\begin{array}{r}3.8 \\
116 \\
128 \\
92 \\
17\end{array}$ & $\begin{array}{l}3.8 \\
4.7 \\
4.7 \\
3.8 \\
3.8\end{array}$ & $\begin{array}{l}5.1 \\
4.7 \\
4.2 \\
3.8 \\
3.8\end{array}$ & $\begin{array}{r}7.7 \\
12 \\
3.4 \\
5.6 \\
5.4\end{array}$ & $\begin{array}{l}4.7 \\
3.8 \\
4.4 \\
3.8 \\
3.8\end{array}$ & $\begin{array}{l}4.7 \\
4.7 \\
4.7 \\
4.7 \\
4.7\end{array}$ & $\begin{array}{l}5.1 \\
5.1 \\
5.1 \\
4.7 \\
4.7\end{array}$ & $\begin{array}{l}5.1 \\
5.1 \\
4.2 \\
78 \\
10\end{array}$ \\
\hline $\begin{array}{l}16 \ldots \ldots \\
17 \ldots \ldots \\
18 \ldots \ldots \\
20 \ldots \ldots\end{array}$ & $\begin{array}{r}3.8 \\
3.8\end{array}$ & $\begin{array}{c}29 \\
58 \\
38 \\
4.4 \\
3.8\end{array}$ & $\begin{array}{l}4.7 \\
4.2 \\
3.8 \\
3.8 \\
3.8\end{array}$ & $\begin{array}{l}3.8 \\
3.8 \\
3.8 \\
3.8 \\
3.8\end{array}$ & $\begin{array}{l}3.4 \\
3.0 \\
2.6 \\
6.0 \\
5.1\end{array}$ & $\begin{array}{r}14 \\
4.9 \\
3.8 \\
3.8 \\
3.8\end{array}$ & $\begin{array}{l}5.1 \\
11 \\
11 \\
5.1 \\
4.2\end{array}$ & $\begin{array}{l}5.1 \\
4.2 \\
4.7 \\
5.6 \\
6.0\end{array}$ & $\begin{array}{c}4.7 \\
4.9 \\
42 \\
48 \\
8.2\end{array}$ & $\begin{array}{l}9.4 \\
9.1 \\
6.5 \\
6.8 \\
6.0\end{array}$ \\
\hline $\begin{array}{l}21 \ldots \ldots \ldots \\
22 . \ldots \ldots \\
23 \ldots \ldots \\
24 \ldots \ldots \\
25 \ldots \ldots\end{array}$ & $\begin{array}{l}\text { 3. } 8 \\
8.8 \\
3.8 \\
3.8 \\
3.8\end{array}$ & $\begin{array}{l}8.8 \\
8.8 \\
4.4 \\
3.8 \\
3.8\end{array}$ & $\begin{array}{l}3.8 \\
3.8 \\
3.8 \\
3.8 \\
3.8\end{array}$ & $\begin{array}{l}3.8 \\
3.8 \\
3.8 \\
3.8 \\
3.8\end{array}$ & $\begin{array}{l}4.7 \\
4.7 \\
4.7 \\
4.4 \\
3.4\end{array}$ & $\begin{array}{l}5.1 \\
3.8 \\
4.0 \\
4.0 \\
4.0\end{array}$ & $\begin{array}{l}4.2 \\
3.4 \\
4.2 \\
3.8 \\
4.7\end{array}$ & $\begin{array}{l}6.5 \\
4.0 \\
6.5 \\
3.8 \\
4.0\end{array}$ & $\begin{array}{l}6.3 \\
4.9 \\
5.1 \\
6.8 \\
5.1\end{array}$ & $\begin{array}{l}6.3 \\
4.9 \\
4.7 \\
4.7 \\
4.7\end{array}$ \\
\hline $\begin{array}{l}26 \\
27 \\
28 \\
29 \\
30 \ldots \ldots \ldots \ldots \\
31 \ldots \ldots \ldots\end{array}$ & $\begin{array}{l}\text { 3. } 8 \\
\text { 3. } \\
6.0 \\
\text { 3. } \\
5.1 \\
\text { 3. } 8\end{array}$ & $\begin{array}{l}3.8 \\
3.8 \\
3.8 \\
3.8 \\
3.8\end{array}$ & $\begin{array}{r}3.8 \\
6.8 \\
10 \\
5.6 \\
3.8 \\
3.8\end{array}$ & $\begin{array}{r}3.8 \\
62 \\
3.8 \\
3.8 \\
4.7\end{array}$ & $\begin{array}{l}2.6 \\
3.4 \\
6.0 \\
3.6 \\
4.7 \\
5.4\end{array}$ & $\begin{array}{r}4.0 \\
5.1 \\
4.7 \\
4.7 \\
4.0 \\
10\end{array}$ & $\begin{array}{l}4.7 \\
4.7 \\
4.9 \\
4.9 \\
4.9\end{array}$ & $\begin{array}{l}4.2 \\
4.2 \\
4.2 \\
4.2 \\
4.0 \\
4.0\end{array}$ & $\begin{array}{r}6.0 \\
4.4 \\
4.2 \\
4.2 \\
4.7 \\
\quad \\
\end{array}$ & $\begin{array}{l}4.7 \\
4.7 \\
4.7 \\
4.7 \\
4.7 \\
5.1\end{array}$ \\
\hline
\end{tabular}


Monthly discharge of South Waiehu Stream near Wailuku, Maui, for 1913.

\begin{tabular}{|c|c|c|c|c|c|}
\hline \multirow{2}{*}{ Month. } & \multicolumn{3}{|c|}{ Discharge in second-feet. } & \multirow{2}{*}{$\begin{array}{l}\text { Run-off } \\
\text { (total in } \\
\text { acre-feet). }\end{array}$} & \multirow{2}{*}{$\begin{array}{l}\text { Accu } \\
\text { racy. }\end{array}$} \\
\hline & Maximum. & Minimum. & Mean. & & \\
\hline 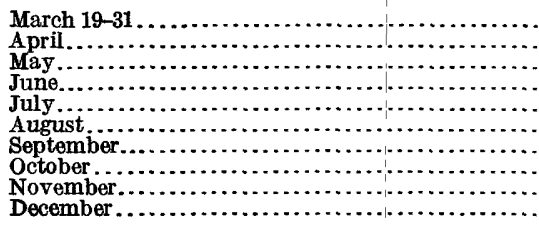 & $\begin{array}{r}8.8 \\
58 \\
128 \\
62 \\
6 \\
14 \\
92 \\
8.8 \\
137 \\
185\end{array}$ & $\begin{array}{l}\text { 3. } \\
3.8 \\
3.8 \\
3.8 \\
2.4 \\
3.4 \\
3.4 \\
3.8 \\
4.0 \\
4.2\end{array}$ & $\begin{array}{l}4.45 \\
11.2 \\
15.1 \\
5.87 \\
3.80 \\
5.45 \\
8.27 \\
5.03 \\
18.3 \\
27.5\end{array}$ & $\begin{array}{r}115 \\
666 \\
928 \\
349 \\
234 \\
335 \\
492 \\
309 \\
1,090 \\
1,690\end{array}$ & $\begin{array}{l}\text { A. } \\
\text { D. } \\
\text { D. } \\
\text { D. } \\
\text { A. } \\
\text { A. } \\
\text { D. } \\
\text { A. } \\
\text { D. } \\
\text { D. }\end{array}$ \\
\hline The period. & . & ... & 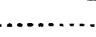 & 6,210 & \\
\hline
\end{tabular}

\section{SOUTH WAIEHU DITCH NEAR WAILUKU, MAUI.}

Location.-About $1 \frac{1}{2}$ miles northwest of Wailuku, one-fourth mile above Waihee canal crossing, in flume 60 feet below intake.

Records available.-July 13, 1912, to December 31, 1913.

Gage.-Vertical staff.

Control.-Fairly permanent.

Diversions.-Ditch diverts all low-water flow.

Discharge measurements.-Made from 2 by 4 at gage.

Accuracy.-Records good.

Discharge measurements of South Waiehu ditch near Wailuku, Maui, in 1912-13.

\begin{tabular}{|c|c|c|c|c|c|c|c|}
\hline Date. & Hydrographer. & $\begin{array}{l}\text { Gage } \\
\text { height. }\end{array}$ & $\begin{array}{c}\text { Dis- } \\
\text { charge. }\end{array}$ & Date. & Hydrographer. & $\begin{array}{c}\text { Gage } \\
\text { height. }\end{array}$ & $\begin{array}{c}\text { Dis- } \\
\text { charge. }\end{array}$ \\
\hline $\begin{array}{l}\text { 1912 } \\
\text { July } 13 \\
\text { July } 16 \\
\text { Sept. } 6 \\
\text { Nov. } 21\end{array}$ & 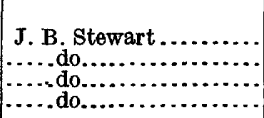 & \begin{tabular}{|r} 
Feet. \\
0.48 \\
.45 \\
.42 \\
.48
\end{tabular} & $\begin{array}{r}\text { Sec. }-f t . \\
4.16 \\
3.79 \\
3.47 \\
4.27\end{array}$ & $\begin{array}{l}1913 \\
\text { Mar. } 15 \\
\text { Mar. } 19 \\
\text { Mar. } 21\end{array}$ & 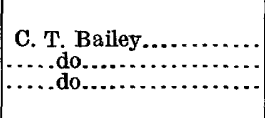 & $\begin{array}{r}\text { Feet. } \\
0.72 \\
.36 \\
.50\end{array}$ & $\begin{array}{r}\text { Sec.-ft. } \\
9.98 \\
2.51 \\
4.57\end{array}$ \\
\hline
\end{tabular}


Daily discharge, in second-feet, of South Waiehu ditch near Wailuku, Maui, for 1918.

\begin{tabular}{|c|c|c|c|c|c|c|c|c|c|c|c|c|}
\hline Day. & Jan. & Feb. & Mar. & Apr. & May. & June. & July. & Aug. & Sept. & Oct. & Nov. & Dec. \\
\hline $\begin{array}{l}1 \ldots \\
2 \ldots \\
3 \ldots \\
4 \ldots \\
5 \ldots\end{array}$ & $\begin{array}{l}3.0 \\
3.0 \\
4.6 \\
3.8 \\
3.0\end{array}$ & $\begin{array}{l}\text { 3. } 0 \\
3.0 \\
3.0 \\
3.0 \\
3.8\end{array}$ & $\begin{array}{l}3.0 \\
3.0 \\
3.0 \\
3.0 \\
3.0\end{array}$ & $\begin{array}{l}2.8 \\
3.0 \\
2.8 \\
3.0 \\
2.8\end{array}$ & $\begin{array}{l}2.8 \\
3.2 \\
2.8 \\
2.8 \\
2.8\end{array}$ & $\begin{array}{l}2.8 \\
2.8 \\
3.0 \\
2.8 \\
2.8\end{array}$ & $\begin{array}{l}3.2 \\
4.6 \\
2.6 \\
2.6 \\
2.6\end{array}$ & $\begin{array}{l}2.8 \\
3.3 \\
3.6 \\
2.6 \\
3.2\end{array}$ & $\begin{array}{r}2.5 \\
15 \\
4.6 \\
2.5 \\
2.5\end{array}$ & $\begin{array}{l}3.0 \\
3.0 \\
3.5 \\
3.2 \\
2.8\end{array}$ & $\begin{array}{l}3.2 \\
5.0 \\
7.3 \\
3.2 \\
2.8\end{array}$ & $\begin{array}{r}4.6 \\
5.0 \\
7.5 \\
13 \\
10\end{array}$ \\
\hline $\begin{array}{r}6 \ldots \ldots \\
7 \ldots \ldots \\
8 \ldots \ldots \\
9 \ldots \ldots \\
10 \ldots \ldots\end{array}$ & $\begin{array}{l}3.0 \\
3.0 \\
3.0 \\
3.0 \\
3.0\end{array}$ & $\begin{array}{l}17 \\
15 \\
19 \\
3.8 \\
3.0\end{array}$ & $\begin{array}{l}3.0 \\
4.6 \\
3.0 \\
3.0 \\
3.0\end{array}$ & $\begin{array}{l}2.8 \\
2.8 \\
2.8 \\
2.8 \\
2.8\end{array}$ & $\begin{array}{l}2.8 \\
2.8 \\
2.8 \\
2.8 \\
2.8\end{array}$ & $\begin{array}{l}2.8 \\
2.8 \\
2.8 \\
2.8 \\
3.3\end{array}$ & $\begin{array}{l}2.6 \\
2.8 \\
2.8 \\
2.6 \\
2.6\end{array}$ & $\begin{array}{l}3.0 \\
3.3 \\
3.2 \\
3.3 \\
4.3\end{array}$ & $\begin{array}{l}2.6 \\
9.4 \\
3.0 \\
2.6 \\
2.6\end{array}$ & $\begin{array}{l}5.9 \\
3.0 \\
3.0 \\
2.8 \\
2.8\end{array}$ & $\begin{array}{c}2.8 \\
5.7 \\
10 \\
9.9 \\
4.6\end{array}$ & $\begin{array}{r}13 \\
9.9 \\
5.3 \\
3.6 \\
3.2\end{array}$ \\
\hline $\begin{array}{l}11 \ldots \ldots \\
12 \ldots \ldots \ldots \\
14 \ldots \ldots \\
15 \ldots \ldots \ldots\end{array}$ & $\begin{array}{l}3.0 \\
3.0 \\
3.0 \\
3.0 \\
3.0\end{array}$ & $\begin{array}{l}\text { 3. } 0 \\
\text { 3. } 0 \\
\text { 3. } 0 \\
\text { 3. } 0 \\
\text { 3. } 0\end{array}$ & $\begin{array}{l}3.0 \\
3.0 \\
3.0 \\
3.0 \\
5.7\end{array}$ & $\begin{array}{r}12 \\
9.9 \\
12 \\
4.3 \\
8.9\end{array}$ & $\begin{array}{c}2.8 \\
6.4 \\
7.1 \\
12 \\
12\end{array}$ & $\begin{array}{l}2.8 \\
3.3 \\
3.3 \\
2.8 \\
2.8\end{array}$ & $\begin{array}{l}2.8 \\
3.2 \\
3.0 \\
3.3 \\
3.3\end{array}$ & $\begin{array}{l}4.1 \\
7.8 \\
2.6 \\
3.5 \\
3.5\end{array}$ & $\begin{array}{l}2.9 \\
2.6 \\
2.9 \\
2.4 \\
2.4\end{array}$ & $\begin{array}{l}2.8 \\
2.8 \\
2.8 \\
3.0 \\
2.8\end{array}$ & $\begin{array}{l}2.9 \\
3.0 \\
3.0 \\
3.0 \\
3.0\end{array}$ & $\begin{array}{l}3.3 \\
3.3 \\
2.8 \\
8.6 \\
6.8\end{array}$ \\
\hline $\begin{array}{l}16 \ldots \ldots \\
17 \ldots \ldots \ldots \\
18 \ldots \ldots \ldots \\
20 \ldots \ldots\end{array}$ & $\begin{array}{l}3.0 \\
3.0 \\
3.0 \\
3.0 \\
3.0\end{array}$ & $\begin{array}{r}4.6 \\
6.8 \\
6.8 \\
12 \\
4.6\end{array}$ & $\begin{array}{l}3.8 \\
4.6 \\
3.8 \\
2.8 \\
2.8\end{array}$ & $\begin{array}{c}10 \\
12 \\
8.4 \\
3.2 \\
2.8\end{array}$ & $\begin{array}{l}3.3 \\
4.6 \\
2.8 \\
2.8 \\
2.8\end{array}$ & $\begin{array}{l}2.8 \\
2.8 \\
2.8 \\
2.8 \\
2.8\end{array}$ & $\begin{array}{l}3.3 \\
3.0 \\
2.6 \\
5.0 \\
3.3\end{array}$ & $\begin{array}{l}9.4 \\
3.2 \\
2.6 \\
2.6 \\
2.6\end{array}$ & $\begin{array}{l}3.0 \\
4.0 \\
5.3 \\
3.3 \\
2.8\end{array}$ & $\begin{array}{l}3.0 \\
2.8 \\
2.8 \\
2.9 \\
3.0\end{array}$ & $\begin{array}{l}3.0 \\
3.2 \\
8.1 \\
7.3 \\
5.3\end{array}$ & $\begin{array}{l}6.4 \\
6.1 \\
4.3 \\
4.4 \\
4.0\end{array}$ \\
\hline 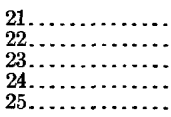 & $\begin{array}{l}3.0 \\
3.0 \\
3.0 \\
3.0 \\
3.0\end{array}$ & $\begin{array}{l}3.0 \\
3.0 \\
3.0 \\
3.0 \\
4.6\end{array}$ & $\begin{array}{l}2.8 \\
6.0 \\
2.8 \\
2.8 \\
2.8\end{array}$ & $\begin{array}{l}6.4 \\
6.4 \\
3.2 \\
2.8 \\
2.8\end{array}$ & $\begin{array}{l}2.8 \\
2.8 \\
2.8 \\
2.8 \\
2.8\end{array}$ & $\begin{array}{l}2.8 \\
2.8 \\
2.8 \\
2.8 \\
2.8\end{array}$ & $\begin{array}{l}2.8 \\
2.5 \\
2.5 \\
3.0 \\
2.8\end{array}$ & $\begin{array}{l}3.3 \\
2.6 \\
2.7 \\
2.7 \\
2.7\end{array}$ & $\begin{array}{l}2.8 \\
2.4 \\
2.7 \\
2.6 \\
2.9\end{array}$ & $\begin{array}{l}3.2 \\
2.7 \\
3.2 \\
2.8 \\
2.8\end{array}$ & $\begin{array}{l}4.1 \\
3.3 \\
3.3 \\
4.1 \\
3.3\end{array}$ & $\begin{array}{l}4.1 \\
3.2 \\
3.0 \\
3.0 \\
3.0\end{array}$ \\
\hline 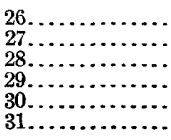 & $\begin{array}{l}3.0 \\
3.0 \\
3.0 \\
3.0 \\
3.0 \\
3.0\end{array}$ & \begin{tabular}{r}
3.0 \\
3.0 \\
3.0 \\
$\cdots . .$. \\
\hdashline$\ldots .$. \\
$\ldots .$.
\end{tabular} & $\begin{array}{l}2.8 \\
2.8 \\
4.3 \\
2.8 \\
5.7 \\
2.8\end{array}$ & $\begin{array}{r}2.8 \\
2.8 \\
2.8 \\
2.8 \\
2.8 \\
\ldots .\end{array}$ & $\begin{array}{l}2.8 \\
4.0 \\
6.4 \\
4.0 \\
2.8 \\
2.8\end{array}$ & $\begin{array}{l}2.8 \\
7.3 \\
2.8 \\
2.8 \\
3.3\end{array}$ & $\begin{array}{l}2.7 \\
2.8 \\
3.5 \\
3.2 \\
3.3 \\
3.2\end{array}$ & $\begin{array}{l}2.7 \\
3.0 \\
3.0 \\
3.0 \\
2.7 \\
6.8\end{array}$ & $\begin{array}{l}2.9 \\
2.8 \\
2.9 \\
3.0 \\
3.0\end{array}$ & $\begin{array}{l}2.8 \\
2.8 \\
2.8 \\
2.8 \\
2.7 \\
2.7\end{array}$ & $\begin{array}{l}4.0 \\
2.9 \\
2.8 \\
2.9 \\
3.0\end{array}$ & $\begin{array}{l}3.0 \\
3.0 \\
3.0 \\
3.0 \\
3.0 \\
3.3\end{array}$ \\
\hline
\end{tabular}

Monthly discharge of South Waiehu ditch near Wailuku, Maui, for 1913.

\begin{tabular}{l} 
Month. \\
\hline
\end{tabular}




\section{NORTH WAIEHU STREAM NEAR WAILUKU, MAUI.}

Location.-One mile above Waihee canal crossing and about $2 \frac{1}{2}$ miles northwest of Wailuku; 50 feet above uppermost diversion.

Records available.-July 9, 1912, to December 31, 1913.

Gage.-Vertical staff.

Control.-Probably shifting.

Discharge measurements.-Made by wading.

Diversions.-None above station.

Accuracy.-Records doubtful.

Cooperation.-Station is maintained in cooperation with Wailuku Sugar Co.

Discharge measurements of North Waiehu Stream near Wailuku, Maui, in 1913.

[C. T. Bailey, hydrographer.]

\begin{tabular}{|c|c|c|c|c|c|}
\hline Date. & $\begin{array}{c}\text { Gage } \\
\text { height. }\end{array}$ & $\begin{array}{l}\text { Dis- } \\
\text { charge. }\end{array}$ & Date. & $\begin{array}{c}\text { Gage } \\
\text { height. }\end{array}$ & $\begin{array}{l}\text { Dis- } \\
\text { charge. }\end{array}$ \\
\hline $\begin{array}{l}\text { Mar. } 14 \ldots \ldots \ldots \\
\text { Apr. } 10 . . . . . .\end{array}$ & $\begin{array}{r}\text { Feet. } \\
0.60 \\
.59\end{array}$ & $\begin{array}{r}\text { Sec.-ft. } \\
3.58 \\
\quad 3.89\end{array}$ & $\begin{array}{l}\text { July } 3 \ldots \ldots \ldots \ldots \\
\text { Aug. } 14 . \ldots \ldots \ldots \ldots\end{array}$ & $\begin{array}{r}\text { Feet. } \\
0.62 \\
.60\end{array}$ & $\begin{array}{r}\text { Sec. }-f t . \\
3.92 \\
4.69\end{array}$ \\
\hline
\end{tabular}

Daily discharge, in second-feet, of North Waiehu Stream near Wailuku, Maui, for 1913.

\begin{tabular}{|c|c|c|c|c|c|c|c|c|c|c|c|c|}
\hline Day. & Jan. & Feb. & Mar. & Apr. & May. & June. & July. & Aug. & Sept. & Oct. & Nov. & Dec. \\
\hline $\begin{array}{l}1 \ldots \\
2 \ldots \\
3 \ldots \\
4 \ldots \\
5 \ldots\end{array}$ & $\begin{array}{l}4.2 \\
4.7 \\
4.7 \\
4.5 \\
4.2\end{array}$ & $\begin{array}{l}4.2 \\
4.2 \\
4.2 \\
4.2 \\
4.2\end{array}$ & $\begin{array}{l}4.2 \\
4.2 \\
4.2 \\
4.2 \\
4.2\end{array}$ & $\begin{array}{l}4.2 \\
4.2 \\
4.2 \\
4.2 \\
4.2\end{array}$ & $\begin{array}{l}4.2 \\
6.5 \\
4.2 \\
4.2 \\
4.2\end{array}$ & $\begin{array}{l}4.2 \\
4.5 \\
4.7 \\
4.7 \\
4.2\end{array}$ & $\begin{array}{l}5.2 \\
7.4 \\
5.5 \\
4.2 \\
4.5\end{array}$ & $\begin{array}{l}4.2 \\
4.7 \\
5.0 \\
4.2 \\
4.2\end{array}$ & $\begin{array}{c}4.5 \\
12 \\
5.5 \\
4.7 \\
4.2\end{array}$ & $\begin{array}{l}4.2 \\
4.5 \\
9.0 \\
6.8 \\
6.5\end{array}$ & $\begin{array}{l}4.5 \\
4.5 \\
4.5 \\
4.5 \\
4.5\end{array}$ & $\begin{array}{l}7.1 \\
6.3 \\
6.5 \\
22 \\
12\end{array}$ \\
\hline $\begin{array}{r}6 \ldots \\
7 \ldots \\
8 \ldots \\
9 \ldots \\
10 \ldots\end{array}$ & $\begin{array}{l}4.2 \\
4.2 \\
4.2 \\
4.2 \\
4.2\end{array}$ & $\begin{array}{c}4.2 \\
10 \\
5.5 \\
5.5 \\
5.5\end{array}$ & $\begin{array}{l}4.2 \\
7.4 \\
4.5 \\
4.2 \\
4.2\end{array}$ & $\begin{array}{l}4.2 \\
4.2 \\
4.2 \\
4.2 \\
3.5\end{array}$ & $\begin{array}{l}4.2 \\
4.2 \\
4.2 \\
4.2 \\
4.5\end{array}$ & $\begin{array}{l}4.2 \\
4.2 \\
4.2 \\
4.2 \\
5.0\end{array}$ & $\begin{array}{l}4.7 \\
4.7 \\
4.7 \\
4.7 \\
4.5\end{array}$ & $\begin{array}{l}4.2 \\
4.2 \\
4.2 \\
4.2 \\
5.2\end{array}$ & $\begin{array}{l}3.9 \\
5.2 \\
4.2 \\
4.0 \\
4.0\end{array}$ & $\begin{array}{l}6.5 \\
6.3 \\
5.8 \\
5.2 \\
5.0\end{array}$ & $\begin{array}{c}4.5 \\
5.5 \\
20 \\
16 \\
7.8\end{array}$ & $\begin{array}{c}14 \\
8.7 \\
7.4 \\
7.4 \\
7.4\end{array}$ \\
\hline $\begin{array}{l}11 \ldots . \\
12 \ldots \\
13 \ldots \\
14 \ldots \\
15 \ldots\end{array}$ & $\begin{array}{l}4.2 \\
4.2 \\
4.2 \\
4.5 \\
4.2\end{array}$ & $\begin{array}{l}5.5 \\
5.5 \\
5.5 \\
5.5 \\
5.5\end{array}$ & $\begin{array}{r}4.2 \\
4.2 \\
4.2 \\
4.2 \\
12\end{array}$ & $\begin{array}{c}12 \\
7.4 \\
6.0 \\
5.2 \\
12\end{array}$ & $\begin{array}{l}4.2 \\
8.7 \\
5.2 \\
4.7 \\
4.7\end{array}$ & $\begin{array}{r}4.5 \\
4.7 \\
7.1 \\
14 \\
6.8\end{array}$ & $\begin{array}{l}4.2 \\
4.2 \\
5.8 \\
4.7 \\
4.5\end{array}$ & $\begin{array}{l}4.2 \\
6.3 \\
4.2 \\
4.5 \\
4.7\end{array}$ & $\begin{array}{l}4.0 \\
4.2 \\
4.2 \\
4.2 \\
4.2\end{array}$ & $\begin{array}{l}4.7 \\
4.2 \\
4.2 \\
4.2 \\
4.2\end{array}$ & $\begin{array}{l}7.1 \\
7.4 \\
6.8 \\
6.3 \\
6.3\end{array}$ & $\begin{array}{r}7.4 \\
7.4 \\
7.4 \\
14 \\
8.4\end{array}$ \\
\hline $\begin{array}{l}16 \ldots \ldots \\
17 \ldots \ldots \ldots \\
19 . \ldots \ldots \\
20 \ldots \ldots\end{array}$ & $\begin{array}{l}4.2 \\
4.2 \\
4.2 \\
4.2 \\
4.2\end{array}$ & $\begin{array}{l}5.5 \\
4.7 \\
7.4 \\
9.4 \\
4.7\end{array}$ & $\begin{array}{r}5.5 \\
4.2 \\
.4 .2 \\
4.2 \\
4.2\end{array}$ & $\begin{array}{l}8.4 \\
7.1 \\
6.3 \\
4.2 \\
4.7\end{array}$ & $\begin{array}{l}5.8 \\
4.5 \\
4.2 \\
4.2 \\
4.2\end{array}$ & $\begin{array}{l}4.7 \\
4.5 \\
4.5 \\
4.7 \\
4.5\end{array}$ & $\begin{array}{l}4.5 \\
4.5 \\
4.5 \\
7.1 \\
4.7\end{array}$ & $\begin{array}{l}8.7 \\
4.2 \\
4.0 \\
4.0 \\
4.0\end{array}$ & $\begin{array}{l}4.2 \\
4.5 \\
5.8 \\
4.5 \\
4.5\end{array}$ & $\begin{array}{l}4.2 \\
4.2 \\
4.2 \\
4.2 \\
4.2\end{array}$ & $\begin{array}{c}6.8 \\
6.8 \\
12 \\
8.1 \\
8.4\end{array}$ & $\begin{array}{l}7.8 \\
7.8 \\
7.8 \\
7.8 \\
7.8\end{array}$ \\
\hline 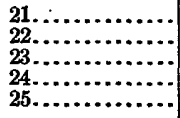 & $\begin{array}{l}4.2 \\
4.2 \\
4.2 \\
4.2 \\
4.2\end{array}$ & $\begin{array}{l}4.2 \\
4.2 \\
4.2 \\
4.2 \\
4.5\end{array}$ & $\begin{array}{l}4.2 \\
8.1 \\
4.7 \\
4.2 \\
4.2\end{array}$ & $\begin{array}{l}5.8 \\
6.3 \\
6.3 \\
4.2 \\
4.2\end{array}$ & $\begin{array}{l}4.2 \\
4.2 \\
4.2 \\
4.2 \\
4.2\end{array}$ & $\begin{array}{l}4.5 \\
4.5 \\
4.5 \\
4.5 \\
4.5\end{array}$ & $\begin{array}{l}4.7 \\
4.7 \\
4.7 \\
4.7 \\
4.7\end{array}$ & $\begin{array}{l}4.0 \\
4.0 \\
4.0 \\
4.0 \\
4.0\end{array}$ & $\begin{array}{l}4.5 \\
4.5 \\
4.5 \\
4.5 \\
4.5\end{array}$ & $\begin{array}{l}4.2 \\
4.2 \\
4.2 \\
4.2 \\
4.2\end{array}$ & $\begin{array}{l}6.5 \\
6.3 \\
6.3 \\
6.8 \\
6.8\end{array}$ & $\begin{array}{l}7.8 \\
7.8 \\
7.8 \\
7.4 \\
7.4\end{array}$ \\
\hline 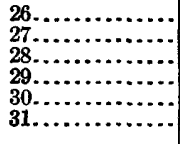 & $\begin{array}{l}4.2 \\
4.2 \\
4.2 \\
4.2 \\
4.2 \\
4.2\end{array}$ & $\begin{array}{r}4.2 \\
4.2 \\
4.2 \\
\cdots \cdots \\
\cdots \cdots\end{array}$ & $\begin{array}{l}4.2 \\
4.2 \\
4.7 \\
4.2 \\
4.2 \\
4.2\end{array}$ & $\begin{array}{l}4.2 \\
4.2 \\
4.2 \\
4.2 \\
4.2\end{array}$ & $\begin{array}{l}4.7 \\
4.2 \\
4.5 \\
4.2 \\
4.2 \\
4.2\end{array}$ & $\begin{array}{l}4.5 \\
6.3 \\
4.7 \\
4.7 \\
6.3\end{array}$ & $\begin{array}{l}4.7 \\
4.2 \\
4.2 \\
4.7 \\
5.2 \\
5.2\end{array}$ & $\begin{array}{r}3.9 \\
3.9 \\
4.0 \\
3.9 \\
3.9 \\
10\end{array}$ & $\begin{array}{l}4.5 \\
4.5 \\
4.5 \\
4.5 \\
4.5\end{array}$ & $\begin{array}{l}4.2 \\
4.2 \\
4.2 \\
4.2 \\
4.2 \\
4.2\end{array}$ & $\begin{array}{l}6.0 \\
6.5 \\
6.3 \\
6.3 \\
6.3\end{array}$ & $\begin{array}{l}7.4 \\
7.4 \\
7.4 \\
7.4 \\
7.4 \\
7.4\end{array}$ \\
\hline
\end{tabular}


Monthly discharge of North Waiehu Stream near Wailuku, Maui, for 1913.

\begin{tabular}{|c|c|c|c|c|c|}
\hline \multirow{2}{*}{ Month. } & \multicolumn{3}{|c|}{ Discharge in second-feet. } & \multirow{2}{*}{$\begin{array}{c}\text { Run-off } \\
\text { (total in } \\
\text { acre-feet). }\end{array}$} & \multirow{2}{*}{$\begin{array}{l}\text { Aceu } \\
\text { racy. }\end{array}$} \\
\hline & Maximum. & Minimum. & Mean. & & \\
\hline 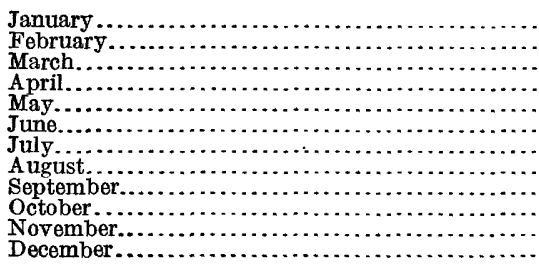 & $\begin{array}{l}4.7 \\
10 \\
12 \\
12 \\
8.7 \\
14 \\
7.4 \\
10 \\
12 \\
9.0 \\
20 \\
22\end{array}$ & $\begin{array}{l}4.2 \\
4.2 \\
4.2 \\
3.5 \\
4.2 \\
4.2 \\
4.2 \\
3.9 \\
3.9 \\
4.2 \\
4.5 \\
6.3\end{array}$ & $\begin{array}{l}\text { 4. } 25 \\
\text { 5. } 17 \\
\text { 4. } 76 \\
\text { 5. } 41 \\
\text { 4. } 58 \\
5.10 \\
4.85 \\
4.60 \\
\text { 4. } 72 \\
\text { 4. } 79 \\
7.21 \\
8.55\end{array}$ & $\begin{array}{l}261 \\
287 \\
293 \\
322 \\
282 \\
303 \\
298 \\
283 \\
281 \\
295 \\
429 \\
526\end{array}$ & $\begin{array}{l}\text { c. } \\
\text { c. } \\
\text { c. } \\
\text { c. } \\
\text { c. } \\
\text { c. } \\
\text { c. } \\
\text { C. } \\
\text { c. } \\
\text { c. }\end{array}$ \\
\hline The year... & 22 & 3.5 & 5.33 & 3,860 & \\
\hline
\end{tabular}

\section{WAIHEE STREAM NEAR WAIHEE, MAUI.}

Iocation.-About 300 feet above Waihee canal intake, 3 miles west of Waihee, and 7 miles northwest of Wailuku.

Records available.-A pril 1 to December 31, 1913.

Gage.-Barrett \& Lawrence water-stage recorder.

Control.-Fairly permanent.

Discharge measurements.-Made from footbridge or by wading.

Diversions.-None above station.

Accuracy.-Records very good except discharge December $5-31$, which was estimated.

Discharge measurements of Waihee Stream near Waihee, Maui, in 1918.

[C. T. Bailey, hydrographer.]

\begin{tabular}{|c|c|c|c|c|c|}
\hline Date. & $\begin{array}{c}\text { Gage } \\
\text { height. }\end{array}$ & $\begin{array}{l}\text { Dis- } \\
\text { charge. }\end{array}$ & Date. & $\begin{array}{l}\text { Gage } \\
\text { height. }\end{array}$ & $\begin{array}{l}\text { Dis- } \\
\text { charge. }\end{array}$ \\
\hline $\begin{array}{l}\text { Apr. } 2 \ldots \ldots \ldots \ldots \\
\text { June } 30 \ldots \ldots \ldots \ldots \\
\text { July } 31 \ldots \ldots \ldots \ldots\end{array}$ & $\begin{array}{r}\text { Feet. } \\
2.75 \\
3.82 \\
2.78\end{array}$ & $\begin{array}{c}\text { Sec. }-f t . \\
42.2 \\
260 \\
45.9\end{array}$ & $\begin{array}{l}\text { Aug. } 13 \ldots \ldots \\
\text { Dec. } 4 \ldots \ldots\end{array}$ & $\begin{array}{l}\text { Feet. } \\
\quad 3.00 \\
\quad 4.08\end{array}$ & $\begin{array}{r}\text { Sec. }-f t . \\
66.5 \\
35.9\end{array}$ \\
\hline
\end{tabular}


ISLAND OF MAUI.

Daily discharge, in second-feet, of Waihee Stream near Waihee, Maui, for 1913.

\begin{tabular}{|c|c|c|c|c|c|c|c|c|c|}
\hline Day. & Apr. & May. & June. & July. & Aug. & Sept. & Oct. & Nov. & Dec. \\
\hline 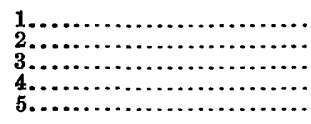 & $\begin{array}{l}44 \\
43 \\
40 \\
40 \\
40\end{array}$ & $\begin{array}{l}97 \\
84 \\
73 \\
58 \\
54\end{array}$ & $\begin{array}{l}49 \\
53 \\
51 \\
51 \\
54\end{array}$ & $\begin{array}{r}80 \\
138 \\
66 \\
21 \\
84\end{array}$ & $\begin{array}{l}38 \\
56 \\
46 \\
38 \\
37\end{array}$ & $\begin{array}{r}46 \\
162 \\
62 \\
50 \\
44\end{array}$ & $\begin{array}{l}42 \\
40 \\
40 \\
40 \\
40\end{array}$ & $\begin{array}{r}45 \\
127 \\
91 \\
55 \\
40\end{array}$ & $\begin{array}{r}56 \\
56 \\
28 \\
584 \\
248\end{array}$ \\
\hline $\begin{array}{r}6 \\
7 \\
8 \\
8 \\
9 \\
10 \ldots \ldots\end{array}$ & $\begin{array}{l}39 \\
39 \\
38 \\
48 \\
44\end{array}$ & $\begin{array}{l}55 \\
74 \\
62 \\
56 \\
53\end{array}$ & $\begin{array}{l}58 \\
55 \\
58 \\
63 \\
68\end{array}$ & $\begin{array}{l}69 \\
68 \\
55 \\
50 \\
48\end{array}$ & $\begin{array}{l}46 \\
42 \\
39 \\
49 \\
68\end{array}$ & $\begin{array}{l}44 \\
54 \\
45 \\
44 \\
43\end{array}$ & $\begin{array}{l}41 \\
42 \\
45 \\
43 \\
41\end{array}$ & $\begin{array}{r}39 \\
97 \\
468 \\
375 \\
97\end{array}$ & $\begin{array}{l}97 \\
57 \\
57 \\
49 \\
46\end{array}$ \\
\hline $\begin{array}{l}11 \\
13 \\
14 \\
15\end{array}$ & $\begin{array}{r}242 \\
91 \\
316 \\
97 \\
248\end{array}$ & $\begin{array}{r}52 \\
112 \\
89 \\
116 \\
97\end{array}$ & $\begin{array}{r}51 \\
106 \\
89 \\
62 \\
95\end{array}$ & $\begin{array}{r}53 \\
116 \\
64 \\
45 \\
47\end{array}$ & $\begin{array}{r}97 \\
188 \\
62 \\
56 \\
68\end{array}$ & $\begin{array}{l}43 \\
43 \\
44 \\
44 \\
44\end{array}$ & $\begin{array}{l}40 \\
42 \\
49 \\
41 \\
41\end{array}$ & $\begin{array}{r}114 \\
127 \\
68 \\
54 \\
51\end{array}$ & $\begin{array}{l}46 \\
44 \\
44 \\
95 \\
46\end{array}$ \\
\hline $\begin{array}{l}16 \ldots \ldots \\
17, \ldots \ldots \\
19 \\
19 \\
20\end{array}$ & $\begin{array}{r}127 \\
116 \\
56 \\
41 \\
35\end{array}$ & $\begin{array}{r}69 \\
112 \\
94 \\
81 \\
49\end{array}$ & $\begin{array}{l}62 \\
56 \\
54 \\
53 \\
55\end{array}$ & $\begin{array}{r}46 \\
52 \\
60 \\
143 \\
136\end{array}$ & $\begin{array}{l}81 \\
49 \\
44 \\
43 \\
44\end{array}$ & $\begin{array}{l}46 \\
78 \\
69 \\
43 \\
42\end{array}$ & $\begin{array}{l}41 \\
41 \\
43 \\
42 \\
42\end{array}$ & $\begin{array}{r}47 \\
46 \\
116 \\
138 \\
80\end{array}$ & $\begin{array}{l}44 \\
62 \\
81 \\
44 \\
43\end{array}$ \\
\hline $\begin{array}{r}21 \\
22 \\
23 \\
24 \\
25 \\
25\end{array}$ & $\begin{array}{l}73 \\
68 \\
46 \\
62 \\
62\end{array}$ & $\begin{array}{l}45 \\
48 \\
51 \\
49 \\
46\end{array}$ & $\begin{array}{l}56 \\
54 \\
47 \\
44 \\
48\end{array}$ & $\begin{array}{l}54 \\
44 \\
51 \\
58 \\
74\end{array}$ & $\begin{array}{l}64 \\
46 \\
46 \\
49 \\
44\end{array}$ & $\begin{array}{l}43 \\
44 \\
44 \\
43 \\
43\end{array}$ & $\begin{array}{l}\mathbf{4 4} \\
45 \\
45 \\
\mathbf{4 4} \\
\mathbf{4 2}\end{array}$ & $\begin{array}{r}81 \\
51 \\
46 \\
71 \\
138\end{array}$ & $\begin{array}{l}43 \\
43 \\
43 \\
42 \\
42\end{array}$ \\
\hline $\begin{array}{l}26 \ldots \ldots \ldots \\
27 \ldots \ldots \ldots \\
28 \ldots \ldots \ldots \\
29 \ldots \ldots \\
30 \ldots \ldots \\
31 \ldots \ldots \ldots\end{array}$ & $\begin{array}{l}37 \\
39 \\
35 \\
33 \\
49\end{array}$ & $\begin{array}{l}67 \\
63 \\
60 \\
55 \\
44 \\
46\end{array}$ & $\begin{array}{r}44 \\
316 \\
127 \\
63 \\
255\end{array}$ & $\begin{array}{l}84 \\
51 \\
77 \\
67 \\
74 \\
44\end{array}$ & $\begin{array}{l}48 \\
56 \\
64 \\
45 \\
51 \\
53\end{array}$ & $\begin{array}{l}43 \\
44 \\
44 \\
43 \\
42\end{array}$ & $\begin{array}{l}42 \\
42 \\
39 \\
39 \\
38 \\
38\end{array}$ & $\begin{array}{l}87 \\
56 \\
52 \\
53 \\
53 \\
\cdots\end{array}$ & $\begin{array}{l}43 \\
44 \\
45 \\
45 \\
46 \\
45\end{array}$ \\
\hline
\end{tabular}

NotE.-Discharge Dec. 5-31 estimated by comparison with record on Tao streaam.

Monthly discharge of Waihee Stream near Waihee, Maui, for 1913.

\begin{tabular}{|c|c|c|c|c|c|}
\hline \multirow{2}{*}{ Month. } & \multicolumn{3}{|c|}{ Discharge in second-feet. } & \multirow{2}{*}{$\begin{array}{c}\text { Run-off } \\
\text { (total in } \\
\text { acre-feet). }\end{array}$} & \multirow{2}{*}{$\begin{array}{l}\text { Accu- } \\
\text { racy. }\end{array}$} \\
\hline & Maximum. & Minimum. & Mean. & & \\
\hline \multirow[t]{2}{*}{ 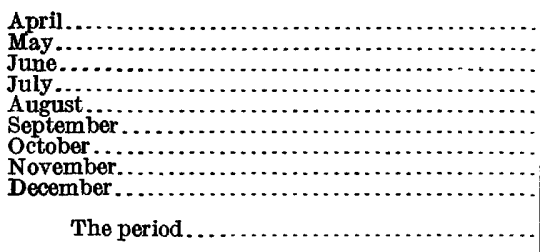 } & $\begin{array}{r}316 \\
116 \\
316 \\
143 \\
188 \\
162 \\
49 \\
468 . \\
584\end{array}$ & $\begin{array}{l}33 \\
44 \\
44 \\
21 \\
37 \\
42 \\
38 \\
40 \\
28\end{array}$ & $\begin{array}{l}76.3 \\
68.1 \\
76.6 \\
68.4 \\
56.7 \\
50.8 \\
41.7 \\
98.8 \\
74.5\end{array}$ & $\begin{array}{l}4,540 \\
4,190 \\
4,560 \\
4,210 \\
3,490 \\
3,020 \\
2,560 \\
5,880 \\
4,580\end{array}$ & \multirow[t]{2}{*}{$\begin{array}{l}\text { A. } \\
\text { A. } \\
\text { A. } \\
\text { A. } \\
\text { A. } \\
\text { A. } \\
\text { A. } \\
\text { A. } \\
\text { D. }\end{array}$} \\
\hline & & $\ldots$. & & 37,000 & \\
\hline
\end{tabular}




\section{SPRECKELS DITCH NEAR WATHEE, MAUI.}

Location.-About 2 miles west of Waihee and about 500 feet below intake.

Records available.-November 16, 1910, to September 30, 1913.

Gage.-Vertical staff.

Control.-Probably permanent.

Discharge measurements.-Made by wading.

Diversion.-Ditch is second diversion from Waihee Stream.

Accuracy.-Records fair.

Discharge measurements of Spreckels ditch near Waihee, Maui, in 1913.

[C. T. Bailey, hydrographer.]

\begin{tabular}{|c|c|c|}
\hline Date. & $\begin{array}{c}\text { Gage } \\
\text { height. }\end{array}$ & $\begin{array}{l}\text { Dis- } \\
\text { charge. }\end{array}$ \\
\hline$\underset{\text { June }}{\text { Apr }} 50$. & $\begin{array}{l}\text { Feet. } \\
0.11 \\
1.80\end{array}$ & $\begin{array}{c}\text { Sec. } f t . \\
0.51 \\
68.9\end{array}$ \\
\hline
\end{tabular}

Monthly discharge of Spreckels ditch near Waihee, Maui, for 1912-13.

\begin{tabular}{|c|c|c|c|c|c|}
\hline \multirow{2}{*}{ Month. } & \multicolumn{3}{|c|}{ Discharge in second-feet. } & \multirow{2}{*}{$\begin{array}{l}\text { Run-off } \\
\text { (total in } \\
\text { acre-feet). }\end{array}$} & \multirow{2}{*}{$\begin{array}{l}\text { Accu- } \\
\text { racy. }\end{array}$} \\
\hline & Maximum. & Minimum. & Mean. & & \\
\hline 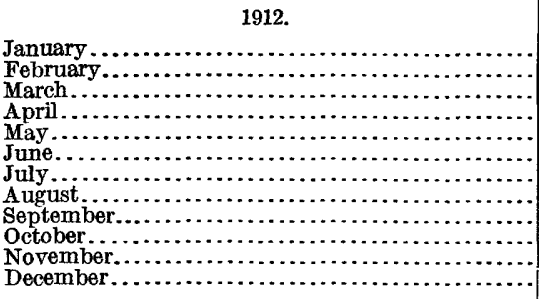 & $\begin{array}{l}14 \\
64 \\
58 \\
82 \\
70 \\
58 \\
73 \\
55 \\
26 \\
43 \\
67 \\
67\end{array}$ & $\begin{array}{r}0.0 \\
1.0 \\
7.2 \\
7.2 \\
1.8 \\
.5 \\
1.0 \\
.0 \\
1.0 \\
1.0 \\
1.6 \\
1.6\end{array}$ & $\begin{array}{c}2.24 \\
18.4 \\
31.4 \\
35.7 \\
13.9 \\
9.04 \\
16.8 \\
10.2 \\
2.90 \\
10.7 \\
19.1 \\
12.8\end{array}$ & $\begin{array}{r}138 \\
1,060 \\
1,930 \\
2,120 \\
855 \\
538 \\
1,030 \\
627 \\
173 \\
658 \\
1,140 \\
787\end{array}$ & $\begin{array}{l}\text { B. } \\
\text { A. } \\
\text { A. } \\
\text { A. } \\
\text { B. } \\
\text { B. } \\
\text { B. } \\
\text { B. } \\
\text { B. } \\
\text { C. } \\
\text { B. } \\
\text { B. }\end{array}$ \\
\hline The year $\ldots \ldots \ldots \ldots \ldots \ldots \ldots \ldots \ldots \ldots \ldots \ldots \ldots$ & 82 & .0 & 15.2 & 11,100 & \\
\hline 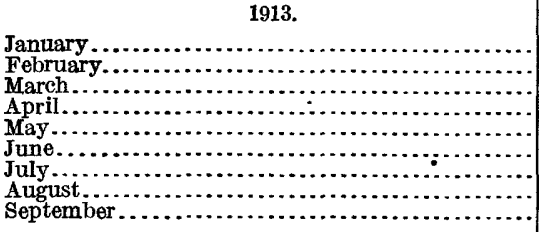 & $\begin{array}{l}67 \\
58 \\
64 \\
67 \\
43 \\
67 \\
67 \\
51 \\
26\end{array}$ & $\begin{array}{r}1.0 \\
1.0 \\
.5 \\
.5 \\
1.6 \\
1.2 \\
.9 \\
1.4 \\
.5\end{array}$ & $\begin{array}{c}9.84 \\
13.6 \\
13.6 \\
23.9 \\
11.8 \\
12.2 \\
9.34 \\
8.78 \\
2.73\end{array}$ & $\begin{array}{r}605 \\
755 \\
836 \\
\mathbf{1}, 420 \\
726 \\
726 \\
574 \\
540 \\
162\end{array}$ & $\begin{array}{l}\text { C. } \\
\text { C. } \\
\text { B. } \\
\text { B. } \\
\text { C. } \\
\text { B. } \\
\text { B. } \\
\text { B. } \\
\text { B. }\end{array}$ \\
\hline The period. ....... & $\theta$ & & & 6,340 & \\
\hline
\end{tabular}

NoTE.-Diteh dry, Jan. 15-18, 21-24, and Aug. 27-30, 1912. 


\section{KAHAKULOA STREAM AT KAHAKULOA, NEAR WAIHEE, MAUI.}

Location-About 13 miles northwest of Wailuku at trail bridge of Kahakuloa, below all diversions.

Records available.-August 27, 1912, to March 31, 1913.

Gage.--Staff gage on left bank, just below trail bridge.

Control.-Probably permanent.

Discharge measurements.-Made by wading.

Accuracy.-Records good.

Station discontinued March 31, 1913, and record continued at new station 3 miles above mouth of stream by water-stage recorder.

Daily discharge, in second-feet, of Kahakuloa Stream at Kahakuloa, near Waihee, Maui, for 1913.

\begin{tabular}{|c|c|c|c|c|c|c|c|c|c|c|c|}
\hline Day. & Jan. & Feb. & Mar. & Day. & Jan. & Feb. & Mar. & Day. & Jan. & Feb. & Mar. \\
\hline $\begin{array}{l}1 \\
2 \ldots \ldots \ldots \\
3 \ldots \ldots \ldots \\
4 \ldots \ldots \ldots \\
5 \ldots \ldots\end{array}$ & $\begin{array}{r}6 \\
6 \\
10 \\
6 \\
5\end{array}$ & $\begin{array}{l}\mathbf{3} \\
\mathbf{3} \\
\mathbf{3} \\
\mathbf{3} \\
\mathbf{3}\end{array}$ & $\begin{array}{l}5 \\
5 \\
5 \\
5 \\
5\end{array}$ & $\begin{array}{l}11 \ldots \ldots \ldots \\
12 \ldots \ldots \ldots \\
13 \ldots \ldots \ldots \\
15 \ldots \ldots \ldots\end{array}$ & $\begin{array}{r}5 \\
5 \\
21 \\
18 \\
10\end{array}$ & $\begin{array}{l}3 \\
3 \\
3 \\
3 \\
4\end{array}$ & $\begin{array}{r}5 \\
5 \\
5 \\
5 \\
20\end{array}$ & $\begin{array}{l}21 \ldots \ldots \ldots \ldots \\
22 \ldots \ldots \ldots \ldots \\
23 \ldots \ldots \ldots \\
25 \ldots \ldots \\
\ldots \ldots \ldots\end{array}$ & $\begin{array}{l}3 \\
5 \\
5 \\
5 \\
5\end{array}$ & $\begin{array}{r}8 \\
6 \\
6 \\
6 \\
18\end{array}$ & $\begin{array}{r}5 \\
25 \\
7 \\
5 \\
10\end{array}$ \\
\hline $\begin{array}{r}6 \ldots \ldots \ldots \ldots \\
7 \ldots \ldots \ldots \ldots \\
8 \ldots \ldots \ldots \ldots \\
10 \ldots \ldots \ldots \ldots \\
10 \ldots \ldots\end{array}$ & $\begin{array}{l}5 \\
5 \\
5 \\
5 \\
5\end{array}$ & $\begin{array}{r}137 \\
36 \\
26 \\
6 \\
5\end{array}$ & $\begin{array}{r}5 \\
18 \\
8 \\
10 \\
5\end{array}$ & $\begin{array}{l}16 \ldots \ldots \\
17 \ldots \ldots \ldots \\
19 \ldots \ldots \\
20 \ldots \ldots \ldots \ldots\end{array}$ & $\begin{array}{l}8 \\
6 \\
4 \\
\mathbf{3} \\
\mathbf{3}\end{array}$ & $\begin{array}{r}10 \\
6 \\
83 \\
39 \\
10\end{array}$ & $\begin{array}{r}9 \\
11 \\
5 \\
5 \\
5\end{array}$ & $\begin{array}{l}26 \ldots \ldots \\
27 \ldots \ldots \\
28 \ldots \ldots \\
29 \ldots \ldots \\
30 \ldots \ldots \\
31 \ldots \ldots\end{array}$ & $\begin{array}{l}5 \\
5 \\
3 \\
3 \\
3 \\
3\end{array}$ & $\begin{array}{r}7 \\
5 \\
5 \\
\cdots \ldots \\
\cdots\end{array}$ & $\begin{array}{l}5 \\
5 \\
9 \\
6 \\
5 \\
5\end{array}$ \\
\hline
\end{tabular}

Monthly discharge of Kahakuloa Stream at Kahakuloa, near Waihee, Maui, for 1913.

\begin{tabular}{|c|c|c|c|c|c|}
\hline \multirow{2}{*}{ Month. } & \multicolumn{3}{|c|}{ Discharge in second-feet. } & \multirow{2}{*}{$\begin{array}{r}\text { Run-off } \\
\text { (total in } \\
\text { acre-feet). }\end{array}$} & \multirow{2}{*}{$\begin{array}{l}\text { Accu- } \\
\text { racy. }\end{array}$} \\
\hline & Maximum. & Minimum. & Mean. & & \\
\hline $\begin{array}{l}\text { January } \ldots \ldots \ldots \\
\text { February } \\
\text { March } \ldots \ldots\end{array}$ & $\begin{array}{r}21 \\
137 \\
25\end{array}$ & $\begin{array}{l}3 \\
3 \\
5\end{array}$ & $\begin{array}{r}6.0 \\
16.1 \\
7.5\end{array}$ & $\begin{array}{l}369 \\
894 \\
461\end{array}$ & $\begin{array}{l}\text { A. } \\
\text { B. }\end{array}$ \\
\hline
\end{tabular}

\section{KAHAKULOA STREAM NEAR HONOKAHAU, MAUI.}

Location.-About 12 miles southeast of Honokahau and 3 miles above mouth of stream.

Records available.-January 22 to August 31, 1913.

Gage.-Stevens water-stage recorder.

Control.-Fairly permanent.

Discharge measurements.-Made from footbridge or by wading.

Diversions. - None above station.

Accuracy.-Records for January, June, and July, good; for other months, poor.

Discharge measurements of Kahakuloa Stream near Honokahau, Maui, in 1913.

[C. T. Bailey, hydrographer.]

\begin{tabular}{|c|c|c|c|c|c|}
\hline Date. & $\begin{array}{c}\text { Gage } \\
\text { height. }\end{array}$ & $\begin{array}{l}\text { Dis- } \\
\text { charge. }\end{array}$ & Date. & $\begin{array}{c}\text { Gage } \\
\text { height. }\end{array}$ & $\begin{array}{l}\text { Dis- } \\
\text { charge. }\end{array}$ \\
\hline $\begin{array}{l}\operatorname{Jan} .23 \ldots \ldots . \\
\text { Mar. } 9 \ldots \ldots\end{array}$ & $\begin{array}{l}\text { Feet. } \\
1.00 \\
1.17\end{array}$ & $\begin{array}{r}\text { Sec.ft. } \\
1.65 \\
3.57\end{array}$ & $\begin{array}{l}\text { May } 21 \ldots \ldots \\
\text { Oet. } 5, \ldots \ldots\end{array}$ & $\begin{array}{l}\text { Feet. } \\
1.10 \\
1.10\end{array}$ & $\begin{array}{r}\text { Sec. fft. } \\
2.46 \\
2.65\end{array}$ \\
\hline
\end{tabular}


Daily discharge, in second-feet, of Kahakuloa Stream near Honokahau, Maui, for 1913.

\begin{tabular}{|c|c|c|c|c|c|c|c|c|}
\hline Day. & Jan. & Feb. & Mar. & Apr. & May. & June. & July. & Aug. \\
\hline 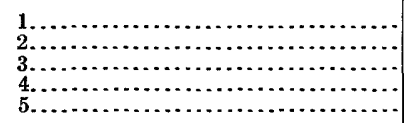 & $\begin{array}{l}\cdots \ldots \ldots \\
\ldots \ldots \ldots \\
\cdots \ldots \ldots \\
\cdots \ldots \ldots \\
\cdots \ldots \ldots \\
\cdots\end{array}$ & $\begin{array}{l}2.4 \\
2.0 \\
1.6 \\
1.6 \\
8.2\end{array}$ & $\begin{array}{l}2.0 \\
2.0 \\
1.9 \\
2.0 \\
1.8\end{array}$ & $\mid \begin{array}{l}\cdots \ldots \ldots \\
\cdots \ldots \ldots \\
\cdots \ldots \ldots \\
\cdots \ldots \ldots \\
\cdots \ldots \ldots \\
\cdots\end{array}$ & $\begin{array}{l}3.2 \\
3.7 \\
3.2 \\
2.8 \\
2.6\end{array}$ & $\begin{array}{r}4.0 \\
3.6 \\
11 \\
9.5 \\
4.4\end{array}$ & $\begin{array}{l}11 \\
19 \\
4.7 \\
3.6 \\
10\end{array}$ & $\begin{array}{r}3.0 \\
11 \\
3.6 \\
3.0 \\
3.4\end{array}$ \\
\hline 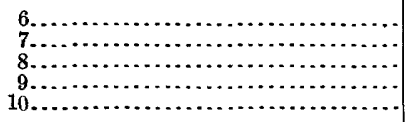 & 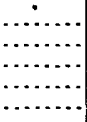 & \begin{tabular}{l|}
38 \\
2.2 \\
2.6 \\
3.0 \\
3.4
\end{tabular} & $\begin{array}{l}1.8 \\
7.2 \\
3.0 \\
4.0 \\
2.3\end{array}$ & $\begin{array}{r}1.7 \\
2.5 \\
2.8\end{array}$ & $\begin{array}{r}2.5 \\
8.5 \\
5.3 \\
25 \\
8.2\end{array}$ & $\begin{array}{l}5.1 \\
5.8 \\
5.3 \\
5.1 \\
7.5\end{array}$ & $\begin{array}{l}5.3 \\
3.2 \\
3.1 \\
2.8 \\
3.1\end{array}$ & $\begin{array}{l}3.8 \\
3.7 \\
8.5 \\
3.1 \\
8.5\end{array}$ \\
\hline $\begin{array}{l}11 \\
12 \ldots \ldots \ldots \\
13 \\
14 \\
15 \\
15 \ldots \ldots\end{array}$ & $\mid \begin{array}{l}\ldots \ldots \ldots \\
\ldots \ldots \ldots \\
\ldots \ldots \ldots \\
\cdots \ldots \ldots \\
\cdots \ldots \ldots\end{array}$ & $\begin{array}{l}3.7 \\
4.1 \\
4.5 \\
4.9 \\
5.3\end{array}$ & $\begin{array}{r}2.1 \\
2.0 \\
2.0 \\
2.3 \\
\cdots\end{array}$ & $\begin{array}{l}59 \\
34 \\
86 \\
26 \\
68\end{array}$ & $\begin{array}{l}14 \\
13 \\
12 \\
11 \\
9.7\end{array}$ & $\begin{array}{c}7.5 \\
5.1 \\
20 \\
9.8 \\
4.2\end{array}$ & $\begin{array}{l}3.7 \\
13 \\
5.5 \\
2.8 \\
2.6\end{array}$ & $\begin{array}{r}11 \\
2.2 \\
3.7 \\
3.1 \\
2.8\end{array}$ \\
\hline 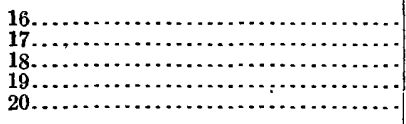 & & $\begin{array}{l}5.7 \\
6.0 \\
6.4 \\
6.8 \\
7.2\end{array}$ & 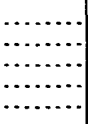 & $\begin{array}{l}35 \\
35 \\
8.2 \\
8.8 \\
6.2\end{array}$ & $\begin{array}{l}8.5 \\
7.3 \\
6.1 \\
4.9 \\
3.7\end{array}$ & $\begin{array}{l}6.9 \\
4.0 \\
3.2 \\
3.2 \\
2.8\end{array}$ & $\begin{array}{l}3.0 \\
3.6 \\
3.1 \\
5.5 \\
2.8\end{array}$ & $\begin{array}{r}2.8 \\
2.8 \\
2.6 \\
2.6 \\
-\end{array}$ \\
\hline 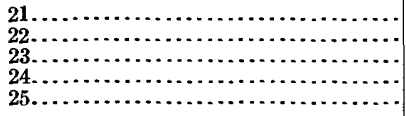 & $\begin{array}{r}1.6 \\
1.6 \\
1.8 \\
2.0\end{array}$ & $\begin{array}{l}3.7 \\
2.5 \\
2.3 \\
2.2 \\
8.2\end{array}$ & 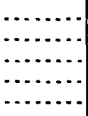 & $\begin{array}{r}32 \\
18 \\
8.5 \\
5.1 \\
15\end{array}$ & $\begin{array}{l}3.1 \\
3.7 \\
5.8 \\
3.8 \\
3.8\end{array}$ & $\begin{array}{l}2.6 \\
3.0 \\
3.0 \\
3.1 \\
5.1\end{array}$ & $\begin{array}{l}2.6 \\
3.0 \\
3.0 \\
3.1 \\
2.8\end{array}$ & $\begin{array}{ll}\ldots \ldots \\
\ldots \ldots \ldots \\
\ldots \ldots \ldots \\
\ldots \ldots \ldots\end{array}$ \\
\hline 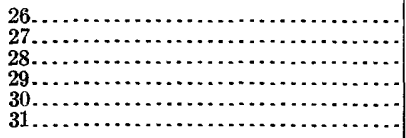 & $\begin{array}{l}1.9 \\
2.0 \\
1.9 \\
1.6 \\
1.5 \\
2.0\end{array}$ & $\begin{array}{c}3.1 \\
2.2 \\
2.1 \\
\ldots . . \\
\end{array}$ & $\begin{array}{l}\ldots . \\
\cdots \\
\cdots \\
\cdots \\
\cdots \\
\cdots\end{array}$ & $\begin{array}{l}5.5 \\
7.5 \\
3.7 \\
3.4 \\
3.2\end{array}$ & $\begin{array}{l}7.5 \\
6.9 \\
5.5 \\
7.2 \\
8.2 \\
4.4\end{array}$ & $\begin{array}{c}3.6 \\
73 \\
23 \\
9.8 \\
22 \\
\ldots \ldots . . .\end{array}$ & $\begin{array}{l}4.7 \\
3.4 \\
3.4 \\
3.4 \\
2.8 \\
2.8\end{array}$ & $\begin{array}{l}\ldots \ldots \\
\ldots \ldots \ldots \\
\ldots \ldots \ldots \\
\ldots \ldots \ldots \\
\ldots \ldots \ldots \\
\ldots \ldots \ldots\end{array}$ \\
\hline
\end{tabular}

NoTE.-Norecord Mar. 15 to Apr. 7 and Aug. 20-31.

Monthly discharge of Kahakuloa Stream near Honokahau, Maui, for 1913.

\begin{tabular}{|c|c|c|c|c|c|}
\hline \multirow{2}{*}{ Month. } & \multicolumn{3}{|c|}{ Discharge in second-feet. } & \multirow{2}{*}{$\begin{array}{l}\text { Run-off } \\
\text { (total in } \\
\text { acre-feet). }\end{array}$} & \multirow{2}{*}{$\begin{array}{l}\text { Accu } \\
\text { racy. }\end{array}$} \\
\hline & Maximum. & Minimum. & Mean. & & \\
\hline 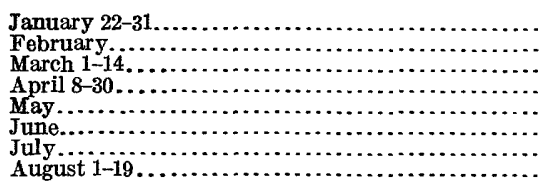 & $\begin{array}{c}2.0 \\
38 \\
7.2 \\
86 \\
25 \\
73 \\
19 \\
11\end{array}$ & $\begin{array}{l}1.5 \\
1.6 \\
1.8 \\
1.7 \\
2.5 \\
2.6 \\
2.6 \\
2.2\end{array}$ & $\begin{array}{c}1.79 \\
5.21 \\
2.60 \\
20.7 \\
6.94 \\
9.14 \\
4.73 \\
4.48\end{array}$ & $\begin{array}{r}36 \\
289 \\
72 \\
944 \\
427 \\
544 \\
291 \\
169\end{array}$ & $\begin{array}{l}\text { A. } \\
\text { D. } \\
\text { D. } \\
\text { D. } \\
\text { D. } \\
\text { A. } \\
\text { A. } \\
\text { D. }\end{array}$ \\
\hline The period..... & $\ldots . .$. & $\ldots \ldots \ldots \ldots$ & $\ldots . .$. & 2,770 & \\
\hline
\end{tabular}

Note.-No record Mar. 15 to Apr. 7. Automatic gage out of order Aug. 20 to Dec. 31, 1913. 


\section{HONOKAHAU STREAM NEAR HONOKAHAU MAUT.}

Location.-On right bank, 1,000 feet above Honokahau ditch intake, about 6 miles southeast of Honokahau post office.

Records available.-March 6 to December 31, 1913.

Gage.--Stevens water-stage recorder.

Control.--Fairly permanent.

Discharge measurements.--Made from cable 400 feet below gage or by wading.

Diversions.-None above station.

Accuracy.-Records good.

Discharge measurements of Honokahau Stream near Honokahau, Maui, in 1913.

[C. T. Bailey, hydrographer.]

\begin{tabular}{|c|c|c|c|c|c|}
\hline Date. & $\begin{array}{c}\text { Gage } \\
\text { height. }\end{array}$ & $\begin{array}{l}\text { Dis- } \\
\text { charge. }\end{array}$ & Date. & $\begin{array}{c}\text { G ge } \\
\text { height. }\end{array}$ & $\begin{array}{c}\text { Dis- } \\
\text { charge. }\end{array}$ \\
\hline $\begin{array}{l}\text { Mar. } 7 \ldots \ldots \ldots \\
8 \text { May } 21 . \ldots \ldots\end{array}$ & $\begin{array}{r}\text { Feet. } \\
1.19 \\
1.07 \\
1.20\end{array}$ & $\begin{array}{r}\text { Sec. }-f t . \\
19.0 \\
17.3 \\
20.0\end{array}$ & $\begin{array}{l}\text { July } 8 \ldots \ldots \\
\text { Aug. } 22 \ldots \ldots \\
\text { Oct. } 4 \ldots \ldots\end{array}$ & $\begin{array}{l}\text { Feet. } \\
1.21 \\
1.18 \\
1.11\end{array}$ & $\begin{array}{r}\text { Sec. }-f t . \\
19.8 \\
19.1 \\
17.0\end{array}$ \\
\hline
\end{tabular}

Daily discharge, in second-feet, of Honokahau Stream near Honokahau, Maui, for 1913.

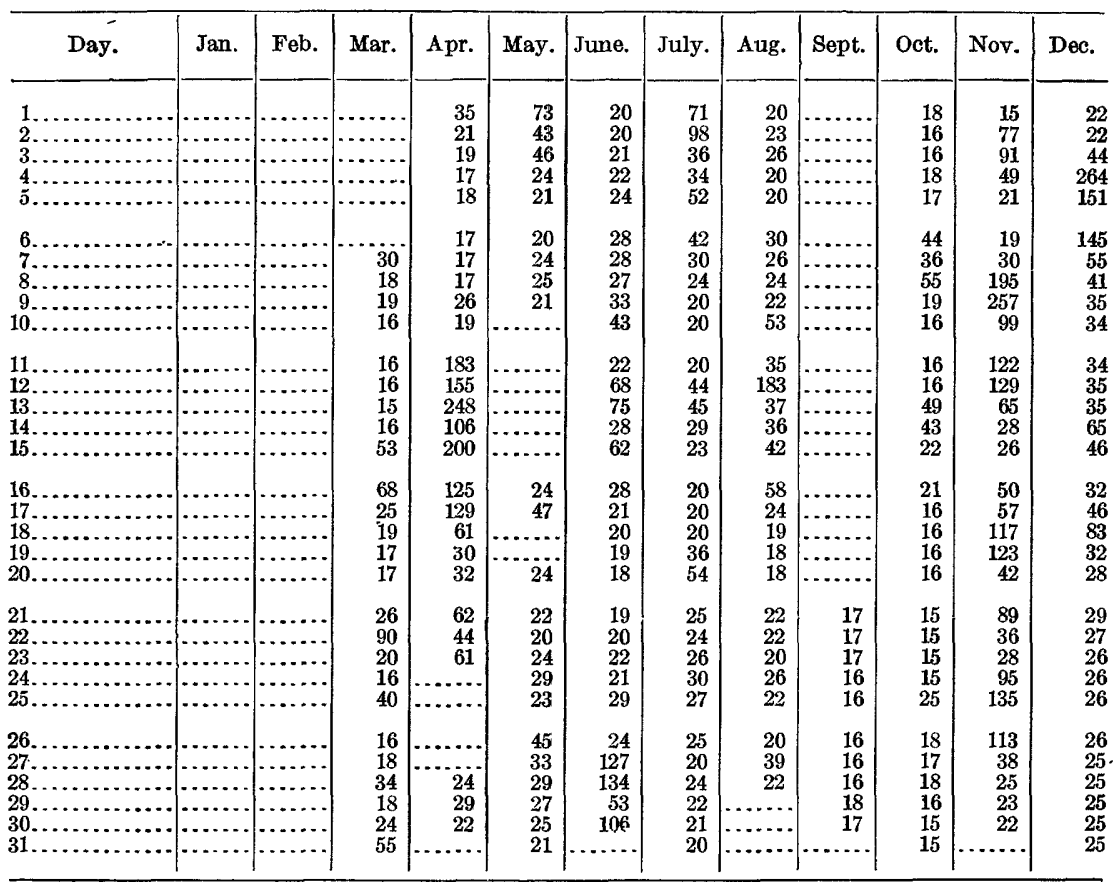

Note.-No record Jan. 1 to Mar. 6, Apr. 24-27, May 10-15, 18-19, and Aug. 29 to Sept. 20. 
Monthly discharge of Honokahau Stream near Honokahau, Maui, for 1913.

\begin{tabular}{|c|c|c|c|c|c|}
\hline \multirow{2}{*}{ Month. } & \multicolumn{3}{|c|}{ Discharge in second-feet. } & \multirow{2}{*}{$\begin{array}{c}\text { Run-off } \\
\text { (total in } \\
\text { acre-feet). }\end{array}$} & \multirow{2}{*}{$\begin{array}{l}\text { Accu- } \\
\text { racy. }\end{array}$} \\
\hline & Maximum. & Minimum. & Mean. & & \\
\hline \multirow[t]{2}{*}{ 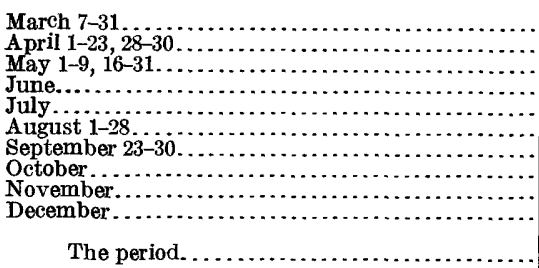 } & $\begin{array}{r}90 \\
248 \\
73 \\
134 \\
98 \\
183 \\
18 \\
55 \\
257 \\
264\end{array}$ & $\begin{array}{l}15 \\
17 \\
20 \\
18 \\
20 \\
18 \\
16 \\
15 \\
15 \\
22\end{array}$ & $\begin{array}{l}28.1 \\
66.1 \\
30.0 \\
39.4 \\
32.3 \\
33.1 \\
16.6 \\
21.6 \\
73.9 \\
49.5\end{array}$ & $\begin{array}{r}1,390 \\
3,400 \\
1,370 \\
2,340 \\
1,990 \\
1,840 \\
329 \\
1,330 \\
4,400 \\
3,040\end{array}$ & \multirow[t]{2}{*}{$\begin{array}{l}\text { B. } \\
\text { B. } \\
\text { B. } \\
\text { B. } \\
\text { B. } \\
\text { B. } \\
\text { B. } \\
\text { B. } \\
\text { B. }\end{array}$} \\
\hline & & & & 21,400 & \\
\hline
\end{tabular}

HONOKAHAU DITCH AT INTAKE, NEAR HONOKAHAU, MAUI.

Location.-About 9 miles by trail above Honokahau, 350 feet below ditch intake. Records available.-February 3, 1907, to September 6, 1913.

Gage.-Vertical staff.

Control.-Probably permanent.

Discharge measurements.-Made from plank at gage.

Diversion.--Ditch diverts total low flow of Honokahau Stream.

Accuracy.-Records good.

Cooperation.-Gage is the property of the Honolua Ranch Co.

Discharge measurements of Honokahau ditch at intake near Honokahau, Maui, in 1913.

[C. T. Bailey, hydrographer.]

\begin{tabular}{|c|c|c|}
\hline Date. & $\begin{array}{c}\text { Gage } \\
\text { height. }\end{array}$ & $\begin{array}{l}\text { Dis- } \\
\text { charge. }\end{array}$ \\
\hline $\begin{array}{l}\text { Mar. } \\
\text { July } 10, \ldots \ldots\end{array}$ & $\begin{array}{c}\text { Feet. } \\
1.88 \\
1.88\end{array}$ & $\begin{array}{r}\text { Sec. } f t . \\
20.5 \\
19.7\end{array}$ \\
\hline
\end{tabular}

Monthly discharge of Honokahau ditch at intake, near Honokahau, Maui, for 1913.

\begin{tabular}{|c|c|c|c|c|c|}
\hline \multirow{2}{*}{ Month. } & \multicolumn{3}{|c|}{ Discharge im second-feet. } & \multirow{2}{*}{$\begin{array}{c}\text { Run-off } \\
\text { (total in } \\
\text { acre-feet). }\end{array}$} & \multirow{2}{*}{$\begin{array}{l}\text { Accu- } \\
\text { racy. }\end{array}$} \\
\hline & Maximum. & Minimum. & Mean. & & \\
\hline 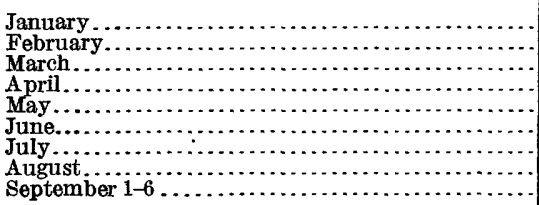 & $\begin{array}{l}43 \\
43 \\
43 \\
43 \\
43 \\
43 \\
43 \\
43 \\
34\end{array}$ & $\begin{array}{l}16 \\
16 \\
16 \\
16 \\
19 \\
19 \\
19 \\
18 \\
18\end{array}$ & $\begin{array}{l}21.8 \\
24.1 \\
22.5 \\
30.4 \\
28.3 \\
27.9 \\
27.3 \\
24.3 \\
23.2\end{array}$ & $\begin{array}{l}1,340 \\
1,340 \\
1,380 \\
1,810 \\
1,740 \\
1,660 \\
1,680 \\
1,490 \\
276\end{array}$ & $\begin{array}{l}\text { A. } \\
\text { A. } \\
\text { A. } \\
\text { A. } \\
\text { A. } \\
\text { A. } \\
\text { A. }\end{array}$ \\
\hline The period. & $\cdots$ & $\ldots \ldots$ & $\ldots \ldots \ldots$ & 12,700 & \\
\hline
\end{tabular}




\section{HONOLUA STREAM NEAR HONOKAHAU, MAUI.}

Location.-About 2 miles south of Honokahau and 300 feet above Honokahau ditch crossing.

Records available.-March 12 to December 31, 1913.

Gage.--Vertical staff.

Control.-Probably shifting.

Discharge measurements.-Made by wading.

Diversions.-None above station.

Accuracy.-Records for low and medium stages good; flood records are little more than estimates.

Discharge measurements of Honolua Stream near Honokahau, Maui, in 1913.

[C. T. Bailey, hydrographer.]

\begin{tabular}{|c|c|c|c|c|c|}
\hline Date. & $\begin{array}{c}\text { Gage } \\
\text { height. }\end{array}$ & $\begin{array}{l}\text { Dis- } \\
\text { charge. }\end{array}$ & Date. & $\begin{array}{c}\text { Gage } \\
\text { height. }\end{array}$ & $\begin{array}{c}\text { Dis- } \\
\text { charge. }\end{array}$ \\
\hline 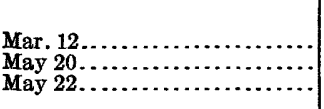 & $\begin{array}{l}\text { Feet. } \\
0.53 \\
.81 \\
.69\end{array}$ & $\begin{array}{r}\text { Sec. } f t . \\
0.74 \\
4.11 \\
2.36\end{array}$ & $\begin{array}{l}\text { July } 10 \ldots \ldots \ldots \\
\text { Aug. } 22, \ldots \ldots \ldots \ldots \\
\end{array}$ & $\begin{array}{l}\text { Feet. } \\
0.55 \\
.46\end{array}$ & $\begin{array}{r}\text { Sec.-ft. } \\
\mathbf{1 . 4 5} \\
.78\end{array}$ \\
\hline
\end{tabular}

Daily discharge, in second-feet, of Honolua Stream near Honokahau, Maui, for 1913.

\begin{tabular}{|c|c|c|c|c|c|c|c|c|c|c|}
\hline Day. & Mar. & Apr. & May. & June. & July. & Aug. & Sept. & Oct. & Nov. & Dec. \\
\hline $\begin{array}{l}1 . . \\
2 . . \\
3 . . \\
4 . \\
5 .\end{array}$ & & $\begin{array}{l}3.0 \\
1.2 \\
1.1 \\
.8 \\
.4\end{array}$ & $\begin{array}{l}2.4 \\
3.7 \\
4.0 \\
2.6 \\
1.9\end{array}$ & $\begin{array}{l}1.2 \\
1.2 \\
1.4 \\
2.4 \\
1.9\end{array}$ & $\begin{array}{l}13 \\
13 \\
6.0 \\
5.2 \\
4.1\end{array}$ & $\begin{array}{r}0.8 \\
.8 \\
4.6 \\
1.0 \\
.8\end{array}$ & $\begin{array}{r}1.4 \\
5.4 \\
6.0 \\
1.5 \\
.8\end{array}$ & $\begin{array}{r}0.4 \\
.4\end{array}$ & $\begin{array}{r}0.6 \\
24^{.7} \\
4.4 \\
3.1\end{array}$ & $\begin{array}{l}1.7 \\
1.5 \\
1.5 \\
85 \\
55\end{array}$ \\
\hline $\begin{array}{l}6 \ldots \\
7 \ldots \\
8 \ldots \\
9 . . . \\
10 \ldots\end{array}$ & $\cdot$ & $\begin{array}{l}.4 \\
.4 \\
.4 \\
.4 \\
.4\end{array}$ & $\begin{array}{l}1.4 \\
1.3 \\
1.6 \\
1.6 \\
1.2\end{array}$ & $\begin{array}{l}2.7 \\
2.4 \\
1.6 \\
2.1 \\
5.6\end{array}$ & $\begin{array}{l}6.9 \\
3.3 \\
2.4 \\
1.7 \\
1.4\end{array}$ & $\begin{array}{r}.8 \\
.8 \\
.8 \\
.7 \\
2.4\end{array}$ & $\begin{array}{r}.8 \\
1.0 \\
.8 \\
.8\end{array}$ & $\begin{array}{r}2.8 \\
4.6 \\
6.0 \\
.8 \\
.8\end{array}$ & $\begin{array}{l}1.5 \\
1.2 \\
40 \\
17 \\
11\end{array}$ & $\begin{array}{c}51 \\
6.9 \\
11 \\
3.8 \\
3.0\end{array}$ \\
\hline 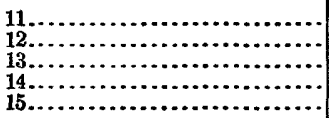 & $\begin{array}{r}0.8 \\
.6 \\
12^{.5}\end{array}$ & $\begin{array}{l}37 \\
22 \\
56 \\
28 \\
51\end{array}$ & $\begin{array}{c}1.2 \\
26 \\
4.4 \\
14 \\
28\end{array}$ & $\begin{array}{c}2.2 \\
6.5 \\
13 \\
2.4 \\
5.6\end{array}$ & $\begin{array}{l}1.4 \\
1.6 \\
7.7 \\
4.1 \\
2.1\end{array}$ & $\begin{array}{r}2.1 \\
43 \\
4.4 \\
5.4 \\
14\end{array}$ & $\begin{array}{l}.8 \\
.8 \\
.8 \\
.6 \\
.6\end{array}$ & $\begin{array}{r}.6 \\
.6 \\
.6 \\
5.2 \\
1.2\end{array}$ & $\begin{array}{l}9.6 \\
8.2 \\
6.2 \\
3.7 \\
3.4\end{array}$ & $\begin{array}{r}5.0 \\
3.0 \\
2.8 \\
25 \\
8.5\end{array}$ \\
\hline 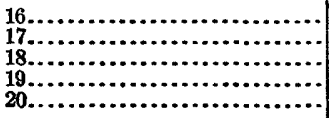 & $\begin{array}{l}15 \\
5.6 \\
2.2 \\
1.4 \\
.7\end{array}$ & $\begin{array}{l}37 \\
27 \\
15 \\
12 \\
9.1\end{array}$ & $\begin{array}{c}8.3 \\
7.4 \\
12 \\
18 \\
5.0\end{array}$ & $\begin{array}{l}2.6 \\
1.6 \\
.9 \\
.7 \\
.6\end{array}$ & $\begin{array}{l}1.5 \\
1.3 \\
1.2 \\
1.0 \\
4.1\end{array}$ & $\begin{array}{l}11 \\
2.6 \\
2.1 \\
1.5 \\
1.3\end{array}$ & $\begin{array}{l}.6 \\
.6 \\
2.2 \\
1.4 \\
1.0\end{array}$ & $\begin{array}{l}1.2 \\
.8 \\
.7 \\
.5 \\
.5\end{array}$ & $\begin{array}{c}3.0 \\
3.0 \\
38 \\
69 \\
3.8\end{array}$ & $\begin{array}{c}5.4 \\
39 \\
6.0 \\
6.9 \\
5.2\end{array}$ \\
\hline $\begin{array}{l}21 \\
22 \\
24 \\
25\end{array} \ldots$ & \begin{tabular}{r|}
1.0 \\
27 \\
3.7 \\
1.1 \\
2.9
\end{tabular} & $\begin{array}{l}16 \\
10 \\
15 \\
7.2 \\
31\end{array}$ & $\begin{array}{l}3.2 \\
2.5 \\
2.4 \\
2.6 \\
2.6\end{array}$ & $\begin{array}{l}.5 \\
.5 \\
.5 \\
.5 \\
.5\end{array}$ & $\begin{array}{l}2.6 \\
1.5 \\
1.4 \\
1.5 \\
2.0\end{array}$ & $\begin{array}{l}.9 \\
.8 \\
.8 \\
.8 \\
.8\end{array}$ & $\begin{array}{l}.8 \\
.6 \\
.5 \\
.5 \\
.5\end{array}$ & $\begin{array}{r}.4 \\
.4 \\
.4 \\
.3\end{array}$ & $\begin{array}{r}3.0 \\
2.2 \\
1.7 \\
3.6 \\
30\end{array}$ & $\begin{array}{l}3 \\
3 \\
3 \\
3 .\end{array}$ \\
\hline $\begin{array}{l}26 \ldots \ldots \ldots \ldots \ldots \ldots \\
27 \ldots \ldots \ldots \ldots \ldots \ldots \ldots \\
28 \ldots \ldots \ldots \ldots \ldots \ldots \ldots \\
30 \ldots \ldots \ldots \ldots \ldots \ldots\end{array}$ & $\begin{array}{l}1.4 \\
1.1 \\
2.2 \\
1.2 \\
.8 \\
3.2\end{array}$ & $\begin{array}{l}7.6 \\
9.1 \\
5.4 \\
5.0 \\
4.0\end{array}$ & $\begin{array}{l}2.4 \\
3.2 \\
3.3 \\
3.2 \\
3.4 \\
1.6\end{array}$ & $\begin{array}{l}1.0 \\
14 \\
24 \\
16 \\
12 \\
\ldots \ldots \ldots\end{array}$ & $\begin{array}{l}1.6 \\
1.4 \\
1.0 \\
.8 \\
.8 \\
.8\end{array}$ & $\begin{array}{l}.8 \\
1.2 \\
5.6 \\
2.1 \\
1.1 \\
6.2\end{array}$ & $\begin{array}{l}.5 \\
.4 \\
.4 \\
.4 \\
.4\end{array}$ & $\begin{array}{r}2.4 \\
.7 \\
.6 \\
.5 \\
.4\end{array}$ & $\begin{array}{c}54 \\
15 \\
3.4 \\
1.9 \\
1.4\end{array}$ & $\begin{array}{l}2 . \\
2 . \\
2 . \\
2\end{array}$ \\
\hline
\end{tabular}


Monthly discharge of Honolua Stream near Honokahau, Maui, for 1913.

\begin{tabular}{|c|c|c|c|c|c|}
\hline \multirow{2}{*}{ Month. } & \multicolumn{3}{|c|}{ Discharge in second-feet. } & \multirow{2}{*}{$\begin{array}{l}\text { Run-off (in } \\
\text { acre-feet). }\end{array}$} & \multirow{2}{*}{$\begin{array}{l}\text { Accu- } \\
\text { racy. }\end{array}$} \\
\hline & Maximum. & Minimum. & Mean. & & \\
\hline \multirow[t]{2}{*}{ 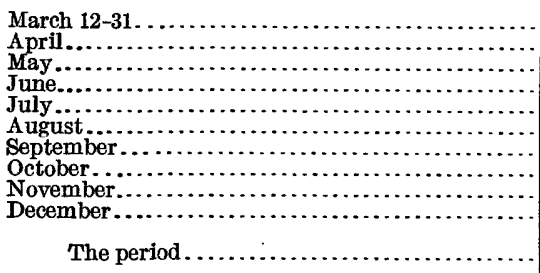 } & $\begin{array}{r}27 \\
56 \\
28 \\
24 \\
13 \\
43 \\
6 \\
24 \\
69 \\
85\end{array}$ & $\begin{array}{r}0.5 \\
.4 \\
1.2 \\
.5 \\
.8 \\
.7 \\
.4 \\
.3 \\
.6 \\
1.5\end{array}$ & $\begin{array}{c}4.22 \\
13.7 \\
5.69 \\
4.27 \\
3.17 \\
3.94 \\
1.12 \\
1.94 \\
12.2 \\
11.6\end{array}$ & $\begin{array}{r}168 \\
815 \\
350 \\
254 \\
195 \\
242 \\
67 \\
119 \\
726 \\
713\end{array}$ & \multirow[t]{2}{*}{$\begin{array}{l}\text { C. } \\
\text { D. } \\
\text { B. } \\
\text { B. } \\
\text { B. } \\
\text { B. } \\
\text { B. } \\
\text { B. } \\
\text { C. } \\
\text { C. }\end{array}$} \\
\hline & $\ldots \ldots \ldots \ldots$ & & $\ldots \ldots \ldots$ & 3,650 & \\
\hline
\end{tabular}

HONOKAWAI STREAM NEAR LAHAINA, MAUT.

Location.--Eight miles northeast of Lahaina, 500 feet below junction of Honokawai and Amalu streams.

Records available.-May 13 to December 31, 1913.

Gage.-Vertical staff.

Control.-Probably permanent.

Discharge measurements.-Made from footbridge or by wading.

Diversions.-Most of the normal flow is diverted into Honokawai ditch half a mile above gage.

Accuracy.-Low-flow records good; flood discharges only estimates.

Discharge measurements of Honokawai Stream near Lahaina, Maui, in 191 s.

[C. T. Bailey, hydrographer.]

\begin{tabular}{|c|c|c|}
\hline Date. & $\begin{array}{c}\text { Gage } \\
\text { height. }\end{array}$ & $\begin{array}{l}\text { Dis- } \\
\text { charge. }\end{array}$ \\
\hline 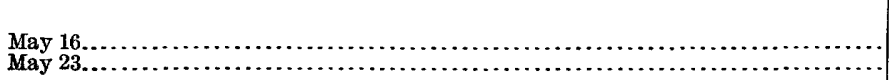 & $\begin{array}{r}\text { Feet. } \\
1.23 \\
.97\end{array}$ & $\begin{array}{r}\text { Sec.-ft. } \\
1.06 \\
.10\end{array}$ \\
\hline
\end{tabular}

Monthly discharge of Honokawai stream near Lahaina, Maui, for 1913.

\begin{tabular}{l} 
Month. \\
\hline
\end{tabular}

NotE.-No discharge during periods as follows: June 1; July 9, 11, 16, 17, 24-31; Aug. 1<9, 11, 18-27, 30; Sept. 1, 2, 4-17, 19-30; Oct. 1-5, 9-12, 15-25, 27-31; Nov. 1, 2, 6, 7, 14, 15, 23, 28-30; Deo. 1-3, 12-14, 16, 19-31. 


\section{HONOKAWAI DITCH NEAR LAHAINA, MAUI.}

Iocation.-Two miles above Pioneer Mill Co.'s power house, and about 7 miles northeast of Lahaina; 250 feet below junction with Amalu wooden flume and 1,000 feet below intake.

Records available.-July 1, 1912, to December 31, 1913.

Gage.-A graduated rod gage, which the observer places in the center of flume each time a reading is taken.

Control.-Probably permanent.

Discharge measurements.-Made in flume near gage.

Diversion.-Ditch diverts all low-water flow from Honokawai and Amalu streams.

Accuracy.-Records good except extremely high discharges.

Cooperation.-Station maintained in cooperation with Pioneer Mill Co.

Discharge measurements of Honokawai ditch near Lahaina, Maui, in 1913.

[C. T. Bailey, hydrographer.]

\begin{tabular}{|c|c|c|}
\hline Date. & $\begin{array}{c}\text { Gage } \\
\text { height. }\end{array}$ & $\begin{array}{c}\text { Dis- } \\
\text { charge. }\end{array}$ \\
\hline $\begin{array}{l}\text { Apr. } 18, \ldots \ldots \ldots \ldots \ldots \ldots \ldots \ldots \\
\text { Aug. } 21 . \ldots \ldots \ldots \ldots \ldots \ldots\end{array}$ & $\begin{array}{r}\text { Feet. } \\
1.20 \\
.92\end{array}$ & $\begin{array}{c}S e c .-f t . \\
10.5 \\
5.24\end{array}$ \\
\hline
\end{tabular}

Daily discharge, in second-feet, of Honokawai ditch near Lahaina, Maui, for 1912-13.

\begin{tabular}{|c|c|c|c|c|c|c|c|c|c|c|c|c|c|}
\hline Day. & July. & Aug. & Sept. & Oct. & Nov. & Dec. & Day. & July. & Aug. & Sept. & Oct. & Nov. & Dec. \\
\hline $\begin{array}{c}1912 . \\
1 \ldots \ldots \ldots \\
2 \ldots \ldots \\
3 \ldots \ldots \\
4 \ldots \ldots \\
5 \ldots \ldots\end{array}$ & $\begin{array}{l}7.9 \\
7.9 \\
8.6 \\
7.5 \\
8.6\end{array}$ & $\begin{array}{l}6.7 \\
6.5 \\
6.5 \\
6.5 \\
6.5\end{array}$ & $\begin{array}{r}6.5 \\
18 \\
7.2 \\
7.5 \\
6.8\end{array}$ & $\begin{array}{r}13 \\
6.8 \\
6.7 \\
6.5 \\
7.2\end{array}$ & $\begin{array}{l}7.0 \\
6.5 \\
6.2 \\
6.0 \\
5.9\end{array}$ & $\begin{array}{l}8.9 \\
12 \\
20 \\
6.7 \\
5.9\end{array}$ & $\begin{array}{r}1912 . \\
16 \ldots \ldots \\
17 \ldots \ldots \\
18 \ldots \ldots \\
19 \ldots \ldots \\
20 \ldots \ldots\end{array}$ & $\begin{array}{r}7.5 \\
6.5 \\
6.5 \\
6.5 \\
12\end{array}$ & $\begin{array}{c}14 \\
7.9 \\
6.7 \\
6.5 \\
6.5\end{array}$ & $\begin{array}{l}6.2 \\
6.2 \\
6.2 \\
6.2 \\
6.4\end{array}$ & $\begin{array}{l}6.4 \\
6.4 \\
6.2 \\
6.2 \\
6.0\end{array}$ & $\begin{array}{c}22 \\
22 \\
23 \\
11 \\
8.7\end{array}$ & $\begin{array}{r}6.5 \\
6.2 \\
5.9 \\
17 \\
8.2\end{array}$ \\
\hline $\begin{array}{r}6 \ldots \ldots \ldots \\
7 \ldots \ldots \ldots \\
8 \ldots \ldots \ldots \\
9 \ldots \ldots \ldots\end{array}$ & $\begin{array}{c}9.3 \\
6.7 \\
17 \\
10 \\
7.9\end{array}$ & $\begin{array}{l}6.5 \\
7.5 \\
7.5 \\
13 \\
16\end{array}$ & $\begin{array}{r}6.8 \\
14 \\
6.7 \\
7.4 \\
6.5\end{array}$ & $\begin{array}{l}6.8 \\
6.2 \\
6.8 \\
6.5 \\
6.5\end{array}$ & $\begin{array}{c}6.2 \\
6.2 \\
6.4 \\
22 \\
8.9\end{array}$ & $\begin{array}{l}5.9 \\
11 \\
13 \\
6.5 \\
6.0\end{array}$ & $\begin{array}{l}21 \ldots \\
22 \ldots \\
23 \ldots \\
24 \ldots \\
25 \ldots\end{array}$ & $\begin{array}{r}8.2 \\
7.0 \\
12 \\
6.8 \\
6.5\end{array}$ & $\begin{array}{l}6.5 \\
8.6 \\
6.8 \\
6.5 \\
6.5\end{array}$ & $\begin{array}{l}7.2 \\
6.8 \\
6.2 \\
6.2 \\
6.2\end{array}$ & $\begin{array}{l}8.6 \\
20 \\
10 \\
8.4 \\
6.5\end{array}$ & \begin{tabular}{|c}
14 \\
8.6 \\
9.1 \\
6.8 \\
6.2
\end{tabular} & $\begin{array}{l}17 \\
22 \\
15 \\
8.7 \\
6.5\end{array}$ \\
\hline $\begin{array}{l}11 \ldots \ldots \ldots \\
12 \ldots \ldots \\
13 \ldots \ldots \\
15 \ldots \ldots\end{array}$ & $\begin{array}{c}6.7 \\
11 \\
7.2 \\
6.7 \\
6.5\end{array}$ & $\begin{array}{r}7.9 \\
8.2 \\
7.9 \\
8.6 \\
18\end{array}$ & $\begin{array}{l}6.5 \\
6.2 \\
6.2 \\
6.2 \\
6.2\end{array}$ & $\begin{array}{r}8.9 \\
7.5 \\
7.2 \\
12 \\
9.3\end{array}$ & $\begin{array}{l}8.2 \\
6.5 \\
6.2 \\
6.2 \\
6.0\end{array}$ & $\begin{array}{l}5.9 \\
5.9 \\
5.9 \\
7.2 \\
7.2\end{array}$ & $\begin{array}{l}26 . \ldots \\
27 \ldots \\
28 \ldots \\
29 \ldots \\
30 \ldots \\
31 \ldots\end{array}$ & $\begin{array}{l}6.5 \\
6.8 \\
7.2 \\
6.7 \\
6.7 \\
6.8\end{array}$ & \begin{tabular}{|c|}
13 \\
9.1 \\
6.7 \\
9.6 \\
8.2 \\
7.2
\end{tabular} & $\begin{array}{c}6.2 \\
6.2 \\
6.7 \\
9.1 \\
10 \\
\cdots \ldots\end{array}$ & $\begin{array}{l}14 \\
15 \\
17 \\
6.5 \\
11 \\
22\end{array}$ & $\begin{array}{r}6.2 \\
5.9 \\
6.2 \\
5.9 \\
5.8 \\
\cdots \cdots\end{array}$ & $\begin{array}{c}17 \\
6.7 \\
12 \\
7.0 \\
14 \\
6.5\end{array}$ \\
\hline
\end{tabular}


Daily discharge, in second-feet, of Honokawaiditch near Lahaina, Maui, for 1912-19-Con.

\begin{tabular}{|c|c|c|c|c|c|c|c|c|c|c|c|c|}
\hline Day. & Jan. & Feb. & Mar. & Apr. & May. & June. & July. & Aug. & Sept. & Oct. & Nov. & Dec. \\
\hline $\begin{array}{c}1913 . \\
1 \ldots \ldots \\
2 \ldots \ldots \\
3 \ldots \ldots \\
4 \ldots \ldots \\
5 \ldots \ldots \ldots\end{array}$ & $\begin{array}{r}6.8 \\
6.4 \\
17 \\
6.2 \\
5.8\end{array}$ & $\begin{array}{l}5.3 \\
5.0 \\
5.0 \\
5.0 \\
4.9\end{array}$ & $\begin{array}{l}5.0 \\
5.0 \\
5.0 \\
5.0 \\
4.9\end{array}$ & $\begin{array}{c}15 \\
6.5 \\
5.6 \\
5.3 \\
5.2\end{array}$ & $\begin{array}{c}12 \\
8.6 \\
10 \\
6.5 \\
6.5\end{array}$ & $\begin{array}{l}4.9 \\
4.9 \\
5.6 \\
5.0 \\
5.0\end{array}$ & $\begin{array}{l}9.5 \\
8.9 \\
8.0 \\
8.6 \\
8.6\end{array}$ & $\begin{array}{l}5.0 \\
5.0 \\
5.0 \\
5.0 \\
5.2\end{array}$ & $\begin{array}{l}5.3 \\
5.0 \\
7.9 \\
5.6 \\
5.2\end{array}$ & $\begin{array}{l}5.3 \\
5.2 \\
5.3 \\
5.4 \\
5.0\end{array}$ & $\begin{array}{c}5.0 \\
5.0 \\
16 \\
14 \\
5.9\end{array}$ & $\begin{array}{r}5.8 \\
5.3 \\
5.0 \\
11 \\
6.2\end{array}$ \\
\hline $\begin{array}{r}6 \ldots \ldots \\
7 \ldots \ldots \\
8 \ldots \ldots \\
10 \ldots \ldots\end{array}$ & $\begin{array}{r}5.8 \\
5.8 \\
8.2 \\
8.6 \\
10\end{array}$ & $\begin{array}{c}10 \\
6.2 \\
17 \\
5.6 \\
5.0\end{array}$ & $\begin{array}{l}4.9 \\
4.9 \\
4.9 \\
4.9 \\
4.9\end{array}$ & $\begin{array}{l}5.0 \\
5.0 \\
4.9 \\
6.8 \\
5.0\end{array}$ & $\begin{array}{l}5.3 \\
5.0 \\
6.2 \\
5.0 \\
5.0\end{array}$ & $\begin{array}{l}6.2 \\
6.5 \\
5.8 \\
6.8 \\
6.8\end{array}$ & $\begin{array}{l}8.2 \\
7.5 \\
6.2 \\
5.6 \\
5.3\end{array}$ & $\begin{array}{l}7.9 \\
5.3 \\
6.8 \\
5.0 \\
7.9\end{array}$ & $\begin{array}{l}5.0 \\
5.0 \\
5.3 \\
5.6 \\
5.3\end{array}$ & $\begin{array}{r}7.9 \\
7.7 \\
15 \\
6.8 \\
5.0\end{array}$ & $\begin{array}{c}5.3 \\
5.2 \\
18 \\
18 \\
16\end{array}$ & $\begin{array}{l}7.9 \\
6.5 \\
5.0 \\
6.5 \\
6.8\end{array}$ \\
\hline $\begin{array}{l}11 \ldots \\
12 \ldots \\
13 \ldots \\
14 \ldots \\
15 \ldots\end{array}$ & $\begin{array}{l}6.5 \\
5.9 \\
23 \\
23 \\
23\end{array}$ & $\begin{array}{l}5.2 \\
5.0 \\
5.0 \\
5.0 \\
6.5\end{array}$ & $\begin{array}{c}4.9 \\
4.8 \\
4.8 \\
4.8 \\
10\end{array}$ & $\begin{array}{l}20 \\
22 \\
22 \\
21 \\
23\end{array}$ & $\begin{array}{r}6.5 \\
12 \\
8.0 \\
8.2 \\
3.8\end{array}$ & $\begin{array}{l}5.3 \\
7.5 \\
8.6 \\
6.7 \\
6.5\end{array}$ & $\begin{array}{l}5.3 \\
7.2 \\
7.5 \\
7.5 \\
6.2\end{array}$ & $\begin{array}{c}5.2 \\
23 \\
7.4 \\
10 \\
9.6\end{array}$ & $\begin{array}{l}5.2 \\
5.9 \\
5.3 \\
5.0 \\
5.3\end{array}$ & $\begin{array}{l}5.3 \\
5.0 \\
11 \\
10 \\
6.4\end{array}$ & $\begin{array}{c}16 \\
19 \\
13 \\
6.5 \\
6.2\end{array}$ & $\begin{array}{l}5.9 \\
5.8 \\
5.6 \\
5.9 \\
6.2\end{array}$ \\
\hline $\begin{array}{l}16 \ldots \ldots \\
17 \ldots \ldots \\
18 \ldots \ldots \\
19 \ldots \ldots \\
20 \ldots \ldots\end{array}$ & $\begin{array}{l}7.7 \\
6.2 \\
6.0 \\
6.2 \\
5.8\end{array}$ & $\begin{array}{c}6.2 \\
5.3 \\
22 \\
17 \\
5.9\end{array}$ & $\begin{array}{c}13 \\
5.9 \\
5.6 \\
5.0 \\
5.0\end{array}$ & $\begin{array}{l}22 \\
21 \\
20 \\
8.2 \\
8.4\end{array}$ & $\begin{array}{l}4.8 \\
6.2 \\
7.0 \\
5.0 \\
5.8\end{array}$ & $\begin{array}{l}6.2 \\
5.6 \\
5.2 \\
5.0 \\
5.0\end{array}$ & $\begin{array}{l}5.4 \\
5.3 \\
5.8 \\
7.9 \\
6.2\end{array}$ & $\begin{array}{c}11 \\
8.2 \\
5.3 \\
5.0 \\
5.0\end{array}$ & $\begin{array}{c}6.2 \\
9.1 \\
12 \\
9.1 \\
5.3\end{array}$ & $\begin{array}{l}5.3 \\
5.0 \\
5.0 \\
5.0 \\
4.9\end{array}$ & $\begin{array}{c}8.6 \\
14 \\
16 \\
16 \\
8.6\end{array}$ & $\begin{array}{r}5.3 \\
6.8 \\
14 \\
8.0 \\
5.9\end{array}$ \\
\hline $\begin{array}{l}21 \ldots \ldots \\
22 \ldots \ldots \\
23 \ldots \ldots \\
24 \ldots \\
25 \ldots\end{array}$ & $\begin{array}{l}5.6 \\
5.6 \\
5.6 \\
5.6 \\
5.6\end{array}$ & $\begin{array}{r}5.9 \\
5.3 \\
5.0 \\
5.0 \\
12\end{array}$ & $\begin{array}{c}12 \\
21 \\
5.6 \\
5.0 \\
12\end{array}$ & $\begin{array}{l}8.6 \\
6.7 \\
8.6 \\
9.6 \\
8.9\end{array}$ & $\begin{array}{l}5.3 \\
5.3 \\
5.4 \\
7.5 \\
7.2\end{array}$ & $\begin{array}{l}5.0 \\
5.0 \\
5.4 \\
5.6 \\
6.8\end{array}$ & $\begin{array}{l}6.2 \\
7.0 \\
6.5 \\
6.2 \\
5.8\end{array}$ & $\begin{array}{l}5.0 \\
5.0 \\
5.0 \\
5.0 \\
5.3\end{array}$ & $\begin{array}{l}5.0 \\
5.0 \\
5.0 \\
5.0 \\
4.8\end{array}$ & $\begin{array}{l}5.0 \\
5.0 \\
5.0 \\
5.2 \\
9.1\end{array}$ & $\begin{array}{c}16 \\
7.4 \\
6.2 \\
12 \\
16\end{array}$ & $\begin{array}{l}5.3 \\
5.3 \\
5.0 \\
5.0 \\
5.0\end{array}$ \\
\hline $\begin{array}{l}26 \ldots \ldots \ldots \\
27 \ldots \ldots \ldots \\
28 \ldots \ldots \ldots \\
29 \ldots \ldots \\
30 \ldots \ldots \\
31 \ldots \ldots\end{array}$ & $\begin{array}{l}5.6 \\
5.6 \\
5.3 \\
5.0 \\
5.0 \\
5.4\end{array}$ & $\begin{array}{r}\mathbf{5 . 8} \\
\mathbf{5 . 2} \\
\mathbf{5 . 0} \\
\mathbf{1} \\
\\
.6\end{array}$ & $\begin{array}{r}5.2 \\
5.3 \\
11 \\
5.3 \\
8.9 \\
14\end{array}$ & $\begin{array}{r}7.2 \\
9.6 \\
8.2 \\
8.6 \\
6.4 \\
\cdots \cdots\end{array}$ & $\begin{array}{l}5.9 \\
5.9 \\
5.9 \\
5.3 \\
5.0 \\
5.0\end{array}$ & $\begin{array}{c}5.9 \\
11 \\
17 \\
7.9 \\
9.5\end{array}$ & $\begin{array}{l}5.3 \\
5.0 \\
5.6 \\
5.0 \\
5.0 \\
5.0\end{array}$ & $\begin{array}{l}5.0 \\
10 \\
11 \\
7.2 \\
5.9 \\
13\end{array}$ & $\begin{array}{r}4.8 \\
4.8 \\
4.8 \\
5.2 \\
5.3 \\
\cdots . . .\end{array}$ & $\begin{array}{l}8.4 \\
5.3 \\
5.0 \\
5.0 \\
5.0 \\
5.0\end{array}$ & $\begin{array}{c}16 \\
12 \\
6.2 \\
5.9 \\
5.3\end{array}$ & $\begin{array}{l}5.3 \\
5.0 \\
5.0 \\
5.0 \\
5.0 \\
5.0\end{array}$ \\
\hline
\end{tabular}

Monthly discharge of Honokawai ditch near Lahaina, Maui, for 1912-13.

\begin{tabular}{|c|c|c|c|c|c|}
\hline \multirow{2}{*}{ Month. } & \multicolumn{3}{|c|}{ Discharge in second-feet. } & \multirow{2}{*}{$\begin{array}{l}\text { Run-off } \\
\text { (total in } \\
\text { acre-feet). }\end{array}$} & \multirow{2}{*}{$\begin{array}{l}\text { Accu- } \\
\text { racy. }\end{array}$} \\
\hline & Maximum. & Minimum. & Mean. & & \\
\hline 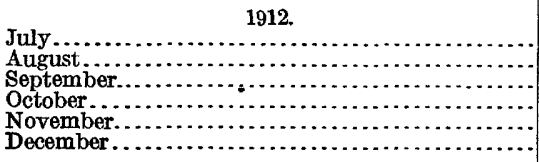 & $\begin{array}{l}17 \\
18 \\
18 \\
20 \\
23 \\
22\end{array}$ & $\begin{array}{l}6.5 \\
6.5 \\
6.2 \\
6.0 \\
5.8 \\
5.9\end{array}$ & $\begin{array}{l}8.05 \\
8.52 \\
7.36 \\
9.29 \\
9.19 \\
9.81\end{array}$ & $\begin{array}{l}495 \\
524 \\
438 \\
571 \\
547 \\
603\end{array}$ & $\begin{array}{l}\text { B. } \\
\text { B. } \\
\text { C. } \\
\text { C. } \\
\text { C. }\end{array}$ \\
\hline The period... & 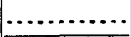 & ..... & ......... & 3,180 & \\
\hline 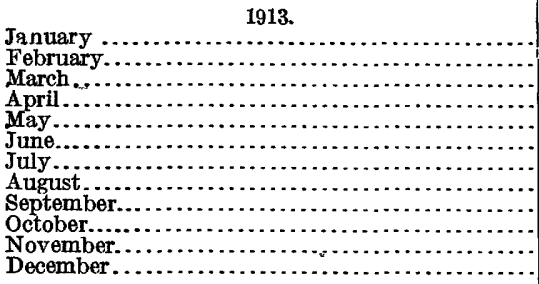 & $\begin{array}{c}23 \\
22 \\
21 \\
23 \\
12 \\
17 \\
9.5 \\
23 \\
12 \\
15 \\
19 \\
14\end{array}$ & $\begin{array}{l}5.0 \\
4.9 \\
4.8 \\
4.9 \\
3.8 \\
4.9 \\
5.0 \\
5.0 \\
4.8 \\
4.9 \\
5.0 \\
5.0\end{array}$ & $\begin{array}{c}8.19 \\
7.19 \\
7.05 \\
11.1 \\
6.49 \\
6.61 \\
6.56 \\
7.26 \\
5.78 \\
6.27 \\
11.2 \\
6.17\end{array}$ & $\begin{array}{l}504 \\
399 \\
433 \\
660 \\
399 \\
393 \\
403 \\
446 \\
344 \\
386 \\
666 \\
379\end{array}$ & $\begin{array}{l}\text { B. } \\
\text { B. } \\
\text { B. } \\
\text { A. } \\
\text { A. } \\
\text { A. } \\
\text { B. } \\
\text { A. } \\
\text { A. } \\
\text { C. } \\
\text { A. }\end{array}$ \\
\hline The year...... & 23 & 3.8 & 7.48 & 5,410 & 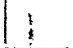 \\
\hline
\end{tabular}


KAHOMA DTTCH AT WEIR, NEAR LAHAINA, MAUI.

Location.-About $3 \frac{1}{2}$ miles east of Lahaina, at portal of the lower of two development tunnels of Pioneer Mill Co.

Records available.-August 1, 1911, to December 31, 1913.

Gage.-Staff.

Discharge measurements.-A 4-foot sharp-crested weir with end contractions; measured discharge from development tunnel and amount diverted by small pipe from stream. Measurements checked by current meter.

Accuracy.-Records very good.

Cooperation.-Records kept by Pioneer Mill Co.

Discharge measurements of Kahoma ditch at weir, near Lahaina, Maui, in 1918.

[C. T. Bailey, hydrographer.]

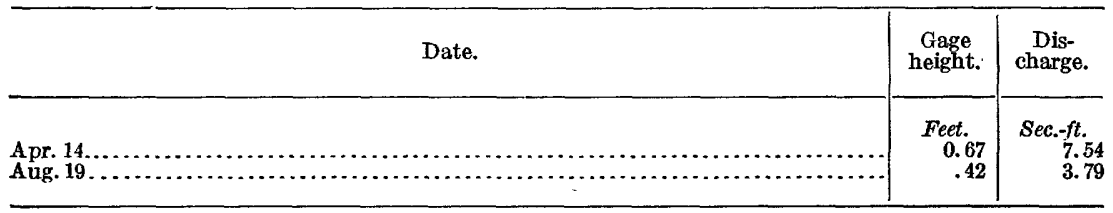

Daily discharge, in second-feet, of Kahoma ditch at weir, near Lahaina, Maui, for 1913.

\begin{tabular}{|c|c|c|c|c|c|c|c|c|c|c|c|c|}
\hline Day. & Jan. & Feb. & Mar. & Apr. & May. & June. & July. & Aug. & Sept. & Oct. & Nov. & Dec. \\
\hline & $\begin{array}{l}3.50 \\
3.24 \\
4.02 \\
3.37 \\
3.12\end{array}$ & $\begin{array}{l}2.76 \\
2.76 \\
2.76 \\
2.64 \\
2.64\end{array}$ & $\begin{array}{l}2.76 \\
2.88 \\
2.76 \\
2.76 \\
2.76\end{array}$ & $\begin{array}{l}6.19 \\
5.58 \\
4.43 \\
3.12 \\
3.12\end{array}$ & $\begin{array}{l}5.58 \\
4.71 \\
4.43 \\
\text { 3.63 } \\
\mathbf{3 . 1 2}\end{array}$ & $\begin{array}{l}3.00 \\
3.00 \\
3.00 \\
3.00 \\
3.00\end{array}$ & $\begin{array}{l}6.19 \\
7.97 \\
7.64 \\
7.80 \\
7.14\end{array}$ & $\begin{array}{l}\text { 3.37 } \\
\text { 3. } 37 \\
\text { 3. } 37 \\
\text { 3.37 } \\
\text { 3.37 }\end{array}$ & $\begin{array}{l}3.76 \\
3.50 \\
4.43 \\
3.50 \\
3.50\end{array}$ & $\begin{array}{l}3.37 \\
3.37 \\
3.37 \\
3.37 \\
\text { 3. } 37\end{array}$ & $\begin{array}{l}3.37 \\
3.37 \\
6.50 \\
6.19 \\
4.43\end{array}$ & $\begin{array}{l}3.76 \\
3.76 \\
3.76 \\
7.47 \\
6.50\end{array}$ \\
\hline $\begin{array}{l}6 \ldots \\
7 \ldots \\
8 \ldots \\
9 \ldots \\
10 \ldots\end{array}$ & $\begin{array}{l}2.88 \\
2.88 \\
3.89 \\
7.14 \\
6.35\end{array}$ & $\begin{array}{l}3.89 \\
7.64 \\
3.89 \\
2.88 \\
2.88\end{array}$ & $\begin{array}{l}2.76 \\
2.76 \\
2.76 \\
2.76 \\
2.76\end{array}$ & $\begin{array}{l}3.00 \\
3.00 \\
2.88 \\
5.58 \\
3.37\end{array}$ & $\begin{array}{l}3.12 \\
3.12 \\
3.12 \\
3.00 \\
3.00\end{array}$ & $\begin{array}{l}3.89 \\
3.63 \\
\text { 3.63 } \\
4.71 \\
5.58\end{array}$ & $\begin{array}{l}5.88 \\
5.29 \\
4.71 \\
3.63 \\
3.50\end{array}$ & $\begin{array}{l}4.71 \\
5.73 \\
4.16 \\
3.63 \\
\text { 3. 50 }\end{array}$ & $\begin{array}{l}\text { 3.37 } \\
\text { 3.37 } \\
\text { 3.37 } \\
\text { 3. 37 } \\
\text { 3.37 }\end{array}$ & $\begin{array}{l}6.82 \\
5.58 \\
6.19 \\
4.43 \\
3.63\end{array}$ & $\begin{array}{l}3.76 \\
3.50 \\
6.82 \\
7.80 \\
6.82\end{array}$ & $\begin{array}{l}6.19 \\
4.57 \\
3.89 \\
3.50 \\
3.50\end{array}$ \\
\hline & $\begin{array}{l}4.29 \\
5.29 \\
7.14 \\
7.14 \\
7.47\end{array}$ & $\begin{array}{l}2.76 \\
2.76 \\
2.76 \\
2.64 \\
5.73\end{array}$ & $\begin{array}{l}2.76 \\
2.76 \\
2.76 \\
2.76 \\
4.43\end{array}$ & $\begin{array}{l}7.80 \\
7.80 \\
7.97 \\
6.82 \\
8.14\end{array}$ & $\begin{array}{l}3.00 \\
5.43 \\
4.85 \\
6.66 \\
6.35\end{array}$ & $\begin{array}{l}3.63 \\
4.71 \\
6.19 \\
4.43 \\
5.58\end{array}$ & $\begin{array}{l}3.37 \\
4.99 \\
5.29 \\
5.29 \\
4.43\end{array}$ & $\begin{array}{l}3.50 \\
7.80 \\
5.58 \\
5.58 \\
5.88\end{array}$ & $\begin{array}{l}3.37 \\
3.63 \\
3.76 \\
3.63 \\
3.63\end{array}$ & $\begin{array}{l}3.50 \\
3.50 \\
5.58 \\
5.73 \\
3.76\end{array}$ & $\begin{array}{l}6.50 \\
6.98 \\
5.58 \\
4.71 \\
4.99\end{array}$ & $\begin{array}{l}3.37 \\
3.37 \\
3.37 \\
3.37 \\
3.37\end{array}$ \\
\hline & $\begin{array}{r}4.43 \\
3.63 \\
3.12 \\
.3 .63 \\
3.12\end{array}$ & $\begin{array}{l}3.00 \\
2.88 \\
7.64 \\
6.66 \\
3.37\end{array}$ & $\begin{array}{l}5.43 \\
5.14 \\
3.89 \\
3.12 \\
2.88\end{array}$ & $\begin{array}{l}7.80 \\
7.97 \\
7.14 \\
5.29 \\
4.99\end{array}$ & $\begin{array}{l}4.43 \\
3.63 \\
6.04 \\
5.73 \\
3.63\end{array}$ & $\begin{array}{l}\text { 4. } 43 \\
\text { 3.37 } \\
\text { 3.37 } \\
\text { 3.12 } \\
\text { 3.12 }\end{array}$ & $\begin{array}{l}3.63 \\
\text { 3. } 63 \\
\text { 3.50 } \\
\text { 5.14 } \\
\text { 5.58 }\end{array}$ & $\begin{array}{l}5.43 \\
4.85 \\
4.02 \\
3.63 \\
3.76\end{array}$ & $\begin{array}{l}4.16 \\
5.58 \\
7.80 \\
5.14 \\
3.76\end{array}$ & $\begin{array}{l}3.76 \\
3.50 \\
3.50 \\
3.50 \\
3.50\end{array}$ & $\begin{array}{l}5.88 \\
6.50 \\
6.50 \\
6.98 \\
5.14\end{array}$ & $\begin{array}{l}3.37 \\
3.37 \\
6.50 \\
4.71 \\
3.89\end{array}$ \\
\hline & $\begin{array}{l}3.12 \\
2.88 \\
2.88 \\
2.88 \\
2.88\end{array}$ & $\begin{array}{l}3.63 \\
2.88 \\
2.76 \\
2.76 \\
5.58\end{array}$ & $\begin{array}{l}6.19 \\
6.82 \\
3.63 \\
3.12 \\
5.14\end{array}$ & $\begin{array}{l}5.73 \\
4.57 \\
5.88 \\
5.29 \\
6.50\end{array}$ & $\begin{array}{l}\text { 3.37 } \\
\text { 3.37 } \\
\text { 3.12 } \\
\text { 6. } 19 \\
\text { 3. } 50\end{array}$ & $\begin{array}{l}3.00 \\
3.00 \\
3.00 \\
3.00 \\
4.43\end{array}$ & $\begin{array}{l}3.89 \\
3.63 \\
4.16 \\
4.71 \\
\text { 3. } 76\end{array}$ & $\begin{array}{l}3.50 \\
3.50 \\
\text { 3. } 50 \\
\text { 3. } 37 \\
4.02\end{array}$ & $\begin{array}{l}\text { 3.50 } \\
\text { 3.377 } \\
\text { 3.37 } \\
\text { 3. 50 } \\
\text { 3. } 37\end{array}$ & $\begin{array}{l}3.50 \\
3.50 \\
3.50 \\
4.71 \\
5.88\end{array}$ & $\begin{array}{l}5.58 \\
4.57 \\
4.43 \\
7.80 \\
7.47\end{array}$ & $\begin{array}{l}3.37 \\
3.37 \\
3.37 \\
3.37 \\
3.37\end{array}$ \\
\hline $\begin{array}{l}26 \ldots \ldots \ldots \\
27 \ldots \ldots \ldots \\
28 \ldots \ldots \ldots \\
29 \ldots \ldots \ldots \\
30 \ldots \ldots \ldots \\
31 \ldots \ldots \ldots\end{array}$ & $\begin{array}{l}2.88 \\
2.88 \\
2.88 \\
2.88 \\
2.88 \\
2.88\end{array}$ & $\begin{array}{l}4.16 \\
4.16 \\
4.16\end{array}$ & $\begin{array}{l}3.12 \\
4.71 \\
6.19 \\
3.63 \\
4.43 \\
6.82\end{array}$ & $\begin{array}{l}4.29 \\
5.58 \\
6.19 \\
4.99 \\
\text { 3. } 63\end{array}$ & $\begin{array}{l}\text { 5. } 88 \\
3.89 \\
\text { 3. 37 } \\
\text { 3.12 } \\
\text { 3. } 12 \\
\text { 3. } 00\end{array}$ & $\begin{array}{l}4.99 \\
5.58 \\
6.98 \\
5.58 \\
6.19\end{array}$ & $\begin{array}{l}3.50 \\
3.50 \\
3.50 \\
3.37 \\
3.37 \\
3.37\end{array}$ & $\begin{array}{l}3.50 \\
3.50 \\
5.58 \\
4.43 \\
4.43 \\
5.88\end{array}$ & $\begin{array}{l}\text { 3. } 37 \\
3.37 \\
3.37 \\
3.37 \\
3.37\end{array}$ & $\begin{array}{l}4.43 \\
3.63 \\
3.50 \\
3.50 \\
3.50 \\
3.50\end{array}$ & $\begin{array}{l}7.14 \\
6.04 \\
4.43 \\
3.89 \\
3.89\end{array}$ & $\begin{array}{l}3.37 \\
3.37 \\
3.24 \\
3.24 \\
3.24 \\
\text { 3.24 }\end{array}$ \\
\hline
\end{tabular}


Monthly discharge of Kahoma ditch at weir, near Lahaina, Maui, for 1913.

\begin{tabular}{|c|c|c|c|c|c|}
\hline \multirow{2}{*}{ Month. } & \multicolumn{3}{|c|}{ Discharge in second-feet. } & \multirow{2}{*}{$\begin{array}{l}\text { Run-off } \\
\text { (total in } \\
\text { acre-feet). }\end{array}$} & \multirow{2}{*}{$\begin{array}{l}\text { Accu- } \\
\text { racy. }\end{array}$} \\
\hline & Maximum. & Minimum. & Mean. & & \\
\hline 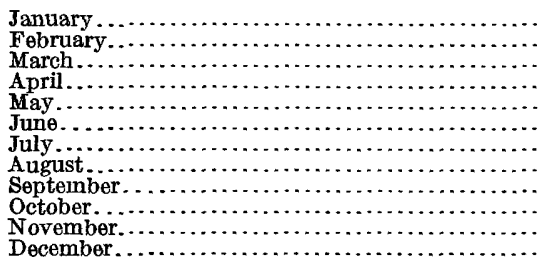 & $\begin{array}{l}7.47 \\
7.64 \\
6.82 \\
8.14 \\
6.66 \\
6.98 \\
7.97 \\
7.80 \\
7.80 \\
6.82 \\
7.80 \\
7.47\end{array}$ & $\begin{array}{l}\text { 2. } 88 \\
2.64 \\
2.76 \\
2.88 \\
\text { 3. } 00 \\
\text { 3. } 00 \\
\text { 3. } 37 \\
\text { 3. } 37 \\
\text { 3. } 37 \\
\text { 3. } 37 \\
\text { 3. } 37 \\
\text { 3. } 24\end{array}$ & $\begin{array}{l}\text { 3.92 } \\
\text { 3. } 75 \\
3.79 \\
5.49 \\
4.15 \\
4.14 \\
4.69 \\
4.32 \\
3.80 \\
4.08 \\
5.58 \\
3.94\end{array}$ & $\begin{array}{l}241 \\
208 \\
233 \\
327 \\
255 \\
246 \\
288 \\
266 \\
226 \\
251 \\
332 \\
242\end{array}$ & $\begin{array}{l}\mathbf{A} \\
\mathbf{A} \\
\mathbf{A} \\
\mathbf{A} \\
\mathbf{A} \\
\mathbf{A} \\
\mathbf{A} \\
\mathbf{A} \\
\mathbf{A} \\
\mathbf{A} \\
\mathbf{A} \\
\mathbf{A}\end{array}$ \\
\hline The year...... & 8.14 & 2.64 & 4.30 & 3,120 & \\
\hline
\end{tabular}

\section{LAHAINALUNA STREAM NEAR LAHAINA, MAUI.}

Location.-One-fourth mile above Lahainaluna Seminary, about $1 \frac{1}{2}$ miles northeast of Lahaina, and 8 feet above Pioneer Mill Co.'s upper ditch intake. Previous to May 7, 200 feet downstream from present location.

Records available.-August 1, 1911, to December 31, 1913.

Gage.-Vertical staff; datum changed May 7, 1913.

Control.-Shifting.

Discharge measurements.-Made by wading.

Diversions.-Most of the normal flow of the stream is diverted into Lahainaluna ditch about 1 mile above the gage.

Accuracy.-Records good except for flood discharges.

Cooperation.-Station maintained in cooperation with Pioneer Mill Co.

Discharge measurements of Lahainaluna Stream near Lahaina, Maui, in 1913.

[C. T. Bailey, hydrographer.]

\begin{tabular}{|c|c|c|c|c|c|}
\hline Date. & $\begin{array}{c}\text { Gajge } \\
\text { height. }\end{array}$ & $\begin{array}{c}\text { Dis- } \\
\text { charge. }\end{array}$ & Date. & $\begin{array}{c}\text { Gage } \\
\text { height. }\end{array}$ & $\begin{array}{c}\text { Dis- } \\
\text { charge. }\end{array}$ \\
\hline 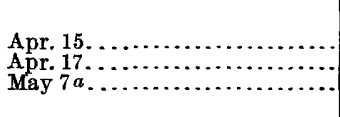 & $\begin{array}{l}\text { Feet. } \\
\quad 1.95 \\
1.45 \\
1.02\end{array}$ & $\begin{array}{l}\text { Sec.-fl. } \\
125 \\
54.7 \\
1.00\end{array}$ & 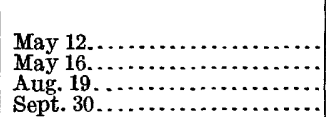 & $\begin{array}{r}\text { Feet. } \\
1.18 \\
1.40 \\
1.18 \\
1.15\end{array}$ & $\begin{array}{r}\text { Sec.-ft. } \\
1.73 \\
3.07 \\
.96 \\
.91\end{array}$ \\
\hline
\end{tabular}

$a$ Gage moved upstream 200 feet; new datum. 
ISLAND OF MAUI.

Daily discharge, in second-feet, of Lahainaluna Stream near Lahaina, Maui, for 1912-13.

\begin{tabular}{|c|c|c|c|c|c|c|c|c|c|c|c|c|}
\hline Day. & Jan. & Feb. & Mar. & Apr. & May. & June. & July. & Aug. & Sept. & Oct. & Nov. & Dec. \\
\hline $\begin{array}{c} \\
1 \ldots \\
2 \ldots \\
3 \ldots \\
4 \ldots \\
5 \ldots\end{array}$ & $\begin{array}{l}2.2 \\
2.3 \\
1.8 \\
1.8 \\
2.0\end{array}$ & $\begin{array}{l}1.2 \\
2.5 \\
2.3 \\
1.5 \\
1.5\end{array}$ & $\begin{array}{l}1.5 \\
3.6 \\
3.9 \\
2.0 \\
1.8\end{array}$ & $\begin{array}{c}7.0 \\
11 \\
6.6 \\
5.6 \\
32\end{array}$ & $\begin{array}{l}1.8 \\
3.4 \\
1.4 \\
2.9 \\
4.4\end{array}$ & $\begin{array}{r}1.5 \\
1.4 \\
1.3 \\
1.3 \\
10\end{array}$ & $\begin{array}{l}2.2 \\
1.6 \\
2.2 \\
3.9 \\
2.0\end{array}$ & $\begin{array}{l}1.1 \\
1.1 \\
1.1 \\
1.1 \\
1.1\end{array}$ & $\begin{array}{r}2.2 \\
14 \\
1.4 \\
1.8 \\
.7\end{array}$ & $\begin{array}{r}3.6 \\
2.8 \\
.9 \\
.5 \\
.5\end{array}$ & $\begin{array}{r}2.0 \\
.7 \\
.7 \\
.6 \\
.8\end{array}$ & $\begin{array}{l}2.5 \\
2.0 \\
2.8 \\
1.5 \\
1.5\end{array}$ \\
\hline $\begin{array}{c}6 \ldots \\
7 \ldots \\
8 \ldots \\
9 \ldots \\
10 \ldots\end{array}$ & $\begin{array}{l}1.6 \\
1.6 \\
2.2 \\
2.0 \\
1.6\end{array}$ & $\begin{array}{l}1.4 \\
1.3 \\
1.3 \\
1.3 \\
2.0\end{array}$ & $\begin{array}{l}1.6 \\
3.1 \\
2.3 \\
4.6 \\
2.5\end{array}$ & $\begin{array}{c}16 \\
46 \\
20 \\
8.8 \\
7.2\end{array}$ & $\begin{array}{l}5.1 \\
1.5 \\
4.2 \\
1.5 \\
1.5\end{array}$ & $\begin{array}{l}1.1 \\
1.1 \\
1.1 \\
2.5 \\
1.4\end{array}$ & $\begin{array}{l}1.1 \\
1.5 \\
1.1 \\
7.6 \\
1.5\end{array}$ & $\begin{array}{l}1.0 \\
1.0 \\
1.4 \\
2.5 \\
4.6\end{array}$ & $\begin{array}{r}16 \\
5.8 \\
.9 \\
1.0 \\
.5\end{array}$ & $\begin{array}{r}.7 \\
.7 \\
1.8 \\
.7 \\
.8\end{array}$ & $\begin{array}{r}.8 \\
.7 \\
1.6 \\
132 \\
3.9\end{array}$ & $\begin{array}{r}.8 \\
3.1 \\
1.0 \\
1.0 \\
1.0\end{array}$ \\
\hline$\ldots$ & $\begin{array}{l}1.8 \\
1.8 \\
1.6 \\
1.6 \\
1.6\end{array}$ & $\begin{array}{l}1.3 \\
1.3 \\
1.3 \\
1.2 \\
1.2\end{array}$ & $\begin{array}{r}22 \\
3.6 \\
3.1 \\
1.5 \\
1.5\end{array}$ & $\begin{array}{l}3.5 \\
3.2 \\
3.1 \\
8.0 \\
1.8\end{array}$ & $\begin{array}{l}1.5 \\
1.4 \\
1.3 \\
1.3 \\
1.3\end{array}$ & $\begin{array}{l}1.3 \\
1.3 \\
1.3 \\
1.5 \\
1.8\end{array}$ & $\begin{array}{l}1.5 \\
1.3 \\
2.2 \\
1.4 \\
1.2\end{array}$ & $\begin{array}{l}8.0 \\
3.1 \\
1.0 \\
1.0 \\
3.5\end{array}$ & $\begin{array}{l}.5 \\
.5 \\
.5 \\
.5 \\
.5\end{array}$ & $\begin{array}{r}.8 \\
.7 \\
1.0 \\
2.3 \\
.7\end{array}$ & $\begin{array}{l}5.1 \\
2.3 \\
1.5 \\
1.0 \\
1.0\end{array}$ & $\begin{array}{r}1.0 \\
.9 \\
.9 \\
.9 \\
1.3\end{array}$ \\
\hline $\begin{array}{l}19 \ldots \ldots \\
20 \ldots\end{array}$ & $\begin{array}{l}1.6 \\
1.6 \\
1.5 \\
1.3 \\
1.3\end{array}$ & $\begin{array}{l}1.2 \\
1.2 \\
1.2 \\
11 \\
46\end{array}$ & $\begin{array}{r}1.5 \\
10 \\
6.4 \\
1.6 \\
2.7\end{array}$ & $\begin{array}{l}1.6 \\
1.9 \\
1.8 \\
1.5 \\
1.5\end{array}$ & $\begin{array}{l}1.3 \\
1.3 \\
1.3 \\
2.7 \\
1.5\end{array}$ & $\begin{array}{l}2.0 \\
1.8 \\
1.3 \\
1.5 \\
1.8\end{array}$ & $\begin{array}{l}1.1 \\
1.3 \\
1.1 \\
1.1 \\
1.0\end{array}$ & $\begin{array}{r}2.0 \\
1.8 \\
.5 \\
.5 \\
.5\end{array}$ & $\begin{array}{l}.5 \\
.7 \\
.7 \\
.9 \\
.8\end{array}$ & $\begin{array}{l}.8 \\
.7 \\
.7 \\
.7 \\
.7\end{array}$ & $\begin{array}{l}3.1 \\
5.6 \\
6.4 \\
4.2 \\
3.9\end{array}$ & $\begin{array}{l}1.3 \\
1.3 \\
1.0 \\
2.5 \\
2.0\end{array}$ \\
\hline $\begin{array}{l}23 \ldots \\
24 \ldots \\
25 \ldots\end{array}$ & $\begin{array}{l}1.3 \\
1.3 \\
1.3 \\
1.3 \\
1.3\end{array}$ & \begin{tabular}{|c|}
124 \\
55 \\
8.0 \\
3.9 \\
3.6
\end{tabular} & $\begin{array}{l}1.5 \\
1.5 \\
1.3 \\
1.3 \\
1.2\end{array}$ & $\begin{array}{l}1.5 \\
3.1 \\
1.6 \\
2.8 \\
8.8\end{array}$ & $\begin{array}{l}6.4 \\
2.8 \\
1.8 \\
2.7 \\
1.5\end{array}$ & $\begin{array}{l}8.0 \\
1.5 \\
1.6 \\
5.1 \\
1.5\end{array}$ & $\begin{array}{l}1.3 \\
1.3 \\
1.3 \\
3.6 \\
1.4\end{array}$ & $\begin{array}{r}.5 \\
2.0 \\
1.0 \\
.5 \\
.5\end{array}$ & $\begin{array}{l}.8 \\
.8 \\
.8 \\
.8 \\
.9\end{array}$ & $\begin{array}{r}3.1 \\
2.8 \\
4.2 \\
1.1 \\
.6\end{array}$ & $\begin{array}{l}4.2 \\
2.5 \\
2.8 \\
.9 \\
1.0\end{array}$ & $\begin{array}{c}16 \\
55 \\
29 \\
3.4 \\
2.4\end{array}$ \\
\hline $\begin{array}{l}26 \ldots \ldots \\
27 \ldots \ldots \\
28 \ldots \ldots \\
29 \ldots \ldots \\
31 \ldots\end{array}$ & $\begin{array}{l}1.3 \\
1.3 \\
1.3 \\
1.2 \\
1.2 \\
1.2\end{array}$ & $\begin{array}{r}3.5 \\
100 \\
6.8 \\
1.8\end{array}$ & $\begin{array}{l}1.2 \\
1.0 \\
1.0 \\
1.4 \\
5.1 \\
6.8\end{array}$ & $\begin{array}{l}3.9 \\
2.0 \\
1.8 \\
1.5 \\
1.8\end{array}$ & $\begin{array}{r}3.6 \\
19 \\
4.4 \\
1.6 \\
1.5 \\
1.5\end{array}$ & $\begin{array}{l}1.5 \\
1.3 \\
1.3 \\
1.3 \\
1.3\end{array}$ & $\begin{array}{l}1.0 \\
2.0 \\
1.1 \\
1.1 \\
1.1 \\
1.0\end{array}$ & $\begin{array}{r}.9 \\
.5 \\
1.1 \\
1.8 \\
2.5\end{array}$ & $\begin{array}{r}.9 \\
.9 \\
.9 \\
1.8 \\
7.2\end{array}$ & $\begin{array}{r}8.0 \\
7.0 \\
2.5 \\
1.3 \\
3.2 \\
78\end{array}$ & $\begin{array}{r}.7 \\
.7 \\
1.4 \\
1.4 \\
1.4\end{array}$ & $\begin{array}{c}14 \\
2.5 \\
2.0 \\
2.4 \\
3.1 \\
2.3\end{array}$ \\
\hline $\begin{array}{l} \\
1 \ldots \\
2 \ldots \\
3 \ldots \\
4 \ldots \\
5 \ldots\end{array}$ & $\begin{array}{l}1.3 \\
1.3 \\
1.2 \\
1.2 \\
1.2\end{array}$ & $\begin{array}{l}1.3 \\
1.3 \\
1.3 \\
1.3 \\
1.3\end{array}$ & $\begin{array}{l}1.3 \\
1.3 \\
1.3 \\
1.3 \\
1.3\end{array}$ & $\begin{array}{l}2.4 \\
2.2 \\
1.6 \\
1.4 \\
1.4\end{array}$ & $\begin{array}{l}1.4 \\
1.4 \\
1.4 \\
1.4 \\
1.4\end{array}$ & $\begin{array}{l}1.6 \\
1.6 \\
1.6 \\
1.6 \\
1.6\end{array}$ & $\begin{array}{c}13 \\
27 \\
6 \\
3.4 \\
26\end{array}$ & $\begin{array}{l}1.6 \\
1.6 \\
1.6 \\
1.6 \\
1.6\end{array}$ & $\begin{array}{r}0.7 \\
.7 \\
1.0 \\
.9 \\
.7\end{array}$ & $\begin{array}{r}0.9 \\
.7 \\
.9 \\
.8 \\
.7\end{array}$ & $\begin{array}{c}0.6 \\
22^{-6} \\
20 \\
1.0\end{array}$ & $\begin{array}{r}2.0 \\
1.7 \\
1.2 \\
24 \\
4.0\end{array}$ \\
\hline $\begin{array}{r}6 \ldots . \\
7 \ldots \\
8 \ldots \\
9 \ldots \\
10 \ldots\end{array}$ & $\begin{array}{l}1.1 \\
1.1 \\
1.2 \\
1.2 \\
1.3\end{array}$ & $\begin{array}{c}1.4 \\
1.4 \\
22 \\
1.4 \\
1.4\end{array}$ & $\begin{array}{l}1.3 \\
1.3 \\
1.3 \\
1.3 \\
1.3\end{array}$ & $\begin{array}{l}1.3 \\
1.3 \\
1.3 \\
2.7 \\
1.4\end{array}$ & $\begin{array}{l}1.4 \\
1.4 \\
1.4 \\
1.4 \\
1.3\end{array}$ & $\begin{array}{l}2.4 \\
2.7 \\
2.3 \\
4.1 \\
7.9\end{array}$ & $\begin{array}{l}6.0 \\
3.9 \\
2.6 \\
1.9 \\
2.0\end{array}$ & $\begin{array}{l}3.2 \\
2.0 \\
1.7 \\
1.6 \\
2.0\end{array}$ & $\begin{array}{l}.6 \\
.6 \\
.7 \\
.6 \\
.7\end{array}$ & $\begin{array}{r}8.6 \\
1.0 \\
15 \\
1.2 \\
.5\end{array}$ & $\begin{array}{l}1.0 \\
2.9 \\
4.8 \\
57 \\
36\end{array}$ & $\begin{array}{l}2.9 \\
2.3 \\
2.1 \\
2.0 \\
1.7\end{array}$ \\
\hline $\begin{array}{l}11 \ldots \\
12 \ldots \\
13 \ldots \\
14 \ldots \\
15 \ldots\end{array}$ & $\begin{array}{c}1.3 \\
1.3 \\
12 \\
12 \\
8.6\end{array}$ & $\begin{array}{l}1.3 \\
1.3 \\
1.3 \\
1.3 \\
1.3\end{array}$ & $\begin{array}{l}1.3 \\
1.2 \\
1.2 \\
1.2 \\
1.5\end{array}$ & $\begin{array}{l}78 \\
44 \\
85 \\
12 \\
88\end{array}$ & $\begin{array}{c}1.3 \\
1.7 \\
3.0 \\
32 \\
12\end{array}$ & $\begin{array}{l}1.7 \\
5.5 \\
6.0 \\
1.8 \\
1.7\end{array}$ & $\begin{array}{l}1.9 \\
4.8 \\
6.3 \\
2.8 \\
2.3\end{array}$ & $\begin{array}{r}1.7 \\
66 \\
2.0 \\
4.0 \\
2.2\end{array}$ & $\begin{array}{r}1.4 \\
.9 \\
2.0 \\
.9 \\
.8\end{array}$ & $\begin{array}{r}.6 \\
32.8 \\
4.8 \\
1.2\end{array}$ & $\begin{array}{c}12 \\
6.3 \\
4.0 \\
1.4 \\
1.4\end{array}$ & $\begin{array}{l}1.4 \\
1.5 \\
1.6 \\
1.6 \\
1.6\end{array}$ \\
\hline $\begin{array}{l}18 \ldots \\
19 \ldots \ldots\end{array}$ & $\begin{array}{l}1.6 \\
1.3 \\
1.1 \\
1.1 \\
1.0\end{array}$ & $\begin{array}{l}1.4 \\
1.3 \\
8.0 \\
7.0 \\
1.4\end{array}$ & $\begin{array}{l}1.4 \\
2.2 \\
1.4 \\
1.4 \\
1.3\end{array}$ & $\begin{array}{c}15 \\
18 \\
5.6 \\
2.4 \\
2.4\end{array}$ & $\begin{array}{l}3.9 \\
3.5 \\
4.6 \\
5.5 \\
2.9\end{array}$ & $\begin{array}{l}1.7 \\
1.5 \\
1.5 \\
1.4 \\
1.4\end{array}$ & $\begin{array}{l}1.9 \\
1.9 \\
1.9 \\
2.0 \\
2.3\end{array}$ & $\begin{array}{r}9.8 \\
2.3 \\
.8 \\
1.0 \\
.9\end{array}$ & $\begin{array}{r}1.5 \\
7.1 \\
30 \\
1.1 \\
.7\end{array}$ & $\begin{array}{l}.6 \\
.6 \\
.7 \\
.6 \\
.7\end{array}$ & $\begin{array}{c}12 \\
17 \\
50 \\
18 \\
2.2\end{array}$ & $\begin{array}{r}1.6 \\
1.6 \\
12.4 \\
2.4 \\
1.6\end{array}$ \\
\hline $\begin{array}{l}21 \ldots \ldots \\
22 \ldots \ldots \\
23 \ldots \ldots \\
24 \ldots \ldots \\
25 \ldots \ldots\end{array}$ & $\begin{array}{l}1.2 \\
1.1 \\
1.1 \\
1.2 \\
1.2\end{array}$ & $\begin{array}{l}1.5 \\
1.4 \\
1.4 \\
1.3 \\
3.6\end{array}$ & $\begin{array}{l}4.3 \\
8.0 \\
1.4 \\
1.3 \\
2.6\end{array}$ & $\begin{array}{l}2.4 \\
2.0 \\
3.6 \\
1.7 \\
6.4\end{array}$ & $\begin{array}{l}1.9 \\
1.9 \\
1.9 \\
3.4 \\
2.0\end{array}$ & $\begin{array}{l}1.4 \\
1.4 \\
1.4 \\
1.8 \\
2.3\end{array}$ & $\begin{array}{l}1.9 \\
2.0 \\
4.4 \\
2.2 \\
2.3\end{array}$ & $\begin{array}{r}.9 \\
.9 \\
.9 \\
1.5 \\
1.0\end{array}$ & $\begin{array}{r}.9 \\
.9 \\
1.0 \\
.8 \\
.8\end{array}$ & $\begin{array}{r}.7 \\
.7 \\
.7 \\
2.9\end{array}$ & $\begin{array}{r}1.6 \\
1.4 \\
1.3 \\
9.8 \\
11\end{array}$ & $\begin{array}{l}1.5 \\
1.5 \\
1.5 \\
1.5 \\
1.5\end{array}$ \\
\hline $\begin{array}{l}26 \ldots \ldots \\
27 \ldots \ldots \\
28 \ldots \ldots \\
29 \\
31 \ldots \ldots\end{array}$ & $\begin{array}{l}1.2 \\
1.2 \\
1.2 \\
1.3 \\
1.3 \\
1.5\end{array}$ & $\begin{array}{l}1.4 \\
1.3 \\
1.3\end{array}$ & $\begin{array}{r}1.4 \\
1.9 \\
4.7 \\
1.4 \\
3.2 \\
10\end{array}$ & $\begin{array}{l}1.8 \\
1.9 \\
2.2 \\
1.6 \\
1.6\end{array}$ & $\begin{array}{l}2.8 \\
2.0 \\
1.7 \\
1.6 \\
1.6 \\
1.6\end{array}$ & $\begin{array}{c}3.9 \\
6.2 \\
21 \\
3.7 \\
20\end{array}$ & $\begin{array}{l}2.0 \\
2.0 \\
1.6 \\
1.5 \\
1.5 \\
1.5\end{array}$ & $\begin{array}{r}.8 \\
.8 \\
1.0 \\
.8 \\
.9 \\
3.1\end{array}$ & $\begin{array}{r}.7 \\
.7 \\
.7 \\
1.0 \\
.8\end{array}$ & $\begin{array}{r}1.4 \\
.7 \\
.7 \\
.6 \\
.6 \\
.6\end{array}$ & $\begin{array}{c}23 \\
4.5 \\
1.7 \\
1.5 \\
2.2\end{array}$ & $\begin{array}{l}1.5 \\
1.5 \\
1.4 \\
1.4 \\
1.4 \\
1.4\end{array}$ \\
\hline
\end{tabular}


Monthly discharge of Lahainaluna Stream near Lahaina, Maui, for 1912-13.

\begin{tabular}{|c|c|c|c|c|c|}
\hline \multirow{2}{*}{ Month. } & \multicolumn{3}{|c|}{ Discharge in second-feet. } & \multirow{2}{*}{$\begin{array}{l}\text { Run-off } \\
\text { (total in } \\
\text { acre-feet). }\end{array}$} & \multirow{2}{*}{$\begin{array}{l}\text { Accu- } \\
\text { racy. }\end{array}$} \\
\hline & Maximum. & Minimum. & Mean. & & \\
\hline 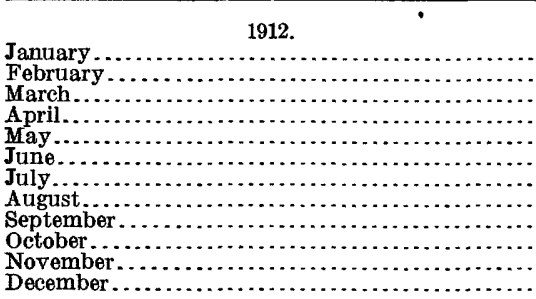 & $\begin{array}{c}2.3 \\
124 \\
22 \\
46 \\
19 \\
10 \\
7.6 \\
8.0 \\
16 \\
78 \\
132 \\
55\end{array}$ & $\begin{array}{r}1.2 \\
1.2 \\
1.0 \\
1.5 \\
1.3 \\
1.1 \\
1.0 \\
.5 \\
.5 \\
.5 \\
.6 \\
.8\end{array}$ & $\begin{array}{c}1.57 \\
13.4 \\
3.36 \\
7.23 \\
2.88 \\
2.09 \\
1.74 \\
1.66 \\
2.21 \\
4.32 \\
6.50 \\
5.24\end{array}$ & $\begin{array}{l}96.5 \\
771 \\
207 \\
430 \\
177 \\
124 \\
107 \\
102 \\
132 \\
266 \\
387 \\
322\end{array}$ & $\begin{array}{l}\text { B. } \\
\text { D. } \\
\text { C. } \\
\text { C. } \\
\text { B. } \\
\text { B. } \\
\text { B. } \\
\text { B. } \\
\text { D. } \\
\text { D. } \\
\text { D. } \\
\text { D. }\end{array}$ \\
\hline The year..... & 132 & .5 & 4. 31 & 3,120 & \\
\hline 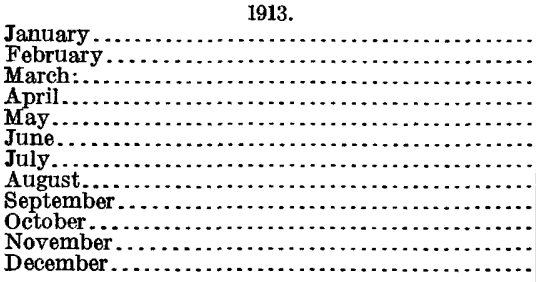 & $\begin{array}{l}12 \\
22 \\
10 \\
88 \\
32 \\
21 \\
27 \\
66 \\
30 \\
32 \\
57 \\
24\end{array}$ & $\begin{array}{r}1.1 \\
1.3 \\
1.2 \\
1.3 \\
1.3 \\
1.4 \\
1.5 \\
.8 \\
.6 \\
.6 \\
.6 \\
1.2\end{array}$ & $\begin{array}{c}2.16 \\
2.60 \\
2.17 \\
13.1 \\
3.44 \\
3.82 \\
4.61 \\
3.93 \\
2.06 \\
3.31 \\
10.9 \\
2.80\end{array}$ & $\begin{array}{l}133 \\
145 \\
133 \\
778 \\
212 \\
227 \\
283 \\
242 \\
123 \\
204 \\
649 \\
172\end{array}$ & $\begin{array}{l}\text { B. } \\
\text { C. } \\
\text { C. } \\
\text { D. } \\
\text { B. } \\
\text { B. } \\
\text { C. } \\
\text { B. } \\
\text { B. } \\
\text { C. } \\
\text { D. } \\
\text { B. }\end{array}$ \\
\hline The year $\ldots \ldots \ldots \ldots \ldots \ldots \ldots \ldots$ & 88 & .6 & 4.56 & 2,070 & \\
\hline
\end{tabular}

\section{LAHAINALUNA DITCH NEAR LAHATNA, MAUT.}

Location.-One and one-half miles east of Lahaina, 200 feet above intake for Lahainaluna School power house.

Records available.-May 6 to December 31, 1913.

Gage.-Vertical staff.

Control.-Probably permanent.

Discharge measurements.-Made by wading.

Diversions.-Ditch diverts nearly all low-water flow from Lahainaluna Stream.

Accuracy.-Records good.

Discharge measurements of Lahainaluna ditch near Lahaina, Maui, in 1913.

[C. T. Bailey, hydrographer.]

\begin{tabular}{|c|c|c|c|c|c|}
\hline Date. & $\begin{array}{c}\text { Gage } \\
\text { height. }\end{array}$ & Discharge. & Date. & $\begin{array}{c}\text { Gage } \\
\text { height. }\end{array}$ & $\begin{array}{l}\text { Dis- } \\
\text { charge. }\end{array}$ \\
\hline 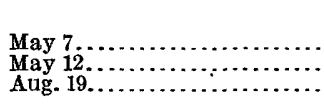 & $\begin{array}{r}\text { Feet. } \\
0.80 \\
.86 \\
.77\end{array}$ & $\begin{array}{r}\text { Sec.-ft. } \\
2.85 \\
3.31 \\
2.04\end{array}$ & $\begin{array}{l}\text { Sept. } 30 \ldots \ldots \ldots \\
\text { Oct. } 3 \ldots \ldots \ldots \ldots\end{array}$ & $\begin{array}{r}\text { Feet. } \\
0.72 \\
.76\end{array}$ & $\begin{array}{r}\text { Sec.-ft. } \\
1.92 \\
1.98\end{array}$ \\
\hline
\end{tabular}


Daily discharge, in second-feet, of Lahainaluna ditch near Lahaina, Maui, for 1913.

\begin{tabular}{|c|c|c|c|c|c|c|c|c|}
\hline Day. & May. & $\operatorname{Jun} \theta$. & July. & Aug. & Sept. & Oct. & Nov. & Dec. \\
\hline $\begin{array}{l}1 \\
2 \\
\mathbf{3} \\
4 \\
\mathbf{1}\end{array}$ & $\cdots$ & $\begin{array}{l}2.7 \\
2.7 \\
3.0 \\
2.7 \\
2.9\end{array}$ & $\begin{array}{l}5.2 \\
5.4 \\
4.3 \\
4.6 \\
5.5\end{array}$ & $\begin{array}{l}2.6 \\
2.7 \\
2.4 \\
2.4 \\
2.4\end{array}$ & $\begin{array}{l}2.9 \\
2.6 \\
2.9 \\
1.9 \\
2.1\end{array}$ & $\begin{array}{l}2.1 \\
1.5 \\
1.6 \\
2.1 \\
1.6\end{array}$ & $\begin{array}{l}1.9 \\
2.0 \\
6.3 \\
5.2 \\
2.6\end{array}$ & $\begin{array}{l}2.6 \\
2.6 \\
2.9 \\
8.1 \\
6.8\end{array}$ \\
\hline $\begin{array}{r}6 \\
7 \\
8 \\
9\end{array}$ & $\begin{array}{l}2.1 \\
2.6 \\
2.9 \\
2.6 \\
2.9\end{array}$ & $\begin{array}{l}3.2 \\
4.3 \\
4.6 \\
5.4 \\
5.5\end{array}$ & $\begin{array}{l}4.9 \\
4.0 \\
2.9 \\
2.6 \\
2.4\end{array}$ & $\begin{array}{l}4.0 \\
4.3 \\
4.0 \\
3.2 \\
4.0\end{array}$ & $\begin{array}{l}2.1 \\
2.1 \\
2.0 \\
2.0 \\
2.4\end{array}$ & $\begin{array}{l}4.9 \\
3.7 \\
6.1 \\
3.6 \\
3.2\end{array}$ & $\begin{array}{l}2.5 \\
1.9 \\
6.1 \\
7.1 \\
4.0\end{array}$ & $\begin{array}{l}5.2 \\
3.4 \\
2.4 \\
2.4 \\
2.4\end{array}$ \\
\hline $\begin{array}{l}11 \\
12 \\
13 \\
14 \\
15\end{array}$ & $\begin{array}{l}2.9 \\
6.1 \\
4.6 \\
5.8 \\
\text { 3. } 2\end{array}$ & $\begin{array}{l}4.3 \\
4.3 \\
5.4 \\
4.0 \\
4.0\end{array}$ & $\begin{array}{l}2.4 \\
4.0 \\
4.6 \\
4.3 \\
3.2\end{array}$ & $\begin{array}{l}2.9 \\
7.4 \\
4.9 \\
5.2 \\
5.0\end{array}$ & $\begin{array}{l}3.2 \\
3.2 \\
3.3 \\
2.9 \\
2.4\end{array}$ & $\begin{array}{l}3.4 \\
2.9 \\
4.8 \\
4.0 \\
2.1\end{array}$ & $\begin{array}{l}4.0 \\
2.6 \\
1.4 \\
2.4 \\
2.1\end{array}$ & $\begin{array}{l}2.9 \\
2.5 \\
2.5 \\
2.6 \\
2.9\end{array}$ \\
\hline $\begin{array}{l}16 \\
17 \\
18 \\
19 \\
20\end{array}$ & $\begin{array}{l}2.1 \\
1.9 \\
2.6 \\
2.4 \\
2.0\end{array}$ & $\begin{array}{l}3.6 \\
2.9 \\
2.9 \\
2.9 \\
2.9\end{array}$ & $\begin{array}{l}2.9 \\
2.7 \\
2.6 \\
2.7 \\
3.4\end{array}$ & $\begin{array}{l}5.8 \\
2.9 \\
2.9 \\
2.4 \\
2.4\end{array}$ & $\begin{array}{l}3.4 \\
5.5 \\
6.8 \\
4.6 \\
3.7\end{array}$ & $\begin{array}{l}2.6 \\
2.4 \\
2.1 \\
2.0 \\
2.1\end{array}$ & $\begin{array}{r}7.4 \\
8.3 \\
10 \\
9.7 \\
6.0\end{array}$ & $\begin{array}{l}2.6 \\
2.7 \\
6.1 \\
4.3 \\
2.6\end{array}$ \\
\hline $\begin{array}{l}21 \\
22 \\
23 \\
24 \\
25\end{array}$ & $\begin{array}{l}2.5 \\
2.4 \\
2.4 \\
2.9 \\
3.2\end{array}$ & $\begin{array}{l}2.9 \\
2.7 \\
3.0 \\
3.4 \\
4.0\end{array}$ & $\begin{array}{l}2.9 \\
3.6 \\
3.7 \\
3.4 \\
2.7\end{array}$ & $\begin{array}{l}2.2 \\
2.1 \\
2.0 \\
3.0 \\
2.1\end{array}$ & $\begin{array}{l}2.9 \\
1.9 \\
1.9 \\
2.1 \\
1.8\end{array}$ & $\begin{array}{l}1.5 \\
1.4 \\
1.4 \\
2.0 \\
4.0\end{array}$ & $\begin{array}{l}6.1 \\
5.2 \\
4.6 \\
7.1 \\
8.0\end{array}$ & $\begin{array}{l}2.6 \\
2.7 \\
2.6 \\
2.9 \\
2.6\end{array}$ \\
\hline 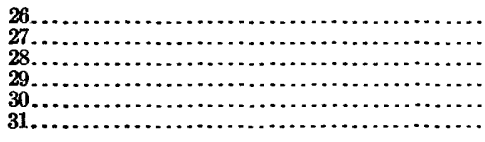 & $\begin{array}{l}3.7 \\
3.4 \\
3.2 \\
3.2 \\
2.9 \\
2.9\end{array}$ & $\begin{array}{r}4.6 \\
4.3 \\
5.7 \\
4.0 \\
4.9\end{array}$ & $\begin{array}{l}2.2 \\
2.1 \\
2.5 \\
2.7 \\
2.6 \\
2.6\end{array}$ & $\begin{array}{l}2.4 \\
2.6 \\
2.6 \\
2.9 \\
2.1 \\
4.8\end{array}$ & $\begin{array}{r}1.9 \\
2.0 \\
1.9 \\
2.1 \\
1.6\end{array}$ & $\begin{array}{l}3.4 \\
2.2 \\
2.1 \\
1.6 \\
1.6 \\
2.1\end{array}$ & $\begin{array}{r}8.5 \\
7.1 \\
3.4 \\
2.9 \\
3.2 \\
\ldots . . .\end{array}$ & $\begin{array}{l}2.5 \\
2.6 \\
2.6 \\
2.6 \\
2.6 \\
2.6\end{array}$ \\
\hline
\end{tabular}

Monthly discharge of Lahainaluna ditch near Lahaina, Maui, for 1913.

\begin{tabular}{|c|c|c|c|c|c|}
\hline \multirow{2}{*}{ Month. } & \multicolumn{3}{|c|}{ Discharge in second-feet. } & \multirow{2}{*}{$\begin{array}{l}\text { Run-off } \\
\text { (total in } \\
\text { acre-fe日t). }\end{array}$} & \multirow{2}{*}{$\begin{array}{l}\text { Accu- } \\
\text { racy. }\end{array}$} \\
\hline & Maximum. & Minimum. & Mean. & & \\
\hline 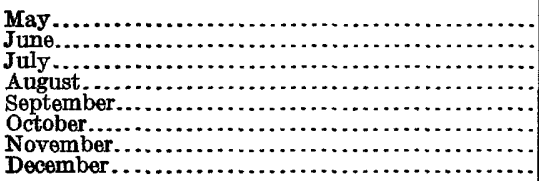 & $\begin{array}{r}6.1 \\
5.7 \\
5.5 \\
7.4 \\
6.8 \\
4.9 \\
10 \\
8.1\end{array}$ & $\begin{array}{l}1.9 \\
2.7 \\
2.1 \\
2.0 \\
1.6 \\
1.4 \\
1.4 \\
2.4\end{array}$ & $\begin{array}{l}3.05 \\
3.79 \\
3.41 \\
3.31 \\
2.74 \\
2.65 \\
4.99 \\
3.21\end{array}$ & $\begin{array}{l}157 \\
226 \\
210 \\
204 \\
163 \\
163 \\
297 \\
197\end{array}$ & $\begin{array}{l}\text { A. } \\
\text { A. } \\
\mathbf{A} . \\
\text { A. } \\
\mathbf{A} . \\
\mathbf{A} . \\
\mathbf{A} . \\
\mathbf{A} .\end{array}$ \\
\hline The period... & $\ldots$ & $\ldots \ldots \ldots \ldots$ & $\ldots \ldots \ldots$ & 1,620 & \\
\hline
\end{tabular}




\section{KAUAULA DITCH NEAR LAHAINA, MAUI.}

Location.-About 100 feet below intake which is uppermost on the stream, about 3 miles east of Lahaina.

Records available.-October 16, 1911, to December 31, 1913.

Gage.-Vertical staff.

Discharge measurements.-Made in flume at gage.

Control.-Probably permanent.

Diversion.-Ditch diverts all low flow of Kauaula Stream.

Accuracy.-Records good.

Cooperation.-Station is maintained in cooperation with Pioneer Mill Co.

Discharge measurements of Kauaula ditch near Lahaina, Maui, in 1913.

[C. T. Bailey, hydrographer.]

\begin{tabular}{|c|c|c|c|c|c|}
\hline Date. & $\begin{array}{c}\text { Gage } \\
\text { height. }\end{array}$ & $\begin{array}{c}\text { Dis- } \\
\text { charge. }\end{array}$ & Date. & $\begin{array}{c}\text { Gage } \\
\text { height. }\end{array}$ & $\begin{array}{l}\text { Dis- } \\
\text { charge. }\end{array}$ \\
\hline Apr. $15 \ldots \ldots \ldots \ldots$ & $\begin{array}{r}\text { Feet. } \\
1.26 \\
.92\end{array}$ & $\begin{array}{c}\text { Sec.-ft. } \\
\quad 14.7 \\
\quad 10.5\end{array}$ & Aug. 19.. & $\begin{array}{l}\text { Feet. } \\
0.84\end{array}$ & Sec.fft. \\
\hline
\end{tabular}

Daily discharge, in second-feet, of Kauaula ditch near Lahaina, Maui, for 1913.

\begin{tabular}{|c|c|c|c|c|c|c|c|c|c|c|c|c|}
\hline Day. & Jan. & Feb. & Mar. & Apr. & May. & June. & July. & Aug. & Sept. & Oct. & Nov. & Dec. \\
\hline $\begin{array}{l}1 \ldots \\
2 \ldots \\
3 \ldots \\
4 \ldots \\
5 \ldots\end{array}$ & $\begin{array}{l}7.5 \\
7.5 \\
8.2 \\
7.5 \\
6.8\end{array}$ & $\begin{array}{l}6.3 \\
6.0 \\
6.0 \\
5.6 \\
5.6\end{array}$ & $\begin{array}{l}6.8 \\
6.6 \\
6.0 \\
6.0 \\
6.0\end{array}$ & $\begin{array}{l}7.5 \\
6.8 \\
6.0 \\
6.0 \\
4.6\end{array}$ & $\begin{array}{l}8.2 \\
9.2 \\
9.2 \\
7.8 \\
7.5\end{array}$ & $\begin{array}{l}6.8 \\
6.4 \\
6.4 \\
6.4 \\
6.0\end{array}$ & $\begin{array}{l}15.0 \\
12.8 \\
12.1 \\
11.4 \\
15.5\end{array}$ & $\begin{array}{l}6.8 \\
6.8 \\
6.8 \\
6.8 \\
6.8\end{array}$ & $\begin{array}{l}7.8 \\
7.7 \\
7.7 \\
7.5 \\
7.2\end{array}$ & $\begin{array}{l}5.9 \\
5.7 \\
5.7 \\
5.7 \\
5.7\end{array}$ & $\begin{array}{r}5.4 \\
5.4 \\
15.0 \\
12.8 \\
8.0\end{array}$ & $\begin{array}{r}10.8 \\
10.5 \\
9.9 \\
16.4 \\
15.5\end{array}$ \\
\hline $\begin{array}{r}6 \ldots \\
7 \ldots \\
8 \ldots \\
9 \ldots \\
10 \ldots\end{array}$ & $\begin{array}{l}6.8 \\
6.4 \\
6.4 \\
6.4 \\
6.4\end{array}$ & $\begin{array}{r}5.6 \\
6.0 \\
11.9 \\
9.2 \\
7.5\end{array}$ & $\begin{array}{l}6.0 \\
6.0 \\
5.6 \\
5.4 \\
5.3\end{array}$ & $\begin{array}{l}4.6 \\
5.0 \\
5.0 \\
6.0 \\
5.3\end{array}$ & $\begin{array}{l}7.5 \\
7.5 \\
6.8 \\
6.4 \\
6.4\end{array}$ & $\begin{array}{r}6.3 \\
6.0 \\
6.0 \\
9.2 \\
11.0\end{array}$ & $\begin{array}{r}9.2 \\
11.0 \\
9.2 \\
8.5 \\
8.2\end{array}$ & $\begin{array}{l}6.9 \\
6.8 \\
6.8 \\
6.8 \\
6.8\end{array}$ & $\begin{array}{l}6.9 \\
6.6 \\
6.6 \\
6.6 \\
6.6\end{array}$ & $\begin{array}{r}13.7 \\
8.0 \\
12.8 \\
7.8 \\
7.2\end{array}$ & $\begin{array}{r}8.0 \\
6.3 \\
16.0 \\
16.4 \\
14.6\end{array}$ & $\begin{array}{l}14.6 \\
13.2 \\
12.1 \\
11.4 \\
10.1\end{array}$ \\
\hline $\begin{array}{l}13 \ldots \ldots \\
14 \ldots \ldots \\
15 \ldots \ldots\end{array}$ & $\begin{array}{r}6.0 \\
6.0 \\
11.9 \\
12.8 \\
14.6\end{array}$ & $\begin{array}{l}7.5 \\
6.8 \\
6.4 \\
6.4 \\
6.0\end{array}$ & $\begin{array}{l}5.2 \\
5.0 \\
4.9 \\
4.9 \\
6.0\end{array}$ & $\begin{array}{l}18.2 \\
18.2 \\
18.2 \\
11.0 \\
18.2\end{array}$ & $\begin{array}{r}6.4 \\
8.0 \\
15.5 \\
12.8 \\
12.8\end{array}$ & $\begin{array}{r}7.5 \\
8.4 \\
10.1 \\
8.4 \\
7.5\end{array}$ & $\begin{array}{l}7.8 \\
7.8 \\
9.9 \\
7.8 \\
7.5\end{array}$ & $\begin{array}{r}6.8 \\
15.5 \\
12.8 \\
8.9 \\
9.6\end{array}$ & $\begin{array}{l}6.6 \\
6.6 \\
6.4 \\
6.4 \\
6.3\end{array}$ & $\begin{array}{r}6.9 \\
6.4 \\
11.0 \\
14.2 \\
7.8\end{array}$ & $\begin{array}{l}14.6 \\
13.7 \\
12.8 \\
12.4 \\
12.3\end{array}$ & $\begin{array}{l}8.9 \\
8.4 \\
8.0 \\
8.4 \\
7.8\end{array}$ \\
\hline $\begin{array}{l}16 \ldots \ldots \\
17 \ldots \ldots \\
19 \ldots \ldots \\
20 \ldots \ldots\end{array}$ & $\begin{array}{r}11.9 \\
9.9 \\
8.9 \\
8.7 \\
8.7\end{array}$ & $\begin{array}{r}6.0 \\
6.3 \\
7.0 \\
15.5 \\
8.0\end{array}$ & $\begin{array}{l}7.8 \\
6.0 \\
6.0 \\
5.3 \\
6.8\end{array}$ & $\begin{array}{l}18.2 \\
18.2 \\
18.2 \\
14.6 \\
12.3\end{array}$ & $\begin{array}{l}12.8 \\
13.4 \\
12.8 \\
12.6 \\
11.0\end{array}$ & $\begin{array}{l}9.2 \\
7.4 \\
6.8 \\
6.4 \\
6.3\end{array}$ & $\begin{array}{l}7.4 \\
7.0 \\
7.0 \\
6.9 \\
6.9\end{array}$ & $\begin{array}{r}13.2 \\
11.0 \\
9.0 \\
8.7 \\
8.4\end{array}$ & $\begin{array}{r}6.6 \\
8.2 \\
12.4 \\
8.9 \\
7.5\end{array}$ & $\begin{array}{l}8.0 \\
6.8 \\
6.6 \\
6.4 \\
5.9\end{array}$ & \begin{tabular}{r}
13.2 \\
15.0 \\
15.0 \\
\hdashline .1 .6 \\
4.6
\end{tabular} & $\begin{array}{r}7.8 \\
7.8 \\
13.7 \\
11.4 \\
8.0\end{array}$ \\
\hline $\begin{array}{l}21 \ldots \ldots \\
22 \ldots \ldots \\
23 \ldots \ldots \\
24 \ldots \ldots \\
25 \ldots \ldots\end{array}$ & $\begin{array}{l}8.7 \\
7.8 \\
7.5 \\
7.2 \\
6.8\end{array}$ & $\begin{array}{r}8.4 \\
7.0 \\
6.8 \\
6.8 \\
12.8\end{array}$ & $\begin{array}{l}6.8 \\
8.4 \\
6.4 \\
6.3 \\
6.0\end{array}$ & $\begin{array}{l}11.0 \\
11.0 \\
14.6 \\
11.0 \\
13.7\end{array}$ & $\begin{array}{r}12.3 \\
11.0 \\
10.6 \\
10.1 \\
9.2\end{array}$ & $\begin{array}{l}6.2 \\
6.0 \\
6.0 \\
5.9 \\
5.7\end{array}$ & $\begin{array}{l}7.0 \\
7.0 \\
7.0 \\
7.2 \\
7.2\end{array}$ & $\begin{array}{l}8.4 \\
8.2 \\
8.2 \\
7.8 \\
7.4\end{array}$ & $\begin{array}{l}7.2 \\
7.0 \\
7.5 \\
7.4 \\
7.0\end{array}$ & $\begin{array}{l}5.7 \\
5.6 \\
5.6 \\
5.6 \\
9.9\end{array}$ & $\begin{array}{l}11.9 \\
11.5 \\
11.0 \\
13.2 \\
15.5\end{array}$ & $\begin{array}{l}7.8 \\
7.5 \\
7.2 \\
7.2 \\
7.0\end{array}$ \\
\hline $\begin{array}{l}26 \ldots \ldots \ldots \\
27 \ldots \ldots \ldots \\
28 \ldots \ldots \ldots \\
29 \ldots \ldots \ldots \\
30 \ldots \ldots \\
31 \ldots \ldots \\
\end{array}$ & $\begin{array}{l}6.4 \\
6.3 \\
6.3 \\
6.0 \\
6.0 \\
6.0\end{array}$ & $\begin{array}{r}8.9 \\
6.3 \\
6.0 \\
\ldots . .\end{array}$ & $\begin{array}{r}6.0 \\
6.0 \\
6.4 \\
6.0 \\
5.6 \\
12.8\end{array}$ & $\begin{array}{r}13.7 \\
11.9 \\
9.2 \\
9.2 \\
8.7\end{array}$ & $\begin{array}{l}8.4 \\
8.4 \\
7.5 \\
7.5 \\
7.2 \\
6.8\end{array}$ & $\begin{array}{r}5.7 \\
5.6 \\
6.9 \\
18.2 \\
13.7 \\
\ldots . .\end{array}$ & $\begin{array}{l}7.0 \\
6.9 \\
6.9 \\
6.9 \\
6.9 \\
6.8\end{array}$ & $\begin{array}{l}7.4 \\
7.4 \\
7.2 \\
7.2 \\
7.2 \\
8.7\end{array}$ & $\begin{array}{l}6.9 \\
6.9 \\
6.8 \\
6.6 \\
6.4\end{array}$ & $\begin{array}{l}5.9 \\
5.9 \\
5.9 \\
5.7 \\
5.7 \\
5.7\end{array}$ & $\begin{array}{l}15.5 \\
13.7 \\
13.3 \\
12.8 \\
11.9\end{array}$ & $\begin{array}{l}7.0 \\
6.9 \\
6.8 \\
6.6 \\
6.4 \\
6.4\end{array}$ \\
\hline
\end{tabular}

NOTE.-No discharge Nov. 19. 
Monthly discharge of Kauaula ditch near Lahaina, Maui, for 1913.

\begin{tabular}{|c|c|c|c|c|c|}
\hline \multirow{2}{*}{ Month. } & \multicolumn{3}{|c|}{ Discharge in second-feet. } & \multirow{2}{*}{$\begin{array}{c}\text { Run-off } \\
\text { (total in } \\
\text { acre-feet). }\end{array}$} & \multirow{2}{*}{$\begin{array}{l}\text { Accu- } \\
\text { racy. }\end{array}$} \\
\hline & Maximum. & Minimum. & Mean. & & \\
\hline 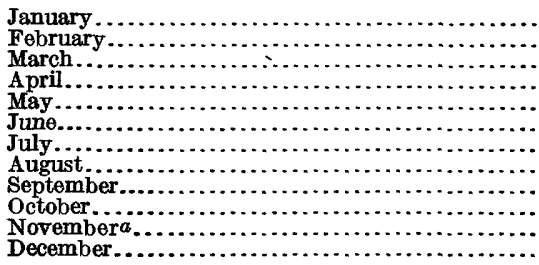 & $\begin{array}{l}14.6 \\
15.5 \\
12.8 \\
18.2 \\
15.5 \\
18.2 \\
15.5 \\
15.5 \\
12.4 \\
14.2 \\
16.4 \\
16.4\end{array}$ & $\begin{array}{l}6.0 \\
5.6 \\
4.9 \\
4.6 \\
6.4 \\
5.6 \\
6.8 \\
6.8 \\
6.3 \\
5.6 \\
4.6 \\
6.4\end{array}$ & $\begin{array}{r}7.88 \\
7.45 \\
6.27 \\
11.2 \\
9.47 \\
7.61 \\
8.57 \\
8.29 \\
7.23 \\
7.27 \\
11.7 \\
9.40\end{array}$ & $\begin{array}{l}485 \\
414 \\
386 \\
666 \\
582 \\
453 \\
527 \\
510 \\
430 \\
447 \\
696 \\
578\end{array}$ & $\begin{array}{l}\text { A. } \\
\text { A. } \\
\text { A. } \\
\text { A. } \\
\text { A. } \\
\text { A. } \\
\text { A. } \\
\text { A. } \\
\text { A. } \\
\text { A. } \\
\text { A. }\end{array}$ \\
\hline The year............ & 18.2 & 4.6 & 8.53 & 6,170 & - \\
\hline
\end{tabular}

$a$ Ditch was dry Nov. 19. Mean is for 29 days.

\section{LAUNIUPOKO STREAM NEAR LAHAINA, MAUI.}

Location.-About 175 feet above Pioneer Mill Co.'s ditch intake, 1 mile above storage reservoir, and about $5 \frac{1}{2}$ miles southeast of Lahaina.

Records available.-July 25, 1911, to December 31, 1913.

Gage.-Vertical staff.

Control.-Probably shifting.

Discharge measurements.-Made by wading.

Diversions.-None above station.

Accuracy.-Records poor.

Cooperation.-Station maintained in cooperation with Pioneer Mill Co.

Discharge measurements of Launiupoko Stream near Lahaina, Maui, in 1913.

[C. T. Bailey, hydrographer.]

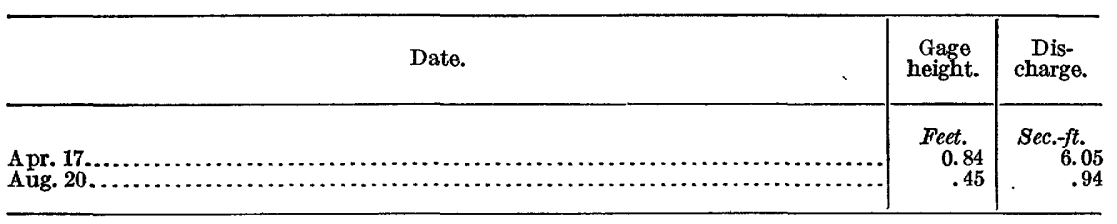

Monthly discharge of Launiupoko Stream near Lahaina, Maui, for 1913.

\begin{tabular}{|c|c|c|c|c|c|}
\hline \multirow{2}{*}{ Month. } & \multicolumn{3}{|c|}{ Discharge in second-feet. } & \multirow{2}{*}{$\begin{array}{l}\text { Run-ofi } \\
\text { (total in } \\
\text { acre-foet). }\end{array}$} & \multirow{2}{*}{$\begin{array}{l}\text { Accu- } \\
\text { racy. }\end{array}$} \\
\hline & Maximum. & Minimum. & Mean. & & \\
\hline 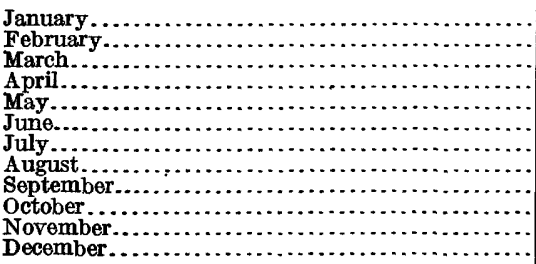 & $\begin{array}{l}5.5 \\
\text { 3.9 } \\
1.4 \\
12 \\
2.5 \\
2.8 \\
2.0 \\
5.5 \\
1.5 \\
3.6 \\
6.9 \\
6.7\end{array}$ & $\begin{array}{r}0.8 \\
.8 \\
.8 \\
.8 \\
.8 \\
.8 \\
.8 \\
.8 \\
.8 \\
.8 \\
.8 \\
.8\end{array}$ & $\begin{array}{r}1.11 \\
1.16 \\
.83 \\
2.86 \\
2.99 \\
1.02 \\
.92 \\
1.14 \\
.82 \\
1.06 \\
2.12 \\
1.08\end{array}$ & $\begin{array}{r}68.2 \\
64.4 \\
51.0 \\
170 \\
184 \\
60.7 \\
56.6 \\
70.1 \\
48.8 \\
65.2 \\
126 \\
66.4\end{array}$ & $\begin{array}{l}\mathrm{D} . \\
\mathrm{D} . \\
\mathrm{D} . \\
\mathrm{D} . \\
\mathrm{D} . \\
\mathrm{D} . \\
\mathrm{D} . \\
\mathrm{D} . \\
\mathrm{D} . \\
\mathrm{D} . \\
\mathrm{D} . \\
\mathrm{D} .\end{array}$ \\
\hline The year...... & 25 & .8 & 1. 43 & 1,030 & \\
\hline
\end{tabular}




\section{OLOWALU STREAM NEAR OLOWALU, MAUI.}

Location.-About 600 feet above Olowalu Sugar Co.'s power house, about 1 mile north of Olowalu.

Record available.-April 26 to December 31, 1913.

Gage.-Vertical staff.

Control.-Fairly permanent.

Discharge measurements.-Made from footbridge or by wading.

Diversions.-Water for power house diverted about $1 \frac{1}{2}$ miles above gage; measured in tailrace called Olowalu ditch No. 1.

Accuracy.-Records fair.

Cooperation.-Station maintained in cooperation with Olowalu Sugar Co.

Discharge measurements of Olowalu Stream near Olowalu, Maui, in 1913.

[C. T. Bailey, hydrographer.]

\begin{tabular}{|c|c|c|c|c|c|}
\hline Date. & $\begin{array}{c}\text { Gage } \\
\text { height. }\end{array}$ & $\begin{array}{c}\text { Dis- } \\
\text { charge. }\end{array}$ & Date. & $\begin{array}{c}\text { Gage } \\
\text { height. }\end{array}$ & $\begin{array}{c}\text { Dis- } \\
\text { charge. }\end{array}$ \\
\hline $\begin{array}{l}\text { Apr. } 26 \ldots \\
\text { May } 15 . . .\end{array}$ & $\begin{array}{c}\text { Feet. } \\
0.83 \\
1.77\end{array}$ & $\begin{array}{r}\text { Sec.-ft. } \\
35.44 \\
55.8\end{array}$ & Aug. $20 \ldots$ & $\begin{array}{l}\text { Feet. } \\
\mathbf{0 . 5 0}\end{array}$ & Sec.fft. \\
\hline
\end{tabular}

Daily discharge, in second-feet, of Olowalu Stream near Olowalu, Maui, for 1918.

\begin{tabular}{|c|c|c|c|c|c|c|c|c|c|}
\hline Day. & Apr. & May. & June. & July. & Aug. & Sept. & Oet. & Nov. & Dec. \\
\hline 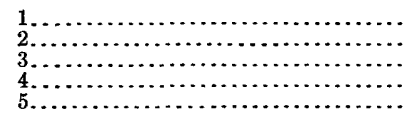 & & $\begin{array}{r}0.4 \\
1.6 \\
.4 \\
.4 \\
.2\end{array}$ & $\begin{array}{r}0.4 \\
.3 \\
2.6 \\
.4 \\
.2\end{array}$ & $\begin{array}{c}21 \\
11 \\
25 \\
11 \\
1.9\end{array}$ & $\ldots .$. & $\begin{array}{r}0.2 \\
.3 \\
\ldots \\
\ldots\end{array}$ & & $\begin{array}{l}32 \\
18 \\
6.3\end{array}$ & $\begin{array}{l}1.2 \\
.4 \\
84^{.3} \\
34\end{array}$ \\
\hline $\begin{array}{r}6 \ldots \\
7 \ldots \\
8 \ldots \\
9 \ldots \\
10 \ldots\end{array}$ & & $\begin{array}{r}.2 \\
\cdots \\
\cdots \cdot\end{array}$ & $\begin{array}{r}.3 \\
8.0 \\
.3 \\
4.0\end{array}$ & $\begin{array}{l}7.6 \\
2.9 \\
2.4 \\
1.9 \\
1.9\end{array}$ & \begin{tabular}{ll}
$\cdots$ & $\ldots$ \\
\hdashline & $\ldots$
\end{tabular} & $\cdots$ & & $\begin{array}{c}.1 \\
402 \\
102 \\
60\end{array}$ & $\begin{array}{l}29 \\
19 \\
12 \\
10 \\
7.6\end{array}$ \\
\hline $\begin{array}{l}11 \ldots \ldots \\
12, \ldots \ldots \\
13 \ldots \ldots \\
14 \ldots \ldots \\
15 \ldots \ldots\end{array}$ & & $\begin{array}{r}46 \\
37 \\
134 \\
86\end{array}$ & \begin{tabular}{r}
.2 \\
\hdashline 7.0 \\
7.0 \\
2.6
\end{tabular} & $\begin{array}{c}.3 \\
\cdots \\
\cdots \\
\cdots \cdots\end{array}$ & $\begin{array}{r}28 \\
.5 \\
2.6 \\
7.3\end{array}$ & $\mid$\begin{tabular}{l}
$\cdots$ \\
$\cdots$ \\
\hdashline$\ldots \ldots$ \\
\hdashline$\ldots \ldots$
\end{tabular} & $\begin{array}{r}1.6 \\
.1\end{array}$ & $\begin{array}{l}35 \\
29 \\
28 \\
15 \\
2.7\end{array}$ & $\begin{array}{r}6.6 \\
5.6 \\
5.0 \\
5.6 \\
.9\end{array}$ \\
\hline $\begin{array}{l}16 \ldots \ldots \\
17 \\
18 \ldots \ldots \\
19 \ldots \ldots\end{array}$ & ...... & $\begin{array}{l}36 \\
24 \\
21 \\
16 \\
13\end{array}$ & $\begin{array}{r}-.4 \\
.8 \\
.2 \\
2.4 \\
.2\end{array}$ & ( & $\begin{array}{c}11 \\
18 \\
4.5 \\
5.6 \\
77\end{array}$ & $\begin{array}{r}4.0 \\
\hdashline\end{array}$ & ….... & $\begin{array}{l}5.3 \\
5.3 \\
16 \\
40 \\
38\end{array}$ & $\begin{array}{r}.2 \\
12 \\
.3 \\
.2\end{array}$ \\
\hline $\begin{array}{l}21 \ldots \ldots \\
22 \ldots \ldots \\
23 \ldots \ldots \\
24 \ldots \ldots \\
25 \ldots \ldots\end{array}$ & $\ldots$ & $\begin{array}{l}11 \\
9.0 \\
5.3 \\
7.0 \\
69\end{array}$ & 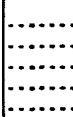 & $\mid \begin{array}{l}\ldots \\
\cdots \\
\cdots \\
\cdots\end{array}$ & $\begin{array}{r}13 \\
39 \\
1.1 \\
.2 \\
.2\end{array}$ & {$\left[\begin{array}{l}\cdots \\
\cdots \\
\cdots\end{array}\right.$} & $\begin{array}{r}7.0 \\
1.1 \\
.2\end{array}$ & $\begin{array}{r}7.6 \\
2.4 \\
.4 \\
1.9 \\
28\end{array}$ & 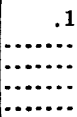 \\
\hline 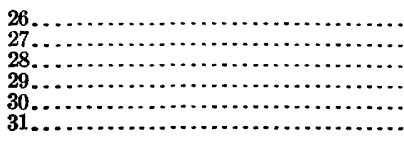 & $\begin{array}{r}2.9 \\
5.1 \\
1.6 \\
1.1 \\
.6\end{array}$ & \begin{tabular}{r}
1.2 \\
\hdashline 107 \\
5.3 \\
1.8 \\
.2
\end{tabular} & $\begin{array}{r}5.6 \\
5.9 \\
126 \\
11\end{array}$ & $\mid$\begin{tabular}{l}
$\cdots$ \\
$\cdots \ldots \ldots$ \\
\hdashline$\ldots \ldots$ \\
$\cdots \cdots$
\end{tabular} & $\begin{array}{r}.2 \\
.2 \\
.2 \\
.2 \\
.2 \\
1.0\end{array}$ & $\left.\begin{array}{l} \\
\cdots \\
\hdashline \\
\hdashline\end{array}\right]$ & $\begin{array}{c}\ldots \ldots . . \\
\cdots \ldots . . \\
\cdots \ldots . . \\
\cdots \ldots . . \\
\cdots\end{array}$ & $\begin{array}{c}50 \\
18 \\
7.0 \\
2.3 \\
4.5\end{array}$ & $\begin{array}{l}\cdots \cdots \\
\cdots \cdots \cdots \\
\cdots \cdots \\
\cdots \cdots \\
\cdots \cdots\end{array}$ \\
\hline
\end{tabular}

Nore.-No discharge for days for which figures are not given; water diverted above station at power house. 
Monthly discharge of Olowalu Stream near Olowalu, Maui, for 1913.

\begin{tabular}{|c|c|c|c|c|c|}
\hline \multirow{2}{*}{ Month. } & \multicolumn{3}{|c|}{ Discharge in second-feet. } & \multirow{2}{*}{$\begin{array}{c}\text { Run-off } \\
\text { (total in } \\
\text { acre-feet). }\end{array}$} & \multirow{2}{*}{$\begin{array}{l}\text { Accu- } \\
\text { racy. }\end{array}$} \\
\hline & Maximum. & Minimum. & Mean. & & \\
\hline \multirow[t]{2}{*}{ 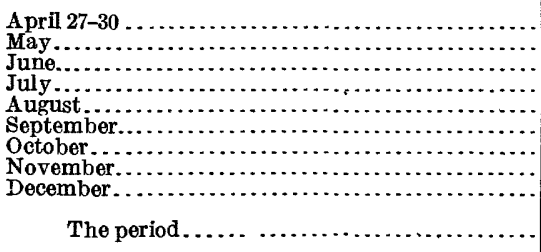 } & $\begin{array}{c}5.1 \\
134 \\
126 \\
25 \\
77 \\
4.0 \\
7.0 \\
102 \\
84\end{array}$ & $\begin{array}{l}(a) \\
(a) 6 \\
(a) \\
(a) \\
(a) \\
(a) \\
(a) \\
(a)\end{array}$ & $\begin{array}{c}2.26 \\
20.4 \\
6.19 \\
3.38 \\
6.77 \\
.15 \\
.32 \\
19.7 \\
7.55\end{array}$ & $\begin{array}{c}22.4 \\
1,250 \\
368 \\
208 \\
416 \\
8.9 \\
19.7 \\
1,170 \\
464\end{array}$ & \multirow[t]{2}{*}{$\begin{array}{l}\text { C. } \\
\text { C. } \\
\text { C. } \\
\text { c. } \\
\text { C. } \\
\text { C. } \\
\text { C. } \\
\text { C. } \\
\text { C. }\end{array}$} \\
\hline & $\cdots$ & & ........... & 3,930 & \\
\hline
\end{tabular}

a Stream dry.

\section{OLOWALU DITCH NO. I NEAR OLOWALU, MAUI.}

Location.-One mile above Olowalu, near Olowalu plantation power house, and about 7 miles east of Lahaina.

Records available.-August 12, 1911, to December 31, 1913.

(Yage.-Vertical staff.

Control.-Probably permanent.

Discharge measurements.-Made in flume.

Accuracy.-Records good.

Cooperation.--Station maintained in cooperation with Olowalu Sugar Co.

Discharge measurements of Olowalu ditch No. 1 near Olowalu, Maui, in 1918.

[C. T. Bailey, hydrographer.]

\begin{tabular}{|c|c|c|}
\hline Date. & $\begin{array}{c}\text { Gage } \\
\text { height. }\end{array}$ & $\begin{array}{c}\text { Dis- } \\
\text { charge. }\end{array}$ \\
\hline $\begin{array}{l}\text { Apr. } 16 \ldots \ldots \ldots \ldots \ldots \ldots \\
\text { Aug. } 20 \ldots \ldots \ldots \ldots\end{array}$ & $\begin{array}{l}\text { Feet. } \\
0.38 \\
.49\end{array}$ & $\begin{aligned} & \text { Sec. }-f t . \\
& 5.31 \\
& 8.04\end{aligned}$ \\
\hline
\end{tabular}

$97764^{\circ}-$ wSP $373-15-9$ 
Daily discharge, in second-feet, of Olowalu ditch No. 1 near Olowalu, Maui, for 1913.

\begin{tabular}{|c|c|c|c|c|c|c|c|c|c|c|c|c|}
\hline Day: & Jan. & Feb. & Mar. & Apr. & May. & June. & July. & Aug. & Sept. & Oct. & Nov. & Dec. \\
\hline $\begin{array}{l}1 \ldots . \\
2 . . . \\
3 \ldots \\
4 \ldots . \\
5 \ldots\end{array}$ & $\begin{array}{l}4.5 \\
4.5 \\
4.5 \\
4.0 \\
4.5\end{array}$ & $\begin{array}{l}4.5 \\
4.0 \\
4.0 \\
4.0 \\
4.0\end{array}$ & $\begin{array}{l}\mathbf{5 . 5} \\
\mathbf{5 . 5} \\
\mathbf{5 . 0} \\
4.5 \\
4.5\end{array}$ & $\begin{array}{l}6.0 \\
5.5 \\
5.0 \\
4.0 \\
4.0\end{array}$ & $\begin{array}{l}8.0 \\
8.5 \\
8.0 \\
8.0 \\
7.0\end{array}$ & $\begin{array}{l}4.5 \\
4.5 \\
5.5 \\
4.5 \\
4.5\end{array}$ & $\begin{array}{l}10 \\
10 \\
10 \\
8.5 \\
8.0\end{array}$ & $\begin{array}{l}5.0 \\
4.5 \\
4.5 \\
4.5 \\
4.5\end{array}$ & $\begin{array}{l}4.5 \\
4.5 \\
4.5 \\
4.5 \\
4.5\end{array}$ & $\begin{array}{l}3.9 \\
4.0 \\
4.0 \\
4.0 \\
4.0\end{array}$ & $\begin{array}{l}3.9 \\
4.0 \\
4.8 \\
5.5 \\
4.8\end{array}$ & $\begin{array}{l}11 \\
11 \\
11 \\
15 \\
13\end{array}$ \\
\hline $\begin{array}{r}6 \ldots . . \\
7 \ldots . \\
8 \ldots . \\
9 \ldots \ldots \\
10 \ldots\end{array}$ & $\begin{array}{l}4.5 \\
4.5 \\
4.5 \\
4.5 \\
4.5\end{array}$ & $\begin{array}{l}6.0 \\
8.0 \\
5.5 \\
4.0 \\
4.5\end{array}$ & $\begin{array}{l}4.5 \\
4.5 \\
4.5 \\
4.5 \\
4.0\end{array}$ & $\begin{array}{l}4.0 \\
4.0 \\
4.0 \\
4.0 \\
4.5\end{array}$ & $\begin{array}{l}6.0 \\
6.0 \\
5.5 \\
5.5 \\
5.5\end{array}$ & $\begin{array}{l}4.5 \\
4.5 \\
4.5 \\
4.5 \\
4.5\end{array}$ & $\begin{array}{l}7.5 \\
5.5 \\
5.5 \\
5.5 \\
5.5\end{array}$ & $\begin{array}{l}4.5 \\
4.5 \\
4.5 \\
4.5 \\
5.0\end{array}$ & $\begin{array}{l}4.5 \\
4.5 \\
4.0 \\
4.0 \\
4.0\end{array}$ & $\begin{array}{l}4.9 \\
4.0 \\
5.5 \\
4.3 \\
4.0\end{array}$ & $\begin{array}{l}4.8 \\
4.8 \\
8.0 \\
4.8 \\
3.8\end{array}$ & $\begin{array}{l}11 \\
11 \\
9.5 \\
7.5 \\
6.8\end{array}$ \\
\hline $\begin{array}{l}11 \ldots \\
12 \ldots \\
13 \ldots \\
14 \ldots \\
15 \ldots\end{array}$ & $\begin{array}{r}4.0 \\
4.0 \\
10 \\
7.5 \\
4.5\end{array}$ & $\begin{array}{l}5.0 \\
4.5 \\
4.5 \\
4.5 \\
.4 .5\end{array}$ & $\begin{array}{l}4.0 \\
4.0 \\
4.0 \\
6.0 \\
6.0\end{array}$ & $\begin{array}{r}9.5 \\
4.5 \\
4.5 \\
9.5 \\
11\end{array}$ & $\begin{array}{c}6.0 \\
5.5 \\
10 \\
8.0 \\
4.5\end{array}$ & $\begin{array}{l}4.0 \\
4.5 \\
5.5 \\
4.5 \\
5.0\end{array}$ & $\begin{array}{l}5.5 \\
7.0 \\
8.0 \\
8.0 \\
7.0\end{array}$ & $\begin{array}{l}4.5 \\
6.0 \\
4.5 \\
8.0 \\
8.0\end{array}$ & $\begin{array}{l}4.0 \\
4.0 \\
4.0 \\
4.0 \\
4.0\end{array}$ & $\begin{array}{l}3.9 \\
3.8 \\
6.5 \\
8.0 \\
5.2\end{array}$ & $\begin{array}{l}3.8 \\
3.0 \\
4.0 \\
4.0 \\
6.0\end{array}$ & $\begin{array}{l}5.5 \\
5.5 \\
4.9 \\
5.2 \\
5.8\end{array}$ \\
\hline 20 & $\begin{array}{l}5.0 \\
4.5 \\
4.0 \\
4.0 \\
3.5\end{array}$ & $\begin{array}{l}4.5 \\
4.5 \\
5.5 \\
8.0 \\
7.0\end{array}$ & $\begin{array}{l}7.5 \\
7.5 \\
5.5 \\
5.0 \\
4.5\end{array}$ & $\begin{array}{r}6.0 \\
4.5 \\
4.0 \\
6.0 \\
11\end{array}$ & $\begin{array}{r}7.0 \\
10 \\
9.5 \\
9.0 \\
6.0\end{array}$ & $\begin{array}{l}4.5 \\
4.5 \\
4.0 \\
4.0 \\
4.0\end{array}$ & $\begin{array}{l}7.0 \\
5.5 \\
5.5 \\
6.0 \\
7.5\end{array}$ & $\begin{array}{l}8.0 \\
7.5 \\
8.0 \\
8.0 \\
7.5\end{array}$ & $\begin{array}{l}4.0 \\
5.0 \\
8.0 \\
6.0 \\
5.0\end{array}$ & $\begin{array}{l}4.3 \\
4.0 \\
4.0 \\
4.0 \\
3.9\end{array}$ & $\begin{array}{l}9.5 \\
11 \\
11 \\
11 \\
9.5\end{array}$ & $\begin{array}{r}7.5 \\
8.0 \\
11 \\
8.0 \\
8.0\end{array}$ \\
\hline $\begin{array}{l}21 \ldots \\
22 \ldots \\
23 \ldots \\
24 \ldots \\
25 \ldots\end{array}$ & $\begin{array}{l}3.0 \\
4.5 \\
4.5 \\
4.5 \\
4.5\end{array}$ & $\begin{array}{l}5.5 \\
4.5 \\
4.0 \\
3.5 \\
7.0\end{array}$ & $\begin{array}{l}5.0 \\
7.5 \\
6.0 \\
5.0 \\
5.0\end{array}$ & $\begin{array}{r}11 \\
7.5 \\
5.0 \\
6.0 \\
8.0\end{array}$ & $\begin{array}{l}5.5 \\
5.5 \\
5.5 \\
5.5 \\
5.5\end{array}$ & $\begin{array}{l}4.0 \\
4.0 \\
4.5 \\
5.5 \\
5.5\end{array}$ & $\begin{array}{l}7.0 \\
5.5 \\
6.0 \\
6.0 \\
5.5\end{array}$ & $\begin{array}{l}7.0 \\
7.0 \\
6.0 \\
5.5 \\
5.5\end{array}$ & $\begin{array}{l}4.5 \\
4.5 \\
4.5 \\
4.0 \\
4.0\end{array}$ & $\begin{array}{l}3.8 \\
4.0 \\
6.8 \\
7.5 \\
6.0\end{array}$ & $\begin{array}{l}11 \\
11 \\
11 \\
11 \\
13\end{array}$ & $\begin{array}{l}8.0 \\
6.8 \\
6.8 \\
6.0 \\
5.5\end{array}$ \\
\hline $\begin{array}{l}26 \ldots \ldots \\
27 \ldots \ldots \\
28 \ldots \ldots \\
29 \ldots \ldots \\
30 \ldots \ldots \\
31 \ldots \ldots\end{array}$ & $\begin{array}{l}4.0 \\
4.0 \\
4.0 \\
4.0 \\
4.0 \\
4.5\end{array}$ & $\begin{array}{l}5.0 \\
4.0 \\
4.5\end{array}$ & $\begin{array}{l}4.5 \\
5.0 \\
6.0 \\
4.5 \\
5.0 \\
8.0\end{array}$ & $\begin{array}{l}8.5 \\
8.5 \\
8.5 \\
8.0 \\
8.0\end{array}$ & $\begin{array}{l}5.5 \\
5.0 \\
5.5 \\
5.5 \\
5.0 \\
5.0\end{array}$ & $\begin{array}{l}5.5 \\
8.0 \\
8.5 \\
9.5 \\
9.5\end{array}$ & $\begin{array}{l}5.5 \\
5.5 \\
5.5 \\
5.0 \\
4.5 \\
4.5\end{array}$ & $\begin{array}{l}5.0 \\
5.0 \\
4.5 \\
4.5 \\
4.5 \\
7.0\end{array}$ & $\begin{array}{l}4.0 \\
4.0 \\
4.0 \\
4.0 \\
4.0\end{array}$ & $\begin{array}{l}5.5 \\
5.2 \\
5.2 \\
5.2 \\
4.8 \\
5.5\end{array}$ & $\begin{array}{l}13 \\
12 \\
11 \\
11 \\
11\end{array}$ & $\begin{array}{l}5.5 \\
5.5 \\
5.5 \\
5.5 \\
5.4 \\
5.2\end{array}$ \\
\hline
\end{tabular}

Monthly discharge of Olowalu ditch No. 1 near Olowalu, Maui, for 1913.

\begin{tabular}{|c|c|c|c|c|c|}
\hline \multirow{2}{*}{ Month. } & \multicolumn{3}{|c|}{ Discharge in second-feet. } & \multirow{2}{*}{$\begin{array}{c}\text { Run-off } \\
\text { (total in } \\
\text { acre-feet). }\end{array}$} & \multirow{2}{*}{$\begin{array}{l}\text { A ccu- } \\
\text { racy. }\end{array}$} \\
\hline & Maximum. & Minimum. & Mean. & & \\
\hline 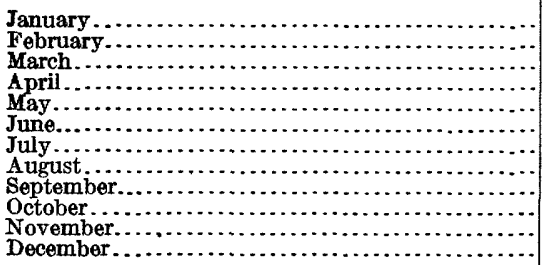 & \begin{tabular}{c|}
10 \\
8.0 \\
8.0 \\
11 \\
10 \\
9.5 \\
10 \\
8.0 \\
8.0 \\
8.0 \\
13 \\
15
\end{tabular} & $\begin{array}{l}3.0 \\
3.5 \\
4.0 \\
4.0 \\
4.5 \\
4.0 \\
4.5 \\
4.5 \\
4.0 \\
3.8 \\
3.0 \\
4.9\end{array}$ & $\begin{array}{l}4.55 \\
4.96 \\
5.24 \\
6.48 \\
6.53 \\
5.17 \\
6.56 \\
5.73 \\
4.43 \\
4.85 \\
7.90 \\
7.80\end{array}$ & $\begin{array}{l}280 \\
275 \\
322 \\
386 \\
402 \\
308 \\
403 \\
352 \\
264 \\
298 \\
470 \\
480\end{array}$ & $\begin{array}{l}\text { B. } \\
\text { B. } \\
\text { B. } \\
\text { B. } \\
\text { B. } \\
\text { B. } \\
\text { B. } \\
\text { B. } \\
\text { B. } \\
\text { B. } \\
\text { B. } \\
\text { B. }\end{array}$ \\
\hline The year......... & 15 & 3.0 & 5.86 & 4,240 & \\
\hline
\end{tabular}




\section{WAIKAPU STREAM NEAR WAIKAPU, MAUI.}

Iocation.-One and one-half miles west of Waikapu and about 5 miles southwest of Wailuku; 500 feet below intake of Palolo ditch.

Fiecords available.-December 1, 1910, to December 31, 1913.

Gage.-Inclined staff.

Control.-Shifting.

Iischarge measurements.-Made by wading.

Iiversions.-Most of the normal flow diverted into the South Side Waikapu and the Palolo ditches.

A.ccuracy.-Records poor on account of insufficient rating.

The following discharge measurement was made by C. T. Bailey:

May 27, 1913: Gage height, 0.34 foot; discharge, 0.20 second-foot.

Monthly discharge of Waikapu Stream near Waikapu, Maui, for 1913.

\begin{tabular}{|c|c|c|c|c|c|}
\hline \multirow{2}{*}{ Month. } & \multicolumn{3}{|c|}{ Discharge in second-feet. } & \multirow{2}{*}{$\begin{array}{c}\text { Run-off } \\
\text { (total in } \\
\text { acre-feet). }\end{array}$} & \multirow{2}{*}{$\begin{array}{l}\text { Accu- } \\
\text { racy. }\end{array}$} \\
\hline & Maximum. & Minimum. & Mean. & & \\
\hline 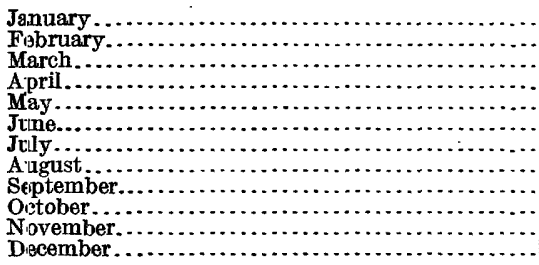 & $\begin{array}{c}50.3 \\
80^{.3} \\
181 \\
27 \\
6.0 \\
25 \\
1.0 \\
149 \\
178\end{array}$ & $\begin{array}{l}0.2 \\
.2 \\
.2 \\
.2 \\
.2 \\
.2 \\
.2 \\
.2 \\
.2 \\
.2 \\
.2 \\
.2\end{array}$ & $\begin{array}{r}0.20 \\
4.70 \\
.21 \\
10.3 \\
8.94 \\
1.60 \\
.53 \\
3.05 \\
.22 \\
.25 \\
9.30 \\
13.6\end{array}$ & $\begin{array}{c}12.3 \\
261 \\
12.9 \\
613 \\
550 \\
95.0 \\
32.6 \\
188 \\
13.1 \\
15.4 \\
553 \\
836\end{array}$ & $\begin{array}{l}\text { D. } \\
\text { D: } \\
\text { D. } \\
\text { D. } \\
\text { D. } \\
\text { D. } \\
\text { D. } \\
\text { D. } \\
\text { D. } \\
\text { D. } \\
\text { D. }\end{array}$ \\
\hline The year. & 181 & .2 & 4. 40 & 3,180 & \\
\hline
\end{tabular}

\section{SOUTH SIDE WAIKAPU DITCH NEAR WAIKAPU, MAUI.}

Location.-One mile below ditch intake, $1 \frac{1}{2}$ miles west of Waikapu, and about $5 \frac{1}{2}$ miles southwest of Wailuku.

Records available.-November 21, 1910, to December 31, 1913.

Gage.-Vertical staff.

Control.-Fairly permanent.

Discharge measurements.-Made from plank over ditch about 400 feet below gage. Accuracy.-Records good.

Discharge measurements of South Side Waikapu ditch near Waikapu, Maui, in 1913. [C. T. Bailey, hydrographer.]

\begin{tabular}{|c|c|c|c|c|c|}
\hline Date. & $\begin{array}{c}\text { Gage } \\
\text { height. }\end{array}$ & $\begin{array}{l}\text { Dis- } \\
\text { charge. }\end{array}$ & Date. & $\begin{array}{c}\text { Gage } \\
\text { height. }\end{array}$ & $\begin{array}{c}\text { Dis- } \\
\text { charge. }\end{array}$ \\
\hline $\begin{array}{l}\text { Apr. } 9 \\
\text { Miry } 27, \ldots \ldots \ldots \ldots\end{array}$ & $\begin{array}{r}\text { Feet. } \\
0.57 \\
.73\end{array}$ & $\begin{aligned} & \text { Sec.-ft. } \\
& \text { 3. } 47 \\
& 6.29\end{aligned}$ & $\begin{array}{l}\text { July } 29 \ldots \ldots \ldots \ldots \ldots \ldots \\
\text { Nov. } 5 \ldots \ldots \ldots \ldots \ldots \ldots\end{array}$ & $\begin{array}{r}\text { Feet. } \\
0.60 \\
.66\end{array}$ & $\begin{array}{r}\text {.Sec. }-f t . \\
3.78 \\
4.86\end{array}$ \\
\hline
\end{tabular}


Daily discharge, in second-feet, of South Side Waikapu ditch near Waikapu, Maui, for 1913.

\begin{tabular}{|c|c|c|c|c|c|c|c|c|c|c|c|c|}
\hline Day. & Jan. & Feb. & Mar. & Apr. & May. & June. & July. & Aug. & Sept. & Oct. & Nov. & Dec. \\
\hline $\begin{array}{l}1 \ldots \\
2 \ldots \\
3 \ldots \\
4 \ldots \\
5 \ldots\end{array}$ & $\begin{array}{l}3.5 \\
4.1 \\
4.5 \\
3.9 \\
3.5\end{array}$ & $\begin{array}{l}2.8 \\
2.8 \\
2.8 \\
2.8 \\
2.8\end{array}$ & $\begin{array}{l}4.8 \\
4.2 \\
4.2 \\
4.1 \\
3.9\end{array}$ & $\begin{array}{l}4.2 \\
4.1 \\
3.9 \\
3.6 \\
3.5\end{array}$ & $\begin{array}{l}5.7 \\
8.8 \\
6.8 \\
7.0 \\
7.6\end{array}$ & $\begin{array}{l}6.0 \\
6.0 \\
6.2 \\
6.0 \\
6.0\end{array}$ & $\begin{array}{l}9.0 \\
9.4 \\
7.5 \\
6.7 \\
5.7\end{array}$ & $\begin{array}{l}4.2 \\
4.8 \\
4.2 \\
4.2 \\
4.2\end{array}$ & $\begin{array}{l}6.0 \\
6.0 \\
5.7 \\
5.4 \\
5.4\end{array}$ & $\begin{array}{l}3.4 \\
3.4 \\
3.1 \\
3.4 \\
3.1\end{array}$ & $\begin{array}{r}2.9 \\
3.1 \\
10.4 \\
9.0 \\
5.4\end{array}$ & $\begin{array}{r}6.0 \\
6.0 \\
5.6 \\
14.5 \\
8.5\end{array}$ \\
\hline $\begin{array}{r}6 \ldots \\
7 \ldots \\
8 \ldots \\
9 \ldots \\
10 \ldots\end{array}$ & $\begin{array}{l}3.1 \\
3.1 \\
2.9 \\
2.8 \\
2.8\end{array}$ & $\begin{array}{r}10.1 \\
5.9 \\
11.0 \\
7.1 \\
5.0\end{array}$ & $\begin{array}{l}3.9 \\
4.2 \\
4.2 \\
3.9 \\
3.9\end{array}$ & $\begin{array}{l}3.5 \\
3.5 \\
3.5 \\
3.5 \\
3.9\end{array}$ & $\begin{array}{l}8.2 \\
8.5 \\
8.3 \\
8.5 \\
8.3\end{array}$ & $\begin{array}{l}6.0 \\
5.9 \\
6.3 \\
6.0 \\
6.7\end{array}$ & $\begin{array}{l}5.9 \\
6.7 \\
5.7 \\
5.1 \\
4.5\end{array}$ & $\begin{array}{l}4.1 \\
3.9 \\
3.8 \\
3.9 \\
7.0\end{array}$ & $\begin{array}{l}5.0 \\
5.7 \\
4.2 \\
4.2 \\
3.9\end{array}$ & $\begin{array}{l}3.6 \\
3.2 \\
4.4 \\
3.4 \\
3.1\end{array}$ & $\begin{array}{r}4.2 \\
3.8 \\
17.3 \\
15.7 \\
10.8\end{array}$ & $\begin{array}{r}6.0 \\
4.5 \\
4.2 \\
.6 \\
7.3\end{array}$ \\
\hline $\begin{array}{l}11 \ldots \\
12 \ldots \\
13 \ldots \\
14 \ldots \\
15 \ldots\end{array}$ & $\begin{array}{l}2.8 \\
2.8 \\
4.1 \\
4.5 \\
4.5\end{array}$ & $\begin{array}{l}5.1 \\
4.2 \\
3.5 \\
3.5 \\
3.5\end{array}$ & $\begin{array}{l}3.9 \\
3.9 \\
3.9 \\
3.9 \\
4.4\end{array}$ & $\begin{array}{r}8.3 \\
11.0 \\
11.3 \\
10.6 \\
13.7\end{array}$ & $\begin{array}{r}8.2 \\
12.6 \\
10.6 \\
11.0 \\
8.0\end{array}$ & $\begin{array}{l}6.3 \\
8.0 \\
6.8 \\
6.0 \\
7.6\end{array}$ & $\begin{array}{l}4.2 \\
7.0 \\
6.2 \\
5.7 \\
5.4\end{array}$ & $\begin{array}{r}4.5 \\
10.8 \\
8.7 \\
6.0 \\
6.3\end{array}$ & $\begin{array}{l}4.4 \\
3.9 \\
4.6 \\
3.9 \\
3.8\end{array}$ & $\begin{array}{l}2.8 \\
2.8 \\
3.9 \\
2.9 \\
3.1\end{array}$ & $\begin{array}{l}8.3 \\
7.1 \\
7.1 \\
6.0 \\
5.2\end{array}$ & $\begin{array}{l}6.7 \\
6.2 \\
5.8 \\
5.4 \\
4.5\end{array}$ \\
\hline $\begin{array}{l}16 \ldots . \\
17 \ldots \\
18 \ldots \\
19 \ldots \\
20 \ldots\end{array}$ & $\begin{array}{l}3.5 \\
3.1 \\
2.8 \\
2.8 \\
2.8\end{array}$ & $\begin{array}{r}3.6 \\
3.5 \\
10.4 \\
6.5 \\
9.0\end{array}$ & $\begin{array}{l}4.5 \\
5.1 \\
4.4 \\
4.1 \\
3.6\end{array}$ & $\begin{array}{r}12.2 \\
11.9 \\
10.8 \\
8.7 \\
7.3\end{array}$ & $\begin{array}{l}6.7 \\
8.7 \\
9.0 \\
9.0 \\
7.8\end{array}$ & $\begin{array}{l}6.2 \\
5.7 \\
5.6 \\
5.1 \\
4.5\end{array}$ & $\begin{array}{l}5.2 \\
5.2 \\
5.2 \\
5.2 \\
5.4\end{array}$ & $\begin{array}{r}10.1 \\
8.3 \\
6.2 \\
5.1 \\
5.4\end{array}$ & $\begin{array}{l}4.2 \\
4.4 \\
7.6 \\
5.1 \\
4.2\end{array}$ & $\begin{array}{l}2.9 \\
2.9 \\
2.8 \\
2.8 \\
2.8\end{array}$ & $\begin{array}{r}6.3 \\
7.3 \\
11.2 \\
13.2 \\
8.3\end{array}$ & $\begin{array}{l}4.2 \\
4.6 \\
5.1 \\
4.2 \\
3.9\end{array}$ \\
\hline $\begin{array}{l}21 \ldots \ldots \\
22 \ldots \ldots \\
23 \ldots \ldots \\
24 \ldots \ldots \\
25 \ldots \ldots\end{array}$ & $\begin{array}{l}2.8 \\
2.8 \\
2.8 \\
2.8 \\
2.8\end{array}$ & $\begin{array}{l}6.5 \\
5.7 \\
5.6 \\
4.2 \\
7.6\end{array}$ & $\begin{array}{l}3.5 \\
5.1 \\
4.2 \\
3.9 \\
3.9\end{array}$ & $\begin{array}{l}8.5 \\
8.2 \\
7.8 \\
7.6 \\
7.3\end{array}$ & $\begin{array}{l}7.1 \\
7.0 \\
6.5 \\
6.0 \\
6.0\end{array}$ & $\begin{array}{l}4.2 \\
4.1 \\
4.1 \\
4.1 \\
4.2\end{array}$ & $\begin{array}{l}5.1 \\
4.5 \\
5.1 \\
5.1 \\
5.7\end{array}$ & $\begin{array}{l}5.7 \\
4.8 \\
5.1 \\
5.1 \\
5.4\end{array}$ & $\begin{array}{l}4.4 \\
3.9 \\
3.9 \\
3.6 \\
3.5\end{array}$ & $\begin{array}{l}2.7 \\
2.7 \\
2.7 \\
2.7 \\
2.8\end{array}$ & $\begin{array}{l}7.0 \\
5.7 \\
6.3 \\
8.3 \\
9.4\end{array}$ & $\begin{array}{l}3.8 \\
2.8 \\
2.8 \\
2.8 \\
2.8\end{array}$ \\
\hline $\begin{array}{l}26 \ldots \\
27 \ldots \\
28 \ldots \\
29 \ldots \\
30 \ldots \\
31 \ldots\end{array}$ & $\begin{array}{l}2.8 \\
2.8 \\
2.8 \\
2.8 \\
2.8 \\
3.5\end{array}$ & $\begin{array}{c}7.3 \\
5.7 \\
5.6 \\
\cdots . . . \\
\cdots . . . \\
\cdots . . .\end{array}$ & $\begin{array}{l}3.6 \\
3.5 \\
5.0 \\
4.1 \\
3.5 \\
5.0\end{array}$ & $\begin{array}{l}6.2 \\
6.3 \\
5.7 \\
5.7 \\
5.9\end{array}$ & $\begin{array}{l}8.8 \\
6.8 \\
6.3 \\
6.0 \\
5.9 \\
5.7\end{array}$ & $\begin{array}{r}3.9 \\
11.2 \\
9.4 \\
8.8 \\
9.4\end{array}$ & $\begin{array}{l}4.8 \\
4.5 \\
3.9 \\
3.9 \\
4.5 \\
4.2\end{array}$ & $\begin{array}{l}5.1 \\
6.2 \\
6.0 \\
5.4 \\
5.4 \\
9.9\end{array}$ & $\begin{array}{l}3.5 \\
3.5 \\
3.5 \\
3.4 \\
3.4\end{array}$ & $\begin{array}{l}2.7 \\
2.7 \\
2.8 \\
3.0 \\
3.1 \\
2.9\end{array}$ & $\begin{array}{r}12.6 \\
8.3 \\
6.8 \\
5.7 \\
8.3\end{array}$ & $\begin{array}{l}2.8 \\
2.7 \\
2.7 \\
2.7 \\
2.5 \\
2.5\end{array}$ \\
\hline
\end{tabular}

Monthly discharge of South Side Waikapu ditch near Waikapu, Maui, for 191 s.

\begin{tabular}{|c|c|c|c|c|c|}
\hline \multirow{2}{*}{ Month. } & \multicolumn{3}{|c|}{ Discharge in second-feet. } & \multirow{2}{*}{$\begin{array}{c}\text { Run-off } \\
\text { (total in } \\
\text { acre-feet). }\end{array}$} & \multirow{2}{*}{$\begin{array}{l}\text { Accu } \\
\text { racy. }\end{array}$} \\
\hline & Maximum. & Minimum. & Mean. & & \\
\hline 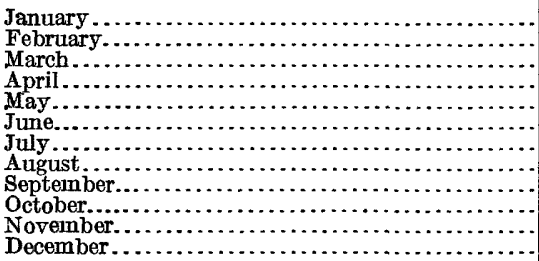 & \begin{tabular}{r|}
4.5 \\
11.0 \\
5.1 \\
13.7 \\
1.6 \\
11.2 \\
9.4 \\
10.8 \\
7.6 \\
4.4 \\
17.3 \\
14.5
\end{tabular} & $\begin{array}{l}2.8 \\
2.8 \\
3 . \\
3.5 \\
5.7 \\
3.9 \\
3.9 \\
3.8 \\
3.4 \\
2.7 \\
2.9 \\
.6\end{array}$ & $\begin{array}{l}3.21 \\
5.50 \\
4.14 \\
7.07 \\
7.79 \\
6.21 \\
5.55 \\
5.80 \\
4.47 \\
3.05 \\
8.03 \\
4.67\end{array}$ & $\begin{array}{l}197 \\
305 \\
255 \\
421 \\
479 \\
370 \\
341 \\
357 \\
266 \\
187 \\
478 \\
287\end{array}$ & $\begin{array}{l}\text { A. } \\
\text { A. } \\
\text { A. } \\
\text { A. } \\
\text { A. } \\
\text { A. } \\
\text { A. } \\
\text { A. } \\
\text { A. } \\
\text { A. } \\
\text { A. } \\
\text { A. }\end{array}$ \\
\hline The year......... & 17.3 & .6 & 5.45 & 3,940 & \\
\hline
\end{tabular}

PALOLO DITCH NEAR WAIKAPU, MAUI.

Location.-Two hundred feet below ditch intake, $1 \frac{1}{2}$ miles west of Waikapu, and about $5 \frac{1}{2}$ miles southwest of Wailuku.

Records available.--November 21, 1910, to December 31, 1913.

Gage.-Vertical staff.

Contro1.-Probably permanent.

Discharge measurements.-Made by wading.

Accuracy.-Records good. 
Discharge measurements of Palolo ditch near Waikapu, Maui, in 1913.

[C. T. Bailey, hydrographer.]

\begin{tabular}{|c|c|c|c|c|c|}
\hline Date. & $\begin{array}{c}\text { Gage } \\
\text { height. }\end{array}$ & $\begin{array}{c}\text { Dis- } \\
\text { charge. }\end{array}$ & Date. & $\begin{array}{c}\text { Gage } \\
\text { height. }\end{array}$ & $\begin{array}{c}\text { Dis- } \\
\text { charge. }\end{array}$ \\
\hline 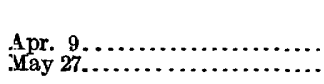 & $\begin{array}{r}\text { Feet. } \\
0.81 \\
.84\end{array}$ & $\begin{array}{r}\text { Sec.-ft. } \\
2.61 \\
2.83\end{array}$ & July $29 .$. & $\begin{array}{c}\text { Feet. } \\
0.80\end{array}$ & sec.-ft. 24 \\
\hline
\end{tabular}

Daily discharge, in second-feet, of Palolo ditch near Waikapu, Maui, for 1913.

\begin{tabular}{|c|c|c|c|c|c|c|c|c|c|c|c|c|}
\hline Day. & Jan. & Feb. & Mar. & Apr. & May. & June. & July. & Aug. & Sept. & Oct. & Nov. & Dec. \\
\hline $\begin{array}{l}1 \ldots . \\
2 \ldots \\
3 \ldots . \\
4 \ldots \\
5 \ldots\end{array}$ & $\begin{array}{l}2.8 \\
2.9 \\
3.1 \\
3.0 \\
3.0\end{array}$ & $\begin{array}{l}2.8 \\
2.6 \\
2.6 \\
2.6 \\
2.6\end{array}$ & $\begin{array}{l}2.8 \\
2.8 \\
2.6 \\
2.5 \\
2.5\end{array}$ & $\begin{array}{l}2.5 \\
2.5 \\
2.5 \\
2.5 \\
2.5\end{array}$ & $\begin{array}{l}2.4 \\
3.1 \\
2.8 \\
2.6 \\
2.5\end{array}$ & $\begin{array}{l}2.6 \\
2.7 \\
2.8 \\
2.6 \\
2.6\end{array}$ & $\begin{array}{l}3.2 \\
3.4 \\
3.1 \\
2.9 \\
2.6\end{array}$ & $\begin{array}{l}2.4 \\
2.6 \\
2.5 \\
2.5 \\
2.5\end{array}$ & $\begin{array}{l}2.6 \\
2.5 \\
2.6 \\
2.5 \\
2.5\end{array}$ & $\begin{array}{l}2.4 \\
2.4 \\
2.4 \\
2.4 \\
2.4\end{array}$ & $\begin{array}{l}2.4 \\
2.5 \\
2.5 \\
2.8 \\
2.5\end{array}$ & $\begin{array}{l}2.6 \\
2.6 \\
2.5 \\
3.5 \\
2.8\end{array}$ \\
\hline $\begin{array}{r}6 \ldots \\
7 \ldots \\
8 \ldots \\
9 \ldots \ldots \\
10 \ldots\end{array}$ & $\begin{array}{l}3.0 \\
3.0 \\
3.0 \\
3.0 \\
3.0\end{array}$ & $\begin{array}{l}4.3 \\
3.5 \\
4.5 \\
3.4 \\
2.8\end{array}$ & $\begin{array}{l}2.5 \\
2.5 \\
2.5 \\
2.5 \\
2.5\end{array}$ & $\begin{array}{l}2.5 \\
2.5 \\
2.5 \\
2.5 \\
2.6\end{array}$ & $\begin{array}{l}2.4 \\
2.5 \\
2.4 \\
2.4 \\
2.4\end{array}$ & $\begin{array}{l}2.6 \\
2.6 \\
2.6 \\
3.0 \\
3.3\end{array}$ & $\begin{array}{l}2.8 \\
3.0 \\
2.6 \\
2.6 \\
2.6\end{array}$ & $\begin{array}{l}2.4 \\
2.4 \\
2.4 \\
2.4 \\
3.7\end{array}$ & $\begin{array}{l}2.5 \\
2.8 \\
2.5 \\
2.5 \\
2.4\end{array}$ & $\begin{array}{l}2.5 \\
2.4 \\
2.5 \\
2.4 \\
2.4\end{array}$ & $\begin{array}{l}2.4 \\
2.4 \\
5.2 \\
3.8 \\
3.0\end{array}$ & $\begin{array}{l}2.9 \\
6.0 \\
5.9 \\
2.7 \\
3.4\end{array}$ \\
\hline $\begin{array}{l}11 \ldots \\
12 \ldots \\
13 \ldots \\
14 \ldots \\
15 \ldots\end{array}$ & $\begin{array}{l}3.0 \\
\text { 3.0 } \\
3.1 \\
3.4 \\
3.2\end{array}$ & $\begin{array}{l}2.8 \\
2.6 \\
2.6 \\
2.6 \\
2.5\end{array}$ & $\begin{array}{l}2.5 \\
2.5 \\
2.5 \\
2.5 \\
2.8\end{array}$ & $\begin{array}{l}4.8 \\
3.9 \\
3.8 \\
3.8 \\
3.9\end{array}$ & $\begin{array}{l}2.4 \\
3.5 \\
3.1 \\
\text { 3.6 } \\
\mathbf{3 . 6}\end{array}$ & $\begin{array}{l}3.0 \\
3.1 \\
2.9 \\
2.7 \\
3.0\end{array}$ & $\begin{array}{l}2.5 \\
3.4 \\
3.0 \\
2.8 \\
2.6\end{array}$ & $\begin{array}{l}2.6 \\
3.9 \\
3.4 \\
2.6 \\
2.7\end{array}$ & $\begin{array}{l}2.5 \\
2.4 \\
2.6 \\
2.4 \\
2.4\end{array}$ & $\begin{array}{l}2.4 \\
2.4 \\
2.5 \\
2.6 \\
2.6\end{array}$ & $\begin{array}{l}2.9 \\
2.8 \\
2.8 \\
2.6 \\
2.5\end{array}$ & $\begin{array}{l}1.4 \\
2.3 \\
2.0 \\
2.2 \\
2.0\end{array}$ \\
\hline $\begin{array}{l}16 . . \\
17 . \\
18 . \\
19 . \\
20 . .\end{array}$ & $\begin{array}{l}3.0 \\
3.0 \\
3.0 \\
2.9 \\
2.8\end{array}$ & $\begin{array}{l}2.6 \\
2.9 \\
3.4 \\
3.4 \\
3.4\end{array}$ & $\begin{array}{l}2.8 \\
2.6 \\
2.5 \\
2.5 \\
2.5\end{array}$ & $\begin{array}{l}3.9 \\
3.9 \\
3.8 \\
3.4 \\
3.1\end{array}$ & $\begin{array}{l}3.4 \\
3.3 \\
3.2 \\
3.3 \\
3.0\end{array}$ & $\begin{array}{l}2.9 \\
2.6 \\
2.5 \\
2.5 \\
2.5\end{array}$ & $\begin{array}{l}2.5 \\
2.5 \\
2.5 \\
2.5 \\
2.7\end{array}$ & $\begin{array}{l}4.2 \\
3.5 \\
2.8 \\
2.6 \\
2.6\end{array}$ & $\begin{array}{l}2.5 \\
2.5 \\
2.9 \\
2.6 \\
2.5\end{array}$ & $\begin{array}{l}2.5 \\
2.4 \\
2.4 \\
2.4 \\
2.4\end{array}$ & $\begin{array}{l}2.6 \\
2.9 \\
3.1 \\
3.3 \\
2.9\end{array}$ & $\begin{array}{l}2.0 \\
2.0 \\
2.1 \\
2.3 \\
2.4\end{array}$ \\
\hline $\begin{array}{l}21 \ldots \ldots \ldots \ldots \\
223 \ldots \ldots \ldots \ldots \\
24 \ldots \ldots \ldots \ldots \\
25 \ldots \ldots \ldots \ldots \\
25 \ldots \ldots\end{array}$ & $\begin{array}{l}2.9 \\
2.8 \\
2.8 \\
2.8 \\
2.8\end{array}$ & $\begin{array}{l}3.2 \\
3.1 \\
3.0 \\
2.8 \\
3.4\end{array}$ & $\begin{array}{l}2.5 \\
2.8 \\
2.6 \\
2.5 \\
2.5\end{array}$ & $\begin{array}{l}3.4 \\
3.1 \\
3.0 \\
2.6 \\
2.6\end{array}$ & $\begin{array}{l}2.9 \\
2.9 \\
2.6 \\
2.6 \\
2.6\end{array}$ & $\begin{array}{l}2.5 \\
2.5 \\
2.5 \\
2.4 \\
2.5\end{array}$ & $\begin{array}{l}2.5 \\
2.5 \\
2.6 \\
2.5 \\
2.6\end{array}$ & $\begin{array}{l}2.6 \\
2.5 \\
2.6 \\
2.6 \\
2.6\end{array}$ & $\begin{array}{l}2.5 \\
2.4 \\
2.4 \\
2.4 \\
2.4\end{array}$ & $\begin{array}{l}2.4 \\
2.4 \\
2.4 \\
2.4 \\
2.5\end{array}$ & $\begin{array}{l}2.6 \\
2.6 \\
2.6 \\
2.6 \\
2.8\end{array}$ & $\begin{array}{l}2.4 \\
2.5 \\
2.5 \\
2.5 \\
2.5\end{array}$ \\
\hline 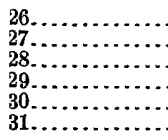 & $\begin{array}{l}2.6 \\
2.6 \\
2.6 \\
2.6 \\
2.6 \\
2.8\end{array}$ & $\begin{array}{r}3.2 \\
3.0 \\
2.8 \\
\cdots \ldots . . \\
\hdashline \cdots . . . \\
\hdashline \cdots . . .\end{array}$ & $\begin{array}{l}2.5 \\
2.5 \\
2.8 \\
2.5 \\
2.5 \\
2.5\end{array}$ & $\begin{array}{l}2.5 \\
2.5 \\
2.4 \\
2.4 \\
2.4\end{array}$ & $\begin{array}{l}3.1 \\
2.8 \\
2.6 \\
2.5 \\
2.5 \\
2.5\end{array}$ & $\begin{array}{l}2.4 \\
3.8 \\
3.6 \\
3.3 \\
3.4\end{array}$ & $\begin{array}{l}2.6 \\
2.5 \\
2.5 \\
2.5 \\
2.6 \\
2.5\end{array}$ & $\begin{array}{l}2.5 \\
2.8 \\
2.6 \\
2.5 \\
2.5 \\
4.0\end{array}$ & $\begin{array}{l}2.4 \\
2.4 \\
2.4 \\
2.4 \\
2.4\end{array}$ & $\begin{array}{l}2.4 \\
2.4 \\
2.9 \\
3.3 \\
2.5 \\
2.4\end{array}$ & $\begin{array}{l}3.5 \\
2.8 \\
2.5 \\
2.5 \\
2.8\end{array}$ & $\begin{array}{l}2.5 \\
2.5 \\
2.5 \\
2.6 \\
2.6 \\
2.6\end{array}$ \\
\hline
\end{tabular}

Monthly discharge of Palolo ditch near Wailuku, Maui, for 1913.

\begin{tabular}{|c|c|c|c|c|c|}
\hline \multirow{2}{*}{ Month. } & \multicolumn{3}{|c|}{ Discharge in second-feet. } & \multirow{2}{*}{$\begin{array}{c}\text { Run-off } \\
\text { (total in } \\
\text { acre-feet). }\end{array}$} & \multirow{2}{*}{$\begin{array}{l}\text { Acçu- } \\
\text { racy. }\end{array}$} \\
\hline & Maximum. & Minimum. & Mean. & & \\
\hline 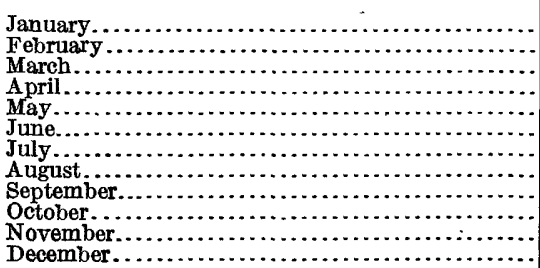 & $\begin{array}{l}3.4 \\
4.5 \\
2.8 \\
4.8 \\
3.6 \\
3.8 \\
3.4 \\
4.2 \\
2.9 \\
3.3 \\
5.2 \\
6.0\end{array}$ & $\begin{array}{l}2.6 \\
2.5 \\
2.5 \\
2.4 \\
2.4 \\
2.4 \\
2.5 \\
2.4 \\
2.4 \\
2.4 \\
2.4 \\
1.4\end{array}$ & $\begin{array}{l}2.91 \\
3.04 \\
2.57 \\
3.01 \\
2.81 \\
2.79 \\
2.70 \\
2.78 \\
2.49 \\
2.48 \\
2.87 \\
2.69\end{array}$ & $\begin{array}{l}179 \\
169 \\
158 \\
179 \\
173 \\
166 \\
166 \\
171 \\
148 \\
152 \\
171 \\
165\end{array}$ & $\begin{array}{l}\text { A. } \\
\text { A. } \\
\text { A. } \\
\text { A. } \\
\text { A. } \\
\text { A. } \\
\text { A. } \\
\text { A. } \\
\text { A. } \\
\text { A. } \\
\text { A. } \\
\text { A. }\end{array}$ \\
\hline The year.$\ldots \ldots \ldots \ldots \ldots \ldots \ldots \ldots \ldots \ldots \ldots$ & 6.0 & 1.4 & 2.76 & 2,000 & \\
\hline
\end{tabular}




\section{haIPUAENa stream NEAR hUELo, maUi (OLD station).}

Location.-About 7 miles by trail east of Huelo post office, about 400 feet below point where Spreckels ditch joins the stream.

Records available.--December 18, 1910, to September 30, 1913.

Gage.-Vertical staff.

Control.-Shifting.

Discharge measurements.-Made by wading.

Diversions.-Combined flow of stream and Spreckels ditch was mẹasured.

Accuracy.-Poor.

Cooperation.-Station is maintained in cooperation with East Maui Ditch Co.

Monthly discharge of Haipuaena Stream near Huelo, Maui, for 1913.

[Old station.]

\begin{tabular}{|c|c|c|c|c|c|}
\hline \multirow{2}{*}{ Month. } & \multicolumn{3}{|c|}{ Discharge in second-feet. } & \multirow{2}{*}{$\begin{array}{l}\text { Run-off } \\
\text { (total in } \\
\text { acre-feet). }\end{array}$} & \multirow{2}{*}{$\begin{array}{c}\text { Accu- } \\
\text { racy. }\end{array}$} \\
\hline & Maximum. & Minimum. & Mean. & & \\
\hline \multirow[t]{2}{*}{ 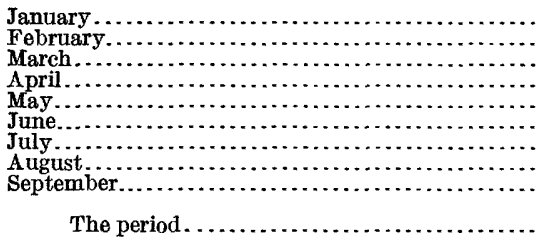 } & $\begin{array}{r}80 \\
100 \\
65 \\
136 \\
79 \\
72 \\
56 \\
96 \\
90\end{array}$ & $\begin{array}{c}9.0 \\
8.5 \\
4.5 \\
14 \\
14 \\
10 \\
7.0 \\
5.5 \\
8.0\end{array}$ & $\begin{array}{l}27.7 \\
20.6 \\
19.4 \\
46.8 \\
23.8 \\
24.6 \\
24.7 \\
25.2 \\
17.8\end{array}$ & $\begin{array}{l}1,700 \\
1,140 \\
1,190 \\
2,780 \\
1,460 \\
1,460 \\
1,520 \\
1,550 \\
1,060\end{array}$ & \multirow[t]{2}{*}{$\begin{array}{l}\text { D. } \\
\text { D. } \\
\text { D. } \\
\text { D. } \\
\text { D. } \\
\text { D. } \\
\text { D. } \\
\text { D. } \\
\text { D. }\end{array}$} \\
\hline & ........... & & ........ & 13,900 & \\
\hline
\end{tabular}

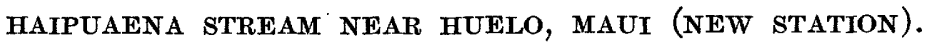

Location.-Seven miles east of Huelo post office, 200 feet above Spreckels ditch inflow.

Records available.-October 19 to December 31, 1913.

Gage.-Friez water-stage recorder.

Control.-Probably permanent.

Discharge measurements.-Made from footbridge or by wading.

Diversions.-None above station.

Accuracy.-Records very good.

Discharge measurements of Haipueno Stream near Huelo, Maui, in 1913.

[New station.]

\begin{tabular}{|c|c|c|c|c|c|c|c|}
\hline Date. & Made by- & $\begin{array}{c}\text { Gage } \\
\text { height. }\end{array}$ & Discharge. & Date. & Made by- & $\begin{array}{c}\text { Gage } \\
\text { height. }\end{array}$ & $\begin{array}{l}\text { Dis- } \\
\text { charge. }\end{array}$ \\
\hline $\begin{array}{l}\text { Oct. } 27 . . \\
\text { Nov. } 4 \ldots \\
\text { Nov. } 8 . .\end{array}$ & 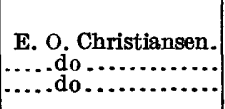 & $\begin{array}{r}\text { Feet. } \\
0.42 \\
1.38 \\
2.46\end{array}$ & $\begin{array}{c}\text { Sec.fft. } \\
2.77 \\
33.6 \\
141\end{array}$ & $\begin{array}{l}\text { Nov. } 12 . \\
\text { Nov. } 13 . \\
\text { Nov. } 18 .\end{array}$ & 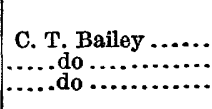 & $\begin{array}{r}\text { Feet. } \\
2.10 \\
1.01 \\
2.72\end{array}$ & $\begin{array}{r}\text { Sec. }-f t . \\
81.0 \\
14.9 \\
161\end{array}$ \\
\hline
\end{tabular}


Daily discharge, in second-feet, of Haipuaena Stream near Huelo, Maui, for 1913.

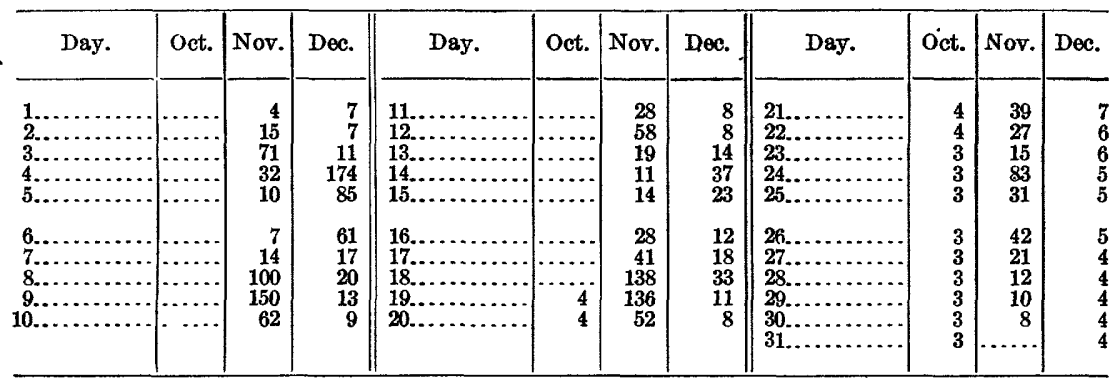

NorE.-Discharge interpolated Nov. 16 and 22.

Monthly discharge of Haipuaena Stream near Huelo, Maui, for 1913.

\begin{tabular}{|c|c|c|c|c|c|}
\hline \multirow{2}{*}{ Month. } & \multicolumn{3}{|c|}{ Discharge in second-foet. } & \multirow{2}{*}{$\begin{array}{l}\text { Run-off } \\
\text { (total in } \\
\text { acre-feet). }\end{array}$} & \multirow{2}{*}{$\begin{array}{l}\text { Accu- } \\
\text { racy. }\end{array}$} \\
\hline & Maximum. & Minimum. & Mean. & & \\
\hline 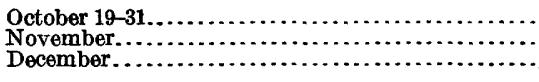 & $\begin{array}{r}4 \\
150 \\
174\end{array}$ & $\begin{array}{l}3 \\
4 \\
4\end{array}$ & $\begin{array}{r}3.3 \\
42.6 \\
20.3\end{array}$ & $\begin{array}{r}85 \\
2,530 \\
1,250\end{array}$ & $\begin{array}{l}\text { A. } \\
\text { A. }\end{array}$ \\
\hline
\end{tabular}

\section{PUOHAKaMoa STREAM NEAR hUElo, MAUt (OLD STATION).}

Location.-About 6 miles by trail east of Huelo post office, immediately below point where Spreckels ditch falls into stream.

Records available.-December 18, 1910, to June 18, 1913.

Gage.-Vertical staff.

Control.-Probably permanent.

Discharge measurements.-Made by wading.

Diversions.-Measurement made of combined flow of stream and Spreckels ditch. Accuracy.-Records poor.

Cooperation.-Station maintained in cooperation with East Maui Ditch Co.

Monthly discharge of Puohakamoa Stream near Huelo, Maui, for 1913.

[Old station.]

\begin{tabular}{|c|c|c|c|c|c|}
\hline \multirow{2}{*}{ Month. } & \multicolumn{3}{|c|}{ Discharge in second-feet. } & \multirow{2}{*}{$\begin{array}{l}\text { Run-off } \\
\text { (total in } \\
\text { acre-feet). }\end{array}$} & \multirow{2}{*}{$\begin{array}{l}\text { Accu- } \\
\text { racy. }\end{array}$} \\
\hline & Maximum. & Minimum. & Mean. & & \\
\hline \multirow{2}{*}{ 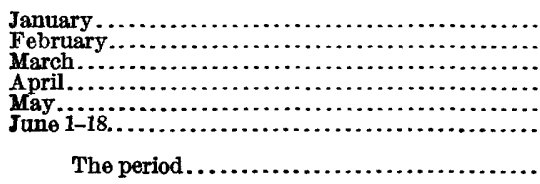 } & $\begin{array}{l}106 \\
169 \\
489 \\
209 \\
177 \\
100\end{array}$ & $\begin{array}{r}16 \\
15 \\
6 \\
20 \\
18 \\
18\end{array}$ & $\begin{array}{l}38.7 \\
35.0 \\
39.1 \\
77.7 \\
36.9 \\
34.3\end{array}$ & $\begin{array}{l}2,380 \\
1,940 \\
2,400 \\
4,620 \\
2,270 \\
1,220\end{array}$ & \multirow[t]{2}{*}{$\begin{array}{l}\text { D } \\
\text { D. } \\
\text { D } \\
\text { D } \\
\text { D. } \\
\text { D. } \\
.\end{array}$} \\
\hline & & & $\ldots \ldots \ldots$ & 14,800 & \\
\hline
\end{tabular}




\section{PUOHAKAMOA STREAM NEAR hUELo, maUt (NEW STATION).}

Location.-Seven miles east of Huelo post office, 150 feet above Spreckels ditch trail. Records available.-June 13 to December 31, 1913.

Gage.-Barrett \& Lawrence water-stage recorder.

Control.-Fairly permanent.

Discharge measurements.-Made from trail bridge or by wading.

Diversions.- Kula pipe line diverts water at an elevation of 4,300 feet.

Accuracy.-Low-water records good; records for high stages only approximate.

Discharge measurements of Puohakamoa Stream near Huelo, Maui, in 1913.

[New station.]

\begin{tabular}{|c|c|c|c|c|c|c|c|}
\hline Date. & Made by- & $\begin{array}{c}\text { Gage } \\
\text { height. }\end{array}$ & $\begin{array}{l}\text { Dis- } \\
\text { charge. }\end{array}$ & Date. & Made by- & $\begin{array}{c}\text { Gage } \\
\text { height. }\end{array}$ & $\begin{array}{c}\text { Dis- } \\
\text { charge. }\end{array}$ \\
\hline $\begin{array}{l}\text { June } 19 . . \\
\text { Oct. } 27 . .\end{array}$ & $\begin{array}{l}\text { C. T. Bailey ....... } \\
\text { E. O. Christiansen. }\end{array}$ & $\begin{array}{r}\text { Feet. } \\
1.26 \\
.94\end{array}$ & $\begin{array}{c}\text { Sec.-ft. } \\
a 7.70 \\
a 6.49\end{array}$ & $\begin{array}{l}\text { Nov. } 12 . \\
\text { Nov. } 13 .\end{array}$ & $\begin{array}{c}\text { C. T. Bailey. } \\
\ldots \ldots . \text { do ........ }\end{array}$ & $\begin{array}{l}\text { Feet. } \\
3.18 \\
2.40\end{array}$ & $\begin{array}{c}\text { Sec.-ft. } \\
130 \\
37.6\end{array}$ \\
\hline
\end{tabular}

a Discharge is the difference between measured discharge of Spreckels ditch above and below Puohakamoa Stream.

Daily discharge, in second-feet, of Puohaleamoa Stream near Huelo, Maui, for 1913.

[New station.]

\begin{tabular}{|c|c|c|c|c|c|c|c|}
\hline Day. & June. & July. & Aug. & Sept. & Oct. & Nov. & Dec. \\
\hline 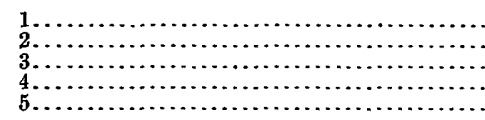 & $\begin{array}{l}\cdots \cdots \cdots \\
\cdots \cdots \cdots \\
\cdots \cdots\end{array}$ & $\begin{array}{l}11 \\
43 \\
21 \\
20 \\
36\end{array}$ & $\begin{array}{l}6.2 \\
6.2 \\
6.1 \\
5.8 \\
5.8\end{array}$ & 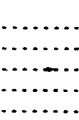 & $\begin{array}{l}6.5 \\
6.0 \\
5.8 \\
6.2 \\
6.2\end{array}$ & $\begin{array}{r}6.6 \\
15 \\
128 \\
68 \\
16\end{array}$ & $\begin{array}{r}14 \\
12 \\
16 \\
366 \\
178\end{array}$ \\
\hline 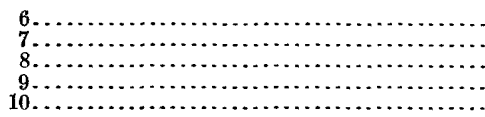 & $\begin{array}{l}-\therefore \ldots . \\
\cdots \cdots \cdots \\
\cdots \cdots \cdots\end{array}$ & $\begin{array}{l}52 \\
21 \\
16 \\
12 \\
10\end{array}$ & $\begin{array}{l}7.1 \\
40 \\
8.4 \\
7.4 \\
7.4\end{array}$ & $\cdots \cdots \cdots$ & $\begin{array}{c}15 \\
27 \\
37 \\
12 \\
8.5\end{array}$ & $\begin{array}{r}11 \\
13 \\
226 \\
305 \\
153\end{array}$ & $\begin{array}{r}147 \\
55 \\
61 \\
34 \\
21\end{array}$ \\
\hline $\begin{array}{r}11 \\
12 \\
13 \\
14 \\
15 \\
15\end{array}$ & $\begin{array}{l}\cdots \cdots \\
53 \\
24 \\
16\end{array}$ & $\begin{array}{l}10 \\
11 \\
11 \\
11 \\
10\end{array}$ & $\begin{array}{l}74 \\
76 \\
17 \\
15 \\
15\end{array}$ & $\begin{array}{ll}\cdots \cdots \cdots \\
\cdots \cdots \cdots \\
\cdots \cdots \cdots\end{array}$ & $\begin{array}{l}8.2 \\
7.6 \\
17 \\
11 \\
9.1\end{array}$ & $\begin{array}{r}79 \\
129 \\
47 \\
22 \\
20\end{array}$ & $\begin{array}{l}17 \\
16 \\
15 \\
79 \\
62\end{array}$ \\
\hline $\begin{array}{r}16 \\
17 \\
18 \\
19 \\
19 \\
20\end{array}$ & $\begin{array}{l}10 \\
8.7 \\
8.1 \\
7.8 \\
7.4\end{array}$ & $\begin{array}{l}9.0 \\
8.7 \\
8.0 \\
7.6 \\
7.8\end{array}$ & $\begin{array}{l}12 \\
9.7 \\
8.6 \\
8.1 \\
7.8\end{array}$ & $\begin{array}{l}13 \\
24 \\
22 \\
10\end{array}$ & $\begin{array}{l}8.0 \\
7.4 \\
7.2 \\
7.0 \\
6.8\end{array}$ & $\begin{array}{r}66 \\
100 \\
267 \\
233 \\
92\end{array}$ & $\begin{array}{l}26 \\
37 \\
91 \\
23 \\
17\end{array}$ \\
\hline $\begin{array}{l}21 \\
22 \\
23 \\
24 \\
25 \\
25\end{array}$ & $\begin{array}{l}7.1 \\
7.0 \\
7.0 \\
7.0 \\
7.0\end{array}$ & $\begin{array}{l}8.1 \\
7.4 \\
7.2 \\
7.2 \\
7.2\end{array}$ & $\begin{array}{l}7.6 \\
7.2 \\
7.0 \\
7.0 \\
6.8\end{array}$ & $\begin{array}{l}8.7 \\
7.8 \\
7.4 \\
7.0 \\
6.7\end{array}$ & $\begin{array}{l}6.9 \\
6.6 \\
6.4 \\
6.3 \\
6.2\end{array}$ & $\begin{array}{r}68 \\
28 \\
31 \\
173 \\
91\end{array}$ & $\begin{array}{l}14 \\
13 \\
12 \\
11 \\
10\end{array}$ \\
\hline 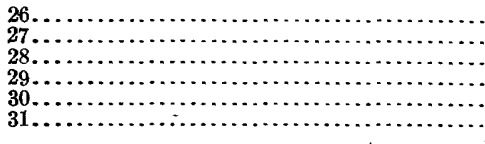 & $\begin{array}{l}\text { 7.0 } \\
\text { 7.0 } \\
7.0 \\
7.0 \\
9.5\end{array}$ & $\begin{array}{l}7.2 \\
7.2 \\
7.0 \\
6.8 \\
6.6 \\
6.5\end{array}$ & $\begin{array}{r}6.8 \\
6.9 \\
7.3 \\
7.0 \\
16 \\
8.8\end{array}$ & $\begin{array}{l}6.5 \\
6.5 \\
6.5 \\
6.4 \\
6.1\end{array}$ & $\begin{array}{l}6.2 \\
6.2 \\
6.2 \\
6.1 \\
6.0 \\
6.0\end{array}$ & $\begin{array}{r}114 \\
57 \\
26 \\
21 \\
16 \\
1 .\end{array}$ & $\begin{array}{l}9.7 \\
9.3 \\
8.9 \\
8.7 \\
8.1 \\
7.8\end{array}$ \\
\hline
\end{tabular}

NoTE.-No record Sept. 1-16; recording instrument out of order. 
Monthly discharge of Puohakamoa Stream near Huelo, Maui, for 1913.

[New station.]

\begin{tabular}{|c|c|c|c|c|c|}
\hline \multirow{2}{*}{ Month. } & \multicolumn{3}{|c|}{ Discharge in second-feet. } & \multirow{2}{*}{$\begin{array}{c}\text { Run-off } \\
\text { (totalin } \\
\text { acre-feet). }\end{array}$} & \multirow{2}{*}{$\begin{array}{l}\text { Accu - } \\
\text { racy. }\end{array}$} \\
\hline & Maximum. & Minimum. & Mean. & & \\
\hline \multirow{2}{*}{ 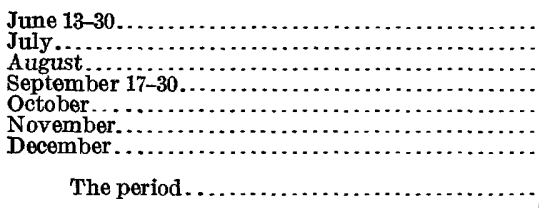 } & $\begin{array}{r}53 \\
52 \\
76 \\
24 \\
37 \\
305 \\
366\end{array}$ & $\begin{array}{l}7.0 \\
6.5 \\
5.8 \\
6.1 \\
5.8 \\
6.6 \\
7.8\end{array}$ & $\begin{array}{c}11.5 \\
13.4 \\
13.9 \\
9.90 \\
9.31 \\
87.4 \\
45.1\end{array}$ & $\begin{array}{r}410 \\
824 \\
. \quad 855 \\
275 \\
572 \\
5,200 \\
2,770\end{array}$ & \multirow[t]{2}{*}{$\begin{array}{l}\text { A. } \\
\text { A. } \\
\text { A. } \\
\text { A. } \\
\text { A. } \\
\text { C. } \\
\text { B. }\end{array}$} \\
\hline & & & $\cdots$ & 10,900 & \\
\hline
\end{tabular}

ALO STREAM NEAR HUELO, MAUI.

Location.-About 5 miles by trail east of Huelo post office, immediately below bridge where old Spreckels ditch trail crosses stream.

Records available.-December 18, 1910, to December 31, 1913.

Gage.-Wertical staff.

Control.-Affected by backwater from inflow of Spreckels ditch.

Discharge measurements.-Made by wading.

Diversions.- None above station.

Accuracy.-Records poor.

Discharge measurements of Alo Stream near Huelo, Maui, for 1913.

[C. T. Bailey, hydrographer.]

\begin{tabular}{|c|c|c|c|c|c|}
\hline Date. & $\begin{array}{c}\text { Gage } \\
\text { height. }\end{array}$ & $\begin{array}{l}\text { Dis- } \\
\text { charge. }\end{array}$ & Date. & $\begin{array}{c}\text { Gage } \\
\text { height. }\end{array}$ & $\begin{array}{c}\text { Dis- } \\
\text { charge. }\end{array}$ \\
\hline $\begin{array}{l}\text { July } 24 \ldots \ldots \\
\text { Nov. } 18 . \ldots \ldots\end{array}$ & $\begin{array}{l}\text { Feet. } \\
1.48 \\
2.58\end{array}$ & $\begin{array}{c}\text { Sec.-ft. } \\
1.45 \\
31.9\end{array}$ & Nov. $20 \ldots$ & $\begin{array}{l}\text { Feet. } \\
2.11\end{array}$ & $\begin{array}{r}\text { Sec.-ft. } \\
18.2\end{array}$ \\
\hline
\end{tabular}

Monthly discharge of Alo Stream near Huelo, Maui, for 1913.

\begin{tabular}{|c|c|c|c|c|c|}
\hline \multirow{2}{*}{ Month. } & \multicolumn{3}{|c|}{ Discharge in second-feet. } & \multirow{2}{*}{$\begin{array}{l}\text { Run-off } \\
\text { (total in } \\
\text { acre-feet). }\end{array}$} & \multirow{2}{*}{$\begin{array}{l}\text { Accu- } \\
\text { racy. }\end{array}$} \\
\hline & Maximum. & Minimum. & Mean. & & \\
\hline \multirow[t]{2}{*}{ 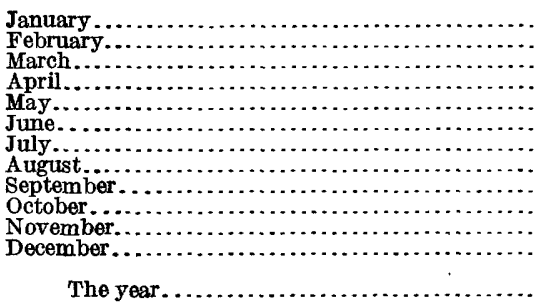 } & $\begin{array}{c}24 \\
61 \\
7.8 \\
51 \\
28 \\
9.4 \\
21 \\
43 \\
11 \\
15 \\
97 \\
110\end{array}$ & $\begin{array}{r}1.1 \\
.8 \\
.5 \\
1.7 \\
1.2 \\
1.2 \\
.7 \\
.5 \\
.7 \\
.4 \\
.7 \\
1.2\end{array}$ & $\begin{array}{c}4.80 \\
4.72 \\
2.65 \\
11.7 \\
3.56 \\
3.33 \\
3.75 \\
4.30 \\
2.04 \\
2.73 \\
22.8 \\
10.7\end{array}$ & $\begin{array}{r}295 \\
262 \\
164 \\
696 \\
219 \\
198 \\
231 \\
264 \\
121 \\
168 \\
1,360 \\
658\end{array}$ & \multirow[t]{2}{*}{$\begin{array}{l}\text { D. } \\
\text { D. } \\
\text { D. } \\
\text { D. } \\
\text { D. } \\
\text { D. } \\
\text { D. } \\
\text { D. } \\
\text { D. } \\
\mathbf{D} \\
\mathbf{D} . \\
\mathbf{D} .\end{array}$} \\
\hline & 110 & .4 & 6.40 & 4,640 & \\
\hline
\end{tabular}




\section{WAIKAMOI STREAM NEAR HUELO, MAUI.}

Location.-Five hundred feet above Spreckels ditch intake and 5 miles east of Huelo post office.

Records available.-December 18, 1910, to December 31, 1913.

Gage.-Friez water-stage recorder installed October 14, 1913, at new datum; staff gage December 18, 1910, to October 13, 1913.

Control.-Probably permanent.

Discharge measurements.-Made from footbridge or by wading.

Diversions.-None above station.

Accuracy.-Records good.

Discharge measurements of Waikamoi Stream near. Huelo, Maui, for 1918.

\begin{tabular}{|c|c|c|c|c|c|c|c|}
\hline Date. & Made by- & $\begin{array}{c}\text { Gage } \\
\text { height. }\end{array}$ & $\begin{array}{l}\text { Dis- } \\
\text { charge. }\end{array}$ & Date. & Made by- & $\begin{array}{c}\text { Gage } \\
\text { height. }\end{array}$ & $\begin{array}{l}\text { Dis- } \\
\text { charge. }\end{array}$ \\
\hline $\begin{array}{l}\text { July } 24 . . . \\
\text { Oct. } 27 a . . \\
\text { Nov. } 4 \ldots\end{array}$ & $\begin{array}{l}\text { C. T. Bailey ........ } \\
\text { E. O.Christiansen, } \\
\ldots \ldots . \text { do ................... }\end{array}$ & $\begin{array}{r}\text { Feet. } \\
1.40 \\
.35 \\
1.49\end{array}$ & $\begin{aligned} \text { Sec. }-f t . \\
3.23 \\
2.15 \\
83.9\end{aligned}$ & $\begin{array}{l}\text { Nov.8... } \\
\text { Nov.13.. } \\
\text { Nov. } 2 . .\end{array}$ & 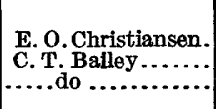 & $\begin{aligned} \text { Feet. } \\
1.80 \\
1.03 \\
1.21\end{aligned}$ & $\begin{array}{r}\text { Sec. } f t . \\
163 \\
26.2 \\
53.4\end{array}$ \\
\hline
\end{tabular}

a Water-stage recorder installed at new datum Oct. 14, 1913.

Daily discharge, in second-feet, of Waikamoi Stream near Huelo, Maui, for 1913.

\begin{tabular}{|c|c|c|c|c|c|c|c|c|c|c|c|c|}
\hline Day. & Jan. & Feb. & Mar. & Apr. & May. & June. & July. & Aug. & Sept. & Oct. & Nov. & Dec. \\
\hline $\begin{array}{l}1 \ldots . \\
2 \ldots \\
3 \ldots \\
4 \ldots \\
5 \ldots\end{array}$ & $\begin{array}{c}10 \\
8.4 \\
7.8 \\
7.0 \\
6.2\end{array}$ & $\begin{array}{l}4.0 \\
3.8 \\
3.0 \\
2.8 \\
3.6\end{array}$ & $\begin{array}{l}2.6 \\
2.4 \\
2.2 \\
2.0 \\
1.8\end{array}$ & $\begin{array}{c}21 \\
14 \\
8.4 \\
6.8 \\
6.4\end{array}$ & $\begin{array}{l}6.8 \\
6.0 \\
7.4 \\
6.8 \\
6.4\end{array}$ & $\begin{array}{l}5.5 \\
4.7 \\
4.7 \\
3.0 \\
6.0\end{array}$ & $\begin{array}{l}25 \\
40 \\
14 \\
15 \\
18\end{array}$ & $\begin{array}{l}2.2 \\
1.9 \\
2.0 \\
1.6 \\
1.4\end{array}$ & $\begin{array}{l}5.7 \\
4.7 \\
4.4 \\
3.3 \\
2.8\end{array}$ & $\cdots$ & $\begin{array}{c}2.8 \\
12 \\
163 \\
67 \\
16\end{array}$ & $\begin{array}{l}11 \\
9.4 \\
12 \\
388 \\
170\end{array}$ \\
\hline $\begin{array}{c}6 \ldots \ldots \\
7 \ldots \ldots \\
8 \ldots \ldots \\
9 \ldots \ldots \\
10 \ldots \ldots\end{array}$ & $\begin{array}{r}5.7 \\
5.0 \\
8.8 \\
9.0 \\
11\end{array}$ & $\begin{array}{l}23 \\
9.2 \\
6.0 \\
4.1 \\
3.6\end{array}$ & $\begin{array}{l}2.0 \\
3.3 \\
2.6 \\
4.1 \\
2.8\end{array}$ & $\begin{array}{l}5.7 \\
5.0 \\
4.4 \\
6.0 \\
4.7\end{array}$ & $\begin{array}{l}5.0 \\
5.0 \\
7.8 \\
5.4 \\
4.7\end{array}$ & $\begin{array}{l}4.7 \\
4.6 \\
4.4 \\
5.2 \\
9.6\end{array}$ & $\begin{array}{l}21 \\
10 \\
9.2 \\
7.4 \\
6.4\end{array}$ & $\begin{array}{l}2.7 \\
4.6 \\
9.6 \\
5.4 \\
7.6\end{array}$ & $\begin{array}{l}2.4 \\
2.7 \\
2.6 \\
2.6 \\
2.4\end{array}$ & & $\begin{array}{l}12 \\
\cdots \cdots \\
250 \\
106\end{array}$ & $\begin{array}{r}143 \\
24 \\
29 \\
18 \\
13\end{array}$ \\
\hline $\begin{array}{l}11 \ldots \ldots \ldots \\
12 \\
13 \ldots \ldots \ldots \\
15 \ldots \ldots \ldots\end{array}$ & $\begin{array}{l}6.4 \\
7.8 \\
22 \\
18 \\
21\end{array}$ & $\begin{array}{l}3.0 \\
2.7 \\
2.4 \\
2.4 \\
2.2\end{array}$ & $\begin{array}{l}1.9 \\
1.0 \\
.9 \\
.8 \\
.8\end{array}$ & $\begin{array}{l}48 \\
60 \\
92 \\
43 \\
70\end{array}$ & $\begin{array}{r}4.1 \\
5.7 \\
7.8 \\
6.8 \\
13\end{array}$ & $\begin{array}{l}6.6 \\
15 \\
15 \\
14 \\
12\end{array}$ & $\begin{array}{r}6.0 \\
7.0 \\
11.0 \\
8.8 \\
6.8\end{array}$ & $\begin{array}{l}5.8 \\
42 \\
16 \\
14 \\
31\end{array}$ & $\begin{array}{l}2.0 \\
3.3 \\
3.0 \\
3.3 \\
3.0\end{array}$ & & $\begin{array}{r}36 \\
-104 \\
30 \\
18 \\
19\end{array}$ & $\begin{array}{l}11 \\
10 \\
9.4 \\
52 \\
36\end{array}$ \\
\hline $\begin{array}{l}16 \ldots \ldots \ldots \\
17 \ldots \ldots \ldots \ldots \\
19 \ldots \ldots \ldots \\
20 \ldots \ldots \ldots\end{array}$ & $\begin{array}{l}14 \\
10 \\
8.4 \\
7.6 \\
6.4\end{array}$ & $\begin{array}{c}5.7 \\
3.6 \\
18 \\
24 \\
9.2\end{array}$ & $\begin{array}{l}5.0 \\
8.0 \\
5.0 \\
5.0 \\
3.6\end{array}$ & $\begin{array}{l}54 \\
71 \\
33 \\
20 \\
15\end{array}$ & $\begin{array}{l}12 \\
10 \\
9.4 \\
9.6 \\
8.4\end{array}$ & $\begin{array}{l}3.0 \\
6.2 \\
5.4 \\
5.0 \\
4.4\end{array}$ & $\begin{array}{l}5.7 \\
4.9 \\
4.3 \\
3.8 \\
3.6\end{array}$ & $\begin{array}{l}11 \\
9.2 \\
7.6 \\
5.8 \\
6.2\end{array}$ & $\begin{array}{l}3.6 \\
7.8 \\
37 \\
17 \\
10\end{array}$ & $\begin{array}{l}6.2 \\
5.2 \\
4.0 \\
4.2 \\
3.8\end{array}$ & $\begin{array}{r}143 \\
108 \\
188 \\
163 \\
58\end{array}$ & $\begin{array}{l}18 \\
24 \\
52 \\
16 \\
14\end{array}$ \\
\hline $\begin{array}{l}21 \ldots \ldots . \\
22 \ldots \ldots \ldots \\
23 \ldots \ldots \\
24 \ldots \ldots \\
25 . \ldots \ldots\end{array}$ & $\begin{array}{r}4.0 \\
6.0 \\
11 \\
4.9 \\
4.7\end{array}$ & $\begin{array}{l}8.0 \\
7.2 \\
6.0 \\
4.9 \\
4.4\end{array}$ & $\begin{array}{r}13 \\
14 \\
7.4 \\
5.7 \\
7.6\end{array}$ & $\begin{array}{l}16 \\
13 \\
17 \\
17 \\
20\end{array}$ & $\begin{array}{l}6.0 \\
5.0 \\
5.0 \\
6.0 \\
4.6\end{array}$ & $\begin{array}{l}3.8 \\
3.6 \\
3.3 \\
3.1 \\
5.4\end{array}$ & $\begin{array}{l}5.7 \\
3.6 \\
3.3 \\
3.6 \\
3.3\end{array}$ & $\begin{array}{l}5.7 \\
4.6 \\
3.8 \\
3.8 \\
3.1\end{array}$ & $\begin{array}{l}7.2 \\
5.4 \\
4.1 \\
2.3 \\
1.4\end{array}$ & $\begin{array}{l}4.0 \\
3.2 \\
2.8 \\
2.7 \\
2.5\end{array}$ & $\begin{array}{r}35 \\
20 \\
22 \\
186 \\
55\end{array}$ & $\begin{array}{l}12 \\
10 \\
9.4 \\
8.5 \\
7.9\end{array}$ \\
\hline $\begin{array}{l}26 \ldots \ldots \\
27 \ldots \ldots \\
28 \ldots \ldots \\
29 \ldots \ldots \\
30 \ldots \ldots \\
31 \ldots \ldots\end{array}$ & $\begin{array}{l}4.4 \\
4.1 \\
3.8 \\
3.3 \\
3.1 \\
3.3\end{array}$ & $\begin{array}{r}3.8 \\
3.3 \\
2.9 \\
\ldots . . \\
\ldots . . \\
\ldots . .\end{array}$ & $\begin{array}{c}5.8 \\
4.9 \\
11 \\
5.8 \\
28 \\
47\end{array}$ & $\begin{array}{c}12 \\
12 \\
9.6 \\
9.0 \\
7.2\end{array}$ & $\begin{array}{r}13 \\
7.6 \\
7.2 \\
7.6 \\
6.4 \\
8.4\end{array}$ & $\begin{array}{r}5.4 \\
8.8 \\
12 \\
8.2 \\
18\end{array}$ & $\begin{array}{l}3.6 \\
3.3 \\
2.9 \\
2.6 \\
2.5 \\
2.4\end{array}$ & $\begin{array}{l}3.3 \\
6.4 \\
5.5 \\
3.8 \\
3.0 \\
6.8\end{array}$ & $\begin{array}{r}1.2 \\
1.2 \\
1.4 \\
1.2 \\
.9\end{array}$ & $\begin{array}{l}2.7 \\
2.7 \\
2.7 \\
2.3 \\
2.0 \\
2.1\end{array}$ & $\begin{array}{c}64 \\
35 \\
21 \\
17 \\
12 \\
\ldots . .\end{array}$ & $\begin{array}{l}7.3 \\
6.8 \\
6.4 \\
6.0 \\
5.6 \\
5.4\end{array}$ \\
\hline
\end{tabular}

NoTE.-No record Oct. 1-14 and Nov. 7-8. 
Monthly discharge of Waikamoi Stream near Huelo, Maui, for 1913.

\begin{tabular}{|c|c|c|c|c|c|}
\hline \multirow{2}{*}{ Month. } & \multicolumn{3}{|c|}{ Discharge in second-feet. } & \multirow{2}{*}{$\begin{array}{l}\text { Run-off } \\
\text { (total in } \\
\text { acre-feet). }\end{array}$} & \multirow{2}{*}{$\begin{array}{l}\text { Accu- } \\
\text { racy. }\end{array}$} \\
\hline & Maximum. & Minimum. & Mean. & & \\
\hline 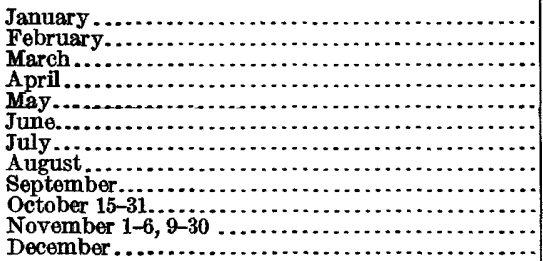 & \begin{tabular}{c|}
22 \\
24 \\
47 \\
92 \\
13 \\
18 \\
40 \\
42 \\
37 \\
7.6 \\
250 \\
388
\end{tabular} & $\begin{array}{l}3.1 \\
2.2 \\
.8 \\
4.4 \\
4.1 \\
3.0 \\
2.4 \\
1.4 \\
.9 \\
2.0 \\
2.8 \\
5.4\end{array}$ & $\begin{array}{r}8.16 \\
6.31 \\
6.71 \\
24.0 \\
7.25 \\
7.01 \\
8.54 \\
7.66 \\
5.00 \\
3.57 \\
70.1 \\
36.9\end{array}$ & $\begin{array}{r}502 \\
350 \\
413 \\
1,430 \\
446 \\
417 \\
525 \\
471 \\
298 \\
110 \\
3,890 \\
2,270\end{array}$ & $\begin{array}{l}\text { B. } \\
\text { B. } \\
\text { B. } \\
\text { B. } \\
\text { B. } \\
\text { B. } \\
\text { B. } \\
\text { B. } \\
\text { B. } \\
\text { A. } \\
\text { A. }\end{array}$ \\
\hline The period.... & & & & 11,100 & \\
\hline
\end{tabular}

OOPUOLA STREAM NEAR HUELO, MAUI.

Location.-About 2 miles by trail east of Huelo post office, about 400 feet above old Spreckels ditch crossing.

Records available.-December 16, 1910, to December 31, 1913.

Gage.--Vertical staff.

Control.-Fairly permanent.

Discharge measurements.-Made by wading.

Diversions.-None above station.

Accuracy.-Records poor on account of only one reading daily.

Discharge measurements of Oopuola Stream near Fuelo, Maui, for 1918.

[C. T. Bailey, hydrographer.]

\begin{tabular}{|c|c|c|}
\hline Date. & $\begin{array}{c}\text { Gage } \\
\text { height. }\end{array}$ & $\begin{array}{l}\text { Dis- } \\
\text { charge. }\end{array}$ \\
\hline $\begin{array}{l}\text { June } 19 \ldots \ldots \\
\text { Nov. } 12 . \ldots \ldots\end{array}$ & $\begin{array}{r}\text { Feet. } \\
1.01 \\
2.29\end{array}$ & $\begin{array}{r}\text { Sec. } f t . \\
0.64 \\
24.0\end{array}$ \\
\hline
\end{tabular}


Daily discharge, in second-feet, of Oopuola Stream near Huelo, Maui, for 1913.

\begin{tabular}{|c|c|c|c|c|c|c|c|c|c|c|c|c|}
\hline Day. & Jan. & Feb. & Mar. & Apr. & May. & June. & July. & Aug. & Sept. & Oct. & Nov. & Dec. \\
\hline $\begin{array}{l}1 \ldots . \\
2 \ldots \\
3 \ldots \\
4 \ldots \\
5 \ldots\end{array}$ & $\begin{array}{l}3.4 \\
5.0 \\
4.8 \\
3.4 \\
2.2\end{array}$ & $\begin{array}{l}0.8 \\
.8 \\
.8 \\
.7 \\
.6\end{array}$ & $\begin{array}{l}0.8 \\
.8 \\
.8 \\
.8 \\
.8\end{array}$ & $\begin{array}{l}16 \\
15 \\
1.2 \\
8.1 \\
6.4\end{array}$ & $\begin{array}{l}1.6 \\
1.2 \\
1.3 \\
1.2 \\
1.1\end{array}$ & $\begin{array}{r}0.8 \\
.8 \\
.8 \\
1.3 \\
.8\end{array}$ & $\begin{array}{c}17 \\
21 \\
12 \\
2.2 \\
2.0\end{array}$ & $\begin{array}{l}0.6 \\
.6 \\
.6 \\
.6 \\
.6\end{array}$ & $\begin{array}{l}0.7 \\
.7 \\
.7 \\
.7 \\
.7\end{array}$ & $\begin{array}{r}0.7 \\
.7 \\
.7 \\
.7 \\
1.4\end{array}$ & $\begin{array}{r}0.7 \\
1.9 \\
3.2 \\
11 \\
3.4\end{array}$ & $\begin{array}{l}4.8 \\
2.0 \\
12 \\
15 \\
32\end{array}$ \\
\hline $\begin{array}{r}6 \ldots \ldots . \\
7 \ldots \ldots \\
8 \ldots \ldots \\
9 \ldots \ldots \\
10 \ldots \ldots\end{array}$ & $\begin{array}{c}.9 \\
12^{.8} \\
12.1\end{array}$ & $\begin{array}{c}35 \\
2.9 \\
10 \\
5.4 \\
.8\end{array}$ & $\begin{array}{l}.8 \\
.8 \\
.8 \\
.8 \\
.8\end{array}$ & $\begin{array}{r}3.6 \\
.9 \\
.9 \\
1.0 \\
.8\end{array}$ & $\begin{array}{l}1.1 \\
.9 \\
.8 \\
.8 \\
.9\end{array}$ & $\begin{array}{r}.8 \\
.8 \\
1.2 \\
1.7 \\
2.2\end{array}$ & $\begin{array}{l}1.8 \\
2.0 \\
1.8 \\
1.6 \\
1.2\end{array}$ & $\begin{array}{r}.7 \\
3.9 \\
1.2 \\
1.0 \\
.9\end{array}$ & $\begin{array}{l}.7 \\
.7 \\
.7 \\
.7 \\
.7\end{array}$ & $\begin{array}{r}2.2 \\
2.9 \\
27 \\
2.0 \\
.8\end{array}$ & $\begin{array}{l}2.4 \\
67^{.8} \\
42 \\
18\end{array}$ & $\begin{array}{l}60 \\
32 \\
4.4 \\
4.1 \\
3.6\end{array}$ \\
\hline $\begin{array}{l}11 \ldots \ldots \\
12 \ldots . \\
13 . \ldots \\
14 \ldots \\
15 \ldots .\end{array}$ & $\begin{array}{l}12 \\
41 \\
70 \\
30 \\
34\end{array}$ & $\begin{array}{r}1.1 \\
.9 \\
.9 \\
.9 \\
.9\end{array}$ & $\begin{array}{l}.8 \\
.8 \\
.8 \\
.8 \\
.8\end{array}$ & $\begin{array}{l}80 \\
18 \\
18 \\
18 \\
21\end{array}$ & $\begin{array}{r}1.1 \\
1.2 \\
1.0 \\
1.0 \\
11\end{array}$ & $\begin{array}{c}1.2 \\
3.1 \\
15 \\
1.4 \\
1.2\end{array}$ & $\begin{array}{l}1.1 \\
.9 \\
1.0 \\
1.2 \\
1.1\end{array}$ & $\begin{array}{r}.8 \\
6.4 \\
6.4 \\
6.7 \\
34\end{array}$ & $\begin{array}{l}.7 \\
.7 \\
.7 \\
.7\end{array}$ & $\begin{array}{r}.8 \\
1.6 \\
2.4 \\
15 \\
1.6\end{array}$ & $\begin{array}{l}25 \\
27 \\
15 \\
10 \\
8.5\end{array}$ & $\begin{array}{l}2.7 \\
3.9 \\
2.6 \\
9.3 \\
16\end{array}$ \\
\hline $\begin{array}{l}16 \ldots \ldots \\
17 \ldots \ldots \\
18 \ldots \ldots \\
19 \ldots \ldots \\
20 \ldots \ldots\end{array}$ & $\begin{array}{r}3.9 \\
2.0 \\
5.0 \\
8.5 \\
12\end{array}$ & $\begin{array}{c}2.4 \\
3.8 \\
12 \\
21 \\
12\end{array}$ & $\begin{array}{r}1.6 \\
2.3 \\
2.0 \\
1.1 \\
.9\end{array}$ & $\begin{array}{l}30 \\
34 \\
24 \\
22 \\
23\end{array}$ & $\begin{array}{l}6.4 \\
5.3 \\
4.0 \\
2.7 \\
1.4\end{array}$ & $\begin{array}{r}1.0 \\
.9 \\
.8 \\
.8 \\
.8\end{array}$ & $\begin{array}{r}1.0 \\
.8 \\
.8 \\
.8 \\
.8\end{array}$ & $\begin{array}{r}5.3 \\
3.4 \\
1.4 \\
1.2 \\
.9\end{array}$ & $\begin{array}{c}1.2 \\
1.2 \\
11 \\
4.4 \\
1.2\end{array}$ & $\begin{array}{r}1.1 \\
1.0 \\
.8 \\
.7 \\
.7\end{array}$ & $\begin{array}{c}9.2 \\
10 \\
67 \\
12 \\
9.0\end{array}$ & $\begin{array}{l}18 \\
19 \\
21 \\
4.4 \\
3.4\end{array}$ \\
\hline $\begin{array}{l}21 \ldots \ldots \\
22 \ldots \ldots \\
23 \ldots \ldots \\
24 \ldots \ldots \\
25 \ldots \ldots \\
\end{array}$ & $\begin{array}{r}10 \\
1.4 \\
1.2 \\
1.1 \\
.8\end{array}$ & $\begin{array}{r}2.0 \\
1.4 \\
3.9 \\
6.4 \\
.8\end{array}$ & $\begin{array}{r}2.4 \\
16 \\
8.4 \\
.9 \\
1.2\end{array}$ & $\begin{array}{l}24 \\
44 \\
16 \\
23 \\
30\end{array}$ & $\begin{array}{l}1.2 \\
1.1 \\
1.0 \\
1.6 \\
9.8\end{array}$ & $\begin{array}{l}.8 \\
.8 \\
.8 \\
.8 \\
.8\end{array}$ & $\begin{array}{l}.9 \\
.7 \\
.7 \\
.7 \\
.7\end{array}$ & $\begin{array}{l}.9 \\
.8 \\
.8 \\
.8 \\
.7\end{array}$ & $\begin{array}{r}1.0 \\
.8 \\
.7 \\
.7\end{array}$ & $\begin{array}{l}.7 \\
.7 \\
.7 \\
.7 \\
.7\end{array}$ & $\begin{array}{l}18 \\
11 \\
14 \\
16 \\
12\end{array}$ & $\begin{array}{l}2.6 \\
1.9 \\
1.8 \\
1.8 \\
1.5\end{array}$ \\
\hline $\begin{array}{l}26 \ldots . . \\
27 . \ldots \\
28 \ldots \\
29 \ldots \\
30 \ldots \\
31 \ldots .\end{array}$ & $\begin{array}{l}.8 \\
.9 \\
.8 \\
.8 \\
.8 \\
.8\end{array}$ & $\begin{array}{r}.8 \\
.8 \\
.8 \\
. .8 . \\
. . .8\end{array}$ & $\begin{array}{r}1.1 \\
.9 \\
2.5 \\
.9 \\
.8 \\
.7\end{array}$ & $\begin{array}{c}27 \\
16 \\
4.4 \\
3.9 \\
2.9\end{array}$ & $\begin{array}{r}18 \\
10 \\
.9 \\
.8 \\
.8 \\
.8\end{array}$ & $\begin{array}{l}.99 \\
21 \\
21 \\
21\end{array}$ & $\begin{array}{l}.7 \\
.7 \\
.7 \\
.7 \\
.7 \\
.7\end{array}$ & $\begin{array}{r}.7 \\
2.9 \\
.8 \\
.8 \\
.7 \\
.8\end{array}$ & $\begin{array}{c}.7 \\
.7 \\
.7 \\
.7 \\
.7\end{array}$ & $\begin{array}{l}.7 \\
.7 \\
.7 \\
.7 \\
.7 \\
.7\end{array}$ & $\begin{array}{r}12 \\
14 \\
14 \\
11 \\
7.9\end{array}$ & $\begin{array}{r}1.2 \\
1.2 \\
1.1 \\
1.0 \\
.9 \\
.9\end{array}$ \\
\hline
\end{tabular}

NOTE.-Discharge estimated for days (usually Sundays) on which no gage heights were recorded.

Monthly discharge of Oopuola Stream near Huelo, Maui, for 1913.

\begin{tabular}{|c|c|c|c|c|c|}
\hline \multirow{2}{*}{ Month. } & \multicolumn{3}{|c|}{ Discharge in second-feet. } & \multirow{2}{*}{$\begin{array}{l}\text { Run-off } \\
\text { (total in } \\
\text { acre-feet). }\end{array}$} & \multirow{2}{*}{$\begin{array}{l}\text { Accu } \\
\text { racy }\end{array}$} \\
\hline & Maximum. & Minimum. & Mean. & & \\
\hline 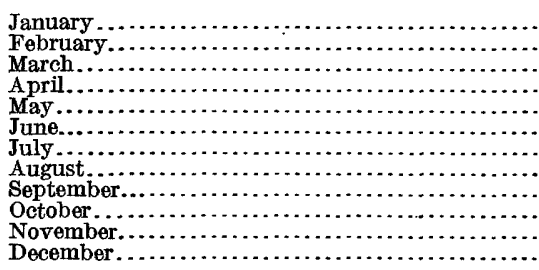 & $\begin{array}{l}70 \\
35 \\
16 \\
80 \\
18 \\
21 \\
21 \\
34 \\
11 \\
27 \\
67 \\
60\end{array}$ & $\begin{array}{r}0.8 \\
.6 \\
.7 \\
.8 \\
.8 \\
.8 \\
.7 \\
.6 \\
.7 \\
.7 \\
.7 \\
.9\end{array}$ & $\begin{array}{r}9.37 \\
4.60 \\
1.80 \\
17.8 \\
2.97 \\
3.61 \\
2.58 \\
2.84 \\
1.23 \\
2.34 \\
15.8 \\
9.24\end{array}$ & $\begin{array}{r}576 \\
259 \\
111 \\
1,060 \\
183 \\
215 \\
159 \\
175 \\
73 \\
144 \\
937 \\
567\end{array}$ & $\begin{array}{l}\mathrm{D} . \\
\mathrm{D} . \\
\mathrm{D} . \\
\mathrm{D} . \\
\mathrm{D} . \\
\mathrm{D} . \\
\mathrm{D}: \\
\mathrm{D} . \\
\mathrm{D} . \\
\mathrm{D} . \\
\mathrm{D} . \\
\mathrm{D} .\end{array}$ \\
\hline The year....... & 80 & .6 & 6.15 & 4,460 & \\
\hline
\end{tabular}




\section{SPRECKELS DITCH AT GAGE NO. 1, NEAR HUELO, MAUI.}

Location.-About $8 \frac{1}{2}$ miles by trail east of Huelo, about 20 feet above bridge which crosses ditch in Ulawina Gulch.

Records available.-December 18, 1910, to September 30, 1913.

Gage.--Vertical staff.

Control.-Probably permanent.

Discharge measurements.-Made by wading.

Accuracy.-Records good.

Cooperation.-Station maintained in cooperation with East Maui Ditch Co.

The following discharge measurement was made by C. T. Bailey:

June 19, 1913: Gage height, 0.40 foot; discharge, 5.94 second-feet.

Monthly discharge of Spreckels ditch at gage No. 1, near Huelo, Maui, for 1913.

\begin{tabular}{|c|c|c|c|c|c|}
\hline \multirow{2}{*}{ Month. } & \multicolumn{3}{|c|}{ Discharge in second-feet. } & \multirow{2}{*}{$\begin{array}{l}\text { Run-off } \\
\text { (total in } \\
\text { acre-feet). }\end{array}$} & \multirow{2}{*}{$\begin{array}{l}\text { Accu- } \\
\text { racy. }\end{array}$} \\
\hline & Maximum. & Minimum. & Mean. & & \\
\hline 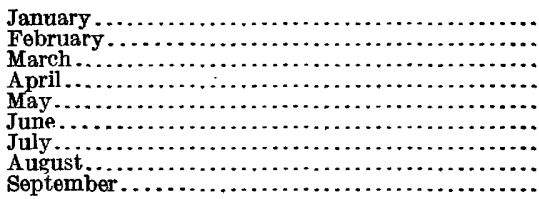 & $\begin{array}{r}40 \\
42 \\
36 \\
48 \\
28 \\
33 \\
44 \\
46 \\
40\end{array}$ & $\begin{array}{l}4.1 \\
3.6 \\
1.6 \\
6.0 \\
5.5 \\
5.1 \\
3.4 \\
2.4 \\
3.7\end{array}$ & $\begin{array}{l}13.4 \\
0.1 \\
29.90 \\
5.8 \\
10.1 \\
11.0 \\
13.5 \\
12.0 \\
8.05\end{array}$ & $\begin{array}{r}824 \\
561 \\
609 \\
1,540 \\
621 \\
655 \\
830 \\
738 \\
479\end{array}$ & $\begin{array}{l}\text { B. } \\
\text { B. } \\
\text { B. } \\
\text { B. } \\
\text { B. } \\
\text { B. } \\
\text { B. } \\
\text { B. } \\
\text { B. }\end{array}$ \\
\hline The period.. & 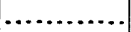 & ....... & $\cdots$ & 6,860 & \\
\hline
\end{tabular}

\section{SPRECKELS DITCH AT GAGE NO. 2, NEAR HUELO, MAUI.}

Location.-About $7 \frac{1}{2}$ miles by trail east of Huelo post office and 100 feet above junction of ditch with Kolea Stream No. 2.

Records available.-November 6, 1911, to September 30, 1913.

Gage.-Vertical staff.

Control.-Probably permanent.

Discharge measurements.-Made from log across ditch near gage.

Accuracy.-Records good.

Cooperation.--Station is maintained in cooperation with East Maui Ditch Co.

The following discharge measurement was made by C. T. Bailey:

June 19, 1913: Gage height, 0.90 foot; discharge, 6.84 second-feet.

Monthly discharge of Spreckels ditch at gage No. 2, near Huelo, Maui, for 1913.

\begin{tabular}{|c|c|c|c|c|c|}
\hline \multirow{2}{*}{ Month. } & \multicolumn{3}{|c|}{ Discharge in second-feet. } & \multirow{2}{*}{$\begin{array}{c}\text { Run-off } \\
\text { (total in } \\
\text { acre-feet). }\end{array}$} & \multirow{2}{*}{$\begin{array}{l}\text { Accu- } \\
\text { racy. }\end{array}$} \\
\hline & Maximum. & Minimum. & Mean. & & \\
\hline 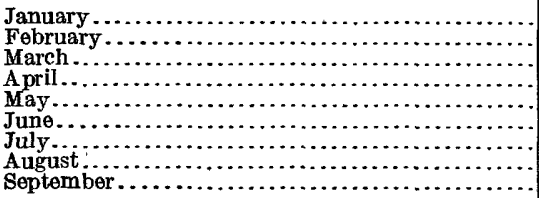 & $\begin{array}{l}36 \\
36 \\
38 \\
38 \\
36 \\
38 \\
35 \\
35 \\
35\end{array}$ & $\begin{array}{r}\mathbf{4} \\
\mathbf{3} \\
\mathbf{2} \\
\mathbf{5} \\
\mathbf{5} \\
\mathbf{4} \\
\mathbf{3} \\
\mathbf{2} \\
\mathbf{3}\end{array}$ & $\begin{array}{r}13.1 \\
8.9 \\
9.3 \\
22.6 \\
10.8 \\
12.9 \\
12.5 \\
12.4 \\
7.5\end{array}$ & $\begin{array}{r}806 \\
\mathbf{4 9 4} \\
\mathbf{5 7 2} \\
\mathbf{1}, 340 \\
664 \\
768 \\
769 \\
762 \\
446\end{array}$ & \multirow[t]{2}{*}{$\begin{array}{l}\text { B. } \\
\text { B. } \\
\text { B. } \\
\text { B. } \\
\text { B. } \\
\text { B. } \\
\text { B. }\end{array}$} \\
\hline The period.. & & & ‥ & 6,620 & \\
\hline
\end{tabular}


SPRECKELS DITCH AT GAGE NO. 3, NEAR RUELO, MAUI.

Location.-About $6 \frac{3}{4}$ miles by trail east of Huelo and 400 feet above junction of ditch with Haipuaena Stream.

Records available.-December 18, 1910, to September 30, 1913.

Gage.--Vertical staff.

Control.-Probably permanent.

Discharge measurements.-Made from log near gage.

Accuracy.-Records good.

Cooperation.-Station maintained in cooperation with East Maui Ditch Co.

The following discharge measurement was made by C. T. Bailey:

June 19, 1913: Gage height, 1.20 feet; discharge, 9.33 second-feet.

Monthly ciischarge of Spreckels ditch at gage No. s, near Huelo, Maui, for 1918.

\begin{tabular}{|c|c|c|c|c|c|}
\hline \multirow{2}{*}{ Month. } & \multicolumn{3}{|c|}{ Discharge in second-feet. } & \multirow{2}{*}{$\begin{array}{l}\text { Rum-off } \\
\text { (total in } \\
\text { acre-feet). }\end{array}$} & \multirow{2}{*}{$\begin{array}{l}\text { Accu- } \\
\text { racy. }\end{array}$} \\
\hline & Maximum. & Minimum. & Mean. & & \\
\hline 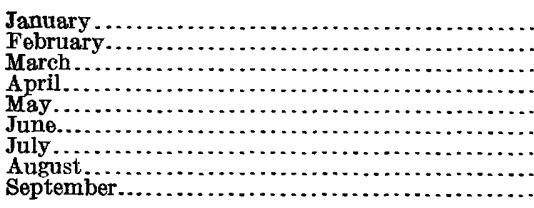 & $\begin{array}{l}40 \\
39 \\
41 \\
45 \\
40 \\
40 \\
38 \\
39 \\
38\end{array}$ & $\begin{array}{l}7.4 \\
6.0 \\
3.6 \\
8.7 \\
7.9 \\
5.8 \\
5.8 \\
4.6 \\
6.0\end{array}$ & $\begin{array}{l}18.3 \\
12.9 \\
13.5 \\
27.4 \\
15.7 \\
16.2 \\
17.0 \\
15.7 \\
11.5\end{array}$ & $\begin{array}{r}1,120 \\
716 \\
830 \\
1,630 \\
965 \\
964 \\
1,050 \\
965 \\
684\end{array}$ & $\begin{array}{l}\text { B. } \\
\text { B. } \\
\text { B. } \\
\text { B. } \\
\text { B. } \\
\text { B. } \\
\text { B. } \\
\text { B. } \\
\text { B. }\end{array}$ \\
\hline The period. & & & & 8,920 & \\
\hline
\end{tabular}

SPRECKELS DITCH AT GAGE NO. 4, NEAR HUELO, MAUI.

Location.-About $6 \frac{1}{1}$ miles by trail east of Huelo and about 250 feet above junction with small tributary of Puohakamoa Stream.

Records available.-December 18, 1910, to September 30, 1913.

Gage.--Vertical staff.

Control.-Probably permanent.

Discharge measurements.-Made from log at gage.

Accuracy.-Records fair.

Cooperation.-Station maintained in cooperation with East Maui Ditch Co.

The following discharge measurement was made by C. T. Bailey:

June 19, 1913: Gage height, 0.90 foot; discharge, 12.2 second-feet.

Monthly discharge of Spreckels ditch at gage No. 4, near Huelo, Maui, for 1913.

\begin{tabular}{|c|c|c|c|c|c|}
\hline \multirow{2}{*}{ Month. } & \multicolumn{3}{|c|}{ Discharge in second-feet. } & \multirow{2}{*}{$\begin{array}{l}\text { Run-off } \\
\text { (total in } \\
\text { acre-feet). }\end{array}$} & \multirow{2}{*}{$\begin{array}{l}\text { Accu } \\
\text { racy. }\end{array}$} \\
\hline & Maximum. & Minimum. & Mean. & & \\
\hline 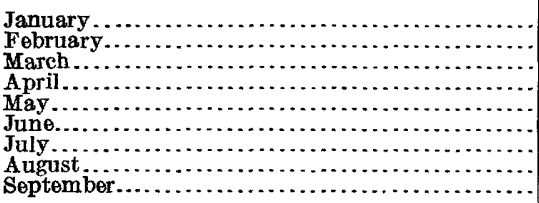 & $\begin{array}{l}38 \\
36 \\
40 \\
44 \\
38 \\
38 \\
36 \\
40 \\
38\end{array}$ & $\begin{array}{r}9.5 \\
7.5 \\
4.0 \\
11.5 \\
9.5 \\
9.5 \\
5.5 \\
4.5 \\
7.0\end{array}$ & $\begin{array}{l}21.2 \\
15.2 \\
14.6 \\
27.8 \\
18.3 \\
18.3 \\
19.0 \\
17.3 \\
13.7\end{array}$ & $\begin{array}{r}1,300 \\
844 \\
898 \\
1,650 \\
1,130 \\
1,090 \\
1,170 \\
1,060 \\
815\end{array}$ & $\begin{array}{l}\text { c. } \\
\text { c. } \\
\text { c. } \\
\text { c. } \\
\text { c. } \\
\text { c. } \\
\text { c. }\end{array}$ \\
\hline The period. . & - & 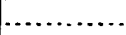 & .... & 10,000 & \\
\hline
\end{tabular}




\section{SPRECKELS DITCH AT GAGE NO. 5, NEAR HUELO, MAUI.}

Location.-About 5 miles east of Huelo and about 500 feet above drop into Alo Stream.

Records available.-November 6, 1911, to September 30, 1913.

Gage.--Vertical staff.

Control.-Probably permanent.

Discharge measurements.-Made from plank at gage.

Accuracy.-Records good.

Cooperation.-Station maintained in cooperation with East Maui Ditch Co.

The following discharge measurement was made by C. T. Bailey:

June 4, 1913: Gage height, 1.10 feet; discharge, 21.6 second-feet.

Monthly discharge of Spreckels ditch at gage No. 5, near Huelo, Maui, for 1913.

\begin{tabular}{|c|c|c|c|c|c|}
\hline \multirow{2}{*}{ Month. } & \multicolumn{3}{|c|}{ Discharge in second-feet. } & \multirow{2}{*}{$\begin{array}{c}\text { Run-off } \\
\text { (total in } \\
\text { acre-feet). }\end{array}$} & \multirow{2}{*}{$\begin{array}{l}\text { Accu- } \\
\text { racy. }\end{array}$} \\
\hline & Maximum. & Minimum. & Mean. & & \\
\hline \multirow[t]{2}{*}{ 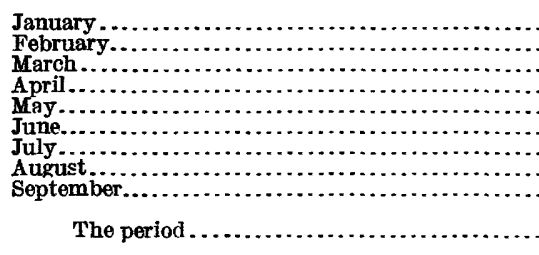 } & $\begin{array}{l}55 \\
54 \\
53 \\
58 \\
51 \\
55 \\
56 \\
66 \\
55\end{array}$ & $\begin{array}{r}16 \\
13 \\
9 \\
19 \\
18 \\
16 \\
13 \\
16 \\
14\end{array}$ & $\begin{array}{l}33.7 \\
24.6 \\
24.3 \\
43.4 \\
30.3 \\
28.2 \\
31.5 \\
30.4 \\
21.7\end{array}$ & $\begin{array}{l}2,070 \\
1,370 \\
1,490 \\
2,580 \\
1,860 \\
1,680 \\
1,940 \\
1,870 \\
1,290\end{array}$ & \multirow[t]{2}{*}{$\begin{array}{l}\text { B. } \\
\text { B. } \\
\text { B. } \\
\text { B. } \\
\text { B. } \\
\text { B. } \\
\text { B. } \\
\text { B. } \\
\text { B. }\end{array}$} \\
\hline & n........... & & & 16,200 & \\
\hline
\end{tabular}

SPRECKELS DITCH AT GAGE NO. 6, NEAR HUELO, MAUI.

Location.-About $3 \frac{1}{2}$ miles east of Huelo and 100 feet below Kolea Stream intake.

Records available.-November 4, 1911, to June 30, 1913.

Gage.-Vertical staff.

Discharge measurements.-Made from log at gage.

Control.-Probably permanent.

Accuracy.--Records good.

Cooperation.--Station maintained in cooperation with East Maui Ditch Co.

The following discharge measurement was made by C. T. Bailey:

June 14, 1913: Gage height, 1.35 feet; discharge, 44.5 second-feet.

Monthly discharge of Spreckels ditch at gage No. 6, near Huelo, Maui, for 1913.

\begin{tabular}{|c|c|c|c|c|c|}
\hline \multirow{2}{*}{ Month. } & \multicolumn{3}{|c|}{ Discharge in second-feet. } & \multirow{2}{*}{$\begin{array}{c}\text { Run-off } \\
\text { (total in } \\
\text { acre-feet). }\end{array}$} & \multirow{2}{*}{$\begin{array}{l}\text { Accu- } \\
\text { racy. }\end{array}$} \\
\hline & Maximum. & Minimum. & Mean. & & \\
\hline \multirow{2}{*}{ 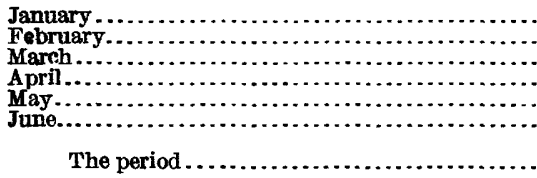 } & $\begin{array}{l}52 \\
46 \\
42 \\
99 \\
50 \\
50\end{array}$ & $\begin{array}{l}30 \\
24 \\
18 \\
34 \\
31 \\
27\end{array}$ & $\begin{array}{l}33.4 \\
30.6 \\
31.8 \\
45.5 \\
42.4 \\
39.7\end{array}$ & $\begin{array}{l}2,050 \\
1,700 \\
1,960 \\
2,710 \\
2,610 \\
2,360\end{array}$ & \multirow[t]{2}{*}{$\begin{array}{l}\text { B. } \\
\text { B. } \\
\text { B. } \\
\text { B. } \\
\text { B. } \\
\text { B. }\end{array}$} \\
\hline & .... & (n............. & ........... & 13,400 & \\
\hline
\end{tabular}


SPRECKELS DITCH AT GAGE NO. 8, NEAR HUELO, MAUI.

Location.-About 1 mile by trail east of Huelo and 250 feet above gate to storage reservoir.

Records available.-November 4, 1911, to September 30, 1913.

Gage.--Vertical staff.

Control.-Probably permanent.

Discharge measurements.-Made from plank.

Accuracy.-Records fair. Discharge estimated for Sundays, when no gage record was obtained.

Cooperation.--Station maintained in cooperation with East Maui Ditch Co.

The following discharge measurement was made by C. T. Bailey:

June 4, 1913: Gage height, 2.10 feet; discharge, 45.1 second-feet.

Monthly discharge of Spreckels ditch at gage No. 8, near Huelo, Maui, for 1913.

\begin{tabular}{|c|c|c|c|c|c|}
\hline \multirow{2}{*}{ Month. } & \multicolumn{3}{|c|}{ Discharge in second-feet. } & \multirow{2}{*}{$\begin{array}{l}\text { Run-off } \\
\text { (total in } \\
\text { acre-feet). }\end{array}$} & \multirow{2}{*}{$\begin{array}{l}\text { Accu- } \\
\text { racy. }\end{array}$} \\
\hline & Maximum. & Minimum. & Mean. & & \\
\hline 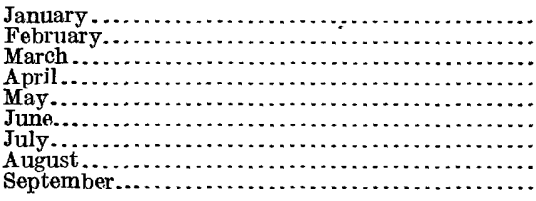 & $\begin{array}{l}62 \\
54 \\
54 \\
68 \\
62 \\
58 \\
59 \\
67 \\
54\end{array}$ & $\begin{array}{l}5.2 \\
1.9 \\
16 \\
22 \\
33 \\
32 \\
13 \\
17 \\
22\end{array}$ & $\begin{array}{l}17.5 \\
22.9 \\
34.1 \\
51.3 \\
45.4 \\
43.9 \\
40.6 \\
38.1 \\
29.8\end{array}$ & $\begin{array}{l}1,080 \\
1,270 \\
2,100 \\
3,050 \\
2,790 \\
2,610 \\
2,500 \\
2,340 \\
1,770\end{array}$ & $\begin{array}{l}\text { C. } \\
\text { C. } \\
\text { C. } \\
\text { C. } \\
\text { C. } \\
\text { C. } \\
\text { C. } \\
\text { C. } \\
\text { C. }\end{array}$ \\
\hline The period .... & $\ldots \ldots$ & $-2-2-2-2$ & $\ldots \ldots$. & 19,500 & \\
\hline
\end{tabular}

NAILIILIHAELE STREAM NEAR HUELO, MAUI.

Location.-About 300 feet above New Hamakua ditch, 3 miles south of Huelo post office.

Records available.-December 9, 1910, to December 31, 1912; October 8 to December 31, 1913.

Gage.-Barrett \& Lawrence water stage recorder.

Control.-Probably permanent.

Discharge measurements.-Made from footbridge.

Diversions.- None above station.

Accuracy.-Records good.

Discharge measurements of Nailiilihaele Stream near Huelo, Maui, in 1913.

\begin{tabular}{|c|c|c|c|c|c|c|c|}
\hline Date. & Made by- & $\begin{array}{c}\text { Gage } \\
\text { height. }\end{array}$ & $\begin{array}{l}\text { Dis- } \\
\text { charge. }\end{array}$ & Date. & Made by- & $\begin{array}{c}\text { Gage } \\
\text { height. }\end{array}$ & $\begin{array}{l}\text { Dis- } \\
\text { charge. }\end{array}$ \\
\hline $\begin{array}{r}\text { Oct. } 2 \ldots \\
9 \ldots \\
\text { Nov. } 3 \ldots\end{array}$ & $\begin{array}{l}\text { E. O.Christiansen.. } \\
\ldots \ldots \text { do } \ldots \ldots \ldots \ldots \\
\ldots \ldots\end{array}$ & $\begin{array}{r}\text { Feet. } \\
0.86 \\
1.17 \\
1.58\end{array}$ & $\begin{array}{c}\text { Sec. }-f t \\
6.89 \\
26.6 \\
52.9\end{array}$ & $\begin{array}{r}\text { Nov.13.. } \\
18 . . \\
19 . .\end{array}$ & 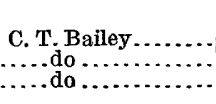 & $\begin{array}{r}\text { Feet. } \\
1.70 \\
2.38 \\
3.30\end{array}$ & $\begin{array}{c}\text { Sec.-ft. } \\
67.5 \\
174 \\
514\end{array}$ \\
\hline
\end{tabular}


Daily discharge, in second-feet, of Nailiilihaele Stream near Huelo, Maui, for 1913.

\begin{tabular}{|c|c|c|c|c|c|c|c|}
\hline Day. & Oct. & Nov. & Dec. & Day. & Oct. & Nov. & Dec. \\
\hline $\begin{array}{l}1 \\
2 \\
3 \\
4 \\
5 \ldots \ldots \cdots \cdots \\
5 \ldots \ldots \cdots \\
\end{array}$ & ...... & $\begin{array}{c}3.6 \\
13 \\
158 \\
104 \\
35\end{array}$ & $\begin{array}{r}32 \\
37 \\
65 \\
674 \\
245\end{array}$ & $\begin{array}{l}16 \ldots \ldots \ldots \\
17 \ldots \ldots \ldots \\
19 \ldots \ldots \ldots \\
20 \ldots \ldots \ldots \ldots\end{array}$ & $\begin{array}{l}14 \\
12 \\
8.5 \\
8.0 \\
8.0\end{array}$ & $\begin{array}{r}45 \\
67 \\
225 \\
275 \\
122\end{array}$ & $\begin{array}{r}46 \\
63 \\
110 \\
49 \\
40\end{array}$ \\
\hline $\begin{array}{r}6 \\
7 \ldots \ldots \\
8 \\
9 \\
9 \\
10 \ldots \ldots\end{array}$ & $\begin{array}{r}156 \\
32 \\
15\end{array}$ & $\begin{array}{r}28 \\
27 \\
290 \\
365 \\
251\end{array}$ & $\begin{array}{r}160 \\
71 \\
91 \\
62 \\
48\end{array}$ & $\begin{array}{l}21 \\
22 \\
23 \\
24 \\
25 \\
25\end{array}$ & $\begin{array}{l}8.0 \\
8.0 \\
7.4 \\
6.8 \\
6.5\end{array}$ & $\begin{array}{r}91 \\
48 \\
38 \\
103 \\
106\end{array}$ & $\begin{array}{l}34 \\
31 \\
27 \\
25 \\
23\end{array}$ \\
\hline 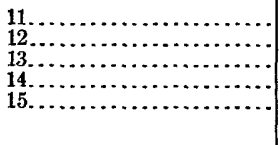 & $\begin{array}{l}14 \\
12 \\
49 \\
42 \\
18\end{array}$ & $\begin{array}{r}111 \\
160 \\
91 \\
44 \\
37\end{array}$ & $\begin{array}{r}41 \\
37 \\
33 \\
144 \\
71\end{array}$ & 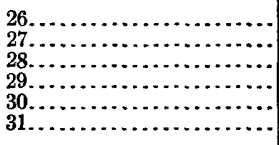 & $\begin{array}{l}5.3 \\
5.0 \\
5.0 \\
3.6 \\
3.6 \\
3.6\end{array}$ & $\begin{array}{r}140 \\
86 \\
52 \\
+\quad 44 \\
36\end{array}$ & $\begin{array}{l}18 \\
13 \\
13 \\
12 \\
12 \\
11\end{array}$ \\
\hline
\end{tabular}

Monthly discharge of Nailiilihaele Stream near Huelo, Maui, for 1913.

\begin{tabular}{|c|c|c|c|c|c|}
\hline \multirow{2}{*}{ - } & \multicolumn{3}{|c|}{ Discharge in second-feet. } & \multirow{2}{*}{$\begin{array}{l}\text { Run-off } \\
\text { (total in } \\
\text { acre-feet). }\end{array}$} & \multirow{2}{*}{$\begin{array}{l}\text { Accu } \\
\text { racy. }\end{array}$} \\
\hline & Maximum. & Minimum. & Mean. & & \\
\hline $\begin{array}{l}\text { October } 8-31 . \ldots \ldots \ldots \\
\text { November............ } \\
\text { December........... }\end{array}$ & $\begin{array}{l}156 \\
365 \\
674\end{array}$ & $\begin{array}{r}3.6 \\
3.6 \\
11.0\end{array}$ & $\begin{array}{c}18.8 \\
106 \\
75.4\end{array}$ & $\begin{array}{r}895 \\
6,310 \\
4,640\end{array}$ & $\begin{array}{l}\text { A. } \\
\text { A. } \\
\text { A. }\end{array}$ \\
\hline
\end{tabular}

\section{KAILUA STREAM NEAR HUELO, MAUI.}

Location.-One mile south of Huelo post office and 800 feet above Spreckels ditch, at 1,250 feet elevation.

Records available.-December 8, 1910, to December 31, 1912; June 17 to December 31, 1913.

Gage.-Barrett \& Lawrence water-stage recorder October 1 to December 31; vertical staff June 17 to September 30.

Control.-Probably permanent.

Discharge measurements.-Made from footbridge or by wading.

Diversions.-Old Hamakua ditch formerly diverted water above station, but that section of ditch is not used at present time.

Accuracy.-Records good October 1 to December 31; poor remainder of period on account of uncertainty of gage heights.

Discharge measurements of Kailua Stream near Huelo, Maui, in 1913.

\begin{tabular}{|c|c|c|c|c|c|c|c|}
\hline Date. & Made by- & $\begin{array}{c}\text { Gage } \\
\text { height. }\end{array}$ & $\begin{array}{c}\text { Dis- } \\
\text { charge. }\end{array}$ & Date. & Made by- & $\begin{array}{c}\text { Gage } \\
\text { height. }\end{array}$ & $\begin{array}{l}\text { Dis- } \\
\text { charge. }\end{array}$ \\
\hline $\begin{array}{l}\text { June } 16 . . \\
\text { Aug. } 26 \ldots \\
\text { Oct. } 2 \ldots \\
9 \ldots\end{array}$ & 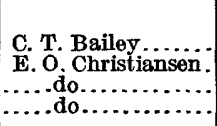 & $\begin{array}{l}\text { Feet. } \\
1.38 \\
1.23 \\
1.14 \\
1.53\end{array}$ & $\begin{array}{c}\text { Sec-ft. } \\
8.51 \\
4.37 \\
2.96 \\
19.4\end{array}$ & Nov. $\begin{array}{r}3 \\
13 \\
18 \\
19\end{array}$ & 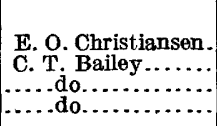 & $\begin{array}{l}\text { Feet. } \\
2.60 \\
2.03 \\
3.42 \\
3.90\end{array}$ & $\begin{aligned} & \text { Sec. }-f t . \\
& 73.8 \\
& 37.4 \\
& 188 \\
& 270\end{aligned}$ \\
\hline
\end{tabular}

$97764^{\circ}-$ WSP $373-15-10$ 
Daily discharge, in second-feet, of Kailua Stream near Huelo, Maui, for 1913.

\begin{tabular}{|c|c|c|c|c|c|c|c|}
\hline Day. & June. & July. & Aug. & Sept. & Oct. & Nov. & Dec. \\
\hline 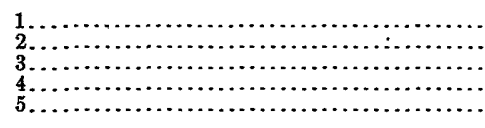 & $\begin{array}{ll}\ldots \ldots \\
\ldots \ldots \ldots \\
\cdots \ldots \ldots \\
\cdots \ldots \ldots\end{array}$ & $\begin{array}{r}45 \\
149 \\
28 \\
47 \\
32\end{array}$ & $\begin{array}{r}11 \\
3.3 \\
2.9 \\
2.9 \\
2.9\end{array}$ & $\begin{array}{l}6.2 \\
5.3 \\
5.3 \\
4.9 \\
4.0\end{array}$ & $\mid \begin{array}{ll}\cdots \ldots \ldots \\
\ldots \ldots \ldots \\
\cdots \cdots \\
\cdots \cdots\end{array}$ & $\begin{array}{l}4.0 \\
30 \\
136 \\
65 \\
22\end{array}$ & $\begin{array}{r}21 \\
20 \\
22 \\
443 \\
262\end{array}$ \\
\hline 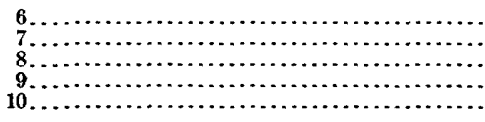 & $\begin{array}{l}\cdots \cdots \\
\cdots \cdots \\
\cdots \cdots \\
\cdots \cdots\end{array}$ & $\begin{array}{r}42 \\
20 \\
11 \\
7.5 \\
8.8\end{array}$ & $\begin{array}{l}4.0 \\
42 \\
12 \\
6.2 \\
6.2\end{array}$ & $\begin{array}{l}3.6 \\
6.2 \\
3.5 \\
3.6 \\
3.6\end{array}$ & $\begin{array}{r}61 \\
13 \\
6.2\end{array}$ & $\begin{array}{r}16 \\
31 \\
216 \\
384 \\
186\end{array}$ & $\begin{array}{r}104 \\
56 \\
45 \\
26 \\
17\end{array}$ \\
\hline $\begin{array}{l}11 \\
12 \\
13 \\
14 \\
15 \\
15\end{array}$ & $\begin{array}{ll}\ldots & \ldots \\
\cdots & \ldots\end{array}$ & $\begin{array}{r}8.8 \\
9.4 \\
17 \\
14 \\
8.8\end{array}$ & $\begin{array}{r}4.9 \\
212 \\
26 \\
19 \\
91\end{array}$ & $\begin{array}{l}3.3 \\
3.3 \\
3.6 \\
3.6 \\
3.6\end{array}$ & $\begin{array}{r}5.5 \\
5.3 \\
12 \\
8.8 \\
8.0\end{array}$ & $\begin{array}{r}70 \\
112 \\
41 \\
22 \\
19\end{array}$ & $\begin{array}{l}13 \\
12 \\
11 \\
61 \\
69\end{array}$ \\
\hline $\begin{array}{l}16 \\
17 \\
18 \\
19 \\
19 \\
20 \\
1\end{array}$ & $\begin{array}{r}7.5 \\
6.7 \\
6.0 \\
5.8\end{array}$ & $\begin{array}{l}7.5 \\
6.2 \\
5.8 \\
5.8 \\
5.8\end{array}$ & $\begin{array}{r}19 \\
10 \\
7.5 \\
7.2 \\
6.7\end{array}$ & $\begin{array}{l}4.9 \\
10 \\
111 \\
24 \\
9.4\end{array}$ & $\begin{array}{l}7.8 \\
7.2 \\
4.0 \\
4.0 \\
4.0\end{array}$ & $\begin{array}{r}46 \\
106 \\
314 \\
290 \\
79\end{array}$ & $\begin{array}{l}2 \\
2 \\
8 \\
2 \\
2\end{array}$ \\
\hline 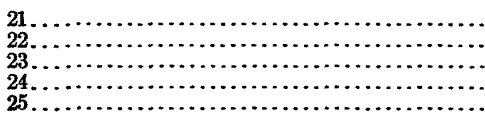 & $\begin{array}{l}5.1 \\
5.1 \\
4.4 \\
4.0 \\
6.0\end{array}$ & $\begin{array}{l}5.3 \\
4.9 \\
4.7 \\
4.7 \\
4.4\end{array}$ & $\begin{array}{l}6.7 \\
6.2 \\
5.8 \\
6.0 \\
5.3\end{array}$ & $\begin{array}{l}6.7 \\
5.3 \\
4.7 \\
4.4 \\
4.4\end{array}$ & $\begin{array}{l}3.3 \\
2.9 \\
2.9 \\
2.9 \\
2.9\end{array}$ & $\begin{array}{r}56 \\
30 \\
25 \\
174 \\
88\end{array}$ & $\begin{array}{l}18 \\
16 \\
1 \\
1 \\
1\end{array}$ \\
\hline 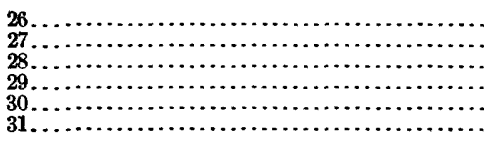 & $\begin{array}{c}6.7 \\
15 \\
12 \\
6.7 \\
38\end{array}$ & $\begin{array}{l}4.9 \\
4.4 \\
4.0 \\
4.0 \\
3.6 \\
3.5\end{array}$ & $\begin{array}{r}5.3 \\
8.3 \\
6.7 \\
5.1 \\
4.4 \\
24\end{array}$ & $\begin{array}{r}3.8 \\
3.6 \\
\mathbf{3 . 6} \\
\mathbf{3 . 6} \\
\mathbf{3 . 5} \\
\ldots \ldots . .\end{array}$ & $\begin{array}{l}2.7 \\
2.7 \\
3.3 \\
3.5 \\
3.8 \\
4.0\end{array}$ & $\begin{array}{r}100 \\
42 \\
33 \\
28 \\
25 \\
\ldots \ldots\end{array}$ & 11 \\
\hline
\end{tabular}

NoTE.-No record Oct. 1-7.

Monthly discharge of Kailua Stream near Huelo, Maui, for 1913.

\begin{tabular}{|c|c|c|c|c|c|}
\hline \multirow{2}{*}{ Month. } & \multicolumn{3}{|c|}{ Discharge in second-feet. } & \multirow{2}{*}{$\begin{array}{l}\text { Run-off (in } \\
\text { acre-feet). }\end{array}$} & \multirow{2}{*}{$\begin{array}{l}\text { Accu- } \\
\text { racy. }\end{array}$} \\
\hline & Maximum. & Minimum. & Mean. & & \\
\hline 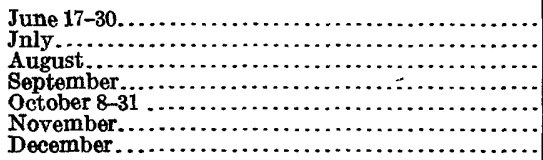 & $\begin{array}{r}38 \\
149 \\
212 \\
111 \\
61 \\
384 \\
443\end{array}$ & $\begin{array}{r}4.0 \\
3.5 \\
2.9 \\
3.3 \\
2.7 \\
4.0 \\
10.0\end{array}$ & $\begin{array}{l}9.21 \\
17.0 \\
18.7 \\
7.57 \\
5.86 \\
93.0 \\
48.6\end{array}$ & $\begin{array}{r}256 \\
1,040 \\
1,150 \\
528 \\
360 \\
5,530 \\
2,990\end{array}$ & \multirow[t]{2}{*}{$\begin{array}{l}\text { B } \\
\text { D. } \\
\text { D. } \\
\text { D. } \\
\text { A. } \\
\text { A. } \\
\text { A. }\end{array}$} \\
\hline The period. & & & $\ldots \ldots \ldots$ & 11,900 & \\
\hline
\end{tabular}

Note.-Water-stage recorder installed October 1.

\section{OANUI STREAM NEAR HUELO, MAUI.}

Iocation.-At New Hamakua ditch crossing, 1 mile south of Huelo post office.

Records available.-December 7, 1910, to December 31, 1911; June 17 to December $31,1913$.

Gage.-Vertical staff in two sections.

Control.-Upper side of New Hamakua ditch forms permanent control.

Discharge measurements.-Made by wading.

Diversions.- None above station.

Accuracy.-Records fair. 
Monthly discharge of Oanui Stream near Huelo, Maui, for 1913.

\begin{tabular}{|c|c|c|c|c|c|}
\hline \multirow{2}{*}{ Month. } & \multicolumn{3}{|c|}{ Discharge in second-feet. } & \multirow{2}{*}{$\begin{array}{l}\text { Run-off } \\
\text { (total in } \\
\text { acre-feet). }\end{array}$} & \multirow{2}{*}{$\begin{array}{l}\text { Accu- } \\
\text { racy. }\end{array}$} \\
\hline & Maximum. & Minimum. & Mean. & & \\
\hline 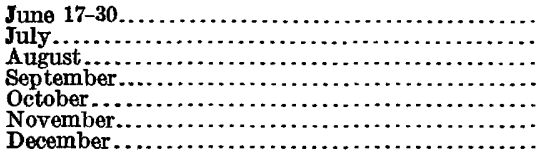 & $\begin{array}{r}10 \\
15 \\
22 \\
8 \\
11 \\
42 \\
72\end{array}$ & $\begin{array}{l}3.5 \\
3.5 \\
3.5 \\
3.5 \\
3.5 \\
3.5 \\
3.5\end{array}$ & $\begin{array}{r}4.46 \\
4.98 \\
5.00 \\
3.75 \\
3.95 \\
12.4 \\
9.87\end{array}$ & $\begin{array}{l}124 \\
306 \\
307 \\
223 \\
243 \\
738 \\
607\end{array}$ & $\begin{array}{l}\text { C. } \\
\text { C. } \\
\text { C. } \\
\text { C. } \\
\text { C. } \\
\text { C. }\end{array}$ \\
\hline The period.... & $\cdots$ & $\cdots$ & n........ & 2,550 & \\
\hline
\end{tabular}

HOOLAWANUI STREAM NEAR HUELO, MAUI.

Location.-One-fourth mile east of Lupi and about 5 miles by trail from Huelo; 300 feet above crossing of New Hamakua ditch.

Records available.-December 12, 1910, to December 31, 1913.

Gage.-Vertical staff; datum lowered 0.20 foot June 6, 1913.

Control.-Probably permanent.

Discharge measurements.-Made by wading.

Diversions.-None above station.

Accuracy.-Records fair.

Cooperation.-Station is maintained in cooperation with East Maui Ditch Co.

Discharge measurements of Hoolawanui Stream near Huelo, Maui, in 1913.

\begin{tabular}{|c|c|c|c|c|c|c|c|}
\hline Date. & Made by- & $\begin{array}{c}\text { Gage } \\
\text { height. }\end{array}$ & $\begin{array}{l}\text { Dis- } \\
\text { charge. }\end{array}$ & Date. & Made by- & $\begin{array}{c}\text { Gage } \\
\text { height. }\end{array}$ & $\begin{array}{l}\text { Dis- } \\
\text { charge. }\end{array}$ \\
\hline $\begin{array}{l}\text { June } 5 . . . \\
\text { Aug. } 27 .\end{array}$ & $\begin{array}{c}\text { C. T. Bailey......... } \\
\ldots . . . \text { do }\end{array}$ & $\begin{array}{r}\text { Feet. } \\
0.30 \\
.34\end{array}$ & $\begin{array}{r}\text { Sec.-ft. } \\
2.84 \\
4.68\end{array}$ & $\begin{array}{l}\text { Oct. } 2 \ldots \\
\text { Nov. } 19\end{array}$ & $\begin{array}{l}\text { E. O. Christiansen. } \\
\text { C. T. Bailey........ }\end{array}$ & $\begin{array}{c}\text { Feet. } \\
0.24 \\
1.02\end{array}$ & $\begin{array}{c}\text { Sec. }-f t . \\
1.79 \\
62.8\end{array}$ \\
\hline
\end{tabular}

Daily discharge, in second-feet, of Hoolawanui Stream near Huelo, Maui, for 1913.

\begin{tabular}{|c|c|c|c|c|c|c|c|c|c|c|c|c|}
\hline Day. & Jan. & Feb. & Mar. & Apr. & May. & June. & July. & Aug. & Sept. & Oct. & Nov. & Dec. \\
\hline $\begin{array}{l}1 \\
2 \ldots \\
3 \ldots \\
5 \ldots \ldots\end{array}$ & $\begin{array}{r}12 \\
9 \\
8 \\
7 \\
6\end{array}$ & $\begin{array}{l}4 \\
4 \\
3 \\
\mathbf{3} \\
\mathbf{3}\end{array}$ & $\begin{array}{l}\mathbf{4} \\
\mathbf{3} \\
\mathbf{3} \\
\mathbf{3} \\
\mathbf{3}\end{array}$ & $\begin{array}{r}15 \\
8 \\
6 \\
5 \\
5\end{array}$ & $\begin{array}{l}9 \\
8 \\
8 \\
7 \\
6\end{array}$ & $\begin{array}{l}2.0 \\
3.0 \\
2.0 \\
6.0 \\
3.0\end{array}$ & $\begin{array}{c}13 \\
32 \\
10 \\
11 \\
9.2\end{array}$ & $\begin{array}{l}2.3 \\
2.3 \\
2.5 \\
2.3 \\
2.3\end{array}$ & $\begin{array}{l}2.6 \\
2.6 \\
2.6 \\
2.5 \\
2.3\end{array}$ & $\begin{array}{l}1.9 \\
1.9 \\
4.0 \\
2.3 \\
1.9\end{array}$ & $\begin{array}{c}1.9 \\
2.1 \\
15 \\
17 \\
5\end{array}$ & $\begin{array}{c}11 \\
9.2 \\
13 \\
112 \\
82\end{array}$ \\
\hline $\begin{array}{r}6 \ldots \ldots \\
7 \ldots \ldots \\
9 \ldots \ldots\end{array}$ & $\begin{array}{r}6 \\
5 \\
10 \\
10 \\
10\end{array}$ & $\begin{array}{r}88 \\
7 \\
5 \\
4 \\
4\end{array}$ & $\begin{array}{l}\mathbf{3} \\
\mathbf{3} \\
\mathbf{3} \\
\mathbf{4} \\
\mathbf{3}\end{array}$ & $\begin{array}{l}5 \\
4 \\
4 \\
4 \\
4\end{array}$ & $\begin{array}{l}6 \\
5 \\
5 \\
5 \\
4\end{array}$ & $\begin{array}{l}3.0 \\
3.0 \\
2.6 \\
2.8 \\
4.0\end{array}$ & $\begin{array}{c}12 \\
.9 .2 \\
8.0 \\
7.0 \\
6.5\end{array}$ & $\begin{array}{l}2.6 \\
5.0 \\
2.6 \\
2.3 \\
2.5\end{array}$ & $\begin{array}{l}2.3 \\
2.6 \\
2.3 \\
2.3 \\
2.3\end{array}$ & $\begin{array}{r}2.5 \\
2.6 \\
19 \\
4.0 \\
3.0\end{array}$ & $\begin{array}{l}\mathbf{5 . 0} \\
4.0 \\
46 \\
94 \\
62\end{array}$ & $\begin{array}{l}62 \\
35 \\
36 \\
24 \\
16\end{array}$ \\
\hline $\begin{array}{l}11 \ldots \ldots \\
13 \ldots \ldots \ldots \\
14 \ldots \ldots\end{array}$ & $\begin{array}{r}7 \\
7 \\
52 \\
44 \\
71\end{array}$ & $\begin{array}{l}\mathbf{3} \\
\mathbf{3} \\
\mathbf{3} \\
\mathbf{3} \\
\mathbf{3}\end{array}$ & $\begin{array}{l}3 \\
3 \\
2 \\
2 \\
2 \\
2\end{array}$ & $\begin{array}{l}40 \\
20 \\
71 \\
50 \\
88\end{array}$ & $\begin{array}{l}4 \\
4 \\
4 \\
5 \\
8\end{array}$ & $\begin{array}{c}2.6 \\
18 \\
10 \\
8.0 \\
6.0\end{array}$ & $\begin{array}{l}6.0 \\
6.0 \\
6.5 \\
6.5 \\
6.0\end{array}$ & $\begin{array}{l}2.3 \\
33 \\
7.5 \\
5.5 \\
30\end{array}$ & $\begin{array}{l}2.3 \\
2.3 \\
2.3 \\
2.3 \\
2.1\end{array}$ & $\begin{array}{l}2.6 \\
2.5 \\
7.0 \\
3.5 \\
2.6\end{array}$ & $\begin{array}{l}48 \\
56 \\
24 \\
17 \\
15\end{array}$ & $\begin{array}{l}15 \\
14 \\
13 \\
40 \\
22\end{array}$ \\
\hline $\begin{array}{l}16 \ldots \ldots \\
17 \ldots \ldots \\
19 \ldots \ldots \\
20 \ldots \ldots\end{array}$ & $\begin{array}{r}13 \\
12 \\
10 \\
10 \\
9\end{array}$ & $\begin{array}{r}4 \\
3 \\
11 \\
48 \\
8\end{array}$ & $\begin{array}{l}8 \\
6 \\
6 \\
4 \\
4\end{array}$ & $\begin{array}{l}82 \\
96 \\
48 \\
30 \\
27\end{array}$ & $\begin{array}{l}6 \\
7 \\
5 \\
5 \\
4\end{array}$ & $\begin{array}{l}5.0 \\
4.0 \\
3.0 \\
3.0 \\
3.0\end{array}$ & $\begin{array}{l}5.0 \\
4.5 \\
4.0 \\
4.0 \\
3.5\end{array}$ & $\begin{array}{l}9.2 \\
8.6 \\
6.5 \\
5.0 \\
5.0\end{array}$ & $\begin{array}{l}2.3 \\
3.5 \\
9.2 \\
5.0 \\
2.6\end{array}$ & $\begin{array}{l}2.6 \\
2.6 \\
2.5 \\
2.3 \\
2.3\end{array}$ & $\begin{array}{l}16 \\
16 \\
66 \\
82 \\
38\end{array}$ & $\begin{array}{l}17 \\
19 \\
39 \\
17 \\
13\end{array}$ \\
\hline $\begin{array}{l}21 \ldots \ldots \\
22 \ldots \ldots \\
23 \ldots \ldots \\
24 \ldots \ldots \\
25 \ldots \ldots\end{array}$ & $\begin{array}{l}8 \\
7 \\
6 \\
5 \\
5\end{array}$ & $\begin{array}{l}7 \\
6 \\
5 \\
5 \\
4\end{array}$ & $\begin{array}{r}5 \\
10 \\
5 \\
4 \\
5\end{array}$ & $\begin{array}{l}30 \\
17 \\
16 \\
16 \\
29\end{array}$ & $\begin{array}{l}\mathbf{3} \\
\mathbf{3} \\
\mathbf{3} \\
\mathbf{3} \\
\mathbf{3}\end{array}$ & $\begin{array}{l}3.0 \\
3.0 \\
3.0 \\
2.8 \\
3.0\end{array}$ & $\begin{array}{l}3.0 \\
3.0 \\
3.0 \\
2.8 \\
2.6\end{array}$ & $\begin{array}{l}5.0 \\
4.0 \\
3.5 \\
3.0 \\
3.0\end{array}$ & $\begin{array}{l}2.6 \\
2.3 \\
2.3 \\
2.3 \\
2.1\end{array}$ & $\begin{array}{l}2.3 \\
2.3 \\
2.1 \\
2.1 \\
2.1\end{array}$ & $\begin{array}{l}29 \\
23 \\
17 \\
44 \\
33\end{array}$ & $\begin{array}{c}12 \\
10 \\
9.2 \\
8.0 \\
8.0\end{array}$ \\
\hline $\begin{array}{l}26 \ldots \ldots \ldots \\
27 \ldots \ldots \ldots \\
28 \ldots \ldots \ldots \\
29 \ldots \ldots \ldots \\
30 \ldots \ldots \ldots \ldots \\
31 \ldots \ldots \ldots \ldots\end{array}$ & $\begin{array}{l}4 \\
4 \\
4 \\
4 \\
5 \\
4\end{array}$ & 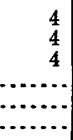 & $\begin{array}{r}\mathbf{3} \\
\mathbf{3} \\
\mathbf{5} \\
4 \\
4 \\
16\end{array}$ & $\begin{array}{l}15 \\
22 \\
14 \\
14 \\
11 \\
\ldots . .\end{array}$ & $\begin{array}{l}6 \\
4 \\
\mathbf{3} \\
\mathbf{3} \\
\mathbf{3} \\
\mathbf{3}\end{array}$ & $\begin{array}{l}4.0 \\
9.2 \\
6.0 \\
4.0 \\
7.0\end{array}$ & $\begin{array}{l}2.6 \\
2.6 \\
2.6 \\
2.5 \\
2.5 \\
2.5\end{array}$ & $\begin{array}{l}3.0 \\
3.5 \\
3.0 \\
2.8 \\
2.6 \\
3.0\end{array}$ & $\begin{array}{l}2.1 \\
2.1 \\
2.1 \\
2.1 \\
2.1\end{array}$ & $\begin{array}{l}2.1 \\
2.1 \\
2.1 \\
2.1 \\
1.9 \\
1.9\end{array}$ & $\begin{array}{l}36 \\
28 \\
20 \\
17 \\
13\end{array}$ & $\begin{array}{l}7.0 \\
6.5 \\
6.0 \\
6.0 \\
5.0 \\
5.0\end{array}$ \\
\hline
\end{tabular}


Monthly discharge of Hoolawanui Stream near Huelo, Maui, for 1913.

\begin{tabular}{|c|c|c|c|c|c|}
\hline \multirow{2}{*}{ Month. } & \multicolumn{3}{|c|}{ Discharge in second-feet. } & \multirow{2}{*}{$\begin{array}{c}\text { Run-off } \\
\text { (total in } \\
\text { acre-feet). }\end{array}$} & \multirow{2}{*}{$\begin{array}{l}\text { Accu } \\
\text { racy. }\end{array}$} \\
\hline & Maximum. & Minimum. & Mean. & & \\
\hline 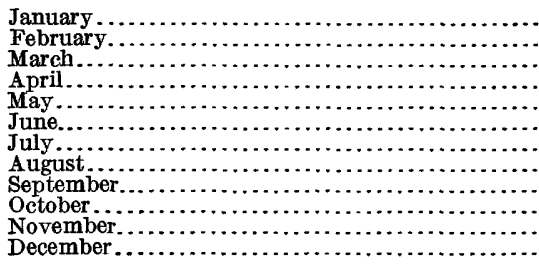 & $\begin{array}{l}71 \\
88 \\
16 \\
96 \\
9.0 \\
18 \\
32 \\
33 \\
9.2 \\
19 \\
94 \\
112\end{array}$ & $\begin{array}{l}4.0 \\
3.0 \\
2.0 \\
4.0 \\
3.0 \\
2.0 \\
2.5 \\
2.3 \\
2.1 \\
1.9 \\
1.9 \\
5.0\end{array}$ & $\begin{array}{r}12.1 \\
9.04 \\
4.39 \\
26.5 \\
4.90 \\
4.63 \\
6.44 \\
5.67 \\
2.68 \\
3.12 \\
29.7 \\
22.3\end{array}$ & $\begin{array}{r}744 \\
502 \\
270 \\
1,580 \\
301 \\
276 \\
396 \\
349 \\
159 \\
192 \\
1,770 \\
1,370\end{array}$ & $\begin{array}{l}\text { C. } \\
\text { C. } \\
\text { B. } \\
\text { B. } \\
\text { B. } \\
\text { B. } \\
\text { C. } \\
\text { B. } \\
\text { B. } \\
\text { B. } \\
\text { B. }\end{array}$ \\
\hline The year....... & 112 & 1.9 & 10.9 & 7,910 & \\
\hline
\end{tabular}

HOOLAWALIILII STREAM NEAR HUELO, MAUI.

Location.-About 400 feet above New Hamakua ditch crossing, $1 \frac{1}{2}$ miles east of Lupi, and about 4 miles above Huelo post office.

Records available.-April 5, 1911, to December 31, 1913.

Gage.-Vertical staff.

Control.-Probably permanent.

Discharge measurements.-Made by wading.

Diversions.-None above station.

Accuracy.-Records good.

Discharge measurements of Hoolawaliilii Stream near Huelo, Maui, in 1913.

\begin{tabular}{|c|c|c|c|c|c|c|c|}
\hline Date. & Made by- & $\begin{array}{c}\text { Gage } \\
\text { height. }\end{array}$ & $\begin{array}{l}\text { Dis- } \\
\text { charge. }\end{array}$ & Date. & Made by- & $\begin{array}{c}\text { Gage } \\
\text { height. }\end{array}$ & $\begin{array}{l}\text { Dis- } \\
\text { charge. }\end{array}$ \\
\hline $\begin{array}{l}\text { June } 6 . . . \\
\text { Aug. } 27 . .\end{array}$ & $\begin{array}{l}\text { C. T. Bailey........ } \\
\text { E. O. Christiansen. }\end{array}$ & $\begin{array}{c}\text { Feet. } \\
0.14 \\
.16\end{array}$ & $\begin{array}{r}\text { Sec.-ft. } \\
3.12 \\
3.66\end{array}$ & $\begin{array}{l}\text { Oct. } 2 . . . \\
\text { Nov. } 19 .\end{array}$ & $\begin{array}{l}\text { E. O. Christiansen. } \\
\text { C. T. Bailey......... }\end{array}$ & $\begin{array}{r}\text { Feet. } \\
0.11 \\
.51\end{array}$ & $\begin{array}{c}\text { Sec.-ft. } \\
2.30 \\
31.2\end{array}$ \\
\hline
\end{tabular}


Daily discharge, in second-feet, of Hoolawaliilii Stream near Huelo, Maui, for 1913.

\begin{tabular}{|c|c|c|c|c|c|c|c|c|c|c|c|c|}
\hline Day. & Jan. & Feb. & Mar. & Apr. & May. & June. & July. & Aug. & Sept. & Oct. & Nov. & Dec. \\
\hline $\begin{array}{l}1 \ldots \\
2 \ldots \\
3 \ldots \\
4 \ldots \\
5 \ldots\end{array}$ & $\begin{array}{l}7.0 \\
5.7 \\
4.9 \\
4.1 \\
3.4\end{array}$ & $\begin{array}{l}2.2 \\
2.2 \\
2.2 \\
1.8 \\
2.6\end{array}$ & $\begin{array}{l}1.8 \\
1.6 \\
1.6 \\
1.6 \\
1.6\end{array}$ & $\begin{array}{l}8.2 \\
3.4 \\
2.6 \\
2.6 \\
2.6\end{array}$ & $\begin{array}{l}4.1 \\
3.8 \\
3.8 \\
3.4 \\
3.4\end{array}$ & $\begin{array}{l}1.4 \\
1.3 \\
1.3 \\
2.2 \\
1.4\end{array}$ & $\begin{array}{c}8.2 \\
16 \\
8.2 \\
8.2 \\
8.2\end{array}$ & $\begin{array}{l}2.6 \\
2.6 \\
2.6 \\
2.6 \\
2.2\end{array}$ & $\begin{array}{l}3.0 \\
3.0 \\
3.4 \\
3.0 \\
2.6\end{array}$ & $\begin{array}{l}2.2 \\
2.2 \\
4.5 \\
2.6 \\
2.6\end{array}$ & $\begin{array}{l}2.2 \\
2.2 \\
4.9 \\
5.7 \\
4.1\end{array}$ & $\begin{array}{l}8.2 \\
7.6 \\
8.2 \\
41 \\
39\end{array}$ \\
\hline $\begin{array}{r}6 \ldots . \\
7 \ldots . \\
8 \ldots \\
9 \ldots \\
10 \ldots\end{array}$ & $\begin{array}{l}3.4 \\
3.0 \\
5.7 \\
5.7 \\
5.7\end{array}$ & $\begin{array}{c}36 \\
24 \\
3.4 \\
3.4 \\
2.6\end{array}$ & $\begin{array}{l}1.8 \\
1.6 \\
2.2 \\
1.6 \\
1.4\end{array}$ & $\begin{array}{l}2.2 \\
2.2 \\
1.8 \\
2.6 \\
1.8\end{array}$ & $\begin{array}{l}3.4 \\
8.0 \\
2.6 \\
2.6 \\
2.2\end{array}$ & $\begin{array}{l}1.4 \\
1.4 \\
1.4 \\
1.4 \\
1.8\end{array}$ & $\begin{array}{l}9.5 \\
7.0 \\
5.7 \\
5.7 \\
4.9\end{array}$ & $\begin{array}{l}2.6 \\
4.5 \\
2.6 \\
2.6 \\
3.0\end{array}$ & $\begin{array}{l}2.6 \\
3.0 \\
3.0 \\
3.4 \\
2.6\end{array}$ & $\begin{array}{l}3.0 \\
3.4 \\
9.5 \\
4.1 \\
3.4\end{array}$ & $\begin{array}{l}3.4 \\
3.0 \\
21 \\
30 \\
23\end{array}$ & $\begin{array}{l}36 \\
26 \\
24 \\
16 \\
11\end{array}$ \\
\hline $\begin{array}{l}11 \ldots . \\
12 \ldots \ldots \\
13 \ldots \ldots \\
14 \ldots \\
15 \ldots\end{array}$ & $\begin{array}{l}4.9 \\
4.1 \\
26 \\
28 \\
28\end{array}$ & $\begin{array}{l}2.6 \\
2.2 \\
2.2 \\
2.2 \\
2.2\end{array}$ & $\begin{array}{l}1.4 \\
1.4 \\
1.4 \\
1.4 \\
1.4\end{array}$ & $\begin{array}{l}32 \\
10 \\
34 \\
22 \\
37\end{array}$ & $\begin{array}{l}1.8 \\
1.8 \\
1.8 \\
2.2 \\
3.0\end{array}$ & $\begin{array}{l}1.4 \\
4.5 \\
5.7 \\
5.3 \\
4.9\end{array}$ & $\begin{array}{l}4.9 \\
4.1 \\
4.9 \\
4.5 \\
4.1\end{array}$ & $\begin{array}{c}2.6 \\
23 \\
5.7 \\
4.9 \\
11\end{array}$ & $\begin{array}{l}2.6 \\
2.6 \\
3.0 \\
2.6 \\
2.6\end{array}$ & $\begin{array}{l}2.6 \\
2.6 \\
7.0 \\
3.8 \\
3.4\end{array}$ & $\begin{array}{l}21 \\
37 \\
19 \\
14 \\
11\end{array}$ & $\begin{array}{c}8.8 \\
8.8 \\
8.2 \\
16 \\
11\end{array}$ \\
\hline $\begin{array}{l}16 \ldots \ldots \\
17 \ldots \ldots \\
18 \ldots . \\
19 \ldots \ldots \\
20 \ldots \ldots\end{array}$ & $\begin{array}{r}22 \\
8.8 \\
6.3 \\
5.3 \\
4.9\end{array}$ & $\begin{array}{r}2.2 \\
2.2 \\
5.7 \\
23 \\
3.4\end{array}$ & $\begin{array}{l}4.5 \\
3.4 \\
3.8 \\
2.2 \\
2.6\end{array}$ & $\begin{array}{l}34 \\
43 \\
26 \\
14 \\
12\end{array}$ & $\begin{array}{l}2.6 \\
2.6 \\
2.6 \\
1.8 \\
1.8\end{array}$ & $\begin{array}{l}3.4 \\
3.4 \\
1.8 \\
1.6 \\
1.6\end{array}$ & $\begin{array}{l}4.1 \\
3.8 \\
3.4 \\
3.4 \\
3.4\end{array}$ & $\begin{array}{l}7.0 \\
4.9 \\
4.9 \\
4.5 \\
4.1\end{array}$ & $\begin{array}{l}2.6 \\
3.4 \\
5.7 \\
3.8 \\
3.4\end{array}$ & $\begin{array}{l}3.4 \\
3.4 \\
3.0 \\
3.0 \\
3.0\end{array}$ & $\begin{array}{l}11 \\
9.5 \\
23 \\
32 \\
21\end{array}$ & $\begin{array}{c}8.2 \\
8.8 \\
16 \\
8.2 \\
8.2\end{array}$ \\
\hline $\begin{array}{l}21 \ldots \ldots \ldots \\
22 \ldots \ldots \ldots \\
23 \ldots \ldots \ldots \\
24 \ldots \ldots\end{array}$ & $\begin{array}{l}4.1 \\
4.1 \\
3.4 \\
3.4 \\
3.4\end{array}$ & $\begin{array}{l}3.0 \\
2.6 \\
2.2 \\
2.2 \\
1.8\end{array}$ & $\begin{array}{l}2.6 \\
4.9 \\
2.6 \\
2.2 \\
2.6\end{array}$ & $\begin{array}{c}13 \\
11 \\
8.2 \\
7.6 \\
16\end{array}$ & $\begin{array}{l}1.6 \\
1.4 \\
1.4 \\
1.6 \\
1.4\end{array}$ & $\begin{array}{l}1.6 \\
1.6 \\
1.6 \\
1.6 \\
1.8\end{array}$ & $\begin{array}{l}3.4 \\
3.4 \\
3.4 \\
3.4 \\
3.4\end{array}$ & $\begin{array}{l}4.5 \\
4.1 \\
4.1 \\
3.4 \\
3.4\end{array}$ & $\begin{array}{l}3.0 \\
3.0 \\
3.0 \\
2.6 \\
2.6\end{array}$ & $\begin{array}{l}3.0 \\
2.6 \\
2.6 \\
2.6 \\
2.6\end{array}$ & $\begin{array}{l}16 \\
13 \\
11 \\
16 \\
15\end{array}$ & $\begin{array}{l}7.6 \\
7.0 \\
5.7 \\
5.3 \\
4.9\end{array}$ \\
\hline $\begin{array}{l}26 \ldots \ldots \ldots \\
27 \ldots \ldots \\
28 \ldots \ldots \\
29 \ldots \ldots \\
30 \ldots \ldots \\
31 \ldots \ldots\end{array}$ & $\begin{array}{l}3.4 \\
3.4 \\
2.6 \\
2.6 \\
2.2 \\
2.2\end{array}$ & 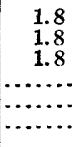 & $\begin{array}{l}1.8 \\
1.8 \\
3.0 \\
2.6 \\
2.6 \\
9.5\end{array}$ & $\begin{array}{c}10 \\
8.2 \\
5.7 \\
5.3 \\
4.1\end{array}$ & $\begin{array}{l}2.6 \\
1.8 \\
1.6 \\
1.4 \\
1.4 \\
1.4\end{array}$ & $\begin{array}{l}1.8 \\
5.3 \\
4.9 \\
3.4 \\
7.6\end{array}$ & $\begin{array}{l}3.0 \\
3.0 \\
2.6 \\
2.6 \\
2.6 \\
2.6\end{array}$ & $\begin{array}{l}3.4 \\
3.8 \\
3.4 \\
3.4 \\
3.4 \\
4.1\end{array}$ & $\begin{array}{r}2.6 \\
2.6 \\
2.6 \\
2.6 \\
2.6 \\
\cdots \cdots\end{array}$ & $\begin{array}{l}2.6 \\
2.6 \\
2.2 \\
2.2 \\
2.2 \\
2.2\end{array}$ & $\begin{array}{l}21 \\
17 \\
13 \\
11 \\
9.5\end{array}$ & $\begin{array}{l}4.5 \\
4.5 \\
4.1 \\
4.1 \\
3.8 \\
3.8\end{array}$ \\
\hline
\end{tabular}

Monthly discharge of Hoolawaliilii Stream near Huelo, Maui, for 1913.

\begin{tabular}{|c|c|c|c|c|c|}
\hline \multirow{2}{*}{ Month. } & \multicolumn{3}{|c|}{ Discharge in second-feet. } & \multirow{2}{*}{$\begin{array}{c}\text { Run-off } \\
\text { (total in } \\
\text { acre-feet). }\end{array}$} & \multirow{2}{*}{$\begin{array}{l}\text { Accu- } \\
\text { racy. }\end{array}$} \\
\hline & Maximum. & Minimum. & Mean. & & \\
\hline 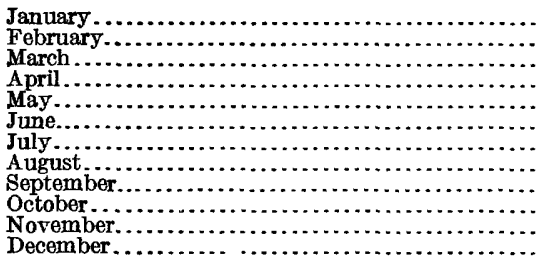 & $\begin{array}{c}28 \\
36 \\
9.5 \\
43 \\
4.1 \\
7.6 \\
16 \\
23 \\
5.7 \\
9.5 \\
37 \\
41\end{array}$ & $\begin{array}{l}2.2 \\
1.8 \\
1.4 \\
1.8 \\
1.4 \\
1.3 \\
2.6 \\
2.2 \\
2.6 \\
2.2 \\
2.2 \\
3.8\end{array}$ & $\begin{array}{r}7.15 \\
5.20 \\
2.47 \\
12.8 \\
2.32 \\
2.66 \\
5.02 \\
4.56 \\
2.97 \\
3.23 \\
14.7 \\
12.1\end{array}$ & $\begin{array}{l}440 \\
289 \\
152 \\
762 \\
143 \\
158 \\
309 \\
280 \\
177 \\
199 \\
875 \\
744\end{array}$ & $\begin{array}{l}\text { B. } \\
\text { B. } \\
\text { B. } \\
\text { C. } \\
\text { B. } \\
\text { B. } \\
\text { B. } \\
\text { B. } \\
\text { B. } \\
\text { B. } \\
\text { B. }\end{array}$ \\
\hline The year............. & 43 & 1.3 & 6.25 & 4,530 & \\
\hline
\end{tabular}

\section{HONOPOU STREAM NEAR HUELO, MAUI.}

Location.-Three-fourths mile northwest of Lupi and about $3 \frac{1}{2}$ miles southwest of Huelo; 200 feet above New Hamakua ditch crossing.

Records available.-December 10, 1910, to December 31, 1913.

Gage.-Vertical staff.

Control.-Probably permanent.

Discharge measurements.-Made by wading.

Diversions.-None above station.

Accuracy.-Records good.

Cooperation.-Station maintained in cooperation with East Maui Ditch Co. 
Discharge measurements of Honopou Stream near Huelo, Maui, in 1913.

\begin{tabular}{|c|c|c|c|c|c|c|c|}
\hline Date. & Made by- & $\begin{array}{c}\text { Gage } \\
\text { height. }\end{array}$ & Discharge. & Date. & Made by- & $\begin{array}{c}\text { Gage } \\
\text { height. }\end{array}$ & $\begin{array}{c}\text { Dis- } \\
\text { charge. }\end{array}$ \\
\hline $\begin{array}{l}\text { June } 5 \ldots \\
\text { Aug. } 27 .\end{array}$ & $\begin{array}{l}\text { C. T. Bailey ........ } \\
\text { E. } 0 . \text { Christiansen. }\end{array}$ & $\begin{array}{r}\text { Feet. } \\
0.10 \\
.20\end{array}$ & $\begin{array}{r}\text { Sec. ftt. } \\
1.55 \\
3.01\end{array}$ & Nov. 19. & C.T.Bailey. & $\begin{array}{l}\text { Feet. } \\
0.79\end{array}$ & Sec.-ft. \\
\hline
\end{tabular}

Daily discharge, in second-feet, of Honopou Stream near Huelo, Maui, for 1912-13.

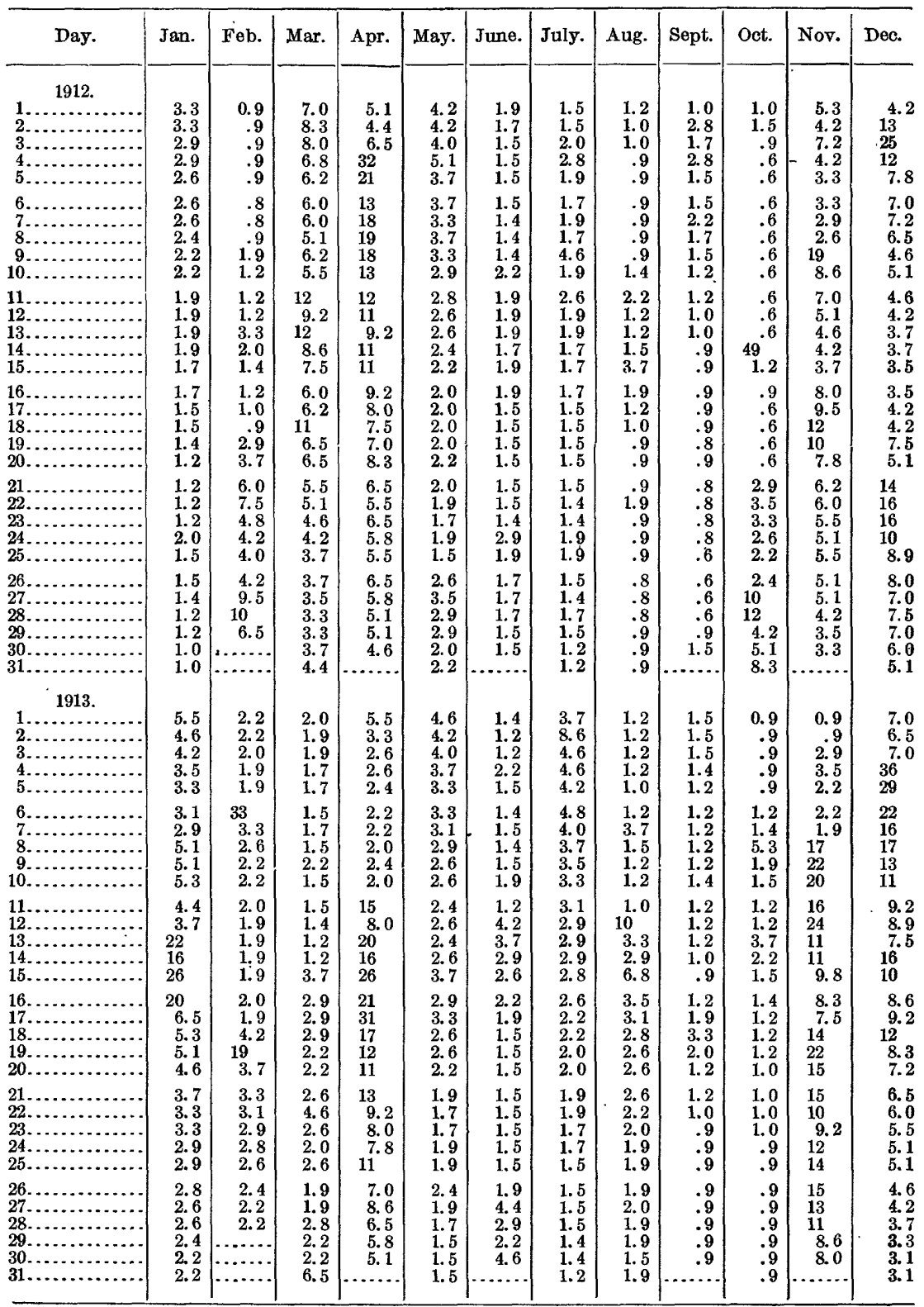


Monthly discharge of Honopou Stream near Huelo, Maui, for 1912-13.

\begin{tabular}{|c|c|c|c|c|c|}
\hline \multirow{2}{*}{ Month. } & \multicolumn{3}{|c|}{ Discharge in second-feet. } & \multirow{2}{*}{$\begin{array}{r}\text { Run-off } \\
\text { (total in } \\
\text { acre-feet). }\end{array}$} & \multirow{2}{*}{$\begin{array}{l}\text { Accu- } \\
\text { racy. }\end{array}$} \\
\hline & Maximum. & Minimum. & Mean. & & \\
\hline 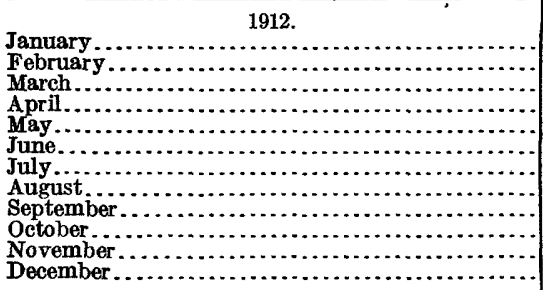 & $\begin{array}{l}3.3 \\
10 \\
12 \\
32 \\
5.1 \\
2.9 \\
4.6 \\
3.7 \\
2.8 \\
49 \\
19 \\
25\end{array}$ & $\begin{array}{r}1.0 \\
.8 .8 \\
3.3 \\
4.4 \\
1.5 \\
1.4 \\
1.2 \\
.8 \\
.6 \\
. .6 \\
2.6 \\
3.5\end{array}$ & $\begin{array}{r}1.87 \\
2.95 \\
6.31 \\
10.0 \\
2.77 \\
1.68 \\
1.79 \\
1.17 \\
1.18 \\
3.85 \\
6.07 \\
7.81\end{array}$ & $\begin{array}{c}115 \\
170 \\
388 \\
595 \\
170 \\
100 \\
110 \\
71.9 \\
70.2 \\
237 \\
361 \\
480\end{array}$ & $\begin{array}{l}\text { B. } \\
\text { B. } \\
\text { B. } \\
\text { A. } \\
\text { B. } \\
\text { B. } \\
\text { B. } \\
\text { B. } \\
\text { C. } \\
\text { C. } \\
\text { B. } \\
\text { B. }\end{array}$ \\
\hline The year...... & 49 & 0.6 & 3. 95 & 2,870 & \\
\hline 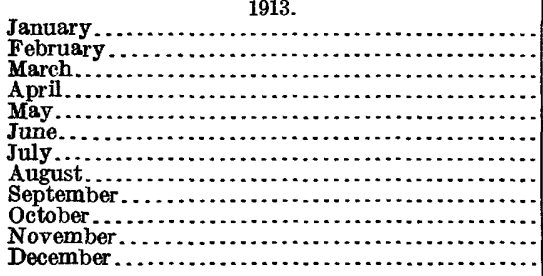 & $\begin{array}{l}26 \\
33 \\
6.5 \\
31 \\
4.6 \\
4.6 \\
8.6 \\
10 \\
3.3 \\
5.3 \\
24 \\
36\end{array}$ & $\begin{array}{r}2.2 \\
1.9 \\
1.2 \\
2.0 \\
1.5 \\
1.2 \\
1.2 \\
1.0 \\
.9 \\
.9 \\
3.9\end{array}$ & $\begin{array}{l}6.04 \\
4.12 \\
2.31 \\
9.54 \\
2.62 \\
2.06 \\
2.83 \\
2.42 \\
1.26 \\
1.35 \\
10.9 \\
10.1\end{array}$ & $\begin{array}{r}371 \\
229 \\
142 \\
568 \\
161 \\
123 \\
174 \\
149 \\
75 \\
83 \\
649 \\
621\end{array}$ & $\begin{array}{l}\text { B. } \\
\text { C. } \\
\text { B. } \\
\text { B. } \\
\text { B. } \\
\text { B. } \\
\text { B. } \\
\text { B. } \\
\text { B. } \\
\text { B. } \\
\text { A. } \\
\text { A. }\end{array}$ \\
\hline The year. & 36 & 0.9 & 4. 62 & 3,340 & \\
\hline
\end{tabular}

\section{MISCELLANEOUS MEASUREMENTS.}

Measurements of streams on the island of Maui at points other than regular gaging stations are listed below:

Miscellaneous discharge measurements on Maui for 1913.

\begin{tabular}{|c|c|c|c|c|}
\hline Date. & Stream. & Locality. & $\begin{array}{c}\text { Gage } \\
\text { height. }\end{array}$ & $\begin{array}{l}\text { Dis- } \\
\text { charge. }\end{array}$ \\
\hline $\begin{array}{lr}\text { Mar. } & 15 \\
\text { Apr. } & 9 \\
& 9\end{array}$ & 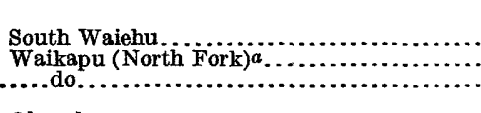 & $\begin{array}{l}\text { Wailuku. } \\
\text { old shops below tumnei portal } \\
300 \text { feet above junction with } \\
\text { South Fork. }\end{array}$ & $\begin{array}{c}\text { Feet. } \\
0.60 \\
0 .\end{array}$ & $\begin{array}{r}\text { Sec. } f t . \\
0.69 \\
2.68 \\
2.73\end{array}$ \\
\hline $\begin{array}{l}16 \\
16\end{array}$ & Olowalu & Olowalu......... & .89 & 51.6 \\
\hline June $\begin{array}{r}16 \\
5\end{array}$ & 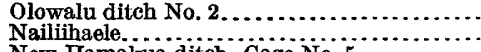 & Hi..dolo.... & $\begin{array}{r}1.35 \\
.81\end{array}$ & $\begin{array}{l}16.9 \\
2.16\end{array}$ \\
\hline $\begin{array}{l}7 \\
7 \\
7 \\
7\end{array}$ & $\begin{array}{l}\text { New Hamakua ditch, Gage No. } 5 \\
\text { New Hamakua ditch, Gage No. 4. } \\
\text { New Hamakua ditch, Gage No. 3. } \\
\text { New Hamakua ditch, Gage No. } 1 .\end{array}$ & $\begin{array}{l}\ldots \text { do } \\
\ldots \text { do }\end{array}$ & $\begin{array}{l}4.80 \\
4.65 \\
2.30 \\
2.80\end{array}$ & $\begin{array}{l}42.4 \\
37.0 \\
31.6 \\
34.3\end{array}$ \\
\hline 7 & New Hamakua ditch. . & Halehaku Weir, Huelo. & .86 & 73.0 \\
\hline $\begin{array}{l}\text { June } 18 \\
\text { July } 21 \\
21\end{array}$ & $\begin{array}{l}\text { Halehaku............ } \\
\text { Lower Springs. . . . } \\
\text { Upper Springs..... }\end{array}$ & Kalepo Gulch, near Kaupo... & .25 & $\begin{array}{r}2.63 \\
.44 \\
.22\end{array}$ \\
\hline 21 & Manawamui... & $\begin{array}{l}\text { Below large springs, n e a } r \\
\text { Kaupo. }\end{array}$ & & 2.03 \\
\hline 21 & .....do... & $\begin{array}{l}\text { A bove large springs, } n \text { e a r } \\
\text { Kaupo. }\end{array}$ & & 1.22 \\
\hline $\begin{array}{ll}\text { July } & 22 \\
22\end{array}$ & $\begin{array}{l}\text { Alelele... } \\
\text { Oheo }\end{array}$ & $\begin{array}{l}\text { Near Kipahulu............... } \\
\text { Wooden bridge near Kipahulu. }\end{array}$ & & $\begin{array}{r}.36 \\
1.17\end{array}$ \\
\hline 22 & $\begin{array}{l}\text { Puaaluu..... } \\
\text { Halalawe... }\end{array}$ & 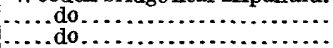 & $\cdots \cdots$ & $\begin{array}{l}2.24 \\
1.86\end{array}$ \\
\hline 22 & Honolewa...... & do. & & 1.29 \\
\hline 23 & Makapipi. & Near Keanä... & $\ldots$ & $\begin{array}{l}1.88 \\
1.71\end{array}$ \\
\hline $\begin{array}{r}23 \\
\text { Sept } 30\end{array}$ & East Wäluaiki. & At do $\ldots \ldots \ldots \ldots \ldots$ & & 7.00 \\
\hline $\begin{aligned} & \text { Sept. } 30 \\
& 30\end{aligned}$ & $\begin{array}{l}\text { Lahaunaluna ditch.. } \\
\ldots . . . \text { do............... }\end{array}$ & $\begin{array}{l}\text { At Intake near Lahaina ....... } \\
\text { Above Intake near Lahaina... }\end{array}$ & & $\begin{array}{l}2.76 \\
4.48\end{array}$ \\
\hline $\begin{array}{l}\text { Apr. } 14 \\
\text { Aug. } 19\end{array}$ & $\begin{array}{l}\text { Kahoma. } \\
\text {.....do... }\end{array}$ & Near Lahaina..................................... & $\begin{array}{r}1.16 \\
.62\end{array}$ & $\begin{array}{r}9.21 \\
.19\end{array}$ \\
\hline
\end{tabular}

a Tributary to Waikapu Stream. 
Miscellaneous discharge measurements on Maui for 1913-Continued.

\begin{tabular}{|c|c|c|c|c|}
\hline Date. & Stream. & Locality. & $\begin{array}{c}\text { Gage } \\
\text { height. }\end{array}$ & $\begin{array}{l}\text { Dis- } \\
\text { charge. }\end{array}$ \\
\hline Apr. 15 & Kauaula. & Near Labaina. & Feet. & Sec.-ft. \\
\hline Api. 29 & .....do.a.. & .....do......... & $\begin{array}{r}1.00 \\
.96\end{array}$ & $\begin{array}{l}00.4 \\
10.8\end{array}$ \\
\hline May 14 & .....do. & .....do.. & 1.44 & 40.6 \\
\hline Aug. 19 & ....do do...... & ....do... & 1.04 & 8.84 \\
\hline Apr. 16 & Ukumehame....... & Near Olowalu................ & 1.40 & 72.8 \\
\hline $\begin{array}{l}21 \\
22\end{array}$ & $\ldots \ldots$ do $\ldots . \ldots \ldots \ldots \ldots$ & 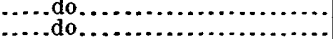 & $\begin{array}{r}.72 \\
1.15\end{array}$ & $\begin{array}{l}11.6 \\
11.8\end{array}$ \\
\hline May 15 & .....do... & ....do..................... & 1.38 & 36.6 \\
\hline Aug. 20 & ....do. do. & $\ldots$ do $\ldots \ldots . . . .$. & .84 & 6.37 \\
\hline $\begin{array}{l}\text { June } 18 \\
\text { Nov. } 18\end{array}$ & Opana ............. & Near Huelo...................... & $\begin{array}{l}1.81 \\
6.02\end{array}$ & $554^{.29}$ \\
\hline & ....do.................. & . & 3.61 & 32 \\
\hline
\end{tabular}

$a$ New gage established May $15,1913 . \quad b$ New datum; gage moved to better location.

\section{ISLAND OF HAWAII.}

\section{GENERAL CONDITIONS.}

The perennial streams of the Island of Hawaii are mainly included in two groups, one comprising an area about 10 miles wide, in the Kohala and Hamakua districts, and the other an area about 16 miles wide in the Hilo district between the South Branch of Wailuku River on the southeast and Maulua Gulch on the northwest.

The low water run-off from the Kohala and Hamakua districts is now practically all diverted into ditches serving the sugar lands of the north coast of the island. Only a small part of the mean run-off from the Hilo district is now being utilized, the greater part being allowed to waste into the sea.

It has been proposed to construct a high-line canal at an elevation of about 2,700 feet and carry this water to serve either the semiarid lands of Kau on the south side of the island, or the dry government lands in the vicinity of Waimea which are now being leased for cattle range.

In September, 1911, a concession was sought, and later granted, by a promotor for the construction of what was generally known as the Kau ditch project, and at the request of the territorial officials the work of investigating the run-off of the Hilo district along the 2,700-foot contour was begun. In September, October, and November, 1911, a foot trail was cut through the swamp and jungle from the South Branch of Wailuku River to the North Branch of Kawainui River, a distance of about 8 miles by trail. Staff gages were established on all appreciable channels, whether of perennial discharge or not.

Eighty-three stations were established and a camp was built about midway on the trail. Lack of funds prohibited further extension of the work and the construction of bridges or cables across the large gulches with the result that the larger streams could not be crossed when in flood. The trail was merely a passage cut through the 
jungle, over and under logs, in and out of gulches, and, for the greater part, from a few inches to several feet deep in swamp muck. Two gage readers were employed who lived in the midway camp and who went over the trail once a day on an average of about four days in the week and read the gages-one man working south and the other north and return. The condition of the trail was such that about eight hours were necessary to cover the 8-mile trip. All supplies for the camp and work were packed in on men's backs from the end of the Kaiwiki homestead road, a distance of about $3 \frac{1}{2}$ miles. One day of the week was allowed the observers for packing in supplies, and one day for rest. Up until May, 1913, when funds became available for the construction of cableways across the larger gulches, many daily records were lost on account of the observers being unable to cross these gulches when in flood. On account of the flashy type of the streams it was found that two readings daily, within a few hours of each other, gave a very poor index of the daily mean gage heights of the streams.

In the spring of 1913 it became apparent that the Kau ditch project would not soon be completed, and this fact, coupled with the high cost of obtaining the unsatisfactory and meager data on surface fluctuation, led to the suspension of the work until funds would be available to construct a dry trail across the entire district, to establish water-stage recorders on the larger streams, and to improve cross sections, controls, and other features. The work was suspended on July 18, 1913.

Ten rain gages read at monthly and bimonthly periods were established at approximately 500-foot elevation intervals from sea level to 5,000 feet, the approximate elevation at which the wet, or forest, zone meets the arid lava slopes of Mauna Kea Mountain. Practically all rainfall above the 5,000-foot contour is absorbed by the porous lava. Rainfall observations were terminated on June 30 , 1913.

\section{HILO GROUP.}

\section{STATIONS AT 2,700-FOOT LEVEL.}

Location.-On trail in forest back of Hilo, at approximately 2,700 feet above sea level. The trail is about 8 miles long and the camp, about midway on the trail, is reached by a foot trail $3 \frac{1}{2}$ miles long from the end of the Kaiwiki homestead road. The camp is about 7 miles west-northwest from Hilo. Beginning at the South Branch of Wailuku River, the stations are numbered from 1 to 18 and from 25 to 87, including stations Nos. 12a and 86a, to the North Branch of Kawainui River. There are no stations numbered 19 to 24 .

Records available.-Stations 1-12, October 6, 1911, to July 18, 1913. Station 12a, November 30, 1912, to July 18, 1913. Stations 13-18, 25-27, October 5, 1911, to July 18, 1913. Stations 28-41, September 28, 1911, to July 18, 1913 . Stations 42-47, September 28, 1911, to July 18, 1913. Stations 48-50, September 31, 1911, to July 18, 1913. Stations 51-52, October 7, 1911, to July 18, 1913. Stations 
53-65, October 10, 1911, to July 19, 1913. Stations 66-68, October 21, 1911, to July 18, 1913. Stations 69-74, November 13, 1911, to July 18, 1913 . Stations $75-86$, November 14, 1911, to July 18, 1913. Station 86 a, November 18, 1912, to July 18, 1913. Station 87, November 14, 1911, to July 18, 1913.

Drainage area.-About 75 square miles for all stations (may be in error 20 per cent). Gages.-Vertical staff read to nearest two-hundredths, twice daily, about 4 days in the week.

Diversions.-All low-water run-off is diverted below the stations, but it is estimated that less than 20 per cent of the mean discharge is being utilized; no diversions above the stations.

Accuracy.-At only 24 of the 83 stations maintained is the discharge sufficient to warrant current-meter measurements and ratings; mean discharge at each of the other 59 stations is one-half second-foot or less; the aggregate mean run-off of these 59 stations for the period January 1 to June 18, 1913, is estimated to have been only 4.2 second-feet. As the mean discharge of the 24 streams rated was 194.0 second-feet, the mean run-off for the 59 estimated stations was less than 2 per cent of the total. For these stations for which ratings have been made and results published rating curves are all fairly well defined for low and medium stages. Discharge for days for which there are no gage-height records was estimated from daily rainfall records at camp near station No. 51.

The total mean run-off for all stations for the period January 1 to July 18, 1913, was 198.2 second-feet. The minimum run-off, which was less than 6 second-feet, occurred in March. The maximum run-off of all stations probably exceeded 5,000 second-feet.

Discharge measurements made at unrated stations on 2,700-foot level near Hilo, Hawaï, during the period Jan. 1 to July 18, 1913.

[E. O. Christiansen, hydrographer.]

\begin{tabular}{|c|c|c|c|c|c|c|c|}
\hline Station No. & Date. & $\begin{array}{c}\text { Gage } \\
\text { height. }\end{array}$ & $\begin{array}{c}\text { Dis- } \\
\text { charge. }\end{array}$ & Station No. & Date. & $\begin{array}{c}\text { Gage } \\
\text { height. }\end{array}$ & $\begin{array}{c}\text { Dis- } \\
\text { charge. }\end{array}$ \\
\hline $\begin{array}{l}39 \ldots \ldots \ldots \ldots \ldots \\
41 \ldots \ldots \ldots \ldots \ldots \\
49 \ldots \ldots \ldots \ldots \ldots \\
50 a \ldots \ldots \ldots\end{array}$ & 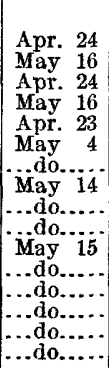 & $\begin{array}{r}\text { Feet. } \\
0.90 \\
.96 \\
.49 \\
1.00 \\
.93 \\
2.38 \\
2.28 \\
2.22 \\
2.24 \\
2.28 \\
2.23 \\
2.26 \\
2.28 \\
2.30 \\
2.32 \\
2.34\end{array}$ & $\begin{array}{r}\text { Sec. } f t . \\
0.23 \\
.44 \\
.80 \\
2.4 \\
.83 \\
1.22 \\
. .12 \\
.06 \\
.08 \\
.11 \\
.07 \\
.10 \\
.12 \\
.12 \\
.13 \\
.18\end{array}$ & $\begin{array}{l}55 \ldots \ldots \ldots \\
59 \ldots \ldots \ldots \ldots \\
70 \ldots \ldots \ldots \\
73 \ldots \ldots \ldots\end{array}$ & 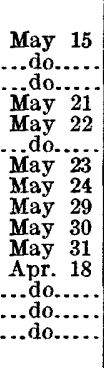 & $\begin{aligned} \text { Feet. } \\
2.36 \\
2.38 \\
2.39 \\
2.19 \\
2.18 \\
2.17 \\
2.16 \\
2.14 \\
2.24 \\
2.20 \\
2.25 \\
1.22 \\
.81 \\
.99 \\
.90\end{aligned}$ & $\begin{array}{r}\text { Sec. } f t . \\
0.21 \\
.23 \\
.25 \\
.04 \\
.03 \\
.03 \\
.03 \\
.02 \\
.07 \\
.05 \\
.08 \\
1.03 \\
.77 \\
1.19 \\
.48\end{array}$ \\
\hline
\end{tabular}

a All measurements on station No. 50 made by the volumetric method.

Discharge measurements of station No. 1 at 2,700-foot level near Hilo, Hawaii, in 1918.

[E. O. Christiansen, hydrographer.]

\begin{tabular}{|c|c|c|c|}
\hline Date. & . & $\begin{array}{c}\text { Gage } \\
\text { height. }\end{array}$ & $\begin{array}{c}\text { Dis- } \\
\text { charge. }\end{array}$ \\
\hline $\begin{array}{l}\text { Apr. } 29 \ldots \ldots \ldots \ldots \ldots \ldots \ldots \ldots \\
\text { May } 24 \ldots \ldots \ldots \ldots \ldots \ldots \ldots \ldots \ldots\end{array}$ & & $\begin{array}{l}\text { Feet. } \\
-0.05 \\
-.50\end{array}$ & $\begin{array}{r}\text { Sec.-ft. } \\
\quad .5 \\
.96\end{array}$ \\
\hline
\end{tabular}


Monthly discharge of station No. 1 at 2,700-foot level near Hilo, Hawaii, for 1913.

\begin{tabular}{|c|c|c|c|c|c|}
\hline \multirow{2}{*}{ Month. } & \multicolumn{3}{|c|}{ Discharge in second-feet. } & \multirow{2}{*}{$\begin{array}{l}\text { Run-off } \\
\text { (total in } \\
\text { acre-feet). }\end{array}$} & \multirow{2}{*}{$\begin{array}{l}\text { Accu- } \\
\text { racy. }\end{array}$} \\
\hline & Maximum. & Minimum. & Mean. & & \\
\hline \multirow{2}{*}{ 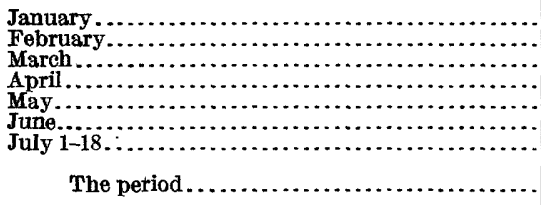 } & $\begin{array}{c}48 \\
12 \\
160 \\
28 \\
146 \\
60\end{array}$ & $\begin{array}{ll}(a) & \\
(a) & \\
& .1 \\
.4 & .6 \\
& .6\end{array}$ & $\begin{array}{c}19.4 \\
3.63 \\
.07 \\
18.6 \\
5.14 \\
25.9 \\
29.5\end{array}$ & $\begin{array}{r}1,190 \\
130 \\
1 \\
1,110 \\
316 \\
1,540 \\
1,050\end{array}$ & \multirow[t]{2}{*}{$\begin{array}{l}\mathrm{D} . \\
\mathrm{D} . \\
\mathrm{D} . \\
\mathrm{D} . \\
\mathrm{D} . \\
\mathrm{D} . \\
\mathrm{D} .\end{array}$} \\
\hline & .. & $\ldots \ldots \ldots$ & $\ldots \ldots$ & 5,340 & \\
\hline
\end{tabular}

a No flow.

Discharge measurements of station No. $s$ at 2,700-foot level near Hilo, Hawaii, in 1913.

[E. O. Christiansen, hydrographer.]

\begin{tabular}{|c|c|c|}
\hline Date. & $\begin{array}{c}\text { Gage } \\
\text { height. }\end{array}$ & $\begin{array}{l}\text { Dis- } \\
\text { charge. }\end{array}$ \\
\hline 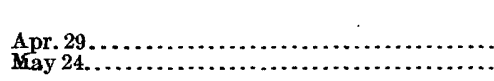 & $\begin{array}{r}\text { Feet. } \\
0.40 \\
.11\end{array}$ & $\begin{array}{r}\text { Sec. } f t . \\
7.02 \\
3.16\end{array}$ \\
\hline
\end{tabular}

Monthly discharge of station No. 3 at 2,700-foot level near Hilo, Hawaii, for 1913.

\begin{tabular}{|c|c|c|c|c|c|}
\hline \multirow{2}{*}{ Month. } & \multicolumn{3}{|c|}{ Discharge in second-feet. } & \multirow{2}{*}{$\begin{array}{c}\text { Run-off } \\
\text { (total in } \\
\text { acre-feet). }\end{array}$} & \multirow{2}{*}{$\begin{array}{l}\text { Accu- } \\
\text { racy. }\end{array}$} \\
\hline & Maximum. & Minimum. & Mean. & & \\
\hline 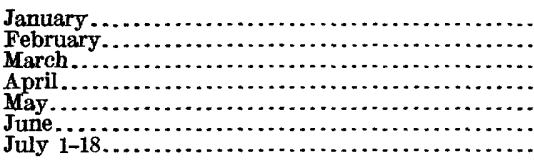 & $\begin{array}{l}15 \\
5.5 \\
5.8 \\
68 \\
11 \\
53 \\
32\end{array}$ & $\begin{array}{l}(a) \\
(a) \\
\\
4.7 \\
4.7 \\
2.5 \\
2.9 \\
2.7\end{array}$ & $\begin{array}{l}10.0 \\
.75 \\
1.74 \\
13.4 \\
4.70 \\
11.8 \\
13.4\end{array}$ & $\begin{array}{r}615 \\
42 \\
107 \\
797 \\
289 \\
702 \\
477\end{array}$ & $\begin{array}{l}\text { D. } \\
\text { D. } \\
\text { D. } \\
\text { D. } \\
\text { D. } \\
\text { D. }\end{array}$ \\
\hline The period. & & & $\ldots$ & 3,030 & \\
\hline
\end{tabular}

$a$ No flow.

Discharge measurements of station No. 12 at 2,700-foot level near Hilo, Hawaii, in 1913.

[E. O. Christiansen, hydrographer.]

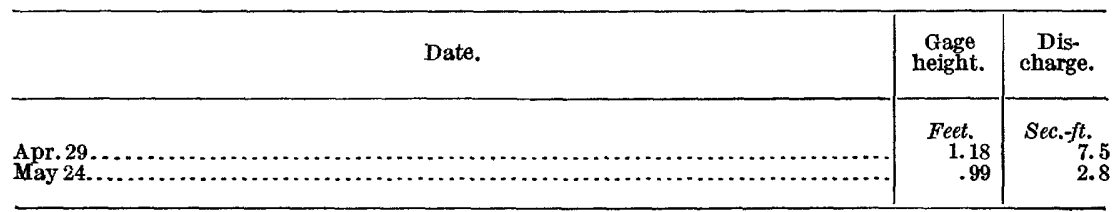


Monthly discharge of station No. 12 at 2,\%00-foot level near Hilo, Hawaii, for 1913.

\begin{tabular}{|c|c|c|c|c|c|}
\hline \multirow{2}{*}{ Month. } & \multicolumn{3}{|c|}{ Discharge in second-feet. } & \multirow{2}{*}{$\begin{array}{l}\text { Run-off } \\
\text { (total in } \\
\text { acre-feet). }\end{array}$} & \multirow{2}{*}{$\begin{array}{l}\text { Accu- } \\
\text { racy. }\end{array}$} \\
\hline & Maximum. & Minimum. & Mean. & & \\
\hline \multirow{2}{*}{ 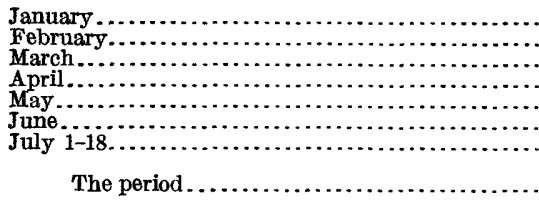 } & $\begin{array}{c}34 \\
2.8 \\
6.1 \\
63 \\
6.1 \\
57 \\
53\end{array}$ & $\begin{array}{r}2.8 \\
.3 \\
.3 \\
3.0 \\
2.0 \\
2.3 \\
2.2\end{array}$ & $\begin{array}{c}12.5 \\
1.03 \\
1.47 \\
12.0 \\
3.48 \\
10.9 \\
13.3\end{array}$ & $\begin{array}{r}769 \\
57 \\
90 \\
714 \\
214 \\
649 \\
474\end{array}$ & \multirow[t]{2}{*}{$\begin{array}{l}\text { D. } \\
\text { D. } \\
\text { D. } \\
\text { D. } \\
\text { D. } \\
\text { D. } \\
\text { D. }\end{array}$} \\
\hline & & & & 2,970 & \\
\hline
\end{tabular}

Discharge measurements of station No. 13 at 2,700-foot level near Hilo, Hawaii, in 1913.

[E. O. Christiansen, hydrographer.]

\begin{tabular}{|c|c|c|}
\hline Date. & $\begin{array}{c}\text { Gage } \\
\text { height. }\end{array}$ & $\begin{array}{c}\text { Dis- } \\
\text { charge. }\end{array}$ \\
\hline $\begin{array}{l}\text { Apr. } 29 \ldots \ldots \ldots \ldots \\
\text { May } 24 . \ldots \ldots \ldots \ldots\end{array}$ & $\begin{array}{l}\text { Feet. } \\
\text { 1. } 05 \\
.74\end{array}$ & $\begin{aligned} & \text { Sec. ft. } \\
& 9.2 \\
& 2.25\end{aligned}$ \\
\hline
\end{tabular}

Monthly discharge of station No. 13 at 2,700-foot level near Hilo, Hawaii, for 1913.

\begin{tabular}{|c|c|c|c|c|c|}
\hline \multirow{2}{*}{ Month. } & \multicolumn{3}{|c|}{ Discharge in second-feet. } & \multirow{2}{*}{$\begin{array}{l}\text { Run-off } \\
\text { (total in } \\
\text { acre-feet). }\end{array}$} & \multirow{2}{*}{$\begin{array}{l}\text { Accu- } \\
\text { racy. }\end{array}$} \\
\hline & Maximum. & Minimum. & Mean. & & \\
\hline \multirow{2}{*}{ 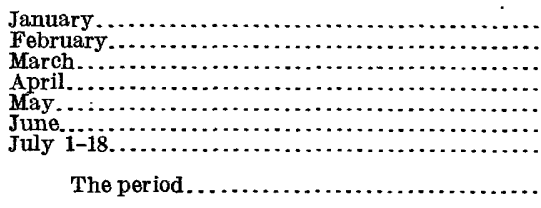 } & $\begin{array}{c}38 \\
3.9 \\
11 \\
54 \\
9.4 \\
47 \\
44\end{array}$ & $\begin{array}{r}4.5 \\
.2 \\
(a) \\
5.1 \\
2.1 \\
3.2 \\
1.9\end{array}$ & $\begin{array}{c}12.8 \\
1.09 \\
2.94 \\
12.8 \\
4.62 \\
11.3 \\
13.6\end{array}$ & $\begin{array}{r}787 \\
60 \\
181 \\
762 \\
284 \\
672 \\
483\end{array}$ & \multirow[t]{2}{*}{$\begin{array}{l}\text { D. } \\
\text { D. } \\
\text { D. } \\
\text { D. } \\
\text { D. } \\
\text { D. } \\
\text { D. }\end{array}$} \\
\hline & $\cdots$ & $\ldots \ldots$ & .......... & 3,230 & \\
\hline
\end{tabular}

a No flow.

Discharge measurements of station No. 15 at 2,\%00-foot level near Hilo, Hawaii, in 1913.

[E. O. Christiansen, hydrographer.]

\begin{tabular}{|c|c|c|}
\hline Date. & $\begin{array}{c}\text { Gage } \\
\text { height. }\end{array}$ & $\begin{array}{l}\text { Dis- } \\
\text { charge. }\end{array}$ \\
\hline $\begin{array}{l}\text { Apr. } 29 \ldots \ldots \ldots \ldots \\
\text { May } 24 . \ldots \ldots \ldots \ldots\end{array}$ & $\begin{array}{r}\text { Feet. } \\
1.26 \\
1.10\end{array}$ & $\begin{array}{r}\text { Sec.-ft. } \\
\mathbf{3 . 4} \\
. \mathbf{6 4}\end{array}$ \\
\hline
\end{tabular}


Monthly discharge of station No. 15 at 2,700-foot level near Hilo, Hawaii, for 1913.

\begin{tabular}{|c|c|c|c|c|c|}
\hline \multirow{2}{*}{ Month. } & \multicolumn{3}{|c|}{ Discharge in second-feet. } & \multirow{2}{*}{$\begin{array}{l}\text { Run-off } \\
\text { (total in } \\
\text { acre-feet). }\end{array}$} & \multirow{2}{*}{$\begin{array}{l}\text { Accu- } \\
\text { racy. }\end{array}$} \\
\hline & Maximum. & Minimum. & Mean. & & \\
\hline \multirow{2}{*}{ 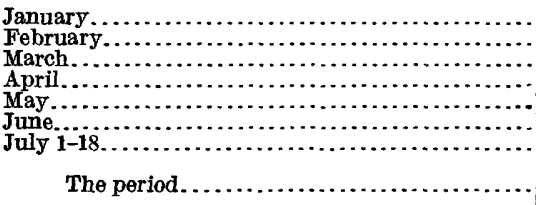 } & $\begin{array}{l}17 \\
.5 \\
4.0 \\
58 \\
3.3 \\
25 \\
29\end{array}$ & (a) $\begin{array}{r}0.7 \\
.2 \\
1.4 \\
.7 \\
.8 \\
.6\end{array}$ & $\begin{array}{r}5.78 \\
.37 \\
.74 \\
9.57 \\
1.31 \\
4.70 \\
6.69\end{array}$ & $\begin{array}{r}355 \\
20 \\
46 \\
569 \\
81 \\
280 \\
238\end{array}$ & \multirow[t]{2}{*}{$\begin{array}{l}\text { D. } \\
\text { D. } \\
\text { D. } \\
\text { D. } \\
\text { D. } \\
\text { D. } \\
\text { D. }\end{array}$} \\
\hline & & & & 1,590 & \\
\hline
\end{tabular}

a No flow.

Discharge measurements of station No. 27 at 2,700-foot level near Hilo, Hawaï, in 1913. [E. O. Christiansen, hydrographer.]

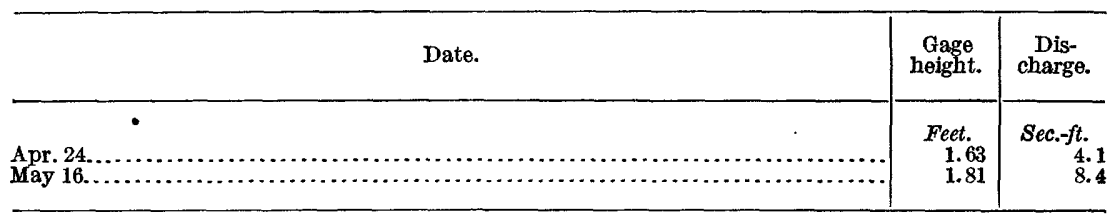

Monthly discharge of station No. 27 at 2,700-foot level near Hilo, Hawaii, for 1913.

\begin{tabular}{|c|c|c|c|c|c|}
\hline \multirow{2}{*}{ Month. } & \multicolumn{3}{|c|}{ Discharge in second-feet. } & \multirow{2}{*}{$\begin{array}{c}\text { Run-off } \\
\text { (total in } \\
\text { acre-feet). }\end{array}$} & \multirow{2}{*}{$\begin{array}{l}\text { Accu- } \\
\text { racy. }\end{array}$} \\
\hline & Maximum. & Minimum. & Mean. & & \\
\hline \multirow{2}{*}{ 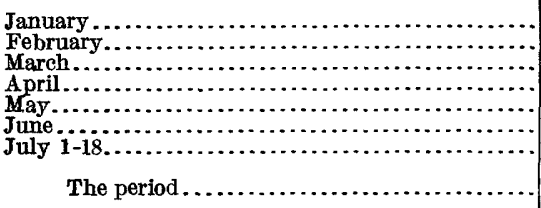 } & $\begin{array}{c}30 \\
2.3 \\
4.0 \\
48 \\
6.2 \\
41 \\
38\end{array}$ & $\begin{array}{r}2.3 \\
.4 \\
.2 \\
2.9 \\
1.2 \\
1.8 \\
1.8\end{array}$ & $\begin{array}{l}7.93 \\
1.00 \\
1.62 \\
8.61 \\
2.70 \\
8.33 \\
1.02\end{array}$ & $\begin{array}{r}488 \\
56 \\
100 \\
512 \\
166 \\
496 \\
365\end{array}$ & \multirow[t]{2}{*}{$\begin{array}{l}\text { D. } \\
\text { D. } \\
\text { D. } \\
\text { D. } \\
\text { D. } \\
\text { D. } \\
\text { D. }\end{array}$} \\
\hline & & & & 2,180 & \\
\hline
\end{tabular}

Discharge measurements of station No. 28 at 2,700-foot level near Hilo, Hawaii, in 1913.

[E. O. Christiansen, hydrographer.]

\begin{tabular}{|c|c|c|}
\hline Date. & $\begin{array}{c}\text { Gage } \\
\text { height. }\end{array}$ & $\begin{array}{l}\text { Dis- } \\
\text { charge. }\end{array}$ \\
\hline 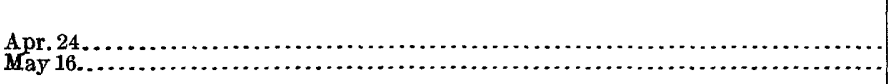 & $\begin{array}{l}\text { Feet. } \\
0.90 \\
1.85\end{array}$ & $\begin{array}{r}\text { Sec. }-f t . \\
10.0 \\
41.5\end{array}$ \\
\hline
\end{tabular}

Monthly discharge of station No. 28 at 2,700-foot level near Hilo, Hawaii, for 1913.

\begin{tabular}{|c|c|c|c|c|c|}
\hline \multirow{2}{*}{ Month. } & \multicolumn{3}{|c|}{ Discharge in second-feet. } & \multirow{2}{*}{$\begin{array}{l}\text { Run-ofi } \\
\text { (total in } \\
\text { acre-feet). }\end{array}$} & \multirow{2}{*}{$\begin{array}{l}\text { Accu- } \\
\text { racy. }\end{array}$} \\
\hline & Maximum. & Minimum. & Mean. & & \\
\hline \multirow{2}{*}{ 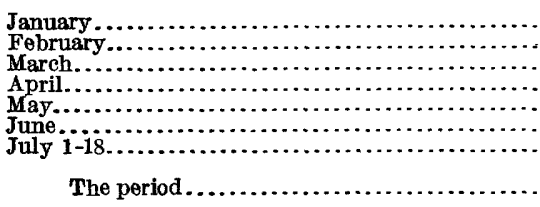 } & $\begin{array}{r}176 \\
6.0 \\
9.8 \\
181 \\
14 \\
135 \\
90\end{array}$ & (a) $\begin{array}{r}8.0 \\
.1 \\
2.0 \\
.4 \\
1.0 \\
.3\end{array}$ & $\begin{array}{c}60.7 \\
2.45 \\
1.27 \\
26.5 \\
3.06 \\
19.7 \\
22.0\end{array}$ & $\begin{array}{r}3,730 \\
136 \\
78 \\
1,580 \\
188 \\
1, \mathbf{1 7 0} \\
785\end{array}$ & \multirow[t]{2}{*}{$\begin{array}{l}D . \\
D . \\
D . \\
D . \\
D . \\
D . \\
D .\end{array}$} \\
\hline & $\cdots$ & & $\cdots$ & 7,670 & \\
\hline
\end{tabular}


Discharge measurements of station No. 31 at 2,700-foot level near Hilo, Hawaii, in 1913.

[E. o. Christiansen, hydrographer.]

\begin{tabular}{|c|c|c|}
\hline Date. & $\underset{\text { height. }}{\text { Gage }}$ & $\begin{array}{c}\text { Dis- } \\
\text { charge. }\end{array}$ \\
\hline $\begin{array}{l}\text { Apr. } 24 \ldots \ldots \ldots \ldots \\
\text { May } 16 . \ldots \ldots \ldots \ldots \ldots \ldots\end{array}$ & $\begin{array}{l}\text { Feet. } \\
0.80 \\
1.05\end{array}$ & $\begin{array}{r}\text { Sec. }-f t . \\
4.76 \\
\cdot 9.44\end{array}$ \\
\hline
\end{tabular}

Monthly discharge of station No. 31 at 2,700-foot level near Hilo, Hawaï, for 1913.

\begin{tabular}{|c|c|c|c|c|c|}
\hline \multirow{2}{*}{ Month. } & \multicolumn{3}{|c|}{ Discharge in second-feet. } & \multirow{2}{*}{$\begin{array}{l}\text { Run-ofi } \\
\text { (total in } \\
\text { acre-feet). }\end{array}$} & \multirow{2}{*}{$\begin{array}{l}\text { Accu } \\
\text { racy. }\end{array}$} \\
\hline & Maximum. & Minimum. & Mean. & & \\
\hline \multirow{2}{*}{ 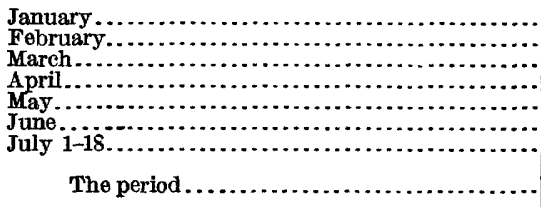 } & $\begin{array}{c}52 \\
2.5 \\
17 \\
115 \\
8.5 \\
34 \\
50\end{array}$ & $\begin{array}{r}2.6 \\
.2 \\
\text { (a) } \\
3.2 \\
1.1 \\
1.7 \\
.9\end{array}$ & $\begin{array}{c}14.0 \\
.76 \\
2.23 \\
18.0 \\
2.94 \\
8.05 \\
11.2\end{array}$ & $\begin{array}{r}861 \\
42 \\
137 \\
1,070 \\
-\quad 181 \\
479 \\
402\end{array}$ & \multirow[t]{2}{*}{$\begin{array}{l}D . \\
D . \\
D . \\
D . \\
D . \\
D . \\
D .\end{array}$} \\
\hline & $\ldots \ldots$ & $\ldots \ldots$ & & 3,170 & \\
\hline
\end{tabular}

$a$ No flow.

Discharge measurements of station No. 34 at 2,700-foot level near Hilo, Hawaii, in 1919. [E. O. Christiansen, hydrographer.]

\begin{tabular}{|c|c|c|}
\hline Date. & $\begin{array}{c}\text { Gage } \\
\text { height. }\end{array}$ & $\begin{array}{l}\text { Dis- } \\
\text { charge. }\end{array}$ \\
\hline $\begin{array}{l}\text { Apr. } 24 \ldots \ldots \ldots \\
\text { May } 16 \ldots \ldots \ldots \ldots\end{array}$ & $\begin{array}{l}\text { Feet. } \\
1.07 \\
1.14\end{array}$ & $\begin{aligned} \text { Sec. }-f t . \\
0.90 \\
1.90\end{aligned}$ \\
\hline
\end{tabular}

Monthly discharge of station No. 34 at 2,700-foot level near Hilo, Hawaï, for 1913.

\begin{tabular}{|c|c|c|c|c|c|}
\hline \multirow{2}{*}{ Month. } & \multicolumn{3}{|c|}{ Discharge in second-feet. } & \multirow{2}{*}{$\begin{array}{c}\text { Run-off } \\
\text { (total in } \\
\text { acre-feet). }\end{array}$} & \multirow{2}{*}{$\begin{array}{l}\text { Accu- } \\
\text { racy. }\end{array}$} \\
\hline & Maximum. & Minimum. & Mean. & & \\
\hline \multirow{2}{*}{ 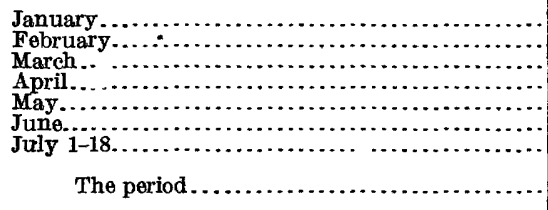 } & $\begin{array}{r}16 \\
16 \\
2.6 \\
24 \\
1.3 \\
7.1 \\
10\end{array}$ & $\begin{array}{r}0.2 \\
.2 \\
.2 \\
.6 \\
.3 \\
.4 \\
.3\end{array}$ & $\begin{array}{r}2.51 \\
.27 \\
.47 \\
3.63 \\
.63 \\
1.35 \\
2.12\end{array}$ & $\begin{array}{r}154 \\
15.0 \\
28.9 \\
216 \\
38.7 \\
80.3 \\
75.6\end{array}$ & \multirow[t]{2}{*}{$\begin{array}{l}\text { D. } \\
\text { D. } \\
\text { D. } \\
\text { D. } \\
\text { D. } \\
\text { D. } \\
\text { D. }\end{array}$} \\
\hline & & & & 608 & \\
\hline
\end{tabular}

Discharge measurements. of station No. 46 at 2,700-foot level near Hilo, Hawaii, in 1913.

[E. O. Christiansen, hydrographer.]

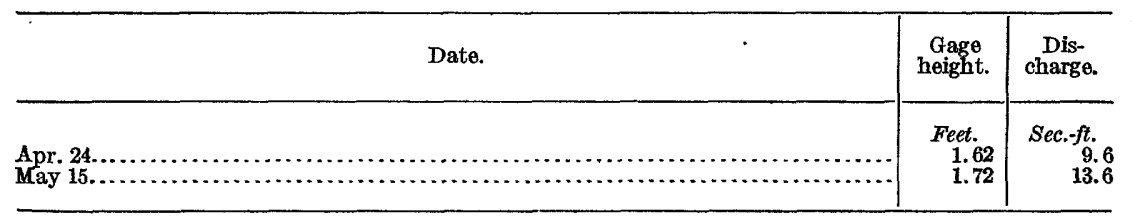


Monthly discharge of station No. 46 at 2,700-foot level near Hilo, Hawaii, for 1913.

\begin{tabular}{|c|c|c|c|c|c|}
\hline \multirow{2}{*}{ Month. } & \multicolumn{3}{|c|}{ Discharge in second-feet. } & \multirow{2}{*}{$\begin{array}{l}\text { Run-off } \\
\text { (total in } \\
\text { acre-feet). }\end{array}$} & \multirow{2}{*}{$\begin{array}{l}\text { Accu } \\
\text { racy. }\end{array}$} \\
\hline & Maximum. & Minimum. & Mean. & & \\
\hline \multirow{2}{*}{ 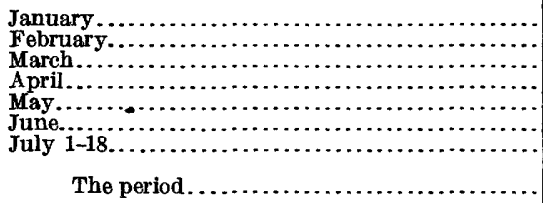 } & $\begin{array}{c}195 \\
4.4 \\
16 \\
188 \\
46 \\
104 \\
82\end{array}$ & (a) $\begin{aligned} & 5.8 \\
&(a) \\
& 6.1 \\
& 3.0 \\
& 4.4 \\
& 2.5\end{aligned}$ & $\begin{array}{c}56.4 \\
1.49 \\
2.85 \\
32.4 \\
9.47 \\
21.7 \\
25.4\end{array}$ & $\begin{array}{r}3,470 \\
83 \\
175 \\
1,930 \\
582 \\
1,290 \\
907\end{array}$ & \multirow[t]{2}{*}{$\begin{array}{l}\text { D. } \\
\text { D. } \\
\text { D. } \\
\text { D. } \\
\text { D. } \\
\text { D. } \\
\text { D. }\end{array}$} \\
\hline & & & & 8,440 & \\
\hline
\end{tabular}

$a$ No flow.

Discharge measurements of station No. 47 at 2,700-foot level near Hilo, Hawaii, in 1913.

[E. O. Christiansen, hydrographer.]

\begin{tabular}{|c|c|c|}
\hline Date. & $\begin{array}{c}\text { Gage } \\
\text { height. }\end{array}$ & $\begin{array}{l}\text { Dis- } \\
\text { charge. }\end{array}$ \\
\hline 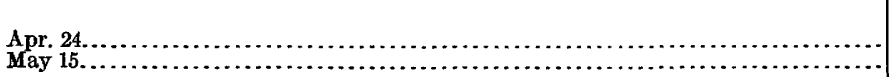 & $\begin{array}{r}\text { Feet. } \\
1.88 \\
1.80\end{array}$ & $\begin{array}{r}\text { Sec.-ft. } \\
3.6 \\
3.0\end{array}$ \\
\hline
\end{tabular}

Monthly discharge of station No. 47 at 2,700-foot level near Hilo, Hawaii, for 1913.

\begin{tabular}{|c|c|c|c|c|c|}
\hline \multirow{2}{*}{ Month. } & \multicolumn{3}{|c|}{ Discharge in second-feet. } & \multirow{2}{*}{$\begin{array}{l}\text { Run-off } \\
\text { (total in } \\
\text { acre-feet). }\end{array}$} & \multirow{2}{*}{$\begin{array}{l}\text { Accu- } \\
\text { racy. }\end{array}$} \\
\hline & Maximum. & Minimum. & Mean. & & \\
\hline \multirow{2}{*}{ 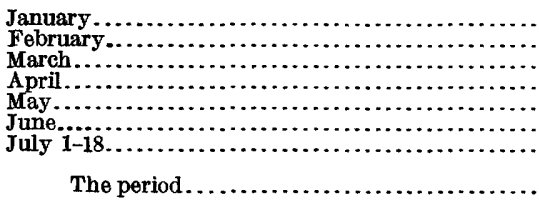 } & $\begin{array}{c}42 \\
1.3 \\
7.0 \\
25 \\
5.3 \\
15 \\
17\end{array}$ & $\begin{array}{r}1.9 \\
.3 \\
.2 \\
2.1 \\
1.2 \\
1.4 \\
1.7\end{array}$ & $\begin{array}{r}10.6 \\
.68 \\
1.44 \\
5.51 \\
2.35 \\
5.00 \\
6.29\end{array}$ & $\begin{array}{r}652 \\
38 \\
88 \\
328 \\
144 \\
298 \\
224\end{array}$ & \multirow[t]{2}{*}{$\begin{array}{l}\mathrm{D} . \\
\mathrm{D}: \\
\mathrm{D}: \\
\mathrm{D}: \\
\mathrm{D}: \\
\mathrm{D}: \\
\mathrm{D} .\end{array}$} \\
\hline & & & $\cdots$ & 1,770 & \\
\hline
\end{tabular}

Discharge measurements of station No. 52 at 2,700-foot level near Hilo, Hawaï, in 1913.

[E. O. Christiansen, hydrographer.]

\begin{tabular}{|c|c|c|}
\hline Date. & $\begin{array}{c}\text { Gage } \\
\text { height. }\end{array}$ & $\begin{array}{l}\text { Dis- } \\
\text { charge. }\end{array}$ \\
\hline $\begin{array}{l}\text { Apr. } 23 \ldots \ldots \ldots \ldots \ldots \ldots \ldots \ldots \ldots \\
\text { May } 21 \ldots \ldots \ldots \ldots \ldots \ldots \ldots \ldots \ldots\end{array}$ & $\begin{array}{r}\text { Feet. } \\
1.06 \\
.81\end{array}$ & $\begin{array}{r}\text { Sec. } f t . \\
21.2 \\
11.8\end{array}$ \\
\hline
\end{tabular}


Monthly discharge of station No. 52 at 2,700-foot level near Hilo, Hawaii, for 1913.

\begin{tabular}{|c|c|c|c|c|c|}
\hline \multirow{2}{*}{ Month. } & \multicolumn{3}{|c|}{ Discharge in second-feet. } & \multirow{2}{*}{$\begin{array}{c}\text { Run-off } \\
\text { (total in } \\
\text { acre-feet). }\end{array}$} & \multirow{2}{*}{$\begin{array}{l}\text { Accu } \\
\text { racy. }\end{array}$} \\
\hline & Maximum. & Minimum. & Mean. & & \\
\hline \multirow{2}{*}{ 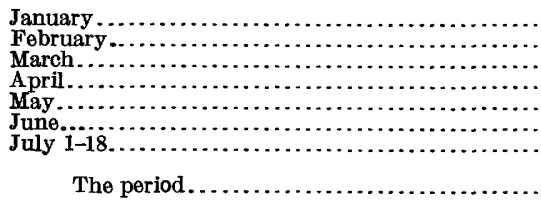 } & $\begin{array}{c}582 \\
7.6 \\
48 \\
219 \\
74 \\
342 \\
155\end{array}$ & $\begin{array}{r}6.2 \\
1.1 \\
16.4 \\
16 \\
6.6 \\
8.9 \\
7.4\end{array}$ & $\begin{array}{c}93.8 \\
3.57 \\
10.1 \\
50.1 \\
17.8 \\
52.6 \\
49.5\end{array}$ & $\begin{array}{r}5,770 \\
198 \\
621 \\
2,980 \\
-1,090 \\
3,130 \\
1,760\end{array}$ & \multirow[t]{2}{*}{$\begin{array}{l}\text { D. } \\
\text { D. } \\
\text { D. } \\
\text { D. } \\
\text { D. } \\
\text { D. } \\
\text { D. }\end{array}$} \\
\hline & & & & 15,500 & \\
\hline
\end{tabular}

Discharge measurements of station No. 54 at 2,700-foot level near Hilo, Hawaii, in 1913. [E. O. Christiansen, hydrographer.]

\begin{tabular}{c|c|c}
\hline Date. & $\begin{array}{c}\text { Gage } \\
\text { height. }\end{array}$ & $\begin{array}{c}\text { Dis- } \\
\text { charge. }\end{array}$ \\
\hline Apr. $18 \ldots \ldots \ldots \ldots \ldots \ldots \ldots \ldots \ldots \ldots \ldots \ldots \ldots \ldots \ldots \ldots \ldots \ldots \ldots \ldots \ldots \ldots \ldots \ldots \ldots \ldots \ldots \ldots \ldots \ldots \ldots \ldots \ldots \ldots \ldots \ldots \ldots$ & $\begin{array}{c}\text { Feet. } \\
0.89\end{array}$ & $\begin{array}{c}\text { Sec.-ft. } \\
1.63\end{array}$ \\
\hline
\end{tabular}

Monthly discharge of station No. 54 at 2,700-foot level near Hilo, Hawaii, for 1913.

\begin{tabular}{l} 
Month. \\
\hline
\end{tabular}

$a$ No flow.

Discharge measurements of station No. 62 at 2,700-foot level near Hilo, Hawaii, in 1913. [E. O. Christiansen, hydrographer.]

\begin{aligned} \hline & \\ \hline & \end{aligned}

Monthly discharge of station No.62 at 2,700-foot level near Hilo, Hawaii, for 1913.

\begin{tabular}{|c|c|c|c|c|c|}
\hline \multirow{2}{*}{ Month. } & \multicolumn{3}{|c|}{ Discharge in second-feet. } & \multirow{2}{*}{$\begin{array}{l}\text { Run-off (in } \\
\text { acre-feet). }\end{array}$} & \multirow{2}{*}{$\begin{array}{l}\text { Accu- } \\
\text { racy. }\end{array}$} \\
\hline & Maximum. & Minimum. & Mean. & & \\
\hline \multirow[t]{2}{*}{ 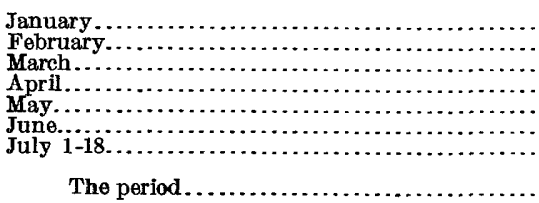 } & $\begin{array}{c}128 \\
2.2 \\
39 \\
100 \\
14 \\
42 \\
61\end{array}$ & $\begin{array}{ll}(a) & 1.1 \\
(a) & \\
5.3 \\
1.5 \\
1.8 \\
1.3\end{array}$ & $\begin{array}{c}24.6 \\
9.95 \\
4.67 \\
20.5 \\
3.82 \\
11.0 \\
14.2\end{array}$ & $\begin{array}{r}1,510 \\
553 \\
287 \\
1,220 \\
235 \\
655 \\
505\end{array}$ & \multirow[t]{2}{*}{$\begin{array}{l}\text { D. } \\
\text { D: } \\
\text { D. } \\
\text { D. } \\
\text { D. } \\
\text { D. } \\
\text { D. }\end{array}$} \\
\hline & & & & 4,960 & \\
\hline
\end{tabular}


Discharge measurements of station No. 68 at 2,700-foot level near Hilo, Hawaï, in 1913.

[E. O. Christiansen, hydrographer.]

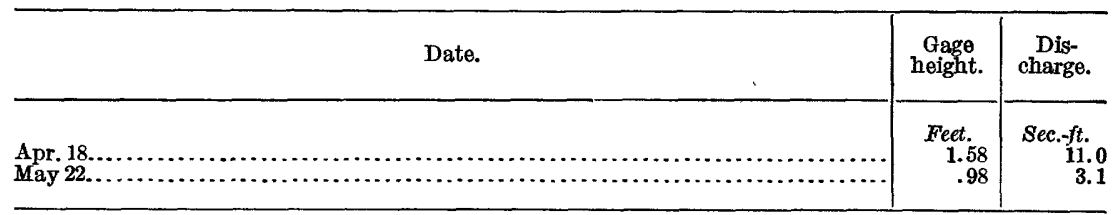

Monthly discharge of station No. 68 at 2,700-foot level near Hilo, Hawaï, for 191 s.

\begin{tabular}{|c|c|c|c|c|c|}
\hline \multirow{2}{*}{ Month. } & \multicolumn{3}{|c|}{ Discharge in second-feet. } & \multirow{2}{*}{$\begin{array}{l}\text { Run-off (in } \\
\text { acre-feet). }\end{array}$} & \multirow{2}{*}{$\begin{array}{l}\text { Accu- } \\
\text { racy. }\end{array}$} \\
\hline & Maximum. & Minimum. & Mean. & & \\
\hline 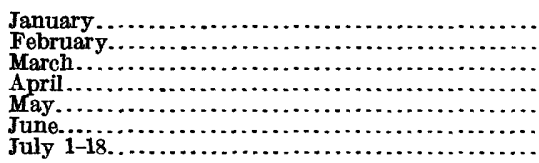 & \begin{tabular}{l|}
42 \\
3.8 \\
26 \\
52 \\
17 \\
29 \\
47
\end{tabular} & $\begin{array}{r}1.9 \\
.8 \\
.5 \\
7.2 \\
2.2 \\
3.0 \\
2.1\end{array}$ & $\begin{array}{l}15.3 \\
1.76 \\
5.37 \\
17.3 \\
6.02 \\
11.5 \\
13.3\end{array}$ & $\begin{array}{r}941 \\
98 \\
330 \\
1,030 \\
370 \\
684 \\
474\end{array}$ & \multirow[t]{2}{*}{$\begin{array}{l}\text { D. } \\
\text { D. } \\
\text { D. } \\
\text { D. } \\
\text { D. } \\
\text { D. } \\
\text { D. }\end{array}$} \\
\hline The period. . & & & $\overline{\ldots . . .}$ & 3,930 & \\
\hline
\end{tabular}

Discharge measurements of station No. 69 at 2,700-foot level near Hilo, Hawaii, in 1913.

[E. O. Christiansen, hydrographer.]

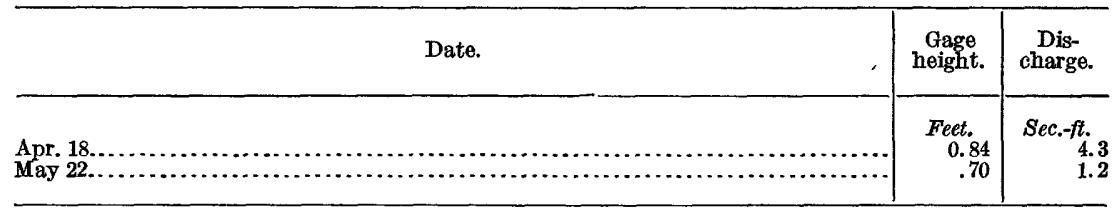

Monthly discharge of station No. 69 at 2,700-foot level near Hilo, Hawaii, for 1913.

\begin{tabular}{l}
\hline \multicolumn{2}{c}{ Month. } \\
\hline
\end{tabular}

Discharge measurements of station No. 74 at 2,700-foot level near Hilo, Hawaii, in 1913.

[E. O. Christiansen, hydrographer.]

\begin{tabular}{|c|c|c|c|}
\hline . & Date. & $\begin{array}{c}\text { Gage } \\
\text { height. }\end{array}$ & $\begin{array}{c}\text { Dis- } \\
\text { charge. }\end{array}$ \\
\hline $\begin{array}{l}\text { Apr. 18.. } \\
\text { May 22.. }\end{array}$ & 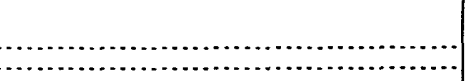 & $\begin{array}{r}\text { Feet. } \\
2.06 \\
1.71\end{array}$ & $\begin{array}{r}s e c .-f t . \\
12.5 \\
4.0\end{array}$ \\
\hline
\end{tabular}


Monthly discharge of station No. 74 at 2,700-foot level near Hilo, Hawaii, for 1913.

\begin{tabular}{|c|c|c|c|c|c|}
\hline \multirow{2}{*}{ Month. } & \multicolumn{3}{|c|}{ Discharge in second-feet. } & \multirow{2}{*}{$\begin{array}{c}\text { Run-off } \\
\text { (total in } \\
\text { acre-feet). }\end{array}$} & \multirow{2}{*}{$\begin{array}{l}\text { Accu } \\
\text { racy. }\end{array}$} \\
\hline & Maximum. & Minimum. & Mean. & & \\
\hline \multirow[t]{2}{*}{ 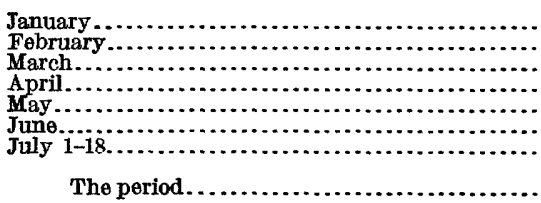 } & $\begin{array}{l}46 \\
4.7 \\
23 \\
52 \\
24 \\
62 \\
56\end{array}$ & $\begin{array}{r}2.9 \\
.8 \\
.4 \\
1.1 \\
3.1 \\
4.1 \\
3.1\end{array}$ & $\begin{array}{c}18.2 \\
2.00 \\
5.46 \\
16.9 \\
7.80 \\
14.1 \\
16.5\end{array}$ & $\begin{array}{r}1,120 \\
111 \\
336 \\
1,010 \\
480 \\
839 \\
589\end{array}$ & \multirow[t]{2}{*}{$\begin{array}{l}\text { D. } \\
\text { D. } \\
\text { D. } \\
\text { D. } \\
\text { D: } \\
\text { D. } \\
\text { D. }\end{array}$} \\
\hline & & & & 4,480 & \\
\hline
\end{tabular}

Discharge measurements of station No. 76 at 2,700-foot level near Hilo, Hawaii, in 1913.

[E. O. Christiansen, hydrographer.]

\begin{tabular}{|c|c|c|}
\hline Date. & $\begin{array}{c}\text { Gage } \\
\text { height. }\end{array}$ & $\begin{array}{c}\text { Dis- } \\
\text { charge. }\end{array}$ \\
\hline Apr. $23 . \ldots \ldots \ldots \ldots \ldots \ldots$ & $\begin{array}{l}\text { Feet. } \\
\quad 1.01\end{array}$ & Sec. $f t$. \\
\hline
\end{tabular}

Monthly discharge of station No. 76 at 2,700-foot level near Hilo, Hawaii, for 1913.

\begin{tabular}{|c|c|c|c|c|c|}
\hline \multirow{2}{*}{ Month. } & \multicolumn{3}{|c|}{ Discharge in second-feet. } & \multirow{2}{*}{$\begin{array}{c}\text { Run-off } \\
\text { (total in } \\
\text { acre-feet). }\end{array}$} & \multirow{2}{*}{$\begin{array}{l}\text { Accu } \\
\text { racy. }\end{array}$} \\
\hline & Maximum. & Minimum. & Mean. & & \\
\hline \multirow{2}{*}{ 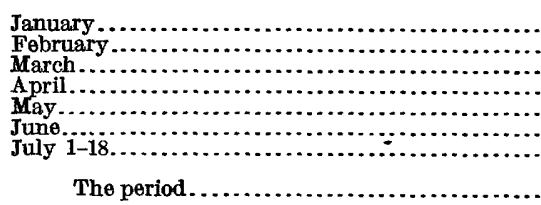 } & $\begin{array}{c}10 \\
1.0 \\
7.8 \\
9.3 \\
2.0 \\
8.7 \\
15\end{array}$ & $\begin{array}{r}0.6 \\
.3 \\
.2 \\
1.0 \\
.6 \\
.7 \\
.5\end{array}$ & $\begin{array}{r}3.90 \\
.48 \\
1.15 \\
2.60 \\
.98 \\
1.96 \\
2.32\end{array}$ & $\begin{array}{c}240 \\
26.6 \\
70.7 \\
155 \\
60.3 \\
117 \\
82.8\end{array}$ & \multirow[t]{2}{*}{$\begin{array}{l}\text { D. } \\
\text { D. } \\
\text { D. } \\
\text { D: } \\
\text { D: } \\
\text { D. } \\
\text { D. }\end{array}$} \\
\hline & & & & 752 & \\
\hline
\end{tabular}

Discharge measurements of station No. 79 at 2,700-foot level near Hilo, Hawaii, in 1913. [E. O. Christiansen, hydrographer.]

\begin{tabular}{|c|c|c|}
\hline Date. & $\begin{array}{c}\text { Gage } \\
\text { height. }\end{array}$ & $\begin{array}{c}\text { Dis- } \\
\text { charge. }\end{array}$ \\
\hline Apr. $23 \ldots \ldots \ldots \ldots$ & $\begin{array}{l}\text { Feet. } \\
0.93\end{array}$ & $\begin{array}{r}\text { sec. } \\
0.83\end{array}$ \\
\hline
\end{tabular}


Monthly discharge of station No. 79 at 2,700-foot level near Hilo, Hawaï, for 1913.

\begin{tabular}{|c|c|c|c|c|c|}
\hline \multirow{2}{*}{ Month. } & \multicolumn{3}{|c|}{ Discharge in second-fe日t. } & \multirow{2}{*}{$\begin{array}{c}\text { Run-off } \\
\text { (total in } \\
\text { acre-feet). }\end{array}$} & \multirow{2}{*}{$\begin{array}{l}\text { Accu- } \\
\text { racy. }\end{array}$} \\
\hline & Maximum. & Minimum. & Mean. & & \\
\hline \multirow{2}{*}{$\begin{array}{l}\text { January } \\
\text { February } \\
\text { March } \\
\text { April } \\
\text { May } \\
\text { June. } \\
\text { July } 1-18 \\
\quad \\
\quad \text { The period }\end{array}$} & $\begin{array}{r}2.5 \\
.2 \\
2.3 \\
3.2 \\
.9 \\
2.4 \\
3.7\end{array}$ & $\begin{array}{l}0.2 \\
.1 \\
.1 \\
.1 \\
.2 \\
.2 \\
.2\end{array}$ & $\begin{array}{r}1.06 \\
.15 \\
.37 \\
1.08 \\
.37 \\
.75 \\
.98\end{array}$ & $\begin{array}{r}65.2 \\
8.3 \\
22.8 \\
64.3 \\
22.8 \\
44.6 \\
33.2\end{array}$ & \multirow[t]{2}{*}{$\begin{array}{l}\text { D. } \\
\text { D. } \\
\text { D. } \\
\text { D. } \\
\text { D. } \\
\text { D. } \\
\text { D. }\end{array}$} \\
\hline & & & & 261 & \\
\hline
\end{tabular}

Discharge measurements of station No. 80 at 2,700-foot level near Hilo, Hawaii, in 1913.

[E. O. Christiansen, hydrographer.]

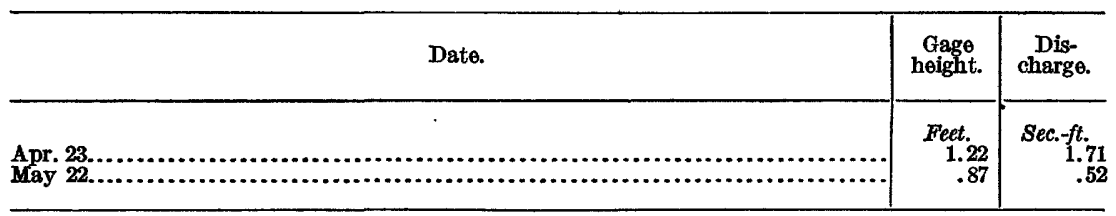

Monthly discharge of station No. 80 at 2,700-foot level near Hilo, Hawaï, for 1913.

\begin{tabular}{|c|c|c|c|c|c|}
\hline \multirow{2}{*}{ Month. } & \multicolumn{3}{|c|}{ Discharge in second-feet. } & \multirow{2}{*}{$\begin{array}{c}\text { Run-off } \\
\text { (total in } \\
\text { acre-foet). }\end{array}$} & \multirow{2}{*}{$\begin{array}{l}\text { Accu- } \\
\text { racy. }\end{array}$} \\
\hline & Maximum. & Minimum. & Mean. & & \\
\hline \multirow{2}{*}{ 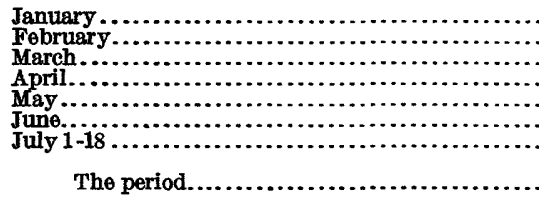 } & $\begin{array}{l}3.6 \\
.6 \\
3.7 \\
4.0 \\
1.6 \\
3.6 \\
4.5\end{array}$ & ${ }^{(a)}{ }^{0.5}$ & $\begin{array}{r}2.04 \\
.38 \\
.67 \\
2.30 \\
.81 \\
1.52 \\
1.81\end{array}$ & $\begin{array}{r}125 \\
21.1 \\
41.2 \\
137 \\
49.8 \\
90.4 \\
64.5\end{array}$ & \multirow[t]{2}{*}{$\begin{array}{l}\text { D. } \\
\text { D: } \\
\text { D: } \\
\text { D: } \\
\text { D. } \\
\text { D. } \\
\text { D. }\end{array}$} \\
\hline & & & & 529 & \\
\hline
\end{tabular}

$a$ No flow.

Discharge measurements of station No. 81 at 2,700-foot level near Hilo, Hawaii, in 1913.

[E. O. Christiansen, hydrographer.]

\begin{tabular}{|c|c|c|}
\hline Date. & $\begin{array}{c}\text { Gage } \\
\text { height. }\end{array}$ & $\begin{array}{c}\text { Dis- } \\
\text { charge. }\end{array}$ \\
\hline Apr. $23 \ldots \ldots \ldots$ & $\begin{array}{l}\text { Feet. } \\
1.00 \\
.69\end{array}$ & $\begin{array}{r}\text { Sec.-ft. } \\
\mathbf{4 . 1} \\
\mathbf{1 . 9}\end{array}$ \\
\hline
\end{tabular}


Monthly discharge of station No. 81 at 2,700-foot level near Hilo, Hawaii, for 1913 ,

\begin{tabular}{|c|c|c|c|c|c|}
\hline \multirow{2}{*}{ Month. } & \multicolumn{3}{|c|}{ Discharge in second-feet. } & \multirow{2}{*}{$\begin{array}{c}\text { Run-off } \\
\text { (total in } \\
\text { acre-feet). }\end{array}$} & \multirow{2}{*}{$\begin{array}{l}\text { Accu- } \\
\text { racy. }\end{array}$} \\
\hline & Maximum. & Minimum. & Mean. & & \\
\hline \multirow[t]{2}{*}{ 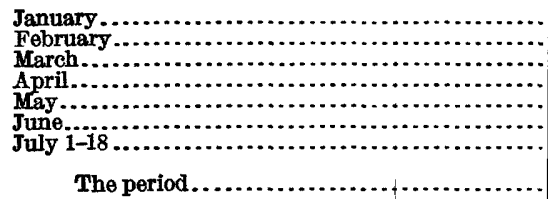 } & $\begin{array}{l}12 \\
1.4 \\
11 \\
15 \\
7.0 \\
21 \\
38\end{array}$ & $\begin{array}{r}1.0 \\
.2 \\
.1 \\
3.3 \\
1.0 \\
1.3 \\
3.3\end{array}$ & $\begin{array}{r}5.98 \\
.59 \\
2.19 \\
7.01 \\
2.44 \\
7.80 \\
11.2\end{array}$ & $\begin{array}{r}368 \\
33 \\
135 \\
417 \\
150 \\
464 \\
399\end{array}$ & \multirow[t]{2}{*}{$\begin{array}{l}\text { D. } \\
\text { D. } \\
\text { D. } \\
\text { D. } \\
\text { D. } \\
\text { D. } \\
\text { D. }\end{array}$} \\
\hline & & & & 1,970 & \\
\hline
\end{tabular}

Discharge measurements of station No. 85 at 2,700-foot level near Hilo, Hawaii, in 1913.

[E. O. Christiansen, hydrographer.]

\begin{tabular}{|c|c|c|}
\hline Date. & $\begin{array}{c}\text { Gage } \\
\text { height. }\end{array}$ & $\begin{array}{l}\text { Dis- } \\
\text { charge. }\end{array}$ \\
\hline 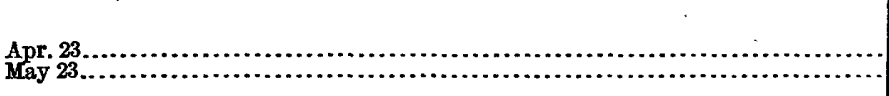 & $\begin{array}{r}\text { Feet. } \\
\mathbf{0 . 8 8} \\
.79\end{array}$ & $\begin{array}{r}\text { Sec.-ft. } \\
1.90 \\
.77\end{array}$ \\
\hline
\end{tabular}

Monthly discharge of station No. 85 at 2,700-foot level near Hilo, Hawaï, for 1913.

\begin{tabular}{|c|c|c|c|c|c|}
\hline \multirow{2}{*}{ Month. } & \multicolumn{3}{|c|}{ Discharge in second-feet. } & \multirow{2}{*}{$\begin{array}{c}\text { Run-off } \\
\text { (total in } \\
\text { acre-feet). }\end{array}$} & \multirow{2}{*}{$\begin{array}{l}\text { Accu- } \\
\text { racy. }\end{array}$} \\
\hline & Maximum. & Minimum. & Mean. & & \\
\hline \multirow{2}{*}{ 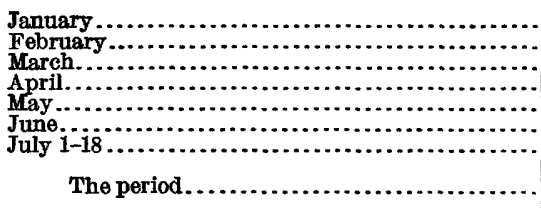 } & $\begin{array}{r}6.9 \\
3.0 \\
7.5 \\
13 \\
2.8 \\
7.3 \\
14\end{array}$ & $\begin{array}{r}1.7 \\
.7 \\
.6 \\
1.6 \\
.5 \\
.6 \\
.6\end{array}$ & $\begin{array}{l}4.07 \\
1.37 \\
2.09 \\
4.78 \\
1.06 \\
2.37 \\
3.68\end{array}$ & $\begin{array}{r}250 \\
76 \\
129 \\
284 \\
65 \\
141 \\
131\end{array}$ & \multirow[t]{2}{*}{$\begin{array}{l}\text { D. } \\
\text { D. } \\
\text { D. } \\
\text { D. } \\
\text { D. } \\
\text { D. } \\
\text { D. }\end{array}$} \\
\hline & & & & 1,080 & \\
\hline
\end{tabular}

Discharge measurements of station No. 86 at 2,700-foot level near Hilo, Hawaii, in 1913.

[E. O. Christiansen, hydrographer.]

\begin{tabular}{|c|c|c|}
\hline Date. & $\begin{array}{c}\text { Gage } \\
\text { height. }\end{array}$ & $\begin{array}{l}\text { Dis- } \\
\text { charge. }\end{array}$ \\
\hline 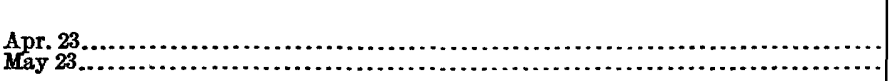 & $\begin{array}{r}\text { Feet. } \\
1.70 \\
1.56\end{array}$ & $\begin{array}{r}\text { sec. } f t . \\
5.1 \\
1.6\end{array}$ \\
\hline
\end{tabular}


Monthly discharge of station No. 86 at 2,700-foot level near Hilo, Hawaï, for 1913 .

\begin{tabular}{|c|c|c|c|c|c|}
\hline \multirow{2}{*}{ Month. } & \multicolumn{3}{|c|}{ Discharge in second-feet. } & \multirow{2}{*}{$\begin{array}{c}\text { Run-off } \\
\text { (total in } \\
\text { acre-feet). }\end{array}$} & \multirow{2}{*}{$\begin{array}{l}\text { Accu- } \\
\text { racy. }\end{array}$} \\
\hline & Maximum. & Minimum. & Mean. & & \\
\hline 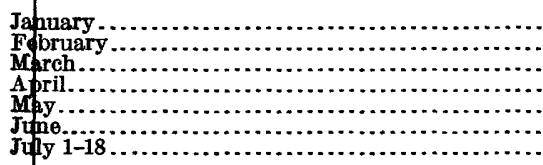 & $\begin{array}{l}12 \\
3.2 \\
12 \\
19 \\
9.3 \\
19 \\
20\end{array}$ & $\begin{array}{r}2.3 \\
.5 \\
.1 \\
4.0 \\
1.4 \\
1.7 \\
1.7\end{array}$ & $\begin{array}{l}6.93 \\
1.58 \\
2.81 \\
8.07 \\
2.76 \\
5.66 \\
6.79\end{array}$ & $\begin{array}{r}426 \\
88 \\
173 \\
480 \\
170 \\
337 \\
242\end{array}$ & $\begin{array}{l}\text { D. } \\
\text { D. } \\
\text { D. } \\
\text { D. } \\
\text { D. } \\
\text { D. }\end{array}$ \\
\hline The period... & & & $\ldots \ldots$ & 1,920 & \\
\hline
\end{tabular}

Discharge measurements of station No. 87 at 2,700-foot level near Hilo, Hawaï, in 1913.

[E. O. Christiansen, hydrographer.]

\begin{tabular}{|c|c|c|}
\hline Date. & $\begin{array}{c}\text { Gage } \\
\text { hoight. }\end{array}$ & $\begin{array}{l}\text { Dis- } \\
\text { charge. }\end{array}$ \\
\hline 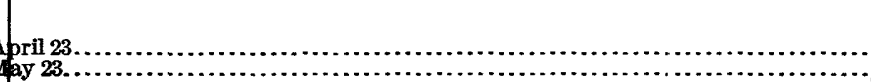 & $\begin{array}{l}\text { Feet. } \\
1.30 \\
1.04\end{array}$ & $\begin{array}{r}\text { Sec. ft. } \\
1.39 \\
.91\end{array}$ \\
\hline
\end{tabular}

Monthly discharge of station No. 87 at 2,700-foot level near Hilo, Hawaï, for 1913.

\begin{tabular}{|c|c|c|c|c|c|}
\hline & \multicolumn{3}{|c|}{ Discharge in second-feet. } & \multirow{2}{*}{$\begin{array}{c}\text { Run-off } \\
\text { (total in } \\
\text { acre-feot). }\end{array}$} & \multirow{2}{*}{$\begin{array}{l}\text { Accu- } \\
\text { racy. }\end{array}$} \\
\hline & Maximum. & Minimum. & Mean. & & \\
\hline \multirow{2}{*}{ 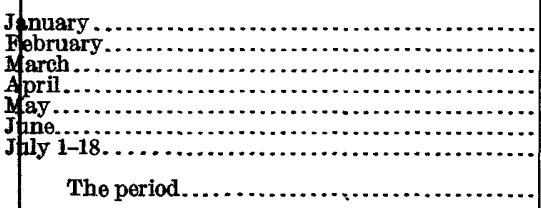 } & $\begin{array}{c}5.5 \\
2.2 \\
4.7 \\
7.9 \\
3.8 \\
8.1 \\
10\end{array}$ & $\begin{array}{r}0.7 \\
.4 \\
.3 \\
1.8 \\
.7 \\
1.0 \\
.9\end{array}$ & $\begin{array}{r}2.80 \\
.74 \\
1.19 \\
3.77 \\
1.61 \\
2.93 \\
3.52\end{array}$ & $\begin{array}{r}172 \\
41 \\
73 \\
224 \\
99 \\
174 \\
126\end{array}$ & \multirow[t]{2}{*}{$\begin{array}{l}\text { D. } \\
\text { D. } \\
\text { D. } \\
\text { D. } \\
\text { D. } \\
\text { D. } \\
\text { D. }\end{array}$} \\
\hline & & & & 909 & \\
\hline
\end{tabular}


WAIIUKU RIVER TEAR HILO, HAWAII.

Location.-About $1 \frac{1}{2}$ miles west of Hilo, a short distance above Hilo Electric Light Co. intake.

Records available.-March 21, 1911, to July 21, 1913.

Gage.-Barrett \& Lawrence water-stage recorder.

Control.-Probably slightly shifting.

Discharge measurements.-Made from cable.

Accuracy.-Records good for January and June; for other months only fair.

Discharge measurements of Wailuku River near Hilo, Hawaii, in 1913.

[E. O. Christiansen, hydrographer.]

\begin{tabular}{|c|c|c|c|c|c|}
\hline Date. & $\begin{array}{c}\text { Gage } \\
\text { height. }\end{array}$ & $\begin{array}{c}\text { Dis- } \\
\text { charge. }\end{array}$ & Date. & $\begin{array}{c}\text { Gage } \\
\text { height. }\end{array}$ & $\begin{array}{l}\text { Dis- } \\
\text { charge. }\end{array}$ \\
\hline 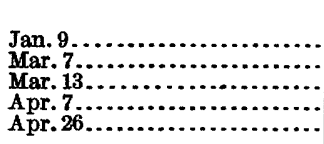 & $\begin{array}{l}\text { Feet. } \\
8.00 \\
5.50 \\
5.23 \\
6.04 \\
6.25\end{array}$ & $\begin{array}{c}\text { Sec.-ft. } \\
703 \\
55.4 \\
32.5 \\
209 \\
254\end{array}$ & 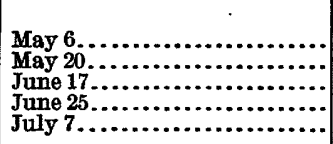 & $\begin{aligned} \text { Feet. } \\
\mathbf{5 . 6 8} \\
\mathbf{5 . 6 4} \\
7.55 \\
\mathbf{6 . 0 0} \\
7.54\end{aligned}$ & $\begin{array}{r}\text { Sec.-ft. } \\
157 \\
152 \\
523 \\
197 \\
567\end{array}$ \\
\hline
\end{tabular}

Daily discharge, in second-feet, of Wailuku River near Hilo, Hawaii, for 1913.

\begin{tabular}{|c|c|c|c|c|c|c|c|}
\hline Day. & Jan. & Feb. & Mar. & Apr. & May. & June. & July. \\
\hline 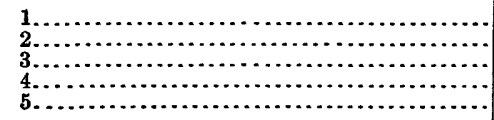 & $\begin{array}{l}484 \\
364 \\
325 \\
265 \\
225\end{array}$ & $\begin{array}{r}127 \\
116 \\
104 \\
91 \\
87\end{array}$ & $\begin{array}{l}88 \\
93 \\
84 \\
71 \\
66\end{array}$ & $\begin{array}{l}350 \\
323 \\
228 \\
165 \\
133\end{array}$ & $\begin{array}{l}190 \\
250 \\
306 \\
254 \\
187\end{array}$ & $\begin{array}{r}94 \\
84 \\
100 \\
97 \\
87\end{array}$ & $\begin{array}{r}700 \\
657 \\
568 \\
479 \\
364\end{array}$ \\
\hline $\begin{array}{r}6 \\
8 \\
8 \\
9 \\
90 \ldots\end{array}$ & $\begin{array}{r}195 \\
187 \\
1,290 \\
1,040 \\
1,250\end{array}$ & $\begin{array}{l}84 \\
89 \\
84 \\
78 \\
69\end{array}$ & $\begin{array}{r}62 \\
122 \\
98 \\
84 \\
70\end{array}$ & $\begin{array}{l}201 \\
216 \\
148 \\
171 \\
187\end{array}$ & $\begin{array}{l}155 \\
134 \\
116 \\
112 \\
106\end{array}$ & $\begin{array}{l}76 \\
72 \\
78 \\
80 \\
76\end{array}$ & $\begin{array}{l}855 \\
614 \\
426 \\
316 \\
264\end{array}$ \\
\hline $\begin{array}{l}11 \\
12 \\
13 \\
14 \\
15 \\
15\end{array}$ & $\begin{array}{l}\mathbf{3}, 350 \\
1,170 \\
2,100 \\
3,090 \\
3,700\end{array}$ & $\begin{array}{l}64 \\
60 \\
52 \\
48 \\
44\end{array}$ & $\begin{array}{r}69 \\
53 \\
107 \\
104 \\
57\end{array}$ & $\begin{array}{r}140 \\
511 \\
1,620 \\
3,110 \\
834\end{array}$ & $\begin{array}{r}94 \\
85 \\
82 \\
103 \\
139\end{array}$ & $\begin{array}{r}69 \\
73 \\
9,400 \\
6,140 \\
3,110\end{array}$ & $\begin{array}{l}222 \\
206 \\
206 \\
162 \\
139\end{array}$ \\
\hline $\begin{array}{l}16 \ldots \ldots \\
17 \\
19 \\
20 \ldots \ldots\end{array}$ & $\begin{array}{r}1,620 \\
1,200 \\
814 \\
1,630 \\
1,450\end{array}$ & $\begin{array}{l}58 \\
84 \\
62 \\
50 \\
48\end{array}$ & $\begin{array}{r}41 \\
43 \\
95 \\
127 \\
80\end{array}$ & $\begin{array}{l}630 \\
484 \\
378 \\
314 \\
264\end{array}$ & $\begin{array}{l}426 \\
250 \\
325 \\
200 \\
150\end{array}$ & $\begin{array}{r}1,260 \\
568 \\
403 \\
314 \\
254\end{array}$ & $\begin{array}{r}117 \\
107 \\
97 \\
88 \\
85\end{array}$ \\
\hline $\begin{array}{l}21 \\
22 \\
23 \\
24 \\
25\end{array}$ & $\begin{array}{r}1,400 \\
1,180 \\
700 \\
525 \\
418\end{array}$ & $\begin{array}{l}\mathbf{4 4} \\
38 \\
38 \\
\mathbf{3 5} \\
\mathbf{3 4}\end{array}$ & $\begin{array}{r}59 \\
140 \\
166 \\
84 \\
66\end{array}$ & $\begin{array}{l}254 \\
269 \\
236 \\
203 \\
275\end{array}$ & $\begin{array}{r}121 \\
98 \\
84 \\
84 \\
84\end{array}$ & $\begin{array}{l}203 \\
175 \\
158 \\
219 \\
203\end{array}$ & 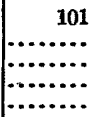 \\
\hline $\begin{array}{r}26 \\
27 \\
28 \\
29 \\
\mathbf{2 0} \\
\mathbf{3 0} \\
\mathbf{3 1}\end{array}$ & $\begin{array}{l}347 \\
283 \\
236 \\
203 \\
172 \\
145\end{array}$ & $\begin{array}{r}34 \\
33 \\
54 \\
\cdots \\
\cdots\end{array}$ & $\begin{array}{r}55 \\
50 \\
203 \\
219 \\
145 \\
568\end{array}$ & $\begin{array}{r}245 \\
348 \\
273 \\
228 \\
211 \\
2\end{array}$ & $\begin{array}{l}82 \\
79 \\
78 \\
79 \\
78 \\
78\end{array}$ & $\begin{array}{l}180 \\
139 \\
127 \\
152 \\
187\end{array}$ & $\begin{array}{ll}\cdots \cdots \cdots \\
\cdots \cdots \cdots \\
\cdots \cdots \cdots\end{array}$ \\
\hline
\end{tabular}


Monthly discharge of Wailuku River near Hilo, Hawaii, for 1913.

\begin{tabular}{|c|c|c|c|c|c|}
\hline \multirow{2}{*}{ Month. } & \multicolumn{3}{|c|}{ Discharge in second-feet. } & \multirow{2}{*}{$\begin{array}{l}\text { Run-off } \\
\text { (total in } \\
\text { acre-foet). }\end{array}$} & \multirow{2}{*}{$\begin{array}{l}\text { Accu- } \\
\text { racy. }\end{array}$} \\
\hline & Maximum. & Minimum. & Mean. & & \\
\hline \multirow{2}{*}{ 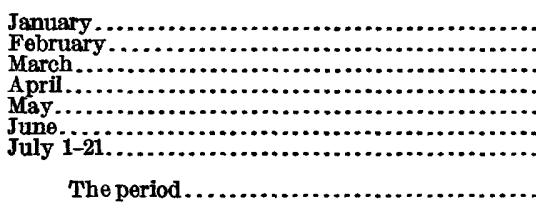 } & $\begin{array}{r}3,700 \\
127 \\
568 \\
3,110 \\
426 \\
9,400 \\
855\end{array}$ & $\begin{array}{r}145 \\
33 \\
41 \\
133 \\
78 \\
69 \\
85\end{array}$ & $\begin{array}{c}1,010 \\
64.6 \\
109 \\
432 \\
149 \\
806 \\
322\end{array}$ & $\begin{array}{r}62,100 \\
3,590 \\
6,700 \\
25,700 \\
9,160 \\
48,000 \\
13,400\end{array}$ & \multirow[t]{2}{*}{$\begin{array}{l}\text { A. } \\
\text { C. } \\
\text { B. } \\
\text { B. } \\
\text { B. } \\
\text { A. } \\
\text { B. }\end{array}$} \\
\hline & $\ldots \ldots \ldots$ & $\ldots \ldots \ldots$ & $\ldots \ldots \ldots$ & 169,000 & \\
\hline
\end{tabular}

HONOLII RIVER AT KAIWIKI, NEAR HILO, HAWAII.

Location.-About one-half mile north of Kaiwiki and 6 miles west of Hilo.

Records available.-June 1, 1911, to March 24, 1913.

Gage.-Barrett \& Lawrence water-stage recorder.

Control.-Probably slightly shifting.

Discharge measurements.-Made from footbridge.

Accuracy.-Records good.

Discharge measurements of Honolii River at Kaiwiki, near Hilo, Hawaii, in 1913.

[E. O. Christiansen, hydrographer.]

\begin{tabular}{|c|c|c|}
\hline Date. & $\begin{array}{c}\text { Gage } \\
\text { height. }\end{array}$ & $\begin{array}{c}\text { Dis- } \\
\text { charge. }\end{array}$ \\
\hline 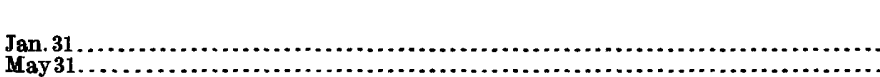 & $\begin{array}{l}\text { Feet. } \\
5.10 \\
5.11\end{array}$ & $\begin{aligned} \text { Sec. }- \text { ft. } \\
18.1 \\
19.4\end{aligned}$ \\
\hline
\end{tabular}

Daily discharge, in second-feet, of Honolii River at Kaiwiki, near Hilo, Hawaii, for 1913.

\begin{tabular}{|c|c|c|c|c|c|c|c|}
\hline Day. & Jan. & Feb. & Mar. & Day. & Jan. & Feb. & Mar. \\
\hline 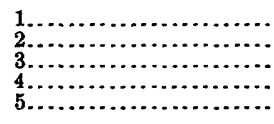 & $\begin{array}{r}153 \\
86 \\
79 \\
56 \\
43\end{array}$ & $\begin{array}{l}16 \\
15 \\
12 \\
11 \\
11\end{array}$ & $\begin{array}{l}3.4 \\
3.4 \\
3.0 \\
3.0 \\
2.8\end{array}$ & $\begin{array}{l}16 \ldots \ldots \\
17 \ldots \ldots \\
18 \ldots \ldots \\
20 \ldots \ldots\end{array}$ & $\begin{array}{l}238 \\
182 \\
155 \\
358 \\
318\end{array}$ & $\begin{array}{l}18 \\
40 \\
18 \\
14 \\
11\end{array}$ & $\begin{array}{r}12 \\
30 \\
86 \\
110 \\
37\end{array}$ \\
\hline 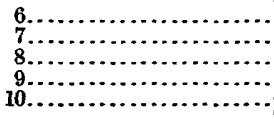 & $\begin{array}{r}42 \\
48 \\
430 \\
315 \\
299\end{array}$ & $\begin{array}{l}16 \\
30 \\
17 \\
13 \\
11\end{array}$ & $\begin{array}{l}2.8 \\
8.6 \\
6.2 \\
3.8 \\
2.8\end{array}$ & 21 24 & $\begin{array}{r}268 \\
202 \\
108 \\
83 \\
65\end{array}$ & $\begin{array}{l}9.4 \\
9.0 \\
7.0 \\
7.0 \\
7.0\end{array}$ & $\begin{array}{r}23 \\
103 \\
80 \\
35\end{array}$ \\
\hline 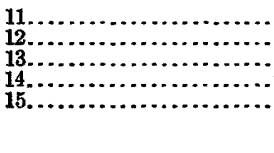 & $\begin{array}{l}724 \\
196 \\
512 \\
840 \\
660\end{array}$ & $\begin{array}{c}11 \\
11 \\
10 \\
8.6 \\
8.6\end{array}$ & $\begin{array}{r}2.4 \\
2.0 \\
1.8 \\
2.0 \\
18\end{array}$ & $\begin{array}{l}26 \\
27 \\
28 \\
30 \\
31\end{array}$ & $\begin{array}{l}48 \\
38 \\
32 \\
27 \\
24 \\
17\end{array}$ & $\begin{array}{r}\mathbf{5 . 0} \\
\mathbf{3 . 0} \\
\mathbf{3 . 0} \\
\cdots \\
\cdots \\
\cdots\end{array}$ & 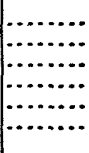 \\
\hline
\end{tabular}

Monthly discharge of Honolii River at Kaiwiki, near Hilo, Hawaii, for 1918.

\begin{tabular}{|c|c|c|c|c|}
\hline \multirow{2}{*}{ Month. } & \multicolumn{3}{|c|}{ Discharge in second-feet. } & \multirow{2}{*}{$\begin{array}{l}\text { Run-off } \\
\text { (total in } \\
\text { acre-feet). }\end{array}$} \\
\hline & Maximum. & Minimum. & Mean. & \\
\hline $\begin{array}{l}\text { January } \\
\text { February } \\
\text { March } 1-24 \ldots \ldots \\
\text { Man. }\end{array}$ & $\begin{array}{r}842 \\
50 \\
110\end{array}$ & $\begin{array}{r}17 \\
3.0 \\
1.8\end{array}$ & $\begin{array}{r}214 \\
12.6 \\
24.2\end{array}$ & $\begin{array}{r}13,200 \\
700 \\
1,150\end{array}$ \\
\hline
\end{tabular}




\section{HAMAKUA GROUP.}

HAMAKUA DITCH AT MATN WEIR, PUUALALA, MEAR WAIMEA, HAWAII.

Location.- Near head of ditch in Lalakea tract, adjacent to forest reserve and close to Kaala Mountain and Pacific Sugar Mill fence. This ditch diverts all run-off from upper headwaters of Waipio.

Records available.-January 1 to December 31, 1913.

Gage.-Water-stage recorder.

Discharge.-Measured by weir consisting of two 5-foot panels, sharp crested, and with good stilling basin above.

Discharge, in million gallons per day, of Hamakua ditch at main weir, at Puualala Waimea, Hawaii, for 1913.

\begin{tabular}{|c|c|c|c|c|c|c|c|c|c|c|c|c|}
\hline Day. & Jan. & Feb. & Mar. & Apr. & May. & June. & July. & Aug. & Sept. & Oct. & Nov. & Dec. \\
\hline $\begin{array}{l}1 \ldots \\
2 \ldots \\
3 \ldots \\
4 \ldots \\
5 \ldots\end{array}$ & $\begin{array}{l}3.35 \\
2.11 \\
4.21 \\
2.89 \\
2.53\end{array}$ & $\begin{array}{r}1.07 \\
.87 \\
.44 \\
.32 \\
.24\end{array}$ & $\begin{array}{r}0.96 \\
.68 \\
.54 \\
.39 \\
.32\end{array}$ & $\begin{array}{l}8.42 \\
7.62 \\
3.86 \\
6.72 \\
3.42\end{array}$ & $\begin{array}{l}2.21 \\
3.84 \\
6.25 \\
5.54 \\
2.94\end{array}$ & $\begin{array}{l}2.09 \\
6.24 \\
7.70 \\
2.55 \\
1.53\end{array}$ & $\begin{array}{c}18.3 \\
16.5 \\
12.7 \\
13.4 \\
8.78\end{array}$ & $\begin{array}{c}1.05 \\
11.8 \\
11.0 \\
2.73 \\
1.39\end{array}$ & $\begin{array}{c}2.66 \\
9.14 \\
10.2 \\
5.76 \\
2.30\end{array}$ & $\begin{array}{r}0.37 \\
.30 \\
.22 \\
.18 \\
.15\end{array}$ & $\begin{array}{c}29.2 \\
22.7 \\
8.51\end{array}$ & $\begin{array}{r}1.25 \\
. .95 \\
1.08 \\
5.06 \\
25.3\end{array}$ \\
\hline $\begin{array}{r}6 \ldots \\
7 \ldots \\
8 \ldots \\
9 \ldots \\
10 \ldots\end{array}$ & $\begin{array}{l}1.45 \\
1.04 \\
.85 \\
1.04 \\
3.70\end{array}$ & $\begin{array}{c}12.1 \\
11.0 \\
2.66 \\
1.45 \\
.83\end{array}$ & $\begin{array}{l}.26 \\
.26 \\
.20 \\
.18 \\
.18\end{array}$ & $\begin{array}{l}5.09 \\
8.37 \\
2.80 \\
9.15 \\
8.02\end{array}$ & $\begin{array}{r}1.56 \\
.93 \\
.64 \\
.61 \\
.52\end{array}$ & $\begin{array}{l}1.14 \\
1.75 \\
4.45 \\
4.96 \\
7.32\end{array}$ & $\begin{array}{c}26.8 \\
20.9 \\
18.5 \\
8.55 \\
6.91\end{array}$ & $\begin{array}{r}.85 \\
.64 \\
.64 \\
1.31 \\
1.45\end{array}$ & $\begin{array}{c}1.79 \\
16.2 \\
5.75 \\
2.50 \\
12.2\end{array}$ & $\begin{array}{r}.23 \\
.32 \\
.99 \\
1.32 \\
.74\end{array}$ & $\begin{array}{c}3.24 \\
1.68 \\
.98 \\
18.7 \\
20.6\end{array}$ & $\begin{array}{c}19.8 \\
9.33 \\
4.55 \\
3.50 \\
2.27\end{array}$ \\
\hline $\begin{array}{l}13 \ldots \\
14 \ldots \\
15 \ldots\end{array}$ & $\begin{array}{c}2.66 \\
1.67 \\
23.0 \\
24.6 \\
18.8\end{array}$ & $\begin{array}{r}.65 \\
.52 \\
.39 \\
4.26 \\
4.89\end{array}$ & $\begin{array}{r}.13 \\
.13 \\
.04 \\
5.61 \\
2.34\end{array}$ & $\begin{array}{l}20.5 \\
24.9 \\
22.9 \\
15.6 \\
10.4\end{array}$ & $\begin{array}{c}.44 \\
6.21 \\
11.1 \\
3.72 \\
12.0\end{array}$ & $\begin{array}{c}3.99 \\
12.7 \\
25.2 \\
20.5 \\
24.8\end{array}$ & $\begin{array}{c}9.86 \\
16.2 \\
16.8 \\
13.8 \\
8.93\end{array}$ & $\begin{array}{l}7.51 \\
21.3 \\
21.2 \\
11.3 \\
19.0\end{array}$ & $\begin{array}{c}7.36 \\
11.6 \\
9.53 \\
3.61 \\
3.52\end{array}$ & $\begin{array}{r}.37 \\
.13 \\
.13 \\
.71 \\
2.88\end{array}$ & $\begin{array}{c}16.1 \\
22.1 \\
17.1 \\
10.0 \\
6.32\end{array}$ & $\begin{array}{r}1.60 \\
1.91 \\
1.47 \\
4.11 \\
24.6\end{array}$ \\
\hline $\begin{array}{l}16 . . \\
17 . . \\
18 . . \\
19 . . \\
20 . .\end{array}$ & $\begin{array}{r}6.38 \\
2.84 \\
1.56 \\
1.26 \\
.95\end{array}$ & $\begin{array}{c}1.46 \\
7.49 \\
10.9 \\
20.6 \\
10.4\end{array}$ & $\begin{array}{l}5.78 \\
8.78 \\
4.43 \\
4.97 \\
2.94\end{array}$ & \begin{tabular}{|c|}
10.6 \\
10.5 \\
10.7 \\
8.41 \\
5.03
\end{tabular} & $\begin{array}{c}17.0 \\
10.7 \\
12.8 \\
17.9 \\
6.59\end{array}$ & \begin{tabular}{|c}
16.9 \\
12.5 \\
4.45 \\
2.90 \\
2.25
\end{tabular} & $\begin{array}{c}8.73 \\
12.7 \\
11.0 \\
5.16 \\
6.08\end{array}$ & $\begin{array}{r}6.85 \\
2.77 \\
1.57 \\
1.08 \\
.83\end{array}$ & $\begin{array}{c}1.69 \\
3.87 \\
14.8 \\
9.65 \\
5.27\end{array}$ & $\begin{array}{r}6.26 \\
1.71 \\
.60 \\
.24 \\
1.60\end{array}$ & $\begin{array}{l}18.5 \\
24.9 \\
30.0 \\
15.0 \\
12.3\end{array}$ & $\begin{array}{c}17.1 \\
14.8 \\
13.5 \\
6.56 \\
3.21\end{array}$ \\
\hline $\begin{array}{l}21 \ldots \\
22 \ldots \\
23 \ldots \\
24 \ldots \\
25 \ldots\end{array}$ & $\begin{array}{r}.74 \\
1.11 \\
.95 \\
.68 \\
.68\end{array}$ & $\begin{array}{c}10.2 \\
8.62 \\
3.54 \\
1.55 \\
2.58\end{array}$ & $\begin{array}{r}11.9 \\
16.7 \\
4.78 \\
3.37 \\
4.06\end{array}$ & $\begin{array}{l}4.36 \\
5.74 \\
7.46 \\
5.95 \\
8.01\end{array}$ & $\begin{array}{l}2.64 \\
1.53 \\
1.42 \\
1.57 \\
2.68\end{array}$ & $\begin{array}{l}1.62 \\
1.37 \\
6.43 \\
8.64 \\
3.94\end{array}$ & $\begin{array}{c}10.1 \\
15.7 \\
6.47 \\
3.70 \\
3.40\end{array}$ & $\begin{array}{r}.92 \\
1.45 \\
.95 \\
1.16 \\
1.14\end{array}$ & $\begin{array}{r}2.79 \\
1.32 \\
.68 \\
.52 \\
.61\end{array}$ & $\begin{array}{r}2.50 \\
1.34 \\
1.87 \\
.95 \\
.37\end{array}$ & $\begin{array}{c}13.5 \\
13.2 \\
9.60 \\
14.1 \\
14.6\end{array}$ & $\begin{array}{r}2.03 \\
1.56 \\
13.3 \\
4.13 \\
1.79\end{array}$ \\
\hline $\begin{array}{l}26 \ldots \ldots \\
27 \ldots \ldots \\
28 \ldots \ldots \\
29 . \ldots \\
30 . \ldots \\
31 \ldots \ldots\end{array}$ & $\begin{array}{l}.40 \\
.46 \\
.55 \\
.37 \\
.30 \\
.24\end{array}$ & $\begin{array}{c}13.8 \\
3.12 \\
1.37 \\
\ldots . . .\end{array}$ & $\begin{array}{c}2.40 \\
4.43 \\
24.6 \\
11.1 \\
13.9 \\
16.2\end{array}$ & $\begin{array}{r}4.65 \\
7.22 \\
8.02 \\
3.82 \\
2.58 \\
. . .\end{array}$ & $\begin{array}{c}20.3 \\
13.6 \\
18.3 \\
13.7 \\
6.22 \\
3.60\end{array}$ & $\begin{array}{c}6.67 \\
23.4 \\
27.5 \\
23.4 \\
22.3 \\
\ldots \ldots .\end{array}$ & $\begin{array}{l}6.45 \\
2.84 \\
5.28 \\
3.04 \\
1.68 \\
.1 .45\end{array}$ & $\begin{array}{r}.68 \\
.62 \\
1.20 \\
2.74 \\
10.7 \\
5.04\end{array}$ & $\begin{array}{l}.60 \\
.30 \\
.26 \\
.32 \\
.30\end{array}$ & $\begin{array}{l}.15 \\
.86 \\
.56\end{array}$ & $\begin{array}{c}12.5 \\
10.3 \\
3.90 \\
2.00 \\
1.68\end{array}$ & $\begin{array}{r}1.25 \\
.85 \\
.70 \\
.52 \\
.40 \\
.30\end{array}$ \\
\hline
\end{tabular}

Nore. -No discharge Oct. 29 to Nov. 2.

Monthly discharge of Hamakua ditch at main weir, Puualala, Waimea, Hawaii, for 1913.

\begin{tabular}{|c|c|c|c|c|}
\hline \multirow{2}{*}{ Month. } & \multicolumn{3}{|c|}{ Discharge in million gallons per } & \multirow{2}{*}{$\begin{array}{l}\text { Run-off } \\
\text { (total in } \\
\text { acre-feet). }\end{array}$} \\
\hline & Maximum. & Minimum. & Mean. & \\
\hline 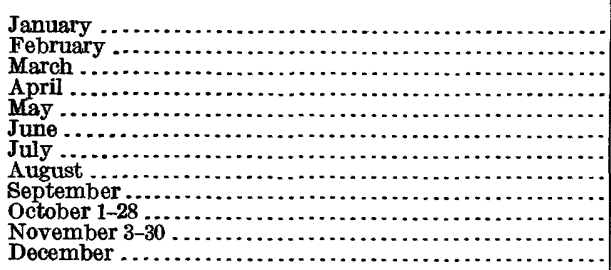 & $\begin{array}{c}24.6 \\
20.6 \\
24.6 \\
24.9 \\
20.3 \\
27.5 \\
26.8 \\
21.3 \\
16.2 \\
6.26 \\
30.0 \\
25.3\end{array}$ & $\begin{array}{r}0.24 \\
.24 \\
.04 \\
2.58 \\
.44 \\
1.14 \\
1.45 \\
.62 \\
.26 \\
.13 \\
.98 \\
.30\end{array}$ & $\begin{array}{c}3.66 \\
4.90 \\
4.92 \\
8.69 \\
6.74 \\
9.70 \\
10.3 \\
4.93 \\
4.90 \\
1.00 \\
13.3 \\
6.09\end{array}$ & $\begin{array}{r}348 \\
422 \\
468 \\
800 \\
641 \\
893 \\
980 \\
469 \\
451 \\
86 \\
\mathbf{1}, \mathbf{1 4 0} \\
\mathbf{5 7 9}\end{array}$ \\
\hline The period & & & ....... & 7,300 \\
\hline
\end{tabular}


NEW HAMAKUA DITCH AT MAIN WEIR NEAR KUKUIHAELE, HAWAII.

Location.-Near head of ditch, about 500 feet south of office of Hawaiian Irrigation Co. (Ltd.), at Kukuihaele.

Records available.-July 18, 1910, to December 31, 1913.

Gage.-Water-stage recorder.

Discharge.--Measured by weir consisting of six 5-foot panels, sharp crested, and with a good stilling basin above. Discharge measurements made in ditch below weir have checked weir discharge to within 2 per cent.

Diversion.-Ditch diverts all run-off from headwaters of Waipio basin below the upper Hamakua ditch.

Cooperation.-Daily-discharge record furnished by the Hawaiian Irrigation Co. (Ltd.).

Discharge, in million gallons per day, of New Hamakua ditch at main weir, Kukuihaele, Hawaii, for 1913.

\begin{tabular}{r|r|r|r|r|r|r|r|r|r|r|r|r}
\hline Day. & Jan. & Feb. & Mar. & Apr. & May. & June. & July. & Aug. & Sept. & Oct. & Nov. & Dec. \\
\hline & & & & & & & & & & & \\
\hline
\end{tabular}


Monthly discharge of New Hamakua ditch at main weir, near Kukuihaele, Hawaii, for 1913.

\begin{tabular}{|c|c|c|c|c|c|}
\hline \multirow{2}{*}{ Month. } & \multicolumn{3}{|c|}{$\begin{array}{l}\text { Discharge in million gallons per } \\
\text { day. }\end{array}$} & \multirow{2}{*}{$\begin{array}{c}\text { Run-off } \\
\text { (total in } \\
\text { acre-feet). }\end{array}$} & \multirow{2}{*}{$\begin{array}{l}\text { Accu- } \\
\text { racy. }\end{array}$} \\
\hline & Maximum. & Minimum. & Mean. & & \\
\hline 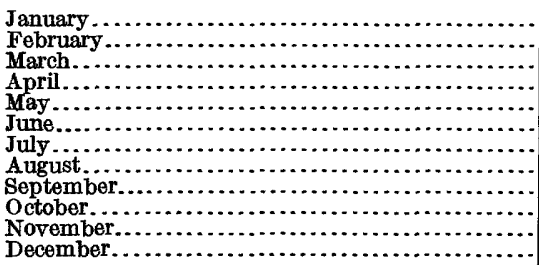 & $\begin{array}{l}34.8 \\
35.0 \\
37.0 \\
34.9 \\
3.4 \\
34.6 \\
44.2 \\
52.1 \\
43.1 \\
30.9 \\
43.8 \\
34.8\end{array}$ & $\begin{array}{l}25.7 \\
25.5 \\
24.6 \\
28.9 \\
26.2 \\
26.9 \\
28.0 \\
12.9 \\
25.5 \\
23.9 \\
23.7 \\
29.0\end{array}$ & \begin{tabular}{l|}
29.7 \\
29.3 \\
28.3 \\
33.0 \\
30.7 \\
30.9 \\
36.7 \\
31.5 \\
31.7 \\
25.5 \\
33.8 \\
32.5
\end{tabular} & $\begin{array}{l}2,830 \\
2,520 \\
2,692 \\
3,040 \\
2,920 \\
2,840 \\
3,490 \\
3,000 \\
2,920 \\
2,430 \\
3,110 \\
3,090\end{array}$ & $\begin{array}{l}\text { A. } \\
\text { A. } \\
\text { A. } \\
\text { A. } \\
\text { A. } \\
\text { A. } \\
\text { A. } \\
\text { A. } \\
\text { A. } \\
\text { A. } \\
\text { A. }\end{array}$ \\
\hline The year..... & 52.1 & 12.9 & 31.1 & 34,900 & \\
\hline
\end{tabular}

KOHALA GROUP.

KOHALA DITCH TMAR KOHALA, HAWAII.

Location of weirs.-The Kohala Ditch Co. (Ltd.) maintains three weirs which measure the discharge carried by the Kohala ditch. The Awini weir is on the southeast side of the east branch of Honokanenui Gulch at an elevation of 1,880 feet. The Niulii weir is about $3 \frac{1}{2}$ miles south of Niulii, Kohala district, at an elevation of 1,000 feet, and about 5 miles below the Awini weir. The Hawi weir is about $1 \frac{1}{2}$ miles south of the Hawi mill near the lower end of the ditch.

Records available.-Daily records at Awini and Niulii weirs kept by Kohala Ditch Co. July 1, 1907, to December 31, 1913. No record of flow at Hawi weir, which has been used mainly as a check on deliveries of water between Niulii and Hawi. The Niulii weir measures the amount of water shown by the Awini weir and also the inflow from the Honokanenui. The record for theHonokenenui is derived by subtracting the flow at the Awini weir from the flow at the Niulii weir.

Gages.-Vertical staffs graduated to hundredths.

Discharge measurements.-Each weir consists of three 5-foot panels with end contractions, sharp-beveled steel crest, and with very slight velocity of approach.

Diversion.-Ditch diverts and delivers to the plantations on the north coast of Hawaii all low-water run-off between the Honokane Gulch and the northwest point of the island below the 2,000-foot contour.

Cooperation.-Daily-discharge record furnished by Kohala Ditch Co. (Ltd.). 
Discharge, in million gallons per day, of Kohala ditch at Awini weir, near Kohala, Hawaii, for 1913.

\begin{tabular}{|c|c|c|c|c|c|c|c|c|c|c|c|c|}
\hline Day. & Jan. & Fob. & Mar. & Apr. & May. & June. & July. & Aug. & Sept. & Oct. & Nov. & Dec. \\
\hline $\begin{array}{l}1 \ldots \\
2 \ldots \\
3 \ldots \\
4 \ldots \\
5 \ldots\end{array}$ & $\begin{array}{l}12.5 \\
11.0 \\
11.0 \\
10.5 \\
11.0\end{array}$ & $\begin{array}{l}9.3 \\
9.8 \\
7.9 \\
5.2 \\
4.4\end{array}$ & $\begin{array}{l}4.8 \\
4.0 \\
3.3 \\
3.7 \\
3.7\end{array}$ & $\begin{array}{r}12.5 \\
11.5 \\
8.4 \\
6.2 \\
6.2\end{array}$ & $\begin{array}{l}7.5 \\
8.8 \\
8.8 \\
7.5 \\
7.5\end{array}$ & $\begin{array}{l}2.3 \\
2.1^{\circ} \\
6.2 \\
6.2\end{array}$ & $\begin{array}{l}16.9 \\
19.5 \\
16.9 \\
14.6 \\
12.5\end{array}$ & $\begin{array}{l}3.3 \\
4.4 \\
8.4 \\
6.2 \\
5.8\end{array}$ & $\begin{array}{l}5.2 \\
3.7 \\
4.8 \\
4.8 \\
3.7\end{array}$ & $\begin{array}{r}0.9 \\
.9 \\
.9 \\
.9 \\
.5\end{array}$ & $\begin{array}{l}11.5 \\
18.3 \\
13.6\end{array}$ & $\begin{array}{r}6.6 \\
9.8 \\
9.8 \\
10.5 \\
20.7\end{array}$ \\
\hline $\begin{array}{r}6 \ldots \ldots \\
7 \ldots \ldots \\
8 \ldots \ldots \\
9 \ldots \ldots \\
10 \ldots \ldots\end{array}$ & $\begin{array}{r}10.5 \\
8.8 \\
9.8 \\
10.5 \\
12.5\end{array}$ & $\begin{array}{r}10.5 \\
14.1 \\
9.8 \\
7.9 \\
4.4\end{array}$ & $\begin{array}{l}3.7 \\
3.7 \\
3.7 \\
3.3 \\
3.0\end{array}$ & $\begin{array}{l}7.5 \\
9.3 \\
6.2 \\
5.2 \\
7.5\end{array}$ & $\begin{array}{l}7.0 \\
6.6 \\
5.2 \\
4.4 \\
4.4\end{array}$ & $\begin{array}{l}4.0 \\
4.8 \\
5.8 \\
5.8 \\
5.8\end{array}$ & $\begin{array}{l}20.7 \\
20.7 \\
16.9 \\
14.6 \\
12.0\end{array}$ & $\begin{array}{l}5.2 \\
4.0 \\
4.0 \\
3.3 \\
4.0\end{array}$ & $\begin{array}{l}3.0 \\
6.2 \\
6.2 \\
5.2 \\
6.2\end{array}$ & $\begin{array}{l}.5 \\
.5 \\
1.5 \\
1.2 \\
2.1\end{array}$ & $\begin{array}{r}10.5 \\
7.0 \\
5.2 \\
20.1 \\
20.1\end{array}$ & $\begin{array}{l}20.7 \\
20.7 \\
20.7 \\
20.1 \\
19.5\end{array}$ \\
\hline $\begin{array}{l}11 \ldots \ldots \\
13 \ldots \ldots \ldots \\
15 \\
13 \ldots \ldots\end{array}$ & $\begin{array}{l}10.5 \\
10.5 \\
20.7 \\
20.7 \\
20.7\end{array}$ & $\begin{array}{l}4.4 \\
3.3 \\
3.3 \\
9.3 \\
8.4\end{array}$ & $\begin{array}{l}2.7 \\
2.7 \\
2.4 \\
2.4 \\
2.7\end{array}$ & $\begin{array}{l}12.5 \\
15.7 \\
15.7 \\
15.7 \\
15.7\end{array}$ & $\begin{array}{r}4.4 \\
6.6 \\
15.7 \\
15.7 \\
15.7\end{array}$ & $\begin{array}{r}5.2 \\
5.2 \\
20.7 \\
20.7 \\
20.7\end{array}$ & $\begin{array}{l}10.5 \\
14.6 \\
16.9 \\
14.6 \\
14.6\end{array}$ & $\begin{array}{r}4.0 \\
16.9 \\
20.7 \\
20.7 \\
16.9\end{array}$ & $\begin{array}{l}7.9 \\
7.0 \\
7.9 \\
6.2 \\
4.8\end{array}$ & $\begin{array}{r}1.5 \\
.9 \\
.7 \\
.5 \\
.5\end{array}$ & $\begin{array}{l}20.1 \\
20.1 \\
15.2 \\
12.0 \\
10.5\end{array}$ & $\begin{array}{l}16.9 \\
14.1 \\
12.5 \\
11.5 \\
20.7\end{array}$ \\
\hline $\begin{array}{l}16 \ldots \ldots . \\
17 \ldots \ldots . \\
18 \ldots \ldots \\
19 \ldots \ldots \\
20 \ldots \ldots\end{array}$ & $\begin{array}{r}13.0 \\
12.5 \\
11.0 \\
9.8 \\
9.8\end{array}$ & $\begin{array}{r}6.6 \\
11.0 \\
11.5 \\
14.1 \\
11.5\end{array}$ & $\begin{array}{l}2.7 \\
2.7 \\
3.3 \\
3.3 \\
3.3\end{array}$ & $\begin{array}{l}15.7 \\
14.6 \\
15.7 \\
14.6 \\
14.6\end{array}$ & $\begin{array}{r}14.6 \\
14.6 \\
11.5 \\
10.5 \\
8.8\end{array}$ & $\begin{array}{r}16.3 \\
13.6 \\
9.8 \\
9.3 \\
6.6\end{array}$ & $\begin{array}{r}12.5 \\
10.5 \\
8.4 \\
8.4 \\
7.5\end{array}$ & $\begin{array}{r}15.2 \\
12.5 \\
7.5 \\
5.8 \\
4.8\end{array}$ & $\begin{array}{r}4.0 \\
3.7 \\
7.9 \\
11.5 \\
7.0\end{array}$ & $\begin{array}{r}1.5 \\
1.2 \\
.9 \\
.7 \\
.5\end{array}$ & $\begin{array}{l}12.0 \\
20.1 \\
20.1 \\
20.1 \\
16.3\end{array}$ & $\begin{array}{l}20.7 \\
20.7 \\
20.7 \\
16.9 \\
14.1\end{array}$ \\
\hline $\begin{array}{l}21 \ldots \ldots \\
22 \ldots \ldots \\
23 \ldots \ldots \\
24 \ldots \ldots \\
25 \ldots \ldots\end{array}$ & $\begin{array}{l}8.4 \\
8.4 \\
7.9 \\
7.9 \\
5.2\end{array}$ & $\begin{array}{l}8.8 \\
8.8 \\
8.4 \\
6.6 \\
6.6\end{array}$ & $\begin{array}{r}7.5 \\
12.5 \\
7.9 \\
4.8 \\
4.0\end{array}$ & \begin{tabular}{r|}
12.5 \\
11.5 \\
12.0 \\
9.8 \\
10.5
\end{tabular} & $\begin{array}{l}7.5 \\
6.2 \\
4.4 \\
4.0 \\
4.0\end{array}$ & $\begin{array}{l}6.6 \\
5.2 \\
4.8 \\
5.2 \\
4.8\end{array}$ & $\begin{array}{l}6.6 \\
7.9 \\
7.5 \\
6.6 \\
5.8\end{array}$ & $\begin{array}{l}4.8 \\
4.0 \\
3.3 \\
2.7 \\
4.0\end{array}$ & $\begin{array}{l}5.2 \\
4.4 \\
3.3 \\
2.7 \\
1.8\end{array}$ & $\begin{array}{l}.5 \\
.5 \\
.5 \\
.5 \\
.5\end{array}$ & $\begin{array}{l}20.1 \\
14.6 \\
13.6 \\
13.6 \\
12.5\end{array}$ & $\begin{array}{l}12.5 \\
10.5 \\
15.7 \\
14.1 \\
12.5\end{array}$ \\
\hline $\begin{array}{l}26 \ldots \\
27 \ldots \ldots \\
28 \ldots \\
29 \ldots \\
30 \ldots \\
31 \ldots \\
\end{array}$ & $\begin{array}{l}5.2 \\
5.2 \\
4.8 \\
4.8 \\
4.8 \\
4.8\end{array}$ & $\begin{array}{r}14.1 \\
8.4 \\
6.6 \\
\ldots . . \\
\ldots . . . \\
\end{array}$ & $\begin{array}{r}4.0 \\
3.3 \\
12.0 \\
10.5 \\
7.5 \\
12.5\end{array}$ & $\begin{array}{r}9.8 \\
14.6 \\
13.0 \\
10.5 \\
9.3\end{array}$ & $\begin{array}{r}12.0 \\
15.7 \\
12.0 \\
13.6 \\
10.5 \\
7.5\end{array}$ & $\begin{array}{r}4.8 \\
14.6 \\
20.7 \\
20.7 \\
20.7\end{array}$ & $\begin{array}{l}6.2 \\
5.8 \\
5.8 \\
5.2 \\
4.4 \\
4.4\end{array}$ & $\begin{array}{l}2.1 \\
2.1 \\
2.1 \\
2.1 \\
5.8 \\
5.8\end{array}$ & $\begin{array}{l}1.8 \\
1.8 \\
1.2 \\
1.2 \\
1.2\end{array}$ & $\begin{array}{r}.5 \\
.3 \\
.2 \\
.2 \\
\ldots . .\end{array}$ & $\begin{array}{r}12.5 \\
11.0 \\
9.8 \\
8.4 \\
7.5\end{array}$ & $\begin{array}{r}10.5 \\
9.8 \\
7.9 \\
6.6 \\
6.2 \\
6.2\end{array}$ \\
\hline
\end{tabular}

Note,-No record June 2, Oct. 29 to Nov. 2.

Monthly discharge of Kohala ditch at Awini weir, near Kohala, Hawaï, for 1913.

\begin{tabular}{|c|c|c|c|c|}
\hline \multirow{2}{*}{ Month. } & \multicolumn{3}{|c|}{$\begin{array}{l}\text { Discharge in million gallons per } \\
\text { per day. }\end{array}$} & \multirow{2}{*}{$\begin{array}{l}\text { Run-off } \\
\text { (total in } \\
\text { acre-feet). }\end{array}$} \\
\hline & Maximum. & Minimum. & Ṃ̨n. & \\
\hline 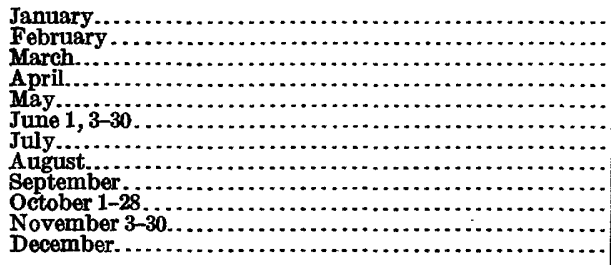 & $\begin{array}{l}20.7 \\
14.1 \\
12.5 \\
15.7 \\
15.7 \\
20.7 \\
20.7 \\
20.7 \\
11.5 \\
20.1 \\
20.7 \\
20.7\end{array}$ & $\begin{array}{l}4.8 \\
3.3 \\
2.4 \\
5.2 \\
4.0 \\
2.1 \\
4.4 \\
2.1 \\
1.2 \\
.2 \\
5.2 \\
6.2\end{array}$ & $\begin{array}{r}10.1 \\
8.39 \\
4.89 \\
1 . .5 \\
9.14 \\
9.63 \\
11.3 \\
6.85 \\
4.72 \\
14.8 \\
14.2\end{array}$ & $\begin{array}{r}961 \\
721 \\
465 \\
1,060 \\
870 \\
857 \\
1,080 \\
652 \\
435 \\
69 \\
1,220 \\
1,340\end{array}$ \\
\hline The period....... & & . & & 9,730 \\
\hline
\end{tabular}


Discharge, in million gallons per day, of Kohala ditch at Nuilii weir, near Kohala, Hawaii, for 1918.

\begin{tabular}{|c|c|c|c|c|c|c|c|c|c|c|c|c|}
\hline Day. & Jan. & Feb. & Mar. & Apr. & May. & June. & July. & Aug. & Sept. & Oct. & Nov. & Dec. \\
\hline $\begin{array}{l}1 \ldots \\
2 \ldots \\
3 \ldots \\
4 \ldots \\
5 \ldots\end{array}$ & $\begin{array}{l}12.8 \\
12.6 \\
12.6 \\
11.5 \\
12.6\end{array}$ & $\begin{array}{l}11.1 \\
11.4 \\
10.1 \\
10.6 \\
10.3\end{array}$ & $\begin{array}{l}9.2 \\
9.2 \\
8.5 \\
8.9 \\
8.9\end{array}$ & $\begin{array}{l}12.8 \\
12.1 \\
11.2 \\
11.8 \\
11.8\end{array}$ & $\begin{array}{l}11.3 \\
13.1 \\
13.1 \\
11.3 \\
11.3\end{array}$ & $\begin{array}{r}6.3 \\
4.0 \\
11.1 \\
11.1\end{array}$ & $\begin{array}{r}10.6 \\
8.0 \\
10.6 \\
12.8 \\
14.9\end{array}$ & $\begin{array}{r}9.9 \\
10.2 \\
12.0 \\
11.8 \\
11.5\end{array}$ & $\begin{array}{l}9.5 \\
8.2 \\
8.5 \\
8.5 \\
8.2\end{array}$ & $\begin{array}{l}6.8 \\
6.3 \\
6.3 \\
6.8 \\
7.3\end{array}$ & $\begin{array}{r}6.0 \\
6.0 \\
14.7 \\
9.1 \\
13.9\end{array}$ & $\begin{array}{r}9.9 \\
13.0 \\
12.2 \\
13.1 \\
6.8\end{array}$ \\
\hline $\begin{array}{r}6 \ldots \\
7 \ldots \\
8 \ldots \\
9 \ldots \\
10 . .\end{array}$ & \begin{tabular}{r|r|}
11.5 & 9.9 \\
10.6 & 12.3 \\
13.6 &
\end{tabular} & $\begin{array}{l}13.9 \\
13.3 \\
11.4 \\
10.1 \\
10.3\end{array}$ & $\begin{array}{l}8.9 \\
8.9 \\
8.9 \\
8.5 \\
8.2\end{array}$ & $\begin{array}{r}11.3 \\
11.1 \\
9.6 \\
9.5 \\
11.3\end{array}$ & $\begin{array}{l}11.0 \\
10.6 \\
10.6 \\
10.3 \\
10.3\end{array}$ & $\begin{array}{r}9.9 \\
9.9 \\
10.0 \\
10.0 \\
10.0\end{array}$ & $\begin{array}{r}6.8 \\
6.8 \\
10.6 \\
12.8 \\
12.4\end{array}$ & $\begin{array}{r}10.6 \\
9.2 \\
9.2 \\
9.2 \\
9.2\end{array}$ & \begin{tabular}{r|}
8.2 \\
10.3 \\
11.1 \\
10.6 \\
11.1
\end{tabular} & $\begin{array}{r}7.3 \\
6.7 \\
7.1 \\
7.4 \\
14.4\end{array}$ & $\begin{array}{r}10.6 \\
9.5 \\
8.1 \\
6.1 \\
7.4\end{array}$ & $\begin{array}{l}6.8 \\
6.8 \\
6.8 \\
7.4 \\
8.0\end{array}$ \\
\hline $\begin{array}{l}11 \ldots \\
12 \ldots \\
13 \ldots \\
14 \ldots \\
15 \ldots\end{array}$ & $\begin{array}{r}10.6 \\
11.5 \\
5.5 \\
6.8 \\
6.8\end{array}$ & $\begin{array}{r}10.3 \\
9.2 \\
9.9 \\
10.3 \\
10.4\end{array}$ & $\begin{array}{l}7.9 \\
7.9 \\
7.5 \\
7.5 \\
7.9\end{array}$ & $\begin{array}{l}13.6 \\
11.7 \\
11.7 \\
11.7 \\
11.7\end{array}$ & $\begin{array}{l}10.3 \\
13.0 \\
11.7 \\
11.7 \\
11.7\end{array}$ & $\begin{array}{l}9.5 \\
9.5 \\
6.8 \\
6.8 \\
6.8\end{array}$ & $\begin{array}{l}11.5 \\
12.8 \\
10.6 \\
12.8 \\
12.8\end{array}$ & $\begin{array}{r}9.2 \\
10.6 \\
6.8 \\
6.8 \\
10.6\end{array}$ & $\begin{array}{r}14.0 \\
11.7 \\
11.6 \\
9.6 \\
8.5\end{array}$ & $\begin{array}{l}8.4 \\
9.0 \\
8.0 \\
7.3 \\
7.3\end{array}$ & $\begin{array}{r}7.4 \\
7.4 \\
12.3 \\
13.3 \\
11.5\end{array}$ & $\begin{array}{r}10.6 \\
13.3 \\
14.9 \\
16.0 \\
6.8\end{array}$ \\
\hline $\begin{array}{l}16 \ldots \ldots \ldots \\
17 \ldots \ldots \ldots \\
18 \ldots \ldots \ldots \ldots \\
190 \ldots \ldots\end{array}$ & $\begin{array}{l}13.1 \\
11.9 \\
11.8 \\
10.6 \\
10.6\end{array}$ & $\begin{array}{l}10.6 \\
12.6 \\
13.8 \\
13.3 \\
13.0\end{array}$ & $\begin{array}{l}7.9 \\
7.9 \\
8.5 \\
8.5 \\
8.5\end{array}$ & $\begin{array}{l}11.7 \\
12.8 \\
11.7 \\
12.8 \\
12.8\end{array}$ & $\begin{array}{l}12.8 \\
12.8 \\
16.0 \\
16.9 \\
14.8\end{array}$ & $\begin{array}{r}11.1 \\
13.9 \\
10.6 \\
10.3 \\
9.1\end{array}$ & $\begin{array}{l}10.3 \\
11.5 \\
13.6 \\
12.8 \\
12.1\end{array}$ & $\begin{array}{r}12.3 \\
12.8 \\
12.1 \\
10.7 \\
9.9\end{array}$ & \begin{tabular}{r|}
7.8 \\
7.5 \\
14.0 \\
16.0 \\
10.2
\end{tabular} & $\begin{array}{l}6.2 \\
6.5 \\
6.8 \\
7.1 \\
7.3\end{array}$ & $\begin{array}{r}11.6 \\
7.4 \\
7.4 \\
7.4 \\
11.1\end{array}$ & $\begin{array}{r}6.8 \\
6.8 \\
6.8 \\
10.6 \\
13.3\end{array}$ \\
\hline 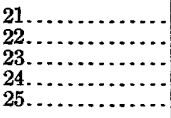 & $\begin{array}{l}10.4 \\
10.4 \\
10.1 \\
10.1 \\
10.6\end{array}$ & $\begin{array}{r}12.3 \\
12.3 \\
11.2 \\
9.9 \\
9.9\end{array}$ & $\begin{array}{r}10.5 \\
14.9 \\
10.1 \\
9.9 \\
9.2\end{array}$ & $\begin{array}{l}13.6 \\
12.1 \\
12.4 \\
12.2 \\
12.3\end{array}$ & $\begin{array}{r}12.9 \\
10.3 \\
10.3 \\
9.2 \\
9.2\end{array}$ & $\begin{array}{r}10.6 \\
11.3 \\
9.9 \\
11.3 \\
11.0\end{array}$ & $\begin{array}{l}12.2 \\
14.0 \\
14.5 \\
13.0 \\
10.7\end{array}$ & $\begin{array}{l}9.9 \\
9.2 \\
9.2 \\
9.2 \\
9.2\end{array}$ & $\begin{array}{l}8.8 \\
8.1 \\
7.2 \\
7.2 \\
6.9\end{array}$ & $\begin{array}{l}7.3 \\
7.3 \\
7.3 \\
8.2 \\
8.2\end{array}$ & $\begin{array}{r}7.4 \\
12.8 \\
13.9 \\
13.9 \\
14.9\end{array}$ & $\begin{array}{l}14.9 \\
15.6 \\
11.7 \\
13.3 \\
12.8\end{array}$ \\
\hline $\begin{array}{l}26 \ldots \\
27 \ldots \\
28 \ldots \\
29 \ldots \\
30 \ldots \\
31 \ldots \\
31 \ldots\end{array}$ & $\begin{array}{r}10.6 \\
10.6 \\
9.9 \\
9.9 \\
9.9 \\
9.9\end{array}$ & $\begin{array}{l}13.3 \\
11.2 \\
10.6 \\
\cdots . . .\end{array}$ & $\begin{array}{r}9.2 \\
9.2 \\
13.3 \\
13.1 \\
12.1 \\
15.8\end{array}$ & $\begin{array}{l}12.2 \\
12.8 \\
13.1 \\
12.3 \\
12.7\end{array}$ & $\begin{array}{l}12.4 \\
11.7 \\
12.4 \\
13.9 \\
11.5 \\
10.5\end{array}$ & $\begin{array}{l}9.9 \\
9.8 \\
6.8 \\
6.8 \\
6.8\end{array}$ & $\begin{array}{l}12.6 \\
11.5 \\
11.5 \\
12.1 \\
11.3 \\
11.3\end{array}$ & $\begin{array}{r}9.1 \\
8.5 \\
8.5 \\
9.1 \\
10.0 \\
10.0\end{array}$ & $\begin{array}{l}7.5 \\
6.9 \\
6.5 \\
7.4 \\
7.4\end{array}$ & $\begin{array}{l}6.7 \\
6.8 \\
7.0 \\
6.6 \\
6.6 \\
6.6\end{array}$ & $\begin{array}{l}14.9 \\
16.5 \\
13.8 \\
12.8 \\
11.3\end{array}$ & $\begin{array}{l}13.1 \\
12.2 \\
11.6 \\
11.4 \\
11.1 \\
11.1\end{array}$ \\
\hline
\end{tabular}

Note.-No discharge June 2.

Monthly discharge of Kohala ditch at Nuilii weir, near Kohala, Hawaii, for 1918.

\begin{tabular}{|c|c|c|c|c|}
\hline \multirow{2}{*}{ Month. } & \multicolumn{3}{|c|}{$\begin{array}{l}\text { Discharge in million gallons per } \\
\text { day. }\end{array}$} & \multirow{2}{*}{$\begin{array}{l}\text { Run-off } \\
\text { (totall in } \\
\text { acre-feet). }\end{array}$} \\
\hline & Maximum. & Minimum. & Mean. & \\
\hline 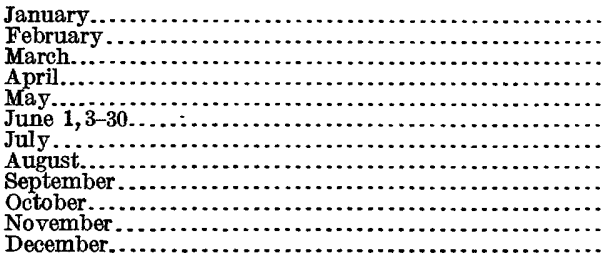 & $\begin{array}{l}13.6 \\
13.9 \\
15.8 \\
13.6 \\
16.9 \\
13.9 \\
14.9 \\
12.8 \\
16.0 \\
14.4 \\
16.5 \\
15.6\end{array}$ & $\begin{array}{l}5.5 \\
9.2 \\
7.5 \\
9.5 \\
9.2 \\
4.0 \\
6.8 \\
6.8 \\
6.5 \\
6.2 \\
6.0 \\
6.8\end{array}$ & $\begin{array}{r}10.7 \\
11.3 \\
9.53 \\
12.0 \\
11.9 \\
9.34 \\
11.7 \\
9.89 \\
9.30 \\
7.38 \\
10.7 \\
10.8\end{array}$ & $\begin{array}{r}1,020 \\
971 \\
907 \\
1,100 \\
1,130 \\
831 \\
1,110 \\
941 \\
856 \\
702 \\
985 \\
1,030\end{array}$ \\
\hline The period (364 days).... & 15.8 & 4.0 & 10.4 & 11,600 \\
\hline
\end{tabular}


KEHENA DITCH AT HONOKANE MAUKA, NEAR NORTH KOHALA, HAWAII.

Location of weirs.-On 22 streams in Kehena district, beginning at head of Honokane Gulch and extending northeast about 8 miles in Kohala Mountains, at elevation of 4,200 to 3,000 feet.

Records available.-April 1, 1912, to March 31, 1913.

Gages.-Vertical staffs; read once daily.

Discharge.-Determined by means of sharp-crested weirs with end contractions.

Diversion.-Ditch diverts most of the water above 2,000-foot contour.

Cooperation.-Daily-discharge record furnished by Kohala Ditch Co. (Ltd.).

Combined daily discharge, in million gallons per day (weirs 1 to 22), of 22 streams supplying Kehena ditch at Honokane Mauka, North Kohala, Hawaii, Apr. 1, 1912, to Mar. 31, 1913.

\begin{tabular}{|c|c|c|c|c|c|c|c|c|c|c|c|c|}
\hline Das & Apr. & May. & June. & July. & Aug. & Sept. & Oct. & Nov. & Dec. & Jan. & Feb. & Mar. \\
\hline $\begin{array}{l}1 . . \\
2 . . \\
3 . . \\
4 . . \\
5 . .\end{array}$ & \begin{tabular}{|l|}
34.23 \\
18.65 \\
54.24 \\
57.50 \\
25.03
\end{tabular} & $\begin{array}{r}5.23 \\
15.10 \\
7.62 \\
10.81 \\
19.96\end{array}$ & $\begin{array}{l}0.83 \\
.57 \\
.28 \\
.11 \\
.09\end{array}$ & $\begin{array}{l}2.96 \\
4.03 \\
5.20 \\
3.72 \\
5.37\end{array}$ & $\begin{array}{r}0.18 \\
.19 \\
.23 \\
.17 \\
.15\end{array}$ & $\begin{array}{r}0.76 \\
33.71 \\
84.81 \\
170.34 \\
9.33\end{array}$ & $\begin{array}{r}7.24 \\
2.25 \\
4.99 \\
3.31 \\
.93\end{array}$ & $\begin{array}{l}9.95 \\
2.73 \\
1.87 \\
1.11 \\
1.77\end{array}$ & $\begin{array}{r}25.78 \\
7.41 \\
2.90 \\
2.00 \\
1.55\end{array}$ & $\begin{array}{l}2.47 \\
1.86 \\
1.50 \\
2.76 \\
1.56\end{array}$ & $\begin{array}{r}0.50 \\
.63 \\
.46 \\
.31 \\
.30\end{array}$ & $\begin{array}{r}0.89 \\
.71 \\
.57 \\
.43 \\
.33\end{array}$ \\
\hline $\begin{array}{r}6 . . \\
7 . . \\
8 . . \\
9 . \\
10 .\end{array}$ & $\begin{array}{r}14.76 \\
119.37 \\
35.19 \\
41.60 \\
28.50\end{array}$ & $\begin{array}{r}26.53 \\
5.36 \\
21.26 \\
11.77 \\
5.06\end{array}$ & $\begin{array}{r}.03 \\
.03 \\
1.45 \\
16.20 \\
11.32\end{array}$ & $\begin{array}{r}3.77 \\
28.23 \\
4.36 \\
11.68 \\
3.05\end{array}$ & $\begin{array}{r}.14 \\
1.29 \\
.87 \\
22.99 \\
20.62\end{array}$ & $\begin{array}{r}3.80 \\
23.61 \\
5.24 \\
2.34 \\
1.12\end{array}$ & $\begin{array}{l}.31 \\
.17 \\
.11 \\
.10 \\
.98\end{array}$ & $\begin{array}{r}1.68 \\
1.24 \\
1.10 \\
21.51 \\
105.51\end{array}$ & $\begin{array}{r}1.26 \\
3.05 \\
10.84 \\
4.34 \\
22.16\end{array}$ & $\begin{array}{l}1.04 \\
.82 \\
1.06 \\
2.06 \\
9.28\end{array}$ & $\begin{array}{r}1.22 \\
5.09 \\
2.33 \\
1.07 \\
.68\end{array}$ & $\begin{array}{l}.29 \\
.24 \\
.19 \\
.18 \\
.17\end{array}$ \\
\hline $\begin{array}{l}11 . \\
12 . \\
13 . \\
14 .\end{array}$ & $\begin{array}{r}16.86 \\
19.93 \\
24.98 \\
9.39 \\
12.18\end{array}$ & $\begin{array}{r}2.19 \\
.97 \\
.99 \\
.92 \\
.53\end{array}$ & $\begin{array}{r}20.22 \\
6.36 \\
10.68 \\
4.78 \\
3.38\end{array}$ & $\begin{array}{l}4.57 \\
3.71 \\
1.60 \\
9.04 \\
2.02\end{array}$ & $\begin{array}{l}10.50 \\
18.50 \\
19.83 \\
16.82 \\
27.82\end{array}$ & $\begin{array}{l}.77 \\
.62 \\
.49 \\
.52 \\
.54\end{array}$ & $\begin{array}{r}.40 \\
.22 \\
.16 \\
7.20 \\
3.48\end{array}$ & $\begin{array}{r}49.26 \\
7.94 \\
3.03\end{array}$ & $\begin{array}{l}3.29 \\
1.93 \\
1.53 \\
1.50 \\
1.28\end{array}$ & $\begin{array}{r}3.54 \\
2.05 \\
10.41 \\
27.97 \\
20.71\end{array}$ & $\begin{array}{r}.42 \\
.28 \\
.16 \\
.51 \\
2.22\end{array}$ & $\begin{array}{l}.17 \\
.17 \\
.17 \\
.14 \\
.14\end{array}$ \\
\hline $\begin{array}{l}16 \\
17 \\
18 \\
19\end{array}$ & $\begin{array}{r}6.64 \\
13.27 \\
3.82 \\
2.04 \\
1.44\end{array}$ & $\begin{array}{r}.53 \\
2.39 \\
1.26 \\
6.05 \\
6.11\end{array}$ & $\begin{array}{r}22.26 \\
13.94 \\
6.58 \\
23.12 \\
8.87\end{array}$ & $\begin{array}{r}.73 \\
1.42 \\
.92 \\
.55 \\
.37\end{array}$ & $\begin{array}{l}5.10 \\
3.04 \\
2.42 \\
7.69 \\
2.64\end{array}$ & $\begin{array}{r}5.90 \\
2.50 \\
.78 \\
.51 \\
.89\end{array}$ & $\begin{array}{l}1.48 \\
2.86 \\
1.25 \\
7.58 \\
1.55\end{array}$ & $\begin{array}{l}41.31 \\
93.77 \\
70.51 \\
39.60 \\
12.19\end{array}$ & $\begin{array}{r}.84 \\
.74 \\
.92 \\
1.09 \\
6.52\end{array}$ & $\begin{array}{r}5.10 \\
2.36 \\
1.54 \\
.96 \\
.74\end{array}$ & $\begin{array}{r}1.06 \\
5.86 \\
7.45 \\
24.81 \\
7.87\end{array}$ & $\begin{array}{r}.13 \\
2.82 \\
2.62 \\
8.20 \\
3.54\end{array}$ \\
\hline $\begin{array}{l}22 \ldots \\
23 \ldots \\
24 \ldots \\
25 \ldots\end{array}$ & \begin{tabular}{|r|}
3.38 \\
5.06 \\
6.94 \\
27.08 \\
13.86
\end{tabular} & $\begin{array}{r}18.91 \\
3.89 \\
1.54 \\
1.14 \\
5.86\end{array}$ & $\begin{array}{r}13.22 \\
4.09 \\
2.97 \\
22.09 \\
4.30\end{array}$ & $\begin{array}{r}.24 \\
.64 \\
4.52 \\
32.66 \\
3.51\end{array}$ & $\begin{array}{c}1.54 \\
.75 \\
.28 \\
.15 \\
.07\end{array}$ & $\begin{array}{r}5.42 \\
1.50 \\
.59 \\
.33 \\
.05\end{array}$ & $\begin{array}{r}3.75 \\
1.34 \\
25.67 \\
33.89 \\
6.37\end{array}$ & $\begin{array}{r}13.50 \\
9.02 \\
12.20 \\
5.83 \\
3.06\end{array}$ & $\begin{array}{r}2.28 \\
12.86 \\
46.28 \\
21.87 \\
5.67\end{array}$ & $\begin{array}{l}.59 \\
.56 \\
.48 \\
.42 \\
.38\end{array}$ & $\begin{array}{l}5.86 \\
6.38 \\
3.51 \\
1.27 \\
3.99\end{array}$ & $\begin{array}{r}8.42 \\
26.49 \\
7.11 \\
3.81 \\
1.23\end{array}$ \\
\hline $\begin{array}{l}26 . . \\
27 . \\
28 . \\
29 . \\
30 .\end{array}$ & $\begin{array}{r}31.01 \\
13.25 \\
7.23 \\
5.95 \\
12.66\end{array}$ & $\begin{array}{r}1.65 \\
14.35 \\
10.02 \\
3.93 \\
4.70 \\
1.84\end{array}$ & $\begin{array}{l}1.77 \\
5.35 \\
1.63 \\
1.17 \\
7.44\end{array}$ & $\begin{array}{r}2.79 \\
3.33 \\
1.46 \\
.78 \\
.44 \\
.26\end{array}$ & $\begin{array}{r}.03 \\
.14 \\
4.90 \\
3.96 \\
1.27 \\
.70\end{array}$ & $\begin{array}{r}.05 \\
.05 \\
.03 \\
.03 \\
13.30\end{array}$ & $\begin{array}{r}3.99 \\
62.69 \\
19.43 \\
23.46 \\
6.39 \\
10.56\end{array}$ & $\begin{array}{l}1.72 \\
1.52 \\
1.32 \\
7.67 \\
2.57\end{array}$ & $\begin{array}{r}10.78 \\
5.08 \\
2.89 \\
6.09 \\
6.14 \\
6.30\end{array}$ & $\begin{array}{l}.28 \\
.25 \\
.31 \\
.23 \\
.15 \\
.12\end{array}$ & $\begin{array}{r}11.18 \\
4.60 \\
2.01 \\
\cdots \ldots . . \\
\ldots \ldots .\end{array}$ & $\begin{array}{r}1.07 \\
1.46 \\
37.46 \\
20.86 \\
7.00 \\
18.24\end{array}$ \\
\hline
\end{tabular}

Combined monthly discharge of weirs Nos. 1 to 22, Kehena ditch, at Honokane Mauka, North Kohala, Hawaii, Apr. 1, 1912, to Mar. 31, 1913.

\begin{tabular}{|c|c|c|c|c|}
\hline \multirow{2}{*}{ Month. } & \multicolumn{3}{|c|}{ Discharge in million gallons per } & \multirow{2}{*}{$\begin{array}{l}\text { Run-off } \\
\text { (total in } \\
\text { acre-feet). }\end{array}$} \\
\hline & Maximum. & Minimum. & Mean. & \\
\hline 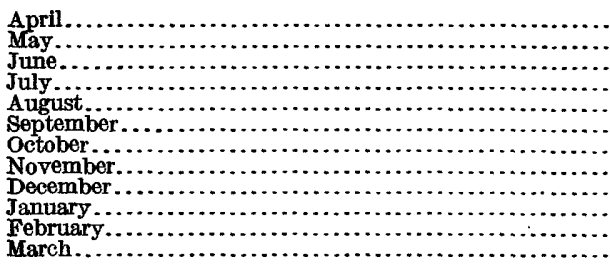 & \begin{tabular}{r|}
119.37 \\
26.53 \\
23.12 \\
32.66 \\
27.82 \\
170.34 \\
62.89 \\
105.51 \\
46.28 \\
27.97 \\
24.81 \\
37.46
\end{tabular} & $\begin{array}{r}1.44 \\
.53 \\
.03 \\
.24 \\
.03 \\
.03 \\
.10 \\
1.10 \\
.74 \\
.12 \\
.16 \\
.13\end{array}$ & $\begin{array}{l}22.2 \\
\mathbf{7 . 0 5} \\
7.17 \\
4.77 \\
\mathbf{5 . 6 5} \\
\mathbf{1 2 . 3} \\
\mathbf{7 . 8 8} \\
\mathbf{1 8 . 8} \\
\mathbf{7 . 3 3} \\
\mathbf{3 . 3 2} \\
\mathbf{3 . 6 5} \\
\mathbf{5 . 0 1}\end{array}$ & $\begin{array}{r}2,040 \\
671 \\
660 \\
454 \\
538 \\
1,130 \\
750 \\
1,730 \\
697 \\
316 \\
314 \\
477\end{array}$ \\
\hline The year... & 170.34 & .03 & 8.74 & 9,780 \\
\hline
\end{tabular}




\section{MISCELLANEOUS MEASUREMENTS.}

Measurements of streams on the island of Hawaii at points other than regular gaging stations are listed below:

Miscellaneous discharge measurements on Hawaii for 1913.

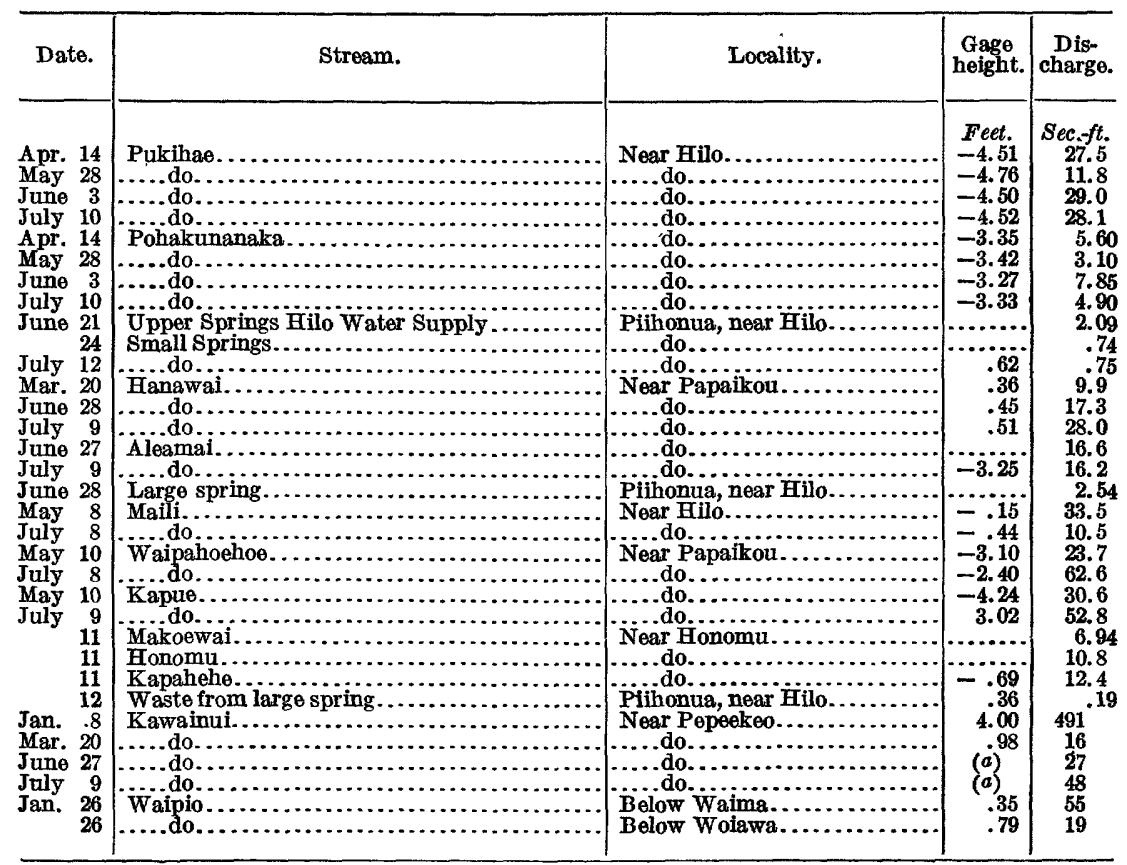

a Staff gage washed out by flood.

RAIN GAGING.

The rainfall of the Hawaiian Islands is extremely variable, ranging from a fow inches at several low-level, leeward localities to more than 600 inches per annum, usually at elevations above 2,000 feet and on the windward sides of the islands. Valleys lying on the same sides of the islands and within a few miles of each other may have a variation in mean annual precipitation of several hundred per cent. The rainfall may also vary greatly in the same valley for different elevations. As a rule the zones of heaviest precipitation are on the windward sides of the islands, 2,000 to 3,000 feet above sea level.

Generally the daily rain gages maintained by the United States Weather Bureau are located at low levels. Lack of funds and the absence of inhabitants at high-level localities have prevented the maintenance of Weather Bureau stations at high levels, although in a number of cases daily records are furnished that bureau by occupants and caretakers of mountain houses and ranches. The data furnisbed by the Weather Bureau are therefore generally of little value in their relation to stream run-off. 
When high levels have been accessible and funds available highlevel rain gages, which are read monthly and bimonthly, have been established by this office and some valuable records obtained. To determine the precipitation of the Territory accurately would require the installation of thousands of gages and the construction of hundreds of miles of trails.

Acknowledgment for cooperation in furnishing rainfall data is due the following: Kauai-Kekaha Sugar Co., Makee Sugar Co., Hawaiian Sugar Co., Grove Farm Plantation, W. F. Sanborn of Princeville Ranch, Kauai Electric Co., and J. McClellan-Oahu, C. C. Bitting, F. Meyer, and Hawaii Preserving Co.; Maui-Wailuku Sugar Co., Honolulu Ranch, Hawaiian Commercial \& Sugar Co., Maui Board of Supervisors, Pioneer Mill Co., and Olowalu Sugar Co.; HawaiiHawaii Mill Co., P. W. P. Bluett, W. S. May, C. F. Clark, C. R. Willard, Alex. Valentine, Honokaa Sugar Co., Pacific Sugar Mill, Hawaiian Irrigation Co., and Kukaiau Ranch Co.

The tables on pages 180-185 show the precipitation at stations maintained by the Geological Survey and precipitation data furnished from private sources which are not included in United States Weather Bureau records, to which those interested in further data are referred.

\section{Rainfall stations in Hawaii.}

KAUAI.

1. Kokee, on mesa one-half mile above Knudsen's camp near head of Kokee Stream and about 19 miles north of Waimea; 3,550 feet above sea level.

2. Puu Lua, near wagon road from Kekaha to Halemanu, about 12 miles north of Waimea; 3.500 feet above sea level.

3. Lehuamakanoi, about 22 miles by road and trail north of Waimea; 3,932 feet above sea level.

4. Paukahana, about 21 miles north of Waimea by road and trail; 3,723 feet above sea level.

5. Waiakoali, about 22 miles by road via Halemanu north of Waimea; 3,450 feet above sea level.

6. Kilohana, near Alakai swamp, about 23 miles by road and trail north-northeast of Waimea; 4,023 feet above sea level.

7. Mohihi, on ridge at head of Mohihi Valley and about 23 miles by road and trail northeast of Waimea; 3,500 feet above sea level.

8. Mohihi-Koaie divide, on ridge ábout 24 miles by road and trail north of Waimea; 3,950 feet above sea level.

9. Waialae, near Kaholuamanu; 14 miles by road and trail north of Waimea, near Waialae gaging station; 3,600 feet above sea level.

10. Hukipo, 3 miles northwest of Waimea; 400 feet above sea level; records furnished by Kekaha Sugar Co.

11. Pali trail, one-half mile mauka of Kekaha ditch where trail crosses and about 2 miles mauka from Waimea; 850 feet above sea level; records furnished by Kekaha Sugar Co.

12. Camp No. 7 , about 2 miles northeast of Waimea; 150 feet above sea level; records furnished by Hawaiian Sugar Co.

13. Waimea, in Mr. J. McClellan's yard; 10 feet above sea level; Mr. McClellan aids in obtaining record. 
14. Kaholuamanu, about 12 miles by road and trail northeast of Waimea; 3,650 feet above sea level.

15. Kahana, near Halekua camp, on ridge about 16 miles by road and trail via Kaholuamanue from Waimea; 3,750 feet above sea level.

16. Keanakua, near Halekua camp, on ridge about 16 miles by road and trail northnortheast of Waimea; 4,450 feet above sea level.

17. Olokele mauka, on ridge on left side of Olokele Stream above intake of Olokele ditch, and about 18 miles by road and trail from Waimea; 2,100 feet above sea level; records furnished by Hawaiian Sugar Co.

18. Waialeale, on summit of ridge at headwaters of Hanapepe, Wainiha, Hanalei, and Olokele streams, and North and South Forks of Wailua River; about 25 miles by road and trail northeast of Waimea; 5,075 feet above sea level.

19. Camp No. 2, about 2 miles northwest of Hanapepe and 7 miles southeast of Waimea; 250 feet above sea level; records furnished by Hawaiian Sugar Co.

20. Hanapepe Valley, on left bank of Hanapepe ditch, 3 miles above Koula, and about 8 miles north of Eleele; 530 feet above sea level; records furnished by Hawaiian Sugar Co.

21. Hiloa-Manawaiopuna divide, on ridge between east and west branches of Hanapepe Stream, about 10 miles northeast of Eleele; 2,080 feet above sea level.

22. Aakukui, near plantation camp, about 5 miles southwest of Lihue; 350 feet above sea level; records furnished by Grove farm.

23. Waiahi, on South Wailua River, near Lihue Electric Co.'s power plant, 7 miles from Lihue; 600 feet above sea level.

24. Hanahanapuni, on ridge near Kauai Electric Co.'s power line, about 10 miles northwest of Lihue; 911 feet above sea level.

25. North Wailua, near Waialeale Pali, in North Kailua Valley, about 15 miles by road and trail northwest of Lihue; 1,250 feet above sea level.

26. Pohakupili, on top of narrow ridge about 6 miles west of Kealia; 2,590 feet above sea level.

27. Puu Eu, on ridge about $6 \frac{1}{2}$ miles west of Kealia; 2,748 feet above sea level.

28. Kaneha, at gate tender's house near the mauka Kaneha reservoir, and about 5 miles northwest of Kealia; 800 feet above sea level; records furnished by Makee Sugar Co.

29. Summit Camp (Wainiha ridge), Hanalei; about 30 feet southwest of house at Summit Camp on power line; 6 miles from Kapaka; 1,900 feet above sea level; gage read by employee of Kauai Electric Co.

30. Kapaka, at Lineman's camp, about 50 feet north of house, and 5 miles south of Hanalei; 635 feet above sea level; gage read by employee of Kauai Electric Co.

31. About 40 feet north of Sanborn's residence, 2 miles from Hanalei; 105 feet above sea level; records furnished by Princeville ranch.

32. About 50 feet below Kauai Electric Co.'s power house, at tailrace, 7 miles west of Hanalei; 125 feet above sea level; records furnished by Kauai Electric Co.

33. Intake of Wainiha Power Co.'s canal, 6 miles back of Hanalei; 700 feet above sea level; records furnished by Kauai Electric Co.

OAHU.

1. Nuuanu Pali, on the water reservation, near the Pali road, about 200 yards toward Honolulu from top of the Pali; 1,200 feet above sea level.

2. Summit of Konahuanui, southeast of Nuuanu Pali, about 6 miles in air line northeast of Honolulu; 3,100 feet above sea level.

3. Near summit of Mount Olympus, back of Manoa Valley, about $6 \frac{1}{2}$ miles in air line northeast of Honolulu; 2,400 feet above sea level.

4. Waiomao, on the property of C. C. Bitting, lot No. 8, Palolo Valley, Honolulu; 600 feet above sea level; records furnished by $\mathrm{C}$. C. Bitting. 
5. Kaau crater, on trail from Mount Olympus, a short distance before it leads down the ridge into Palolo Valley and about $3 \frac{1}{2}$ miles north of Kaimuki car line; 1,700 feet above sea level.

6. On the ridge between the Waiahole and Waiawa portals of the Waiahole Water Co.'s tunnel, near Waiahole, Waikane, Oahu; 2,150 feet above sea level.

7. Makaha, near Waianae, on property of Waianae plantation; 1,300 feet above sea level; records furnished by F. Meyer, manager Waianae plantation.

8. About 150 yards downstream on left bank of North Fork of Kaukonahua stream from Waialua Agricultural Co.'s ditch intake, on trail 8 miles north of Wahiawa; 1,200 feet above sea level.

9. Near the office of the Hawaiian Preserving Co., Wahiawa; 940 feet above sea level; records are furnished by Hawaiian Preserving Co.

\section{MAUI.}

1. In H. B. Penhallow's yard, Wailuku; 390 feet above sea level; records furnished by Wailuku Sugar Co.

2. Yard at Wailuku Sugar Co.'s office, Wailuku; 175 feet above sea level; records furnished by Wailuku Sugar Co.

3. Right bank of Iao Stream at the gaging station, 20 feet from the north anchorage of the cable; 3 miles west of Wailuku; 830 feet above sea level.

4. Iao Valley on small plateau or tableland between north and south branches of Iao Stream, about 1 mile above the junction; about 4 miles west of Wailuku; 1,500 feet above sea level.

5. About 1,000 feet below small cave in Iao Valley, on ridge between north and south branches of Iao Stream; about $5 \frac{1}{2}$ miles west of Wailuku; 1,720 feet above sea level.

6. Waiehu, at T. Burlem's house on old Spreckels ditch just south of South Waiehu Gulch, 2 miles northwest of Wailuku; 200 feet above sea level; records furnished by Hawaiian Commercial \& Sugar Co.

7. Waihee, on roof of building formerly used as plantation office; $3 \frac{1}{2}$ miles from Wailuku; 125 feet above sea level; records furnished by Wailuku Sugar Co.

8. Waihee Gulch, on right bank of the stream, about 100 feet below lower development tunnel; about $5 \frac{1}{2}$ miles from mouth of gulch and 3 miles above intake of Waihee canal and the Waihee gaging station; 1,500 feet above sea level.

9. Rim of extinct crater of Mount Eke; 14 miles by trail from Honokahau; 4,500 feet above sea level.

10. Honokahau Gulch at ditchman's house on left bank of stream, 150 feet below ditch intake; about 8 miles from Honokahau; 800 feet above sea level; records furnished by Honolua Ranch Co.

11. Camp on ridge between Honokahau and Kahakuloa gulches beside trail leading to top of Mount Eke; about 12 miles from Honokahau; 2,300 feet above sea level.

12. Honokahau ditch about one-half mile south of Honokahua Gulch and about 4 miles from Honokahau; 760 feet above sea level; records furnished by Honolua Ranch Co.

13. Honokawai Gulch at junction with Amalu Stream; on trestle supporting Honokawai flume about 1,000 feet below intake; about $3 \frac{1}{2}$ miles from Kaanapali and $7 \frac{1}{2}$ miles from Lahaina; 1,500 feet above sea level; records furnished by Pioneer Mill Co.

14. West slope of Puu Kukui, about one-half mile south of Honokawai Gulch; about $4 \frac{1}{2}$ miles east of Kaanapali and $8 \frac{1}{2}$ miles from Lahaina; 2,500 feet above sea level; records furnished by Pioneer Mill Co.

15. West slope of Puu Kukui at top of left bank of Honokawai Gulch; about 6 miles east of Kaanapali and 10 miles from Lahaina; 5,000 feet above sea level; records furnished by the Pioneer Mill Co.

$97764^{\circ}-$ wSP $373-15-12$ 
16. Olowalu Sugar Co.'s mill in Olowalu; 10 feet above sea level; records furnished by Olowalu Sugar Co.

17. Olowalu Gulch (mauka) on right bank of Olowalu Stream about $3 \frac{1}{2}$ miles north of Olowalu; 600 feet above sea level; records furnished by Olowalu Sugar Co.

18. Waikapu Gulch, on left bank of the South Branch of Waikapu Stream, about 4 miles by trail from Waikapu and 6 miles from Wailuku; 1,535 feet above sea level.

19. Olinda, on Kula pipe line; 4 miles east of Makawao; 4,000 feet above sea level; records furnished by Board of Supervisors, County of Maui.

20. Waikamoi Gulch, on Kula pipe line at reservoir; 3 miles from Olinda and 7 miles from Makawao; 4,200 feet above sea level; records furnished by Board of Supervisors, County of Maui.

21. Puohakamoa, on Kula pipe line about 1,000 feet below intake at Puohakamoa Gulch; 4 miles east of Olinda and 8 miles from Makawao; 4,300 feet above sea level; records furnished by Board of Supervisors, County of Maui.

22. Camp No. 1 of Hawaiian Commercial \& Sugar Co., near Spreckelsville, 5 miles east of Kahului; 50 feet above sea level; records furnished by Hawaiian Commercial \& Sugar Co.

23. Camp No. 7 of Hawaiian Commercial \& Sugar Co., near Kihei, about 5 miles south of Puunene; 50 feet above sea level; records furnished by Hawaiian Commercial \& Sugar Co.

24. Puunene, at Hawaiian Commercial \& Sugar Co.'s office; 50 feet above sea level; records furnished by Hawaiian Commercial \& Sugar Co.

\section{HAWAII.}

1. Near flume at camp No. 8 of Waiakea plantation, near Waiakea; 1,500 feet above sea level; records furnished by Waiakea Plantation Co.

2. Camp No. 6, at plantation camp back of Waiakea; 800 feet above sea level; records furnished by Waiakea plantation.

3. United States Engineer's office at Hilo breakwater, Hilo; 15 feet above sea level; records furnished by United States Engineers' office.

4. Piihonua, at office and store of Hawaii Mill Co., near Hilo; 915 feet above sea level; records furnished by Hawaii Mill Co.

5. Hilo, in rear of J. T. Lewis's residence, Waianuenue Street; 60 feet above sea level.

6. Station No. 1, about 50 feet in rear of Alex. Valentine's house at Wainaku camp of Hilo Sugar Co.; 200 feet above sea level; records June 30 to November 30 furnished by Alex. Valentine, of Hilo Sugar Co.

7. Station No. 2, at Antone Mogordo's blacksmith shop near Kaiwiki road, and about 1 mile above the Wainaku camp of the Hilo Sugar Co.; 1,000 feet above sea level.

8. Station No. 3, near Kaiwiki, on trail from mauka end of Kaiwiki road to 2,700-foot camp, and about 600 feet above end of road; at lower edge of timber; 1,500 feet above sea level.

9. Station No. 4, on trail from mauka end of Kaiwiki road to 2,700-foot camp, in timber belt; 2,000 feet above sea level.

10. Station No. 5 , about $3 \frac{1}{2}$ miles above Kaiwiki, ${ }^{1}$ near Hilo; 2,650 feet above sea level.

11. Station No. 6, at camp, on east slope of Mauna Kea, in heavy timber belt; 3,000 feet above sea level.

12. Station No. 7, on east slope of Mauna Kea, in heavy timber belt; 3,500 feet above sea level.

1 Gage originally located at elevation of 2,500 feet by H. R. Schulz; later moved to elevation of 2,650 feet; thought to be 2,500 feet by C. H. Pierce, July 1,1911 , 
13. Station No. 8, on east slope of Mauna Kea, in heavy timber belt; 4,000 feet above sea level.

14. Station No. 9, on east slope of Mauna Kea, in heavy timber belt; 4,500 feet above sea level.

15. Station No. 10, on east slope of Mauna Kea, in heavy timber belt; 5,000 feet above sea level.

16. Umikoa, on property of Kukaiau Ranch Co. (Ltd.), near ranch house; 3,400 feet above sea level; records furnished by Kukaiau Ranch Co. (Ltd.).

17. Puukihe, on top of Kihe hill on the side of Mauna Kea, about 10 miles south of Kukaiau; 7,822 feet above sea level; records furnished by Kukaiau Ranch Co. (Ltd.).

18. Reservoir No. 13, on land of Honokaa Sugar Co., near Honokaa; 1,480 feet above sea level; records furnished by the civil engineers' office of Honokaa Sugar Co. and Pacific Sugar Mill.

19. Near Honokaa, at office of civil engineers for Pacific Sugar Mill and Honokaa Sugar Co.; 1,120 feet above sea level; records furnished by civil engineers.

20. Ahualoa homesteads, at ditch tender's house, near the Parker ranch, Honokaa; 2,551 feet above sea level; records furnished by civil engineers' office of Honokaa Sugar Co. and Pacific Sugar Mill.

21. Waima, in Waipio Valley along the line of lower Hamakua ditch, near Kukuihaele; 980 feet above sea level; records furnished by Hawaiian Irrigation Co.

22. Lower Koiawe, near the line of the lower Hamakua ditch in Waipio Valley near Kukuihaele; 1,000 feet above sea level; records furnished by Hawaiian Irrigation Co.

23. Upper Koiawe, along the line of the upper Hamakua ditch, Waipio Valley, near Kukuihaele; 3,350 feet above sea level; records furnished by Hawaiian Irrigation Co.

24. Alakahi-Waipio, in Waipio Valley, Kohala Mountains, along the line of the lower Hamakua ditch, near Kukuihaele; 1,030 feet above sea level; records furnished. by Hawaiian Sugar Co.

25. Alakahi-Waipio, in Waipio Valley, Kohala Mountains, near the line of the upper Hamakua ditch; 3,870 feet above sea level; records furnished by Hawaiian Irrigation Co.

26. Lower Kawainui, in Waipio Valley, near the line of the lower Hamakua ditch, near Kukuihaele; 1,040 feet above sea level; records furnished by Hawaiian Irrigation Co.

27. Upper Kawainui, in the Kohala Mountains, near the line of the upper Hamakua ditch, near Kukuihaele; 4,080 feet above sea level; records furnished by Hawaiian Irrigation Co.

28. Honokane mauka; in the Kohala Mountains, near the intake of the Kehena ditch, near Kohala; 3,800 feet above sea level; records furnishod by Kohala Ditch Co.

29. Kaauhuhu, on property of W. S. May, about 3 miles northwest of Hawi; 1,400 feet above sea level; records furnished by W. S. May. 


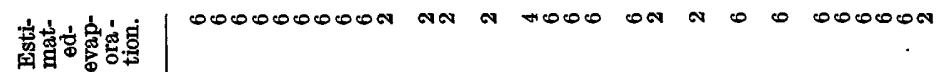
|

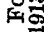

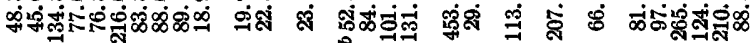

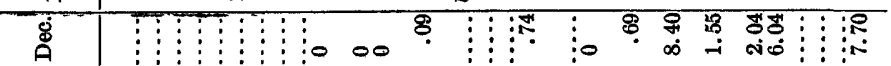

है

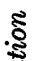

(1)

密

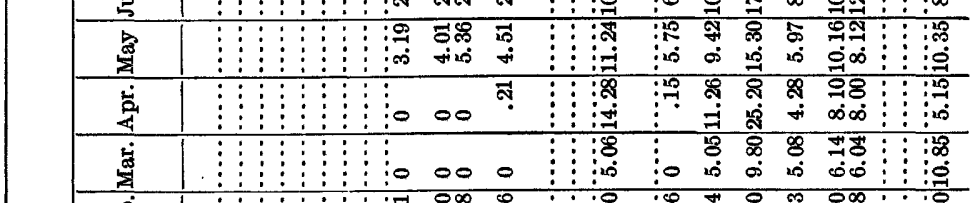




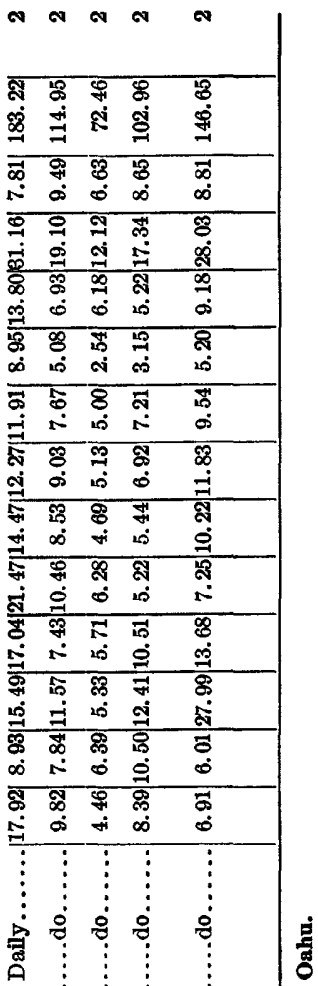

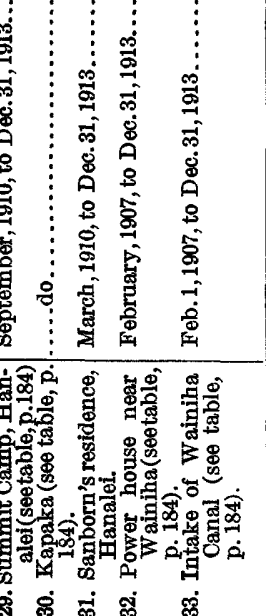

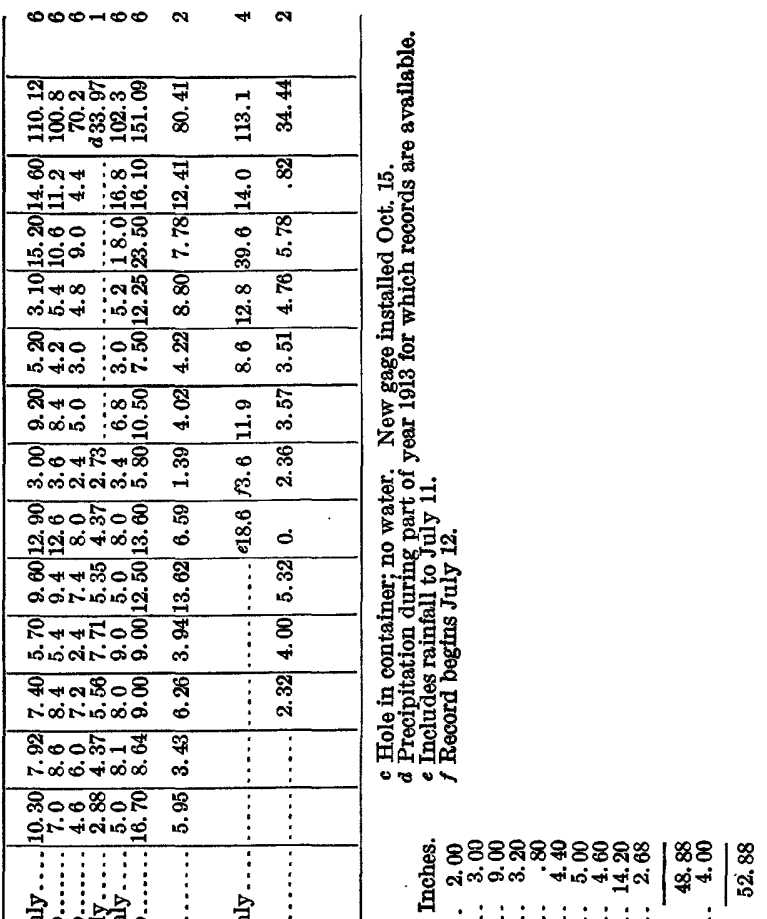

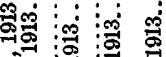

लिळं मे ले

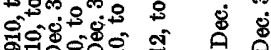

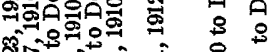
सित= $\Rightarrow$ क

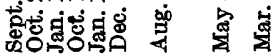

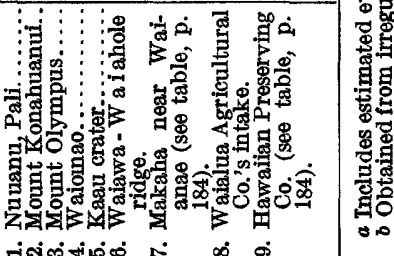

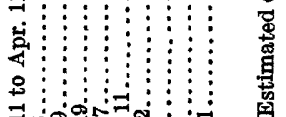
(1)

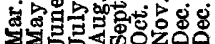




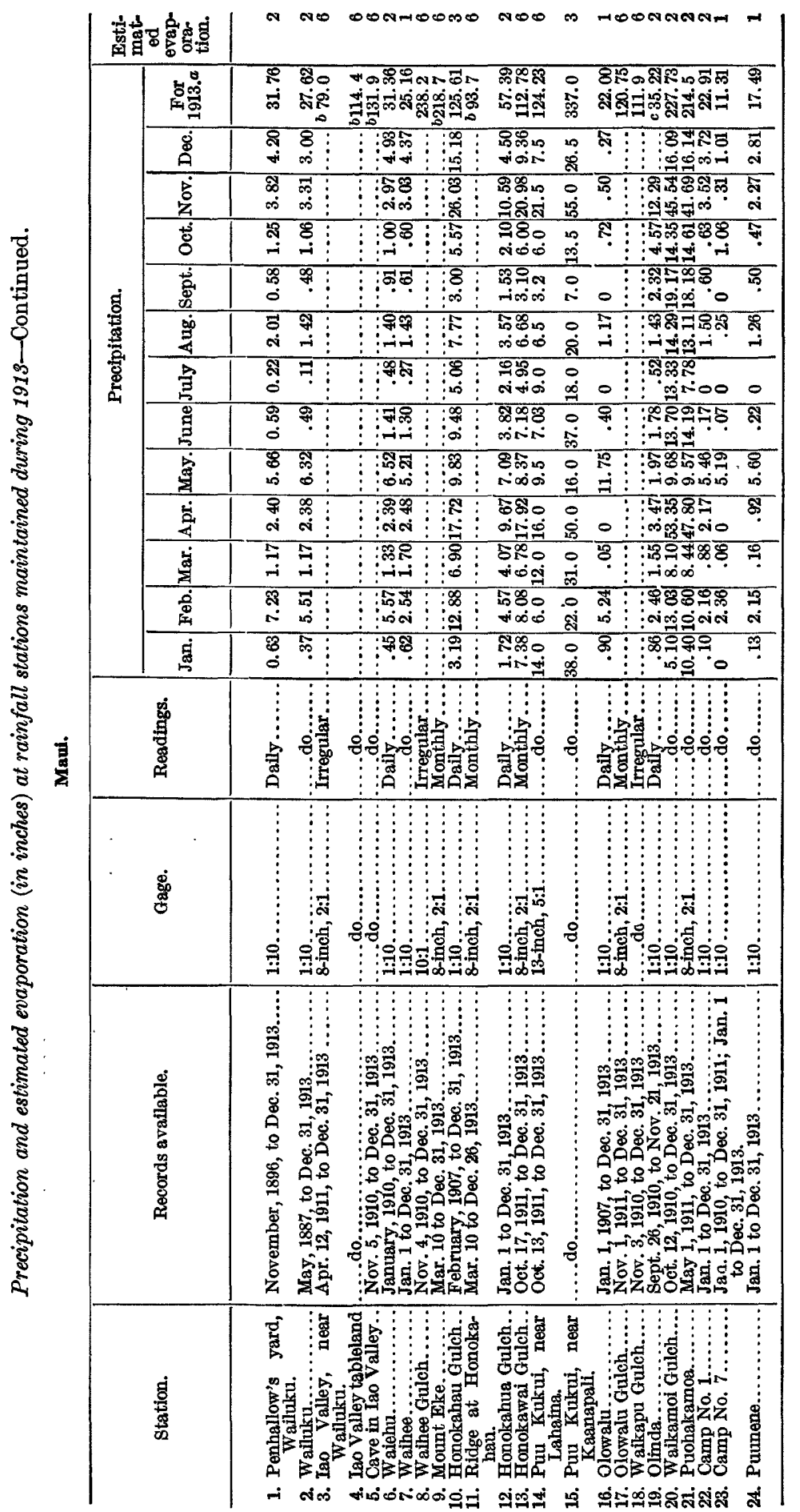


ס

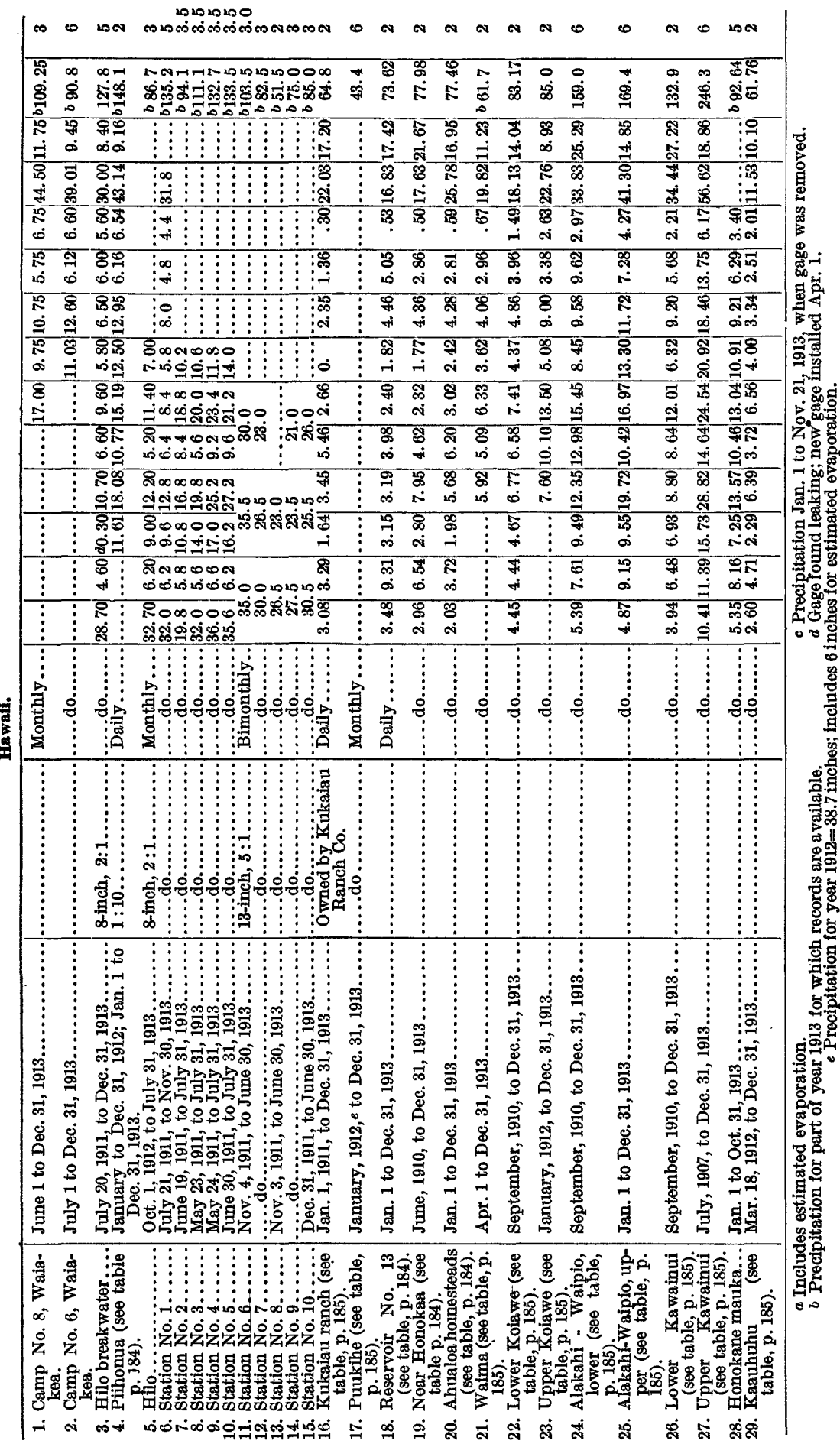


Maximum precipitation, in inches, at rainfall stations maintained during 1913.

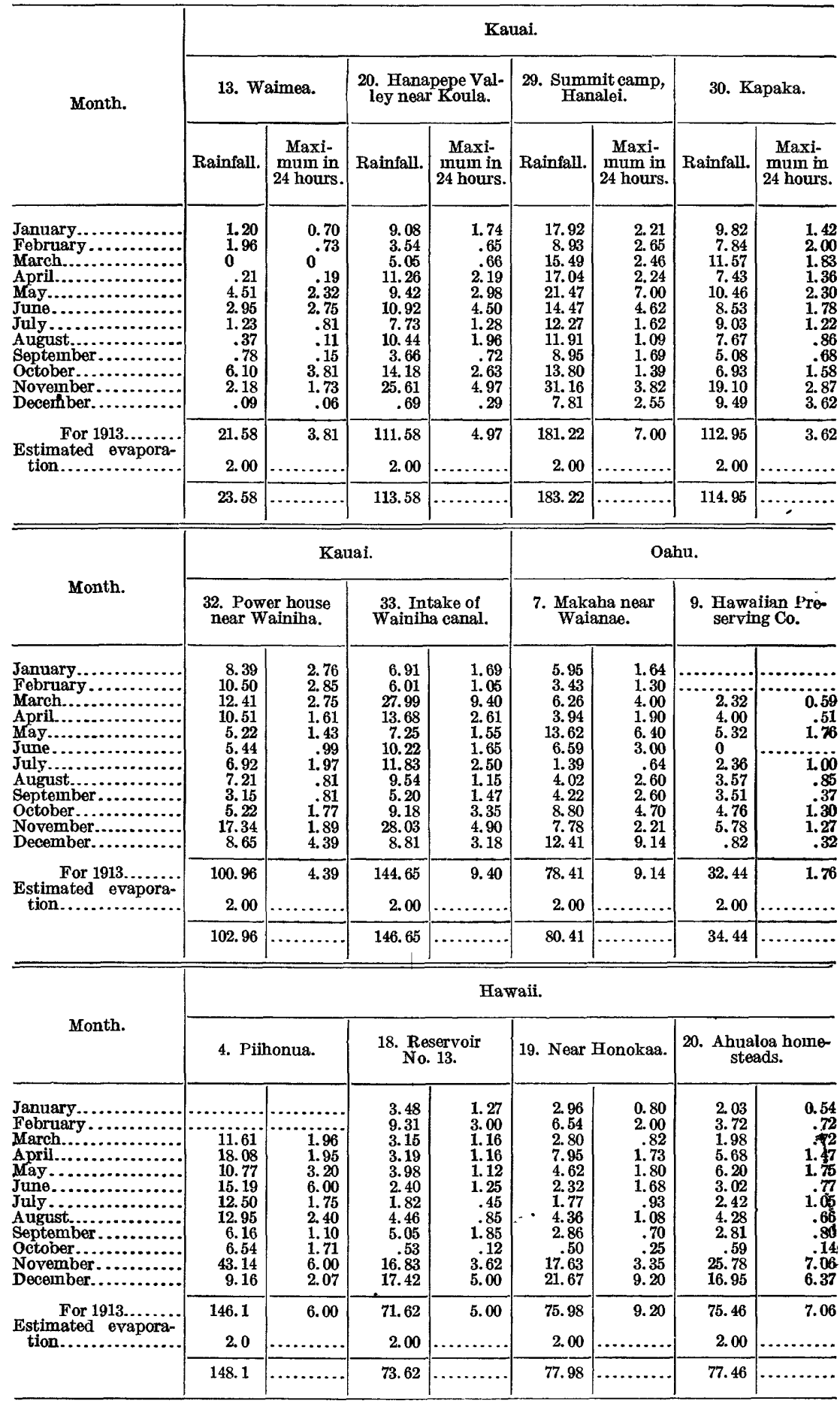


Maximum precipitation, in inches, at rainfall stations maintained during 191s-Contd.

\begin{tabular}{|c|c|c|c|c|c|c|c|c|}
\hline \multirow{2}{*}{ 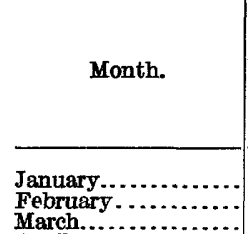 } & \multicolumn{8}{|c|}{ Hawail. } \\
\hline & \multicolumn{2}{|c|}{ 21. Waima. } & \multicolumn{2}{|c|}{ 22. Lower Koiawe. } & \multicolumn{2}{|c|}{ 23. Upper Koiawe. } & \multicolumn{2}{|c|}{$\begin{array}{l}\text { 24. Alakahi-Waipio, } \\
\text { lower. }\end{array}$} \\
\hline 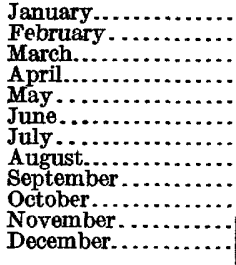 & $\begin{array}{r}5.92 \\
5.09 \\
6.33 \\
3.62 \\
4.06 \\
2.96 \\
.67 \\
19.82 \\
11.23\end{array}$ & $\begin{array}{r}1.20 \\
.95 \\
1.50 \\
1.00 \\
1.30 \\
.88 \\
.25 \\
5.20 \\
6.20\end{array}$ & $\begin{array}{r}4.45 \\
4.44 \\
4.67 \\
6.77 \\
6.58 \\
7.41 \\
4.37 \\
4.86 \\
3.96 \\
1.49 \\
18.13 \\
14.04\end{array}$ & $\begin{array}{r}1.14 \\
1.22 \\
.75 \\
1.36 \\
.95 \\
1.55 \\
1.10 \\
.90 \\
.96 \\
.40 \\
4.20 \\
4.30\end{array}$ & $\begin{array}{r}7.60 \\
10.10 \\
13.50 \\
5.08 \\
9.00 \\
3.38 \\
2.63 \\
22.76 \\
8.93\end{array}$ & $\begin{array}{r}1.00 \\
2.00 \\
2.10 \\
.60 \\
1.70 \\
.57 \\
.65 \\
4.55 \\
2.35\end{array}$ & $\begin{array}{r}5.39 \\
7.61 \\
9.49 \\
12.35 \\
12.98 \\
15.45 \\
8.45 \\
9.58 \\
9.62 \\
2.97 \\
33.83 \\
25.29\end{array}$ & $\begin{array}{l}1.57 \\
1.58 \\
1.83 \\
3.10 \\
1.75 \\
2.89 \\
1.75 \\
1.74 \\
3.52 \\
.49 \\
6.92 \\
9.25\end{array}$ \\
\hline \multirow[t]{2}{*}{$\begin{array}{c}\text { For } 1913 \ldots . . . . . \\
\text { Estimated evapora- } \\
\text { tion.................... }\end{array}$} & $\begin{array}{r}59.70 \\
2.00 \\
\end{array}$ & 6.20 & $\begin{array}{r}81.17 \\
2.00 \\
\end{array}$ & 4.30 & $\begin{array}{r}82.98 \\
2.00 \\
\end{array}$ & 4.55 & $\begin{array}{r}153.0 \\
6.0 \\
\end{array}$ & $\begin{array}{l}9.25 \\
\cdots \cdots \\
\end{array}$ \\
\hline & 61.7 & & 83.17 & & 85.0 & - & 159.0 & $\cdots$ \\
\hline \multirow{2}{*}{ Month. } & \multicolumn{8}{|c|}{ Hawaii. } \\
\hline & \multicolumn{2}{|c|}{$\begin{array}{l}\text { 25. Alakahi-Wai- } \\
\text { pio, upper. }\end{array}$} & \multicolumn{2}{|c|}{$\begin{array}{l}\text { 26. Lower Kawai- } \\
\text { nui. }\end{array}$} & \multicolumn{2}{|c|}{$\begin{array}{l}\text { 27. Upper Kawai- } \\
\text { nui. }\end{array}$} & \multicolumn{2}{|c|}{ 29. Kaauhuhu. } \\
\hline 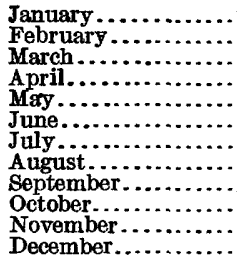 & $\begin{array}{r}4.87 \\
9.15 \\
9.55 \\
19.72 \\
10.42 \\
16.97 \\
13.30 \\
11.72 \\
7.28 \\
4.27 \\
41.30 \\
14.85 \\
\end{array}$ & $\begin{array}{l}1.60 \\
1.65 \\
2.10 \\
3.65 \\
2.01 \\
2.60 \\
2.21 \\
2.30 \\
1.40 \\
.60 \\
8.50 \\
4.55\end{array}$ & $\begin{array}{r}3.94 \\
6.48 \\
6.93 \\
8.80 \\
8.64 \\
12.01 \\
6.32 \\
9.20 \\
5.68 \\
2.21 \\
34.44 \\
27.22 \\
\end{array}$ & $\begin{array}{r}0.97 \\
1.22 \\
1.40 \\
1.60 \\
1.15 \\
2.20 \\
1.20 \\
1.80 \\
1.50 \\
.45 \\
8.00 \\
11.03 \\
\end{array}$ & $\begin{array}{r}10.41 \\
11.39 \\
15.73 \\
28.82 \\
14.64 \\
24.54 \\
20.92 \\
18.46 \\
13.75 \\
6.17 \\
56.62 \\
18.86 \\
\end{array}$ & $\begin{array}{r}3.90 \\
1.75 \\
3.63 \\
4.50 \\
2.10 \\
3.05 \\
3.63 \\
3.32 \\
3.15 \\
.73 \\
13.32 \\
5.87 \\
\end{array}$ & $\begin{array}{r}2.60 \\
4.71 \\
2.29 \\
6.39 \\
3.72 \\
6.56 \\
4.00 \\
3.34 \\
2.51 \\
2.01 \\
11.53 \\
10.10 \\
\end{array}$ & $\begin{array}{r}1.17 \\
.66 \\
.45 \\
1.32 \\
.89 \\
1.26 \\
1.90 \\
. .98 \\
1.02 \\
.45 \\
2.02 \\
1.94 \\
\end{array}$ \\
\hline \multirow[t]{2}{*}{$\begin{array}{c}\text { For } 1913 \ldots \ldots \ldots \\
\text { Estimated evapora- } \\
\text { tion. }\end{array}$} & $\begin{array}{r}163.4 \\
6.0\end{array}$ & 8.50 & $\begin{array}{r}131.87 \\
2.00\end{array}$ & 11.03 & $\begin{array}{r}240.3 \\
6.0\end{array}$ & 13.32 & $\begin{array}{r}59.76 \\
2.00\end{array}$ & 2.02 \\
\hline & 169.4 & & 133.9 & & 246.3 & & 61.76 & n........... \\
\hline
\end{tabular}

Precipitation at rainfall stations maintained on Hawaii during 1911 and 1912.

\begin{tabular}{|c|c|c|c|}
\hline \multirow{2}{*}{ Month. } & \multicolumn{2}{|c|}{ 16. Kukaiau ranch. } & 17. Puukihe. \\
\hline & 1911 & 1912 & 1912 \\
\hline 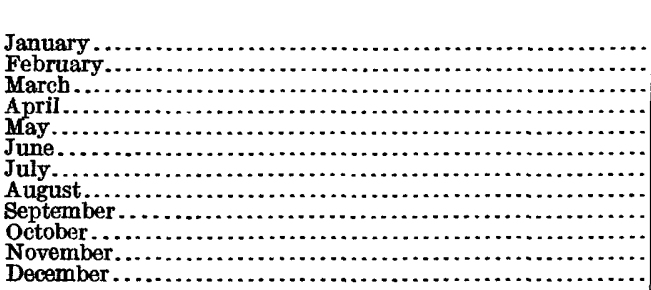 & $\begin{array}{r}\text { Inches. } \\
\mathbf{1 2 . 4 9} \\
\mathbf{4 3 . 4 4} \\
\mathbf{3 . 3 0} \\
\mathbf{1 1 . 7 4} \\
\mathbf{9 . 4 9} \\
\mathbf{3 . 2 4} \\
\mathbf{2 . 5 3} \\
\mathbf{3 . 6 9} \\
\mathbf{3 . 7 7} \\
\mathbf{3 . 3 9} \\
\mathbf{4 . 7 2} \\
\mathbf{5 . 7 1}\end{array}$ & $\begin{array}{r}\text { Inches. } \\
0 \\
22.04 \\
10.61 \\
13.15 \\
1.93 \\
.11 \\
.67 \\
1.42 \\
1.47 \\
4.55 \\
6.53 \\
1.51\end{array}$ & \begin{tabular}{|c}
\multicolumn{2}{|c}{ Inches. } \\
$\ldots \ldots$ \\
$\ldots \ldots$ \\
$\cdots$ \\
$\cdots$
\end{tabular} \\
\hline Year...... & $a 107.5$ & $a 64.0$ & $\overline{b 8.7}$ \\
\hline
\end{tabular}

$a$ Two inches should be added for estimated evaporation.

$b$ Includes 6 inches estimated evaporation. 


\section{INDEX.}

A.

Page.

Acre-foot, definition of..................... 15

Akulikulí Spring near Kealia, Kauai....... 60-61

Alo Stream near Huelo, Maui................ 137

Anahola ditch at Kiokala, near Kealia, Kauai 64-65

Anahola River above dam at Kiokala, near

Kealia, Kauai.

$63-64$

Appropriation, character and purpose of.... 7-8

Austin, H. A. R., work of................ 22

Authority for work

\section{B.}

Bailey, C. T., work of..................... 22

Bitting, C. C., cooperation of.............. 175

Bluett, P. W. P., cooperation of........... 175

Bridge station, definition of............... 14

c.

Cable station, definition of

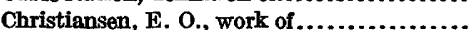

Clark, C. F., cooperation of ..................

Computations, accuracy of

methods of obtaining................. 17-20

Conservation fund, levying of.............. $7-8$

Cooperation, terms of...................... 8-9

Cross-section, determination of ............ 11

Current-meter, description of.............. 11

\section{D.}

Definition of terms ...................... 15-16

Discharge, methods of computing........... 17-20

Discharge measurements, accuracy of........ 20-21

tables of, explanation of................ ' 21

Division of work....................... 22

Dort, J. C., work of....................... 22

Dort, S. W., work of.................... 22

E.

East Branch or Fork. See name of main stream.

East Maui, list of stations on .............. 25-26

Eleele, Kauai, Hanapepe River near........ 37-38

Hanapepe diteh near................ 40-41

Hiloa ditch near ....................... 38-40

Equivalents, convenient................... 15

G.

Gages, method of installation............... 14

Gaging stations, classes of ................... 10

kinds used........................... 13-14

list of................................. 22-27

records of........................... 27-151

Gallons per minute, definition of............. is

Goo, E. E., work of.................... 22

Goo, R. M. S., work of.................. 22

Grove Farm Plantation, cooperation of...... 175
H.

Page.

Haipuaena Stream near Huelo, Maui (old and new stations).............. 134-135

Halekua Stream near Waimea, Kauai....... 32-33 Hamakua ditch at main weir, Puualala, near Waimea, Hawaii.

Hamakua group, Hawaii, stream-flow measurements on................. 168-170

Hanalei, Kauai, Kuna ditch near........... 68-69

Hanalei River near Hanalei, Kauai.......... 67-68

Hanamaulu ditch near Lihue, Kauai........ 48-49

Hanamaulu River at Kapaia near Lihue.... 45-46

Hanapepe ditch near Eleele, Kauai........ 40-41 near Hanapepe, Kauai................ 41-42

Hanapepe River at Koula, near Eleele, Kauai 37-38

Hardy, W. V., work of .................... 22

Hawaii, conditions in various districts of. 152-153

list of stations on . ................... 26-27

miscellaneous measurements on......... 174

rainfall stations on................... 178-179

stream-flow records on................ 153-174

table showing precipitation on........... 183

Hawaii Mill Co., cooperation of............. 175

Hawaii Preserving Co., cooperation of...... 175

Hawaiian Commercial \& Sugar Co., cooperation of........................ 175

Hawaiian Irrigation Co., cooperation of...... 175

Hawaiian Sugar Co., cooperation of......... 175 Hilo, Hawaii, Honolil River at Kaiwiki, near. $\quad 167$

? stations at 2,700-foot level, near ........ 153-165

Wailuku River near.................. 166-167

Hiloa ditch near Eleele, Kauai............. 38-40

Honoka Sugar Co., cooperation of......... 175

Honokahau, Mani, Honolua Stream near.. 117-118

Kahakuloa Stream near ... ............ 113-114

Honokahau ditch at intake, near Honokahau, Maui.

Honokahau Stream near Honokahau, Maui....................... 115-116

Honokawai ditch near Lahaina, Maui..... 119-120

Honokawai Stream near Lahaina, Maui..... 118

Honolii River at Kaiwiki, near Hilo, Hawaii. 167

Honolua Stream near Honokahau, Maui... 117-118

Honolulu, Oahu, East Branch of Manoa Stream at upper Manoa Valley, near.......................... 82-83

Kahuawai Spring near.................. 79

Kalihi Stream at Kioi Pool, near........ 74

Lulumaha ditch at upper Nuuanu reservoir, near.................... 76-77

Manoa Stream near................... 80-82

Nuuanu Stream at Luakaha weir in upper Nuuanu Valley, near............ 75 below reservoir No. 2 wasteway, near 75-76 
Honolulu, Oahu, Panoa Stream at upe.

Pauoa Valley, near ............. 78-79

Waiomao Stream at upper Palolo Valley, near ....................... 85-86

West Branch of Manoa Stream at upper Manoa Valley, near............. 84-85

Honolulu Ranch, cooperation of............ 175

Honopou Stream near Huelo, Maui........ 149-151

Hoolawaliilii Stream near Huelo, Maui.... 148-149

Hoolawanui Stream near Huelo, Maui..... 147-148

Huelo, Maui, Alo Stream near............. 137

Haipuaena Stream near (old and new stations)................... 134-135

Honopou Stream near................ 149-151

Hoolawaliilii Stream near............ 148-149

Hoolawanui Stream near.............. 147-148

Kailua Stream near................. 145-146

Nailiilihaele Stream near............. 144-145

Oanui Stream near.................. 146-147

Oopuola Stream near................ 139-140

Puohakamoa Stream near (old and new stations) .................... 135-137

Spreckels ditch near................. 141-144

Waikamoi Stream near................ 138-139

Huleia River near Lihue, Kauai............. 43-44

I.

Tao Stream near Wailuku, Maui ........... 104-105 Integration method, use of ................ 12-13

Investigations, authority for............... $7-8$

scope of.

$\mathrm{K}$.

Kaehulua Stream at Kuhinoa weir, near Kealia, Kauai............... 57-58

Kaehulua Stream, North Fork, at Kainahola weir, near Kealia, Kauai........ 58-59

Kaohulua Stream, South Fork, at Wainamuamu weir, near Kealia, Kauai. 56-57

Kahakuloa Stream near Honokahua, Maui. .113-114

at Kahakuloa, near Waihee, Maui...... 113

Kahanaiki Stream, North Branch, in Kailua Valley, near Kailua, Oahu...... 96-97

Kahanaiki Stream, South Branch, in Kailua Valley, near Kailua, Oahu.......

Kaheaku, John, work of....................

Kahoma ditch at weir, near Lahaina, Maui. 121-122

Kahuawai Spring at upper Pauoa Valley, near Honolulu, Oahu.............

Kailua, Oahu, Kaimi Stream in Kailua Valley, near..................... 91-92

Kamakalepo Stream in Kailua Valley, near........................... 93-94

Makawao Stream in Kailua Valley, near. 89-90

North Branch of Kahanaiki Stream in Kailua Valley, near............ 96-97

Pohakea Stream in Kailua Valley, near. 94-95

South Branch of Kahanaiki Stream in Kailua Valley, near............. 96

Kailua Stream near Huelo, Maui. . ........ 145-146 Kaimi Stream in Kailua Valley, near Kailua, Oahu......................... 91-92

Kainahola weir near Kealia, Kauai, North Fork of Kaehulua Stream at..... 58-59
Kaiwiki near Hilo, Hawaii, Honolii River at.......................... 167

Kalihj Stream at Kioi Pool, near Honolulu, Oahu........................ 74

Kalihiwai River near Kilauea, Kauai....... 65-66 Kamakalepo Stream in Kailua Valley, near Kailua, Oahu................ 93-94

Kanaha ditch near Lihue, Kauai........... 51-53

Kaneha ditch near Kealia, Kauai............ 62-63

Kapaa River near Kealia, Kauai........... 59-60

Kapahi ditch at Kapahi, near Kealia, Kauai. 61-62 Kapaia near Lihue, Kauai, Hanamaulu

River at...................... 45-46

Kauai, list of stations on . . ................ 22-23

miscellaneous measurements on......... 73

rainfall stations on . ................ 175-176

stream-flow records on................. 27-73

table showing precipitation on......... 180-181

Kauai Electric Co., cooperation of........... $\quad 175$

Kauaula ditch near Lahaina, Maui. ....... 126-127

Kaukonahua Stream, Left Branch of North Fork, near Wahiawa, Oahu.... 100-101

Right Branch of North Fork, near Wahiawa, Oahu.................. 99-100

South Fork, near Wahiawa, Oahu....... 102

Kawaikoi Stream near Waimea, Kauai...... 28-29

Kealia, Kauai, Akulikuli Spring near....... 60-61

Anahola ditch at Kiokala, near......... 64-65

Anahola River above dam at Kiokala, near.......................6 63-64

Kaøhulua Stream at Kuhinoa weir, near. 57-58

Kaneha ditch near.................... 62-63

Kapaa River near..................... 59-60

Kapahi ditch at Kapahi, near........... 61-62

Konohiki Stream at Makakualele woir (mauka), near................. 55-56

North Fork of Keahulua Stream at Katnahola weir, near............. 58-59

South Fork of Kaehulua Stream at Wajnamiramu weir, near.......... 56-57

Kehena ditch at Honokane Mauka, near North Kohala, Hawaii.......... 173

Kekaha Sugar Co., cooperation of........... $\quad 175$

Kennedy, H. M., work of ................ 22

Kilauea, Kauai, Kalihiwai River near....... 65-66

Kimble, Howard, work of. ................ 22

Kioi Pool near Honolulu, Oahu, Kalihi Stream at............ 74

Kiokala, near Kealia, Kauai, Anahola ditch at............................ 64-65

Anahola River above dam at........... 63-64 Kohala ditch near Kohala, Hawaii........ 170-172 Kohala group, Hawaii, stream-flow records for ....................... 170-172

Konohiki Stream at Makakualele weir (mauka), near Kealia, Kauai.... 55-56 Koula, Kauai, Hanapepe ditch at......... 40-41

Hanapepe River at................... 37-38 Kuhinoa weir near Kealia, Kauai, Kaehulua

Stream at................... 57-58

Kukaiau Ranch Co., cooperation of.......... 175 Kukuihaele, Hawaii, New Hamakua diteh at main weir near. ............ 169-170 Kuna ditch near Hanalei, Kauai. .......... 68-69 
L.

Page.

Lahaina, Maui, Honokawai ditch near... 119-120

Honokawai Stream near.............. 118

Kahoma ditch at weir, near.......... 121-122

Kauaula ditch near.................. 126-127

Lahamaluna ditch near ............... 124-125

Lahainaluna Stream near............ 122-124

Launiupoko Stream near............... 127

Lahainaluna ditch near Lahaina, Maui.... 124-125

Lahainaluna Stream near Lahaina, Maui.. 122-124

Launiupoko Stream near Lahaina, Maui..... 127

Lihue, Kauai, East Branch of North Fork of

Wailua River near............. 53-55

Hanamaulu ditch near................. 48-49

Hanamaulu River at Kapaia, near...... 45-46

Huleia River near..................... 43-44

Kanaha ditch near.................. 51-53

North Fork of Wailua River near....... 50-51

South Fork of Wailua River above

Waiehu Falls near.............. 46-47

Lihue ditch near Lilhue, Kauai.............. 49-50

Luakaha weir in upper Nuuanu Valley, near Honolulu, Oahu, Nuuanu Stream at.............................

Lulumaha ditch at upper Nuuanu reservoir, near Honolulu, Oahu.

M.

Makaknalele weir (mauka) near Kealia, Kauai, Konohiki Stream at...... 55-56

Makawao ditch at Makawao flume, near Waimanalo, Oahu...............

Makawao Stream in Kailua Valley, near Kailua, Oahu................. 89-90

Makaweli, Kauai, Olokele ditch at Tunnel No. 12 , near................... 33-34

Olokele ditch at weir, near ............. 35-36

Makaweli River near Waimea, Kauai........ 31-32

Makee Sugar Co., cooperation of............ 175

Maniania ditch near Wailuku, Maui......... 105

Manianiaula, near Waikane, Oahu, Waiahole Stream at.................... 97-98

Manoa Stream at College of Hawaii, near Honolulu, Oahu............... 80-82

at upper Manoa Valley, near Honolulu, Oahu........................ 80

Manoa Stream, East Branch, at upper Manoa Valley, near Honolulu, Oahu.... 82-83

Manoa Stream, West Branch, at upper Manoa Valley, near Honolulu, Oahu.... 84-85

Man's water, definition of................. 16

Maui, list of stations on.................. 24-26

'miscellaneous measurements on....... 151-152

rainfall stations on ................. 177-178

stream-flow records on................ 104-152

table showing precipitation on.......... 182

Mani Board of Supervisiors, cooperation of.. 175

May, W. 8., cooperation of ............... $\quad 175$

McClellan, J., cooperation of............... 175

Measurements, stream-flow, methods of obtaining....................... $9-10$

Meyer, F., cooperation of.................. 175

Million gallons, definition of ................ 15

Miner's inch, definition of ................. 15

Miscellarreous measurements on Hawaii..... 174 on Kauai. cellaneous measurements on Mati. .... 151-152 on Oahu. ..........................

Multiple-point method, use of.............. 12

N.

Nailiilihaele Stream near Huelo, Maui..... 144-145

New Hamakua ditch at main weir near $\mathrm{Ku}$ kuihaele, Hawaii............. 169-170

North Branch or Fork. See name of main stream.

North Kohala, Hawaii, Kehena ditch at Honokane Mauka, near........ 173

North Waiehu Stream near Wailuku, Maui. 109-110 Nuuanu reservoir, upper, near Honolulu, Oahu, Lulumaha ditch at....... 76-77

Nuuanu Stream at Luakaha weir in upper Nuuanu Valley, near Honolulu, Oahu.

below reservoir No. 2 wasteway, near Honolulu, Oahu.............75-76

o.

Oahu, list of stations on.................. 23-24

miscellaneous measurements on......... 103

ptinfall stations on . . . . . . . . . . . 176-177

'stream-flow records on................ 74-103

table showing precipitation on........... 181

Oanui Stream near Huelo, Maui.......... 146-147 Olokele ditch at Tunnel No. 12, Makaweli,

Kauai....................... 33-34

at weir near Makaweli, Kauai.......... 35-36

Olowalu diteh No. 1 near Olowalu, Maui. . 129-130

Olowalu Stream near Olowalu, Maui...... 128-129

Olowalu Sugar Co., cooperation of........... 175

Oopuola Stream near Huelo, Maui......... 139-140

P.

Pacific Sugar Mill, cooperation of............ 175

Palolo ditch near Waikapu, Maui......... 132-133

Palolo Valley, upper, near Honolulu, Oahu, Waiomao Stream at........... 85-86

Pauoa Stream at upper Pauoa Valley, near Honolulu, Oahu.............. 78-79

Pauoa Valley, upper, near Honolulu, Oahu, Kahuawai Spring at............ 79

Pioneer Mill Co., cooperation of............. 175

Pohakea Stream in Kailua Valley, near Kailua, Oahu.................... 94-95

Poowaiomahaihai ditch near Waimea, Kauai. 36-37

Princeville ranch (W. F. Sanborn), cooperation of........................ 175

Puohakamoa Stream near Huelo, Maui (old and new stations) ........... 135-137

Puualala, near Waimea, Hawaii, Hamakua ditch at main weir............. 168

R.

Rainfall stations, table showing maximum precipitation................ 184-185

table showing precipitation and evaporation....................... 180-183

Rainfall stations in Hawaii, list of........ 175-179

Rain gaging, explanation of. ............ 174-175

Rating table, use of . ..................... 17-18

Hbasis of............................. 21 
Page.

Run-off, definition of... method of obtaining

S.

Sanborn, W. F., cooperation of.

Scope of work.

Second-foot, definition of ..................

Second-feet per square mile, definition of....

Single-point method, use of...................

Soundings, method of obtaining..............

South Branch or Fork. See name of main stream.

South Side Waikapu ditch near Waikapu, Maui...................... 131-132

South Waiehu ditch near Wailuku, Maui . . 107-108 South Waiehu Stream near Wailuku, Maui. 106-107 Spreckels ditch near Huelo, Maui. ........ 141-144 near Waihee, Maui.................. 112

Stream measurement, accuracy of .......... 21 field methods of . .................... 9-10 mothods of computing............... 17-20

\section{T.}

Tables, explanation of 20-21

Terms, definition of 15-16

V.

Valentine, Alex., cooperation of.

Velocity-area measurements, methods of mak ing $11-14$

W:

$\checkmark$ Wading station, definition of . .............

Wahiawa, Oahu, Left Branch of North Fork of Kaukonahua Stream, near.. 100-101

Wahiawa, Oahu, Right Branch of North Fork of Kaukonahua Stream near......................99-100

Wabiawa, Oahu, South Fork of Kaukonahua Stream near.................... 102

Waiahole Stream at Manianiaula, near Waikans, Oahu . .................. 97-98

Waialae River near Waimea, Kauai . . . . . . . 29-30

Waiehu Falls, near Lihue, Kauai, South Fork of Wailua River above......... 46-47

Waiehu ditch, South, near Wailuku, Maui. 107-108

Waiehu Stream, North, near Wailuku, Maui...................... 109-110

Waiehu Stream, South, near Wailuku, Maui.................... 106-107

Waihee Stream near Waihee, Maui.
Waihee, Maui, Kahakuloa Stream at Kaha-

kuloa, near.................. 113

Spreckels ditch near. . ................... 112

Waikamoi Stream near Huelo, Maui....... 138-139

Waikane, Oahu, Waiahole Stream at Mani-

aniaula, near................. 97-98

Waikapu, Maui, Palolo ditch near........ 132-133

South Side Waikapu ditch near...... 131-132

Waikapu Stream near Waikapu, Maui. . . . . 131

Wailua River, East Branch of North Fork, near Lihue, Kauai............... 53-55

Wailua River, North Fork, near Lihue, Kauai.......................50-51

Wailua River, South Fork, above Waiehu Falls, near Lihue, Kauai. ....... 46-47

Wailuku, Maui, Iao Stream near......... 104-105

Maniania ditch near.................... 105

North Waiehu Stream near........... 109-110

South Waiehu ditch near............. 107-108

South Waiehu Stream near........... 106-107

Wailuku River near Hilo, Hawaii. . . . . . . . 166-167

Wailuku Sugar Co., cooperation of........... 175

Waimanalo, Oahu, Makawao ditch at Makawao flume, near............... 88

Waimanalo ditch below main reservoir on the Waimanalo plantation, near Waimanalo, Oahu............. 86-88

Waimea, Hawaii, Hamakua ditch at main weir, Puualala, near........... 168

Waimea, Kauai, Halekia Stream near . .. 32-33

Kawaikoi Stream near................. 28-29

Makaweli River near..................... 31-32

Poowaiomahaihai ditch near.......... 36-37

Waialae River near... . . . . . . . . . . . . ... 29-30

Waimea ditch near Waimea, Kauai........ 31

Waimea River near Waimea, Kauai. . . . . . . 27-28

Wainamuamu weir near Kealia, Kauai, South Fork of Kaehulua Stream at. ......................... 56-57

Wainiha RIver (East and West channels) at power house, near Wainiha, Kauai . ................... 69-73

Waiomao Stream at upper Palolo Valloy, near Honolulu, Oahu.......... 85-86

Water-stage recorder, description of. ........ 14

Weir measurements, advantages of . ........ 10

West Branch or Fork. See name of main stream.

West Maui, list of stations on............. 24-25

White, G. R., work of . ................. 22

Willard, C. R., cooperation of ........... 175

Wood, B. D., work of.................... 22 